\title{
CÁlCULO INTEGRAL
}

RICARDO SIU KOOCHOY

CARLOS ANDALUZ ZƯNIGA

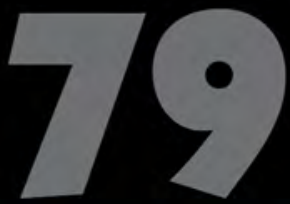

Apuntes de Estudio 



\section{CÁLCULO INTEGRAL}

RICARDO SIU KOOCHOY

CARLOS ANDALUZ ZÚÑ̃IGA

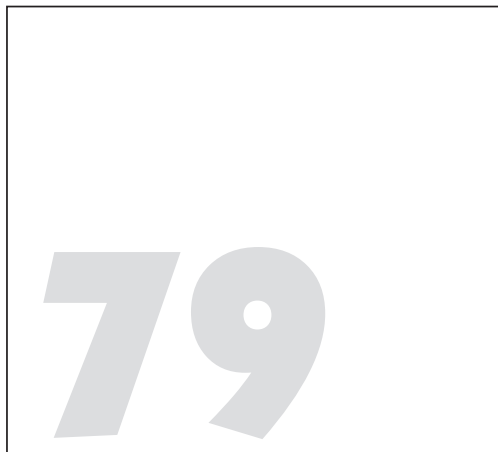

Apuntes de Estudio

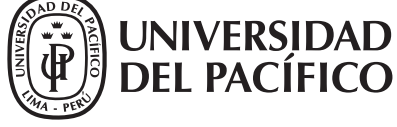


(C) Universidad del Pacífico

Avenida Salaverry 2020

Lima 11, Perú

www.up.edu.pe

\section{CÁLCULO INTEGRAL}

Ricardo Siu Koochoy y Carlos Andaluz Zúñiga

$1^{a}$ edición versión e-book: diciembre 2014

Diseño gráfico: Ícono Comunicadores

ISBN: 978-9972-57-311-8

doi: http://dx.doi.org/10.21678/978-9972-57-311-8

\section{BUP}

Siu Koochoy, Ricardo.

Cálculo integral / Ricardo Siu Koochoy, Carlos Andaluz Zúñiga. -- 1a edición. -- Lima : Universidad del Pacífico, 2014.

1 recurso electrónico (436 p.) -- (Apuntes de estudio ; 79)

1. Cálculo integral -- Problemas, ejercicios, etc.

I. Andaluz Zúñiga, Carlos

II. Universidad del Pacífico (Lima)

515.43 (SCDD)

Miembro de la Asociacion Peruana de Editoriales Universitarias y de Escuelas Superiores (Apesu) y miembro de la Asociación de Editoriales Universitarias de América Latina y el Caribe (Eulac).

La Universidad del Pacífico no se solidariza necesariamente con el contenido de los trabajos que publica. Prohibida la reproducción total o parcial de este documento por cualquier medio sin permiso de la Universidad del Pacífico.

Derechos reservados conforme a Ley. 


\section{A nuestros padres}

A María Isabel, Suclen, Meylin y Nina R. S. K.

A Yuli, Fátima y Carlos C. A. Z. 



\section{ÍNDICE}

PRÓLOGO

I. Derivadas y antiderivadas

1. Derivadas de funciones con una variable

2. Antiderivadas de funciones con una variable

14

II. Integral indefinida 17

1. Diferencial de funciones con una variable

2. Integral indefinida

III. Fórmulas de integración inmediata $\quad 23$

1. Reglas de integración $\quad 23$

2. Fórmulas de integración inmediata 24

2.1 Regla de las potencias 24

2.2 Funciones exponenciales $\quad 27$

2.3 Funciones trigonométricas $\quad 29$

2.4 Funciones que contienen $a u^{2}+a^{2} \quad 32$

2.5 Funciones que contienen $a u^{2}-a^{2} \quad 36$

2.6 Funciones que contienen a $a^{2}-u^{2} \quad 39$

IV. Métodos de integración $\quad 43$

1. Integración por sustitución $\quad 43$

2. Integración por partes $\quad 45$

3. Integración por sustitución trigonométrica 48 
4. Integración por descomposición en fracciones parciales

5. Integración de ciertas fracciones irracionales

5.1 Integrales del tipo $\int P\left[(a x+b)^{m_{1} / n_{1}},(a x+b)^{m_{2}} / n_{2}, \ldots\right] d x$

5.2 Integrales del tipo $\int \frac{d x}{(x-\alpha)^{n} \sqrt{a x^{2}+b x+c}}$

V. Integral definida

1. Integral definida como límite de una suma 57

2. Propiedades de la integral definida 64

3. Teorema fundamental del cálculo 64

\begin{tabular}{ll} 
VI. Integrales impropias & 69 \\
\hline
\end{tabular}

\begin{tabular}{ll} 
1. Integral impropia & 69 \\
\hline
\end{tabular}

1.1 Integral impropia con límite de integración no finito 69

1.2 Integral impropia con integrando no continuo 73

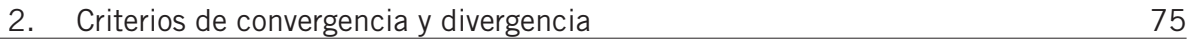

2.1 Criterio de comparación directa $\quad 75$

2.2 Criterio de comparación de límites 77

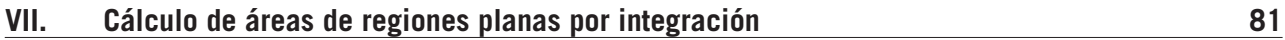

\begin{tabular}{ll} 
1. Área bajo una curva & 81 \\
\hline
\end{tabular}

2. Área comprendida entre dos curvas que no se cruzan $\quad 86$

3. Área comprendida entre dos curvas que se cruzan $\quad 89$

4. Área de regiones mediante integración $\quad 91$

\begin{tabular}{ll} 
VIII. Aplicaciones de la integral & 95 \\
\hline
\end{tabular}

1. Problemas geométricos $\quad 95$

2. Problemas económicos $r$

3. Problemas financieros 102

4. Problemas estadísticos 104

5. Problemas sociales 106

6. Problemas biológicos 108

7. Problemas físicos 110

IX. Integración numérica

1. Reglas para aproximar el valor de $\int_{a}^{b} f_{(x)} d x$

1.1 Regla del punto medio

1.2 Regla del trapecio

116

1.3 Regla de Simpson

118 
\begin{tabular}{lr}
$X$. Integrales dobles & 139 \\
\hline
\end{tabular}

1. Integrales dobles 139

2. Integrales reiteradas 140

3. Cálculo del área de una región plana 144

Anexo 1: Sumatorias 148

Anexo 2: Fórmulas de derivación 153

$\begin{array}{ll}\text { Amenidad } 1 & 155\end{array}$

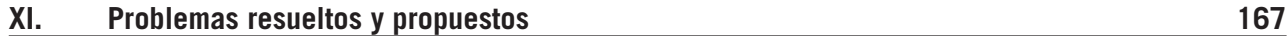

1. Problemas resueltos y propuestos del capítulo I 167

2. Problemas resueltos y propuestos del capítulo II 173

3. Problemas resueltos y propuestos del capítulo III 187

4. Problemas resueltos y propuestos del capítulo IV 201

5. Problemas resueltos y propuestos del capítulo V 254

6. Problemas resueltos y propuestos del capítulo $\mathrm{VI} \quad 290$

7. Problemas resueltos y propuestos del capítulo VII 311

8. Problemas resueltos y propuestos del capítulo VIII 370

9. Problemas resueltos y propuestos del capítulo X 412

$\begin{array}{ll}\text { Amenidad } 2 & 423\end{array}$ 



\section{PRÓLOGO}

El presente Apuntes de Estudio trata temas relacionados con el Cálculo Integral de funciones con una o dos variables. Está basado en las clases dictadas por los profesores Ricardo Siu Koochoy y Carlos Andaluz Zúñiga en la Universidad del Pacífico.

En la primera parte, se define la integración como operación inversa a la diferenciación, se detallan las fórmulas de integración inmediata y se explican diferentes métodos de integración. En la segunda parte, se cubren los temas de integrales impropias, aplicaciones de las integrales definidas y las integrales dobles. En la última parte, se presenta una colección de 520 problemas resueltos y propuestos de los diferentes capítulos.

Confiamos en que el lector llegue a comprender los temas tratados en el libro, toda vez que estos se presentan de manera simple y clara, y que pueda resolver adecuadamente los problemas relativos al Cálculo Integral.

Los autores 



\section{DERIVADAS Y ANTIDERIVADAS}

\section{DERIVADAS DE FUNCIONES CON UNA VARIABLE}

De acuerdo con el Cálculo Diferencial, se tiene que la derivada de una función $y=f_{(x)}$ con respecto a la variable $x$, evaluada en $x_{p}$, tal que $y_{p}=f_{\left(x_{p}\right)}$, representa:

i. La pendiente de la recta tangente a la gráfica de la función $y=f_{(x)}$ trazada en el punto $P_{\left(x_{p}, y_{p}\right)}$.

ii. El límite de la razón promedio de cambio $\frac{f\left(x_{p}+h\right)-f\left(x_{p}\right)}{h}$ cuando el incremento $h$ de la variable independiente tiende a cero.

Por otro lado, dada una función $y=f_{(x)}$, su derivada se denota como:

$$
\frac{d y}{d x}=\frac{d f}{d x}=f^{\prime}(x)
$$

Por ejemplo:

$$
\begin{aligned}
& f_{(x)}=x^{4} \Rightarrow f^{\prime}(x)=\frac{d\left(x^{4}\right)}{d x}=4 x^{3} \\
& g_{(x)}=x^{4}+4 \Rightarrow g_{(x)}^{\prime}=\frac{d\left(x^{4}+4\right)}{d x}=4 x^{3}+0=4 x^{3}
\end{aligned}
$$




$$
\begin{aligned}
& h_{(x)}=\frac{2 x+3}{x-1}=\frac{2(x-1)+5}{x-1}=2+5(x-1)^{-1},(x \neq 1) \Rightarrow \\
& h_{(x)}^{\prime}=\frac{d\left(\frac{2 x+3}{x-1}\right)}{d x}=0+5(-1)(x-1)^{-2}=\frac{-5}{(x-1)^{2}} \\
& j_{(x)}=\cos \left(5 x^{2}\right) \Rightarrow j^{\prime}{ }_{(x)}=\frac{d \cos \left(5 x^{2}\right)}{d x}=-\left[\operatorname{sen}\left(5 x^{2}\right)\right](10 x)=-10 x \operatorname{sen}\left(5 x^{2}\right) \\
& k_{(x)}=[\ln (2 x)]^{2} \Rightarrow k_{(x)}^{\prime}=\frac{d[\ln (2 x)]^{2}}{d x}=2[\ln (2 x)] \frac{2}{2 x}=\frac{2 \ln (2 x)}{x}
\end{aligned}
$$

\section{ANTIDERIVADAS DE FUNCIONES CON UNA VARIABLE}

Ciertas aplicaciones del Cálculo requieren resolver el siguiente problema: dada la derivada de una función, se trata de encontrar dicha función. Es decir, conocida $f^{\prime}{ }_{(x)}$, se debe encontrar $f_{(x)}$. En el acápite anterior se obtuvo que:

$$
f^{\prime}(x)=\frac{d x^{4}}{d x}=4 x^{3}
$$

Dada la derivada de una función $f_{(x)}, f^{\prime}{ }_{(x)}=4 x^{3}$, se llega a la conclusión de que $f_{(x)}=x^{4}$, y se afirma que $f_{(x)}=x^{4}$ es una antiderivada o primitiva de $4 x^{3}$.

Además, del acápite anterior se obtuvo que:

$$
g^{\prime}(x)=\frac{d\left[x^{4}+4\right]}{d x}=4 x^{3}
$$

Dada la derivada de una función $g_{(x)}, g^{\prime}{ }_{(x)}=4 x^{3}$, se concluye con que $g_{(x)}=x^{4}+4$ y se afirma que $g_{(x)}=x^{4}+4$ es una antiderivada o primitiva de $4 x^{3}$.

De los dos ejemplos anteriores, dada la derivada de una función $F_{(x)}, F_{(x)}^{\prime}=4 x^{3}$, se concluye con que $F_{(x)}=x^{4}+C$ es la antiderivada o primitiva de $F_{(x)}^{\prime}$, en donde $C$ es una constante.

Del mismo modo, y con referencia al acápite anterior, se tiene que:

$$
\text { la antiderivada de } F^{\prime}(x)=\frac{-5}{(x-1)^{2}} \text { es } \frac{2 x+3}{x-1}+C,(x \neq 1) \text {. }
$$

la antiderivada de $F_{(x)}^{\prime}=-10 x \operatorname{sen} 5 x^{2}$ es $\cos \left(5 x^{2}\right)+C$.

la antiderivada de $F_{(x)}^{\prime}=\frac{2 \ln (2 x)}{x}$ es $[\ln (2 x)]^{2}+C$. 
En general, la antiderivada o primitiva de $F^{\prime}{ }_{(x)}$ es una función de la forma $F_{(x)}+C$, dado que la derivada de $F_{(x)}+C$ es $F_{(x)}^{\prime}+0=F_{(x)}^{\prime}$.

\section{Ejemplo 1:}

Si la derivada de la función $F_{(x)}=10 a^{4} x^{5}$ es $F_{(x)}^{\prime}=50 a^{4} x^{4}$, entonces la antiderivada de $F^{\prime}{ }_{(x)}$ es $10 a^{4} x^{5}+C$.

\section{Ejemplo 2:}

Si la derivada de la función $F_{(x)}=\sqrt{3 p x^{3}}$ es $F^{\prime}(x)=\sqrt{3 p}\left(\frac{3}{2}\right) x^{1 / 2}=\frac{3 \sqrt{3 p x}}{2}$, entonces la primitiva
de $F^{\prime}{ }_{(x)}$ es $\sqrt{3 p x^{3}}+C$.

\section{Ejemplo 3:}

Si la derivada de la función $F_{(x)}=x(x+a)(x+b)=x^{3}+(a+b) x^{2}+a b x$ es $F_{(x)}^{\prime}=3 x^{2}+2(a+b)$ $x+a b$, la antiderivada de $F_{(x)}^{\prime}$ es $x(x+a)(x+b)+C$.

\section{Ejemplo 4:}

Si la derivada de la función $F_{(x)}=\tan (q x)$ es $F_{(x)}^{\prime}=q \sec ^{2}(q x)$, la primitiva de $F_{(x)}^{\prime}$ es $\tan (q x)+C$.

\section{Ejemplo 5:}

Si la derivada de la función $F_{(x)}=a e^{-b x}$ es $F_{(x)}^{\prime}=-a b e^{-b x}$, la antiderivada de $F_{(x)}^{\prime}$ es $a e^{-b x}+C$.

\section{Ejemplo 6:}

Si la derivada de la función $F_{(x)}=\log _{9} x^{9}=9 \log _{9} x$ es $F_{(x)}^{\prime}=\frac{9}{x} \log _{9}$ e, entonces la primitiva de $F_{(x)}^{\prime}$ es $\log _{9} x^{9}+C$ 



\section{INTEGRAL INDEFINIDA}

\section{DIFERENCIAL DE FUNCIONES CON UNA VARIABLE}

Del Cálculo Diferencial se tiene que el diferencial de una función $y=f_{(x)}$ viene dado por:

$$
d y=\left(\frac{d y}{d x}\right) d x=f^{\prime}(x) d x
$$

Si $y=f_{(x)}=x^{6}+C \rightarrow f_{(x)}^{\prime}=6 x^{5} \Rightarrow d y=6 x^{5} d x$.

Conocido el diferencial $d y=6 x^{5} d x$, la operación que permite encontrar todas las soluciones de dicha ecuación, $y=f_{(x)}$, (es decir, la antiderivada de $f_{(x)}^{\prime}$ ), se denomina integración indefinida y se denota con el símbolo integral: $\int$.

Es decir:

$$
y=\int d y=\int \underbrace{6 x^{5}}_{f^{\prime}(x)} d x=x^{6}+C
$$

Esto es:

$$
y=f_{(x)}=x^{6}+C
$$




\section{INTEGRAL INDEFINIDA}

En general, dado el diferencial de $y=f_{(x)}, d y=f_{(x)}^{\prime} d x$, la función $y$ se obtiene a partir de:

$y=f_{(x)}=\int d y=\int f^{\prime}(x) d x$, y se afirma que $y$ es la integral indefinida o la antiderivada o la primitiva de $f_{(x)}^{\prime}$

Ejemplo 7:

Si la derivada de $y=f_{(x)}$ es $f_{(x)}^{\prime}=4$, hallar la integral indefinida de $f_{(x)}^{\prime}$.

Se tiene que $d y=f_{(x)}^{\prime} d x=4 d x$ y que la integral indefinida de $f_{(x)}^{\prime}$ es $y=\int d y=\int 4 d x$. Como $f_{(x)}^{\prime}=4$ significa que la pendiente de la recta tangente a $f_{(x)}$ es 4 , y que la ecuación de la recta debe ser $y=4 x+C$. De donde $y=\int 4 d x=4 x+C$ es la integral indefinida de $f_{(x)}^{\prime}$.

Ejemplo 8:

Conocida la derivada de $y=f_{(x)}, f_{(x)}^{\prime}=x$, encontrar la antiderivada o primitiva de $f_{(x)}^{\prime}$. que:

$$
\frac{d x^{n}}{d x}=n x^{n-1}
$$

De lo anterior, se desprende que:

$$
\begin{aligned}
d x^{n} & =n x^{n-1} d x \\
\int d x^{n} & =\int n x^{n-1} d x \\
x^{n}+C & =\int n x^{n-1} d x
\end{aligned}
$$

Si consideramos $n=2$ :

$$
\begin{aligned}
& \int 2 x^{2-1} d x=x^{2}+C^{\prime} \\
& \int 2 x d x=x^{2}+C^{\prime} \\
& 2 \int x d x=x^{2}+C^{\prime}
\end{aligned}
$$




$$
\begin{aligned}
& \int x d x=\frac{x^{2}}{2}+\frac{C^{\prime}}{2} \\
& \int x d x=\frac{x^{2}}{2}+C
\end{aligned}
$$

La antiderivada o primitiva de $f_{(x)}^{\prime}=x$ es $\frac{x^{2}}{2}+C$.

\section{Ejemplo 9:}

Dados $y=f_{(x)} \wedge d y=30 x^{5} d x$, determinar la integral indefinida $\int 30 x^{5} d x$.

Reemplacemos $n=6$ en la fórmula $(\alpha)$ del ejemplo anterior:

$$
x^{6}+C=\int 6 x^{6-1} d x=\int 6 x^{5} d x
$$

Multiplicando por 5:

$$
5 \int 6 x^{5} d x=\int 30 x^{5} d x=5\left(x^{6}+C\right)=5 x^{6}+C^{1}
$$

Ejemplo 10:

Encontrar la integral indefinida $\int e^{a x^{2}+b x+d}(2 a x+b) d x$.

Como $\frac{d e^{u}}{d x}=e^{u} \frac{d u}{d x}$, se tiene que el diferencial de $e^{u}$ es $d e^{u}=\left(\frac{d e^{u}}{d x}\right) d x=\left[e^{u} \frac{d u}{d x}\right] d x \rightarrow e^{u}\left(\frac{d u}{d x}\right) d x=e^{u} d u$.

Integrando ambos miembros:

$$
\begin{aligned}
\int d e^{u} & =\int e^{u} d u \\
e^{u}+C & =\int e^{u} d u
\end{aligned}
$$

Si consideramos que:

$$
\begin{gathered}
u=a x^{2}+b x+d \\
d u=(2 a x+b) d x \\
\int e^{u} d u=\int e^{a x^{2}+b x+d}(2 a x+b) d x \\
=e^{a x^{2}+b x+d}+C
\end{gathered}
$$




\section{Ejemplo 11:}

Obtener la antiderivada siguiente:

$$
\int \frac{6}{3 x+5} d x
$$

Se tiene que:

$$
\frac{d \ln u}{d x}=\frac{1}{u} \frac{d u}{d x}
$$

El diferencial $d \ln u$ es $d \ln u=\left(\frac{1}{u} \frac{d u}{d x}\right) d x=\frac{1}{u} d u$, de donde:

$$
\begin{aligned}
& \int d \ln u=\int \frac{1}{u} d u \\
& \ln u+C=\int \frac{1}{u} d u
\end{aligned}
$$

Hagamos $u=3 x+5 \Rightarrow d u=3 d x$.

20 Reemplacemos en $(\beta)$ :

$$
\int \frac{1}{u} d u=\int \frac{1}{3 x+5}(3 d x)=\int \frac{3}{3 x+5} d x=\ln (3 x+5)+C
$$

Entonces:

$$
\int \frac{6}{3 x+5} d x=2 \int \frac{3}{3 x+5} d x=2 \ln (3 x+5)+C^{\prime}
$$

\section{Ejemplo 12:}

Halle la primitiva siguiente:

$$
\int(\cos 10 x) d x
$$

Como $\frac{d \operatorname{sen} u}{d x}=-\cos u \frac{d u}{d x}$,

$$
d \operatorname{sen} u=\left(-\cos u \frac{d u}{d x}\right) d x=-\cos u d u
$$


Entonces:

$$
\begin{aligned}
& \int-\cos u d u=\int d \operatorname{sen} u \\
& -\int \cos u d u=\operatorname{sen} u+C
\end{aligned}
$$

Si $u=10 x \Rightarrow d u=10 d x$, de donde:

$$
-\int(\cos 10 x)(10 d x)=-10 \int \cos 10 x d x=\operatorname{sen} 10 x+C
$$

Finalmente:

$$
\int(\cos 10 x) d x=\frac{-1}{10} \operatorname{sen} 10 x+C^{\prime}
$$





\section{FII RMULAS DE INTEGRACIÓN INMEDIATA}

\section{REGLAS DE INTEGRACIÓN}

Conforme a lo establecido en el capítulo anterior, se desprenden las siguientes reglas de integración:
a) $\int\left[f_{(x)} \pm g_{(x)}\right] d x=\int f_{(x)} d x \pm \int g_{(x)} d x$
b) $\int\left[K f_{(x)}\right] d x=K \int f_{(x)} d x$
c) $\int 0 d x=C$
d) $\int K d x=K x+C$
e) $\int d f_{(x)}=\int \frac{d f_{(x)}}{d x} d x=f_{(x)}+C$
f) Si $f^{\prime}(x)=g_{(x)}$, entonces $\int g_{(x)} d x=f_{(x)}+C$ 
Ejemplo 13:

$$
\int\left[4 x^{2}+2 x-6\right] d x=4 \int x^{2} d x+2 \int x d x-\int 6 d x=4 \int x^{2} d x+2 \int x d x-6 x+C \text { por a) y por d) }
$$

Ejemplo 14:

$$
\int 4 d x=4 \int d x=4 x+C \text { por b) y por d) }
$$

Ejemplo 15:

$$
\text { Si } \frac{d e^{2 x^{2}}}{d x}=e^{2 x^{2}} 4 x \text {, entonces } \int \underbrace{e^{2 x^{2}} 4 x d x}_{d\left(e^{2 x^{2}}\right)}=e^{2 x^{2}}+C \text { por e) }
$$

Ejemplo 16:

$$
\int d\left(a x^{2}+b\right)=a x^{2}+b+C^{\prime}=a x^{2}+C \text { por e) }
$$

Se lo puede comprobar de esta manera:

$$
d\left(a x^{2}+b\right)=2 a x d x \Rightarrow \int d\left(a x^{2}+b\right)=\int 2 a x d x=2 a \int x d x=2 a\left(\frac{x^{2}}{2}\right)+C=a x^{2}+C
$$

\section{FÓRMULAS DE INTEGRACIÓN INMEDIATA}

En las siguientes fórmulas se considerará $u=f_{(x)}$ y $c, n, a=$ constantes.

\subsection{REGLA DE LAS POTENCIAS}
a) $\int u^{n} d u=\frac{u^{n+1}}{n+1}+C$
$(n \neq-1)$
b) $\int u^{-1} d u=\int \frac{d u}{u}=\ln |u|+C$

Nota: en el libro se asumirá que $u$ es mayor que cero y, por lo tanto, se prescindirá del símbolo del valor absoluto.

Ejemplo 17:

$$
\int x^{9} d x=\frac{x^{9+1}}{9+1}+C=\frac{x^{10}}{10}+C
$$

porque según a) $u=x \rightarrow d u=d x$ y $n=9$. 
Ejemplo 18:

$$
\int \frac{4}{x^{2}} d x=4 \int x^{-2} d x=4 \frac{x^{-2+1}}{-2+1}+C=4\left(\frac{x^{-1}}{-1}\right)+C=\frac{-4}{x}+C
$$

porque según a) $u=x \rightarrow d u=d x$ y $n=-2$.

Ejemplo 19:

$$
\int \frac{6}{x} d x=6 \int \frac{d x}{x}=6 \ln x+C
$$

porque según b) $u=x \rightarrow d u=d x$.

Ejemplo 20:

$$
\begin{gathered}
I=\int(n x)^{\frac{1-n}{n}} d x \\
I=n^{\frac{1-n}{n}} \int x^{\frac{1-n}{n}} d x \\
I=n^{\frac{1}{n}-1} \int x^{\frac{1}{n}-1} d x \\
I=n^{\frac{1}{n}-1} \frac{x^{\frac{1}{n}-1+1}}{\frac{1}{n}-1+1}+C \\
I=n^{\frac{1}{n}-1} \frac{x^{1 / n}}{1 / n}+C=n^{1 / n} x^{1 / n}+C \\
I=\sqrt[n]{n x}+C
\end{gathered}
$$

Ejemplo 21:

$$
\begin{gathered}
I=\int(\sqrt{x}+1)(x-\sqrt{x}+1) d x \\
I=\int(x \sqrt{x}-x+\sqrt{x}+x-\sqrt{x}+1) d x \\
I=\int(x \sqrt{x}+1) d x \\
I=\int\left(x^{3 / 2}+1\right) d x
\end{gathered}
$$




$$
I=\frac{x^{5 / 2}}{5 / 2}+x+C=\frac{2}{5} x^{5 / 2}+x+C
$$

Ejemplo 22:

$$
I=\int\left(3 x^{2}-2 x+4\right)^{3}(3 x-1) d x
$$

Consideremos:

$$
u=3 x^{2}-2 x+4
$$

$$
d u=(6 x-2) d x \rightarrow \frac{d u}{2}=(3 x-1) d x
$$

Entonces:

$$
\begin{gathered}
I=\int u^{3} \frac{d u}{2} \\
I=\frac{1}{2} \int u^{3} d u=\frac{1}{2} \cdot \frac{u^{4}}{4}+C=\frac{u^{4}}{8}+C
\end{gathered}
$$

De donde:

26

$$
I=\frac{\left(3 x^{2}-2 x+4\right)^{4}}{8}+C
$$

Ejemplo 23:

$$
\begin{gathered}
I=\int \frac{x d x}{\sqrt{x^{2}-4}} \\
I=\frac{1}{2} \int \frac{2 x d x}{\sqrt{x^{2}-4}}
\end{gathered}
$$

Si $u=x^{2}-4 \rightarrow d u=(2 x) d x$

$$
I=\frac{1}{2} \int \frac{d u}{\sqrt{u}}=\frac{1}{2} \int u^{-1 / 2} d u=\frac{1}{2} \frac{u^{1 / 2}}{1 / 2}+C=u^{1 / 2}+C=\sqrt{x^{2}-4}+C
$$

Ejemplo 24:

$$
\begin{gathered}
I=\int \frac{-5}{(x-1)^{2}} d x \\
I=-5 \int \underbrace{(x-1)^{-2}}_{u} \underbrace{n}_{d u} d x=-5 \frac{(x-1)^{-2+1}}{-2+1}+C
\end{gathered}
$$


APUNTES DE ESTUDIO

$$
\begin{aligned}
& I=\frac{-5}{-1}(x-1)^{-1}+C \\
& I=\frac{5}{x-1}+C
\end{aligned}
$$

2.2 FUNCIONES EXPONENCIALES

a) $\int a^{x} d x=\frac{a^{x}}{\ln a}+C$

b) $\int e^{x} d x=e^{x}+C$

Ejemplo 25:

Demostrar la fórmula a) a partir de la b):

$$
\int a^{x} d x=\int\left(e^{\ln a}\right)^{x} d x=\int e^{x \cdot \ln a} d x
$$

Si $x \cdot \ln a=u \Rightarrow d u=1 . \ln a \cdot d x \Rightarrow d x=\frac{d u}{\ln a}$

Entonces:

27

$$
\int a^{x} d x=\int e^{u} \frac{d u}{\ln a}=\frac{1}{\ln a} \int e^{u} d u=\frac{1}{\ln a} e^{u}=\frac{1}{\ln a} e^{x \cdot \ln a}=\frac{1}{\ln a}\left(e^{\ln a}\right)^{x}=\frac{1}{\ln a} a^{x}
$$

Ejemplo 26:

$$
\int x \cdot 8^{x^{2}} \cdot d x=\int 8^{x^{2}}(x d x)=\frac{1}{2} \int 8^{x^{2}}(2 x d x)=\frac{1}{2} \frac{8^{x^{2}}}{\ln 8}+C=\frac{8^{x^{2}}}{2 \ln 8}+C
$$

Ejemplo 27:

$$
\int\left(e^{2 t}-e^{-2 t}\right) d t=\frac{1}{2} \int\left(e^{2 t}\right)(2 d t)+\frac{1}{2} \int\left(e^{-2 t}\right)(-2 d t)=\frac{1}{2} e^{2 t}+\frac{1}{2} e^{-2 t}+C
$$

Ejemplo 28:

$$
\begin{aligned}
& I=\int 4^{\overbrace{}^{u-3 x}} d x=\frac{1}{-3} \int 4^{2-3 x} \underbrace{(-3 d x)}_{d u} \\
& I=-\frac{1}{3} \cdot \frac{4^{2-3 x}}{\ln 4}+C
\end{aligned}
$$

Ejemplo 29:

$$
I=\int a e^{-m x} d x=a \int e^{-m x} d x
$$


Si $u=-m x \Rightarrow d u=-m \cdot d x \Rightarrow d x=\frac{-d u}{m}$

$$
I=a \int e^{u}\left(\frac{-d u}{m}\right)=-\frac{a}{m} \int e^{u} \cdot d u=-\frac{a}{m} e^{u}+C=-\frac{a}{m} e^{-m x}+C
$$

Ejemplo 30:

$$
\begin{gathered}
I=\int\left(e^{x / b}+e^{-x / b}\right)^{2} d x \\
I=\int\left[e^{\frac{2 x}{b}}+2 e^{0}+e^{-\frac{2 x}{b}}\right] d x \\
I=\frac{e^{\frac{2 x}{b}}}{2 / b}+2 x+\frac{e^{-\frac{2 x}{b}}}{(-2 / b)}+C \\
I=\frac{b}{2} e^{2 x / b}-\frac{b}{2} e^{-2 x / b}+2 x+C
\end{gathered}
$$

28 Ejemplo 31:

$$
\begin{gathered}
I=\int \frac{b^{2 x}-1}{\sqrt{b^{x}}} d x \\
I=\int \frac{b^{2 x}}{b^{x / 2}} d x-\int \frac{1}{b^{x / 2}} d x \\
I=\int b^{\frac{3 x}{2}} d x-\int b^{-\frac{x}{2}} d x \\
I=\frac{1}{3 / 2} \int b^{\frac{3 x}{2}}\left(\frac{3 d x}{2}\right)-\frac{1}{\ln b} \int \frac{3 x}{(-1 / 2)} \int b^{\frac{-x}{2}}\left(\frac{-1}{2} d x\right) \\
\ln b
\end{gathered}
$$

Ejemplo 32:

$$
\begin{gathered}
I=\int \frac{\left(b^{x}-d^{x}\right)^{2}}{b^{x} d^{x}} d x \\
I=\int \frac{b^{2 x}}{b^{x} d^{x}} d x-\int \frac{2 b^{x} d^{x}}{b^{x} d^{x}} d x+\int \frac{d^{2 x}}{b^{x} d^{x}} d x
\end{gathered}
$$




$$
\begin{gathered}
I=\int \frac{b^{x}}{d^{x}} d x-2 \int d x+\int \frac{d^{x}}{b^{x}} d x \\
I=\int\left(\frac{b}{d}\right)^{x} d x-2 x+\int\left(\frac{d}{b}\right)^{x} d x \\
I=\frac{(b / d)^{x}}{\ln (b / d)}-2 x+\frac{(d / b)^{x}}{\ln (d / b)}+C,(b \neq d, b d>0)
\end{gathered}
$$

\subsection{FUNCIONES TRIGONOMÉTRICAS}
a) $\int \operatorname{sen} x d x=-\cos x+C$
b) $\int \cos x d x=\operatorname{sen} x+C$
c) $\int \tan x d x=\int \frac{\operatorname{sen} x}{\cos x} d x=-\ln \cos x+C$
d) $\int \cot x d x=\int \frac{\cos x}{\operatorname{sen} x} d x=\ln \operatorname{sen} x+C$
e) $\int \sec ^{2} x d x=\tan x+C$
f) $\int \csc ^{2} x d x=-\cot x+C$
g) $\int \sec x d x=\ln (\tan x+\sec x)+C$
h) $\int \csc x d x=\ln (\csc x-\cot x)+C$

Ejemplo 33:

Comprobar la fórmula g).

$$
\frac{d \ln (\tan x+\sec x)}{d x}=\frac{1}{\tan x+\sec x}\left[\sec ^{2} x+\sec x \cdot \tan x\right]=\frac{\sec x(\sec x+\tan x)}{\tan x+\sec x}=\sec x
$$

Ejemplo 34:

$$
\int \operatorname{sen}(a+b x) d x=\frac{1}{b} \int[\operatorname{sen} \underbrace{(a+b x)}_{u}] \underbrace{[b d x]}_{d u}=-\frac{1}{b} \cos (a+b x)+C
$$

Ejemplo 35:

$$
\begin{gathered}
I=\int(\operatorname{sen} a x+\cos a x)^{2} d x \\
I=\int\left[\operatorname{sen}^{2} a x+2 \operatorname{sen} a x \cdot \cos a x+\cos ^{2} a x\right] d x
\end{gathered}
$$




$$
\begin{gathered}
I=\int[1+2 \operatorname{sen} a x \cdot \cos a x] d x \\
I=\int[1+\operatorname{sen} 2 a x] d x \\
I=\int d x+\frac{1}{2 a} \int(\operatorname{sen} 2 a x)(2 a d x) \\
I=x-\frac{\cos 2 a x}{2 a}+C
\end{gathered}
$$

Ejemplo 36:

$$
I=\int \operatorname{sen}^{2} x d x
$$

Se sabe que: $\cos 2 x=\cos ^{2} x-\operatorname{sen}^{2} x$

$$
\begin{aligned}
& \cos 2 x=\left(1-\operatorname{sen}^{2} x\right)-\operatorname{sen}^{2} x \\
& \cos 2 x=1-2 \operatorname{sen}^{2} x \quad \\
& \quad I=\int \operatorname{sen}^{2} x d x=\int \frac{1-\cos 2 x}{2} d x
\end{aligned}
$$

30

$$
\begin{gathered}
I=\frac{1}{2} \int d x-\frac{1}{4} \int(\cos 2 x)(2 d x) \\
I=\frac{x}{2}-\frac{1}{4} \operatorname{sen} 2 x+C
\end{gathered}
$$

Ejemplo 37:

$$
I=\int \frac{\tan \sqrt{x}}{\sqrt{x}} d x
$$

Si $u=\sqrt{x} \Rightarrow d u=\frac{1}{2 \sqrt{x}} d x \Rightarrow \frac{d x}{\sqrt{x}}=2 d u$

$$
\begin{gathered}
I=\int \tan u(2 d u)=2 \int \tan u d u \\
I=2 \int \frac{\operatorname{sen} u}{\cos u} d u \\
I=-2 \int \frac{-\operatorname{sen} u}{\cos u} d u \\
I=-2 \ln (\cos u)+C \\
I=-2 \ln \cos \sqrt{x}+C
\end{gathered}
$$


Ejemplo 38:

$$
I=\int \cot ^{2} a x d x
$$

Se sabe que: $\operatorname{sen}^{2} x+\cos ^{2} x=1$

(Dividiendo entre $\operatorname{sen}^{2} x$ ): $1+\cot ^{2} x=\csc ^{2} x$

$$
\begin{gathered}
I=\int \cot ^{2} a x d x=\int\left[\csc ^{2} a x-1\right] d x \\
I=\frac{1}{a} \int \operatorname{acsc}^{2} a x d x-\int d x=\frac{1}{a}\left(-\cot ^{2} a x\right)-x+C
\end{gathered}
$$

Ejemplo 39:

$$
I=\int \tan ^{3} \frac{x}{3} \cdot \sec ^{2} \frac{x}{3} \cdot d x
$$

$\operatorname{Si} u=\tan \frac{x}{3} \Rightarrow d u=\sec ^{2} \frac{x}{3} \cdot\left(\frac{1}{3}\right) d x$

$$
\begin{gathered}
I=\int u^{3} 3 d u=3 \int u^{3} d u \\
I=3 \frac{u^{4}}{4}+C=\frac{3}{4} \tan ^{4} \frac{x}{3}+C
\end{gathered}
$$

Ejemplo 40:

$$
\begin{gathered}
I=\int \cot ^{2 / 3} x \cdot \frac{1}{\operatorname{sen}^{2} x} \cdot d x \\
I=\int \cot ^{2 / 3} x \cdot \csc ^{2} x \cdot d x \\
I=-\int \underbrace{(\cot x)^{2 / 3}}_{u} \underbrace{\left(-\csc ^{2} x d x\right)}_{d u} \\
I=-\frac{(\cot x)^{2 / 3+1}}{\frac{2}{3}+1}+C \\
\therefore I=\frac{-3}{5}(\cot x)^{5 / 3}+C
\end{gathered}
$$




\subsection{FUNCIONES QUE CONTIENEN A $u^{2}+a^{2}$}
a) $\int \frac{d u}{u^{2}+a^{2}}=\frac{1}{a} \arctan \frac{u}{a}+C$.
b) $\int \frac{d u}{\sqrt{u^{2}+a^{2}}}=\ln \left[u+\sqrt{u^{2}+a^{2}}\right]+C$.
C) $\int \sqrt{u^{2}+a^{2}} d u=\frac{1}{2} u \sqrt{u^{2}+a^{2}}+\frac{1}{2} a^{2} \ln \left[u+\sqrt{u^{2}+a^{2}}\right]+C$.

\section{Ejemplo 41:}

Comprobar la fórmula a). Bastará demostrar que la derivada de $\frac{1}{a} \arctan \frac{x}{a}+C$ es $\frac{1}{x^{2}+a^{2}}$.

Recordemos que $\frac{d}{d x} \arctan w=\frac{1}{1+w^{2}} \frac{d w}{d x}$.

$$
\frac{d}{d x}\left\{\frac{1}{a} \arctan \frac{x}{a}+C\right\}=\frac{1}{a} \frac{\frac{1}{a}}{1+\left(\frac{x}{a}\right)^{2}}=\frac{1 / a^{2}}{1+\frac{x^{2}}{a^{2}}}=\frac{1}{x^{2}+a^{2}} .
$$

Ejemplo 42:

32 Comprobar la fórmula b).

La derivada de $\ln \left[x+\sqrt{x^{2}+a^{2}}\right]+C$ debería ser $\frac{1}{\sqrt{x^{2}+a^{2}}}$ para que la fórmula b) quede demostrada.

$$
\frac{d\left\{\ln \left[x+\sqrt{x^{2}+a^{2}}\right]+C\right\}}{d x}=\frac{1+\frac{2 x}{2 \sqrt{x^{2}+a^{2}}}}{x+\sqrt{x^{2}+a^{2}}}=\frac{2\left[\sqrt{x^{2}+a^{2}}+x\right]}{2\left[x+\sqrt{x^{2}+a^{2}}\right] \sqrt{x^{2}+a^{2}}}=\frac{1}{\sqrt{x^{2}+a^{2}}}
$$

Ejemplo 43:

Comprobar la fórmula c).

Análogamente:

$$
\begin{gathered}
\frac{d}{d x}\left\{\frac{1}{2} x \sqrt{x^{2}+a^{2}}+\frac{1}{2} a^{2} \ln \left[x+\sqrt{x^{2}+a^{2}}\right]+C\right\}= \\
\frac{1}{2}\left[1 \sqrt{x^{2}+a^{2}}+x \frac{2 x}{2 \sqrt{x^{2}+a^{2}}}+a^{2} \frac{1+\frac{2 x}{2 \sqrt{x^{2}+a^{2}}}}{x+\sqrt{x^{2}+a^{2}}}\right]= \\
\frac{1}{2}\left[\sqrt{x^{2}+a^{2}}+\frac{x^{2}}{\sqrt{x^{2}+a^{2}}}+\frac{a^{2}}{\sqrt{x^{2}+a^{2}}}\right]=
\end{gathered}
$$




$$
\begin{gathered}
\frac{1}{2}\left[\sqrt{x^{2}+a^{2}}+\frac{x^{2}+a^{2}}{\sqrt{x^{2}+a^{2}}}\right]=\frac{1}{2}\left[\sqrt{x^{2}+a^{2}}+\sqrt{x^{2}+a^{2}}\right]= \\
=\sqrt{x^{2}+a^{2}}
\end{gathered}
$$

Ejemplo 44:

$$
\begin{gathered}
I=\int \frac{d x}{4 x^{2}+9}=\int \frac{d x}{(2 x)^{2}+3^{2}} \\
u=2 x \rightarrow d u=2 d x \rightarrow d x=\frac{d u}{2} \\
I=\int \frac{\frac{1}{2} d u}{u^{2}+3^{2}}=\frac{1}{2} \int \frac{d u}{u^{2}+3^{2}} \\
I=\frac{1}{2} \cdot \frac{1}{3} \arctan \frac{u}{3}+C \\
I=\frac{1}{6} \arctan \frac{2 x}{3}+C
\end{gathered}
$$

Ejemplo 45:

$$
\begin{gathered}
I=\int \frac{x d x}{x^{4}+3}=\int \frac{x d x}{\left(x^{2}\right)^{2}+(\sqrt{3})^{2}} \\
u=x^{2} \rightarrow d u=2 x d x \rightarrow x d x=\frac{d u}{2} \\
I=\int \frac{\frac{d u}{2}}{u^{2}+(\sqrt{3})^{2}}=\frac{1}{2} \int \frac{d u}{u^{2}+(\sqrt{3})^{2}} \\
I=\frac{1}{2} \cdot \frac{1}{\sqrt{3}} \arctan \frac{u}{\sqrt{3}}+C \\
I=\frac{1}{2 \sqrt{3}} \arctan \frac{x^{2}}{\sqrt{3}}+C
\end{gathered}
$$

Ejemplo 46:

$$
\begin{gathered}
I=\int \frac{\sec x \cdot \tan x \cdot d x}{4 \sec ^{2} x+9}=\int \frac{\sec x \cdot \tan x \cdot d x}{(2 \sec x)^{2}+3^{2}} \\
u=2 \sec x \rightarrow d u=2 \sec x \cdot \tan x \cdot d x
\end{gathered}
$$




$$
\begin{gathered}
I=\int \frac{\frac{d u}{2}}{u^{2}+3^{2}}=\frac{1}{2} \int \frac{d u}{u^{2}+3^{2}} \\
I=\frac{1}{2} \cdot \frac{1}{3} \arctan \frac{u}{3}+C \\
I=\frac{1}{6} \arctan \left(\frac{2 \sec x}{3}\right)+C
\end{gathered}
$$

Ejemplo 47:

$$
\begin{gathered}
I=\int \frac{d x}{2 x^{2}+2 x+5}=\int \frac{2 d x}{4 x^{2}+4 x+10} \\
I=\int \frac{2 d x}{(2 x+1)^{2}+3^{2}} \\
u=2 x+1 \rightarrow d u=2 d x \\
I=\int \frac{d u}{u^{2}+3^{2}}=\frac{1}{3} \arctan \frac{u}{3}+C
\end{gathered}
$$

$$
I=\frac{1}{3} \arctan \frac{2 x+1}{3}+C
$$

Ejemplo 48:

$$
\begin{aligned}
& I=\int \frac{d x}{\sqrt{4 x^{2}+9}}=\int \frac{d x}{\sqrt{(2 x)^{2}+3^{2}}} \\
& u=2 x \rightarrow d u=2 d x \rightarrow d x=\frac{d u}{2} \\
& I=\int \frac{\frac{d u}{2}}{\sqrt{u^{2}+3^{2}}}=\frac{1}{2} \int \frac{d u}{\sqrt{u^{2}+3^{2}}} \\
& I=\frac{1}{2} \ln \left[u+\sqrt{u^{2}+3^{2}}\right]+C \\
& I=\frac{1}{2} \ln \left[2 x+\sqrt{4 x^{2}+9}\right]+C
\end{aligned}
$$

Ejemplo 49:

$$
I=\int \frac{x+2}{\sqrt{x^{2}+9}} d x=\int \frac{x d x}{\sqrt{x^{2}+9}}+\int \frac{2 d x}{\sqrt{x^{2}+9}}
$$




$$
\begin{gathered}
I=\frac{1}{2} \int \frac{2 x d x}{\sqrt{x^{2}+9}}+2 \int \frac{d x}{\sqrt{x^{2}+9}} \\
I=\frac{1}{2} \int \underbrace{\left(x^{2}+9\right)^{-1 / 2}}_{u} \underbrace{(2 x d x)}_{d u}+2 \int \frac{d x}{\sqrt{x^{2}+9}} \\
I=\frac{\frac{1}{2}\left(x^{2}+9\right)^{-1 / 2+1}}{-\frac{1}{2}+1}+2 \ln \left[x+\sqrt{x^{2}+9}\right]+C \\
I=\sqrt{x^{2}+9}+2 \ln \left[x+\sqrt{x^{2}+9}\right]+C
\end{gathered}
$$

Ejemplo 50:

$$
I=\int \frac{d x}{\sqrt{1+4 e^{2 x}}}
$$

Escribámoslo así:

$$
\begin{gathered}
I=\int \frac{d x}{\sqrt{e^{2 x} \frac{1+4 e^{2 x}}{e^{2 x}}}} \\
I=\int \frac{d x}{\sqrt{e^{2 x}} \sqrt{e^{-2 x}+4}} \\
I=\int \frac{e^{-x} d x}{\sqrt{e^{-2 x}+4}}=\int \frac{e^{-x} d x}{\sqrt{\left(e^{-x}\right)^{2}+2^{2}}} \\
I=\int \frac{-d u}{\sqrt{u^{2}+2^{2}}}=-\ln \left[u+\sqrt{u^{2}+4}\right]+C \\
I=-\ln \left[e^{-x}+\sqrt{e^{-2 x}+4}\right]+C
\end{gathered}
$$

Ejemplo 51:

$$
\begin{gathered}
I=\int \sqrt{3 x^{2}+5} d x=\int \sqrt{(\sqrt{3} x)^{2}+(\sqrt{5})^{2}} d x \\
u=\sqrt{3} x \rightarrow d u=\sqrt{3} d x
\end{gathered}
$$




$$
\begin{gathered}
I=\int \sqrt{u^{2}+(\sqrt{5})^{2}}\left(\frac{d u}{\sqrt{3}}\right) \\
I=\frac{1}{\sqrt{3}}\left[\frac{1}{2} u \sqrt{u^{2}+5}+\frac{5}{2} \ln \left[u+\sqrt{u^{2}+5}\right]\right]+C \\
I=\frac{1}{2} x \sqrt{3 x^{2}+5}+\frac{5 \sqrt{3}}{6} \ln \left[\sqrt{3} x+\sqrt{3 x^{2}+5}\right]+C
\end{gathered}
$$

Ejemplo 52:

$$
\begin{gathered}
I=\int \sqrt{4 x^{2}-4 x+5} d x \\
I=\frac{1}{2} \int \sqrt{(2 x-1)^{2}+2^{2}}(2 d x) \\
u=2 x-1 \rightarrow \mathrm{du}=2 \mathrm{dx} \\
I=\frac{1}{2} \int \sqrt{u^{2}+2^{2}} d u \\
I=\frac{1}{2}\left[\frac{1}{2} u \sqrt{u^{2}+4}+\frac{2^{2}}{2} \ln \left[u+\sqrt{u^{2}+4}\right]\right]+C \\
I=\frac{1}{4} u \sqrt{u^{2}+4}+\ln \left[u+\sqrt{u^{2}+4}\right]+C \\
I=\frac{1}{4}(2 x-1) \sqrt{4 x^{2}-4 x+5}+\ln \left[(2 x-1)+\sqrt{4 x^{2}-4 x+5}\right]+C
\end{gathered}
$$

36

\subsection{FUNCIONES QUE CONTIENEN A $u^{2}-a^{2}$}
a) $\int \frac{d u}{u^{2}-a^{2}}=\frac{1}{2 a} \ln \frac{u-a}{u+a}+C$.
b) $\int \frac{d u}{\sqrt{u^{2}-a^{2}}}=\ln \left[u+\sqrt{u^{2}-a^{2}}\right]+C$.
c) $\int \frac{d u}{u \sqrt{u^{2}-a^{2}}}=\frac{1}{a} \operatorname{arcsec} \frac{u}{a}+C$.

Ejemplo 53:

Comprobar la fórmula a).

La derivada de $f_{(u)}=\frac{1}{2 a} \ln \frac{u-a}{u+a}+C$ deberá ser $\frac{1}{u^{2}-a^{2}}$. 
Entonces:

$$
\begin{aligned}
& \frac{d f_{(u)}}{d u}=\frac{1}{2 a} \cdot \frac{\frac{(u+a)(1)-(u-a)(1)}{(u+a)^{2}}}{\frac{u-a}{u+a}} \\
& \frac{d f_{(u)}}{d u}=\frac{1}{2 a} \cdot \frac{2 a}{(u+a)(u-a)}=\frac{1}{u^{2}-a^{2}}
\end{aligned}
$$

\section{Ejemplo 54:}

Comprobar la fórmula c).

La derivada de $f_{(u)}=\frac{1}{a} \operatorname{arcsec} \frac{u}{a}+C$ deberá ser $\frac{1}{u \sqrt{u^{2}-a^{2}}}$.

Recordemos que $\frac{d}{d x}(\operatorname{arcsec} w)=\frac{1}{w \sqrt{w^{2}-1}} \cdot \frac{d w}{d x}$.

Entonces:

$$
\begin{gathered}
\frac{d f_{(u)}}{d u}=\frac{1}{a} \cdot \frac{1}{\frac{u}{a} \sqrt{\left(\frac{u}{a}\right)^{2}-1}} \cdot\left(\frac{1}{a}\right) \\
\frac{d f_{(u)}}{d u}=\frac{1}{a} \cdot \frac{1}{u \sqrt{\frac{u^{2}-a^{2}}{a^{2}}}}=\frac{1}{u \sqrt{u^{2}-a^{2}}}
\end{gathered}
$$

Ejemplo 55:

$$
\begin{gathered}
I=\int \frac{d x}{x^{2}+6 x+8}=\int \frac{d x}{(x+3)^{2}-1^{2}} \\
I=\frac{1}{2(1)} \ln \frac{(x+3)-1}{(x+3)+1}+C \\
I=\frac{1}{2} \ln \frac{x+2}{x+4}+C
\end{gathered}
$$

Ejemplo 56:

$$
I=\int \frac{2 x-3}{4 x^{2}-11} d x
$$


Descompongamos "I" en dos integrales:

$$
\begin{gathered}
I=\int \frac{2 x-3}{4 x^{2}-11} d x=\int \frac{2 x d x}{4 x^{2}-11}-\int \frac{3 d x}{4 x^{2}-11} \\
I=\frac{1}{4} \int \frac{8 x d x}{4 x^{2}-11}-\frac{3}{2} \int \frac{2 d x}{(2 x)^{2}-(\sqrt{11})^{2}} \\
I=\frac{1}{4} \ln \left(4 x^{2}-11\right)-\frac{3}{2} \cdot \frac{1}{2 \sqrt{11}} \cdot \ln \frac{2 x-\sqrt{11}}{2 x+\sqrt{11}}+C
\end{gathered}
$$

Ejemplo 57:

$$
I=\int \frac{x+2}{\sqrt{x^{2}+2 x+3}} d x
$$

Análogamente:

$$
\begin{gathered}
I=\frac{1}{2} \int \frac{2(x+2)}{\sqrt{x^{2}+2 x-3}} d x \\
I=\frac{1}{2}\left[\int \frac{2 x+2}{\sqrt{x^{2}+2 x-3}} d x+\int \frac{2 d x}{\sqrt{x^{2}+2 x-3}}\right] \\
I=\frac{1}{2}\left[\int\left(x^{2}+2 x-3\right)^{-1 / 2}(2 x+2) d x+2 \int \frac{d x}{\sqrt{(x+1)^{2}-2^{2}}}\right] \\
I=\sqrt{x^{2}+2 x-3}+\ln \left[x+1+\sqrt{x^{2}+2 x-3}\right]+C
\end{gathered}
$$

Ejemplo 58:

$$
I=\int \frac{d x}{\sqrt{e^{4 x}-1}}
$$

Escribámoslo así:

$$
I=\frac{1}{2} \int \frac{2 e^{2 x} d x}{e^{2 x} \sqrt{\left(e^{2 x}\right)^{2}-1}}
$$

Si $u=e^{2 x} \rightarrow d u=2 e^{2 x} d x$ 
Entonces:

$$
\begin{gathered}
I=\frac{1}{2} \int \frac{d u}{u \sqrt{u^{2}-1}} \\
I=\frac{1}{2} \cdot \frac{1}{1} \cdot \operatorname{arcsec} \frac{u}{1}+C \\
I=\frac{1}{2} \operatorname{arcsec} e^{2 x}+C
\end{gathered}
$$

\subsection{FUNCIONES QUE CONTIENEN A $a^{2}-u^{2}$}
a) $\int \frac{d u}{a^{2}-u^{2}}=\frac{1}{2 a} \ln \frac{a+u}{a-u}+C$.
b) $\int \sqrt{a^{2}-u^{2}} d u=\frac{1}{2} u \sqrt{a^{2}-u^{2}}+\frac{1}{2} a^{2} \operatorname{arcsen} \frac{u}{a}+C$.
c) $\int \frac{d u}{\sqrt{a^{2}-u^{2}}}=\operatorname{arcsen} \frac{u}{a}+C$.

Ejemplo 59:

Comprobar la fórmula b).

Recordemos que $\frac{d(\operatorname{arcsen} w)}{d x}=\frac{1}{\sqrt{1-w^{2}}} \frac{d w}{d x}$.

La derivada de la expresión del segundo miembro de la fórmula b) es:

$$
\begin{aligned}
& \frac{1}{2}(1) \sqrt{a^{2}-u^{2}}+\frac{1}{2} u\left(\frac{-u}{\sqrt{a^{2}-u^{2}}}\right)+\frac{1}{2} a^{2} \cdot \frac{1}{\sqrt{1-(u / a)^{2}}} \cdot \frac{1}{a} \\
& \frac{\sqrt{a^{2}-u^{2}}}{2}-\frac{u^{2}}{2 \sqrt{a^{2}-u^{2}}}+\frac{a^{2}}{2 \sqrt{a^{2}-u^{2}}}=\frac{\sqrt{a^{2}-u^{2}}}{2}+\frac{a^{2}-u^{2}}{2 \sqrt{a^{2}-u^{2}}}
\end{aligned}
$$

De donde:

$$
\frac{\sqrt{a^{2}-u^{2}}}{2}+\frac{\sqrt{a^{2}-u^{2}}}{2}=\sqrt{a^{2}-u^{2}}
$$


Ejercicio 60:

Comprobar la fórmula c).

Análogamente, la derivada del segundo miembro es:

$$
\frac{d}{d u}\left(\operatorname{arcsen} \frac{u}{a}\right)=\frac{1}{\sqrt{1-\left(\frac{u}{a}\right)^{2}}} \frac{1}{a}
$$

De donde se obtiene:

$$
\frac{1}{a \sqrt{1-\left(\frac{u}{a}\right)^{2}}}=\frac{1}{\sqrt{a^{2}-u^{2}}}
$$

Ejemplo 61:

$$
I=\int \frac{d x}{4 x-x^{2}}=\int \frac{d x}{2^{2}-(x-2)^{2}}
$$

40

$$
\begin{gathered}
I=\frac{1}{2(2)} \ln \frac{2+(x-2)}{2-(x-2)}+C \\
I=\frac{1}{4} \ln \frac{x}{4-x}+C
\end{gathered}
$$

Ejemplo 62:

$$
I=\int \frac{d x}{\sec x\left(25-16 \operatorname{sen}^{2} x\right)}
$$

Escribámoslo:

$$
I=\int \frac{\cos x d x}{25-16 \operatorname{sen}^{2} x}=\int \frac{\cos x d x}{5^{2}-(4 \operatorname{sen} x)^{2}}
$$

Si $u=4 \operatorname{sen} x \Rightarrow d u=4 \cos x d x$.

Entonces:

$$
I=\frac{1}{4} \int \frac{4 \cos x d x}{5^{2}-(4 \operatorname{sen} x)^{2}}=\frac{1}{4} \int \frac{d u}{5^{2}-u^{2}}
$$


De donde:

$$
I=\frac{1}{4} \cdot \frac{1}{2(5)} \cdot \ln \frac{5+u}{5-u}+C
$$

Finalmente:

$$
I=\frac{1}{40} \cdot \ln \frac{5+4 \operatorname{sen} x}{5-4 \operatorname{sen} x}+C
$$

Ejemplo 63:

$$
\begin{gathered}
I=\int \sqrt{3-2 x-x^{2}} d x \\
I=\int \sqrt{4-\left(x^{2}+2 x+1\right)} d x \\
I=\int \sqrt{2^{2}-(x+1)^{2}} d x \\
I=\frac{1}{2}(x+1) \sqrt{2^{2}-(x+1)^{2}}+2 \operatorname{arcsen} \frac{x+1}{2}+C
\end{gathered}
$$

Ejemplo 64:

$$
I=\int \frac{d x}{x \sqrt{20 x^{2}+8 x-1}}
$$

Hagamos un cambio de variable: $x=\frac{1}{u} \rightarrow d x=\frac{-d u}{u^{2}}$

Entonces:

$$
\begin{gathered}
\int \frac{-\frac{d u}{u^{2}}}{\frac{1}{u} \sqrt{\frac{20}{u^{2}}+\frac{8}{u}-1}} \\
I=-\int \frac{d u}{\sqrt{20+8 u-u^{2}}} \\
I=-\int \frac{d u}{\sqrt{6^{2}-(u-4)^{2}}} \\
I=-\operatorname{arcsen} \frac{u-4}{6}+C
\end{gathered}
$$




$$
\begin{aligned}
& I=-\operatorname{arcsen} \frac{1 / x-4}{6}+C \\
& I=-\operatorname{arcsen} \frac{1-4 x}{6 x}+C
\end{aligned}
$$




\section{IV MÉTODOS DE INTEGRACIÓN}

\section{INTEGRACIÓN POR SUSTITUCIÓN}

En el ejemplo 10 del capítulo II, para encontrar la integral $I=\int e^{a x^{2}+b x+d} \cdot(2 a x+b) d x$, a partir de la fórmula $\int e^{u} d u=e^{u}+C$, se efectuó el cambio de variable $\begin{aligned} & u=a x^{2}+b x+d \\ & d u=(2 a x+b) d x\end{aligned}$.

A esta forma de calcular la integral se le denomina método de sustitución. Así pues:

$$
\begin{gathered}
I=\int e^{a x^{2}+b x+d} \cdot(2 a x+b) \cdot d x=\int e^{u} \cdot d u \\
I=\int e^{u} d u=e^{u}+C=e^{a x^{2}+b x+d}+C
\end{gathered}
$$

Ejemplo 65:

$$
I=\int \frac{d x}{x\left[1-\ln ^{2} x\right]}
$$

Si $u=\ln x \rightarrow d u=\frac{d x}{x}$

$$
I=\int \frac{d x / x}{1-\ln ^{2} x}=\int \frac{d u}{1-u^{2}}
$$




$$
\begin{aligned}
& I=\frac{1}{2(1)} \ln \frac{1+u}{1-u}+C \\
& I=\frac{1}{2} \ln \frac{1+\ln x}{1-\ln x}+C
\end{aligned}
$$

Ejemplo 66:

$$
I=\int \frac{2 d x}{x \sqrt{x+4}}
$$

Si $x+4=u^{2} \Rightarrow d x=2 u d u$

$$
I=\int \frac{2(2 u d u)}{\left(u^{2}-4\right) u}=4 \int \frac{d u}{u^{2}-4}
$$

De donde:

$$
I=4 \frac{1}{2(2)} \ln \frac{u-2}{u+2}+C
$$

$$
I=\ln \frac{\sqrt{x+4}-2}{\sqrt{x+4}+2}+C
$$

Ejemplo 67:

$$
I=\int \frac{e^{x} d x}{\sqrt{e^{x}+1}-1}
$$

Si $e^{x}+1=u^{2} \rightarrow e^{x} d x=2 u d u$

Entonces:

$$
I=\int \frac{2 u d u}{u-1}=2 \int \frac{u d u}{u-1}
$$

De donde:

$$
\begin{gathered}
I=2\left[\int \frac{u-1}{u-1} d u+\int \frac{1 d u}{u-1}\right] \\
I=2[u+\ln (u-1)]+C
\end{gathered}
$$

Finalmente:

$$
I=2 \sqrt{e^{x}+1}+2 \ln \left[\sqrt{e^{x}+1}-1\right]+C
$$


Ejemplo 68:

$$
I=\int \sqrt{\frac{\ln \left(x+\sqrt{x^{2}+1}\right)}{1+x^{2}}} d x
$$

Si $u=\ln \left(x+\sqrt{x^{2}+1}\right)$

$$
d u=\frac{1+\frac{2 x}{2 \sqrt{x^{2}+1}}}{x+\sqrt{x^{2}+1}} d x
$$

De donde:

$$
d u=\frac{1}{\sqrt{x^{2}+1}} d x
$$

Entonces:

$$
\begin{gathered}
I=\int \frac{\sqrt{\ln \left(x+\sqrt{x^{2}+1}\right)}}{\sqrt{x^{2}+1}} d x \\
I=\int \sqrt{u} d u \\
I=\frac{2 u^{3 / 2}}{3}+C \\
I=\frac{2}{3}\left[\ln \left(x+\sqrt{x^{2}+1}\right)\right]^{3 / 2}+C
\end{gathered}
$$

\section{INTEGRACIÓN POR PARTES}

Sean $u$ y $v$ dos funciones cualesquiera de $x$, entonces:

$$
\begin{aligned}
& d(u v)=u d v+v d u \\
& u d v=d(u v)-v d u
\end{aligned}
$$

De donde:

$$
\int u d v=\int d(u v)-\int v d u
$$

Con lo cual:

$$
\int u d v=u v-\int v d u
$$


A este método se le denomina integración por partes y lo aplicaremos a la integral $\int \ln x d x$. En $\int \ln x d x$, hagamos:

$$
\begin{array}{cc}
u=\ln x & v=x \\
\downarrow & \uparrow \\
d u=\frac{1}{x} d x & d v=d x
\end{array}
$$

Entonces:

$$
\int \ln x d x=(\ln x)(x)-\int(x)\left(\frac{1}{x} d x\right)
$$

Con lo cual:

$$
\begin{aligned}
& \int \ln x d x=x \ln x-\int d x \\
& \int \ln x d x=x \ln x-x+C
\end{aligned}
$$

46

Ejemplo 69:

$$
I=\int e^{\sqrt{x}} \sqrt{x} d x
$$

Hagamos: $z=\sqrt{x} \Rightarrow d z=\frac{1}{2 \sqrt{x}} d x$

$$
\begin{gathered}
d x=2 \sqrt{x} d z \\
\sqrt{x} d x=2 x d z
\end{gathered}
$$

De donde:

$$
\begin{aligned}
& I=\int e^{z}(2 x d z) \\
& I=2 \int e^{z} z^{2} d z
\end{aligned}
$$

Se tiene que:

$$
\begin{array}{cc}
u=z^{2} & v=e^{z} \\
\downarrow & \uparrow \\
d u=2 z d z & d v=e^{z} d z
\end{array}
$$

Entonces:

$$
I=2\left[e^{z} z^{2}-2 \int e^{z} z d z\right]
$$


Hagamos:

$$
\begin{array}{cc}
u=z & v=e^{z} \\
\downarrow & \uparrow \\
d u=d z & d v=e^{z} d z
\end{array}
$$

Se tendrá que:

$$
\begin{gathered}
I=2\left[e^{z} z^{2}-2\left\{e^{z} z-\int e^{z} d z\right\}\right] \\
I=2 e^{z} z^{2}-4 e^{z} z+4 e^{z}+C
\end{gathered}
$$

De donde:

$$
I=2 e^{\sqrt{x}} x-4 e^{\sqrt{x}} \sqrt{x}+4 e^{\sqrt{x}}+C
$$

Ejemplo 70:

Demostrar que se cumple:

$$
\int x^{n+1} f^{\prime \prime}(x) d x=x^{n+1} f^{\prime}(x)-(n+1) x^{n} f_{(x)}+n(n+1) \int x^{n-1} f_{(x)} d x
$$

En la integral $I=\int x^{n+1} f^{\prime \prime}(x) d x$, hagamos que:

$$
\begin{array}{cc}
u=x^{n+1} & v=f^{\prime}(x) \\
\downarrow & \uparrow \\
d u=(n+1) x^{n} d x & d v=f^{\prime \prime}{ }_{(x)} d x
\end{array}
$$

Entonces:

$$
\begin{array}{ccc}
I= & x^{n+1} f^{\prime}(x)-\int(n+1) x^{n} f_{(x)}^{\prime} d x \\
u=x^{n} & v=f_{(x)} \\
& \downarrow & \uparrow \\
d u & =n x^{n-1} d x & d v=f^{\prime}(x)
\end{array}
$$

De donde:

$$
I=x^{n+1} f^{\prime}(x)-(n+1)\left[x^{n} f_{(x)}-\int n x^{n-1} f_{(x)} d x\right]
$$

Finalmente:

$$
I=x^{n+1} f^{\prime}(x)-(n+1) x^{n} f_{(x)}+n(n+1) \int x^{n-1} f_{(x)} d x
$$




\section{INTEGRACIÓN POR SUSTITUCIÓN TRIGONOMÉTRICA}

Si el integrando contiene expresiones como $u^{2}+a^{2}, u^{2}-a^{2} 0 a^{2}-u^{2}$, siendo $u$ una función de $x$ y a una constante, se aconseja efectuar un cambio de variable conveniente para aplicar alguna identidad trigonométrica.

En el caso de $u^{2}+a^{2}$, conviene hacer $u=a \tan \theta$, de modo que:

$$
u^{2}+a^{2}=a^{2} \tan ^{2} \theta+a^{2}=a^{2}\left(\tan ^{2} \theta+1\right)=a^{2} \sec ^{2} \theta
$$

En el caso de $u^{2}-a^{2}$, conviene hacer $u=a \sec \theta$, de modo que:

$$
u^{2}-a^{2}=a^{2} \sec ^{2} \theta-a^{2}=a^{2}\left(\sec ^{2} \theta-1\right)=a^{2} \tan ^{2} \theta
$$

En el caso de $a^{2}-u^{2}$, conviene hacer $u=a \cos \theta$, de modo que:

$$
a^{2}-u^{2}=a^{2}-a^{2} \cos ^{2} \theta=a^{2}\left(1-\cos ^{2} \theta\right)=a^{2} \operatorname{sen}^{2} \theta
$$

Como ejemplo de aplicación demostraremos que $\int \frac{d u}{u^{2}+a^{2}}=\frac{1}{a} \arctan \frac{u}{a}+C$

Se efectúa el cambio de variable $u=a \tan \theta \Rightarrow d u=a \sec ^{2} \theta d \theta$

48

$$
\theta=\arctan \frac{u}{a}
$$

Entonces:

$$
\int \frac{d u}{u^{2}+a^{2}}=\int \frac{a \sec ^{2} \theta d \theta}{a^{2} \tan ^{2} \theta+a^{2}}=\frac{1}{a} \int \frac{\sec ^{2} \theta}{\sec ^{2} \theta} d \theta=\frac{1}{a} \int d \theta=\frac{1}{a} \theta+C
$$

Con lo cual:

$$
\int \frac{d u}{u^{2}+a^{2}}=\frac{1}{a}\left(\arctan \frac{u}{a}\right)+C
$$

\section{Ejemplo 71:}

Encontrar

$$
\int \frac{x^{3} d x}{\sqrt{x^{2}+4}}
$$

Sea $x=2 \tan \theta \rightarrow d x=2 \sec ^{2} \theta d \theta$

Entonces:

$$
\int \frac{x^{3} d x}{\sqrt{x^{2}+4}}=\int \frac{\left(8 \tan ^{3} \theta\right)\left(2 \sec ^{2} \theta d \theta\right)}{\sqrt{4\left(\tan ^{2} \theta+1\right)}}=8 \int \frac{\tan ^{3} \theta \sec ^{2} \theta d \theta}{\sec \theta}
$$




$$
\begin{gathered}
\int \frac{x^{3} d x}{\sqrt{x^{2}+4}}=8 \int \tan ^{3} \theta \sec \theta d \theta=8 \int\left(\tan ^{2} \theta\right)(\tan \theta \sec \theta) d \theta \\
\int \frac{x^{3} d x}{\sqrt{x^{2}+4}}=8 \int\left(\sec ^{2} \theta-1\right)(\sec \theta \tan \theta d \theta)=8\left[\frac{\sec ^{3} \theta}{3}-\sec \theta\right]+C
\end{gathered}
$$

Como $\tan \theta=\frac{x}{2} \rightarrow \sec \theta=\sqrt{\tan ^{2} \theta+1}=\sqrt{\frac{x^{2}}{4}+1}=\frac{1}{2} \sqrt{x^{2}+4}$, la integral es:

$$
\begin{gathered}
8\left[\frac{1}{3(8)}\left(x^{2}+4\right)^{3 / 2}-\frac{1}{2}\left(x^{2}+4\right)^{1 / 2}\right]+C \\
\frac{1}{3}\left(x^{2}+4\right)^{3 / 2}-4\left(x^{2}+4\right)^{1 / 2}+C
\end{gathered}
$$

Ejemplo 72:

Encontrar

$$
\int \frac{1}{x^{2} \sqrt{4-x^{2}}} d x
$$

Sea $x=2 \operatorname{sen} \theta \rightarrow d x=2 \cos \theta d \theta$

Entonces:

$$
\begin{gathered}
\int \frac{1}{x^{2} \sqrt{4-x^{2}}} d x=\int \frac{2 \cos \theta d \theta}{\left(4 \operatorname{sen}^{2} \theta\right) \sqrt{4\left(1-\operatorname{sen}^{2} \theta\right)}}=\frac{1}{4} \int \frac{\cos \theta d \theta}{\operatorname{sen}^{2} \theta \cos \theta} \\
\int \frac{1}{x^{2} \sqrt{4-x^{2}}} d x=\frac{1}{4} \int \csc ^{2} \theta d \theta=-\frac{1}{4} \cot \theta+C
\end{gathered}
$$

Como $\cot \theta=\frac{\cos \theta}{\operatorname{sen} \theta}=\frac{\sqrt{1-(x / 2)^{2}}}{x / 2}=\frac{\sqrt{4-x^{2}}}{x}$, la respuesta es:

$$
-\frac{1}{4} \cdot \frac{\sqrt{4-x^{2}}}{x}+C
$$

\section{INTEGRACIÓN POR DESCOMPOSICIÓN EN FRACCIONES PARCIALES}

Si el integrando consiste en un cociente de dos polinomios, $P_{(x)}$ y $Q_{(x)}$, que sean polinomios enteros, tal que el grado de $P_{(x)}$ es menor que el grado de $Q_{(x)}$, vale decir si se debe integrar una 
fracción racional propia $\frac{P_{(x)}}{Q_{(x)}}$, se recomienda descomponer en fracciones parciales e integrar separadamente cada una de ellas.

Recordemos que se pueden presentar los cuatro casos siguientes:

a) Factor lineal simple $\frac{A}{x-a}+\frac{B}{x-b}+\ldots+\frac{E}{x-e}$

b) Factor lineal repetido $\frac{A}{x-a}+\frac{B}{(x-a)^{2}}+\ldots+\frac{Z}{(x-a)^{n}}$

c) Factor cuadrático simple $\frac{A x+B}{x^{2}+b x+c}+\frac{C x+D}{x^{2}+d x+e}+\ldots$

d) Factor cuadrático repetido $\frac{A x+B}{x^{2}+b x+c}+\frac{C x+D}{\left(x^{2}+b x+c\right)^{2}}+\ldots$

Una vez descompuesto en fracciones parciales, se procede a integrar cada una de ellas. Por 50 ejemplo, en el caso de fracciones lineales:

$$
\begin{aligned}
& \int \frac{A}{x-a} d x=A \int \frac{d x}{x-a}=A \ln (x-a)+C \\
& \int \frac{B}{(x-a)^{2}} d x=B \int(x-a)^{-2} d x=\frac{B(x-a)^{-1}}{-1}+C=\frac{-B}{(x-a)}+C \\
& \int \frac{C}{(x-a)^{3}} d x=C \int(x-a)^{-3} d x=\frac{C(x-a)^{-2}}{-2}+C^{\prime}=\frac{-C}{2(x-a)^{2}}+C^{\prime}
\end{aligned}
$$

En el caso de los factores cuadráticos, supongamos el siguiente ejemplo:

$$
\int \frac{A x+B}{x^{2}+b x+c} d x=\int \frac{x+1}{x^{2}+4 x+8} d x=\frac{1}{2} \int \frac{2 x+2}{x^{2}+4 x+8} d x=\frac{1}{2} \int\left[\frac{2 x+4}{x^{2}+4 x+8}-\frac{2}{(x+2)^{2}+2^{2}}\right] d x
$$

De donde:

$$
\int \frac{x+1}{x^{2}+4 x+8} d x=\frac{1}{2}\left[\ln \left(x^{2}+4 x+8\right)-\frac{2}{2} \arctan \frac{x+2}{2}\right]+C
$$

Ejemplo 73:

$$
I=\int \frac{1}{x^{2}-a x} d x
$$


Se descompone:

$$
\begin{aligned}
\frac{1}{x^{2}-a x} & =\frac{1}{x(x-a)}=\frac{A}{x}+\frac{B}{x-a} \\
1 & =A(x-a)+B x \\
1 & =(A+B) x-A a
\end{aligned}
$$

De donde:

$$
\left\{\begin{array}{l}
A+B=0 \rightarrow B=1 / a \\
-A a=1 \rightarrow A=-1 / a
\end{array}\right.
$$

Entonces:

$$
\begin{gathered}
I=\int \frac{-1 / a}{x} d x+\int \frac{1 / a}{x-a} d x \\
I=-\frac{1}{a} \ln x+\frac{1}{a} \ln (x-a)+C \\
I=\frac{1}{a} \ln \frac{x-a}{x}+C
\end{gathered}
$$

Ejemplo 74:

$$
\int \frac{x^{3}+4 x+2}{\left(x^{2}+4\right)^{2}} d x
$$

Se descompone:

$$
\begin{gathered}
\frac{x^{3}+4 x+2}{\left(x^{2}+4\right)^{2}}=\frac{A x+B}{x^{2}+4}+\frac{C x+D}{\left(x^{2}+4\right)^{2}} \\
\frac{x^{3}+4 x+2}{\left(x^{2}+4\right)^{2}}=\frac{(A x+B)\left(x^{2}+4\right)+(C x+D)}{\left(x^{2}+4\right)^{2}}
\end{gathered}
$$

De donde: $x^{3}+0 x^{2}+4 x+2=A x^{3}+B x^{2}+4 A x+C x+4 B+D$

$$
\begin{aligned}
& \text { Igualamos los } \\
& \text { coeficientes } \\
& \text { respectivos: }
\end{aligned} \quad\left\{\begin{array}{l}
1=A \\
0=B \\
4=4 A+C \\
2=4 B+D
\end{array}\right.
$$


Se obtiene $A=1, B=0, C=0$ y $D=2$.

Entonces:

$$
\begin{gathered}
\int \frac{x^{3}+4 x+2}{\left(x^{2}+4\right)^{2}} d x=\int \frac{x d x}{x^{2}+4}+\int \frac{2 d x}{\left(x^{2}+4\right)^{2}} \\
\int \frac{x^{3}+4 x+2}{\left(x^{2}+4\right)^{2}} d x=\frac{1}{2} \ln \left(x^{2}+4\right)+2 \int \frac{d x}{\left(x^{2}+4\right)^{2}}
\end{gathered}
$$

Por separado:

52

$$
\begin{array}{ll}
\int \frac{d x}{\left(x^{2}+4\right)^{2}}=\int \frac{2 \sec ^{2} \theta d \theta}{\left[4\left(\tan ^{2} \theta+1\right)\right]^{2}} \quad \begin{array}{ll}
x=2 \tan \theta \\
\end{array} \\
\int \frac{d x}{\left(x^{2}+4\right)^{2}}=\frac{1}{8} \int \frac{\sec ^{2} \theta d \theta}{\sec ^{4} \theta}=\frac{1}{8} \int \cos ^{2} \theta d \theta
\end{array}
$$$$
\int \frac{d x}{\left(x^{2}+4\right)^{2}}=\frac{1}{8} \int\left[\frac{1+\cos 2 \theta}{2}\right] d \theta=\frac{1}{16}\left[\theta+\frac{1}{2} \operatorname{sen} 2 \theta\right]
$$

$$
\text { Como } \tan \theta=\frac{x}{2}
$$

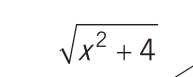

2

$$
\left\{\begin{array}{l}
\theta=\arctan \frac{x}{2} \\
\operatorname{sen} 2 \theta=2 \operatorname{sen} \theta \cos \theta=2 \frac{x}{\sqrt{x^{2}+4}} \cdot \frac{2}{\sqrt{x^{2}+4}}=\frac{4 x}{x^{2}+4} \\
\int \frac{d x}{\left(x^{2}+4\right)^{2}}=\frac{1}{16}\left[\arctan \frac{x}{2}+\frac{1}{2} \cdot \frac{4 x}{x^{2}+4}\right]
\end{array}\right.
$$

Finalmente:

$$
\int \frac{x^{3}+4 x+2}{\left(x^{2}+4\right)^{2}} d x=\frac{1}{2} \ln \left(x^{2}+4\right)+\frac{1}{8} \arctan \frac{x}{2}+\frac{x}{4\left(x^{2}+4\right)}+C
$$




\section{INTEGRACIÓN DE CIERTAS FRACCIONES IRRACIONALES}

5.1 INTEGRALES DEL TIPO $\int \boldsymbol{P}\left[(\boldsymbol{a}+\boldsymbol{b})^{m_{1} / m_{1}},(\boldsymbol{a x}+\boldsymbol{b})^{m_{2} / n_{2}}, \ldots\right] d x$

Se resuelven efectuando el cambio de variable:

$a x+b=z^{n}$, en donde " $\mathrm{n}$ " es el mínimo común múltiplo de $n_{1}, n_{2}, \ldots$

Ejemplo 75:

$$
\int \frac{\sqrt{x+1}+2}{(x+1)^{2}-\sqrt{x+1}} d x
$$

Consideremos:

$$
x+1=z^{2} \rightarrow d x=2 z d z
$$

Entonces:

$$
\int \frac{\sqrt{x+1}+2}{(x+1)^{2}-\sqrt{x+1}} d x=\int \frac{z+2}{z^{4}-z}(2 z d z)=2 \int \frac{z+2}{z^{3}-1} d z
$$

Por fracciones parciales:

$$
\frac{z+2}{z^{3}-1}=\frac{1}{z-1}-\frac{1 z+1}{z^{2}+z+1}
$$

De donde:

$$
\begin{gathered}
\int \frac{\sqrt{x+1}+2}{(x+1)^{2}-\sqrt{x+1}} d x=2 \int\left[\frac{1}{z-1}-\frac{1}{2}\left\{\frac{2 z+1}{z^{2}+z+1}+\frac{1}{\left(z+\frac{1}{2}\right)^{2}+\left(\frac{\sqrt{3}}{2}\right)^{2}}\right\}\right] \\
\int \frac{\sqrt{x+1}+2}{(x+1)^{2}-\sqrt{x+1}} d x=2 \ln (z-1)-\ln \left(z^{2}+z+1\right)-\frac{1}{\sqrt{3} / 2} \arctan \frac{z+1 / 2}{\sqrt{3} / 2}+C \\
\int \frac{\sqrt{x+1}+2}{(x+1)^{2}-\sqrt{x+1}} d x=2 \ln (\sqrt{x+1}-1)-\ln (x+2+\sqrt{x+1})-\frac{2 \sqrt{3}}{3} \arctan \frac{2 \sqrt{x+1}+1}{\sqrt{3}}+C
\end{gathered}
$$




\subsection{INTEGRALES DEL TIPO $\int \frac{d x}{(x-\alpha)^{n} \sqrt{a x^{2}+b x+c}}$}

Se resuelven valiéndose de la sustitución:

$$
\frac{1}{x-\alpha}=u
$$

Ejemplo 76:

$$
\int \frac{d x}{x^{5} \sqrt{x^{2}-1}}
$$

Asumamos:

$$
u=\frac{1}{x} \Rightarrow x=\frac{1}{u} \Rightarrow d x=-\frac{d u}{u^{2}}
$$

Entonces:

$$
\int \frac{d x}{x^{5} \sqrt{x^{2}-1}}=\int \frac{-\frac{d u}{u^{2}}}{\frac{1}{u^{5}} \cdot \sqrt{\frac{1}{u^{2}}-1}}=-\int \frac{u^{4} d u}{\sqrt{1-u^{2}}}
$$

54 Hagamos:

$$
1-u^{2}=z^{2} \rightarrow u=\sqrt{1-z^{2}} \Rightarrow d u=\frac{1}{2} \frac{(-2 z) d z}{\sqrt{1-z^{2}}}=\frac{-z d z}{\sqrt{1-z^{2}}}
$$

De donde:

$$
\int \frac{d x}{x^{5} \sqrt{x^{2}-1}}=-\int \frac{\left(1-z^{2}\right)^{2}\left(\frac{-z d z}{\sqrt{1-z^{2}}}\right)}{z}=\int\left(1-z^{2}\right)^{3 / 2} d z
$$

Sea $z=\cos \theta \Rightarrow d z=-\operatorname{sen} \theta d \theta$

Con lo cual:

$$
\begin{gathered}
\int \frac{d x}{x^{5} \sqrt{x^{2}-1}}=\int\left(1-\cos ^{2} \theta\right)^{3 / 2}(-\operatorname{sen} \theta d \theta)=-\int \operatorname{sen}^{4} \theta d \theta \\
\int \frac{d x}{x^{5} \sqrt{x^{2}-1}}=-\int\left(\operatorname{sen}^{2} \theta\right)^{2} d \theta=-\int\left[\frac{1-\cos 2 \theta}{2}\right]^{2} d \theta=-\frac{1}{4} \int\left(1-2 \cos 2 \theta+\cos ^{2} 2 \theta\right) d \theta \\
\int \frac{d x}{x^{5} \sqrt{x^{2}-1}}=-\frac{1}{4}\left[\theta-\operatorname{sen} 2 \theta+\int\left(\frac{1-\cos 4 \theta}{2}\right) d \theta\right]
\end{gathered}
$$




$$
\begin{gathered}
\int \frac{d x}{x^{5} \sqrt{x^{2}-1}}=-\frac{1}{4} \theta+\frac{1}{4} \operatorname{sen} 2 \theta-\frac{1}{8} \theta-\frac{1}{32} \operatorname{sen} 4 \theta+C \\
\int \frac{d x}{x^{5} \sqrt{x^{2}-1}}=-\frac{3}{8} \theta+\frac{1}{4} \operatorname{sen} 2 \theta-\frac{1}{32} \operatorname{sen} 4 \theta+C
\end{gathered}
$$

A partir de $\left\{\begin{array}{l}\cos \theta=z \\ z^{2}=1-u^{2} \rightarrow \cos \theta=z=\sqrt{1-u^{2}}=\sqrt{1-\frac{1}{x^{2}}}=\frac{1}{x} \sqrt{x^{2}-1} \\ u=\frac{1}{x}\end{array}\right.$

y del gráfico

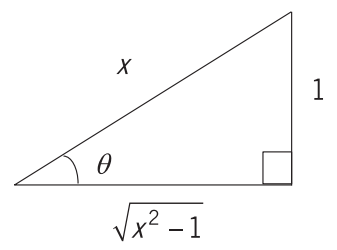

se tiene que:

$$
\begin{gathered}
\theta=\operatorname{arcsen} \frac{1}{x} \\
\operatorname{sen} 2 \theta=2 \operatorname{sen} \theta \cos \theta=2\left(\frac{1}{x}\right)\left(\frac{\sqrt{x^{2}-1}}{x}\right)=\frac{2 \sqrt{x^{2}-1}}{x^{2}} \\
\cos 2 \theta=1-2 \operatorname{sen}^{2} \theta=1-2\left(\frac{1}{x}\right)^{2}=\frac{x^{2}-2}{x^{2}} \\
\operatorname{sen} 4 \theta=2 \operatorname{sen} 2 \theta \cos 2 \theta=2\left(\frac{2 \sqrt{x^{2}-1}}{x^{2}}\right)\left(\frac{x^{2}-2}{x^{2}}\right)=\frac{4\left(x^{2}-2\right) \sqrt{x^{2}-1}}{x^{4}}
\end{gathered}
$$

Finalmente:

$$
\begin{aligned}
& \int \frac{d x}{x^{5} \sqrt{x^{2}-1}}=-\frac{3}{8} \operatorname{arcsen} \frac{1}{x}+\frac{1}{4}\left(\frac{2 \sqrt{x^{2}-1}}{x^{2}}\right)-\frac{1}{32}\left(\frac{4\left(x^{2}-2\right) \sqrt{x^{2}-1}}{x^{4}}\right)+C \\
& \int \frac{d x}{x^{5} \sqrt{x^{2}-1}}=-\frac{3}{8} \operatorname{arcsen} \frac{1}{x}+\left(\frac{1}{4 x^{4}}+\frac{3}{8 x^{2}}\right) \sqrt{x^{2}-1}+C
\end{aligned}
$$



V

INTEGRAL DEFINIDA

\section{INTEGRAL DEFINIDA COMO LÍMITE DE UNA SUMA}

Sea $y=f_{(x)}$ una función continua definida en el intervalo $a \leq x \leq b$ (asúmase que $a=x_{0}$ y $b=x_{n}$ ).

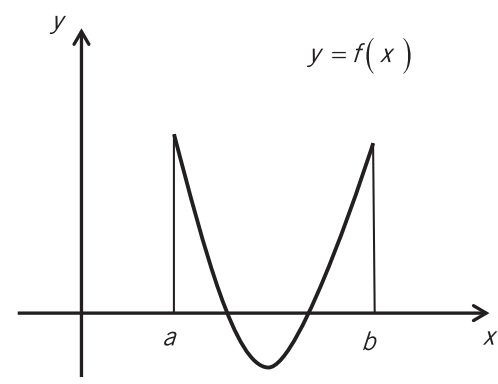

Consideremos que dicho intervalo está dividido en $n$ subintervalos $\left(\Delta x_{0}, \Delta x_{1}, \ldots, \Delta x_{n-1}\right)$ mediante los puntos $x_{1}, x_{2}, \ldots, x_{n-1}$.

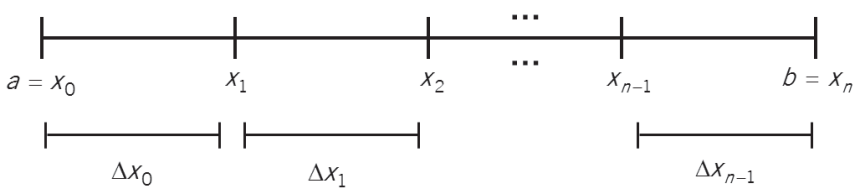


Escojamos $\varepsilon_{0}, \varepsilon_{1}, \ldots, \varepsilon_{\mathrm{n}-1}$, tal que:

$$
\begin{gathered}
a=x_{0} \leq \varepsilon_{0} \leq x_{1} \\
x_{1} \leq \varepsilon_{1} \leq x_{2} \\
\cdots \\
x_{n-1} \leq \varepsilon_{n-1} \leq x_{n}=b
\end{gathered}
$$

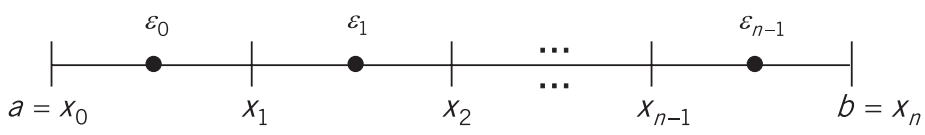

La suma de la forma

$$
f_{\left(\varepsilon_{0}\right)} \cdot \Delta x_{0}+f_{\left(\varepsilon_{1}\right)} \cdot \Delta x_{1}+\ldots+f_{\left(\varepsilon_{n-1}\right)} \cdot \Delta x_{n-1}
$$

recibe el nombre de suma integral de la función $f$ en $[a, b]$.

Dicha suma integral corresponde a la suma algebraica de las áreas de los rectángulos mostrados a continuación:

\section{8}

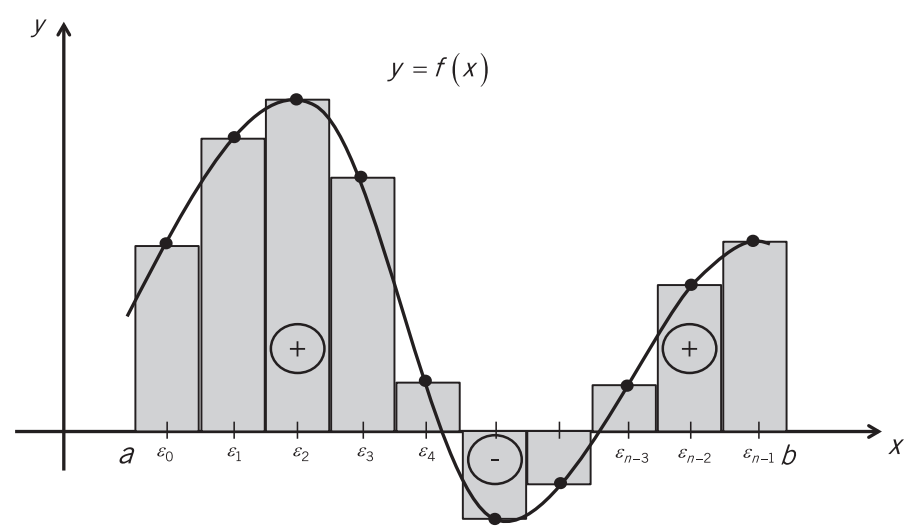

La suma integral de $f$ en $[a, b]$ se puede escribir como:

$$
S_{n}=\sum_{i=0}^{n-1} f_{\left(\varepsilon_{i}\right)} \cdot \Delta x_{i}
$$

Definición: la integral definida de la función $f_{(x)}$ entre los límites $x=a$ y $x=b$ es:

$$
\lim _{n \rightarrow \infty} S_{n}=\lim _{n \rightarrow \infty} \sum_{i=0}^{n-1} f_{\left(\varepsilon_{i}\right)} \cdot \Delta x_{i}
$$
Por convención, se empleará el símbolo $\int_{a}^{b} f_{(x)} d x$ para representar a la integral definida de $f_{(x)}$ con
respecto a $x$, desde $x=a$ hasta $x=b$. 
Es decir:

$$
\int_{a}^{b} f_{(x)} d x=\lim _{n \rightarrow \infty} \sum_{i=0}^{n-1} f_{\left(\varepsilon_{i}\right)} \cdot \Delta x_{i}
$$

\section{Ejemplo 77:}

Considere la función constante $y=f_{(x)}=c$ y evalúe $\int_{a}^{b} f_{(x)} d x$.

Dividamos el intervalo de $[a, b]$ en subintervalos de i) igual longitud y ii) diferente longitud, para evaluar $\int_{a}^{b} c d x$

i). Intervalos de igual longitud:

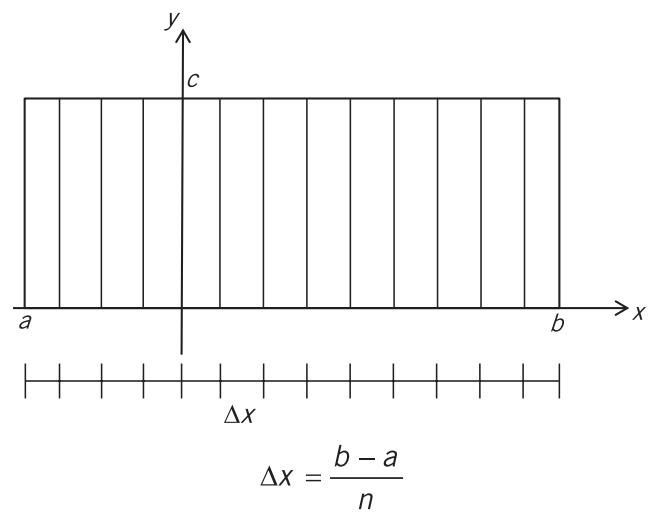

$\varepsilon_{i}$ puede ser cualquier valor tal que $x_{i} \leq \varepsilon_{i} \leq x_{i+1}$, ya que $f_{\left(\varepsilon_{i}\right)}=c \forall i \quad 0 \leq i \leq n-1$.

$$
\begin{gathered}
\int_{a}^{b} c d x=\lim _{n \rightarrow \infty} \sum_{i=0}^{n-1} f_{\left(\varepsilon_{i}\right)} \Delta x_{i}=\lim _{n \rightarrow \infty} \sum_{i=0}^{n-1} f_{\left(\varepsilon_{i}\right)}\left(\frac{b-a}{n}\right) \\
\int_{a}^{b} c d x=(b-a) \lim _{n \rightarrow \infty} \frac{1}{n}\left[f_{\left(\varepsilon_{0}\right)}+f_{\left(\varepsilon_{1}\right)}+\ldots+f_{\left(\varepsilon_{n-1}\right)}\right] \\
\int_{a}^{b} c d x=(b-a) \lim _{n \rightarrow \infty} \frac{1}{n}[c+c+\ldots+c] \\
\int_{a}^{b} c d x=(b-a) \lim _{n \rightarrow \infty} \frac{1}{n}[n c]=(b-a) \lim _{n \rightarrow \infty} c=c(b-a)
\end{gathered}
$$


ii). Intervalos de diferente longitud:

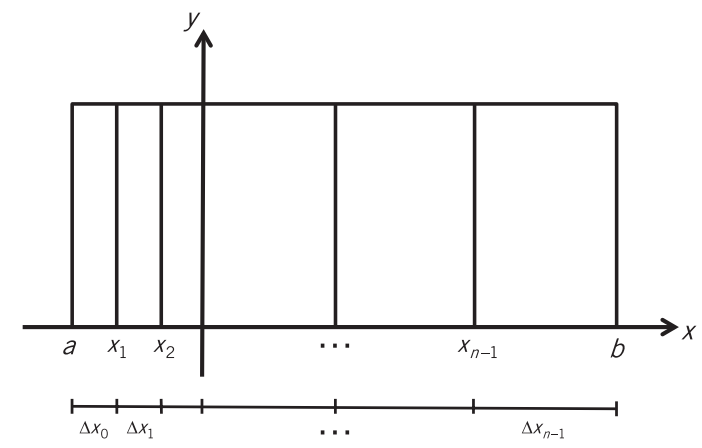

$$
\left.\begin{array}{c}
\Delta x_{0}=x_{1}-a \\
\Delta x_{1}=x_{2}-x_{1} \\
\downarrow \\
\begin{array}{c}
\Delta x_{n-2}=x_{n-1}-x_{n-2} \\
\Delta x_{n-1}=b-x_{n-1}
\end{array}
\end{array}\right\} \begin{aligned}
& \text { Sumándolos } \\
& \sum_{i=0}^{n-1} \Delta x_{i}=b-a
\end{aligned}
$$

60

$$
\begin{gathered}
\int_{a}^{b} c d x=\lim _{n \rightarrow \infty} \sum_{i=0}^{n-1} f_{\left(\varepsilon_{i}\right)} \cdot \Delta x_{i} \\
\int_{a}^{b} c d x=\lim _{n \rightarrow \infty}\left[f_{\left(\varepsilon_{0}\right)} \cdot \Delta x_{0}+f_{\left(\varepsilon_{1}\right)} \cdot \Delta x_{1}+\ldots+f_{\left(\varepsilon_{n-1}\right)} \cdot \Delta x_{n-1}\right] \\
\text { Como } f_{\left(\varepsilon_{i}\right)}=c \quad \forall i \quad 0 \leq i \leq n-1 \\
\int_{a}^{b} c d x=\lim _{n \rightarrow \infty}\left[c \sum_{i=0}^{n-1} \Delta x_{i}\right]=c \lim _{n \rightarrow \infty} \sum_{i=0}^{n-1} \Delta x_{i}=c(b-a)
\end{gathered}
$$

\section{Ejemplo 78:}

Sea $y=f_{(x)}=x$ tal que $x \in[2,6]$. Dividir el intervalo $[2,6]$ en $n$ subintervalos de igual longitud $y$ $\operatorname{considerar} \varepsilon_{i}$ en el extremo izquierdo del respectivo subintervalo para evaluar $\int_{2}^{6} x d x$. 


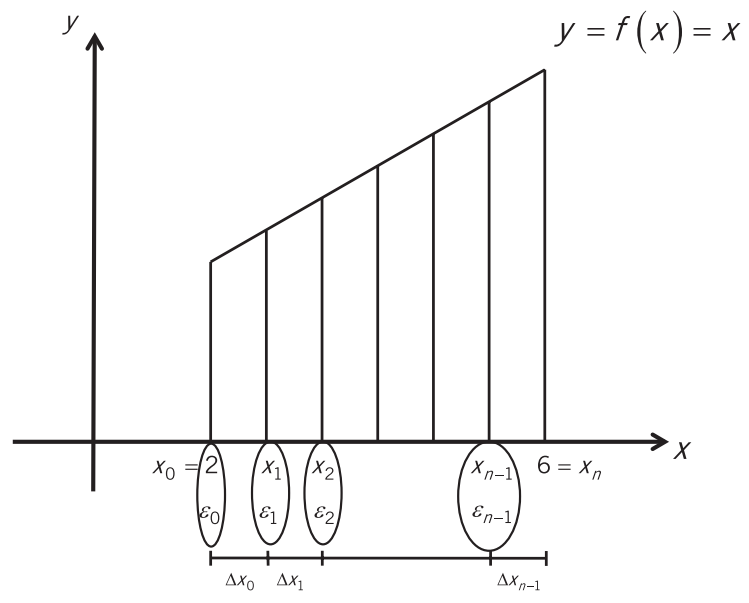

Del gráfico:

$$
\begin{aligned}
\Delta x_{0}=\Delta x_{1}= & \ldots=\Delta x_{n-1}=\frac{6-2}{n}=\frac{4}{n} \\
\varepsilon_{0}= & 2 \\
\varepsilon_{1}= & 2+\Delta x \\
\varepsilon_{2}= & 2+2 \Delta x \\
& \cdots \\
\varepsilon_{n-1} & =2+(n-1) \Delta x
\end{aligned}
$$

Se tiene que:

$$
\begin{aligned}
& \int_{2}^{6} x d x=\lim _{n \rightarrow \infty} \sum_{i=0}^{n-1} f_{\left(\varepsilon_{i}\right)} \cdot \Delta x_{i}=\lim _{n \rightarrow \infty} \sum_{i=0}^{n-1}\left(f_{\left(\varepsilon_{i}\right)} \cdot \frac{4}{n}\right) \\
& \int_{2}^{6} x d x=4 \lim _{n \rightarrow \infty}\left[\frac{1}{n}\left(f_{\left(\varepsilon_{0}\right)}+f_{\left(\varepsilon_{1}\right)}+\ldots+f_{\left(\varepsilon_{n-1}\right)}\right)\right] \\
& \int_{2}^{6} x d x=4 \lim _{n \rightarrow \infty}\left[\frac{1}{n}\{2+(2+\Delta x)+\ldots+(2+(n-1) \Delta x)\}\right] \\
& \int_{2}^{6} x d x=4 \lim _{n \rightarrow \infty} \frac{1}{n}\left[2(n)+\Delta x \cdot \frac{(n-1) n}{2}\right] \\
& \int_{2}^{6} x d x=4 \lim _{n \rightarrow \infty} \frac{1}{n}\left[2 n+\frac{(n-1) n}{2} \cdot \frac{4}{n}\right]
\end{aligned}
$$




$$
\begin{aligned}
& \int_{2}^{6} x d x=4 \lim _{n \rightarrow \infty}\left[2+\frac{2(n-1)}{n}\right] \\
& \int_{2}^{6} x d x=4 \lim _{n \rightarrow \infty}\left[2+2-\frac{2}{n}\right]=4(4)=16
\end{aligned}
$$

Ejemplo 79:

Comprobar que $\int_{1}^{4} x^{2} d x=21$

Consideremos subintervalos de igual longitud y escojamos los puntos medios de cada intervalo como los respectivos $\varepsilon_{i}$.

62

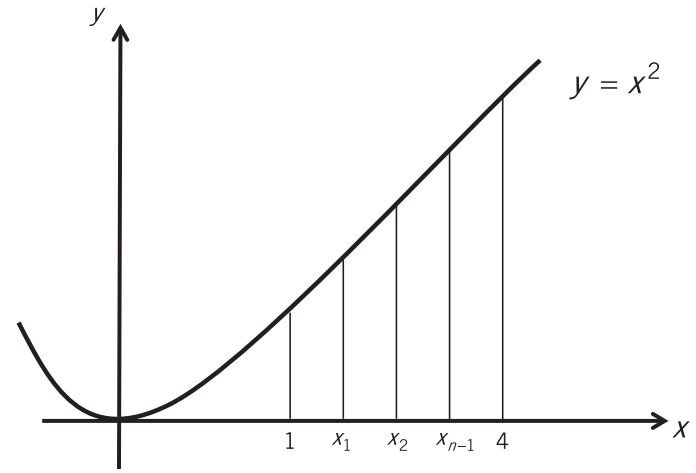

Consideremos:

$$
\begin{aligned}
& \begin{array}{llllllllll}
\bullet & \bullet & & \bullet & & & & & \bullet & \bullet \\
\hline \varepsilon_{0} & x_{1} & \varepsilon_{1} & x_{2} & x_{n-2} & \varepsilon_{n-2} & x_{n-1} & \varepsilon_{n-1} & x_{n}=4
\end{array} \\
& \Delta x=\frac{4-1}{n}=\frac{3}{n} \\
& \varepsilon_{0}=x_{0}+\frac{1}{2} \Delta x=1+\frac{1}{2}\left(\frac{3}{n}\right)=1+\frac{3}{2 n}=1+1\left(\frac{3}{2 n}\right)
\end{aligned}
$$

Nótese que $1=2(0)+1$

$$
\varepsilon_{1}=x_{0}+\frac{3}{2} \Delta x=1+\frac{3}{2}\left(\frac{3}{n}\right)=1+\frac{9}{2 n}=1+3\left(\frac{3}{2 n}\right)
$$

Nótese que $3=2(1)+1$ 


$$
\varepsilon_{n-1}=x_{0}+\frac{2(n-1)+1}{2}\left(\frac{3}{n}\right)=1+\frac{2 n-1}{2}\left(\frac{3}{n}\right)=1+(2 n-1)\left(\frac{3}{2 n}\right)
$$

Nótese que $2 n-1=2(n-1)+1$.

De donde:

$$
\begin{gathered}
\int_{1}^{4} x^{2} d x=\lim _{n \rightarrow \infty} \sum_{i=0}^{n-1} f_{\left(\varepsilon_{i}\right)} \cdot \Delta x_{i}=\lim _{n \rightarrow \infty} 3 \sum_{i=0}^{n-1} \frac{f_{\left(\varepsilon_{i}\right)}}{n} \\
\int_{1}^{4} x^{2} d x=3 \lim _{n \rightarrow \infty} \frac{1}{n}\left[\left(1+1\left(\frac{3}{2 n}\right)\right)^{2}+\left(1+3\left(\frac{3}{2 n}\right)\right)^{2}+\ldots+\left(1+(2 n-1)\left(\frac{3}{2 n}\right)\right)^{2}\right] \\
\int_{1}^{4} x^{2} d x=3 \lim _{n \rightarrow \infty} \frac{1}{n}\left[1+2\left(\frac{3}{2 n}\right)+\left(\frac{3}{2 n}\right)^{2}+\right. \\
1+2(3)\left(\frac{3}{2 n}\right)+9\left(\frac{3}{2 n}\right)^{2}+ \\
+\ldots+ \\
\left.1+2(2 n-1)\left(\frac{3}{2 n}\right)+(2 n-1)^{2}\left(\frac{3}{2 n}\right)^{2}\right]
\end{gathered}
$$

Téngase en cuenta que:

$$
\begin{aligned}
& \sum_{i=1}^{n}(2 i-1)=n^{2} \\
& \sum_{i=1}^{n}(2 i-1)^{2}=\frac{4 n^{3}-n}{3}
\end{aligned}
$$

\section{Entonces:}

$$
\begin{aligned}
& \int_{1}^{4} x^{2} d x=3 \lim _{n \rightarrow \infty} \frac{1}{n}\left[n(1)+2\left(\frac{3}{2 n}\right)\left[n^{2}\right]+\left(\frac{3}{2 n}\right)^{2}\left(\frac{4 n^{3}-n}{3}\right)\right] \\
& \int_{1}^{4} x^{2} d x=3 \lim _{n \rightarrow \infty}\left[1+3+\frac{9}{4}\left(\frac{1}{3}\right)\left[4-\frac{1}{n^{2}}\right]\right] \\
& \int_{1}^{4} x^{2} d x=3 \lim _{n \rightarrow \infty}\left[7-\frac{3}{4 n^{2}}\right]=3(7-0)=21
\end{aligned}
$$




\section{PROPIEDADES DE LA INTEGRAL DEFINIDA}

Si $f_{(x)}$ y $g_{(x)}$ son funciones continuas en el intervalo de integración $a \leq x \leq b$, se cumple que:
a) $\int_{a}^{a} f_{(x)} d x=0$
b) $\int_{a}^{b} f_{(x)} d x=-\int_{b}^{a} f_{(x)} d x$
c) $\int_{a}^{b} c f_{(x)} d x=c \int_{a}^{b} f_{(x)} d x$
(c: constante)
d) $\int_{a}^{b}\left[f_{(x)}+g_{(x)}\right] d x=\int_{a}^{b} f_{(x)} d x+\int_{a}^{b} g_{(x)} d x$
e) $\int_{a}^{c} f_{(x)} d x+\int_{c}^{b} f_{(x)} d x=\int_{a}^{b} f_{(x)} d x . \quad(a<c<b)$

\section{TEOREMA FUNDAMENTAL DEL CÁLCULO}

64 El teorema fundamental del cálculo, Ilamado Regla de Newton-Leibniz o Regla de Barrow, permite calcular el valor de una integral definida a partir de la antiderivada. Consta de dos partes que serán enunciadas y demostradas a continuación.

Parte 1: Si $f$ es una función continua en $[a, b]$, entonces la función $F_{(x)}=\int_{a}^{x} f_{(t)} d t$ tiene una derivada en cada punto $x$ de $[a, b]$ y se cumple que:

$$
\frac{d F}{d x}=\frac{d}{d x} \int_{a}^{x} f_{(t)} d t=f_{(x)}
$$

\section{Demostración:}

Se sabe que la derivada de la función $F_{(x)}$ es:

$$
\begin{aligned}
& \frac{d F}{d x}=\lim _{h \rightarrow 0} \frac{F_{(x+h)}-F_{(x)}}{h} \\
& \frac{d F}{d x}=\lim _{h \rightarrow 0} \frac{\int_{a}^{x+h} f_{(t)} d t-\int_{a}^{x} f_{(t)} d t}{h} \\
& \frac{d F}{d x}=\lim _{h \rightarrow 0} \frac{\int_{x}^{x+h} f_{(t)} d t}{h} \quad \text { (a partir de la propiedad 2e) }
\end{aligned}
$$




$$
\frac{d F}{d x}=\lim _{h \rightarrow 0}\{\underbrace{\left\{\frac{1}{h} \int_{x}^{x+h} f_{(t)} d t\right\}}_{\begin{array}{c}
\text { Valor promedio de la } \\
\text { función } f \text { desde } x \\
\text { hasta } x+h
\end{array}}
$$

Según el Teorema del valor medio para integrales definidas, la expresión (1) se puede escribir como:

$$
\frac{d F}{d x}=\lim _{h \rightarrow 0} f_{(c)}, \text { en donde } c \in[x, x+h]
$$

En la medida en que $h$ tienda a 0 , el valor de c se aproxima al valor de $x$ y como $f$ es continua, se cumple que $f_{(c)}$ tiende a $f_{(x)}$. Con lo cual:

$$
\frac{d F}{d x}=\lim _{h \rightarrow 0} f_{(c)}=f_{(x)}
$$

Parte 2: Si f es una función continua en cada punto de $[a, b]$, y si $F_{(x)}$ es una antiderivada de $f_{(x)}$ en $[a, b]$, se cumple que:

$$
\int_{a}^{b} f_{(x)} d x=F_{(b)}-F_{(a)}
$$

\section{Demostración:}

La parte 1 del teorema fundamental indica que:

$$
\frac{d F}{d x}=\frac{d}{d x} \int_{a}^{x} f_{(t)} d t
$$

Integrando ambos miembros se obtiene:

$$
F_{(x)}=\int_{a}^{x} f_{(t)} d t \text { es una antiderivada cualquiera de } f_{(x)} \text {. }
$$

Por lo tanto, se puede afirmar que:

$$
\int_{a}^{x} f_{(t)} d t=F_{(x)}+C
$$

Escribamos:

$$
\int_{a}^{b} f_{(x)} d x=\int_{a}^{b} f_{(x)} d x-0=\int_{a}^{b} f_{(x)} d x-\int_{a}^{a} f_{(x)} d x
$$

Con lo cual se llega a:

$$
\int_{a}^{b} f_{(x)} d x=F_{(b)}-F_{(a)}
$$


Ejemplo 80:

Calcular

$$
\begin{aligned}
& \int_{1}^{4} x^{2} d x \\
& \int_{1}^{4} x^{2} d x=\left.\frac{x^{3}}{3}\right|_{1} ^{4}=\frac{1}{3}\left(4^{3}-1^{3}\right)=\frac{63}{3}=21
\end{aligned}
$$

Ejemplo 81:

Calcular

$$
\begin{aligned}
& \int_{0}^{1} x^{3} \sqrt{x^{4}+3} d x \\
& \int_{0}^{1} x^{3} \sqrt{x^{4}+3} d x=\int_{0}^{1}\left(x^{4}+3\right)^{1 / 2}\left(x^{3} d x\right)=\frac{1}{4} \int_{0}^{1}\left(x^{4}+3\right)^{1 / 2}\left(4 x^{3} d x\right) \\
& \int_{0}^{1} x^{3} \sqrt{x^{4}+3} d x=\left.\frac{1}{4} \cdot \frac{\left(x^{4}+3\right)^{3 / 2}}{3 / 2}\right|_{0} ^{1}=\frac{1}{6}\left[4^{3 / 2}-3^{3 / 2}\right]
\end{aligned}
$$

66 De donde:

$$
\int_{0}^{1} x^{3} \sqrt{x^{4}+3} d x=\frac{8-\sqrt{27}}{6}
$$

Ejemplo 82:

Calcular

$$
\int_{e^{2}}^{e^{4}} \ln u d u
$$

Se sabe que $\int \ln u d u=u \ln u-u+C$

Entonces:

$$
\begin{gathered}
\int_{e^{2}}^{e^{4}} \ln u d u=u \ln u-\left.u\right|_{e^{2}} ^{e^{4}}=\left(e^{4} \ln e^{4}-e^{4}\right)-\left(e^{2} \ln e^{2}-e^{2}\right) \\
\int_{e^{2}}^{e^{4}} \ln u d u=\left(4 e^{4}-e^{4}\right)-\left(2 e^{2}-e^{2}\right)=3 e^{4}-e^{2}
\end{gathered}
$$

Ejemplo 83:

Calcular

$$
\int_{\frac{\pi}{2}}^{\pi} \frac{4 \cos x}{(\operatorname{sen} x+1)^{2}} d x
$$




$$
\begin{aligned}
& \int_{\frac{\pi}{2}}^{\pi} \frac{4 \cos x}{(\operatorname{sen} x+1)^{2}} d x=4 \int_{\frac{\pi}{2}}^{\pi}(\operatorname{sen} x+1)^{-2}(\cos x d x)=\left.4 \frac{(\operatorname{sen} x+1)^{-1}}{-1}\right|_{\pi / 2} ^{\pi} \\
& \int_{\frac{\pi}{2}}^{\pi} \frac{4 \cos x}{(\operatorname{sen} x+1)^{2}} d x=-4\left[\frac{1}{\operatorname{sen} \pi+1}-\frac{1}{\operatorname{sen} \frac{\pi}{2}+1}\right]=-4\left[1-\frac{1}{2}\right]=-2
\end{aligned}
$$

\section{Ejemplo 84:}

Calcular $\int_{\ln 2}^{\ln 4} \frac{e^{x}-e^{-x}}{e^{x}+e^{-x}} d x$

Sea $u=e^{x}+e^{-x} \rightarrow d u=\left[e^{x}+e^{-x}(-1)\right] d x=\left[e^{x}-e^{-x}\right] d x$

Si $x=\ln 2 \rightarrow u=e^{\ln 2}+e^{-\ln 2}=e^{\ln 2}+e^{\ln 1 / 2}=2+1 / 2=5 / 2$

$$
x=\ln 4 \rightarrow u=e^{\ln 4}+e^{-\ln 4}=e^{\ln 4}+e^{\ln 1 / 4}=4+1 / 4=17 / 4
$$

\section{Entonces:}

$$
\int_{\ln 2}^{\ln 4} \frac{e^{x}-e^{-x}}{e^{x}+e^{-x}} d x=\int_{\frac{5}{2}}^{\frac{17}{4}} \frac{d u}{u}=\left.\ln u\right|_{5 / 2} ^{17 / 4}=\ln \frac{17 / 4}{5 / 2}=\ln \frac{17}{10}
$$





\section{VI \\ INTEGRALES IMPROPIAS}

Sea $y=f_{(x)}$ una función continua definida en el intervalo $a \leq x \leq b$. Como se explicó en el capítulo V, la integral definida es $\int_{a}^{b} f_{(x)} d x$.

\section{INTEGRAL IMPROPIA}

Se denomina integral impropia a $\int_{a}^{b} f_{(x)} d x$ en cualquiera de los dos casos siguientes:

i) Por lo menos uno de los dos límites de integración ( $a$ y/o b) no es finito.

ii) El integrando $f_{(x)}$ tiene un punto de discontinuidad, por lo menos, en el intervalo $a \leq x \leq b$.

\subsection{INTEGRAL IMPROPIA CON LÍMITE DE INTEGRACIÓN NO FINITO}

La integral $\int_{a}^{b} f_{(x)} d x$ es impropia en cualquiera de los tres casos siguientes, y se indica el modo de calcularla.

i) $\int_{-\infty}^{b} f_{(x)} d x=\lim _{a \rightarrow-\infty} \int_{a}^{b} f_{(x)} d x$.

ii) $\int_{a}^{\infty} f_{(x)} d x=\lim _{b \rightarrow \infty} \int_{a}^{b} f_{(x)} d x$. 
iii) $\int_{-\infty}^{\infty} f_{(x)} d x=\lim _{a \rightarrow-\infty} \int_{a}^{c} f_{(x)} d x+\lim _{b \rightarrow \infty} \int_{c}^{b} f_{(x)} d x \quad(c \in R)$.

Si al calcular estas integrales impropias el resultado es finito, se afirma que la integral es convergente; en caso contrario, la integral es divergente.

Ejemplo 85:

$$
\begin{gathered}
I=\int_{1}^{\infty} \frac{d x}{x^{2}} \\
I=\lim _{b \rightarrow \infty} \int_{1}^{b} \frac{d x}{x^{2}}=\left.\lim _{b \rightarrow \infty} \frac{-1}{x}\right|_{1} ^{b} \\
I=\lim _{b \rightarrow \infty}\left(-\frac{1}{b}+1\right)=1-0=1
\end{gathered}
$$

La integral impropia $\int_{1}^{\infty} \frac{d x}{x^{2}}$ converge a 1 .

Ejemplo 86:

70

$$
I=\int_{-\infty}^{1} e^{x} d x
$$

$$
\begin{gathered}
I=\lim _{a \rightarrow-\infty} \int_{a}^{1} e^{x} d x=\left.\lim _{a \rightarrow-\infty} e^{x}\right|_{a} ^{1} \\
I=\lim _{a \rightarrow-\infty}\left(e^{1}-e^{a}\right) \\
I=\lim _{a \rightarrow-\infty} e-\lim _{a \rightarrow-\infty} e^{a} \\
I=e-0=e
\end{gathered}
$$

La integral $\int_{-\infty}^{1} e^{x} d x$ converge a $e$.

Ejemplo 87:

$$
\begin{gathered}
I=\int_{-\infty}^{\infty} \frac{d x}{1+4 x^{2}} \\
I=\lim _{a \rightarrow-\infty} \int_{a}^{c} \frac{d x}{1+4 x^{2}}+\lim _{b \rightarrow \infty} \int_{c}^{b} \frac{d x}{1+4 x^{2}} \\
I=\lim _{a \rightarrow-\infty} \frac{1}{2} \int_{a}^{c} \frac{2 d x}{1+(2 x)^{2}}+\lim _{b \rightarrow \infty} \frac{1}{2} \int_{c}^{b} \frac{2 d x}{1+(2 x)^{2}}
\end{gathered}
$$




$$
\begin{gathered}
I=\frac{1}{2}\left[\left.\lim _{a \rightarrow-\infty} \frac{1}{1} \arctan \frac{2 x}{1}\right|_{a} ^{c}+\left.\lim _{b \rightarrow \infty} \frac{1}{1} \arctan \frac{2 x}{1}\right|_{c} ^{b}\right] \\
I=\frac{1}{2}\left[\lim _{a \rightarrow-\infty}(\arctan 2 c-\arctan 2 a)+\lim _{b \rightarrow \infty}(\arctan 2 b-\arctan 2 c)\right] \\
I=\frac{1}{2}\left\{\left[\arctan 2 c-\left(-\frac{\pi}{2}\right)\right]+\left[\left(\frac{\pi}{2}\right)-\arctan 2 c\right]\right\} \\
I=\frac{1}{2}\left\{\frac{\pi}{2}+\frac{\pi}{2}\right\}=\frac{\pi}{2}
\end{gathered}
$$

La integral $\int_{-\infty}^{\infty} \frac{d x}{1+4 x^{2}}$ converge a $\frac{\pi}{2}$.

Ejemplo 88:

$$
I=\int_{-\infty}^{0} x e^{x} d x
$$

Calculemos la integral indefinida $\int x e^{x} d x$ por el método de integración por partes:

$$
\begin{array}{cc}
u=x & v=e^{x} \\
\downarrow & \uparrow \\
d u=d x & d v=e^{x} d x
\end{array}
$$

Entonces:

$$
\int x e^{x} d x=x e^{x}-\int e^{x} d x=x e^{x}-e^{x}+C
$$

De donde:

$$
\begin{aligned}
& I=\lim _{a \rightarrow-\infty} \int_{a}^{0} x e^{x} d x=\left.\lim _{a \rightarrow-\infty} e^{x}(x-1)\right|_{a} ^{0} \\
& I=\lim _{a \rightarrow-\infty}\left[e^{0}(0-1)-e^{a}(a-1)\right] \\
& I=-1-\underbrace{\lim _{a \rightarrow-\infty} e^{a}(a-1)}_{I_{1}}
\end{aligned}
$$

Calculemos $I_{1}=\lim _{a \rightarrow-\infty} e^{a}(a-1)$

Hagamos $d=-a$ 
Si $a \rightarrow-\infty$, entonces $d \rightarrow \infty$.

Entonces:

$$
\begin{gathered}
l_{1}=\lim _{a \rightarrow-\infty} e^{a}(a-1)=\lim _{d \rightarrow \infty} e^{-d}(-d-1) \\
I_{1}=-\lim _{d \rightarrow \infty} \frac{d+1}{e^{d}}
\end{gathered}
$$

Según la regla de L'Hopital:

$$
I_{1}=-\lim _{d \rightarrow \infty} \frac{1+0}{e^{d}}=-\lim _{d \rightarrow \infty} \frac{1}{e^{d}}=0
$$

Finalmente:

$$
I=-1-I_{1}=-1-0=-1
$$

Ejemplo 89:

$$
\begin{aligned}
& I=\int_{-\infty}^{0} \frac{d x}{x-3} \\
& I=\lim _{a \rightarrow-\infty} \int_{a}^{0} \frac{d x}{x-3}
\end{aligned}
$$

$$
\begin{aligned}
& I=\left.\lim _{a \rightarrow-\infty} \ln [x-3]\right|_{a} ^{0} \\
& I=\lim _{a \rightarrow-\infty}\{\ln |0-3|-\ln |a-3|\} \\
& I=\ln 3-\underbrace{\lim \ln |a-3|}_{\infty}
\end{aligned}
$$

Se desprende que la integral es divergente.

Ejemplo 90:

$$
I=\int_{1}^{\infty} \ln x d x
$$

Como $\int \ln x d x=x(\ln x-1)+C$, se tendrá que:

$$
\begin{aligned}
& I=\lim _{b \rightarrow \infty} \int_{1}^{b} \ln x d x=\left.\lim _{b \rightarrow \infty} x(\ln x-1)\right|_{1} ^{b} \\
& I=\lim _{b \rightarrow \infty}\{b(\ln b-1)-1(\ln 1-1)\} \\
& I=\underbrace{\left(\lim _{b \rightarrow \infty} b\right)}_{\infty} \underbrace{\left\{\lim _{b \rightarrow \infty}(\ln b-1)\right\}}_{\infty}+1
\end{aligned}
$$


Se deduce que la integral es divergente.

\subsection{INTEGRAL IMPROPIA CON INTEGRANDO NO CONTINUO}

La integral $\int_{a}^{b} f_{(x)} d x$ es impropia en cualquiera de los tres casos siguientes, y se indica el modo de calcularla:

i) Si $f_{(x)}$ es discontinua solo en $x=a$.

$$
\int_{a}^{b} f_{(x)} d x=\lim _{\varepsilon \rightarrow 0^{+}} \int_{a+\varepsilon}^{b} f_{(x)} d x
$$

ii) Si $f_{(x)}$ es discontinua solo en $x=b$.

$$
\int_{a}^{b} f_{(x)} d x=\lim _{\varepsilon \rightarrow 0^{+}} \int_{a}^{b-\varepsilon} f_{(x)} d x
$$

iii) Si $f_{(x)}$ es discontinua solo en $x=c .(c \in[a, b])$.

$$
\int_{a}^{b} f_{(x)} d x=\lim _{\varepsilon \rightarrow 0^{+}} \int_{a}^{c-\varepsilon} f_{(x)} d x+\lim _{\varepsilon^{\prime} \rightarrow 0^{+}} \int_{c+\varepsilon^{\prime}}^{b} f_{(x)} d x
$$

Si al calcular estas integrales impropias el resultado es finito, se afirma que la integral es convergente; de lo contrario, la integral es divergente.

Ejemplo 91:

$$
I=\int_{0}^{1 / 2} \frac{d x}{x(\ln x)^{2}}
$$

Como el integrando $\frac{1}{x(\ln x)^{2}}$ no es continuo en $x=0$ que pertenece al intervalo de integración $[0,1 / 2]$, se puede escribir que:

$$
\begin{aligned}
& I=\lim _{\varepsilon \rightarrow 0^{+}} \int_{0+\varepsilon}^{1 / 2} \frac{d x}{x(\ln x)^{2}} \\
& I=\lim _{\varepsilon \rightarrow 0^{+}} \int_{0+\varepsilon}^{1 / 2} \underbrace{(\ln x)^{-2}}_{u} \underbrace{\left.\frac{d x}{x}\right)}_{d u} \\
& I=\left.\lim _{\varepsilon \rightarrow 0^{+}} \frac{(\ln x)^{-2+1}}{-2+1}\right|_{0+\varepsilon} ^{1 / 2}
\end{aligned}
$$




$$
\begin{aligned}
& I=\lim _{\varepsilon \rightarrow 0^{+}}-\left.\frac{1}{\ln x}\right|_{0+\varepsilon} ^{1 / 2} \\
& I=\lim _{\varepsilon \rightarrow 0^{+}}\left[-\frac{1}{\ln 1 / 2}-\left(\frac{-1}{\ln \varepsilon}\right)\right]
\end{aligned}
$$

Como $\lim _{\varepsilon \rightarrow 0^{+}} \ln \varepsilon=-\infty$,

$$
\begin{aligned}
& I=-\frac{1}{\ln 1 / 2}-(0) \\
& I=+\frac{1}{\ln 2}
\end{aligned}
$$

\section{Ejemplo 92:}

74

$$
I=\int_{0}^{4} \frac{d x}{(x-2)^{2 / 3}}
$$

Como el integrando $\frac{1}{(x-2)^{2 / 3}}$ es discontinuo en $x=2$ que pertenece al intervalo de integración $[0,4]$, se tiene que:

$$
\begin{gathered}
I=\lim _{\varepsilon \rightarrow 0^{+}} \int_{0}^{2-\varepsilon} \frac{d x}{(x-2)^{2 / 3}}+\lim _{\varepsilon^{\prime} \rightarrow 0^{+}} \int_{2+\varepsilon^{\prime}}^{4} \frac{d x}{(x-2)^{2 / 3}} \\
I=\left.\lim _{\varepsilon \rightarrow 0^{+}} 3(x-2)^{1 / 3}\right|_{0} ^{2-\varepsilon}+\left.\lim _{\varepsilon^{\prime} \rightarrow 0^{+}} 3(x-2)^{1 / 3}\right|_{2+\varepsilon^{\prime}} ^{4} \\
I=3 \lim _{\varepsilon \rightarrow 0^{+}}\left[(-\varepsilon)^{1 / 3}-(-2)^{1 / 3}\right]+3 \lim _{\varepsilon^{\prime} \rightarrow 0^{+}}\left[2^{1 / 3}-\left(\varepsilon^{\prime}\right)^{1 / 3}\right] \\
I=3\left[0-(-2)^{1 / 3}\right]+3\left[2^{1 / 3}-0\right] \\
I=3(2)^{1 / 3}+3(2)^{1 / 3}=6 \sqrt[3]{2}
\end{gathered}
$$




\section{CRITERIOS DE CONVERGENCIA Y DIVERGENCIA}

Si no es posible evaluar una integral impropia en forma directa, se puede determinar la convergencia o divergencia de la integral mediante dos criterios: el criterio de comparación directa o el criterio de comparación de límites.

\subsection{CRITERIO DE COMPARACIÓN DIRECTA}

Si $f_{(x)}$ y $g_{(x)}$ son funciones continuas en $\left[a, \infty\left[\right.\right.$ tal que $0 \leq f_{(x)} \leq g_{(x)} \forall x>a$, entonces:

a) Si $\int_{a}^{\infty} g_{(x)} d x$ converge $\Rightarrow \int_{a}^{\infty} f_{(x)} d x$ converge.

b) Si $\int_{a}^{\infty} f_{(x)} d x$ diverge $\Rightarrow \int_{a}^{\infty} g_{(x)} d x$ diverge.

\section{Ejemplo 93:}

Analizar la convergencia de $\int_{1}^{\infty} \frac{\operatorname{sen}^{2} x}{x^{2}} d x$.

Se tiene que $-1 \leq \operatorname{sen} x \leq 1 \Rightarrow 0 \leq \operatorname{sen}^{2} x \leq 1$.

Como $x^{2}>0, \forall x \geq 1$, entonces $0 \leq \frac{\operatorname{sen}^{2} x}{x^{2}} \leq \frac{1}{x^{2}}$.

Según el ejemplo 85, $\int_{1}^{\infty} \frac{1}{x^{2}} d x$ converge al valor de 1. Por lo tanto $\int_{1}^{\infty} \frac{\operatorname{sen}^{2} x}{x^{2}} d x$ es convergente.

\section{Ejemplo 94:}

Demostrar que $\int_{0}^{\infty} e^{-x^{2}} d x$ es convergente.

Se tiene que $\int_{0}^{\infty} e^{-x^{2}} d x=\int_{0}^{1} e^{-x^{2}} d x+\int_{1}^{\infty} e^{-x^{2}} d x$

Averigüemos si ambas integrales son convergentes:

$$
\begin{gathered}
\text { Si } 0 \leq x \leq 1 \rightarrow 0 \leq x^{2} \leq 1 \rightarrow 0 \geq-x^{2} \geq-1 \Rightarrow e^{0} \geq e^{-x^{2}} \rightarrow 1 \geq e^{-x^{2}} \\
\qquad \int_{0}^{1} e^{-x^{2}} d x \leq \int_{0}^{1} 1 d x \\
\int_{0}^{1} e^{-x^{2}} d x \leq 1 \rightarrow \int_{0}^{1} e^{-x^{2}} d x \text { es convergente. }
\end{gathered}
$$


Si $\quad x \geq 1 \rightarrow x^{2} \geq x \rightarrow e^{x^{2}} \geq e^{x} \rightarrow \frac{1}{e^{x}} \geq \frac{1}{e^{x^{2}}}$

De donde $e^{-x} \geq e^{-x^{2}}>0$.

Calculemos:

$$
\begin{aligned}
& \int_{1}^{\infty} e^{-x} d x=\lim _{b \rightarrow \infty} \int_{1}^{b} e^{-x} d x=-\lim _{b \rightarrow \infty} \int_{1}^{b} e^{-x}(-d x) \\
& \int_{1}^{\infty} e^{-x} d x=-\left.\lim _{b \rightarrow \infty} e^{-x}\right|_{1} ^{b}=-\lim _{b \rightarrow \infty}\left(e^{-b}-e^{-1}\right)=-\left(0-\frac{1}{e}\right)=\frac{1}{e}
\end{aligned}
$$

Como $\int_{1}^{\infty} e^{-x} d x$ converge a $\frac{1}{e}$, entonces $\int_{1}^{\infty} e^{-x^{2}} d x$ es convergente.

Con lo cual, $\int_{0}^{\infty} e^{-x^{2}} d x$ es convergente.

Ejemplo 95:

Demostrar que $\int_{1}^{\infty} \frac{1}{\sqrt{x^{2}+4}} d x$ es divergente.

76

Si $x \geq 1$, se cumple que $\frac{1}{x} \leq \frac{1}{\sqrt{x^{2}+4}}$.

Analicemos la integral:

$$
\begin{aligned}
& \int_{1}^{\infty} \frac{1}{x} d x=\lim _{b \rightarrow \infty} \int_{1}^{b} \frac{1}{x} d x=\left.\lim _{b \rightarrow \infty} \ln x\right|_{1} ^{b} \\
& \int_{1}^{\infty} \frac{1}{x} d x=\lim _{b \rightarrow \infty}[\ln b-\ln 1] \text { no es finito. }
\end{aligned}
$$

De donde, si $\int_{1}^{\infty} \frac{1}{x} d x$ es divergente, también lo será $\int_{1}^{\infty} \frac{1}{\sqrt{x^{2}+4}} d x$.

Ejemplo 96:

Demostrar que $\int_{1}^{\infty} \frac{x^{2}+2}{x^{3}} d x$ es divergente.

Si $x \geq 1 \rightarrow \frac{x^{2}+2}{x^{3}}>\frac{x^{2}}{x^{3}}=\frac{1}{x}$.

Del ejemplo anterior: $\int_{1}^{\infty} \frac{1}{x} d x$ es divergente. Entonces $\int_{1}^{\infty} \frac{x^{2}+2}{x^{3}} d x$ también será divergente. 


\subsection{CRITERIO DE COMPARACIÓN DE LÍMITES}

Si $f_{(x)}$ y $g_{(x)}$ son funciones positivas y continuas en $\left[a, \infty\right.$ [ y si $\lim _{x \rightarrow \infty} \frac{f_{(x)}}{g_{(x)}}=L(0<\mathrm{L}<\infty)$, entonces: $\int_{a}^{\infty} f_{(x)} d x$ y $\int_{a}^{\infty} g_{(x)} d x$ convergen ambas o divergen ambas.

Además, si $L=0$, se cumplirá que $f_{(x)} \leq g_{(x)}$ a partir de cierto $x$ y si $\int_{a}^{\infty} g_{(x)} d x$ es convergente, entonces $\int_{a}^{\infty} f_{(x)} d x$ también lo será.

Ejemplo 97:

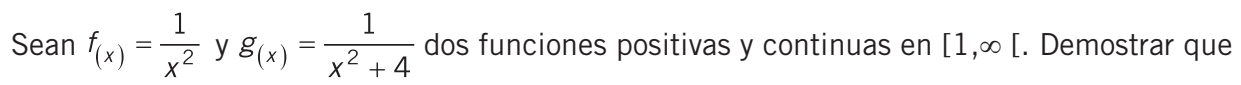
$\int_{1}^{\infty} \frac{1}{x^{2}} d x y \int_{1}^{\infty} \frac{1}{x^{2}+4} d x$ convergen.

Como $\lim _{x \rightarrow \infty} \frac{f(x)}{g_{(x)}}=\lim _{x \rightarrow \infty} \frac{\frac{1}{x^{2}}}{\frac{1}{x^{2}+4}}=\lim _{x \rightarrow \infty} \frac{x^{2}+4}{x^{2}}=\lim _{x \rightarrow \infty} \frac{1+\frac{4}{x^{2}}}{1}=\frac{1+0}{1}=1$,

las dos integrales siguientes deberán ser ambas convergentes o ambas divergentes.

Así pues:

$$
\begin{aligned}
& \int_{1}^{\infty} \frac{1}{x^{2}} d x=\lim _{b \rightarrow \infty} \int_{1}^{b} \frac{1}{x^{2}} d x=\left.\lim _{b \rightarrow \infty} \frac{-1}{x}\right|_{1} ^{b} \\
& \int_{1}^{\infty} \frac{1}{x^{2}} d x=\lim _{b \rightarrow \infty}\left(\frac{-1}{b}+\frac{1}{1}\right)=1 \\
& \therefore \quad \int_{1}^{\infty} \frac{1}{x^{2}} d x \text { es convergente. }
\end{aligned}
$$

Por otro lado:

$$
\begin{aligned}
& \int_{1}^{\infty} \frac{d x}{x^{2}+4}=\lim _{b \rightarrow \infty} \int_{1}^{b} \frac{d x}{x^{2}+4}=\left.\lim _{b \rightarrow \infty} \frac{1}{2} \arctan \frac{x}{2}\right|_{1} ^{b} \\
& \int_{1}^{\infty} \frac{d x}{x^{2}+4}=\frac{1}{2} \lim _{b \rightarrow \infty}\left(\arctan \frac{b}{2}-\arctan \frac{1}{2}\right)=\frac{1}{2}\left[\frac{\pi}{2}-0.464\right]=0.553 \\
& \therefore \quad \int_{1}^{\infty} \frac{d x}{x^{2}+4} \text { también es convergente. }
\end{aligned}
$$




\section{Ejemplo 98:}

Analizar la convergencia de la integral $\int_{1}^{\infty} \frac{\sqrt{x+1}}{x^{2}} d x$.

Consideremos $f_{(x)}=\frac{\sqrt{x+1}}{x^{2}}$ y $g_{(x)}=\frac{\sqrt{x}}{x^{2}}=\frac{1}{x^{3 / 2}}$.

Las dos funciones son positivas y continuas en $[1, \infty[$.

Como $\lim _{x \rightarrow \infty} \frac{f_{(x)}}{g_{(x)}}=\lim _{x \rightarrow \infty} \frac{\frac{\sqrt{x+1}}{x^{2}}}{\frac{1}{x^{3 / 2}}}=\lim _{x \rightarrow \infty} \frac{\sqrt{x+1}}{\sqrt{x}}=\lim _{x \rightarrow \infty} \sqrt{1+\frac{1}{x}}=1$,

analicemos $\int_{1}^{\infty} \frac{1}{x^{3 / 2}} d x$

78

$$
\begin{gathered}
\int_{1}^{\infty} \frac{d x}{x^{3 / 2}}=\lim _{b \rightarrow \infty} \int_{1}^{b} \frac{d x}{x^{3 / 2}}=\left.\lim _{b \rightarrow \infty} \frac{x^{-1 / 2}}{-1 / 2}\right|_{1} ^{b} \\
\int_{1}^{\infty} \frac{d x}{x^{3 / 2}}=-2 \lim _{b \rightarrow \infty}\left[\frac{1}{\sqrt{b}}-\frac{1}{1}\right]=-2(0-1)=2
\end{gathered}
$$

De donde: $\int_{1}^{\infty} \frac{d x}{x^{3 / 2}}$ y $\int_{1}^{\infty} \frac{\sqrt{x+1}}{x^{2}} d x$ ambas son convergentes.

\section{Ejemplo 99:}

Analizar la convergencia de la integral $\int_{1}^{\infty} \frac{d x}{\sqrt{x^{2}+1}}$.

Consideremos $f_{(x)}=\frac{1}{\sqrt{x^{2}+1}}$ y $g_{(x)}=\frac{1}{x}$, ambas positivas $\mathrm{y}$ continuas en $[1, \infty[$.

Calculemos:

$$
\lim _{x \rightarrow \infty} \frac{g_{(x)}}{f_{(x)}}=\lim _{x \rightarrow \infty} \frac{\frac{1}{x}}{\frac{1}{\sqrt{x^{2}+1}}}=\lim _{x \rightarrow \infty} \sqrt{1+\frac{1}{x^{2}}}=1
$$

Como $\int_{1}^{\infty} \frac{1}{x} d x$ es divergente, entonces $\int_{1}^{\infty} \frac{d x}{\sqrt{x^{2}+1}}$ también lo será. 


\section{Ejemplo 100:}

Determinar si la integral $\int_{e}^{\infty} \frac{\ln z}{z} d z$ es divergente.

Supongamos $f_{(z)}=\frac{1}{z}$ y $g_{(z)}=\frac{\ln z}{z}$, dos funciones positivas y continuas en $[1, \infty[$.

Calculemos:

$$
\lim _{z \rightarrow \infty} \frac{f_{(z)}}{g_{(z)}}=\lim _{z \rightarrow \infty} \frac{\frac{1}{z}}{\frac{\ln z}{z}}=\lim _{z \rightarrow \infty} \frac{1}{\ln z}=0
$$

Entonces, a partir de $z>e$ :

$$
f_{(z)}=\frac{1}{z}<g_{(z)}=\frac{\ln z}{z}
$$

Por el criterio de comparación directa, como $\int_{e}^{\infty} \frac{d z}{z}=\lim _{b \rightarrow \infty} \int_{e}^{b} \frac{d z}{z}=\left.\lim _{b \rightarrow \infty} \ln z\right|_{e} ^{b}$ es divergente, se concluye con que $\int_{e}^{\infty} \frac{\ln z}{z} d z$ es igualmente divergente. 



\section{VII \\ CÁLCULO DE ÁREAS DE REGIONES PLANAS POR INTEGRACIOON}

\section{1. ÁREA BAJO UNA CURVA}

Sea $y=f_{(x)}$ una función continua no negativa en el intervalo $a \leq x \leq b$.

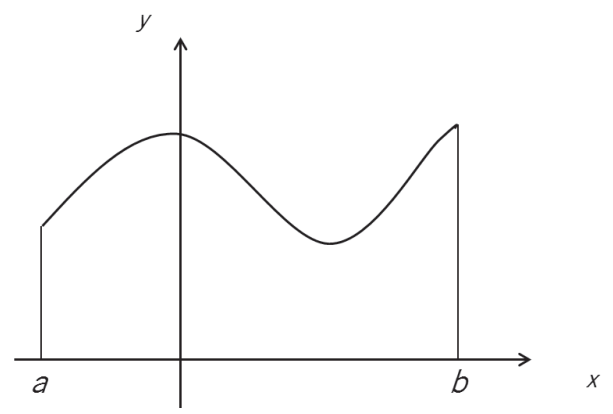

Analicemos qué representa gráficamente la integral definida de la función $f_{(x)}$ entre los límites $x=a$ y $x=b$.

$$
\int_{a}^{b} f_{(x)} d x=\lim _{n \rightarrow \infty} \sum_{i=0}^{n-1} f_{\left(\varepsilon_{i}\right)} \cdot \Delta x_{i} \quad f_{\left(\varepsilon_{i}\right)} \cdot \Delta x_{i}=\text { Área del rectángulo sombreado a }
$$


Por ello, grafiquemos $y=f_{(x)}$ :

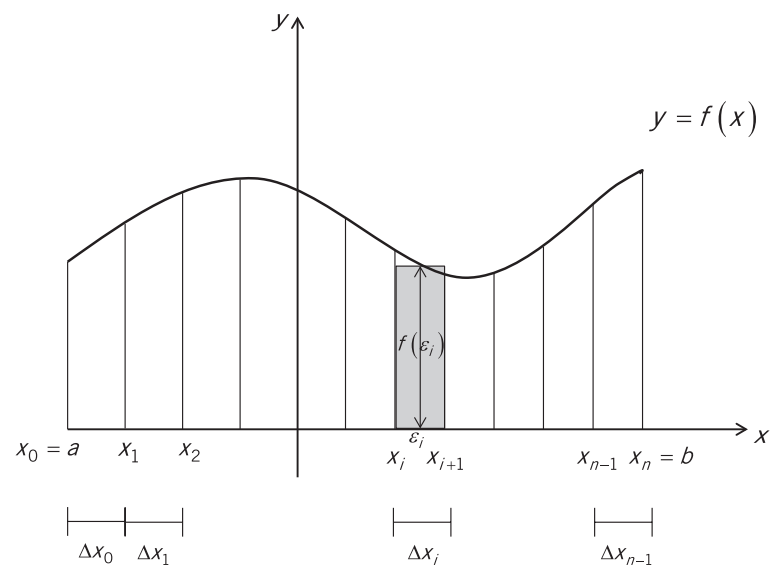

El término $f_{\left(\varepsilon_{j}\right)} \cdot \Delta x_{i}$ corresponde al área del rectángulo mostrado en la figura. Cuando $\mathrm{n} \rightarrow \infty$, el número de subintervalos aumenta y su ancho $\left(\Delta x_{i}\right)$ disminuye de forma tal que la suma de las 82 áreas de los rectángulos se aproxima al área bajo la curva $y=f_{(x)}$ y por encima del eje $x$.

Así pues:

$$
\int_{a}^{b} f_{(x)} d x=A
$$

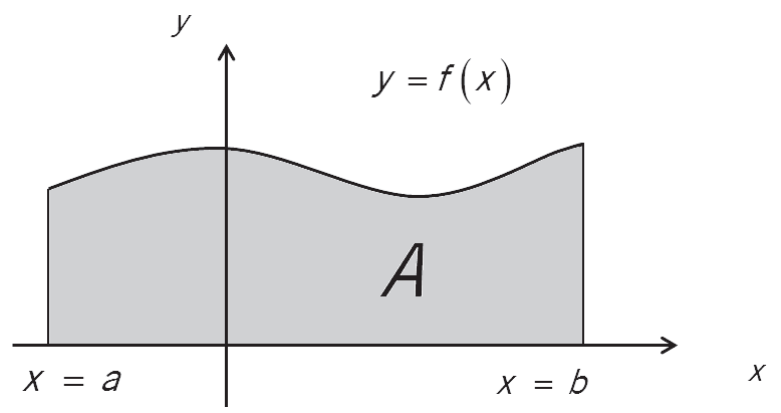

Si la función $y=f_{(x)}$ es continua y no positiva en el intervalo $a \leq x \leq b$, el término $\overbrace{f_{\left(\varepsilon_{i}\right)}^{(-)}}^{(+)} \cdot \overbrace{\Delta x_{i}}^{+)}$será negativo y $-f_{\left(\varepsilon_{i}\right)} \cdot \Delta x_{i}$ corresponderá al área comprendida por encima de $y=f_{(x)}$ y por debajo del eje $x$. 
De donde:

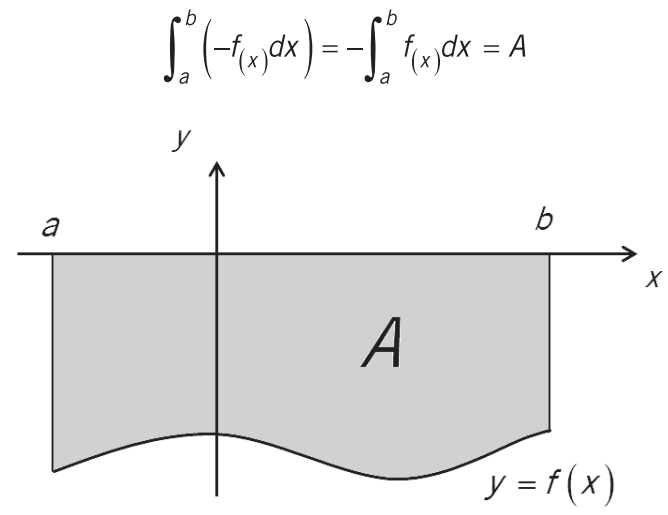

Ejemplo 101:

Encontrar el área situada bajo la curva $y=x^{2}$ y por encima del eje $x$, comprendida entre las rectas $x=-2$ y $x=4$.

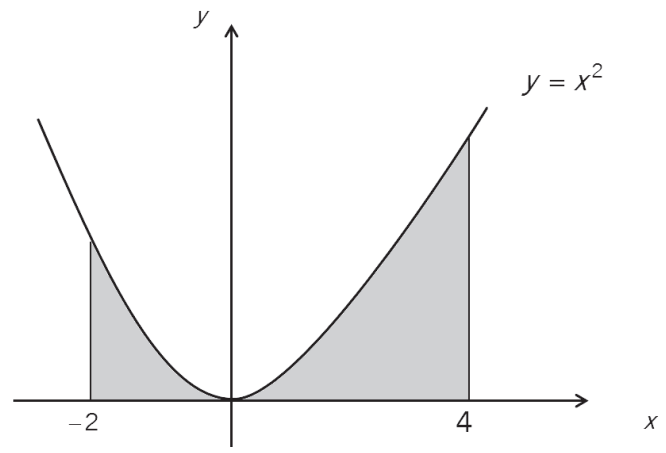

El área comprendida entre $y=x^{2}$ y $y=0$ (eje $x$ ) es:

$$
\begin{gathered}
A=\int_{-2}^{4} f_{(x)} d x=\int_{-2}^{4} x^{2} d x \\
A=\left.\frac{x^{3}}{3}\right|_{-2} ^{4}=\frac{64}{3}-\frac{(-8)}{3}=24 u n^{2}
\end{gathered}
$$

Nota:

Las áreas $A_{1}$ y $A_{2}$ mostradas en la figura siguiente están en relación de 1 a 2 . 


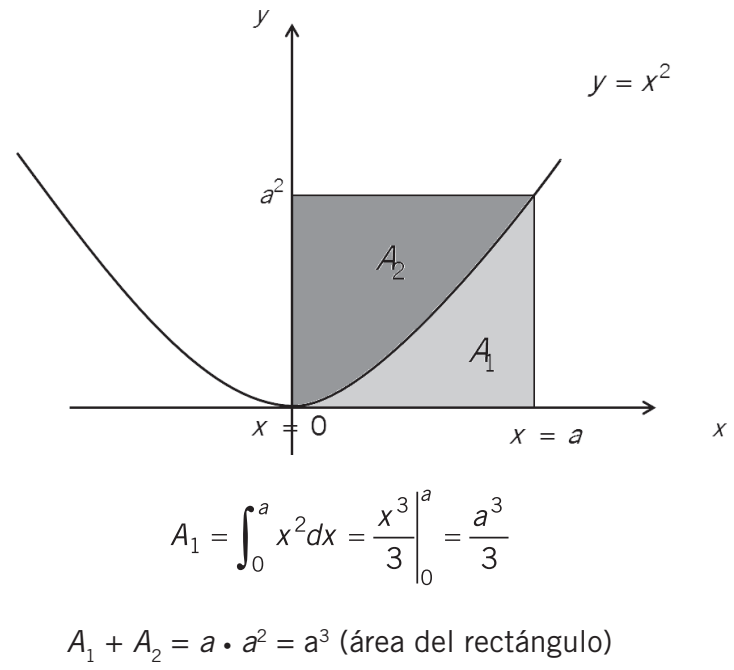

Entonces:

84

$$
\begin{aligned}
& A_{1}=a^{3} / 3 \\
& A_{2}=2 a^{3} / 3
\end{aligned} \quad \frac{A_{1}}{A_{2}}=\frac{1}{2}
$$

El resultado del ejemplo anterior coincide con el del siguiente procedimiento:

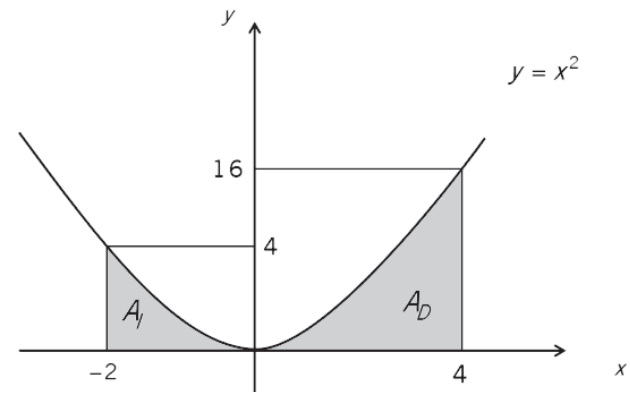

$$
\begin{aligned}
& A_{I}=\frac{1}{3}(2)(4)=\frac{8}{3} \\
& A_{D}=\frac{1}{3}(4)(16)=\frac{64}{3} \\
& \therefore A_{T}=8 / 3+64 / 3=24
\end{aligned}
$$

Ejemplo 102:

Calcular el área sombreada en la figura: 


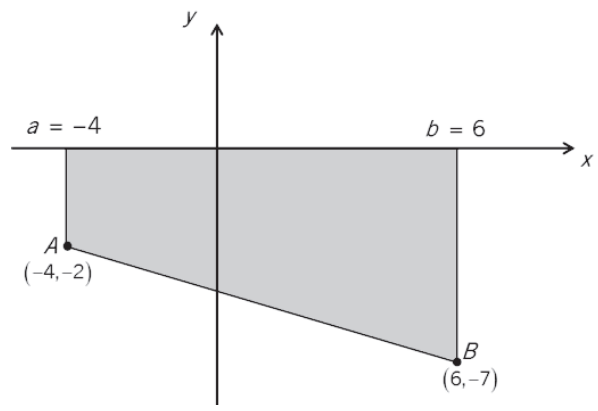

La ecuación de la recta que pasa por $A$ y $B$ es:

$$
\begin{gathered}
y-(-7)=\frac{-2-(-7)}{-4-(6)}(x-6) \\
y+7=\frac{-1}{2}(x-6) \\
y=\frac{-1}{2} x-4
\end{gathered}
$$

El área sombreada es:

$$
\begin{gathered}
A=-\int_{-4}^{6}\left[\frac{-1}{2} x-4\right] d x \\
A=\int_{-4}^{6}\left(\frac{1}{2} x+4\right) d x \\
A=\frac{x^{2}}{4}+\left.4 x\right|_{-4} ^{6} \\
A=\left(\frac{36}{4}+24\right)-\left(\frac{16}{4}-16\right) \\
A=45 \text { un }^{2}
\end{gathered}
$$

Este resultado coincide con el área del trapecio.

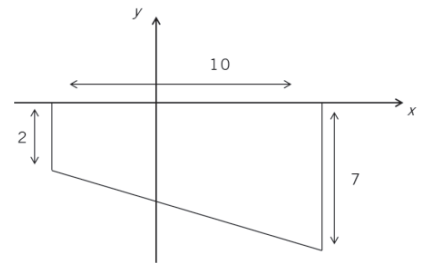

$$
\begin{gathered}
A=\frac{1}{2}(2+7) 10 \\
A=45 u n^{2}
\end{gathered}
$$




\section{2. ÁREA COMPRENDIDA ENTRE DOS CURVAS QUE NO SE CRUZAN}

Sean $y=f_{(x)}$ e $y=g_{(x)}$ dos funciones cuyas gráficas no se cruzan en el intervalo $a \leq x \leq b .\left(f_{(x)} \geq g_{(x)}\right.$ $\forall x \in[a, b])$.

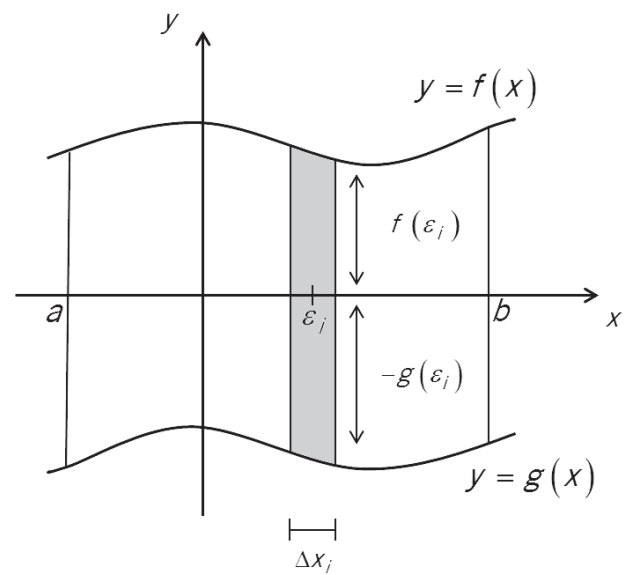

86

El área del rectángulo sombreado es:

$$
\left[f_{\left(\varepsilon_{i}\right)}-g_{\left(\varepsilon_{i}\right)}\right] \Delta x_{i}
$$

El área comprendida entre $y=f_{(x)}$ e $y=g_{(x)}$ es:

$$
\begin{gathered}
A=\lim _{n \rightarrow \infty} \sum_{i=0}^{n-1}\left[f_{\left(\varepsilon_{i}\right)}-g_{\left(\varepsilon_{i}\right)}\right] \Delta x_{i} \\
A=\lim _{n \rightarrow \infty} \sum_{i=0}^{n-1} f_{\left(\varepsilon_{i}\right)} \Delta x_{i}-\lim _{n \rightarrow \infty} \sum_{i=0}^{n-1} g_{\left(\varepsilon_{i}\right)} \Delta x_{i}
\end{gathered}
$$

Es decir:

$$
\begin{aligned}
& A=\int_{a}^{b} f_{(x)} d x-\int_{a}^{b} g_{(x)} d x \\
& A=\int_{a}^{b}[f(x)-g(x)] d x
\end{aligned}
$$

Ejemplo 103:

Determinar el área mostrada en la figura siguiente: 


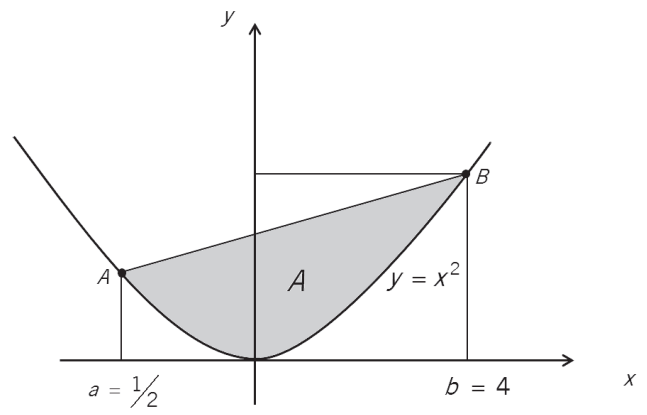

Encontramos la ecuación de la recta que pasa por $A$ y $B$.

$$
\begin{gathered}
A_{(-1 / 2 ; 1 / 4)} \quad y \quad B_{(4 ; 16)} \\
y-\frac{1}{4}=\frac{16-1 / 4}{4-(-1 / 2)}(x+1 / 2) \\
y-\frac{1}{4}=\frac{63 / 4}{9 / 2}(x+1 / 2) \\
y-\frac{1}{4}=\frac{7}{2}(x+1 / 2) \\
y=\frac{7}{2} x+2
\end{gathered}
$$

El área pedida es:

$$
\begin{gathered}
A=\int_{-1 / 2}^{4}\left[\left(\frac{7}{2} x+2\right)-\left(x^{2}\right)\right] d x \\
A=\frac{7}{4} x^{2}+2 x-\left.\frac{x^{3}}{3}\right|_{-1 / 2} ^{4} \\
A=\left(28+8-\frac{64}{3}\right)-\left(\frac{7}{16}-1+\frac{1}{24}\right) \\
A=\frac{44}{3}-\left(-\frac{25}{48}\right)=\frac{243}{16} u n^{2}
\end{gathered}
$$

Ejemplo 104:

Hallar el área comprendida entre la recta que pasa por $A$ y $B$ y la curva $y=x^{3}$, situada a la derecha de $x=x_{A}$ y a la izquierda de $x=x_{B}$. 


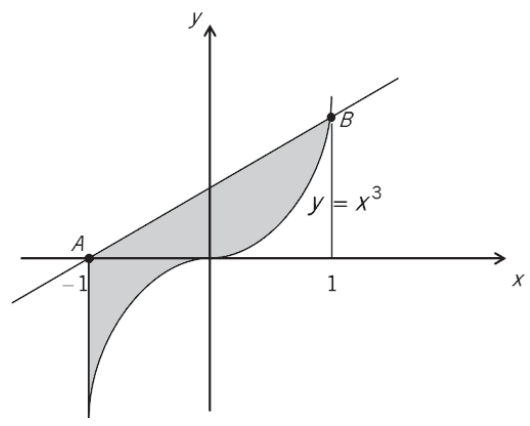

La ecuación de la recta que pasa por $A_{(-1,0)}$ y $B_{(1,1)}$ es:

$$
\begin{gathered}
y-0=\frac{1-0}{1-(-1)}(x+1) \\
y=\frac{1}{2} x+\frac{1}{2}
\end{gathered}
$$

El área pedida es:

88

$$
\begin{gathered}
A=\int_{-1}^{1}\left[\left(\frac{1}{2} x+\frac{1}{2}\right)-x^{3}\right] d x \\
A=\frac{x^{2}}{4}+\frac{1}{2} x-\left.\frac{x^{4}}{4}\right|_{-1} ^{1} \\
A=\left(\frac{1}{4}+\frac{1}{2}-\frac{1}{4}\right)-\left(\frac{1}{4}-\frac{1}{2}-\frac{1}{4}\right) \\
A=1 \text { un }^{2}
\end{gathered}
$$

Nótese que el área pedida es $A_{1}=A_{2}+A_{3}$

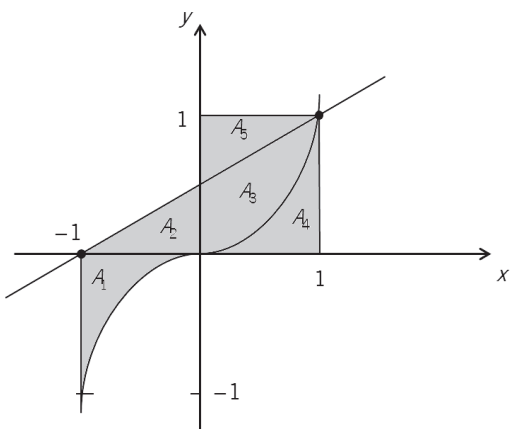




$$
A_{T}=A_{1}+A_{2}+A_{3}
$$

Como $A_{4}=A_{1}$ y $A_{5}=A_{2}$, se puede escribir $A_{T}=A_{4}+A_{5}+A_{3}$, que corresponde al área de un cuadrado de 1 un. de lado.

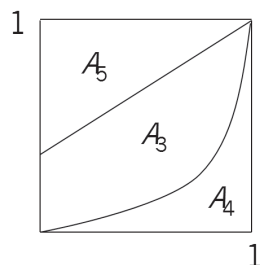

$$
\therefore A_{T}=1(1)=1 u n^{2}
$$

\section{3. ÁREA COMPRENDIDA ENTRE DOS CURVAS QUE SE CRUZAN}

Sean $y=f_{(x)}$ e $y=g_{(x)}$ dos funciones cuyas gráficas se cruzan en el intervalo $a \leq x \leq b$.
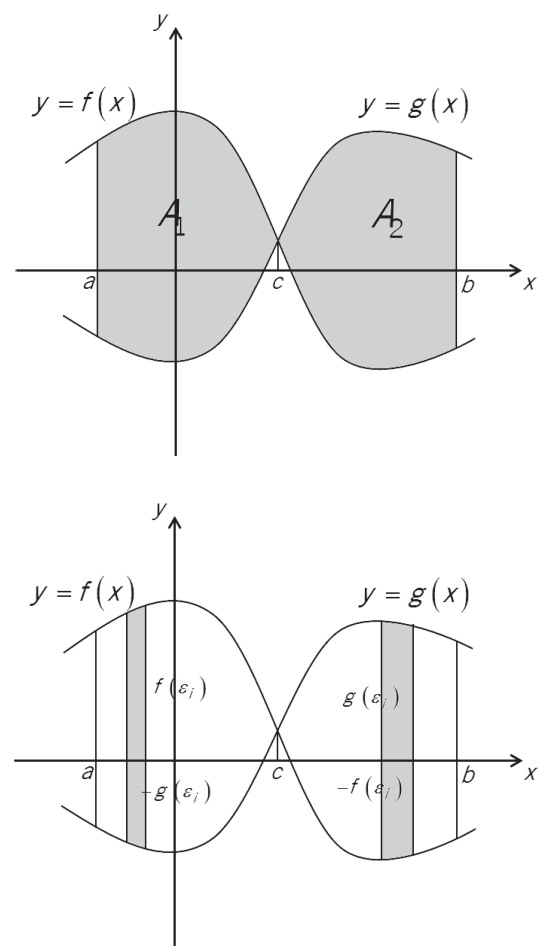
De acuerdo con el acápite anterior:

$$
\begin{aligned}
& A_{1}=\int_{a}^{c}\left[f_{(x)}-g_{(x)}\right] d x \\
& A_{2}=\int_{c}^{b}\left[g_{(x)}-f_{(x)}\right] d x
\end{aligned}
$$

Con lo cual, el área pedida es:

$$
\begin{gathered}
A_{T}=A_{1}+A_{2} \\
A_{T}=\int_{a}^{c}\left[f_{(x)}-g_{(x)}\right] d x+\int_{c}^{b}\left[g_{(x)}-f_{(x)}\right] d x
\end{gathered}
$$

Ejemplo 105:

Determinar el área de la región acotada por la recta $y=x-1$ y por la curva $y=(x-1)^{3}$.

La región cuya área se requiere calcular es:

90

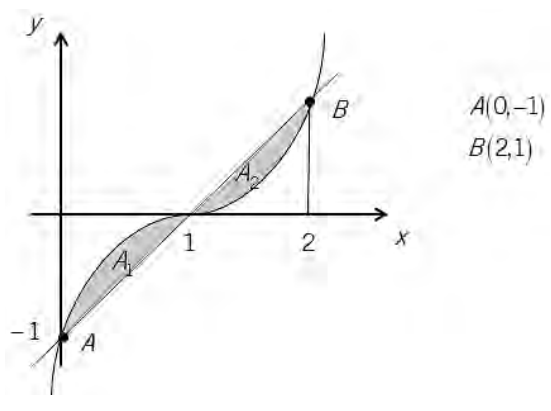

El área pedida es:

$$
\begin{gathered}
A_{T}=A_{1}+A_{2}=\int_{0}^{1}\left[(x-1)^{3}-(x-1)\right] d x+\int_{1}^{2}\left[(x-1)-(x-1)^{3}\right] d x \\
A_{T}=\left.\left[\frac{(x-1)^{4}}{4}-\frac{(x-1)^{2}}{2}\right]\right|_{0} ^{1}+\left.\left[\frac{(x-1)^{2}}{2}-\frac{(x-1)^{4}}{4}\right]\right|_{1} ^{2} \\
A_{T}=\left(0-0-\frac{1}{4}+\frac{1}{2}\right)+\left(\frac{1}{2}-\frac{1}{4}-0+0\right)=0.5 \mathrm{un}^{2}
\end{gathered}
$$


Ejemplo 106:

Encontrar el área de la región limitada por las curvas $y=x^{2}$ e $y=1 / x$ comprendida entre $x=1 / 2 y$ $x=2$.

El gráfico correspondiente es:

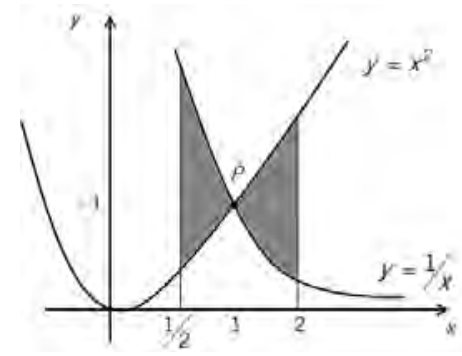

El área pedida es:

$$
\begin{gathered}
A_{T}=A_{1}+A_{2}=\int_{1 / 2}^{1}\left(\frac{1}{x}-x^{2}\right) d x+\int_{1}^{2}\left(x^{2}-\frac{1}{x}\right) d x \\
A_{T}=\left.\left(\ln x-\frac{x^{3}}{3}\right)\right|_{1 / 2} ^{1}+\left.\left(\frac{x^{3}}{3}-\ln x\right)\right|_{1} ^{2} \\
A_{T}=\left(0-\frac{1}{3}+\ln 2+\frac{1}{24}\right)+\left(\frac{8}{3}-\ln 2-\frac{1}{3}+0\right) \\
A_{T}=\frac{47}{24} \mathrm{un}^{2}
\end{gathered}
$$

\section{4. ÁREA DE REGIONES MEDIANTE INTEGRACIÓN}

En ciertos casos es conveniente considerar a los elementos diferenciales del área como franjas horizontales de ancho $d y$, tal como se muestra a continuación:
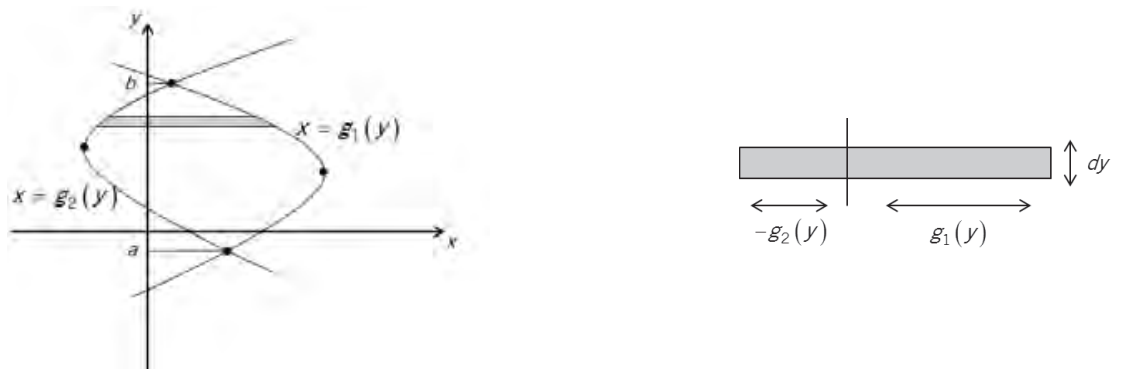
La franja horizontal, de ancho $d y$, tiene área:

$$
d A=\left[g_{1(y)}-g_{2(y)}\right] d y
$$

Entonces, el área de la región comprendida entre las curvas $x=g_{1(y)}$ y $x=g_{2(y)}$ es:

$$
A=\int_{a}^{b} d A=\int_{a}^{b}\left[g_{1(y)}-g_{2(y)}\right] d y
$$

Ejemplo 107:

Determinar el área de la región encerrada por la recta $y=-x$ y la parábola $x=2-y^{2}$.

Se tiene que:

92

$$
\begin{gathered}
x=2-y^{2} \\
(y-0)^{2}=-(x-2) \\
V(2,0)
\end{gathered}
$$

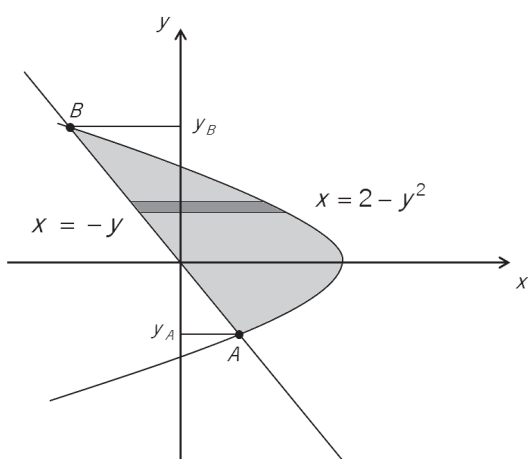

El área pedida es:

$$
\int_{y_{A}}^{y_{B}}\left[\left(2-y^{2}\right)-(-y)\right] d y
$$

Los puntos $A$ y $B$ de intersección de las dos curvas son $A_{(1,-1)}$ y $B_{(-2,2)}$.

Es decir:

$$
\begin{aligned}
& A=\int_{-1}^{2}\left[2-y^{2}+y\right] d y \\
& A=\left.\left(2 y-\frac{y^{3}}{3}+\frac{y^{2}}{2}\right)\right|_{-1} ^{2}
\end{aligned}
$$




$$
\begin{gathered}
A=\left(4-\frac{8}{3}+2\right)-\left(-2+\frac{1}{3}+\frac{1}{2}\right) \\
A=\frac{10}{3}-\left(\frac{-7}{6}\right)=\frac{27}{6}=\frac{9}{2} u n^{2}
\end{gathered}
$$

Ejemplo 108:

¿Cuál es el área de la región comprendida entre las parábolas $x=y^{2}-4$ y $x=8-2 y^{2}$ ?

Las parábolas se cortan cuando:

$$
\begin{gathered}
y^{2}-4=8-2 y^{2} \\
3 y^{2}=12
\end{gathered}
$$

$$
\mathrm{y}= \pm 2 \succ \begin{aligned}
& A(0,-2) \\
& B(0,2)
\end{aligned}
$$

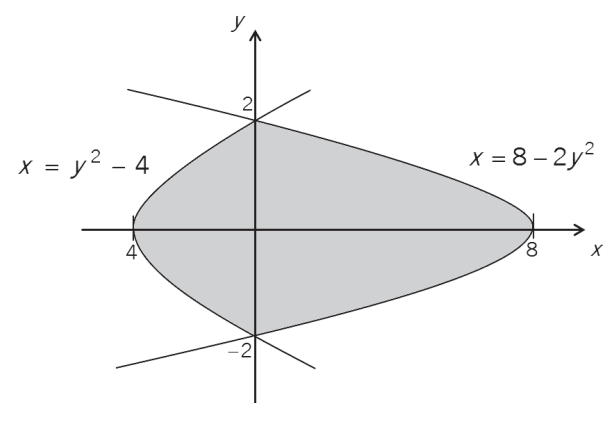

El área solicitada es:

$$
\begin{gathered}
A=\int_{-2}^{2}\left[\left(8-2 y^{2}\right)-\left(y^{2}-4\right)\right] d y \\
A=\int_{-2}^{2}\left(-3 y^{2}+12\right) d y \\
A=-y^{3}+\left.12 y\right|_{-2} ^{2} \\
A=(-8+24)-(8-24)=32 u n^{2}
\end{gathered}
$$





\section{VIII \\ APLICACIONES DE LA INTEGRAL}

En los casos en que se conoce la rapidez de variación de una función y se requiere encontrar dicha función, se debe emplear el Cálculo Integral. Las aplicaciones pueden estar referidas a situaciones de naturaleza geométrica, económica, financiera, estadística, física, biológica, social, entre otras.

\section{PROBLEMAS GEOMÉTRICOS}

Conviene recordar que dada una función $y=f_{(x)}$, la derivada $\left.\frac{d y}{d x}\right|_{x_{p}}$ representa la pendiente de la recta tangente a la curva de ecuación $y=f_{(x)}$ en el punto $P$ de abscisa $X_{P}$.

Ejemplo 109:

Encontrar la ecuación de la familia de curvas cuya pendiente en un punto dado sea igual y de signo contrario al triple de la abscisa en dicho punto. Determinar la curva de la familia que pasa por el punto $(1,1 / 2)$.

Se tiene que $\frac{d y}{d x}=-3 x$

El diferencial

$$
d y=\left(\frac{d y}{d x}\right) d x
$$




$$
d y=-3 x d x
$$

Entonces:

$$
\int d y=\int-3 x d x
$$

Método I: Con la integral indefinida.

$$
\begin{gathered}
y=\int d y=\int-3 x d x \\
y=-\frac{3 x^{2}}{2}+C
\end{gathered}
$$

Como $(1,1 / 2)$ pertenece a la curva:

$$
\frac{1}{2}=\frac{-3}{2}(1)^{2}+C \Rightarrow C=2
$$

La ecuación de la curva de la familia que pasa por $(1,1 / 2)$ es $y=-\frac{3 x^{2}}{2}+2$

Método II: Con la integral definida.

96

Como $(1,1 / 2)$ pertenece a la curva, se tendrá que:

$$
\begin{gathered}
\text { valor de } y \rightarrow \int_{1 / 2}^{y} d y=\int_{1}^{x}-3 x d x \\
\left.y\right|_{1 / 2} ^{y}=-\left.\frac{3 x^{2}}{2}\right|_{1} ^{x}
\end{gathered}
$$

De donde:

$$
\begin{gathered}
y-\frac{1}{2}=-\frac{3}{2}\left(x^{2}\right)+\frac{3}{2}(1)^{2} \\
y=\frac{-3}{2} x^{2}+2
\end{gathered}
$$

Ejemplo 110:

Determinar la ecuación de una curva que pasa por el punto $P(1,0)$, en el que la pendiente es igual a 4 , y que se cumple que $y^{\prime \prime}=6 x-8$ en cualquiera de sus puntos. 
Se tiene que:

$$
y^{\prime \prime}=\frac{d^{2} y}{d x^{2}}=\frac{d\left[\frac{d y}{d x}\right]}{d x}=\frac{d y^{\prime}}{d x}=6 x-8
$$

El diferencial

$$
\begin{gathered}
d y^{\prime}=\frac{d y^{\prime}}{d x} d x \\
d y^{\prime}=(6 x-8) d x
\end{gathered}
$$

Entonces:

$$
\int d y^{\prime}=\int(6 x-8) d x
$$

Como en el punto $P(1,0)$ se cumple que $\frac{d y}{d x}=4$,

$$
\begin{aligned}
& \text { valor de } y^{\prime} \rightarrow 4 \int_{4}^{y^{\prime}} d y^{\prime}=\int_{1}^{x}(6 x-8) d x \\
&\left.y^{\prime}\right|_{4} ^{y^{\prime}}=3 x^{2}-\left.8 x\right|_{1} ^{x}
\end{aligned}
$$

De donde:

$$
\begin{gathered}
y^{\prime}-4=\left(3 x^{2}-8 x\right)-\left(3(1)^{2}-8(1)\right) \\
y^{\prime}=\frac{d y}{d x}=3 x^{2}-8 x+9
\end{gathered}
$$

Por otro lado, si la curva pasa por $P(1,0)$, se tendrá que:

$$
\begin{gathered}
\int_{0}^{y} d y=\int_{1}^{x}\left(3 x^{2}-8 x+9\right) d x \\
\left.y\right|_{0} ^{y}=x^{3}-4 x^{2}+\left.9 x\right|_{1} ^{x}
\end{gathered}
$$

Finalmente:

$$
\begin{gathered}
y-0=\left(x^{3}-4 x^{2}+9 x\right)-(1-4+9) \\
y=x^{3}-4 x^{2}+9 x-6
\end{gathered}
$$

Ejemplo 111:

Encontrar la ecuación de la familia de curvas ortogonales a la familia de parábolas $y^{2}=2 x+C$. 
La familia de curvas ortogonales a una familia dada de curvas es aquella en la que cada una de ellas corta en un ángulo recto a cualquier curva de la familia dada.

La familia dada $y^{2}=2 x+C$ tiene una pendiente que se obtiene de $2 y y^{\prime}=2 \rightarrow y^{\prime}=\frac{d y}{d x}=\frac{1}{y}$.

La familia de curvas ortogonales tendrá pendiente:

$$
\frac{d y}{d x}=\frac{-1}{(1 / y)}=-y
$$

Con lo cual:

$$
d y=\frac{d y}{d x} d x=-y d x
$$

De donde:

$$
\begin{gathered}
\frac{d y}{-y}=d x \\
-\int \frac{d y}{y}=\int d x \\
-\ln y=x+C
\end{gathered}
$$

$$
\begin{gathered}
\ln y=-x-C \\
y=e^{-x-C}=e^{-C} e^{-x}=C^{\prime} e^{-x}
\end{gathered}
$$

\section{PROBLEMAS ECONÓMICOS}

Con frecuencia se conocen la utilidad marginal (UM), el ingreso marginal (IM) y el costo marginal (CM). Se pueden encontrar las funciones $U$, I y $C$ a partir de:

$$
\begin{aligned}
& U=\int(\mathrm{UM}) d q \\
& I=\int(\mathrm{IM}) d q \\
& C=\int(\mathrm{CM}) d q
\end{aligned}
$$

Otra de las aplicaciones de la integral a este tipo de situaciones es la referida al cálculo del excedente de los productores y al de los consumidores.

Mayoritariamente, la cantidad total que los consumidores gastan en un artículo difiere de la cantidad total que estarían dispuestos a gastar. A la diferencia entre esta última cantidad y la primera de ellas se le conoce como excedente de los consumidores y representa el ahorro total de los consumidores. 
Así pues, si $p=f_{(q)}$ es la función demanda del artículo y los consumidores adquieren $q_{0}$ artículos al precio $p_{0}$ por unidad, el excedente de los consumidores está representado por el área sombreada en la figura siguiente, tal como se demostrará a continuación:

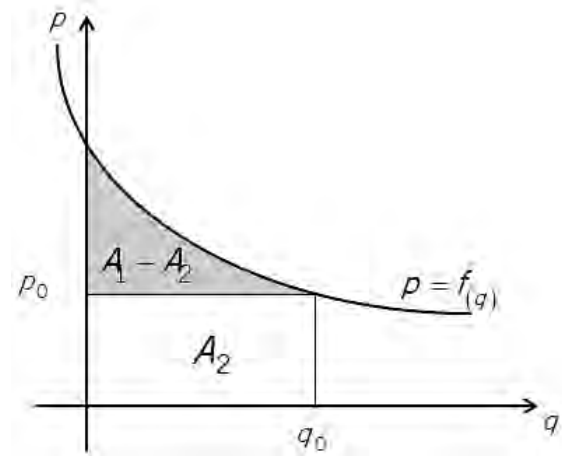

El área sombreada se calcula como:

$$
\begin{gathered}
A=\int_{0}^{q_{0}}\left[f_{(q)}-p_{0}\right] d q \\
A=\int_{0}^{q_{0}} f_{(q)} d q-\int_{0}^{q_{0}} p_{0} d q \\
A=\int_{0}^{q_{0}} f_{(q)} d q-p_{0}\left[q_{0}-0\right] \\
A=\underbrace{\int_{0}^{q_{0}} f_{(q)} d q}_{A_{1}}-\underbrace{p_{0} q_{0}}_{A_{2}} \\
A=A_{1}-A_{2}
\end{gathered}
$$

En donde:

$A_{1}$ representa la cantidad total que los consumidores estaban dispuestos a pagar por adquirir $q_{0}$ unidades.

$A_{2}$ representa la cantidad total que los consumidores han gastado.

Con lo cual:

$$
\mathrm{EC}=\int_{0}^{q_{0}}\left[f_{(q)}-p_{0}\right] d q
$$


De forma análoga, si $p=g_{(q)}$ es la función de oferta del artículo, el excedente de los productores al vender $q_{0}$ artículos al precio $p_{0}$ por unidad es la ganancia total de los productores dado que $p_{0}$ excede al precio al que estaban dispuestos a vender los artículos.

Es decir:

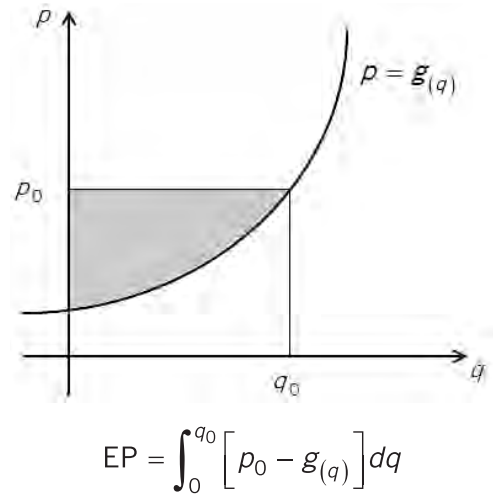

Ejemplo 112:

100 Sea $p=150-2 q-3 q^{2}$ dólares por unidad la función de demanda de los consumidores. Encontrar el excedente de los consumidores si el precio de mercado se fija en $p_{0}=117$.

La gráfica de $p=-3 q^{2}-2 q+150$ es una parábola de eje vertical cuyo vértice se encuentra en:

$$
v\left(\frac{-(-2)}{2(-3)}, \frac{4(-3)(150)-(-2)^{2}}{4(-3)}\right)
$$

Es decir: $v\left(-\frac{1}{3}, \frac{451}{3}\right)$

Encontremos el valor de $q$ tal que $p_{0}=\$ 117$.

$$
\begin{gathered}
117=-3 q^{2}-2 q+150 \\
3 q^{2}+2 q-33=0 \\
(3 q+11)(q-3)=0 \rightarrow q=3
\end{gathered}
$$

El gráfico correspondiente con el excedente del consumidor (EC) se muestra a continuación: 
De donde:

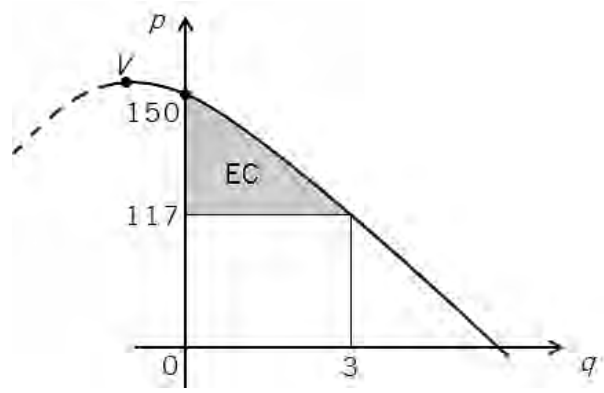

$$
\begin{gathered}
\mathrm{EC}=\int_{0}^{3}\left[\left(-3 q^{2}-2 q+150\right)-117\right] d q \\
\mathrm{EC}=-q^{3}-q^{2}+\left.33 q\right|_{0} ^{3} \\
\mathrm{EC}=(-27-9+99)-(0)={ }^{\$} 63
\end{gathered}
$$

Ejemplo 113:

La ecuación de demanda para un producto es $p=2^{11-q}$ y la ecuación de oferta es $p=2^{q+1}$, en donde $p$ es el precio por unidad (en cientos de dólares) cuando $q$ unidades se demandan 0 se ofrecen. Encontrar el millar de unidades más cercano al excedente de los productores bajo equilibrio del mercado.

La gráfica de las funciones dadas se muestra a continuación:

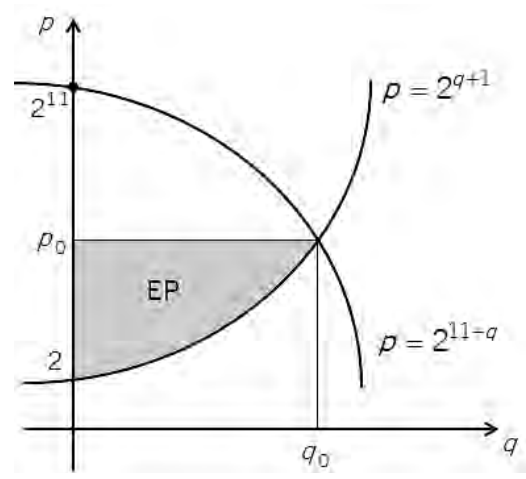

El excedente de los productores bajo equilibrio del mercado se calcula como:

$$
\mathrm{EP}=\int_{0}^{q_{0}}\left[p_{0}-2^{q+1}\right] d q \text { cientos de dólares }
$$


Encontremos el punto de equilibrio:

$$
2^{11-q_{0}}=2^{q_{0}+1} \rightarrow 11-q_{0}=q_{0}+1 \rightarrow q_{0}=5 \text { unidades }
$$

Si $q_{0}=5 \rightarrow p_{0}=2^{11-5}=2^{6}=64$ cientos de dólares por unidad.

De donde:

$$
\begin{gathered}
\mathrm{EP}=\int_{0}^{5}\left[64-2^{q+1}\right] d q \\
\mathrm{EP}=64 q-\left.\frac{2^{q+1}}{\ln 2}\right|_{0} ^{5} \\
\mathrm{EP}=\left(320-\frac{64}{\ln 2}\right)-\left(0-\frac{2}{\ln 2}\right) \\
\mathrm{EP}=320-\frac{62}{\ln 2}=320-89.45=230.55 \text { cientos de dólares }
\end{gathered}
$$

Vale decir EP = 23.055 dólares, que corresponde a 23 millones de dólares como aproximación.

\section{2}

\section{PROBLEMAS FINANCIEROS}

Dos aplicaciones de la integral a las finanzas están referidas al valor presente o futuro de un flujo de ingresos y al monto de una anualidad, como se explica a continuación.

El valor futuro total o acumulado de un flujo de ingresos de $R_{(t)}$ dólares por año al cabo de $T$ años, que ganan intereses en una tasa $r$ anual compuesta en forma continua, viene dado por:

$$
A=e^{r T} \int_{0}^{T} R_{(t)} e^{-r t} d t
$$

El valor presente de un flujo de ingresos de $R_{(t)}$ dólares por año durante $T$ años, que ganan intereses con una tasa anual $r$ compuesta de forma continua, está dado por:

$$
V P=\int_{0}^{T} R_{(t)} e^{-r t} d t
$$

Ejemplo 114:

Suponga que se espera que una inversión genere ingresos a razón de $R_{(t)}=200,000$ dólares por año durante los próximos 5 años. Encuentre el valor total acumulado de este flujo de ingresos al cabo de 5 años si se asume que los ingresos se reinvierten en una empresa que rinde intereses a la tasa del $8 \%$ anual compuesta continuamente. 
Se tiene que: $\quad R_{(t)}=200,000$ dólares

$r=0.08$

$T=5$ años

Entonces:

$$
\begin{gathered}
A=e^{0.08(5)} \int_{0}^{5} 200,000 e^{-0.08 t} d t \\
A=200,\left.000 e^{0.40}\left(\frac{e^{-0.08 t}}{-0.08}\right)\right|_{0} ^{5} \\
A=\frac{-200,000 e^{0.40}}{0.08}\left[e^{-0.40}-e^{0}\right] \\
A=2,500,000\left[e^{0.4}-1\right]=1,229,562 \text { dólares }
\end{gathered}
$$

Una anualidad es una serie de pagos $P$ durante $T$ años. Si se considera que se efectúan $m$ pagos por año y que la tasa de interés compuesta en forma continua es $r$ anual, el monto de la anualidad ( $A$, suma de los pagos más los intereses generados) se calcula con la fórmula del valor futuro total, asumiendo un flujo constante de $R_{(t)}=m P$ dólares por año. Así pues:

$$
\begin{gathered}
A=e^{r T} \int_{0}^{T} m P e^{-r t} d t \\
A=\left.m P e^{r t}\left(-\frac{e^{-r t}}{r}\right)\right|_{0} ^{T} \\
A=m P e^{r t}\left[-\frac{e^{-r t}}{r}+\frac{e^{0}}{r}\right]
\end{gathered}
$$

Con lo cual:

$$
A=\frac{m P}{r}\left(e^{r t}-1\right)
$$

Ejemplo 115:

Si usted depositara US $\$ 200$ cada 2 meses en una cuenta de ahorros que paga $10 \%$ por año, con interés compuesto en forma continua, estime la cantidad que tendrá acumulada en su cuenta al cabo de 12 años.

De acuerdo con la fórmula de la anualidad:

$$
\begin{aligned}
& P=200 \text { dólares } \\
& T=12 \text { años }
\end{aligned}
$$


Se tiene que:

$$
\begin{aligned}
& r=0.10 \text { anual } \\
& m=6 \text { pagos por año }
\end{aligned}
$$

$$
\begin{gathered}
A=\frac{6(200)}{0.10}\left[e^{0.10(12)}-1\right] \\
A={ }^{\$} 27,841.40
\end{gathered}
$$

\section{PROBLEMAS ESTADÍSTICOS}

La aplicación más frecuente de las integrales en la Estadística es el cálculo de las probabilidades de las variables aleatorias continuas, a partir de sus funciones de densidad de probabilidad.

Sea $x$ una variable aleatoria continua (por ejemplo, $x$ puede representar el tiempo de espera en un paradero de ómnibus), si la función de densidad de probabilidad es $f_{(x)}$, la probabilidad de que $x$ esté comprendida entre los valores $a$ y $b$ viene dada por:

$$
P_{(a \leq x \leq b)}=\int_{a}^{b} f_{(x)} d x
$$

Así pues, en el caso de que $x$ sea el tiempo de espera en el paradero, $P_{\left(2^{\prime} \leq x \leq 5^{\prime}\right)}$ representa la 104 probabilidad de que el tiempo de espera en el paradero esté comprendido entre 2' y 5'.

\section{Ejemplo 116:}

Se aplica un examen de 4 horas de duración a todos los candidatos a vendedores en una cadena minorista. Se ha encontrado que el tiempo $x$ en horas necesario para rendir el examen es aleatorio y tiene una función de densidad de probabilidad dada por:

$$
f_{(x)}=\frac{80-x^{3}}{256}
$$

a) Comprobar que el área bajo la gráfica de $f_{(x)}$ es 1 .

b) Encontrar la probabilidad de que terminen de rendir la prueba en un tiempo comprendido entre 1 y 4 horas.

a) El área bajo la curva de ecuación $f_{(x)}=\frac{80-x^{3}}{256}$ comprendida entre $x=0$ y $x=4$ es:

$$
\begin{aligned}
& A=\int_{0}^{4} \frac{80-x^{3}}{256} d x=\left.\frac{1}{256}\left[80 x-\frac{x^{4}}{4}\right]\right|_{0} ^{4} \\
& A=\frac{1}{256}\left[80(4)-4^{3}-0\right]=\frac{1}{256}[256]=1
\end{aligned}
$$


Es decir, la probabilidad de que los candidatos demoren entre 0 y 4 horas en rendir el examen es $100 \%$.

b) Se pide:

$$
\begin{gathered}
P_{(1 \leq x \leq 4)}=\int_{1}^{4} f_{(x)} d x=\int_{1}^{4}\left(\frac{80-x^{3}}{256}\right) d x \\
P=\left.\frac{1}{256}\left(80 x-\frac{x^{4}}{4}\right)\right|_{1} ^{4}=\frac{1}{256}\left(320-64-80+\frac{1}{4}\right) \\
P=\frac{1}{256}(176.25)=0.6885=68.85 \%
\end{gathered}
$$

Ejemplo 117:

En cierta ciudad, la función de densidad de probabilidad para que la duración de una llamada telefónica seleccionada al azar sea $x$ minutos es:

$$
f_{(x)}=\left\{\begin{array}{cc}
\frac{1}{3} e^{-x / 3} & x \geq 0 \\
0 & x<0
\end{array}\right.
$$

Encontrar la probabilidad de que la duración de una llamada escogida al azar:

a) esté entre 1 y 2 minutos.

b) sea 5 minutos cuando menos.

a) Se pide:

$$
\begin{aligned}
& P_{(1 \leq x \leq 2)}=\int_{1}^{2} \frac{1}{3} e^{-x / 3} d x=-\int_{1}^{2} e^{-x / 3}\left(-\frac{1}{3} d x\right) \\
& P_{(1 \leq x \leq 2)}=-\left.e^{-x / 3}\right|_{1} ^{2}=-e^{-2 / 3}+e^{-1 / 3}=0.203
\end{aligned}
$$

b) Se pide:

$$
\begin{gathered}
P_{(5 \leq x<\infty)}=\lim _{b \rightarrow \infty} \int_{5}^{b} \frac{1}{3} e^{-x / 3} d x \\
P_{(5 \leq x<\infty)}=-\left.\lim _{b \rightarrow \infty} e^{-x / 3}\right|_{5} ^{b} \\
P_{(5 \leq x<\infty)}=-\lim _{b \rightarrow \infty}\left(\frac{1}{e^{b / 3}}-e^{-5 / 3}\right)
\end{gathered}
$$




$$
P_{(5 \leq x<\infty)}=-\left(0-e^{-5 / 3}\right)=\frac{1}{e^{5 / 3}}=0.188
$$

\section{PROBLEMAS SOCIALES}

Si se conoce la rapidez de crecimiento poblacional, es posible estimar el tamaño futuro de la población.

Igualmente, a partir de la velocidad a la que se propaga determinado rumor, se puede aproximar cuántas personas se han enterado del mismo en determinado momento. 0 , a partir de la rapidez de expansión de un virus, es posible estimar el porcentaje de personas infectadas.

Ejemplo 118:

Se estima que dentro de $t$ meses la población de cierta ciudad crecerá a razón de $8 t+9 t^{2 / 3}$ personas por mes. Si la población actual es 20,000, ¿cuál será la población dentro de 27 meses?

Sea $P_{(t)}$ la población al cabo de $t$ meses, entonces:

$$
\frac{d P}{d t}=8 t+9 t^{2 / 3}
$$

Entonces:

$$
d P=\frac{d P}{d t} \cdot d t=\left(8 t+9 t^{2 / 3}\right) d t
$$

Integremos con límites definidos:

$$
\begin{gathered}
\int_{20,000}^{P_{(t)}} d P=\int_{0}^{t}\left(8 t+9 t^{2 / 3}\right) d t \\
P_{(t)}-20,000=\left.\left(4 t^{2}+9 \frac{t^{5 / 3}}{5 / 3}\right)\right|_{0} ^{t} \\
P_{(t)}=20,000+4 t^{2}+\frac{27}{5} t^{5 / 3}
\end{gathered}
$$

Cuando $t=27$ :

$$
\begin{gathered}
P_{(27)}=20,000+4(27)^{2}+\frac{27}{5}(\sqrt[3]{27})^{5} \\
P_{(27)}=20,000+2,916+1,312=24,228 \text { personas }
\end{gathered}
$$




\section{Ejemplo 119:}

Una comunidad de $A$ personas es susceptible de contagiarse de cierto virus, el cual se propaga con una rapidez que es conjuntamente proporcional al número de personas que han contraído dicho virus y al número de ellas que aún no lo han contraído. El $10 \%$ de $A$ tenía el virus inicialmente y 3 semanas después, el $25 \%$ de $A$ se había infectado. ¿Qué porcentaje de $A$ se habrá infectado después de 3 semanas más?

Sea $N_{(t)}$ el número de personas que han contraído el virus en el tiempo $t$. Se cumple que:

$$
\frac{d N}{d t}=K N(A-N)
$$

De donde:

$$
\begin{gathered}
d N=\frac{d N}{d t} \cdot d t=K N(A-N) d t \\
\frac{d N}{N(A-N)}=K d t \Rightarrow \int \frac{d N}{N(A-N)}=\int K d t
\end{gathered}
$$

Por fracciones parciales:

$$
\frac{1}{N(A-N)}=\frac{1 / A}{N}+\frac{1 / A}{A-N}
$$

Entonces:

$$
\int \frac{d N}{N(A-N)}=\frac{1}{A} \int \frac{d N}{N}+\frac{1}{A} \int \frac{d N}{A-N}=\int K d t
$$

Como $N=0.10 A$ para $t=0$ :

$$
\begin{aligned}
& \frac{1}{A} \int_{0.1 A}^{N} \frac{d N}{N}-\frac{1}{A} \int_{0.1 A}^{N} \frac{d N}{N-A}=K \int_{0}^{t} d t \\
& \left.\frac{1}{A} \ln N\right|_{0.1 A} ^{N}-\left.\frac{1}{A} \ln (N-A)\right|_{0.1 A} ^{N}=\left.K t\right|_{0} ^{t} \\
& \frac{1}{A} \ln \frac{N}{0.1 A}-\frac{1}{A} \ln \frac{N-A}{-0.9 A}=K(t-0) \\
& \frac{1}{A} \ln \frac{N / 0.1 A}{(N-A) /-0.9 A}=K t \\
& \frac{1}{A} \ln \frac{-9 N}{N-A}=K t \Rightarrow \frac{-9 N}{N-A}=e^{A K t}
\end{aligned}
$$


Cálculo de $K$ :

Cuando $t=3, N=0.25 A$; en (1):

$$
\frac{-9(0.25 A)}{0.25 A-A}=\frac{-2.25 A}{-0.75 A}=3=e^{A(K) 3}
$$

Cuando $t=6, N=A_{(6)}$; en (1):

$$
\frac{-9 N_{(6)}}{N_{(6)}-A}=e^{A(K) 6}=\left[e^{A(K) 3}\right]^{2}
$$

(2) en (3):

$$
\frac{-9 N_{(6)}}{N_{(6)}-A}=(3)^{2} \Rightarrow-N_{(6)}=N_{(6)}-A
$$

De donde:

$$
2 N_{(6)}=A
$$

108

$$
N_{(6)}=\frac{A}{2}=50 \% \text { de } A
$$

\section{PROBLEMAS BIOLÓGICOS}

Es posible describir el crecimiento de una colonia de bacterias si se sabe que la razón de crecimiento de las mismas es proporcional al número de bacterias en cada momento.

En ese caso se encuentra la ley de crecimiento exponencial. A saber, si $Q_{(t)}$ es la cantidad de bacterias en el tiempo $t$ y $Q_{(0)}$ es la cantidad inicial de bacterias, se debe cumplir que:

$$
\frac{d Q_{(t)}}{d t}=K Q \quad(K>0)
$$

De donde:

$$
\begin{gathered}
d Q=\frac{d Q}{d t} \cdot d t=K Q d t \\
\frac{d Q}{Q}=K d t \Rightarrow \int_{Q_{0}}^{Q_{(t)}} \frac{d Q}{Q}=\int_{0}^{t} K d t \\
\left.\ln Q\right|_{Q_{0}} ^{Q_{(t)}}=\left.K t\right|_{0} ^{t} \\
\ln Q_{(t)}-\ln Q_{0}=K(t-0)
\end{gathered}
$$




$$
\ln \frac{Q_{(t)}}{Q_{0}}=K t \Rightarrow Q_{(t)}=Q_{0} e^{K t}
$$

Ejemplo 120:

Los siguientes datos los recolectó un investigador durante los 10 primeros minutos de un experimento.

\begin{tabular}{c|c|c}
\hline Tiempo & 0 & $10^{\prime}$ \\
\hline Número de bacterias & 5,000 & 8,000 \\
\hline
\end{tabular}

Asuma que el número de bacterias crece exponencialmente y encuentre cuántas bacterias habrá al cabo de 30 minutos.

De acuerdo con $Q_{(t)}=Q_{0} e^{k t}$ y con los datos dados:

$$
\begin{aligned}
& Q_{(10)}=5,000 e^{10 k}=8,000 \\
& Q_{(30)}=5,000 e^{30 k}
\end{aligned}
$$

De (1):

$$
e^{10 K}=\frac{8,000}{5,000}=1.6
$$

En (2):

$$
\begin{gathered}
Q_{(30)}=5,000\left(e^{10 K}\right)^{3} \\
Q_{(30)}=5,000(1.6)^{3} \\
Q_{(30)}=20,480 \text { bacterias }
\end{gathered}
$$

Se corrobora que el crecimiento exponencial implica que, para intervalos iguales, el porcentaje de variación es el mismo. Así pues:

$$
\begin{aligned}
& Q_{(0)}=5,000 \text { bacterias } \\
& Q_{(10)}=5,000 \times 1.6=8,000 \text { bacterias } \\
& Q_{(20)}=8,000 \times 1.6=12,800 \text { bacterias } \\
& Q_{(30)}=12,800 \times 1.6=20,480 \text { bacterias }
\end{aligned}
$$




\section{Ejemplo 121:}

Los registros de salud pública indican que la velocidad de crecimiento del número de organismos al cabo de $t$ minutos viene dada por:

$$
80(76)(1.2) \frac{e^{-1.2 t}}{\left(4+76 e^{-1.2 t}\right)^{2}} \quad \frac{\text { miles de organismos }}{\text { minuto }}
$$

Encontrar el número de organismos al cabo de 2 minutos si había 1,000 organismos inicialmente.

Se tiene que:

$$
\begin{aligned}
& \frac{d Q_{(t)}}{d t}=80(76)(1.2) \frac{e^{-1.2 t}}{\left(4+76 e^{-1.2 t}\right)^{2}} \\
& d Q_{(t)}=80(76)(1.2) \frac{e^{-1.2 t}}{\left(4+76 e^{-1.2 t}\right)^{2}} d t
\end{aligned}
$$

Integrando con límites definidos:

110

$$
\begin{gathered}
\int_{1}^{Q_{(t)}} d Q_{(t)}=\int_{0}^{t} 80(76)(1.2) \frac{e^{-1.2 t}}{\left(4+76 e^{-1.2 t}\right)^{2}} d t \\
Q_{(t)}-1=\left.\frac{80}{4+76 e^{-1.2 t}}\right|_{0} ^{t} \\
Q_{(t)}=1+\left(\frac{80}{4+76 e^{-1.2 t}}-\frac{80}{4+76 e^{0}}\right)
\end{gathered}
$$

De donde:

$$
Q_{(t)}=1+\frac{80}{4+76 e^{-1.2 t}}-1=\frac{80}{4+76 e^{-1.2 t}}
$$

Con lo cual:

$$
Q_{(2)}=\frac{80}{4+76 e^{-1.2(2)}}=7.343 \text { miles de organismos }
$$

\section{PROBLEMAS FÍSICOS}

Están referidos a un sinnúmero de situaciones relacionadas con la distancia, velocidad, aceleración, masa, centro de masa, trabajo, presión, temperatura, entre otros. 


\section{Ejemplo 122:}

Una partícula se mueve a lo largo de una línea recta de tal forma que la velocidad $v$ (metros/ segundo) a los $t$ segundos es:

$$
v=\frac{t+3}{t^{2}+3 t+2}
$$

Calcule la distancia recorrida por la partícula a partir del tiempo en que $t=0$ hasta cuando $t=2$.

La distancia recorrida se calcula como:

$$
d=\int_{0}^{2} v d t=\int_{0}^{2} \frac{t+3}{t^{2}+3 t+2} d t=\int_{0}^{2} \frac{t+3}{(t+1)(t+2)} d t
$$

Es decir:

$$
\begin{gathered}
d=\int_{0}^{2} \frac{2}{t+1} d t-\int_{0}^{2} \frac{1}{t+2} d t \\
d=\left.2 \ln (t+1)\right|_{0} ^{2}-\left.\ln (t+2)\right|_{0} ^{2} \\
d=2 \ln \frac{3}{1}-\ln \frac{4}{2} \\
d=2 \ln 3-\ln 2=\ln \frac{9}{2}
\end{gathered}
$$

La distancia recorrida en los 2 primeros segundos es 1.504 metros.

Ejemplo 123:

La medida de la densidad lineal en cualquier punto de una barra de 6 metros de largo varía de forma directamente proporcional a la distancia del punto a un punto externo en la línea de la barra situado a 4 metros de un extremo, en donde la densidad es $3 \mathrm{~kg} / \mathrm{m}$. Encontrar la masa total de la barra dada y la ubicación del centro de masa.

Coloquemos la barra lineal según se muestra en la figura:

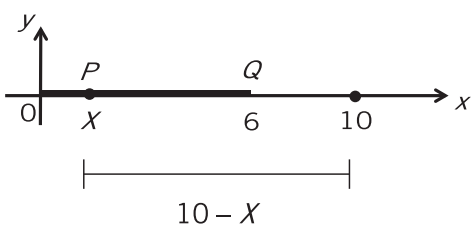


La densidad lineal $\rho_{(x)}$ del punto $P$ que está situado a una distancia $x$ del origen es:

$$
\rho_{(x)}=K(10-x)
$$

La densidad lineal del punto $Q$ es $3 \mathrm{~kg} / \mathrm{m}$, entonces:

$$
\rho_{(6)}=K(10-6)=4 K=3 \Rightarrow K=\frac{3}{4}
$$

Si la densidad lineal es $\rho_{(x)}=\frac{3}{4}(10-x)$, la masa es:

$$
\begin{gathered}
M=\int_{0}^{6} \rho_{(x)}=\frac{3}{4} \int_{0}^{6}(10-x) d x \\
M=\left.\frac{3}{4}\left[10 x-\frac{x^{2}}{2}\right]\right|_{0} ^{6} \\
M=\frac{3}{4}(60-18-0)=31.5 \mathrm{~kg} .
\end{gathered}
$$

112 El centro de masa se encuentra a $X_{M}$ del origen $O$ y se lo calcula como:

$$
X_{M}=\frac{\int_{0}^{6} x \rho_{(x)} d x}{\int_{0}^{6} \rho_{(x)} d x}=\frac{\frac{3}{4} \int_{0}^{6} x(10-x) d x}{M}
$$

Es decir:

$$
X_{M}=\frac{\left.\frac{3}{4}\left[5 x^{2}-\frac{x^{3}}{3}\right]\right|_{0} ^{6}}{31.5}=\frac{1}{42}[180-72-0]=2.57 \text { metros }
$$




\section{REGLAS PARA APROXIMAR EL VALOR DE $\int_{a}^{b} f_{(x)} d x$} En el capítulo V se indicó que de acuerdo con el Teorema Fundamental del Cálculo, $\int_{a}^{b} f_{(x)} d x=\left.g_{(x)}\right|_{a} ^{b}$,
siendo $g_{(x)}$ una antiderivada o primitiva de $f_{(x)}$.

Sin embargo, ¿qué sucedería si no fuera posible encontrar la antiderivada de $f_{(x)}$ ? (Por ejemplo, trate de calcular $\left.\int \sqrt{x^{4}+1} d x \circ \int e^{-x^{2} / 2} d x\right)$. Al no ser posible determinar $\int f_{(x)} d x$, no se podrá encontrar el valor de la integral definida $\int_{a}^{b} f_{(x)} d x$ de acuerdo con el Teorema Fundamental del
Cálculo.

En el capítulo VII se señaló que la integral $\int_{a}^{b} f_{(x)} d x$ corresponde al área bajo la curva $y=f_{(x)}$ comprendida entre las rectas $x=a$ y $x=b$, siendo $f_{(x)} \geq 0, \forall x \in[a, b]$. Por lo tanto, bastará con dar un valor aproximado del área, mediante métodos numéricos.

Los métodos más utilizados para la integración numérica son:

- La regla del punto medio

- La regla del trapecio

- La regla de Simpson 
En los tres métodos, si se trata de evaluar $\int_{a}^{b} f_{(x)} d x$, se debe descomponer la región limitada por la gráfica de $y=f_{(x)}$, el eje $x$ y las rectas $y=a$ e $y=b$ en " $n$ " franjas verticales, como se muestra a continuación, de preferencia de ancho constante. Así pues:

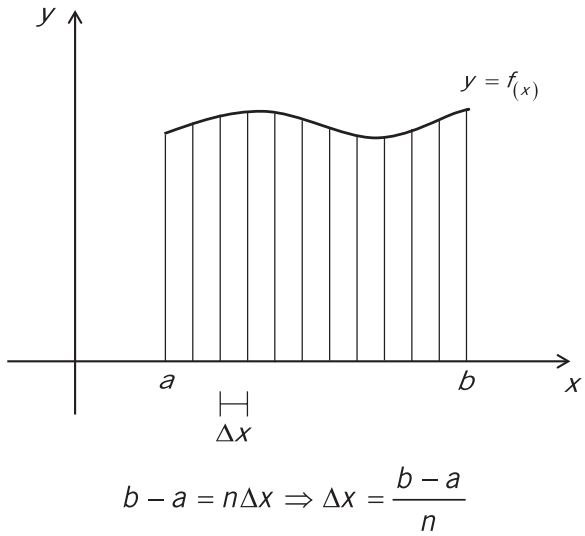

1.1 REGLA DEL PUNTO MEDIO
114 Dividamos la región correspondiente en " $n$ " franjas verticales de ancho constante $\Delta x=\frac{b-a}{n} y$ ubiquemos el punto medio $c_{i}$ de cada franja.

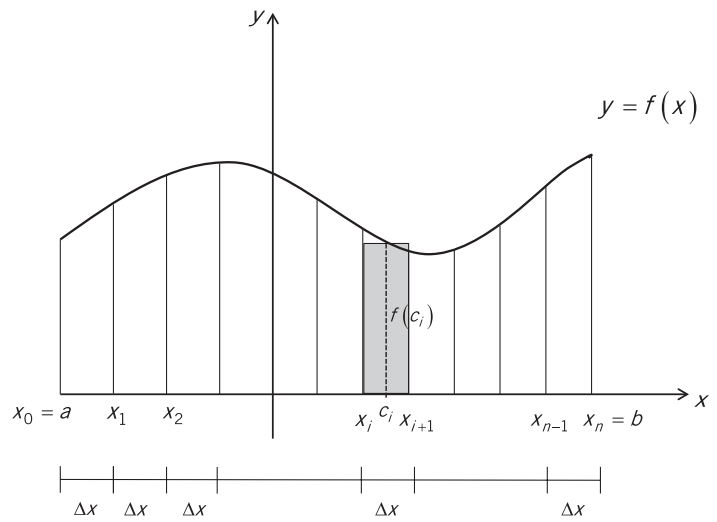

La figura anterior es similar a la mostrada en el capítulo VII. El área del elemento diferencial rectangular es $f_{(c i)} \Delta x$ aproximadamente. Con lo cual:

$$
A \approx \sum_{i=0}^{n-1} f_{\left(c_{i}\right)} \Delta x
$$


De donde:

$$
\int_{a}^{b} f_{(x)} d x \approx \sum_{i=0}^{n-1} f_{\left(c_{i}\right)} \Delta x
$$

Ejemplo 124:

Encontrar $\int_{3}^{9} x^{2} d x$ con la regla del punto medio. Asuma 12 franjas verticales.

Se tiene que:

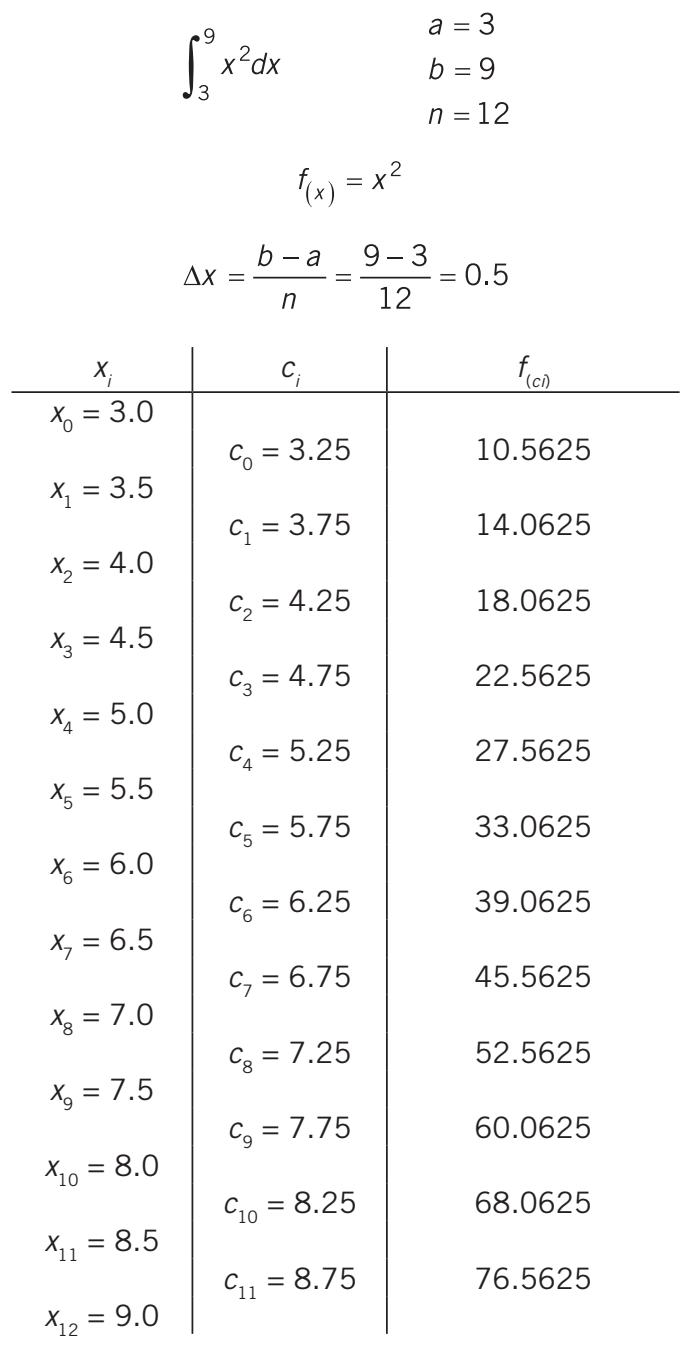


Con lo cual:

$$
\begin{gathered}
\int_{3}^{9} x^{2} d x \approx f_{\left(c_{0}\right)} \Delta x+f_{(c 1)} \Delta x+\ldots+f_{\left(c_{11}\right)} \Delta x \\
\int_{3}^{9} x^{2} d x \approx \Delta x \sum_{i=0}^{11} f_{\left(c_{i}\right)} \\
\int_{3}^{9} x^{2} d x \approx 0.5[467.75]=233.875
\end{gathered}
$$

El valor exacto de la integral es:

$$
\left.\frac{x^{3}}{3}\right|_{3} ^{9}=\frac{729}{3}-\frac{27}{3}=234
$$

\subsection{REGLA DEL TRAPECIO}

En lugar de considerar un elemento diferencial rectangular, como en la regla anterior, asumamos un elemento diferencial trapezoidal como el que se muestra a continuación.

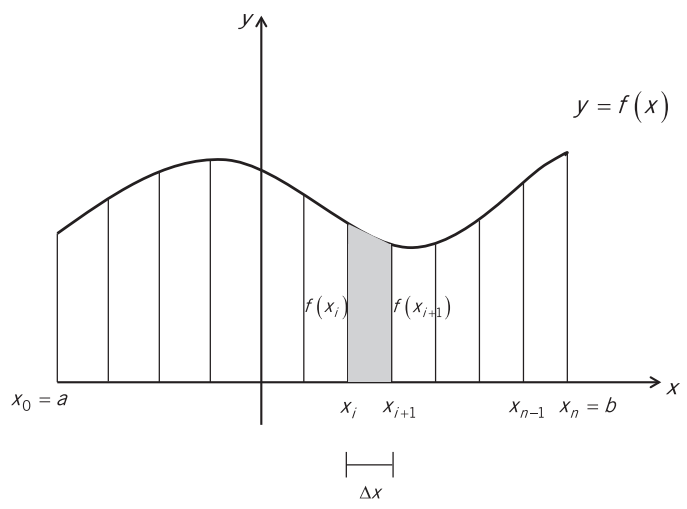

Se cumple que el área aproximada de dicho elemento es:

$$
\frac{\left[f_{\left(x_{i}\right)}+f_{\left(x_{i+1}\right)}\right]}{2} \Delta x
$$

Con lo cual, el área total aproximada será:

$$
A \approx\left[\frac{f_{\left(x_{0}\right)}+f_{\left(x_{1}\right)}}{2}+\frac{f_{\left(x_{1}\right)}+f_{\left(x_{2}\right)}}{2}+\frac{f_{\left(x_{2}\right)}+f_{\left(x_{3}\right)}}{2}+\ldots+\frac{f_{\left(x_{n-1}\right)}+f_{\left(x_{n}\right)}}{2}\right] \Delta x
$$


De donde:

$$
\begin{gathered}
A \approx\left[\frac{f_{\left(x_{0}\right)}+2 f_{\left(x_{1}\right)}+2 f_{\left(x_{2}\right)}+\ldots+2 f_{\left(x_{n-1}\right)}+f_{\left(x_{n}\right)}}{2}\right] \Delta x \\
A \approx\left[\frac{f_{\left(x_{0}\right)}+f_{\left(x_{n}\right)}}{2}+\sum_{i=1}^{n-1} f_{\left(x_{i}\right)}\right] \Delta x
\end{gathered}
$$

Finalmente:

$$
\int_{a}^{b} f_{(x)} d x \approx\left[\frac{f_{(a)}+f_{(b)}}{2}+\sum_{i=1}^{n-1} f_{\left(x_{i}\right)}\right] \Delta x
$$

Ejemplo 125:

Aproximar el valor de $\int_{-8}^{-2} \frac{d x}{x}$ con la regla del trapecio. Considere 12 intervalos de ancho constante.

Se tiene:

$$
\int_{-8}^{-2}\left(\frac{1}{x}\right) d x \quad f_{(x)}=\frac{1}{x} \quad \begin{aligned}
& b \\
& n=-2 \\
& n=12
\end{aligned} \quad \Delta x=\frac{b-a}{n}=0.5
$$

\begin{tabular}{c|c|c}
$i$ & $x_{i}$ & $f_{\left(x_{i}\right)}$ \\
\hline 0 & -8.0 & -0.12500 \\
1 & -7.5 & -0.13333 \\
2 & -7.0 & -0.14286 \\
3 & -6.5 & -0.15385 \\
4 & -6.0 & -0.16666 \\
5 & -5.5 & -0.18182 \\
6 & -5.0 & -0.20000 \\
7 & -4.5 & -0.22222 \\
8 & -4.0 & -0.25000 \\
9 & -3.5 & -0.28571 \\
10 & -3.0 & -0.33333 \\
11 & -2.5 & -0.40000 \\
12 & -2.0 & -0.50000
\end{tabular}

De donde:

$$
\int_{-8}^{-2} \frac{d x}{x} \approx\left[\frac{f_{(-8)}+f_{(-2)}}{2}+\sum_{i=1}^{11} f_{\left(x_{i}\right)}\right] \Delta x
$$




$$
\int_{-8}^{-2} \frac{d x}{x} \approx[-0.3125+(-2.46978)] 0.5=-1.39114
$$

El valor exacto de la integral es:

$$
\int_{-8}^{-2} \frac{d x}{x}=\ln |x|_{-8}^{-2}=\ln 2-\ln 8=\ln 2-3 \ln 2=-2 \ln 2=-1.38629
$$

\subsection{REGLA DE SIMPSON}

En el subacápite anterior se analizó la regla del trapecio, la cual aproxima los tramos cortos de la gráfica de la curva $y=f_{(x)}$ mediante segmentos de recta.

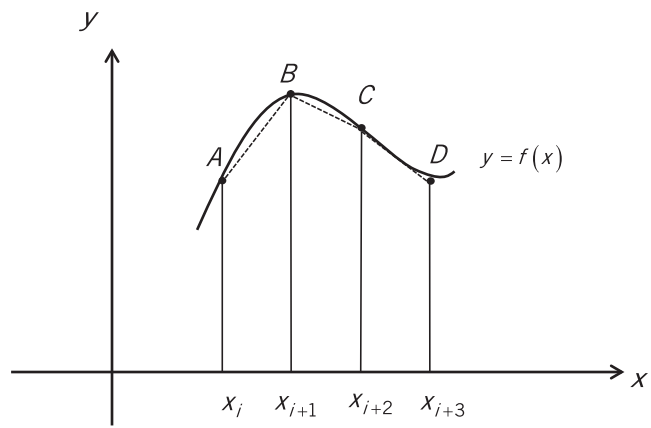

Un método que proporciona una mejor estimación de la integral definida es la regla de Simpson, la cual considera un polinomio de orden superior que pasa por los puntos $A\left(x_{i}, y_{i}\right), B\left(x_{i+1}, y_{i+1}\right)$ y $C\left(x_{i+2}, y_{i+2}\right)$.

Consideremos en primer lugar un polinomio de grado 2 que corresponde a la ecuación de una parábola, y en segundo lugar un polinomio de Lagrange de segundo orden.

\subsubsection{Polinomio cuadrático}

Analicemos una subregión cualquiera de ancho $2 h$ y consideremos la función cuadrática $y=g_{(x)}=$ $a x^{2}+b x+c$ que pasa por los puntos $A, B$ y $C$ que pertenecen a la función $y=f_{(x)}$.

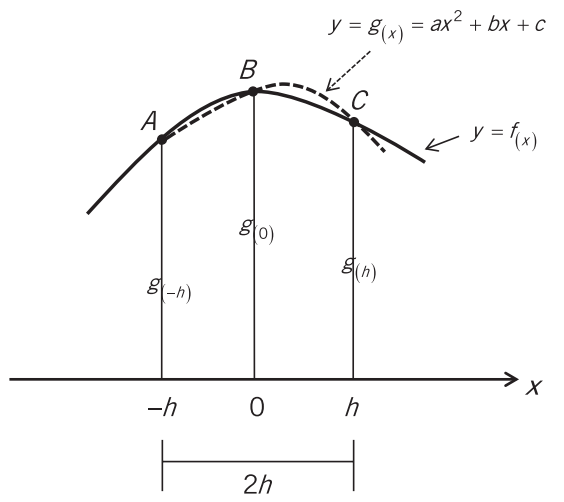


El área de la subregión mostrada comprendida entre el arco de la parábola, el eje $x$ y las rectas vértices $x=h \wedge x=h$, viene dada por:

$$
A \approx \int_{-h}^{h} g_{(x)} d x=\int_{-h}^{h}\left(a x^{2}+b x+c\right) d x=\frac{a x^{3}}{3}+\frac{b x^{2}}{2}+\left.c x\right|_{-h} ^{h}
$$

De donde:

$$
\begin{gathered}
A \approx \frac{1}{3} a h^{3}+\frac{1}{2} b h^{2}+c h-\left(-\frac{1}{3} a h^{3}+\frac{1}{2} b h^{2}-c h\right) \\
A \approx \frac{2}{3} a h^{3}+2 c h=\frac{h}{3}\left[2 a h^{2}+6 c\right]
\end{gathered}
$$

Comparemos el gráfico anterior con el de una subregión genérica:

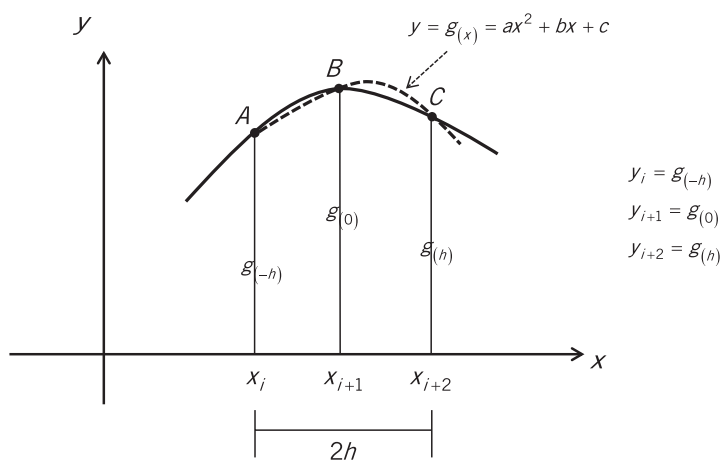

Se desprende que:

$$
\begin{gathered}
y_{i}=g_{\left(x_{i}\right)}=g_{(-h)}=a(-h)^{2}+b(-h)+c=a h^{2}-b h+c \ldots \\
y_{i+1}=g_{\left(x_{i+1}\right)}=g_{(0)}=a(0)^{2}+b(0)+c=c \\
y_{i+2}=g_{\left(x_{i+2}\right)}=g_{(h)}=a(h)^{2}+b(h)+c=a h^{2}+b h+c \ldots
\end{gathered}
$$

De $1+3$ :

$$
y_{i}+y_{i+2}=2 a h^{2}+2 c
$$

De $(\alpha)$ :

$$
A \approx \frac{h}{3}\left[2 a h^{2}+6 c\right]=\frac{h}{3}\left[\left(2 a h^{2}+2 c\right)+4 c\right]
$$


(2) y (4) en $(\beta)$ :

$$
A \approx \frac{h}{3}\left[y_{i}+y_{i+2}+4 y_{i+1}\right]
$$

Con lo cual, el área de la subregión genérica de ancho $2 h$ es:

$$
A \approx \frac{h}{3}\left[y_{i}+4 y_{i+1}+y_{i+2}\right]
$$

Si tenemos en cuenta que el área de toda la región ha sido dividida en $n$ subintervalos de ancho $2 h$ constante, encontraremos la fórmula siguiente:

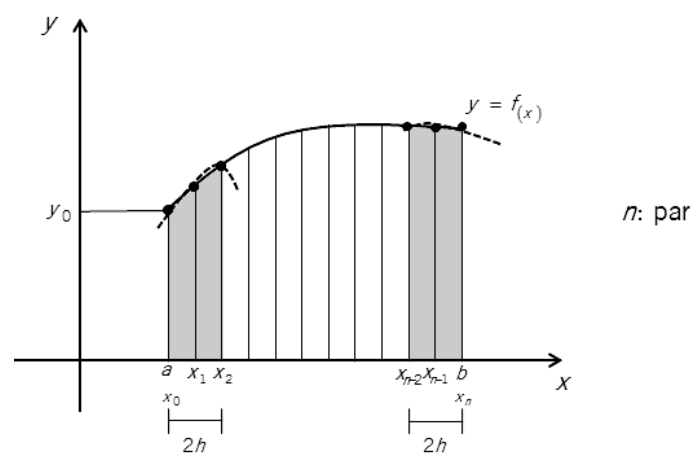

$$
\begin{gathered}
A \approx \frac{h}{3}\left[\left(y_{0}+4 y_{1}+y_{2}\right)+\left(y_{2}+4 y_{3}+y_{4}\right)+\ldots+\left(y_{2 k-2}+4 y_{2 k-1}+y_{2 k}\right)+\ldots+\left(y_{n-2}+4 y_{n-1}+y_{n}\right)\right] \\
A \approx \frac{h}{3}\left[y_{0}+4\left(y_{1}+y_{3}+y_{5}+\ldots+y_{n-1}\right)+2\left(y_{2}+y_{4}+y_{6}+\ldots+y_{n-2}\right)+y_{n}\right]
\end{gathered}
$$

O mejor:

$$
\int_{a}^{b} f_{(x)} d x \approx \frac{h}{3}[\underbrace{4 \sum_{y_{\text {impares }}}^{n / 2} y_{2 k-1}}_{y_{\text {izquierdo }} y_{0}}+\underbrace{2 \sum_{k=2}^{n / 2} y_{2 k-2}}_{y_{\text {pares }}}+\underbrace{y_{n}}_{y_{\text {derecho }}}]
$$

\subsubsection{Polinomio de Lagrange de $2^{\circ}$ orden}

Analicemos una subregión cualquiera de ancho $2 h$ y consideremos el polinomio de Lagrange $g_{(x)}$ que cumpla con:

$$
g_{(a)}=f_{(a)} ; g_{\left(x_{m}\right)}=f_{\left(x_{m}\right)} \text { y } g_{(b)}=f_{(b)}
$$




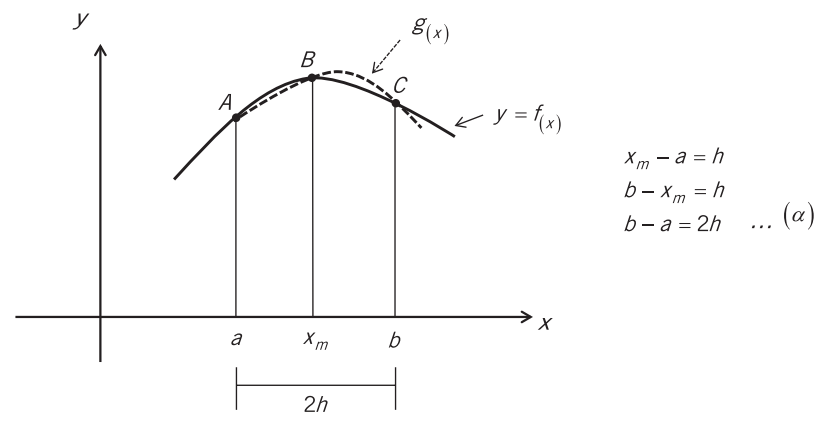

Sea $g_{(x)}=f_{(a)} \frac{\left(x-x_{m}\right)(x-b)}{\left(a-x_{m}\right)(a-b)}+f_{\left(x_{m}\right)} \frac{(x-a)(x-b)}{\left(x_{m}-a\right)\left(x_{m}-b\right)}+f_{(b)} \frac{(x-a)\left(x-x_{m}\right)}{(b-a)\left(b-x_{m}\right)}$

Si tenemos en cuenta a $(\alpha)$ :

$$
g_{(x)}=f_{(a)} \frac{\left(x-x_{m}\right)(x-b)}{(-h)(-2 h)}+f_{\left(x_{m}\right)} \frac{(x-a)(x-b)}{h(-h)}+f_{(b)} \frac{(x-a)\left(x-x_{m}\right)}{2 h(h)}
$$

De donde:

$$
g_{(x)}=\frac{f_{(a)}}{2 h^{2}}\left(x-x_{m}\right)(x-b)-\frac{f_{\left(x_{m}\right)}}{h^{2}}(x-a)(x-b)+\frac{f_{(b)}}{2 h^{2}}(x-a)\left(x-x_{m}\right)
$$

Con lo cual:

$$
A \approx \int_{a}^{b} g_{(x)} d x
$$

$$
A \approx \frac{f_{(a)}}{2 h^{2}} \underbrace{\int_{a}^{b}\left(x-x_{m}\right)(x-b) d x}_{l_{1}}-\frac{2 f_{\left(x_{m}\right)}}{2 h^{2}} \underbrace{\int_{a}^{b}(x-a)(x-b) d x}_{I_{2}}+\frac{f_{(b)}}{2 h^{2}} \underbrace{\int_{a}^{b}(x-a)\left(x-x_{m}\right) d x}_{I_{3}} \ldots(\beta)
$$

Las integrales de $I_{1}, I_{2}$ e $I_{3}$ corresponden a una integral semejante a:

$$
I=\int(x-p)(x-q) d x
$$

Integrando por partes:

$$
\begin{array}{cc}
u=x-p & v=\frac{(x-q)^{2}}{2} \\
\downarrow & \uparrow \\
d u=d x & d v=(x-q) d x
\end{array}
$$


De donde:

$$
\begin{aligned}
& I=\frac{(x-p)(x-q)^{2}}{2}-\frac{1}{2} \int(x-q)^{2} d x \\
& I=\frac{(x-p)(x-q)^{2}}{2}-\frac{1}{6}(x-q)^{3}+C \\
& I=\frac{(x-q)^{2}}{6}[3(x-p)-(x-q)]
\end{aligned}
$$

Calculemos las integrales $I_{1}, I_{2}$ e $I_{3}$ :

$$
\begin{aligned}
& I_{1}=\left.\frac{(x-b)^{2}}{6}\left[3\left(x-x_{m}\right)-(x-b)\right]\right|_{a} ^{b} \\
& I_{1}=0-\frac{(a-b)^{2}}{6}\left[3\left(a-x_{m}\right)-(a-b)\right]
\end{aligned}
$$

Si tenemos en cuenta a $(\alpha)$ :

122

$$
I_{1}=-\frac{(-2 h)^{2}}{6}[3(-h)-(-2 h)]=\frac{-2 h^{2}}{3}[-h]=\frac{2 h^{3}}{3}
$$

Análogamente:

$$
\begin{aligned}
& I_{2}=\left.\frac{(x-b)^{2}}{6}[3(x-a)-(x-b)]\right|_{a} ^{b} \\
& I_{2}=0-\frac{(a-b)^{2}}{6}[3(0)-(a-b)] \\
& I_{2}=-\frac{(-2 h)^{2}}{6}[-(-2 h)]=-\frac{4 h^{3}}{3}
\end{aligned}
$$

Del mismo modo:

$$
\begin{gathered}
I_{3}=\left.\frac{\left(x-x_{m}\right)^{2}}{6}\left[3(x-a)-\left(x-x_{m}\right)\right]\right|_{a} ^{b} \\
I_{3}=\frac{\left(b-x_{m}\right)^{2}}{6}\left[3(b-a)-\left(b-x_{m}\right)\right]-\frac{\left(a-x_{m}\right)^{2}}{6}\left[3(0)-\left(a-x_{m}\right)\right]
\end{gathered}
$$




$$
\begin{gathered}
I_{3}=\frac{h^{2}}{6}[3(2 h)-h]-\frac{(-h)^{2}}{6}[-(-h)] \\
I_{3}=\frac{5 h^{3}}{6}-\frac{h^{3}}{6}=\frac{4 h^{3}}{6}=\frac{2 h^{3}}{3}
\end{gathered}
$$

Reemplazando finalmente en $(\beta)$ :

$$
\begin{gathered}
A \approx \frac{f_{(a)}}{2 h^{2}} \frac{2 h^{3}}{3}-\frac{f_{\left(x_{m}\right)}}{h^{2}}\left(\frac{-4 h^{3}}{3}\right)+\frac{f_{(b)}}{2 h^{2}} \frac{2 h^{3}}{3} \\
A \approx \frac{h}{3}\left[f_{(a)}+4 f_{\left(x_{m}\right)}+f_{(b)}\right]
\end{gathered}
$$

Si se tuviera en cuenta el área de toda la región que ha sido dividida en $n$ subdivisiones de ancho $2 h$ constante, Ilegaremos a la fórmula siguiente:

$$
A \approx \frac{h}{3}\left[f_{\left(x_{0}\right)}+4 \sum_{k=1}^{n / 2} f_{\left(x_{2 k-1}\right)}+2 \sum_{k=2}^{n / 2} f_{\left(x_{2 k-2}\right)}+f_{\left(x_{n}\right)}\right]
$$

Es decir:

$$
\int_{a}^{b} f_{(x)} d x \approx \frac{h}{3}\left[f_{\left(x_{0}\right)}+4\left(f_{\left(x_{1}\right)}+f_{\left(x_{3}\right)}+\ldots+f_{\left(x_{n-1}\right)}\right)+2\left(f_{\left(x_{2}\right)}+f_{\left(x_{4}\right)}+\ldots f_{\left(x_{n-2}\right)}\right)+f_{\left(x_{n}\right)}\right]
$$

\section{Ejemplo 126:}

Hacer un valor aproximado de $\int_{a}^{4 a}\left(a x^{3}+2 a^{3} x\right) d x$ con la regla de Simpson. Considere $n=12$.

Se tiene que:

$$
\int_{a}^{4 a} \underbrace{}_{f_{(x)}\left(a x^{3}+2 a^{3} x\right)} d x \quad \begin{aligned}
& x_{0}=a \\
& x_{12}=4 a \\
& \Delta x=\frac{x_{12}-x_{0}}{12}=0.25 a
\end{aligned}
$$




\begin{tabular}{c|c|c}
$i$ & $x_{i}$ & $f_{\left(x_{i}\right)}$ \\
\hline 0 & $a$ & $3 a^{4}$ \\
1 & $1.25 a$ & $4.453125 a^{4}$ \\
2 & $1.5 a$ & $6.375 a^{4}$ \\
3 & $1.75 a$ & $8.859375 a^{4}$ \\
4 & $2 a$ & $12 a^{4}$ \\
5 & $2.25 a$ & $15.890625 a^{4}$ \\
6 & $2.5 a$ & $20.625 a^{4}$ \\
7 & $2.75 a$ & $26.296875 a^{4}$ \\
8 & $3 a$ & $33 a^{4}$ \\
9 & $3.25 a$ & $40.828125 a^{4}$ \\
10 & $3.5 a$ & $49.875 a^{4}$ \\
11 & $3.75 a$ & $60.234375 a^{4}$ \\
12 & $4 a$ & $72 a^{4}$
\end{tabular}

De donde:

124

$$
\begin{gathered}
\int_{a}^{4 a}\left(a x^{3}+2 a^{3} x\right) d x \approx \frac{0.25 a}{3}\left[f_{\left(x_{0}\right)}+4 \sum_{k=1}^{6} f_{\left(x_{2 k-1}\right)}+2 \sum_{k=2}^{6} f_{\left(x_{2 k-2}\right)}+f_{\left(x_{12}\right)}\right] \\
\int_{a}^{4 a}\left(a x^{3}+2 a^{3} x\right) d x \approx \frac{0.25 a}{3}\left[3 a^{4}+4\left(149.0625 a^{4}\right)+2\left(129.375 a^{4}\right)+72 a^{4}\right] \\
\int_{a}^{4 a}\left(a x^{3}+2 a^{3} x\right) d x \approx \frac{a}{12}\left[930 a^{4}\right]=77.50 a^{5}
\end{gathered}
$$

El valor exacto de la integral es:

$$
a \frac{x^{4}}{4}+\left.a^{3} x^{2}\right|_{a} ^{4 a}=\frac{a}{4}\left(256 a^{4}\right)+a^{3}\left(16 a^{2}\right)-\frac{a}{4}\left(a^{4}\right)-a^{3}\left(a^{2}\right)=78.75 a^{5}
$$

Ejemplo 127:

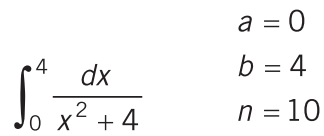

$$
\begin{aligned}
& \Delta x=\frac{b-a}{n}=\frac{4-0}{10}=0.4
\end{aligned}
$$




\section{Regla del punto medio:}

\begin{tabular}{c|c|c}
$c_{i}$ & $x_{i}$ & $f_{\left(c_{i}\right)}$ \\
\hline$x_{0}=0$ & $c_{0}=0.2$ & $f_{(0.2)}=0.247525$ \\
$x_{1}=0.4$ & $c_{1}=0.6$ & $f_{(0.6)}=0.229358$ \\
$x_{3}=1.2$ & $c_{2}=1.0$ & $f_{(1.0)}=0.200000$ \\
$x_{4}=1.6$ & $c_{3}=1.4$ & $f_{(1.4)}=0.167785$ \\
$x_{5}=2.0$ & $c_{4}=1.8$ & $f_{(1.8)}=0.138122$ \\
$x_{6}=2.4$ & $c_{5}=2.2$ & $f_{(2.2)}=0.113122$ \\
$x_{7}=2.8$ & $c_{6}=2.6$ & $f_{(2.6)}=0.092937$ \\
$x_{8}=3.2$ & $c_{7}=3.0$ & $f_{(3.0)}=0.076923$ \\
$x_{9}=3.6$ & $c_{8}=3.4$ & $f_{(3.4)}=0.364267$ \\
$x_{10}=4.0$ & $c_{9}=3.8$ & $f_{(3.8)}=0.054230$ \\
& &
\end{tabular}

La suma de Riemann es:

$$
\left[f_{(0.2)}+f_{(0.6)}+\ldots+f_{(3.4)}+f_{(3.8)}\right] \cdot \Delta x
$$

Es decir: $1.384269(0.4)=0.553708$

\section{Regla del trapecio:}

$$
\begin{gathered}
n=10 \\
\Delta x=0.4
\end{gathered}
$$




\begin{tabular}{c|c}
$x_{i}$ & $f_{\left(x_{i}\right)}$ \\
\hline$a=x_{0}=0$ & $f_{(0.0)}=0.250000$ \\
$x_{1}=0.4$ & $f_{(0.4)}=0.240385$ \\
$x_{2}=0.4$ & $f_{(0.8)}=0.215517$ \\
$x_{3}=0.4$ & $f_{(1.2)}=0.183824$ \\
$x_{4}=0.4$ & $f_{(1.6)}=0.152439$ \\
$x_{5}=0.4$ & $f_{(2.0)}=0.125000$ \\
$x_{6}=0.4$ & $f_{(2.4}=0.102459$ \\
$x_{7}=0.4$ & $f_{(2.8)}=0.084459$ \\
$x_{8}=0.4$ & $f_{(3.2)}=0.070225$ \\
$x_{9}=0.4$ & $f_{(3.6)}=0.058962$ \\
$b=x_{10}=4.0$ & $f_{(4.0)}=0.050000$
\end{tabular}

El valor aproximado de la integral es:

$$
\begin{gathered}
{\left[\frac{f_{(0)}+f_{(4)}}{2}+\sum_{k=1}^{9} f_{(0.4 k)}\right] \Delta x} \\
{\left[\frac{0.25+0.05}{2}+1.233270\right](0.4)}
\end{gathered}
$$

126

Es decir: $1.38327(0.4)=0.553308$

\section{Regla de Simpson:}

$$
\begin{aligned}
& a=x_{0}=0 \\
& b=x_{10}=4 \\
& n=10 \\
& \Delta x=\frac{b-a}{n}=\frac{4-0}{10}=0.4
\end{aligned}
$$

Se tiene que:

$$
\int_{0}^{4} \frac{d x}{x^{2}+4} \approx \frac{\Delta x}{3}\left[f_{\left(x_{0}\right)}+4 \sum_{k=1}^{5} f_{\left(x_{2 k-1}\right)}+2 \sum_{k=2}^{5} f_{\left(x_{2 k-2}\right)}+f_{\left(x_{10}\right)}\right]
$$

Como:

$$
\begin{gathered}
\sum_{k=1}^{5} f_{\left(x_{2 k-1}\right)}=f_{\left(x_{1}\right)}+f_{\left(x_{3}\right)}+f_{\left(x_{5}\right)}+f_{\left(x_{7}\right)}+f_{\left(x_{9}\right)}=0.692630 \\
\sum_{k=1}^{5} f_{\left(x_{2 k-2}\right)}=f_{\left(x_{2}\right)}+f_{\left(x_{4}\right)}+f_{\left(x_{6}\right)}+f_{\left(x_{8}\right)}=0.540640
\end{gathered}
$$


Entonces:

$$
\begin{gathered}
\int_{0}^{4} \frac{d x}{x^{2}+4} \approx \frac{0.4}{3}[0.25+4(0.692630)+2(0.540640)+0.05] \\
\int_{0}^{4} \frac{d x}{x^{2}+4} \approx \frac{0.4}{3}[4.1518]=0.553573
\end{gathered}
$$

Calculemos el valor de la integral:

$$
\int_{0}^{4} \frac{d x}{x^{2}+4}=\left.\frac{1}{2} \arctan \frac{x}{2}\right|_{0} ^{4}=\frac{1}{2}[\arctan 2-\arctan 0]
$$

El resultado es $\frac{1}{2} \arctan 2=0.553574358$.

La tabla siguiente muestra los resultados obtenidos:

\begin{tabular}{|cc|}
\hline Regla del punto medio & 0.553708 \\
Regla del trapecio & 0.553308 \\
Regla de Simpson & 0.553573 \\
\hline $\int_{0}^{4} \frac{d x}{x^{2}+4}$ & 0.553574 \\
\hline
\end{tabular}

Ejemplo 128:

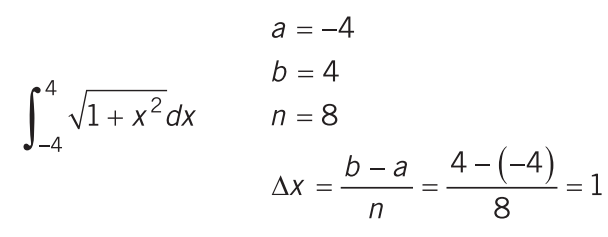

\section{Regla del punto medio:}

\begin{tabular}{c|c|c|c}
$i$ & $x_{i}$ & $c_{i}$ & $f_{\left(c_{i}\right)}$ \\
\hline 0 & -4 & -3.5 & 3.640055 \\
1 & -3 & -2.5 & 2.692582 \\
2 & -2 & -1.5 & 1.802776 \\
3 & -1 & -0.5 & 1.118034 \\
4 & 0 & 0.5 & 1.118034 \\
5 & 1 & 1.5 & 1.802776 \\
6 & 2 & 2.5 & 2.692582 \\
7 & 3 & 3.5 & 3.640055 \\
8 & 4 & &
\end{tabular}


La suma de Riemann es:

$$
\sum_{i=0}^{7} f_{\left(c_{i}\right)} \Delta x=(18.506894)(1)=18.506894
$$

\section{Regla del trapecio:}

128

$$
\int_{-4}^{4} \sqrt{1+x^{2}} d x \quad \begin{aligned}
& n=8 \\
& \Delta x=10
\end{aligned}
$$

\begin{tabular}{c|c|c}
$i$ & $x_{i}$ & $f_{\left(x_{i}\right)}$ \\
\hline 0 & -4 & 4.123106 \\
1 & -3 & 3.162278 \\
2 & -2 & 2.236068 \\
3 & -1 & 1.414214 \\
4 & 0 & 1.000000 \\
5 & 1 & 1.414214 \\
6 & 2 & 2.236068 \\
7 & 3 & 3.162278 \\
8 & 4 & 4.123106
\end{tabular}

El valor aproximado de la integral es:

$$
\begin{aligned}
& {\left[\frac{f_{(-4)}+f_{(4)}}{2}+\sum_{k=1}^{7} f_{(-4+k)}\right] \Delta x} \\
& {[4.123106+14.62512](1)}
\end{aligned}
$$

Es decir:

18.748226

\section{Regla de Simpson:}

$$
\begin{aligned}
& \begin{array}{ll}
\int_{-4}^{4} \sqrt{1+x^{2}} d x & b=x_{0}=-4 \\
& b=x_{8}=4 \\
n=8
\end{array} \\
& \Delta x=\frac{b-a}{n}=\frac{4-(-4)}{8}=1
\end{aligned}
$$

Se tiene que:

$$
\int_{-4}^{4} \sqrt{1+x^{2}} d x \approx \frac{\Delta x}{3}\left[f_{\left(x_{0}\right)}+4 \sum_{k=1}^{4} f_{\left(x_{2 k-1}\right)}+2 \sum_{k=2}^{4} f_{\left(x_{2 k-2}\right)}+f_{\left(x_{8}\right)}\right]
$$




$$
\begin{gathered}
\int_{-4}^{4} \sqrt{1+x^{2}} d x \approx \frac{1}{3}[4.123106+4(9.152984)+2(5.472136)+4.123106] \\
\int_{-4}^{4} \sqrt{1+x^{2}} d x \approx \frac{1}{3}[55.80242]=18.600810
\end{gathered}
$$

Calculamos el valor de la integral:

$$
\begin{gathered}
\int_{-4}^{4} \sqrt{1+x^{2}} d x=\left.\frac{1}{2}\left[x \sqrt{1+x^{2}}+\ln \left(x+\sqrt{1+x^{2}}\right)\right]\right|_{-4} ^{4} \\
\int_{-4}^{4} \sqrt{1+x^{2}} d x=\frac{1}{2}[4 \sqrt{17}+\ln (4+\sqrt{17})+4 \sqrt{17}-\ln (-4+\sqrt{17})] \\
\int_{-4}^{4} \sqrt{1+x^{2}} d x=16.492423+\frac{2.094713+2.094713}{2}=18.587136
\end{gathered}
$$

Comparemos los resultados obtenidos:

\begin{tabular}{|ll|}
\hline Regla del punto medio & 18.506894 \\
Regla del trapecio & 18.748226 \\
Regla de Simpson & 18.600810 \\
\hline $\int_{-4}^{4} \sqrt{1+x^{2}} d x$ & 18.587136 \\
\hline
\end{tabular}

Ejemplo 129:

$$
\int_{2}^{10} x \ln x d x \quad \begin{aligned}
& a=2 \\
& b=10 \\
& n=8 \\
& \Delta x=\frac{b-a}{n}=\frac{10-2}{8}=1
\end{aligned}
$$




\section{Regla del punto medio:}

130

\begin{tabular}{c|c|c}
$X_{i}$ & $c_{i}$ & $f_{\left(c_{i}\right)}$ \\
\hline$X_{0}=2$ & $c_{0}=2.5$ & $f_{(2.5)}=2.290727$ \\
$X_{1}=3$ & $c_{1}=3.5$ & $f_{(3.5)}=4.384670$ \\
$X_{3}=5$ & $c_{2}=4.5$ & $f_{(4.5)}=6.768348$ \\
$X_{4}=6$ & $c_{3}=5.5$ & $f_{(5.5)}=9.376115$ \\
$X_{5}=7$ & $c_{4}=6.5$ & $f_{(6.5)}=12.166714$ \\
$X_{6}=8$ & $c_{5}=7.5$ & $f_{(7.5)}=15.111773$ \\
$X_{7}=9$ & $c_{6}=8.5$ & $f_{(8.5)}=18.190562$ \\
$X_{8}=10$ & $c_{7}=9.5$ & $f_{(9.5)}=21.387272$
\end{tabular}

La suma de Riemann es:

$$
\sum_{i=1}^{8} f_{\left(c_{1.5+i}\right)} \Delta x=(89.676181)(1)=89.676181
$$

\section{Regla del trapecio:}

\begin{tabular}{c|c|c}
\multicolumn{2}{l}{$\int_{2}^{10} x \ln x d x$} & $\begin{array}{c}n=8 \\
\Delta x=1\end{array}$ \\
$i$ & $x_{i}$ & $f_{\left(x_{i}\right)}$ \\
\hline 0 & 2 & 1.386294 \\
1 & 3 & 3.295837 \\
2 & 4 & 5.545178 \\
3 & 5 & 8.047189 \\
4 & 6 & 10.750557 \\
5 & 7 & 13.621371 \\
6 & 8 & 16.635532 \\
7 & 9 & 19.775021 \\
8 & 10 & 23.025851
\end{tabular}


El valor aproximado de la integral es:

$$
\begin{gathered}
{\left[\frac{f_{(2)}+f_{(10)}}{2}+\sum_{k=1}^{7} f_{(2+k)}\right] \Delta x} \\
{[12.206072+77.670685](1)=89.876757}
\end{gathered}
$$

\section{Regla de Simpson:}

$$
\int_{2}^{10} x \ln x d x \quad \begin{aligned}
& a=2=x_{0} \\
& b=10=x_{8} \\
& n=8 \\
& \Delta x=1
\end{aligned}
$$

Se tiene que:

$$
\int_{2}^{10} x \ln x d x \approx \frac{\Delta x}{3}\left[f_{\left(x_{0}\right)}+4 \sum_{k=1}^{4} f_{\left(x_{2 k-1}\right)}+2 \sum_{k=2}^{4} f_{\left(x_{2 k-2}\right)}+f_{\left(x_{8}\right)}\right]
$$

Es decir:

$$
\begin{gathered}
\int_{2}^{10} x \ln x d x \approx \frac{\Delta x}{3}[1.386294+4(44.739418)+2(32.931267)+23.025851] \\
\int_{2}^{10} x \ln x d x \approx \frac{1}{3}(269.232330)=89.744110
\end{gathered}
$$

Calculemos el valor de la integral:

$$
\begin{array}{lc}
\int_{2}^{10} x \ln x d x & \\
u=\ln x & v=\frac{x^{2}}{2} \\
\downarrow & \uparrow \\
d u=\frac{d x}{x} & d v=x d x
\end{array}
$$

\section{Entonces:}

$$
\begin{gathered}
\int_{2}^{10} x \ln x d x=\left.\frac{x^{2}}{2} \ln x\right|_{2} ^{10}-\int_{2}^{10} \frac{x}{2} d x \\
\int_{2}^{10} x \ln x d x=50 \ln 10-2 \ln 2-\frac{1}{4}(100-4)
\end{gathered}
$$




$$
\int_{2}^{10} x \ln x d x=115.129255-1.386294-24=89.742961
$$

Comparemos los resultados anteriores:

\begin{tabular}{|ll|}
\hline Regla del punto medio & 89.676181 \\
Regla del trapecio & 89.876757 \\
Regla de Simpson & 89.744110 \\
\hline $\int_{2}^{10} x \ln x d x$ & 89.742961 \\
\hline
\end{tabular}

Ejemplo 130:

$$
\begin{array}{ll}
a & =2 \\
& b=5 \\
\int_{2}^{5} \frac{e^{x}}{x} d x \quad & n=12 \\
\Delta x & =\frac{b-a}{n}=\frac{5-2}{12}=0.25
\end{array}
$$

\section{Regla del punto medio:}

132

\begin{tabular}{c|c|c} 
& $c_{i}$ & $f_{\left(c_{i}\right)}$ \\
\hline$X_{0}=0$ & $c_{0}=2.125$ & 3.940187 \\
$X_{1}=2.25$ & $c_{1}=2.375$ & 4.526742 \\
$X_{3}=2.75$ & $c_{2}=2.625$ & 5.258885 \\
$X_{4}=3.00$ & $c_{3}=2.875$ & 6.165365 \\
$X_{5}=3.25$ & $c_{4}=3.125$ & 7.283166 \\
$X_{6}=3.50$ & $c_{5}=3.375$ & 8.659047 \\
$X_{7}=3.75$ & $c_{6}=3.625$ & 10.351648 \\
$X_{8}=4.00$ & $c_{7}=3.875$ & 12.434245 \\
$X_{9}=4.25$ & $c_{8}=4.125$ & 14.998257 \\
& $c_{9}=4.375$ & 18.157678 \\
\hline
\end{tabular}




$$
\begin{array}{l|l|l}
X_{10}=4.50 & c_{10}=4.625 & 22.054654 \\
X_{11}=4.75 & c_{11}=4.875 & 26.866493 \\
X_{12}=5.00 & &
\end{array}
$$

La suma de Riemann es:

$$
\sum_{i=0}^{11} f_{\left(c_{i}\right)} \cdot \Delta x=(140.69636)(0.25)=35.174090
$$

\section{Regla del trapecio:}

\begin{tabular}{c|c|c}
\multicolumn{3}{|c}{$n=12$} \\
\multicolumn{3}{|c}{$\Delta x=0.25$} \\
$i$ & $x_{i}$ & $f_{\left(x_{i}\right)}$ \\
\hline 0 & 2.00 & 3.694528 \\
1 & 2.25 & 4.216771 \\
2 & 2.50 & 4.872998 \\
3 & 2.75 & 5.688230 \\
4 & 3.00 & 6.695179 \\
5 & 3.25 & 7.935489 \\
6 & 3.50 & 9.461558 \\
7 & 3.75 & 11.338955 \\
8 & 4.00 & 13.649538 \\
9 & 4.25 & 16.495391 \\
10 & 4.50 & 20.003807 \\
11 & 4.75 & 24.333534 \\
12 & 5.00 & 29.682632
\end{tabular}

El valor aproximado de la integral es:

$$
\left[\frac{f_{(2)}+f_{(5)}}{2}+\sum_{k=1}^{11} f_{(2+0.25 k)}\right] \Delta x
$$

$[16.68858+124.69144](0.25)$

Es decir:

$141.38002(0.25)=35.345005$ 


\section{Regla de Simpson:}

$$
\begin{aligned}
& a=x_{0}=2 \\
& b=x_{12}=5 \\
& n=12 \\
& \Delta x=\frac{b-a}{n}=\frac{5-2}{12}=0.25
\end{aligned}
$$

Se tiene que:

$$
\int_{2}^{5} \frac{e^{x}}{x} d x \approx \frac{\Delta x}{3}\left[f_{\left(x_{0}\right)}+4 \sum_{k=1}^{6} f_{\left(x_{2 k-1}\right)}+2 \sum_{k=2}^{6} f_{\left(x_{2 k-2}\right)}+f_{\left(x_{12}\right)}\right]
$$

Como:

$$
\begin{aligned}
& \sum_{k=1}^{6} f_{\left(x_{2 k-1}\right)}=70.00837 \\
& \sum_{k=2}^{6}\left(x_{2 k-2}\right) \quad 54.68308
\end{aligned}
$$

Entonces:

134

$$
\begin{gathered}
\int_{2}^{5} \frac{e^{x}}{x} d x \approx \frac{0.25}{3}[3.694528+4(70.00837)+2(54.68308)+29.682632] \\
\int_{2}^{5} \frac{e^{x}}{x} d x \approx \frac{0.25}{3}[422.77679]=35.231396
\end{gathered}
$$

Comparemos los resultados:

\begin{tabular}{|ll}
\hline Regla del punto medio & 35.174090 \\
Regla del trapecio & 35.345005 \\
Regla de Simpson & 35.231396 \\
\hline
\end{tabular}

Ejemplo 131:

$$
\begin{aligned}
& a=\frac{\pi}{4} \text { radianes }=45^{\circ} \\
& \int_{\pi / 4}^{5 \pi / 12} \operatorname{sen} x d x \quad b=\frac{5 \pi}{12} \text { radianes }=75^{\circ} \\
& n=15 \\
& \Delta x=\frac{b-a}{n}=\frac{75^{\circ}-45^{\circ}}{15}=2^{\circ} \\
& \Delta x=2^{\circ} \times \frac{\pi \text { radianes }}{180^{\circ}}=\frac{\pi}{90} \text { radianes }
\end{aligned}
$$




\section{Regla del punto medio:}

\begin{tabular}{c|c|c|c}
$i$ & $x_{i}$ & $c_{i}$ & $f_{\left(c_{i}\right)}$ \\
\hline 0 & $45^{\circ}$ & $46^{\circ}$ & 0.719340 \\
1 & $47^{\circ}$ & $48^{\circ}$ & 0.743145 \\
2 & $49^{\circ}$ & $50^{\circ}$ & 0.766044 \\
3 & $51^{\circ}$ & $52^{\circ}$ & 0.788011 \\
4 & $53^{\circ}$ & $54^{\circ}$ & 0.809017 \\
5 & $55^{\circ}$ & $56^{\circ}$ & 0.829038 \\
6 & $57^{\circ}$ & $58^{\circ}$ & 0.848048 \\
7 & $59^{\circ}$ & $60^{\circ}$ & 0.866025 \\
8 & $61^{\circ}$ & $62^{\circ}$ & 0.882948 \\
9 & $63^{\circ}$ & $64^{\circ}$ & 0.898794 \\
10 & $65^{\circ}$ & $66^{\circ}$ & 0.913545 \\
11 & $67^{\circ}$ & $68^{\circ}$ & 0.927184 \\
12 & $69^{\circ}$ & $70^{\circ}$ & 0.939693 \\
13 & $71^{\circ}$ & $72^{\circ}$ & 0.951057 \\
14 & $73^{\circ}$ & $74^{\circ}$ & 0.961262 \\
15 & $75^{\circ}$ & &
\end{tabular}

La suma de Riemann es:

Regla del trapecio:

$$
\sum_{i=0}^{14} f_{\left(c_{i}\right)} \cdot \Delta x=(12.843151)(\pi / 90)=0.4483105
$$

$$
\int_{\pi / 4}^{5 \pi / 12} \operatorname{sen} x d x \quad \begin{aligned}
& n=15 \\
& \Delta x=2^{\circ}=\frac{\pi}{90} \text { radianes }
\end{aligned}
$$

\begin{tabular}{c|c|c}
$i$ & $x_{i}$ & $f_{\left(x_{i}\right)}$ \\
\hline 0 & $45^{\circ}$ & 0.707107 \\
1 & $47^{\circ}$ & 0.731354 \\
2 & $49^{\circ}$ & 0.754709 \\
3 & $51^{\circ}$ & 0.777146 \\
4 & $53^{\circ}$ & 0.798636 \\
5 & $55^{\circ}$ & 0.819152 \\
6 & $57^{\circ}$ & 0.838671 \\
7 & $59^{\circ}$ & 0.857167 \\
8 & $61^{\circ}$ & 0.874620 \\
9 & $63^{\circ}$ & 0.891007 \\
10 & $65^{\circ}$ & 0.906308 \\
11 & $67^{\circ}$ & 0.920505 \\
12 & $69^{\circ}$ & 0.933580 \\
13 & $71^{\circ}$ & 0.945519 \\
14 & $73^{\circ}$ & 0.956305 \\
15 & $75^{\circ}$ & 0.965926
\end{tabular}


El valor aproximado de la integral es:

$$
\begin{aligned}
& {\left[\frac{f_{\left(45^{\circ}\right)}+f_{\left(75^{\circ}\right)}}{2}+\sum_{k=1}^{14} f_{\left(45^{\circ}+2 k\right)}\right] \Delta x} \\
& {[0.836517+12.004679]\left(\frac{\pi}{90}\right)}
\end{aligned}
$$

Es decir:

$$
(12.841196) \frac{\pi}{90}=0.448242
$$

\section{Regla de Simpson:}

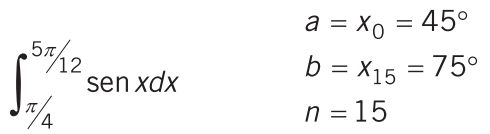

$$
\begin{aligned}
& \Delta x=\frac{75^{\circ}-45^{\circ}}{15}=2^{\circ}=\frac{\pi}{90} \text { radianes }
\end{aligned}
$$

136

Se tiene que:

$$
\int_{\pi / 4}^{5 \pi / 12} \operatorname{sen} x d x \approx \frac{\Delta x}{3}\left[f_{\left(x_{0}\right)}+4\left\{f_{\left(x_{1}\right)}+f_{\left(x_{3}\right)}+\ldots+f_{\left(x_{13}\right)}\right\}+2\left\{f_{\left(x_{2}\right)}+f_{\left(x_{4}\right)}+\ldots+f_{\left(x_{14}\right)}\right\}+f_{\left(x_{15}\right)}\right]
$$

Es decir:

$$
\begin{gathered}
\int_{\pi / 4}^{5 \pi / 12} \operatorname{sen} x d x \approx \frac{\pi / 90}{3}\left[f_{\left(45^{\circ}\right)}+4\left\{f_{\left(47^{\circ}\right)}+f_{\left(51^{\circ}\right)}+\ldots+f_{\left(71^{\circ}\right)}\right\}+2\left\{f_{\left(49^{\circ}\right)}+f_{\left(53^{\circ}\right)}+\ldots+f_{\left(73^{\circ}\right)}\right\}+f_{\left(75^{\circ}\right)}\right] \\
\int_{\pi / 4}^{5 \pi / 12} \operatorname{sen} x d x \approx \frac{\pi}{270}[0.707107+4(5.941850)+2(6.062829)+0.965926] \\
\int_{\pi / 4}^{5 \pi / 12} \operatorname{sen} x d x \approx \frac{\pi}{270}(37.566091)=0.437101
\end{gathered}
$$

Calculemos el valor de la integral:

$$
\begin{aligned}
& \int_{\pi / 4}^{5 \pi / 12} \operatorname{sen} x d x=-\left.\cos x\right|_{\pi / 4} ^{5 \pi / 12}=-\cos 75^{\circ}+\cos 45^{\circ} \\
& \int_{\pi / 4}^{5 \pi / 12} \operatorname{sen} x d x=-0.258819+0.707107=0.448288
\end{aligned}
$$


Comparemos los resultados obtenidos:

\begin{tabular}{|cc|}
\hline Regla del punto medio & 0.4483105 \\
Regla del trapecio & 0.448242 \\
Regla de Simpson & 0.437101 \\
\hline $\int_{\pi / 4}^{5 \pi / 12} \operatorname{sen} x d x$ & 0.448288 \\
\hline
\end{tabular}

Ejemplo 132:

La distribución normal o curva normal se define como sigue:

$$
f_{(x)}=\frac{1}{\sigma \sqrt{2 \pi}} e^{-\frac{1}{2} \cdot \frac{(x-\mu)^{2}}{\sigma^{2}}},
$$

en donde $\mu$ y $\sigma$ son constantes arbitrarias, y constituye uno de los ejemplos más importantes de una distribución de probabilidad continua. La gráfica de $f_{(x)}$ para $\mu=0$ y $\sigma=1$ es:

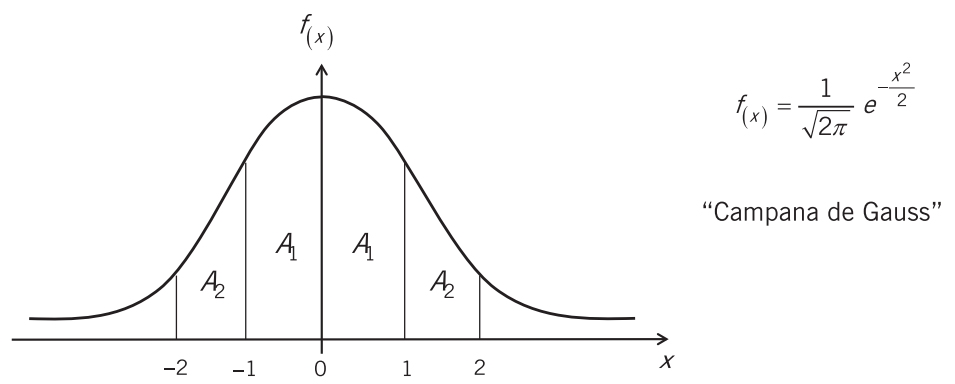

En las tablas de las áreas de la curva normal estándar de los libros de Estadística figuran los siguientes resultados:

$$
A_{1}=0.3413 \text { y } A_{1}+A_{2}=0.4772
$$

Emplee métodos numéricos para determinar $A_{1}$ y $A_{1}+A_{2}$.

Se tiene que $f_{(x)}=\frac{1}{\sqrt{2 \pi}} e^{-\frac{x^{2}}{2}} \rightarrow F_{(x)}=\sqrt{2 \pi} f_{(x)}=e^{-\frac{x^{2}}{2}}$.

Apliquemos el método del trapecio para encontrar $A_{1}$ y el método de Simpson para encontrar $A_{1}$ $+A_{2}$. 


\begin{tabular}{c|c|c}
$i$ & $x_{i}$ & $f_{\left(x_{i}\right)}$ \\
\hline 0 & 0 & 1.000000 \\
1 & 0.25 & 0.969233 \\
2 & 0.50 & 0.882496 \\
3 & 0.75 & 0.754840 \\
4 & 1.00 & 0.606531 \\
5 & 1.25 & 0.457834 \\
6 & 1.50 & 0.324652 \\
7 & 1.75 & 0.216265 \\
8 & 2.00 & 0.135335
\end{tabular}

De acuerdo con la regla del trapecio:

$$
\begin{gathered}
A_{1}=\int_{0}^{1} \frac{e^{-x^{2} / 2}}{\sqrt{2 \pi}} d x \approx \frac{1}{\sqrt{2 \pi}}\left[\frac{F_{\left(x_{0}\right)}+F_{\left(x_{4}\right)}}{2}+F_{\left(x_{1}\right)}+F_{\left(x_{2}\right)}+F_{\left(x_{3}\right)}\right]\left(\frac{1-0}{4}\right) \\
A_{1}=\int_{0}^{1} \frac{e^{-x^{2} / 2}}{\sqrt{2 \pi}} d x \approx \frac{1}{\sqrt{2 \pi}}\left(\frac{1}{4}\right)\left[\frac{1.606531}{2}+2.606569\right]
\end{gathered}
$$

138

$$
A_{1} \approx \frac{1}{4 \sqrt{2 \pi}}(3.4098345)=0.340082
$$

Según la regla de Simpson:

$$
\begin{gathered}
A_{2}=\int_{0}^{2} \frac{e^{-x^{2} / 2}}{\sqrt{2 \pi}} d x \approx \frac{1}{3}\left(\frac{2-0}{8}\right) \frac{1}{\sqrt{2 \pi}}\left[F_{\left(x_{0}\right)}+4\left[F_{\left(x_{1}\right)}+F_{\left(x_{3}\right)}+\ldots\right]+2\left[F_{\left(x_{2}\right)}+F_{\left(x_{4}\right)}+\ldots\right]+F_{\left(x_{8}\right)}\right] \\
A_{2} \approx \frac{1}{12 \sqrt{2 \pi}}[1.00+4(2.398172)+2(1.813679)+0.135335] \\
A_{2} \approx \frac{1}{12 \sqrt{2 \pi}}(14.355373)=0.47725
\end{gathered}
$$


$X$

INTEGRALES DOBLES

\section{INTEGRALES DOBLES}

Sea $F_{(x, y)}$ una función definida en una región cerrada $R$ del plano XOY. Se subdivide $\mathrm{R}$ en $n$ regiones $\Delta R_{K}$ de área $\Delta A_{K}, K=1,2, \ldots, n$. Consideremos $\left(\alpha_{K}, \beta_{K}\right)$, un punto cualquiera de $\Delta R_{K}$.

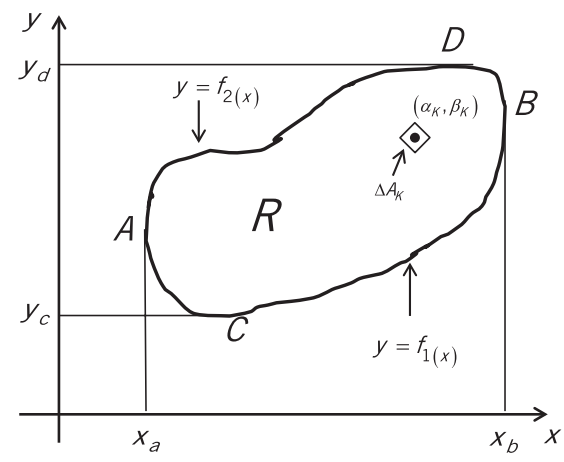

Se forma la suma:

$$
\sum_{K=1}^{n} F_{\left(\alpha_{k}, \beta_{K}\right)} \cdot \Delta A_{K}
$$


Consideremos $\lim _{n \rightarrow \infty} \sum_{K=1}^{n} F_{\left(\alpha_{K}, \beta_{K}\right)} \cdot \Delta A_{K}$ de modo que la máxima dimensión lineal de cada $\Delta R_{K}$ tiende a O. Si dicho límite existe, lo denotaremos por $\iint_{R} F_{(x, y)} d A$ y lo denominaremos integral doble de $F_{(x, y)}$ sobre la región $\mathrm{R}$.

\section{INTEGRALES REITERADAS}

Si asumimos que el elemento diferencial de área $\Delta A_{K}$ es un cuadrado de lados $d x$ y $d y$, paralelos a los ejes coordenados, entonces la integral doble será:

$$
\iint_{R} F_{(x, y)} d x d y
$$

Vale decir:

$$
\int_{x=x_{a}}^{x=x_{b}} \underbrace{\left\{\int_{y=f_{1(x)}}^{y=f_{2}(x)} F_{(x, y)} d y\right\}}_{\substack{\text { se asume que " } x " \\ \text { es constante }}} d x
$$

$$
\int_{y=y_{c}}^{y=y_{d}} \underbrace{\left\{\int_{x=g_{1(y)}}^{x=g_{2}(y)} F_{(x, y)} d x\right\}}_{\substack{\text { se asume que "y" } \\ \text { es constante }}} d y
$$

Lo que implica que una integral doble se puede calcular expresándola como dos integrales simples reiteradas.

Ejemplo 133:

Encontrar $\iint_{R} x y d x d y$, siendo $R$ la región mostrada:

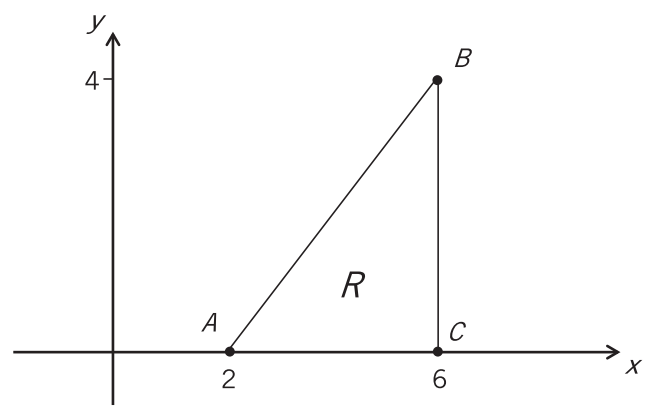


La recta que pasa por $A$ y $B$ tiene ecuación $y-4=1(x-6)$

$$
y=f_{(x)}=x-2
$$

Entonces:

$x$ varía desde 2 hasta 6 .

$y$ varía desde $y=0$ hasta $y=x-2$.

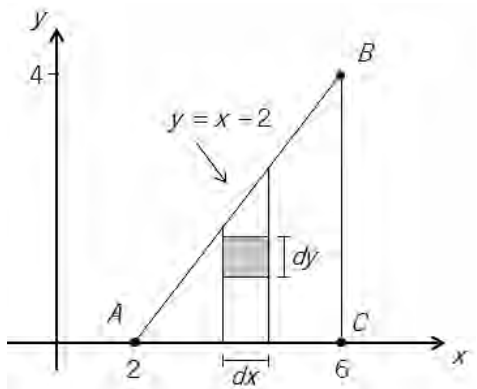

$$
\begin{aligned}
& \iint_{R} x y d x d y=\int_{2}^{6}\left[\int_{0}^{x-2} y d y\right] x d x \\
& \iint_{R} x y d x d y=\left.\int_{2}^{6} \frac{y^{2}}{2}\right|_{0} ^{x-2}(x d x)=\frac{1}{2} \int_{2}^{6}\left[(x-2)^{2}-0^{2}\right](x d x) \\
& \iint_{R} x y d x d y=\left.\frac{1}{2}\left(\frac{x^{4}}{4}-\frac{4 x^{3}}{3}+2 x^{2}\right)\right|_{2} ^{6}=\frac{1}{2}\left[(324-288+72)-\left(4-\frac{32}{3}+8\right)\right]=\frac{160}{3}
\end{aligned}
$$

Ejemplo 134:

Resolver el ejemplo anterior cambiando el orden de integración.

Sea:

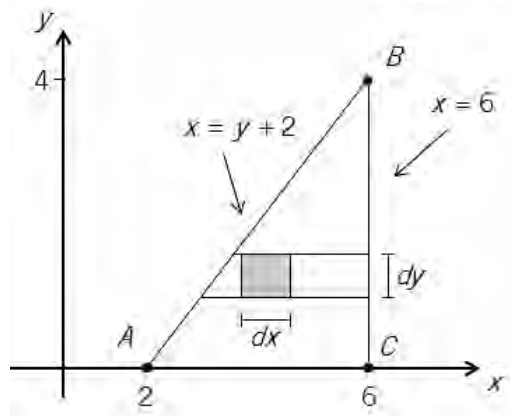




\section{Entonces:}

$y$ varía desde 0 hasta 4 .

$x$ varía desde $x=y+2$ hasta $x=6$.

$$
\begin{gathered}
\iint_{R} x y d x d y=\int_{0}^{4}\left[\int_{y+2}^{6} x d x\right](y d y) \\
\iint_{R} x y d x d y=\left.\int_{0}^{4} \frac{x^{2}}{2}\right|_{y+2} ^{6}(y d y)=\frac{1}{2} \int_{0}^{4}\left[6^{2}-(y+2)^{2}\right](y d y) \\
\iint_{R} x y d x d y=\left.\frac{1}{2}\left[16 y^{2}-\frac{4 y^{3}}{3}-\frac{y^{4}}{4}\right]\right|_{0} ^{4} \\
\iint_{R} x y d x d y=\frac{1}{2}\left[\left(256-\frac{256}{3}-64\right)-(0)\right]=\frac{160}{3}
\end{gathered}
$$

142

Ejemplo 135:

Calcular $\iint_{R}\left(x^{2}+y^{2}\right) d x d y$ siendo $R$ la región limitada por $y=x$ y $y^{2}=2 x$.

La región $R$ es:
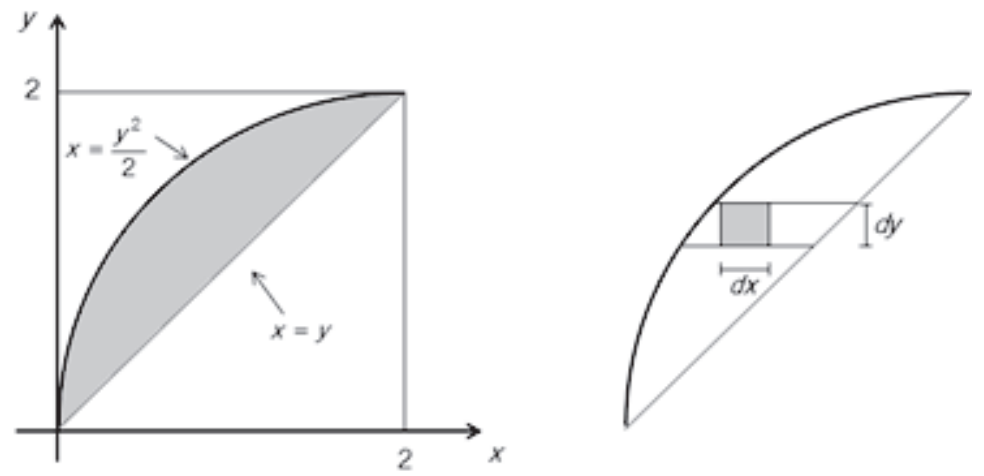

Se pide:

$$
\iint_{R}\left(x^{2}+y^{2}\right) d x d y=\int_{0}^{2}\left[\int_{y^{2} / 2}^{y}\left(x^{2}+y^{2}\right) d x\right] d y
$$


Es decir:

$$
\begin{aligned}
& \iint_{R}\left(x^{2}+y^{2}\right) d x d y=\left.\int_{0}^{2}\left(\frac{x^{3}}{3}+y^{2} x\right)\right|_{y^{2} / 2} ^{y} d y=\int_{0}^{2}\left[\frac{y^{3}}{3}+y^{3}-\frac{y^{6}}{24}-\frac{y^{4}}{2}\right] d y \\
& \iint_{R}\left(x^{2}+y^{2}\right) d x d y=\frac{y^{4}}{12}+\frac{y^{4}}{4}-\frac{y^{7}}{168}-\left.\frac{y^{5}}{10}\right|_{0} ^{2}=\frac{16}{12}+4-\frac{128}{168}-\frac{32}{10}=\frac{48}{35}
\end{aligned}
$$

Ejemplo 136:

Resolver el ejemplo anterior con otro orden de integración.
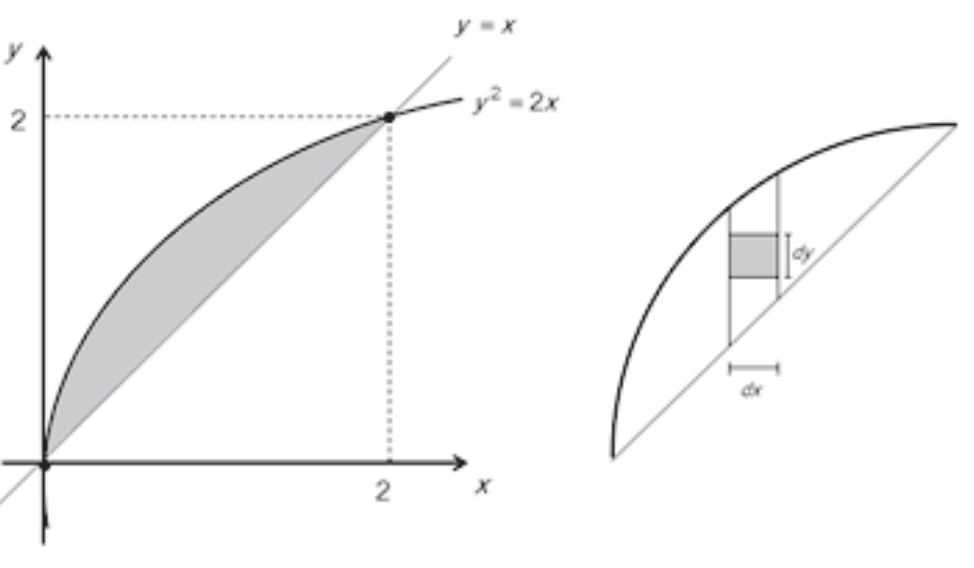

Se pide:

$$
\iint_{R}\left(x^{2}+y^{2}\right) d x d y=\int_{0}^{2}\left[\int_{x}^{\sqrt{2 x}}\left(x^{2}+y^{2}\right) d y\right] d x
$$

Es decir:

$$
\begin{gathered}
\iint_{R}\left(x^{2}+y^{2}\right) d x d y=\left.\int_{0}^{2}\left(x^{2} y+\frac{y^{3}}{3}\right)\right|_{x} ^{\sqrt{2 x}} d x=\int_{0}^{2}\left[\sqrt{2} x^{5 / 2}+\frac{1}{3}(2 \sqrt{2}) x^{3 / 2}-x^{3}-\frac{1}{3} x^{3}\right] d x \\
\iint_{R}\left(x^{2}+y^{2}\right) d x d y=\left.\left(\frac{\sqrt{2} x^{7 / 2}}{7 / 2}+\frac{2 \sqrt{2}}{3(5 / 2)} x^{5 / 2}-\frac{x^{4}}{4}-\frac{1}{12} x^{4}\right)\right|_{0} ^{2}
\end{gathered}
$$




$$
\begin{gathered}
\iint_{R}\left(x^{2}+y^{2}\right) d x d y=\frac{2 \sqrt{2}}{7}(2)^{7 / 2}+\frac{4}{15} \sqrt{2}(2)^{5 / 2}-\frac{1}{4}(2)^{4}-\frac{1}{12}(2)^{4} \\
\iint_{R}\left(x^{2}+y^{2}\right) d x d y=\frac{2}{7}(16)+\frac{4}{15}(8)-4-\frac{4}{3}=\frac{48}{35}
\end{gathered}
$$

\section{CÁLCULO DEL ÁREA DE UNA REGIÓN PLANA}

El área de una región plana $\mathrm{R}$ se puede calcular con ayuda de las integrales dobles. Basta con asumir que $F_{(x, y)}=1$ en la fórmula del acápite 1. Entonces:

$$
\iint_{R} F_{(x, y)} d x d y=\iint_{R} d x d y
$$

Por ejemplo, si $x$ varía desde a hasta $b, y$ variará desde $y=f_{(x)}$ hasta $y=g_{(x)}$.

$$
A=\iint_{R} d x d y=\int_{a}^{b}\left[\int_{f_{(x)}}^{g(x)} d y\right] d x
$$

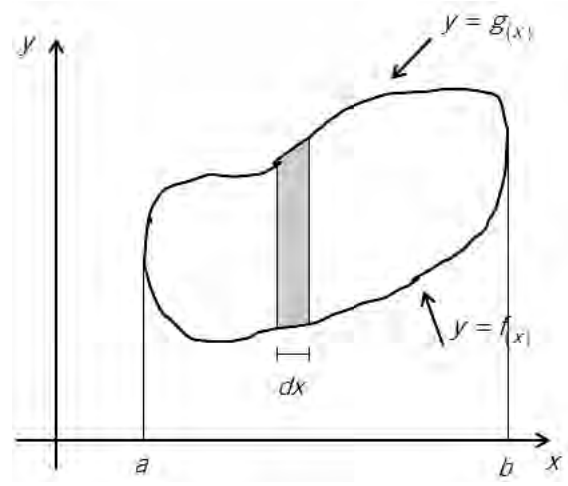

De esta manera, la integral doble sobre $\mathrm{R}$ cubre toda la región y se evalúa el área de la región mediante la integral doble.

Ejemplo 137:

Encontrar el área situada bajo la curva $y=x^{2}$ y por encima del eje $x$, comprendida entre las rectas $x=-2$ y $x=4$. (Véase el ejemplo 101). 


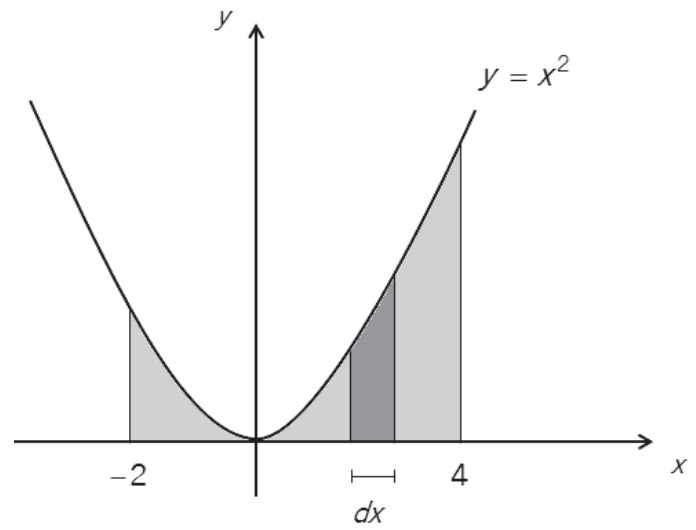

La región está limitada verticalmente por las rectas $x=-2$ y $x=4$, mientras que el elemento diferencial vertical de ancho $d x$ está limitado inferiormente por la recta $y=0$ y superiormente por la curva $y=x^{2}$.

Entonces:

$$
\begin{gathered}
A=\iint_{R} d x d y=\int_{-2}^{4}\left[\int_{0}^{x^{2}} d y\right] d x \\
A=\left.\int_{-2}^{4} y\right|_{0} ^{x^{2}} d x=\int_{-2}^{4}\left(x^{2}-0\right) d x=\left.\frac{x^{3}}{3}\right|_{-2} ^{4}
\end{gathered}
$$

Con lo cual:

$$
A=\frac{1}{3}\left(4^{3}-(-2)^{3}\right)=24 u n^{2}
$$

Ejemplo 138:

Encontrar el área sombreada en la figura (véase el ejemplo 102):

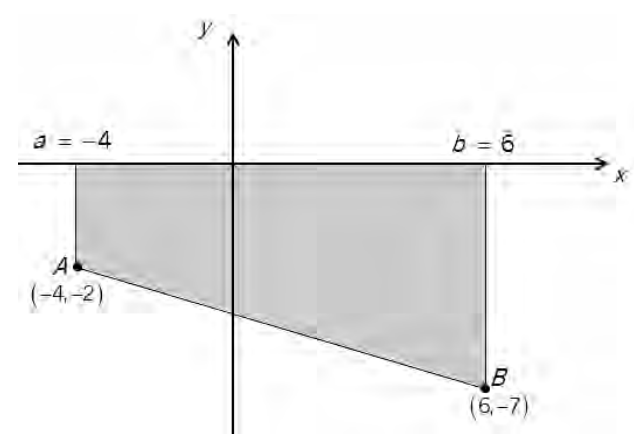


Si se escoge el elemento diferencial vertical, $x$ varía desde -4 hasta 6 , mientras que y varía desde $y=-\frac{1}{2} x-4$ (ecuación de la recta que pasa por $A$ y $B$ ) y el eje $x(y=0)$.

Con lo cual:

$$
\begin{gathered}
A=\iint_{R} d x d y=\int_{-4}^{6}\left[\int_{-\frac{1}{2} x-4}^{0} d y\right] d x \\
A=\left.\int_{-4}^{6} y\right|_{-\frac{1}{2} x-4} ^{0} d x=\int_{-4}^{6}\left[0+\frac{1}{2} x+4\right] d x \\
A=\frac{x^{2}}{4}+\left.4 x\right|_{-4} ^{6}=(9+24)-(4-16)=45 \mathrm{un}^{2}
\end{gathered}
$$

Ejemplo 139:

Determinar el área mostrada en la figura siguiente (véase el ejemplo 103):

146

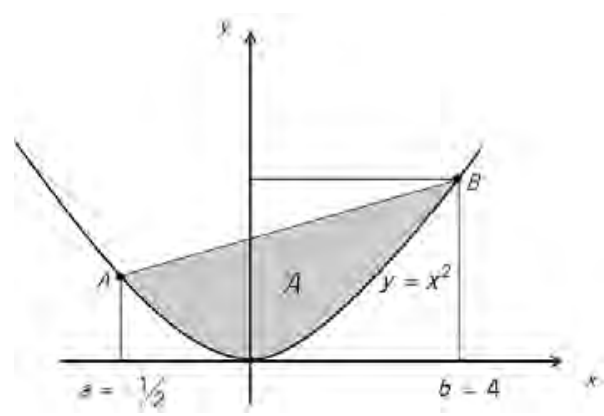

La ecuación de la recta que pasa por $A\left(\frac{-1}{2}, \frac{1}{4}\right)$ y $B(4,16)$ es:

$$
\begin{gathered}
y-16=\frac{16-\frac{1}{4}}{4+\frac{1}{2}}(x-4) \\
y-16=\frac{7}{2}(x-4) \rightarrow y=\frac{7}{2} x+2
\end{gathered}
$$


El área pedida es:

$$
\begin{gathered}
A=\iint_{R} d x d y=\int_{-1 / 2}^{4}\left[\int_{x^{2}}^{\frac{7}{2} x+2} d y\right] d x \\
A=\left.\int_{-1 / 2}^{4} y\right|_{x^{2}} ^{\frac{7}{2} x+2} d x=\int_{-1 / 2}^{4}\left[\frac{7}{2} x+2-x^{2}\right] d x
\end{gathered}
$$

La integral coincide con la del ejemplo 103. Por lo tanto, $A=\frac{243}{16} \mathrm{un}^{2}$.

Ejemplo 140:

Hallar el área comprendida entre la recta que pasa por A y B y la curva $y=x^{3}$, situada a la derecha de $x=x_{A}$ y a la izquierda de $x=x_{B}$ (véase el ejemplo 104).

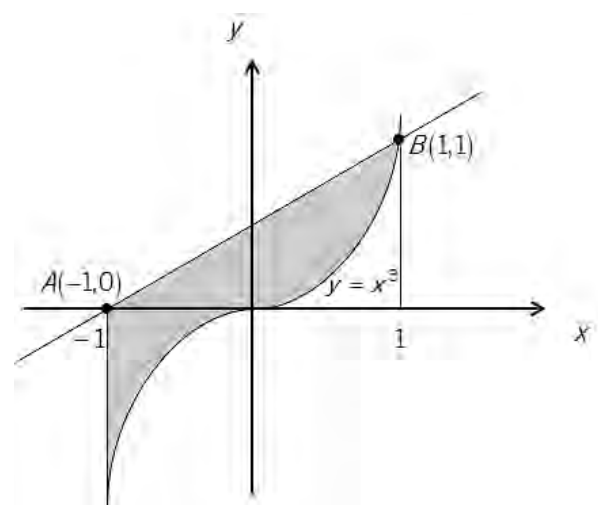

La ecuación de la recta que pasa por A y B es $y=\frac{1}{2} x+\frac{1}{2}$.

El área pedida es: $\int_{-1}^{1}\left[\int_{x^{3}}^{\frac{1}{2} x+\frac{1}{2}} d y\right] d x$

Es decir: $\int_{-1}^{1}\left(\frac{1}{2} x+\frac{1}{2}-x^{3}\right) d x$

La integral coincide con la del ejemplo 104. Por ende, $A=1$ un $^{2}$. 


\section{ANEXO 1: SUMATORIAS}

Consideremos la variable $x$ que depende del subíndice $i$ que toma valores enteros consecutivos donde $i=1$ hasta $i=n$. Por ejemplo:

$$
x_{i}=3 i+2, i=1 \text { hasta } i=80
$$

Entonces: $\quad x_{1}=3(1)+2=5$

$$
\begin{gathered}
x_{2}=3(2)+2=8 \\
x_{3}=3(3)+2=11 \\
\cdots \\
x_{80}=3(80)+2=242
\end{gathered}
$$

Si se requiere abreviar la suma $x_{1}+x_{2}+x_{3}+\ldots+x_{80}$ y calcularlo más sencillamente, es posible utilizar la sumatoria, la cual es un operador matemático representado por la letra griega sigma mayúscula: $\Sigma$. Esto es:

$$
\sum_{i=1}^{80} x_{i}=x_{1}+x_{2}+x_{3}+\ldots+x_{80}
$$

en donde el subíndice $i$ empieza con el valor que aparece en la parte inferior de $\Sigma$ y aumenta de uno en uno hasta llegar al valor que aparece en la parte superior de $\Sigma$.

Por la propiedad $\sum_{i=m}^{n}\left(x_{i}+y_{i}\right)=\sum_{i=m}^{n} x_{i}+\sum_{i=m}^{n} y_{i}$ se puede escribir:

$$
\sum_{i=1}^{80} x_{i}=\sum_{i=1}^{80}(3 i+2)=3 \sum_{i=1}^{80} i+\sum_{i=1}^{80} 2
$$

La sumatoria $\sum_{i=1}^{80} i$ corresponde a la suma de los 80 primeros números naturales $(1+2+3+\ldots$ +80) y se puede emplear la fórmula $\sum_{i=1}^{n} i=\frac{n(n+1)}{2}$. Entonces: $\sum_{i=1}^{80} i=\frac{80(80+1)}{2}=3,240$.

La sumatoria $\sum_{i=1}^{80} 2$ equivale a la suma $\underbrace{2+2+2+\ldots 2}_{80 \text { veces }}$ y se puede utilizar la fórmula $\sum_{i=m}^{n} K=K(n-m+1)$. Es decir: $\sum_{i=1}^{80} 2=2(80-1+1)=2(80)=160$. 
De donde:

$$
\sum_{i=1}^{80}(3 i+2)=3 \sum_{i=1}^{80} i+\sum_{i=1}^{80} 2=3(3,240)+(160)=9,880
$$

Ejemplos:

a) $6+8+10+\ldots+100=\sum_{i=3}^{50}(2 i)$
b) $16+25+36+\ldots+100=\sum_{i=4}^{10}\left(i^{2}\right)$

c) $8+16+32+\ldots+4,096$

$$
2^{3}+2^{4}+2^{5}+\ldots+2^{12}=\sum_{i=3}^{12}\left(2^{i}\right)
$$

d) $\frac{3}{16}+\frac{4}{25}+\frac{5}{36}+\ldots+\frac{15}{256}$

$$
\frac{3}{4^{2}}+\frac{4}{5^{2}}+\frac{5}{6^{2}}+\ldots+\frac{15}{16^{2}}=\sum_{i=4}^{16} \frac{i-1}{i^{2}}
$$

Ejemplo:

Calcular $\underbrace{22+25+28+\ldots}_{120 \text { sumandos }}$

Encontremos $x_{i}$ :

Como la diferencia entre dos términos consecutivos cualesquiera es 3 y el subíndice aumenta de uno en uno, conviene que aparezca el término $3 i$. De donde:

$$
\begin{aligned}
& x_{i}=3 i+a \\
& x_{1}=3(1)+a=22 \rightarrow a=19 \\
& \therefore \quad x_{i}=3 i+19
\end{aligned}
$$

Se pide:

$$
\sum_{i=1}^{120} x_{i}=\sum_{i=1}^{120}(3 i+19)=3 \sum_{i=1}^{120} i+19 \sum_{i=1}^{120} 1
$$

(Se aplicaron las propiedades $\sum_{i=m}^{n} K x_{i}=K \sum_{i=m}^{n} x_{i}$ y $\sum_{i=m}^{n} K=K \sum_{i=m}^{n} 1$ ) 
Entonces:

$$
\begin{aligned}
& \sum_{i=1}^{120} x_{i}=3 \frac{120(121)}{2}+19(120-1+1) \\
& \sum_{i=1}^{120} x_{i}=21,780+2,280=24,060
\end{aligned}
$$

Ejemplo:

Calcular $\underbrace{22+25+28+\ldots}_{120 \text { sumandos }}$ con valores inicial y final del subíndice que sean diferentes a los empleados en el ejercicio anterior.

Es posible escribir:

$$
\begin{array}{cc}
x_{1}=22 & x_{1}=7+3(5) \\
x_{2}=25 & x_{2}=7+3(6) \\
\ldots & \ldots \\
& x_{i}=7+3 i
\end{array}
$$

150 Entonces la suma pedida considerará valores de $i$ desde $i=5$ hasta $i=124$.

$$
\begin{gathered}
\sum_{i=5}^{124}(7+3 i)=7 \sum_{i=5}^{124} 1+3 \sum_{i=5}^{124} i \\
\sum_{i=5}^{124}(7+3 i)=7[124-5+1]+3\left[\sum_{i=1}^{124} i-\sum_{i=1}^{4} i\right] \\
\sum_{i=5}^{124}(7+3 i)=7(120)+3\left[\frac{124(125)}{2}-\frac{4(5)}{2}\right] \\
\sum_{i=5}^{124}(7+3 i)=840+3(7,740)=24,060
\end{gathered}
$$

\section{PROPIEDADES DE LAS SUMATORIAS}

Se señalarán algunas propiedades ya vistas y otras de igual importancia.

$$
\begin{array}{ll}
\text { P1) } & \sum_{i=m}^{n}\left(x_{i}+y_{i}\right)=\sum_{i=m}^{n} x_{i}+\sum_{i=m}^{n} y_{i} \\
\text { P2) } & \sum_{i=m}^{n} K x_{i}=K \sum_{i=m}^{n} x_{i}
\end{array}
$$




$$
\begin{aligned}
& \text { P3) } \sum_{i=m}^{n} 1=n-m+1 \\
& \text { P4) } \sum_{i=m}^{n} x_{i}=\sum_{i=m}^{p} x_{i}+\sum_{i=p+1}^{n} x_{i} \\
& \text { P5) } \sum_{i=m}^{n} x_{i}=\sum_{i=m+K}^{n+K} x_{i-K} \\
& \text { P6) } \sum_{i=1}^{n} i=\frac{n(n+1)}{2} \\
& \text { P7) } \sum_{i=1}^{n} 2 i=n(n+1) \\
& \text { P8) } \sum_{i=1}^{n}(2 i-1)=n^{2} \\
& \text { P9) } \sum_{i=1}^{n}(4 i-1)=n(2 n+1) \\
& \text { P10) } \sum_{i=1}^{n} i=(i+1)=\frac{n(n+1)(n+2)}{3} \\
& \text { P11) } \sum_{i=1}^{n} \frac{1}{i(i+1)}=\frac{n}{n+1} \\
& \text { P12) } \sum_{i=1}^{n} i^{2}=\frac{n(n+1)(2 n+1)}{6} i^{3}=\left[\frac{n(n+1)}{2}\right]^{2} \\
& \sum_{i=1}^{n} i^{4}=\frac{n(n+1)(2 n+1)\left(3 n^{2}+3 n-1\right)}{30} \\
& \text { P13 }
\end{aligned}
$$

Ejemplo:

Comprobar la propiedad 5 en el cálculo de la suma:

$$
6^{2}+7^{2}+8^{2}+\ldots+24^{2}
$$


Se deberá verificar que:

$$
\sum_{i=6}^{24} i^{2}=\sum_{i=6-5}^{24-5}(i+5)^{2}
$$

Es decir:

$$
\sum_{i=6}^{24} i^{2}=\sum_{i=1}^{19}(i+5)^{2}
$$

Calculemos:

$$
\begin{gathered}
\sum_{i=6}^{24} i^{2}=\sum_{i=1}^{24} i^{2}-\sum_{i=1}^{5} i^{2} \\
\sum_{i=6}^{24} i^{2}=\frac{24(25)(49)}{6}-\frac{5(6)(11)}{6} \\
\sum_{i=6}^{24} i^{2}=4,900-55=4,845
\end{gathered}
$$

Calculemos:

\section{2}

$$
\begin{gathered}
\sum_{i=1}^{19}(i+5)^{2}=\sum_{i=1}^{19} i^{2}+\sum_{i=1}^{19} 10 i+\sum_{i=1}^{19} 25 \\
\sum_{i=1}^{19}(i+5)^{2}=\frac{19(20)(39)}{6}+10 \frac{19(20)}{2}+25(19) \\
\sum_{i=1}^{19}(i+5)^{2}=2,470+1,900+475=4,845
\end{gathered}
$$




\section{ANEXO 2: FÓRMULAS DE DERIVACIÓN}

\section{REGLAS DE DERIVACIÓN}

Sean las funciones diferenciables $f_{(x)}, g_{(x)}$ y $h_{(x)}$ y la constante $C$ :

1. $\frac{d\left[f_{(x)} \pm g_{(x)}\right]}{d x}=\frac{d f_{(x)}}{d x} \pm \frac{d g_{(x)}}{d x}=f^{\prime}(x)^{ \pm} g^{\prime}(x)$

2. $\frac{d\left[C f_{(x)}\right]}{d x}=C \frac{d f_{(x)}}{d x}=C f^{\prime}(x)$

3. $\frac{d\left[f_{(x)} \cdot g_{(x)}\right]}{d x}=f_{(x)} \cdot \frac{d g_{(x)}}{d x}+g_{(x)} \cdot \frac{d f_{(x)}}{d x}=f_{(x)} g^{\prime}(x)+g_{(x)} f^{\prime}(x)$

4. $\frac{d\left[\frac{f_{(x)}}{g_{(x)}}\right]}{d x}=\frac{g_{(x)} \cdot \frac{d f_{(x)}}{d x}-f_{(x)} \cdot \frac{d g_{(x)}}{d x}}{\left[g_{(x)}\right]^{2}}=\frac{g_{(x)} f^{\prime}(x)-f_{(x)} g^{\prime}(x)}{\left[g_{(x)}\right]^{2}} \quad\left(g_{(x)} \neq 0\right)$

5. Si $y=f_{(u)} \operatorname{con} u=g_{(x)}$,

$$
\left.\frac{d y}{d x}=\frac{d y}{d u} \cdot \frac{d u}{d x}=f^{\prime}(u) \cdot \frac{d u}{d x}=f^{\prime} g_{(x)}\right) \cdot g_{(x)}^{\prime}
$$

6. Si $y=f_{(x)} \rightarrow x=f_{(y)}^{-1}$ y se cumple que $\frac{d y}{d x}=\frac{1}{d x / d y}$.

7. Si $x=f_{(t)}$ e $y=g_{(t)}$, se cumple que

$$
\frac{d y}{d x}=\frac{d y / d t}{d x / d t}=\frac{f^{\prime}(t)}{g^{\prime}(t)}
$$

\section{DERIVADA DE FUNCIONES USUALES}

Sea $u=f_{(x)}$ y las constantes $n$ y a.

1. $\frac{d}{d x}(C)=0$

2. $\frac{d}{d x} u^{n}=n u^{n-1} \frac{d u}{d x}$ 
3. $\frac{d}{d x} a^{u}=a^{u} \ln a \frac{d u}{d x}$

4. $\frac{d}{d x} e^{u}=e^{u} \frac{d u}{d x}$

5. $\frac{d}{d x} \log _{a} u=\frac{1}{u} \log _{a} e \frac{d u}{d x} \quad(a>0, a \neq 1)$

6. $\frac{d}{d x} \ln u=\frac{1}{u} \frac{d u}{d x}$

7. $\frac{d}{d x} \operatorname{sen} u=\cos u \frac{d u}{d x}$

8. $\frac{d}{d x} \cos u=-\operatorname{sen} u \frac{d u}{d x}$

9. $\frac{d}{d x} \tan u=\sec ^{2} u \frac{d u}{d x}$

10. $\frac{d}{d x} \cot u=-\csc ^{2} u \frac{d u}{d x}$

154 11. $\frac{d}{d x} \sec u=\sec u \tan u \frac{d u}{d x}$

12. $\frac{d}{d x} \csc u=-\csc u \cot u \frac{d u}{d x}$

13. $\frac{d}{d x} \operatorname{arcsen} u=\frac{1}{\sqrt{1-u^{2}}} \frac{d u}{d x}$

14. $\frac{d}{d x} \arccos u=\frac{-1}{\sqrt{1-u^{2}}} \frac{d u}{d x}$

15. $\frac{d}{d x} \arctan u=\frac{1}{1+u^{2}} \frac{d u}{d x}$

16. $\frac{d}{d x} \operatorname{arccot} u=\frac{-1}{1+u^{2}} \frac{d u}{d x}$

17. $\frac{d}{d x} \operatorname{arcsec} u= \pm \frac{1}{u \sqrt{u^{2}-1}} \frac{d u}{d x} \quad \begin{cases}+ \text { si } & u>1 \\ - \text { si } & u<-1\end{cases}$

18. $\frac{d}{d x} \operatorname{arccsc} u=\mp \frac{1}{u \sqrt{u^{2}-1}} \frac{d u}{d x} \quad \begin{cases}- \text { si } & u>1 \\ + \text { si } & u<-1\end{cases}$ 


\section{AMENIDAD 1}

En esta sección se encontrará la integral indefinida de cinco funciones, cada una de las cuales se obtendrá de dos o tres formas distintas.

Se aplicarán diferentes métodos de integración:

- Sustitución algebraica.

- Sustitución trigonométrica.

- Integración por partes.

- Por descomposición en fracciones parciales.

Asimismo, se las hallará de forma:

- Directa.

- Previa división.

- Previa factorización.

- Previa multiplicación y división.

1. a) Calcule la siguiente integral de dos maneras diferentes:

$$
\int\left(3 e^{3 u}+6 e^{2 u}+3 e^{u}\right) d u
$$

b) ¿Qué relación guardan entre sí las constantes de integración?

\section{Solución:}

a) De forma directa:

$$
\begin{gathered}
\int\left(3 e^{3 u}+6 e^{2 u}+3 e^{u}\right) d u=\int e^{3 u}(3 d u)+3 \int e^{2 u}(2 d u)+3 \int e^{u} d u \\
\int\left(3 e^{3 u}+6 e^{2 u}+3 e^{u}\right) d u=e^{3 u}+3 e^{2 u}+3 e^{u}+C_{1}
\end{gathered}
$$

$a_{\text {iij }}$ Previa factorización:

$$
\begin{aligned}
& \int\left(3 e^{3 u}+6 e^{2 u}+3 e^{u}\right) d u=3 \int\left(e^{2 u}+2 e^{u}+1\right)\left(e^{u} d u\right) \\
& \int\left(3 e^{3 u}+6 e^{2 u}+3 e^{u}\right) d u=3 \int\left(e^{u}+1\right)^{2}\left(e^{u} d u\right) \\
& \int\left(3 e^{3 u}+6 e^{2 u}+3 e^{u}\right) d u=3 \frac{\left(e^{u}+1\right)^{3}}{3}+C_{2} \\
& \int\left(3 e^{3 u}+6 e^{2 u}+3 e^{u}\right) d u=\left(e^{u}+1\right)^{3}+C_{2} \\
& \int\left(3 e^{3 u}+6 e^{2 u}+3 e^{u}\right) d u=e^{3 u}+3 e^{2 u}+3 e^{u}+1+C_{2}
\end{aligned}
$$


b) Comparando ambos resultados, se obtiene que $C_{1}=C_{2}+1$.

2. a) Encuentre de dos formas distintas la siguiente integral:

$$
\int \frac{x d x}{2 x+4}
$$

b) ¿Son iguales las dos constantes de integración?

\section{Solución:}

a.) Previa división:

$$
\begin{array}{l|lc}
\frac{-x-2}{-2} & \frac{2 x+4}{1 / 2} & \frac{x}{2 x+4}=\frac{1}{2}-\frac{2}{2 x+4} \\
& \int \frac{x d x}{2 x+4}=\int\left[\frac{1}{2}-\frac{1}{x+2}\right] d x=\frac{1}{2} x-\ln (x+2)+C_{1}
\end{array}
$$

$a_{\mathrm{ii}}$ ) Con cambio de variable:

$$
\text { Sea } u=2 x+4 \rightarrow d u=2 d x
$$

$$
\begin{aligned}
& \int \frac{x d x}{2 x+4}=\int \frac{\left(\frac{u-4}{2}\right)\left(\frac{d u}{2}\right)}{u}=\frac{1}{4} \int \frac{u-4}{u} d u \\
& \int \frac{x d x}{2 x+4}=\frac{1}{4} \int\left[1-\frac{4}{u}\right] d u \\
& \int \frac{x d x}{2 x+4}=\frac{1}{4}[u-4 \ln u]+C_{2} \\
& \int \frac{x d x}{2 x+4}=\frac{u}{4}-\ln u+C_{2} \\
& \int \frac{x d x}{2 x+4}=\frac{2 x+4}{4}-\ln (2 x+4)+C_{2} \\
& \int \frac{x d x}{2 x+4}=\frac{x}{2}+1-\ln 2-\ln (x+2)+C_{2} \\
& \int \frac{x d x}{2 x+4}=\frac{1}{2} x-\ln (x+2)+1-\ln 2+C_{2}
\end{aligned}
$$

b) De la comparación de los dos resultados obtenidos, solo se llega a $C_{1}=C_{2}$ si $1-\ln 2=0$ $\Rightarrow \ln 2=1$, lo cual no es cierto. Por lo tanto, $C_{1} \neq C_{2}$. 
3. a) Obtenga $\int \frac{x^{3} d x}{\sqrt{x^{2}+1}}$ de tres maneras distintas.

b) ¿Difieren entre sí las tres constantes de integración que se obtienen?

\section{Solución:}

$\left.a_{i}\right)$ Integración por partes:

$$
\begin{array}{cc}
u=x^{2} & v=\sqrt{x^{2}+1} \\
\downarrow & \uparrow \\
d u=2 x d x & d v=\frac{x d x}{\sqrt{x^{2}+1}} \\
\int \frac{x^{3} d x}{\sqrt{x^{2}+1}}=x^{2} \sqrt{x^{2}+1}-\int 2 x \sqrt{x^{2}+1} d x \\
\int \frac{x^{3} d x}{\sqrt{x^{2}+1}}=x^{2} \sqrt{x^{2}+1}-\frac{2\left(x^{2}+1\right)^{3 / 2}}{3}+C_{1}
\end{array}
$$

$\mathrm{a}_{\mathrm{ii}}$ ) Cambio de variable trigonométrico:

$$
\begin{gathered}
x=\tan \theta \rightarrow d x=\sec ^{2} \theta d \theta \\
x^{2}+1=\tan ^{2} \theta+1=\sec ^{2} \theta \\
\int \frac{x^{3} d x}{\sqrt{x^{2}+1}}=\int \frac{\tan ^{3} \theta\left(\sec ^{2} \theta d \theta\right)}{\sec \theta}=\int \tan ^{3} \theta \sec \theta d \theta \\
\int \frac{x^{3} d x}{\sqrt{x^{2}+1}}=\int \underbrace{\left[\sec ^{2} \theta-1\right]}_{\tan ^{2} \theta}[\sec \theta \tan \theta d \theta] \\
\int \frac{x^{3} d x}{\sqrt{x^{2}+1}}=\frac{\sec ^{3} \theta}{3}-\sec \theta+C_{2} \\
\int \frac{x^{3} d x}{\sqrt{x^{2}+1}}=\frac{1}{3} \sqrt{\left(x^{2}+1\right)^{3}}-\sqrt{x^{2}+1}+C_{2} \\
\int \frac{x^{3} d x}{\sqrt{x^{2}+1}}=\frac{1}{3}\left(x^{2}+1\right)^{3 / 2}-\sqrt{x^{2}+1}+C_{2}
\end{gathered}
$$


$a_{\text {iii }}$ ) Cambio de variable algebraico:

$$
\begin{gathered}
x^{2}+1=u^{2} \rightarrow 2 x d x=2 u d u \rightarrow x d x=u d u \\
\int \frac{x^{3} d x}{\sqrt{x^{2}+1}}=\int \frac{x^{2}(x d x)}{\sqrt{x^{2}+1}}=\int \frac{\left(u^{2}-1\right)(u d u)}{u} \\
\int \frac{x^{3} d x}{\sqrt{x^{2}+1}}=\int\left(u^{2}-1\right) d u=\frac{u^{3}}{3}-u+C_{3} \\
\int \frac{x^{3} d x}{\sqrt{x^{2}+1}}=\frac{1}{3}\left(x^{2}+1\right)^{3 / 2}-\sqrt{x^{2}+1}+C_{3}
\end{gathered}
$$

Obsérvese que los resultados $\mathrm{a}_{\mathrm{ii}}$ ) y $\mathrm{a}_{\mathrm{iii}}$ ) son idénticos. Por ende, $C_{2}=C_{3}$.

b) Finalmente, comprobemos que la diferencia entre los resultados obtenidos es una constante y averigüemos dicha constante. Restemos el resultado de las partes $\mathrm{a}_{\mathrm{ii}}$ ) $0 \mathrm{a}_{\mathrm{iii}}$ ) del resultado de la parte $a_{i}$ ).

158

$$
\begin{aligned}
& \Delta C=C_{2}-C_{1}=\left[x^{2} \sqrt{x^{2}+1}-\frac{2}{3}\left(x^{2}+1\right)^{3 / 2}\right]-\left[\frac{1}{3}\left(x^{2}+1\right)^{3 / 2}-\sqrt{x^{2}+1}\right] \\
& \Delta C=C_{2}-C_{1}=\sqrt{x^{2}+1}\left(x^{2}+1\right)-\frac{3}{3}\left(x^{2}+1\right)^{3 / 2} \\
& \Delta C=C_{2}-C_{1}=\sqrt{x^{2}+1}\left[x^{2}+1-\left(x^{2}+1\right)\right]=0
\end{aligned}
$$

Como $C_{2}-C_{1}=0$ y $C_{2}=C_{3} \Rightarrow C_{1}=C_{2}=C_{3}$. Ello significa que las tres constantes no difieren entre sí y que los tres resultados son coincidentes:

$$
\begin{gathered}
x^{2} \sqrt{x^{2}+1}-\frac{2}{3}\left(x^{2}+1\right)^{3 / 2}+C \\
0 \\
\frac{1}{3}\left(x^{2}+1\right)^{3 / 2}-\sqrt{x^{2}+1}+C
\end{gathered}
$$

4. a) Halle $\int \frac{x d x}{x^{4}+1}$ de tres formas diferentes.

b) ¿En cuánto difieren entre sí las constantes de integración halladas?

c) Mostrar cálculos que comprueben lo hallado en b). 


\section{Solución:}

$a_{\mathrm{i}}$ ) Cambio de variable algebraico:

$$
\begin{gathered}
u=x^{2} \rightarrow d u=2 x d x \\
\int \frac{x d x}{x^{4}+1}=\int \frac{\frac{1}{2} d u}{u^{2}+1}=\frac{1}{2} \int \frac{d u}{u^{2}+1} \\
\int \frac{x d x}{x^{4}+1}=\frac{1}{2} \arctan u+C_{1}=\frac{1}{2} \arctan x^{2}+C_{1}
\end{gathered}
$$

$\mathrm{a}_{\mathrm{ii}}$ ) Cambio de variable trigonométrico:

$$
\begin{gathered}
x^{2}=\tan \theta \rightarrow 2 x d x=\sec ^{2} \theta d \theta \\
x^{4}+1=\tan ^{2} \theta+1=\sec ^{2} \theta \\
\int \frac{x d x}{x^{4}+1}=\int \frac{\frac{1}{2} \sec ^{2} \theta d \theta}{\sec ^{2} \theta}=\frac{1}{2} \int d \theta=\frac{1}{2} \theta \\
\int \frac{x d x}{x^{4}+1}=\frac{1}{2} \arctan x^{2}+C_{2}
\end{gathered}
$$

$a_{i i i}$ ) Descomposición en fracciones parciales:

Factoricemos $x^{4}+1$

$$
\begin{gathered}
x^{4}+2 x^{2}+1-2 x^{2} \\
\left(x^{2}+1\right)^{2}-(\sqrt{2} x)^{2} \\
\left(x^{2}+1+\sqrt{2} x\right)\left(x^{2}+1-\sqrt{2} x\right) \\
\left(x^{2}+\sqrt{2} x+1\right)\left(x^{2}-\sqrt{2} x+1\right)
\end{gathered}
$$

Entonces:

$$
\begin{aligned}
& \frac{x}{x^{4}+1}=\frac{A x+B}{x^{2}+\sqrt{2} x+1}+\frac{C x+D}{x^{2}-\sqrt{2} x+1} \\
& \frac{x}{x^{4}+1}=\frac{(A x+B)\left(x^{2}-\sqrt{2} x+1\right)+(C x+D)\left(x^{2}+\sqrt{2} x+1\right)}{x^{4}+1} \\
& x=(A+C) x^{3}+(B-\sqrt{2} A+D+\sqrt{2} C) x^{2}+(A-\sqrt{2} B+C+\sqrt{2} D) x+(B+D)
\end{aligned}
$$


Igualemos los coeficientes correspondientes:

$$
\begin{array}{ll}
x^{3}: & 0=A+C \\
x^{2}: & 0=B+D-\sqrt{2}(A+C) \\
x^{1}: & 1=A+C-\sqrt{2}(B-D) \\
x^{0}: & 0=B+D
\end{array}
$$

De (1):

$$
A+C=0 \rightarrow C=-A
$$

(1) en (2):

$$
0=B+D-\sqrt{2}(0) \rightarrow 0=B+D \rightarrow D=-B
$$

Como $B+D=0$ coincide con la ecuación (4), esta sería una ecuación redundante y el sistema de ecuaciones tendría infinitas soluciones.

(1) y (5) en (3) :

$$
\begin{aligned}
1=0-\sqrt{2}(B+B) \rightarrow B & =\frac{-1}{2 \sqrt{2}} \\
D & =\frac{1}{2 \sqrt{2}}
\end{aligned}
$$

Las soluciones del sistema son:

$$
\begin{aligned}
& A=K \in \mathrm{R} \\
& B=\frac{-1}{2 \sqrt{2}} \\
& C=-K \in \mathrm{R} \\
& D=\frac{1}{2 \sqrt{2}}
\end{aligned}
$$

Por simplicidad, escojamos $A=0$ y $C=0$.

\section{Entonces:}

$$
\begin{aligned}
& \int \frac{x d x}{x^{4}+1}=\int \frac{-\frac{1}{2 \sqrt{2}} d x}{x^{2}+\sqrt{2} x+1}+\int \frac{\frac{1}{2 \sqrt{2}} d x}{x^{2}-\sqrt{2} x+1} \\
& \int \frac{x d x}{x^{4}+1}=-\frac{1}{2 \sqrt{2}} \int \frac{d x}{\left(x+\frac{\sqrt{2}}{2}\right)^{2}+\left(\frac{\sqrt{2}}{2}\right)^{2}}+\frac{1}{2 \sqrt{2}} \int \frac{d x}{\left(x-\frac{\sqrt{2}}{2}\right)^{2}+\left(\frac{\sqrt{2}}{2}\right)^{2}}
\end{aligned}
$$




$$
\begin{aligned}
& \int \frac{x d x}{x^{4}+1}=-\frac{1}{2 \sqrt{2}} \cdot \frac{1}{\frac{\sqrt{2}}{2}} \arctan \frac{x+\frac{\sqrt{2}}{2}}{\frac{\sqrt{2}}{2}}+\frac{1}{2 \sqrt{2}} \cdot \frac{1}{\frac{\sqrt{2}}{2}} \arctan \frac{x-\frac{\sqrt{2}}{2}}{\frac{\sqrt{2}}{2}}+C_{3} \\
& \int \frac{x d x}{x^{4}+1}=-\frac{1}{2} \arctan \frac{\sqrt{2} x+1}{1}+\frac{1}{2} \arctan \frac{\sqrt{2} x-1}{1}+C_{3} \\
& \int \frac{x d x}{x^{4}+1}=\frac{1}{2} \arctan (\sqrt{2} x-1)-\frac{1}{2} \arctan (\sqrt{2} x+1)+C_{3}
\end{aligned}
$$

b) De $\mathrm{a}_{\mathrm{i}}$ ) y $\mathrm{a}_{\mathrm{ij}}$ ) se desprende que $C_{1}=C_{2}$. Ahora, comparemos los resultados $\mathrm{a}_{\mathrm{i}}$ ) y $\mathrm{a}_{\mathrm{iii}}$ ):

$$
\begin{aligned}
& \text { a) } \frac{1}{2} \underbrace{\arctan x^{2}}_{\alpha}+C_{1} \\
& \text { a } \left._{\mathrm{iii}}\right) \frac{1}{2} \underbrace{[\arctan (\sqrt{2} x-1)-\arctan (\sqrt{2} x+1)]}_{\beta}+C_{3}
\end{aligned}
$$

De $\left.\mathrm{a}_{\mathrm{i}}\right) \quad \tan \alpha=\tan \left(\arctan x^{2}\right)=x^{2}$

De $\left.\mathrm{a}_{\mathrm{iii}}\right) \tan \beta=\tan [\arctan (\sqrt{2} x-1)-\arctan (\sqrt{2} x+1)]$

$$
\begin{aligned}
& \tan \beta=\frac{(\sqrt{2} x-1)-(\sqrt{2} x+1)}{1+(\sqrt{2} x-1)(\sqrt{2} x+1)}=\frac{-2}{1+\left(2 x^{2}-1\right)} \\
& \tan \beta=\frac{-2}{2 x^{2}}=\frac{-1}{x^{2}} \Rightarrow \cot \beta=-x^{2}
\end{aligned}
$$

Como $\tan \alpha=x^{2} y \cot \beta=-x^{2}$, se concluye $\operatorname{con}$ que $\cot \beta=-\tan \alpha y \operatorname{como} \cot \beta=-\tan \left(\frac{\pi}{2}+\beta\right)$, se deduce que $\alpha=\frac{\pi}{2}+\beta$.

Como se pide hallar la diferencia entre $C_{1}$ y $C_{3}$, igualemos los resultados obtenidos en $\mathrm{a}_{\mathrm{i}}$ ) y $\mathrm{a}_{\mathrm{iij}}$ ).

$$
\begin{aligned}
& \frac{1}{2} \alpha+C_{1}=\frac{1}{2} \beta+C_{3} \\
& C_{1}-C_{3}=\frac{1}{2} \beta-\frac{1}{2} \alpha \\
& C_{1}-C_{3}=\frac{1}{2} \beta-\frac{1}{2}\left(\frac{\pi}{2}+\beta\right)
\end{aligned}
$$


Con lo cual:

$$
C_{1}-C_{3}=\frac{-\pi}{4}=-45^{\circ}
$$

c) Se encontró que:

$$
\begin{aligned}
& \int \frac{x d x}{x^{4}+1}=\frac{1}{2} \arctan x^{2}+C_{1} \\
& \int \frac{x d x}{x^{4}+1}=\frac{1}{2}[\arctan (\sqrt{2} x-1)-\arctan (\sqrt{2} x+1)]+C_{3}
\end{aligned}
$$

Tabulemos para determinar:

$$
C_{1}-C_{3}=\frac{1}{2}[\arctan (\sqrt{2} x-1)-\arctan (\sqrt{2} x+1)]-\frac{1}{2} \arctan x^{2}
$$

\begin{tabular}{cc|c|c|c|c|c}
\hline & $x$ & $\arctan (\sqrt{2} x-1)$ & $\arctan (\sqrt{2} x+1)$ & $\begin{array}{c}\arctan (\sqrt{2} x-1) \\
-\arctan (\sqrt{2} x+1)\end{array}$ & $\arctan x^{2}$ & $C_{1}-C_{3}$ \\
\hline 1 & $22.5^{\circ}$ & $67.5^{\circ}$ & $-45^{\circ}$ & $45^{\circ}$ & $-45^{\circ}$ \\
& 2 & $61.32^{\circ}$ & $75.36^{\circ}$ & $-14.04^{\circ}$ & $75.96^{\circ}$ & $-45^{\circ}$ \\
& 162 & $79.20^{\circ}$ & $-6.34^{\circ}$ & $83.66^{\circ}$ & $-45^{\circ}$ \\
& 3 & $72.86^{\circ}$ & $81.46^{\circ}$ & $-3.58^{\circ}$ & $86.42^{\circ}$ & $-45^{\circ}$ \\
& 4 & $77.88^{\circ}$ & $82.94^{\circ}$ & $-2.29^{\circ}$ & $87.71^{\circ}$ & $-45^{\circ}$ \\
& 5 & $80.65^{\circ}$ & $83.98^{\circ}$ & $-1.59^{\circ}$ & $88.41^{\circ}$ & $-45^{\circ}$
\end{tabular}

$\therefore$ Se comprueba lo hallado en $\mathrm{b}$ ).

5. a) Determinar la siguiente integral:

$$
\int \sec \theta d \theta
$$

b) Comprobar que $\int \sec \theta d \theta=\frac{1}{2} \ln \frac{1+\operatorname{sen} \theta}{1+\operatorname{sen} \theta}+C$.

c) Verificar que $\int \sec \theta d \theta=\ln \tan \left(\frac{\pi}{4}+\frac{\theta}{2}\right)+C$.

d) Mostrar que las tres integrales dadas anteriormente se cumplen para $\theta=0^{\circ}, 30^{\circ}, 45^{\circ}$ y $60^{\circ}$. 


\section{Solución:}

a) Previa multiplicación y división:

$$
\begin{aligned}
& \int \sec \theta d \theta=\int \sec \theta \cdot \frac{\sec \theta+\tan \theta}{\sec \theta+\tan \theta} d \theta \\
& \int \sec \theta d \theta=\int \frac{\sec ^{2} \theta+\sec \theta \tan \theta}{\tan \theta+\sec \theta} d \theta
\end{aligned}
$$

Como d $(\tan \theta+\sec \theta)=\left(\sec ^{2} \theta+\sec \theta \tan \theta\right) d \theta$,

$$
\int \sec \theta d \theta=\ln (\tan \theta+\sec \theta)+C
$$

b) $\int \sec \theta d \theta=\int \frac{d \theta}{\cos \theta}=\frac{1}{2} \int \frac{2 d \theta}{\cos \theta}=\frac{1}{2} \int \frac{2 \cos \theta d \theta}{\cos ^{2} \theta}$

$$
\begin{aligned}
& \int \sec \theta d \theta=\frac{1}{2} \int \frac{2 \cos \theta d \theta}{1-\operatorname{sen}^{2} \theta}=\frac{1}{2} \int \frac{2 \cos \theta d \theta}{(1+\operatorname{sen} \theta)(1-\operatorname{sen} \theta)} \\
& \int \sec \theta d \theta=\frac{1}{2} \int \frac{\frac{2 \cos \theta d \theta}{\frac{(1-\operatorname{sen} \theta)^{2}}{1+\operatorname{sen} \theta}}}{1-\operatorname{sen} \theta}
\end{aligned}
$$

$$
\int \sec \theta d \theta=\frac{1}{2} \int \frac{\frac{(1-\operatorname{sen} \theta)(\cos \theta)-(1+\operatorname{sen} \theta)(-\cos \theta)}{(1-\operatorname{sen} \theta)^{2}}}{\frac{1+\operatorname{sen} \theta}{1-\operatorname{sen} \theta}} d \theta
$$

Con lo cual:

$$
\int \sec \theta d \theta=\frac{1}{2} \ln \frac{1+\operatorname{sen} \theta}{1-\operatorname{sen} \theta}+C
$$

c) Se debe verificar que se cumple:

$$
\begin{aligned}
& \frac{1}{2} \ln \frac{1+\operatorname{sen} \theta}{1-\operatorname{sen} \theta}=\ln \tan \left(\frac{\pi}{4}+\frac{\theta}{2}\right) \\
& \ln \frac{1+\operatorname{sen} \theta}{1-\operatorname{sen} \theta}=\ln \tan ^{2}\left(\frac{\pi}{4}+\frac{\theta}{2}\right)
\end{aligned}
$$


Escribamos:

$$
\begin{aligned}
& \text { In } \tan ^{2}\left(\frac{\pi}{4}+\frac{\theta}{2}\right)=\ln \left(\frac{1+\tan \frac{\theta}{2}}{1-\tan \frac{\theta}{2}}\right)^{2} \\
& \text { In } \tan ^{2}\left(\frac{\pi}{4}+\frac{\theta}{2}\right)=\ln \frac{1+2 \tan \frac{\theta}{2}+\tan ^{2} \frac{\theta}{2}}{1-2 \tan \frac{\theta}{2}+\tan ^{2} \frac{\theta}{2}} \\
& \text { In } \tan ^{2}\left(\frac{\pi}{4}+\frac{\theta}{2}\right)=\ln \frac{\sec ^{2} \frac{\theta}{2}+2 \tan \frac{\theta}{2}}{\sec ^{2} \frac{\theta}{2}-2 \tan \frac{\theta}{2}}
\end{aligned}
$$

Como:

$$
\cos \theta=2 \cos ^{2} \frac{\theta}{2}-1 \rightarrow \cos ^{2} \frac{\theta}{2}=\frac{1+\cos \theta}{2}
$$

164

$$
\begin{aligned}
& \cos \theta=1-2 \operatorname{sen}^{2} \frac{\theta}{2} \rightarrow \operatorname{sen}^{2} \frac{\theta}{2}=\frac{1-\cos \theta}{2} \\
& \ln \tan ^{2}\left(\frac{\pi}{4}+\frac{\theta}{2}\right)=\ln \frac{\frac{2}{1+\cos \theta}+2 \sqrt{\frac{1-\cos \theta}{1+\cos \theta}}}{1+\cos \theta}-2 \sqrt{\frac{1-\cos \theta}{1+\cos \theta}} \\
& \ln \tan ^{2}\left(\frac{\pi}{4}+\frac{\theta}{2}\right)=\ln \frac{2+2 \sqrt{1-\cos \theta} \sqrt{1+\cos \theta}}{2-2 \sqrt{1-\cos \theta}} \sqrt{1+\cos \theta} \\
& \ln \tan ^{2}\left(\frac{\pi}{4}+\frac{\theta}{2}\right)=\ln \frac{1+\sqrt{1-\cos ^{2} \theta}}{1-\sqrt{1-\cos ^{2} \theta}}=\ln \frac{1+\operatorname{sen} \theta}{1-\operatorname{sen} \theta}
\end{aligned}
$$

Lo cual corresponde a lo pedido.

d) Se debe comprobar que $\ln (\tan \theta+\sec \theta)=\frac{1}{2} \ln \frac{1+\operatorname{sen} \theta}{1-\operatorname{sen} \theta}=\ln \tan \left(\frac{\pi}{4}+\frac{\theta}{2}\right)$

Es decir: $\tan \theta+\sec \theta=\sqrt{\frac{1+\operatorname{sen} \theta}{1-\operatorname{sen} \theta}}=\tan \left(\frac{\pi}{4}+\frac{\theta}{2}\right)$ 


\begin{tabular}{c|c|c|c|c|c|c|c|c}
\hline & $\tan \theta$ & $\sec \theta$ & $\operatorname{sen} \theta$ & $\left(\frac{\pi}{4}+\frac{\theta}{2}\right)$ & $\tan \theta+\sec \theta$ & $\frac{1+\operatorname{sen} \theta}{1-\operatorname{sen} \theta}$ & $\sqrt{\frac{1+\operatorname{sen} \theta}{1-\operatorname{sen} \theta}}$ & $\tan \left(\frac{\pi}{4}+\frac{\theta}{2}\right)$ \\
\hline $0^{\circ}$ & 0 & 1 & 0 & $45^{\circ}$ & 1 & 1 & 1 & 1 \\
$30^{\circ}$ & $\sqrt{3} / 3$ & $2 \sqrt{3} / 3$ & $1 / 2$ & $60^{\circ}$ & $\sqrt{3}$ & 3 & $\sqrt{3}$ & $\sqrt{3}$ \\
$45^{\circ}$ & 1 & $\sqrt{2}$ & $\sqrt{2} / 2$ & $67.5^{\circ}$ & $1+\sqrt{2}$ & $\frac{2+\sqrt{2}}{2-\sqrt{2}}$ & $1+\sqrt{2}$ & $1+\sqrt{2}$ \\
$60^{\circ}$ & $\sqrt{3}$ & 2 & $\sqrt{3} / 2$ & $75^{\circ}$ & $2+\sqrt{3}$ & $\frac{2+\sqrt{3}}{2-\sqrt{3}}$ & $2+\sqrt{3}$ & $2+\sqrt{3}$
\end{tabular}

Queda mostrado que las tres integrales indefinidas dadas son coincidentes para $\theta=0^{\circ}, 30^{\circ}, 45^{\circ}$ y $60^{\circ}$. 



\section{XI}

\section{PROBLEMAS RESUELTOS $Y$ PROPUESTOS}

\section{PROBLEMAS RESUELTOS Y PROPUESTOS DEL CAPÍTULO I}

1. Encontrar $d y / d x$ a partir de $y=\sqrt{1+\sqrt{x}}$.

Solución:

Si $y=\left(1+x^{1 / 2}\right)^{1 / 2}$, según la fórmula de derivación de una potencia se llega a:

$$
\frac{d x}{d y}=\frac{1}{2}\left(1+x^{1 / 2}\right)^{-1 / 2}\left(\frac{1}{2 x^{1 / 2}}\right)=\frac{1}{4 \sqrt{x} \sqrt{1+\sqrt{x}}}
$$

2. Calcular la derivada de la función $y=\frac{a^{2}+x^{2}}{a^{2}-x^{2}}$ en el punto de abscisa $a / 2$.

Solución:

Según la fórmula de derivación de un cociente, se obtiene que:

$$
y^{\prime}(x)=\frac{2 x\left(a^{2}-x^{2}\right)-(-2 x)\left(a^{2}+x^{2}\right)}{\left(a^{2}-x^{2}\right)^{2}}=\frac{4 a^{2} x}{\left(a^{2}-x^{2}\right)^{2}}
$$


De donde:

$$
y_{(a / 2)}^{\prime}=\frac{4 a^{2}(a / 2)}{\left(a^{2}-\frac{a^{2}}{4}\right)^{2}}=\frac{2 a^{3}}{\frac{9}{16} a^{4}}=\frac{32}{9 a}
$$

3. Derivar $f_{(x)}=x \cdot \operatorname{sen} \frac{a}{x}$ y obtener $f^{\prime}\left(\frac{4 a}{\pi}\right)$.

\section{Solución:}

Se sabe que:

$$
\begin{aligned}
f^{\prime}(x) & =1 \cdot \operatorname{sen} \frac{a}{x}+x \cdot \cos \frac{a}{x} \cdot\left(\frac{-a}{x^{2}}\right) \\
f^{\prime}(x) & =\operatorname{sen} \frac{a}{x}-\frac{a}{x} \cos \frac{a}{x}
\end{aligned}
$$

De donde:

$$
\begin{aligned}
f^{\prime}(4 a / \pi) & =\operatorname{sen} \frac{a}{4 a / \pi}-\frac{a}{4 a / \pi} \cos \frac{a}{4 a / \pi} \\
f^{\prime}(4 a / \pi) & =\operatorname{sen} \frac{\pi}{4}-\frac{\pi}{4} \cos \frac{\pi}{4}=\frac{\sqrt{2}}{2}\left[1-\frac{\pi}{4}\right]
\end{aligned}
$$

4. Dada la función $y=x^{2} \cdot 10^{2 x}$, calcular $y^{\prime}(1)$.

\section{Solución:}

Se tiene que:

$$
\frac{d y}{d x}=2 x \cdot 10^{2 x}+x^{2} \cdot 10^{2 x} \cdot(2) \cdot \ln 10
$$

De donde:

$$
\left.\frac{d y}{d x}\right|_{(x=1)}=2 \cdot 10^{2}+10^{2}(2) \ln 10=200(1+\ln 10)
$$

5. Encontrar la derivada de $g_{(x)}=\sqrt{\frac{\sec x-\tan x}{\sec x+\tan x}}$ simplificando previamente.

\section{Solución:}


Escribamos:

$$
\frac{\sec x-\tan x}{\sec x+\tan x}=\frac{\frac{1}{\cos x}-\frac{\operatorname{sen} x}{\cos x}}{\frac{1}{\cos x}+\frac{\operatorname{sen} x}{\cos x}}=\frac{1-\operatorname{sen} x}{1+\operatorname{sen} x}
$$

De donde:

$$
\begin{gathered}
g_{(x)}=\sqrt{\frac{1-\operatorname{sen} x}{1+\operatorname{sen} x}} \\
g_{(x)}^{\prime}=\frac{1}{2 \sqrt{\frac{1-\operatorname{sen} x}{1+\operatorname{sen} x}}} \cdot \frac{-\cos x(1+\operatorname{sen} x)-\cos x(1-\operatorname{sen} x)}{(1+\operatorname{sen} x)^{2}}
\end{gathered}
$$

Simplificando:

$$
\begin{aligned}
& g_{(x)}^{\prime}=\frac{-2 \cos x}{2(1+\operatorname{sen} x)^{2}} \cdot \frac{\sqrt{1+\operatorname{sen} x}}{\sqrt{1-\operatorname{sen} x}} \cdot \frac{\sqrt{1-\operatorname{sen} x}}{\sqrt{1-\operatorname{sen} x}} \\
& g_{(x)}^{\prime}=\frac{-\cos x \sqrt{1-\operatorname{sen}^{2} x}}{(1+\operatorname{sen} x)^{2}(1-\operatorname{sen} x)}=\frac{-\cos x(\cos x)}{(1+\operatorname{sen} x)^{2}(1-\operatorname{sen} x)} \\
& g_{(x)}^{\prime}=\frac{-\left(1-\operatorname{sen}^{2} x\right)}{(1+\operatorname{sen} x)^{2}(1-\operatorname{sen} x)}=\frac{-(1+\operatorname{sen} x)}{(1+\operatorname{sen} x)^{2}}=\frac{-1}{1+\operatorname{sen} x}
\end{aligned}
$$

6. Derivar la función del problema 23:

$$
f_{(x)}=6 \ln x-\ln (x+1)-\frac{9}{x+1}+C
$$

\section{Solución:}

Se tiene que:

$$
\begin{aligned}
& f_{(x)}^{\prime}=\frac{6}{x}-\frac{1}{x+1}+\frac{9}{(x+1)^{2}} \\
& f_{(x)}^{\prime}=\frac{6\left(x^{2}+2 x+1\right)-\left(x^{2}+x\right)+9 x}{x(x+1)^{2}}
\end{aligned}
$$


Es decir:

$$
f^{\prime}(x)=\frac{5 x^{2}+20 x+6}{x(x+1)^{2}}=\frac{5 x^{2}+20 x+6}{x^{3}+2 x^{2}+x}
$$

7. Encontrar la derivada de la función del problema 34:

$$
f_{(x)}=\frac{1}{2} \tan ^{2} x+3 \ln (\tan x)-\frac{3}{2 \tan ^{2} x}-\frac{1}{4 \tan ^{4} x}+C
$$

\section{Solución:}

Tenemos que:

$$
\begin{aligned}
& f_{(x)}^{\prime}=\frac{2}{2}(\tan x) \sec ^{2} x+\frac{3 \sec ^{2} x}{\tan x}+\frac{3 \sec ^{2} x}{\tan ^{3} x}+\frac{1 \sec ^{2} x}{\tan ^{5} x}+0 \\
& f_{(x)}^{\prime}=\sec ^{2} x\left[\tan x+\frac{3}{\tan x}+\frac{3}{\tan ^{3} x}+\frac{1}{\tan ^{5} x}\right] \\
& f_{(x)}^{\prime}=\frac{\sec ^{2} x}{\tan ^{5} x}\left[\tan ^{6} x+3 \tan ^{4} x+3 \tan ^{2} x+1\right]
\end{aligned}
$$

170

O mejor:

$$
\begin{aligned}
& f^{\prime}(x)=\frac{\sec ^{2} x}{\tan ^{5} x}\left[\left(\tan ^{2} x+1\right)^{3}\right] \\
& f^{\prime}(x)=\frac{\left(\sec ^{2} x\right)\left(\sec ^{2} x\right)^{3}}{\tan ^{5} x}=\frac{\sec ^{8} x}{\tan ^{5} x}
\end{aligned}
$$

De donde:

$$
f^{\prime}(x)=\frac{\frac{1}{\cos ^{8} x}}{\frac{\operatorname{sen}^{5} x}{\cos ^{5} x}}=\frac{1}{\operatorname{sen}^{5} x \cos ^{3} x}
$$

8. Derivar la función del problema 101.

$$
f_{(x)}=-2 \sqrt{1-x}+\sqrt{x-x^{2}}+\operatorname{arcsen} \sqrt{1-x}+C
$$

\section{Solución:}

Se tiene que:

$$
f^{\prime}(x)=\frac{-2(-1)}{2 \sqrt{1-x}}+\frac{(1-2 x)}{2 \sqrt{x-x^{2}}}+\frac{\frac{1(-1)}{2 \sqrt{1-x}}}{\sqrt{1-(1-x)}}
$$




$$
\begin{aligned}
& f^{\prime}(x)=\frac{1}{\sqrt{1-x}}+\frac{1-2 x}{2 \sqrt{x} \sqrt{1-x}}-\frac{1}{2 \sqrt{x} \sqrt{1-x}} \\
& f^{\prime}(x)=\frac{2 \sqrt{x}+(1-2 x)-1}{2 \sqrt{x} \sqrt{1-x}} \\
& f^{\prime}(x)=\frac{2 \sqrt{x}-2 x}{2 \sqrt{x} \sqrt{1-x}}=\frac{\sqrt{x}-x}{\sqrt{x} \sqrt{1-x}}=\frac{1-\sqrt{x}}{\sqrt{1-x}} \\
& f^{\prime}(x)=\frac{1-\sqrt{x}}{\sqrt{1-x}}=\frac{1-\sqrt{x}}{(\sqrt{1+\sqrt{x}})(\sqrt{1-\sqrt{x}})}=\frac{\sqrt{1-\sqrt{x}}}{\sqrt{1+\sqrt{x}}}
\end{aligned}
$$

9. Hallar la derivada de la función del problema 114:

$$
f_{(\theta)}=\frac{e^{a \theta}(a \operatorname{sen} b \theta-b \cos b \theta)}{a^{2}+b^{2}}+C
$$

\section{Solución:}

Obtenemos que:

$$
\begin{aligned}
& f^{\prime}(\theta)=\frac{1}{a^{2}+b^{2}}\left[a e^{a \theta}(a \operatorname{sen} b \theta-b \cos b \theta)+e^{a \theta}\left(a b \cos b \theta+b^{2} \operatorname{sen} b \theta\right)\right] \\
& f^{\prime}(\theta)=\frac{e^{a \theta}}{a^{2}+b^{2}}\left[a^{2} \operatorname{sen} b \theta-a b \cos b \theta+a b \cos b \theta+b^{2} \operatorname{sen} b \theta\right]
\end{aligned}
$$

Con lo cual:

$$
f^{\prime}(\theta)=\frac{e^{a \theta}\left(a^{2}+b^{2}\right)(\operatorname{sen} b \theta)}{a^{2}+b^{2}}=e^{a \theta} \operatorname{sen} b \theta
$$

10. Comprobar la respuesta del problema 160:

$$
f(x)=\frac{6}{7} x^{7 / 6}-\frac{6}{5} x^{5 / 6}-\frac{3}{2} x^{2 / 3}+2 x^{1 / 2}+3 x^{1 / 3}-6 x^{1 / 6}-3 \ln \left(x^{1 / 3}+1\right)+6 \arctan x^{1 / 6}+C
$$

\section{Solución:}

Derivamos $f_{(x)}$ :

$$
\begin{aligned}
& f^{\prime}(x)=x^{1 / 6}-x^{-1 / 6}-x^{-1 / 3}+x^{-1 / 2}+x^{-2 / 3}-x^{-5 / 6}-\frac{3\left(\frac{1}{3}\right) x^{-2 / 3}}{x^{1 / 3}+1}+\frac{6\left(\frac{1}{6}\right) x^{-5 / 6}}{1+x^{1 / 3}} \\
& f^{\prime}(x)=\frac{x^{1 / 6}-x^{-1 / 6}-x^{-1 / 3}+x^{-1 / 2}+x^{-2 / 3}-x^{-5 / 6}+x^{1 / 2}-x^{1 / 6}-1+x^{-1 / 6}+x^{-1 / 3}-x^{-1 / 2}}{1+x^{1 / 3}}-\frac{x^{-2 / 3}-x^{-5 / 6}}{1+x^{1 / 3}}
\end{aligned}
$$


Simplificamos:

$$
f^{\prime}(x)=\frac{x^{1 / 2}-1}{1+x^{1 / 3}}
$$

Verificar que la derivada de las funciones dadas a continuación, y que aparecen en los problemas cuya numeración se señala, corresponden a la función cuya antiderivada se encontró en el respectivo problema.

11. $f_{(x)}=\frac{1}{2[x(\ln x-1)]^{2}}+C$

(Problema 104)

12. $f_{(x)}=2 \sqrt{x+9}+3 \cdot \ln \frac{\sqrt{x+9}-3}{\sqrt{x+9}+3}+C$

(Problema 109)

13. $f_{(x)}=\frac{1}{2} \ln \left(x^{2}+\sqrt{1+x^{4}}\right)+C$

(Problema 112)

14. $f_{(x)}=2\left[\frac{-\sqrt{x-1}}{\sqrt{x-1}+1}+\ln (\sqrt{x-1}+1)\right]+C$

(Problema 117)

172 15. $f_{(x)}=\frac{x^{2}}{2} \operatorname{arcsen} x+\frac{1}{4} x \sqrt{1-x^{2}}-\frac{1}{4} \operatorname{arcsen} x+C$

(Problema 124)

16. $f_{(x)}=\frac{1}{3} e^{x^{3}}\left(x^{3}-1\right)+C$

(Problema 125)

17. $f_{(z)}=-\frac{5}{6} \arctan \frac{z}{3}+\frac{1}{2} \arctan z+C$,

(Problema 144)

en donde $z=\tan \frac{x}{2}$.

18. $f_{(x)}=\ln \frac{\tan \frac{x}{2}-5}{\tan \frac{x}{2}-3}+C$

(Problema 146)

19. $f_{(u)}=\frac{2 u}{u^{3}-1}+\frac{1}{3} \ln \frac{u^{2}+u+1}{(u-1)^{2}}+\frac{2 \sqrt{3}}{3} \arctan \frac{2 u+1}{\sqrt{3}}+C$,

(Problema 159)

en donde $u=\sqrt[3]{\frac{x+1}{x-1}}$.

20. $f_{(x)}=\frac{1}{4} \ln \frac{5-\operatorname{sen} x}{1-\operatorname{sen} x}+C$

(Problema 188) 


\section{PROBLEMAS RESUELTOS Y PROPUESTOS DEL CAPÍTULO II}

21. Si $\int(a+b x)^{n} d x=\frac{(a+b x)^{n+1}}{C}+C \quad(n \neq-1)$, hallar el valor de $C$.

\section{Solución:}

Derivando ambos miembros, se obtiene:

$$
(a+b x)^{n}=\frac{1}{C}(n+1)(a+b x)^{n}(b)
$$

De donde:

$$
C=b(n+1)
$$

22. Si se cumple que:

$$
\int \frac{x d x}{(2+3 x)^{3}}=K\left[-\frac{1}{2+3 x}+\frac{1}{(2+3 x)^{2}}\right]+C
$$

encontrar el valor de $K$.

Solución:

Derivemos ambos miembros con relación a $x$ :

$$
\frac{x}{(2+3 x)^{3}}=K\left[\frac{3}{(2+3 x)^{2}}-\frac{2(3)}{(2+3 x)^{3}}\right]
$$

De donde:

$$
\begin{aligned}
& x=K[3(2+3 x)-6] \\
& x=6 K+9 K x-6 K=9 K x
\end{aligned}
$$

Con lo cual:

$$
K=\frac{1}{9}
$$

23. Al integrar $\int \frac{5 x^{2}+20 x+6}{x^{3}+2 x^{2}+x} d x$ se obtuvo $6 K \ln x-\ln (x+1)-\frac{9 K}{x+1}+C$. Hallar el valor de $K$.

\section{Solución:}

Se debe cumplir que: 


$$
\frac{5 x^{2}+20 x+6}{x\left(x^{2}+2 x+1\right)}=\frac{6 K}{x}-\frac{1}{x+1}+\frac{9 K}{(x+1)^{2}}
$$

Es decir:

$$
\frac{5 x^{2}+20 x+6}{x(x+1)^{2}}=\frac{6 K(x+1)^{2}-x(x+1)+9 K x}{x(x+1)^{2}}
$$

O mejor:

$$
5 x^{2}+20 x+6=6 K(x+1)^{2}-x(x+1)+9 K x
$$

Si $x=0$ :

$$
0+0+6=6 K(1)^{2}-0+0
$$

De donde:

$$
K=1
$$

24. Si $\int f_{(x)} d x=\frac{-x}{2\left(x^{2}+a^{2}\right)}+\frac{1}{2 a} \arctan \left(\frac{x}{a}\right)+C$, halle $f(a)$.

\section{Solución:}

Derivemos ambos miembros:

$$
\begin{aligned}
& f_{(x)}=\frac{-1\left(x^{2}+a^{2}\right)+x(2 x)}{2\left(x^{2}+a^{2}\right)^{2}}+\frac{1}{2 a} \cdot \frac{1}{1+\left(\frac{x}{a}\right)^{2}} \cdot \frac{1}{a} \\
& f_{(x)}=\frac{x^{2}-a^{2}}{2\left(x^{2}+a^{2}\right)^{2}}+\frac{1}{2 a^{2}} \cdot \frac{a^{2}}{x^{2}+a^{2}} \\
& f_{(x)}=\frac{x^{2}-a^{2}}{2\left(x^{2}+a^{2}\right)^{2}}+\frac{1}{2\left(x^{2}+a^{2}\right)}
\end{aligned}
$$

De donde:

$$
f_{(a)}=0+\frac{1}{2\left(a^{2}+a^{2}\right)}=\frac{1}{4 a^{2}}
$$

25. Comprobar la fórmula siguiente:

$$
\int \frac{d x}{x^{2}(a+b x)^{2}}=-\frac{a+2 b x}{a^{2} x(a+b x)}+\frac{2 b}{a^{3}} \ln \frac{a+b x}{x}+C
$$




\section{Solución:}

Reescribamos el segundo miembro:

$$
-\frac{a+2 b x}{a^{3} x+a^{2} b x^{2}}+\frac{2 b}{a^{3}} \ln \left(\frac{a}{x}+b\right)
$$

Derivémoslo con relación a $x$ :

$$
-\frac{2 b\left(a^{3} x+a^{2} b x^{2}\right)-(a+2 b x)\left(a^{3}+2 a^{2} b x\right)}{\left(a^{3} x+a^{2} b x^{2}\right)^{2}}+\frac{2 b}{a^{3}} \cdot \frac{\frac{-a}{x^{2}}}{\frac{a}{x}+b}
$$

Simplificando:

$$
\frac{a^{2}+2 b^{2} x^{2}+2 a b x}{a^{2}\left(a x+b x^{2}\right)^{2}}-\frac{2 b}{a^{2}\left(a x+b x^{2}\right)}
$$

Desarrollemos después de multiplicar el sustraendo por $\frac{a x+b x^{2}}{a x+b x^{2}}$ :

$$
\begin{aligned}
& \frac{a^{2}+2 b^{2} x^{2}+2 a b x-2 a b x-2 b^{2} x^{2}}{a^{2}\left(a x+b x^{2}\right)^{2}} \\
& \frac{a^{2}}{a^{2} x^{2}(a+b x)^{2}}=\frac{1}{x^{2}(a+b x)^{2}}
\end{aligned}
$$

Con lo cual, se obtiene la derivada de $\int \frac{d x}{x^{2}(a+b x)^{2}}$.

26. Demostrar $\int\left(u^{2}-a^{2}\right)^{m} d u=\frac{u\left(u^{2}-a^{2}\right)^{m}}{2 m+1}-\frac{2 m a^{2}}{2 m+1} \int\left(u^{2}-a^{2}\right)^{m-1} d u$, donde $m \neq-\frac{1}{2}$.

Solución:

La derivada del primer miembro es:

$$
\left(u^{2}-a^{2}\right)^{m}
$$

La derivada del segundo miembro es:

$$
\frac{1}{2 m+1}\left[\left(u^{2}-a^{2}\right)^{m}+u m\left(u^{2}-a^{2}\right)^{m-1}(2 u)\right]-\frac{2 m a^{2}}{2 m+1}\left(u^{2}-a^{2}\right)^{m-1}
$$




$$
\begin{gathered}
\frac{\left(u^{2}-a^{2}\right)^{m}}{2 m+1}\left[1+\frac{2 u^{2} m}{u^{2}-a^{2}}-\frac{2 m a^{2}}{u^{2}-a^{2}}\right] \\
\frac{\left(u^{2}-a^{2}\right)^{m}}{2 m+1}\left[1+\frac{2 m\left(u^{2}-a^{2}\right)}{u^{2}-a^{2}}\right]=\frac{\left(u^{2}-a^{2}\right)^{m}}{2 m+1}(1+2 m)=\left(u^{2}-a^{2}\right)^{m}
\end{gathered}
$$

Se comprueba, pues, que se cumple la fórmula de reducción.

27. Comprobar la siguiente antiderivada:

$$
\int \sqrt{u^{2} \pm a^{2}} d u=\frac{u}{2} \sqrt{u^{2} \pm a^{2}} \pm \frac{a^{2}}{2} \ln \left(u+\sqrt{u^{2} \pm a^{2}}\right)+C
$$

\section{Solución:}

Derivemos el segundo miembro:

$$
\frac{1}{2} \sqrt{u^{2} \pm a^{2}}+\frac{u}{2} \cdot \frac{u}{\sqrt{u^{2} \pm a^{2}}} \pm \frac{a^{2}}{2} \cdot \frac{1+\frac{u}{\sqrt{u^{2} \pm a^{2}}}}{u+\sqrt{u^{2} \pm a^{2}}}
$$

$$
\begin{gathered}
\frac{1}{2} \sqrt{u^{2} \pm a^{2}}+\frac{u^{2}}{2 \sqrt{u^{2} \pm a^{2}}} \pm \frac{a^{2}}{2} \cdot \frac{\sqrt{u^{2} \pm a^{2}}+u}{\sqrt{u^{2} \pm a^{2}}\left(u+\sqrt{u^{2} \pm a^{2}}\right)} \\
\frac{1}{2} \sqrt{u^{2} \pm a^{2}}+\frac{u^{2} \sqrt{u^{2} \pm a^{2}}}{2\left(u^{2} \pm a^{2}\right)} \pm \frac{a^{2}}{2} \cdot \frac{\sqrt{u^{2} \pm a^{2}}}{u^{2} \pm a^{2}} \\
\frac{1}{2} \sqrt{u^{2} \pm a^{2}}+\frac{1}{2} \cdot \frac{\left(u^{2} \pm a^{2}\right) \sqrt{u^{2} \pm a^{2}}}{u^{2} \pm a^{2}}=\frac{1}{2} \sqrt{u^{2} \pm a^{2}}+\frac{1}{2} \sqrt{u^{2} \pm a^{2}}=\sqrt{u^{2} \pm a^{2}}
\end{gathered}
$$

Este resultado coincide con la derivada del primer miembro.

28. Comprobar la siguiente antiderivada:

$$
\int \frac{d x}{(x-3) \sqrt{x+1}}=\frac{1}{2} \ln \frac{\sqrt{x+1}-2}{\sqrt{x+1}+2}+C
$$

\section{Solución:}

La derivada de $\frac{1}{2} \ln \frac{\sqrt{x+1}-2}{\sqrt{x+1}+2}$ es: 


$$
\frac{1}{2} \cdot \frac{\frac{1}{2 \sqrt{x+1}(\sqrt{x+1}+2)-\frac{1}{2 \sqrt{x+1}}(\sqrt{x+1}-2)}}{(\sqrt{x+1}+2)^{2}}=\frac{1}{2} \cdot \frac{\frac{1}{2 \sqrt{x+1}}(4)}{(\sqrt{x+1}-2} \frac{\sqrt{x+1}-2)(\sqrt{x+1}+2)}{\sqrt{x+1}+2}
$$

Es decir:

$$
\frac{2}{2} \cdot \frac{1}{(x+1-4) \sqrt{x+1}}=\frac{1}{(x-3) \sqrt{x+1}}
$$

Este resultado coincide con la derivada del primer miembro.

29. Comprobar la siguiente fórmula de reducción:

$$
\int \frac{\operatorname{sen}^{m} x}{\cos ^{n} x} d x=\frac{\operatorname{sen}^{m+1} x}{(n-1) \cos ^{n-1} x}-\frac{m-n+2}{n-1} \int \frac{\operatorname{sen}^{m} x}{\cos ^{n-2} x} d x
$$

\section{Solución:}

Derivemos el primer miembro:

Derivemos el segundo miembro:

$$
\frac{\operatorname{sen}^{m} x}{\cos ^{n} x}
$$

$$
\begin{gathered}
\frac{1}{n-1} \frac{(m+1) \operatorname{sen}^{m} x \cos x \cos ^{n-1} x-(n-1) \cos ^{n-2} x(-\operatorname{sen} x) \operatorname{sen}^{m+1} x}{\cos ^{2 n-2} x}-\frac{(m-n+2)}{n-1} \frac{\operatorname{sen}^{m} x}{\cos ^{n-2} x} \\
\frac{1}{n-1}\left[\frac{(m+1) \operatorname{sen}^{m} x \cos ^{n} x+(n-1) \cos ^{n-2} x \operatorname{sen}^{m+2} x}{\cos ^{2 n-2} x}-\frac{(m-n+2) \operatorname{sen}^{m} x}{\cos ^{n-2} x}\right] \\
\frac{1}{n-1}\left[\frac{\operatorname{sen}^{m} x}{\cos ^{n} x}\right]\left[\frac{(m+1) \cos ^{n} x+(n-1) \cos ^{n-2} x\left(1-\cos ^{2} x\right)}{\cos ^{n-2} x}-\frac{m-n+2}{\cos ^{-2} x}\right] \\
\frac{1}{n-1}\left[\frac{\operatorname{sen}^{m} x}{\cos ^{n} x}\right]\left[(m+1) \cos ^{2} x+(n-1)\left(1-\cos ^{2} x\right)-(m-n+2) \cos ^{2} x\right] \\
\frac{1}{n-1}\left(\frac{\operatorname{sen}^{m} x}{\cos ^{n} x}\right)\left[(m+1-n+1-m+n-2) \cos ^{2} x+n-1\right] \\
\frac{1}{n-1} \frac{\operatorname{sen}^{m} x}{\cos ^{n} x}[n-1]=\frac{\operatorname{sen}^{m} x}{\cos ^{n} x}
\end{gathered}
$$


Con lo cual, se comprueba la fórmula de reducción.

30. Encontrar el valor de la constante $K$ en la siguiente fórmula de reducción:

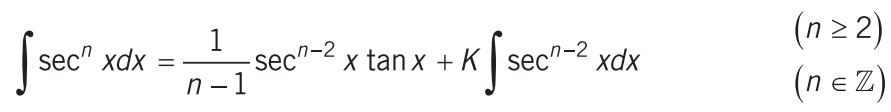

\section{Solución:}

Derivemos ambos miembros con relación a $x$ :

$$
\sec ^{n} x=\frac{1}{n-1} \sec ^{n-2} x \sec ^{2} x+\frac{1}{n-1}(n-2) \sec ^{n-3} x(\sec x \tan x) \tan x+K \sec ^{n-2} x
$$

Escribámoslo así:

$$
\begin{aligned}
& (n-1) \sec ^{n} x=\sec ^{n} x+(n-2) \sec ^{n-2} x \tan ^{2} x+K(n-1) \sec ^{n-2} x \\
& (n-2) \sec ^{n} x=(n-2) \sec ^{n-2} x \tan ^{2} x+K(n-1) \sec ^{n-2} x \\
& (n-2)\left(\sec ^{n-2} x\right)\left(1+\tan ^{2} x\right)=(n-2) \sec ^{n-2} x \tan ^{2} x+K(n-1) \sec ^{n-2} x \\
& (n-2)+(n-2) \tan ^{2} x=(n-2) \tan ^{2} x+K(n-1)
\end{aligned}
$$

De donde:

$$
\begin{aligned}
n-2 & =K(n-1) \\
K & =\frac{n-2}{n-1}
\end{aligned}
$$

31. Se sabe que para cualquier número entero positivo $n$, se cumple que:

$$
\int \cos ^{n} x d x=\frac{1}{n} \cos ^{n-1} x \operatorname{sen} x+f(n) \int \cos ^{n-2} x d x
$$

Encontrar el valor de $f_{(6)}$.

\section{Solución:}

Derivemos ambos miembros con respecto a $x$ :

$$
\cos ^{n} x=\frac{1}{n}(n-1) \cos ^{n-2} x(-\operatorname{sen} x)(\operatorname{sen} x)+\frac{1}{n} \cos ^{n-1} x(\cos x)+f_{(n)} \cos ^{n-2} x
$$


De donde:

$$
\begin{gathered}
\left(1-\frac{1}{n}\right) \cos ^{n} x=\left[\frac{n-1}{n}\left(-\operatorname{sen}^{2} x\right)+f_{(n)}\right] \cos ^{n-2} x \\
\frac{n-1}{n} \cos ^{n-2} x \cos ^{2} x=\left[-\frac{n-1}{n} \operatorname{sen}^{2} x+f_{(n)}\right] \cos ^{n-2} x \\
\frac{n-1}{n}\left(1-\operatorname{sen}^{2} x\right)=\left[f_{(n)}-\frac{n-1}{n} \operatorname{sen}^{2} x\right]
\end{gathered}
$$

Con lo cual:

$$
\frac{n-1}{n}=f_{(n)}
$$

Finalmente:

$$
f_{(6)}=\frac{5}{6}
$$

32. Halle $g(0,1,2)$ si:

$$
\int x^{m}\left(a+b x^{n}\right)^{p} d x=\frac{x^{m+1}\left(a+b x^{n}\right)^{p}}{n p+m+1}+g(n, m, p) \int x^{m}\left(a+b x^{n}\right)^{p-1} d x
$$

\section{Solución:}

La derivada del primer miembro es:

$$
x^{m}\left(a+b x^{n}\right)^{p}
$$

La derivada del segundo miembro es:

$$
\begin{gathered}
\frac{1}{n p+m+1}\left[(m+1) x^{m}\left(a+b x^{n}\right)^{p}+p\left(x^{m+1}\right)\left(a+b x^{n}\right)^{p-1}\left(n b x^{n-1}\right)\right]+g(n, m, p) x^{m}\left(a+b x^{n}\right)^{p-1} \\
x^{m}\left(a+b x^{n}\right)^{p}\left[\frac{m+1}{n p+m+1}+\frac{n b p x^{n}}{\left(a+b x^{n}\right)(n p+m+1)}+\frac{g(n, m, p)}{a+b x^{n}}\right] \ldots(\beta)
\end{gathered}
$$

Para que se cumpla la fórmula de reducción dada, se requiere que $(\alpha)=(\beta)$, es decir:

$$
\frac{m+1}{n p+m+1}+\frac{n b p x^{n}}{\left(a+b x^{n}\right)(n p+m+1)}+\frac{g(n, m, p)}{a+b x^{n}}=1
$$

Reemplacemos $n=0, m=1$ y $p=2$ :

$$
\frac{2}{2}+\frac{0}{2(a+b)}+\frac{g(0,1,2)}{a+b}=1
$$


De donde:

$$
g(0,1,2)=0
$$

33. Si $\int x^{m} e^{n x} d x=\frac{1}{n} x^{m} e^{n x}+k \int x^{m-1} e^{n x} d x$, halle el valor de $k$ cuando $m=2 n$.

\section{Solución:}

Si $m=2 n$, se tendrá:

$$
\int x^{2 n} e^{n x} d x=\frac{1}{n} x^{2 n} e^{n x}+k \int x^{2 n-1} e^{n x} d x
$$

Derivemos ambos miembros con relación a $x$ :

$$
x^{2 n} e^{n x}=\frac{1}{n} 2 n x^{2 n-1} e^{n x}+\frac{1}{n} x^{2 n}\left(n e^{n x}\right)+k x^{2 n-1} e^{n x}
$$

Dividamos ambos miembros entre $x^{2 n-1} e^{n x}$ :

$$
x=2+x+k \Rightarrow k=-2
$$

34. Calcular $K$ si se sabe que:

$$
\int \frac{d x}{\operatorname{sen}^{5} x \cos ^{3} x}=\frac{1}{2} \tan ^{2} x+3 \ln \tan x-\frac{k}{2} \tan ^{-2} x-\frac{1}{4} \tan ^{-4} x+C
$$

\section{Solución:}

Derivemos ambos miembros con relación a $x$ :

$$
\frac{1}{\operatorname{sen}^{5} x \cdot \cos ^{3} x}=\frac{1}{2}(2)(\tan x) \sec ^{2} x+\frac{3 \sec ^{2} x}{\tan x}-\frac{k}{2}(-2) \frac{\sec ^{2} x}{\tan ^{3} x}-\frac{1}{4}(-4) \frac{\sec ^{2} x}{\tan ^{5} x}
$$

Multipliquemos ambos miembros por $\cos ^{2} x 0 \frac{1}{\sec ^{2} x}$ :

$$
\frac{1}{\operatorname{sen}^{5} x \cdot \cos x}=\frac{\operatorname{sen} x}{\cos x}+\frac{3 \cos x}{\operatorname{sen} x}+\frac{K \cos ^{3} x}{\operatorname{sen}^{3} x}+\frac{\cos ^{5} x}{\operatorname{sen}^{5} x}
$$

Multipliquemos ambos miembros por $\operatorname{sen}{ }^{5} x \cdot \cos x$ :

$$
1=\operatorname{sen}^{6} x+3 \operatorname{sen}^{4} x \cos ^{2} x+K \operatorname{sen}^{2} x \cos ^{4} x+\cos ^{6} x
$$

Como

$$
\operatorname{sen}^{2} x+\cos ^{2} x=1 \rightarrow\left(\operatorname{sen}^{2} x+\cos ^{2} x\right)^{3}=1^{3}
$$


Es decir:

$$
\operatorname{sen}^{6} x+3 \operatorname{sen}^{4} x \cos ^{2} x+3 \operatorname{sen}^{2} x \cos ^{4} x+\cos ^{6} x=1
$$

De $(\alpha)$ y $(\beta)$, se deduce que:

$$
K=3
$$

35. Si $\int x^{m}(a x+b)^{n} d x=\frac{x^{m}(a x+b)^{n+1}}{k}-\frac{m b}{k} \int x^{m-1}(a x+b)^{n} d x$, halle el valor de la constante $k$.

\section{Solución:}

Derivando ambos miembros:

$$
x^{m}(a x+b)^{n}=\frac{m x^{m-1}(a x+b)^{n+1}}{k}+\frac{a(n+1) x^{m}(a x+b)^{n}}{k}-\frac{m b x^{m-1}(a x+b)^{n}}{k}
$$

Multipliquemos ambos miembros por $\frac{k}{x^{m-1}(a x+b)^{n}}$.

$$
\begin{gathered}
k x=m(a x+b)+a(n+1) x-m b \\
k x=a m x+b m+a n x+a x-b m \\
k x=a(m+n+1) x
\end{gathered}
$$

De donde:

$$
k=a(m+n+1)
$$

36. Si se cumple que $\int \frac{b c-a d}{(c+d x)^{2}} d x=\frac{a+e x}{f+d x}+C$, calcular $2 e-3 f$.

\section{Solución:}

Derivemos ambos miembros con relación a $x$ :

$$
\begin{gathered}
\frac{b c-a d}{(c+d x)^{2}}=\frac{e(f+d x)-d(a+e x)}{(f+d x)^{2}} \\
\frac{b c-a d}{(c+d x)^{2}}=\frac{e f-a d}{(f+d x)^{2}}
\end{gathered}
$$

Por simple inspección, se obtiene que:

$$
c=f \text { y } b=e
$$


De donde:

$$
2 e-3 f=2 b-3 c
$$

37. A partir de $\int K \csc ^{2} 2 x d x=\tan x-\cot x+C$, encontrar el valor de $K$.

Solución:

Si derivamos ambos miembros, se obtiene que:

$$
\frac{k}{\operatorname{sen}^{2} 2 x}=\sec ^{2} x+\csc ^{2} x=\frac{1}{\cos ^{2} x}+\frac{1}{\operatorname{sen}^{2} x}
$$

De donde:

$$
\frac{k}{\operatorname{sen}^{2} 2 x}=\frac{\operatorname{sen}^{2} x+\cos ^{2} x}{\operatorname{sen}^{2} x \cos ^{2} x}=\frac{1}{(\operatorname{sen} x \cos x)^{2}}
$$

Con lo cual:

$$
K(\operatorname{sen} x \cos x)^{2}=\operatorname{sen}^{2} 2 x
$$

\section{2}

$$
\begin{gathered}
K\left[\frac{\operatorname{sen} 2 x}{2}\right]^{2}=\operatorname{sen}^{2} 2 x \\
\therefore K=4
\end{gathered}
$$

38. Si $\int \frac{\operatorname{sen}^{3} x}{\cos ^{4} x} d x=\frac{K}{\cos ^{3} x}-\frac{1}{\cos x}+C$, obtener el valor de $K$.

\section{Solución:}

A partir del dato, se concluye con que:

$$
\frac{\operatorname{sen}^{3} x}{\cos ^{4} x}=\frac{-3 k(-\operatorname{sen} x)}{\cos ^{4} x}+\frac{-\operatorname{sen} x}{\cos ^{2} x}
$$

De donde:

$$
\begin{gathered}
\operatorname{sen}^{3} x=3 K \operatorname{sen} x-\operatorname{sen} x \cos ^{2} x \\
\operatorname{sen}^{2} x+\cos ^{2} x=3 K \\
3 K=1 \rightarrow K=1 / 3
\end{gathered}
$$


39. ¿Cuánto es $f_{(a / 2)}$ a partir de $\int f_{(x)} d x=(a+x) \sqrt{a-x}+C$ ?

\section{Solución:}

Se tiene que:

$$
\begin{aligned}
& f_{(x)}=\frac{-(a+x)}{2 \sqrt{a-x}}+\sqrt{a-x} \\
& f_{(x)}=\frac{-a-x+2(a-x)}{2 \sqrt{a-x}}=\frac{a-3 x}{2 \sqrt{a-x}}
\end{aligned}
$$

De donde:

$$
f(a / 2)=\frac{a-\frac{3 a}{2}}{2 \sqrt{a-\frac{a}{2}}}=\frac{-a / 2}{2 \sqrt{a / 2}}=\frac{-\sqrt{2 a}}{4}
$$

40. Conocido $\int \frac{(\alpha-\beta) \operatorname{sen} 2 x}{2 \sqrt{\alpha \operatorname{sen}^{2} x+\beta \cos ^{2} x}} d x=\left(\alpha \operatorname{sen}^{2} x+\beta \cos ^{2} x\right)^{n}+C$, determinar el valor de $n$.

\section{Solución:}

Tenemos que:

$$
\begin{gathered}
\frac{(\alpha-\beta) \operatorname{sen} 2 x}{2 \sqrt{\alpha \operatorname{sen}^{2} x+\beta \cos ^{2} x}}=n\left(\alpha \operatorname{sen}^{2} x+\beta \cos ^{2} x\right)^{n-1}(2 \alpha \operatorname{sen} x \cos x-2 \beta \cos x \operatorname{sen} x) \\
\frac{(\alpha-\beta) \operatorname{sen} 2 x}{2 \sqrt{\alpha \operatorname{sen}^{2} x+\beta \cos ^{2} x}}=\frac{n(\alpha-\beta)(2 \operatorname{sen} x \cos x)}{\left(\alpha \operatorname{sen}^{2} x+\beta \cos ^{2} x\right)^{1-n}}
\end{gathered}
$$

De donde:

$$
\begin{aligned}
& \frac{1}{2 n}=\left(\alpha \operatorname{sen}^{2} x+\beta \cos ^{2} x\right)^{\frac{1}{2}-1+n} \\
& \frac{1}{2 n}=\left(\alpha \operatorname{sen}^{2} x+\beta \cos ^{2} x\right)^{n-1 / 2}
\end{aligned}
$$

Como el primer miembro no depende de $x$, se tiene que $n-\frac{1}{2}=0 \Rightarrow n=\frac{1}{2}$, que cumple con que $\frac{1}{2(1 / 2)}=1=\left(\alpha \operatorname{sen}^{2} x+\beta \cos ^{2} x\right)^{0}$. 
41. Si $\int f_{(x)} d x=\frac{-x}{2\left(x^{2}+a^{2}\right)}+\frac{1}{2 a} \arctan \left(\frac{x}{a}\right)+C$, halle $f(2 a)$.

R.: $\frac{4}{25 a^{2}}$

42. Si se cumple que:

$$
\int \frac{x^{2} d x}{3+2 x}=K\left[\frac{(3+2 x)^{2}}{2}-6(3+2 x)+9 \ln (3+2 x)\right]+C
$$

calcular el valor de $K$.

R.: $\frac{1}{8}$

43. La integral de $\frac{5 x^{2}+6 x+2}{(x+2)\left(x^{2}+2 x+5\right)}$ es

$$
K \ln (x+2)+\frac{3 K}{4} \ln \left(x^{2}+2 x+5\right)-\frac{7 K}{4} \arctan \frac{x+1}{2}+C .
$$

Encontrar el valor de $K$.

184
R.: 2

44. A partir de la fórmula $\int x(a+b x)^{n} d x=A(a+b x)^{n+2}-B(a+b x)^{n+1}+C(n \neq-1,-2)$, determinar $B / A$.
R.: $\frac{a(n+2)}{n+1}$

45. Si $\int g_{(x)} d x=\frac{-1}{a} \ln \frac{a+\sqrt{a^{2}+x^{2}}}{x}+C$, encontrar $a^{2} g_{(3 a)}$.
R.: $\frac{1}{3 \sqrt{10}}$

46. Encontrar $K$ para que se cumpla la fórmula:

$$
\int(\ln x)^{2} d x=x(\ln x)^{2}-2 x \ln x+K x+C
$$

R.: 2 
47. Si se cumple que:

$$
\int x^{n} \ln x d x=x^{k}\left[\frac{\ln x}{n+1}-\frac{1}{K^{2}}\right]
$$

determinar el valor de la constante $K$.

R.: $n+1$

48. Comprobar que se verifica:

$$
\int \frac{d x}{x(a+b x)^{2}}=\frac{1}{a(a+b x)}-\frac{1}{a^{2}} \ln \frac{a+b x}{x}+C
$$

49. Comprobar la fórmula de reducción:

$$
\int \operatorname{sen}^{n} x d x=\frac{-1}{n} \operatorname{sen}^{n-1} x \cos x+\frac{n-1}{n} \int \operatorname{sen}^{n-2} x d x
$$

50. Halle $K$, si $\int x^{m}(\ln x)^{n} d x=\frac{x^{m+1}(\ln x)^{n}}{m+1}+K \int x^{m}(\ln x)^{n-1} d x$.

$$
\text { R.: }-\frac{n}{m+1}
$$

51. Demostrar $\int \operatorname{sen}^{m} u \cdot \cos ^{n} u \cdot d u=\frac{\operatorname{sen}^{m+1} u \cos ^{n-1} u}{m+n}+\frac{n-1}{m+n} \int \operatorname{sen}^{m} u \cdot \cos ^{n-2} u \cdot d u$.

52. Halle $g(1,1,1)$ si se cumple:

$$
\int x^{m}\left(a+b x^{n}\right)^{p} d x=\frac{x^{m+1}\left(a+b x^{n}\right)^{p}}{n p+m+1}+g(n, m, p) \int x^{m}\left(a+b x^{n}\right)^{p-1} d x
$$

R.: $a / 3$

53. Halle $g(0,1, p)$ si:

$$
\int x^{m}\left(a+b x^{n}\right)^{p} d x=\frac{x^{m+1}\left(a+b x^{n}\right)^{p}}{n p+m+1}+g(m, n, p) \int x^{m}\left(a+b x^{n}\right)^{p-1} d x
$$

$$
\text { R.: } \frac{a p}{p+1}
$$

54. Si $\int f(x) d x=e^{\frac{1-x}{1+x}} \ln \left(1+x^{2}\right)-2 \arctan x+C$, halle $f(1)$.

$$
\text { R.: }-\frac{1}{2} \ln 2
$$


55. Si $\int f_{(x)} d x=\frac{-1}{4} \sqrt{1+\frac{4}{x^{2}}}+C$, encontrar $f_{(2)}$.

R.: $\frac{\sqrt{2}}{16}$

56. Dada $\int F_{(x)} d x=\frac{2 x^{2}-2 x+3}{20} \sqrt{3+4 x}+C$, hallar $F_{(0.25)}$.

R.: $\frac{1}{32}$

57. Calcular $g_{(a)}$ si se tiene que:

$$
\int g_{(x)} d x=\frac{1}{2 b^{3}}(a+b x)^{2}-\frac{2 a}{b^{3}}(a+b x)+\frac{a^{2}}{b^{3}} \ln (a+b x)+C
$$

R.: $\frac{a}{b+1}$

58. A partir de $\int G_{(x)} d x=\frac{-3}{20}\left(1-x^{2}\right)^{2 / 3}\left(2 x^{2}+3\right)+C$, obtener $G_{(1 / 2)}$.

R.: $\frac{\sqrt[3]{36}}{24}$

59. Sea $\int h_{(\theta)} d \theta=\frac{e^{a \theta}(a \operatorname{sen} b \theta-b \cos b \theta)}{a^{2}+b^{2}}+C$, determinar $h_{\left(\frac{\pi}{2 b}\right)}$.

R.: $e^{\frac{a \pi}{2 b}}$

60. Calcular $H_{(\pi / 4)}$ si se sabe que $\int H_{(\theta)} d \theta=\frac{\sqrt{15}}{15} \arctan \left(\frac{\sqrt{3} \tan \theta}{\sqrt{5}}\right)+C$.

R.: 0.25 


\section{PROBLEMAS RESUELTOS Y PROPUESTOS DEL CAPÍTULO III}

61. Encontrar la integral de la función $f_{(x)}=a x^{2 / n}-b \sqrt[n]{x}+\frac{c}{\sqrt[3]{x^{n}}}$.

Solución:

A partir de la fórmula $\frac{d u^{n}}{d x}=n u^{n-1} \frac{d u}{d x}$, se obtiene:

$$
\int\left(a x^{2 / n}-b x^{1 / n}+c x^{-n / 3}\right) d x=\frac{a x^{\frac{2}{n}+1}}{\frac{2}{n}+1}-\frac{b x^{\frac{1}{n}+1}}{\frac{1}{n}+1}+\frac{c x^{-\frac{n}{3}+1}}{-\frac{n}{3}+1}
$$

De donde:

$$
\frac{a n x^{\frac{n+2}{n}}}{n+2}-\frac{b n x^{\frac{n+1}{n}}}{n+1}+\frac{3 c}{(3-n) x^{\frac{n-3}{3}}}+C
$$

62. Calcular $\int \frac{2 x^{2}}{\left(1-8 x^{3}\right)^{4}} d x$.

Solución:

Escribamos:

$$
\int \frac{2 x^{2}}{\left(1-8 x^{3}\right)^{4}} d x=\int 2 x^{2}\left(1-8 x^{3}\right)^{-4} d x=\frac{-1}{12} \int\left(1-8 x^{3}\right)^{-4}\left(-24 x^{2} d x\right)
$$

De donde:

$$
\int \frac{2 x^{2}}{\left(1-8 x^{3}\right)^{4}} d x=\frac{-1}{12} \cdot \frac{\left(1-8 x^{3}\right)^{-3}}{-3}+C=\frac{1}{36\left(1-8 x^{3}\right)^{3}}+C
$$

63. Obtener $\int x^{2} \sqrt{x+1} d x$ a partir del cambio de variable $y=x+1$.

\section{Solución:}

Si $y=x+1 \rightarrow x=y-1 \Rightarrow d x=d y$.

La integral pedida se reduce a:

$$
\int x^{2} \sqrt{x+1} d x=\int(y-1)^{2} \sqrt{y} d y=\int\left(y^{2}-2 y+1\right) y^{1 / 2} d y
$$


Con lo cual:

$$
\int x^{2} \sqrt{x+1} d x=\int\left(y^{5 / 2}-2 y^{3 / 2}+y^{1 / 2}\right) d y=\frac{2 y^{7 / 2}}{7}-\frac{4 y^{5 / 2}}{5}+\frac{2 y^{3 / 2}}{3}+C
$$

La respuesta es:

$$
\frac{2}{7} \sqrt{(x+1)^{7}}-\frac{4}{5} \sqrt{(x+1)^{5}}+\frac{2}{3} \sqrt{(x+1)^{3}}+C
$$

64. Si $\int \frac{a e^{\theta}+b}{a e^{\theta}-b} d \theta=F_{(\theta)}+C$, halle $F_{(0)}$.

\section{Solución:}

Escribamos:

De donde:

$$
\begin{aligned}
& \int \frac{a e^{\theta}+b}{a e^{\theta}-b} d \theta=\int \frac{a e^{\theta} d \theta}{a e^{\theta}-b}+\int \frac{b d \theta}{a e^{\theta}-b} \\
& \int \frac{a e^{\theta}+b}{a e^{\theta}-b} d \theta=\int \frac{a e^{\theta} d \theta}{a e^{\theta}-b}+\int \frac{b e^{-\theta} d \theta}{a-b e^{-\theta}} \\
& \int \frac{a e^{\theta}+b}{a e^{\theta}-b} d \theta=\underbrace{\ln \left(a e^{\theta}-b\right)+\ln \left(a-b e^{-\theta}\right)}_{F_{(\theta)}}+C
\end{aligned}
$$

$$
F_{(0)}=\ln (a-b)+\ln (a-b)=2 \ln (a-b)
$$

Otro modo de resolverlo:

Escribamos:

$$
\begin{aligned}
& \int \frac{a e^{\theta}+b}{a e^{\theta}-b} d \theta=\int \frac{a e^{\theta}-b+2 b}{a e^{\theta}-b} d \theta=\int \frac{a e^{\theta}-b}{a e^{\theta}-b} d \theta+2 \int \frac{b}{a e^{\theta}-b} d \theta \\
& \int \frac{a e^{\theta}+b}{a e^{\theta}-b} d \theta=\int d \theta+2 \int \frac{b e^{-\theta} d \theta}{a-b e^{-\theta}}=\theta+2 \int \frac{b e^{-\theta} d \theta}{a-b e^{-\theta}} \\
& \int \frac{a e^{\theta}+b}{a e^{\theta}-b} d \theta=\underbrace{\theta+2 \ln \left(a-b e^{-\theta}\right)}_{F_{(\theta)}}+C
\end{aligned}
$$

Entonces:

$$
F_{(0)}=0+2 \ln (a-b)=2 \ln (a-b)
$$


65. Encontrar $\int \sec ^{2} \theta \tan \theta d \theta$ de dos formas distintas y averiguar en cuánto se diferencian las dos constantes de integración.

\section{Solución:}

Primera forma:

$$
\int \sec ^{2} \theta \tan \theta d \theta=\int \underbrace{(\tan \theta)}_{u} \underbrace{\left(\sec ^{2} \theta d \theta\right)}_{d u}=\frac{(\tan \theta)^{2}}{2}+C_{1}
$$

Segunda forma:

$$
\int \sec ^{2} \theta \tan \theta d \theta=\int \underbrace{(\sec \theta)}_{u} \underbrace{(\sec \theta \tan \theta d \theta)}_{d u}=\frac{(\sec \theta)^{2}}{2}+C_{2}
$$

La diferencia entre las constantes se obtiene de igualar:

$$
\begin{gathered}
\frac{(\tan \theta)^{2}}{2}+C_{1}=\frac{(\sec \theta)^{2}}{2}+C_{2} \\
C_{1}-C_{2}=\frac{1}{2}[\underbrace{\sec ^{2} \theta}_{1+\tan ^{2} \theta}-\tan ^{2} \theta]=\frac{1}{2}
\end{gathered}
$$

66. Si $\int \frac{d x}{x^{2} \sqrt{a^{2}-x^{2}}}=F_{(x)}+C$, halle $F\left(\frac{a}{2}\right)$.

\section{Solución:}

Calculemos $\int \frac{d x}{x^{2} \sqrt{a^{2}-x^{2}}}$ mediante una sustitución trigonométrica:

$$
x=a \operatorname{sen} \theta \rightarrow d x=a \cos \theta d \theta
$$

Entonces:

$$
\begin{gathered}
\int \frac{d x}{x^{2} \sqrt{a^{2}-x^{2}}}=\int \frac{a \cos \theta d \theta}{\left(a^{2} \operatorname{sen}^{2} \theta\right) \sqrt{a^{2}\left(1-\operatorname{sen}^{2} \theta\right)}}=\frac{1}{a^{2}} \int \frac{d \theta}{\operatorname{sen}^{2} \theta} \\
\int \frac{d x}{x^{2} \sqrt{a^{2}-x^{2}}}=\frac{1}{a^{2}} \int \csc ^{2} \theta d \theta=\underbrace{\frac{1}{a^{2}}(-\cot \theta)}_{F_{(\theta)}}+C
\end{gathered}
$$


De donde:

$$
\begin{gathered}
F_{(\theta)}=\frac{-1}{a^{2}} \cot \theta \\
F_{(x)}=\frac{-1}{a^{2}} \cot \left(\operatorname{arcsen} \frac{x}{a}\right)
\end{gathered}
$$

Con lo cual:

$$
\begin{gathered}
F_{(a / 2)}=\frac{-1}{a^{2}} \cot \left(\operatorname{arcsen} \frac{a / 2}{a}\right)=\frac{-1}{a^{2}} \cot (\operatorname{arcsen} 1 / 2) \\
F_{(a / 2)}=\frac{-1}{a^{2}} \cot \frac{\pi}{6}=\frac{-1}{a^{2}}(\sqrt{3})=\frac{-\sqrt{3}}{a^{2}}
\end{gathered}
$$

67. Si $\int \tan ^{4} u d u=\frac{\tan ^{\alpha} u}{\alpha}-\frac{\tan ^{\beta} u}{\beta}+u+C$, halle $\alpha-\beta$.

\section{Solución:}

Escribamos:

$$
\begin{gathered}
\int \tan ^{4} u d u=\int\left(\tan ^{2} u \cdot \tan ^{2} u\right) d u=\int\left(\sec ^{2} u-1\right) \tan ^{2} u d u \\
\int \tan ^{4} u d u=\int\left(\sec ^{2} u \cdot \tan ^{2} u-\tan ^{2} u\right) d u=\int\left[\tan ^{2} u \cdot \sec ^{2} u-\left(\sec ^{2} u-1\right)\right] d u \\
\int \tan ^{4} u d u=\frac{\tan ^{3} u}{3}-\tan u+u+C
\end{gathered}
$$

De donde:

$$
\alpha=3 \text { y } \beta=1
$$

Con lo cual:

$$
\alpha-\beta=2
$$

68. Obtener: $\int \frac{d x}{\operatorname{sen} x \cos x}$.

\section{Solución:}

Escribamos:

$$
\begin{gathered}
\int \frac{d x}{\operatorname{sen} x \cos x}=\int \frac{\cos ^{2} x+\operatorname{sen}^{2} x}{\operatorname{sen} x \cos x} d x=\int \frac{\frac{\cos ^{2} x+\operatorname{sen}^{2} x}{\cos ^{2} x}}{\frac{\operatorname{sen} x \cos x}{\cos ^{2} x}} d x= \\
\int \frac{d x}{\operatorname{sen} x \cos x}=\int \frac{\frac{\cos x(\cos x)-\operatorname{sen} x(-\operatorname{sen} x)}{\cos ^{2} x}}{\frac{\operatorname{sen} x}{\cos x}} d x
\end{gathered}
$$


Al analizar la última integral, se llega a la conclusión de que la respuesta es:

$$
\ln \frac{\operatorname{sen} x}{\cos x}+C \circ \ln \tan x+C
$$

69. Encontrar: $\int \frac{d x}{1-\cos x}$.

\section{Solución:}

Se tiene que:

$$
\begin{gathered}
\cos x=\cos ^{2} \frac{x}{2}-\operatorname{sen}^{2} \frac{x}{2} \\
\cos x=\left(1-\operatorname{sen}^{2} \frac{x}{2}\right)-\operatorname{sen}^{2} \frac{x}{2}=1-2 \operatorname{sen}^{2} \frac{x}{2}
\end{gathered}
$$

De donde:

$$
1-\cos x=2 \operatorname{sen}^{2} \frac{x}{2}
$$

Entonces:

$$
\int \frac{d x}{1-\cos x}=\int \frac{d x}{2 \operatorname{sen}^{2} \frac{x}{2}}=\frac{1}{2} \int \csc ^{2} \frac{x}{2} d x
$$

Con lo cual:

$$
\int \frac{d x}{1-\cos x}=-\frac{1}{2} \cot \frac{x}{2}+C
$$

70. Integrar $I=\int \operatorname{sen}^{3} x \cdot \sec x \cdot d x$.

\section{Solución:}

Escribamos:

$$
\begin{gathered}
I=\int \operatorname{sen}^{2} x \cdot \frac{\operatorname{sen} x}{\cos x} d x=\int\left(1-\cos ^{2} x\right) \tan x d x \\
I=\int \tan x d x-\int \cos ^{2} x \tan x d x \\
I=\int \frac{\operatorname{sen} x}{\cos x} d x-\int \cos ^{2} x \cdot \frac{\operatorname{sen} x}{\cos x} d x \\
I=-\int \frac{-\operatorname{sen} x}{\cos x} d x-\frac{1}{2} \int \operatorname{sen} 2 x d x \\
I=-\ln (\cos x)+\frac{1}{4} \cos 2 x+C \\
I=\ln (\sec x)+\frac{1}{4} \cos 2 x+C
\end{gathered}
$$


71. Resolver: $\int \frac{x+7}{\sqrt{x^{2}+4 x+13}} d x$

\section{Solución:}

Escribamos:

$$
\begin{gathered}
\int \frac{x+7}{\sqrt{x^{2}+4 x+13}} d x=\int\left(x^{2}+4 x+13\right)^{-1 / 2}(x+7) d x \\
\int \frac{x+7}{\sqrt{x^{2}+4 x+13}} d x=\frac{1}{2} \int[(2 x+4)+10]\left(x^{2}+4 x+13\right)^{-1 / 2} d x
\end{gathered}
$$

Descompongamos en dos integrales:

$$
\begin{aligned}
& \frac{1}{2} \int(2 x+4)\left(x^{2}+4 x+13\right)^{-1 / 2} d x+5 \int \frac{1}{\sqrt{(x+2)^{2}+3^{2}}} d x \\
& \frac{1}{2} \cdot \frac{\left(x^{2}+4 x+13\right)^{1 / 2}}{1 / 2}+5 \ln \left[x+2+\sqrt{x^{2}+4 x+13}\right]+C
\end{aligned}
$$

192

La respuesta es:

$$
\sqrt{x^{2}+4 x+13}+5 \ln \left[x+2+\sqrt{x^{2}+4 x+13}\right]+C
$$

72. Hallar la integral:

$$
\int \frac{x}{\sqrt{x^{2}+p x+p^{2}}} d x
$$

\section{Solución:}

Escribamos:

$$
\begin{aligned}
& \int \frac{x}{\sqrt{x^{2}+p x+p^{2}}} d x=\int\left(x^{2}+p x+p^{2}\right)^{-1 / 2}(x d x)=\frac{1}{2} \int\left(x^{2}+p x+p^{2}\right)^{-1 / 2}[2 x+p-p] d x \\
& \int \frac{x}{\sqrt{x^{2}+p x+p^{2}}} d x=\frac{1}{2} \int\left(x^{2}+p x+p^{2}\right)^{-1 / 2}(2 x+p) d x-\frac{p}{2} \int \frac{d x}{\sqrt{\left(x+\frac{p}{2}\right)^{2}+\left(\frac{\sqrt{3} p}{2}\right)^{2}}} \\
& \int \frac{x}{\sqrt{x^{2}+p x+p^{2}}} d x=\frac{1}{2} \cdot \frac{\left(x^{2}+p x+p^{2}\right)^{1 / 2}}{1 / 2}-\frac{p}{2} \ln \left[\left(x+\frac{p}{2}\right)+\sqrt{x^{2}+p x+p^{2}}\right]+C \\
& \int \frac{x}{\sqrt{x^{2}+p x+p^{2}}} d x=\sqrt{\left(x^{2}+p x+p^{2}\right)}-\frac{p}{2} \ln \left[\left(x+\frac{p}{2}\right)+\sqrt{x^{2}+p x+p^{2}}\right]+C
\end{aligned}
$$


73. Si $\int \frac{d x}{x^{2}+a x+b x+a b}=F_{(x)}+C$, halle $F_{(0)}$, sabiendo que $b=\frac{a}{2}$.

\section{Solución:}

Como:

$$
\int \frac{d x}{x^{2}+3 b x+2 b^{2}}=\int \frac{d x}{\left(x+\frac{3 b}{2}\right)^{2}-\left(\frac{b}{2}\right)^{2}}=\frac{1}{2\left(\frac{b}{2}\right)} \ln \frac{x+\frac{3 b}{2}-\frac{b}{2}}{x+\frac{3 b}{2}+\frac{b}{2}}+C
$$

De donde:

$$
\begin{aligned}
& F_{(x)}=\frac{1}{b} \ln \frac{x+b}{x+2 b} \\
& F_{(0)}=\frac{1}{b} \ln \frac{b}{2 b}=\frac{1}{b} \ln \frac{1}{2}=\ln \sqrt[b]{1 / 2}
\end{aligned}
$$

74. Calcular: $\int \frac{e^{t}}{e^{2 t}+a e^{t}+b e^{t}+a b} d t$

\section{Solución:}

Escribamos:

$\int \frac{e^{t} d t}{e^{2 t}+(a+b) e^{t}+a b}=\int \frac{e^{t} d t}{\left(e^{t}+\frac{a+b}{2}\right)^{2}-\left(\frac{a+b}{2}\right)^{2}+a b}=\int \frac{e^{t} d t}{\left(e^{t}+\frac{a+b}{2}\right)^{2}-\left(\frac{a-b}{2}\right)^{2}}$

Si $e^{t}+\frac{a+b}{2}=u \Rightarrow d u=e^{t} d t$

Entonces:

$$
\begin{aligned}
& \int \frac{e^{t} d t}{e^{2 t}+(a+b) e^{t}+a b}=\frac{1}{2\left(\frac{a-b}{2}\right)} \ln \frac{e^{t}+\frac{a+b}{2}-\frac{a-b}{2}}{e^{t}+\frac{a+b}{2}+\frac{a-b}{2}}+C \\
& \int \frac{e^{t} d t}{e^{2 t}+(a+b) e^{t}+a b}=\frac{1}{a-b} \ln \frac{e^{t}+b}{e^{t}+a}+C
\end{aligned}
$$

75. Evaluar: $\int \frac{d x}{x \sqrt{1+x+x^{2}}}$ con un cambio de variable inicial adecuado.

\section{Solución:}

Sea $x=\frac{1}{t} \Rightarrow d x=\frac{-1}{t^{2}} d t$ 
Entonces:

$$
\begin{aligned}
& \int \frac{d x}{x \sqrt{1+x+x^{2}}}=\int \frac{-\frac{d t}{t^{2}}}{\frac{1}{t} \sqrt{1+\frac{1}{t}+\frac{1}{t^{2}}}}=-\int \frac{d t}{\sqrt{t^{2}+t+1}} \\
& \int \frac{d x}{x \sqrt{1+x+x^{2}}}=-\int \frac{d t}{\sqrt{(t+1 / 2)^{2}+(\sqrt{3} / 2)^{2}}} \\
& \int \frac{d x}{x \sqrt{1+x+x^{2}}}=-\ln \left[(t+1 / 2)+\sqrt{(t+1 / 2)^{2}+(\sqrt{3} / 2)^{2}}\right]+C
\end{aligned}
$$

La respuesta es:

$$
-\ln \left[(t+1 / 2)+\sqrt{t^{2}+t+1}\right]+C=-\ln \left[\left(\frac{1}{x}+\frac{1}{2}\right)+\sqrt{\frac{1}{x^{2}}+\frac{1}{x}+1}\right]+C
$$

76. Si $\int \frac{d x}{(1+x)^{3 / 2}+(1+x)^{1 / 2}}=F_{(x)}+C$ y $F_{\left(x_{0}\right)}=\frac{\pi}{3}$, halle $x_{0}$.

\section{Solución:}

Se tiene que:

$$
\int \frac{d x}{(1+x)^{3 / 2}+(1+x)^{1 / 2}}=\int \frac{d x}{(1+x)^{1 / 2}[(1+x)+1]}=\int \frac{d x}{(1+x)^{1 / 2}(x+2)}
$$

Sea $1+x=u^{2} \Rightarrow d x=2 u d u$

Entonces:

$$
\begin{gathered}
\int \frac{d x}{(1+x)^{1 / 2}(x+2)}=\int \frac{2 u d u}{u\left(u^{2}-1+2\right)}=2 \int \frac{d u}{u^{2}+1} \\
\int \frac{d x}{(1+x)^{1 / 2}(x+2)}=\underbrace{2\left(\frac{1}{1}\right) \arctan \frac{u}{1}}_{F_{(u)}}+C
\end{gathered}
$$

De donde:

$$
\begin{aligned}
& F_{(x)}=2 \arctan \sqrt{1+x} \\
& F_{\left(x_{0}\right)}=2 \arctan \sqrt{1+x_{0}}=\frac{\pi}{3}
\end{aligned}
$$




$$
\begin{aligned}
& \arctan \sqrt{1+x_{0}}=\frac{\pi}{6} \\
& \sqrt{1+x_{0}}=\tan \frac{\pi}{6}=\frac{\sqrt{3}}{3} \\
& 1+x_{0}=\frac{3}{9}=\frac{1}{3} \\
& \therefore \quad x_{0}=-2 / 3
\end{aligned}
$$

77. Si $\int \frac{1+3 x^{2}}{x^{2}\left(1+x^{2}\right)} d x=-\frac{1}{x}+f_{(x)}+C$, halle $f_{(1)}$.

\section{Solución:}

Descompongamos el integrando de la siguiente manera:

$$
\frac{1+3 x^{2}}{x^{2}\left(1+x^{2}\right)}=\frac{1+x^{2}}{x^{2}\left(1+x^{2}\right)}+\frac{2 x^{2}}{x^{2}\left(1+x^{2}\right)}=\frac{1}{x^{2}}+\frac{2}{1+x^{2}}
$$

Entonces:

$$
\int \frac{1+3 x^{2}}{x^{2}\left(1+x^{2}\right)} d x=\int\left[\frac{1}{x^{2}}+\frac{2}{1+x^{2}}\right] d x=-\frac{1}{x}+2 \arctan x+C
$$

De donde:

$$
f_{(x)}=2 \arctan x
$$

Con lo cual:

$$
f_{(1)}=2 \arctan 1=2(\pi / 4)=\pi / 2
$$

78. Usar el cambio de variable $u=x+\frac{1}{x}$ en

$$
\int \sqrt{x^{2}+\frac{1}{x^{2}}+11}\left(\frac{x^{2}-1}{x^{2}}\right) d x=F_{(x)}+C \text { para evaluar } F_{(1)} .
$$

\section{Solución:}

Sea:

$$
\begin{gathered}
u=x+\frac{1}{x} \Rightarrow d u=\left(1-\frac{1}{x^{2}}\right) d x \Rightarrow d u=\frac{x^{2}-1}{x^{2}} d x \\
u^{2}=x^{2}+2+\frac{1}{x^{2}} \Rightarrow x^{2}+\frac{1}{x^{2}}=u^{2}-2
\end{gathered}
$$


Entonces se tiene que:

$$
\begin{aligned}
& \int \sqrt{x^{2}+\frac{1}{x^{2}}+11}\left(\frac{x^{2}-1}{x^{2}}\right) d x=\int \sqrt{\left(u^{2}-2\right)+11}(d u) \\
& \int \sqrt{x^{2}+\frac{1}{x^{2}}+11}\left(\frac{x^{2}-1}{x^{2}}\right) d x=\int \sqrt{u^{2}+9} d u=\frac{1}{2} u \sqrt{u^{2}+9}+\frac{1}{2}(9) \ln \left[u+\sqrt{u^{2}+9}\right]+C \\
& \text { Si } x=1 \rightarrow u=1+\frac{1}{1}=2
\end{aligned}
$$

De donde:

$$
\begin{gathered}
F_{(2)}=\frac{1}{2}(2) \sqrt{13}+\frac{9}{2} \ln [2+\sqrt{13}] \\
F_{(2)}=3.6056+7.7570=11.3626
\end{gathered}
$$

79. Integrar: $I=\int \frac{4 e^{2 x}}{e^{2 x}-2 e^{x}-3} d x$.

\section{Solución:}

Escribámoslo así:

$$
\begin{gathered}
I=2 \int \frac{2 e^{2 x}}{e^{2 x}-2 e^{x}-3} d x \\
I=2\left[\int \frac{\left(2 e^{2 x}-2 e^{x}\right)}{e^{2 x}-2 e^{x}-3} d x+\int \frac{2 e^{x}}{e^{2 x}-2 e^{x}-3} d x\right] \\
I=2\left[\ln \left(e^{2 x}-2 e^{x}-3\right)+2 \int \frac{e^{x}}{\left(e^{x}-1\right)^{2}-2^{2}} d x\right]
\end{gathered}
$$

Finalmente:

$$
\begin{gathered}
I=2\left[\ln \left(e^{2 x}-2 e^{x}-3\right)+2\left(\frac{1}{2(2)}\right) \ln \frac{e^{x}-1-2}{e^{x}-1+2}\right] \\
I=2 \cdot \ln \left(e^{2 x}-2 e^{x}-3\right)+\ln \frac{e^{x}-3}{e^{x}+1}+C
\end{gathered}
$$

80. Integrar: $I=\int \frac{(x+3) d x}{\sqrt{x^{2}+4 x-5}}$. 


\section{Solución:}

Escribámoslo así:

$$
\begin{gathered}
I=\frac{1}{2} \int \frac{2 x+4+2}{\sqrt{x^{2}+4 x-5}} d x \\
I=\frac{1}{2} \int \frac{2 x+4}{\sqrt{x^{2}+4 x-5}} d x+\int \frac{d x}{\sqrt{(x+2)^{2}-3^{2}}}
\end{gathered}
$$

De donde:

$$
\begin{gathered}
I=\frac{1}{2} \frac{\left(x^{2}+4 x-5\right)^{1 / 2}}{1 / 2}+\ln \left[x+2+\sqrt{x^{2}+4 x-5}\right]+C \\
I=\sqrt{x^{2}+4 x-5}+\ln \left[x+2+\sqrt{x^{2}+4 x-5}\right]+C
\end{gathered}
$$

81. Integrar: $\int \frac{d x}{(\operatorname{sen} x+\cos x)^{2}}$. Sugerencia: hagau $=\operatorname{sen} 2 x$.

R.: $-\frac{1}{2} \sqrt{\frac{1-\operatorname{sen} 2 x}{1+\operatorname{sen} 2 x}}+C$

82. Integrar: $I=\int \frac{\ln (x+1)-\ln x}{x(x+1)} d x$.

$$
\text { R.: }-\frac{1}{2} \ln ^{2} \frac{x+1}{x}+C
$$

83. Integrar: $I=\int \frac{\arctan (\sqrt{x})}{\sqrt{x^{3}+2 x^{2}+x}} d x$.

$$
\mathrm{R} .:(\arctan \sqrt{x})^{2}+C
$$

84. Integrar: $\int \frac{\ln (\ln x)+1}{x \ln x} d x$.

$$
\mathrm{R} .: \frac{1}{2}[\ln (\ln x)+1]^{2}+C
$$


85. Integrar: $\int \frac{x^{3}}{\left(x^{2}+8\right)^{3 / 2}} d x$.

$$
\text { R.: } \frac{8}{\sqrt{x^{2}+8}}+\sqrt{x^{2}+8}+C
$$

86. Integrar: $\int \frac{d x}{\sqrt{x+a}+\sqrt{x-b}}$.

$$
\text { R.: } \frac{2}{3(a+b)}\left[(x+a)^{3 / 2}-(x-b)^{3 / 2}\right]+C
$$

87. Integrar: $\int \frac{d x}{x \cdot \cos ^{2}(1+\ln x)}$.

R.: $\tan (1+\ln x)+C$

88. Integrar: $\int \frac{1+\cos ^{2} x}{1+\cos 2 x} d x$.

198

$$
\mathrm{R} .: \frac{1}{2}(\tan x+x)+C
$$

89. Integrar: $\int \frac{\ln x \sqrt{\ln ^{2} x+4}}{x} d x$.

$$
\text { R.: } \frac{1}{3}\left(\ln ^{2} x+4\right)^{3 / 2}+C
$$

90. Resolver: $\int \cos ^{4} 3 x d x$.

$$
\text { R.: } \frac{3 x}{8}+\frac{\operatorname{sen} 6 x}{12}+\frac{\operatorname{sen} 12 x}{96}+C
$$

91. Resolver: $\int \frac{d x}{\operatorname{sen}^{2} x \cos ^{2} x}$.

$$
\text { R.: }-2 \cot 2 x+C
$$


92. Señalar $g_{(1)}$ si se conoce que $\int \frac{\sqrt{x^{2}+1}}{x^{2}} d x=g_{(x)}+C$.

$$
\text { R.: }-\sqrt{2}+\ln (1+\sqrt{2})
$$

93. Si $\int \frac{d x}{\sqrt[n]{x}}=K x g_{(x)}+C$, halle $g_{\left(2^{n}\right)}$.

\section{R.: $2^{-1}$}

94. Si $\int \frac{d x}{x^{2}+a^{2}}=h_{(x)}+C$, halle $h_{(a)}$.

$$
\text { R.: } \frac{\pi}{4 a}
$$

95. Si $\int \frac{\ln x}{x\left(1+\ln ^{2} x\right)} d x=F_{(x)}+C$, entonces $F_{(1)}$ es igual a:

$$
\text { R.: } 0
$$

96. Si $\int \frac{d u}{u \sqrt{a^{2}-u^{2}}}=F_{(u)}+C$, entonces $F\left(\frac{a}{2}\right)$ es igual a:

$$
R .: \ln \sqrt[a]{2-\sqrt{3}}
$$

97. Si $\int\left(n^{K} x\right)^{\frac{1}{n}-1} d x=n^{2-n} x^{1 / n}$, determinar el valor de $K$.

R.: $n$

98. Integrar: $\int \frac{x}{a+b x} d x$.

$$
\text { R.: } \frac{1}{b} x-\frac{a}{b^{2}} \ln (a+b x)+\frac{a}{b^{2}}+C \circ \frac{1}{b} x-\frac{a}{b^{2}} \ln (a+b x)+C^{\prime}
$$

99. Integrar: $\int \frac{x^{2}}{\sqrt{1-x^{2}}} d x$.

$$
\text { R.: }-\frac{1}{2} \arccos x-\frac{1}{2} x \sqrt{1-x^{2}}+C
$$


100. Integrar: $\int \frac{d x}{3 x^{2}-x+1}$.

$$
\text { R.: } \frac{2 \sqrt{11}}{11} \arctan \frac{6 x-1}{\sqrt{11}}+C
$$




\section{PROBLEMAS RESUELTOS Y PROPUESTOS DEL CAPÍTULO IV}

101. Integrar: $\int \sqrt{\frac{1-\sqrt{x}}{1+\sqrt{x}}} d x$.

Solución:

$$
\begin{aligned}
& \int \sqrt{\frac{1-\sqrt{x}}{1+\sqrt{x}}} d x=\int \sqrt{\frac{1-\sqrt{x}}{1+\sqrt{x}} \cdot \frac{1-\sqrt{x}}{1-\sqrt{x}}} d x \\
&=\int \frac{(1-\sqrt{x})}{\sqrt{1-x}} d x \\
&=\int \frac{1}{\sqrt{1-x}} d x-\int \frac{\sqrt{x}}{\sqrt{1-x}} d x \\
& I=-\int(1-x)^{-1 / 2}(-1) d x-\underbrace{\int \frac{\sqrt{x}}{\sqrt{1-x}} d x}_{J} \\
& I=-2 \sqrt{1-x}-J
\end{aligned}
$$

Calculemos $\mathrm{J}$ :

$u^{2}=1-x, 2 u d u=-d x$

$$
\begin{aligned}
J & =\int \frac{\sqrt{1-u^{2}}}{u} \cdot(-2 u d u) \\
J & =-2 \int \sqrt{1-u^{2}} d u \\
& =-2\left[\frac{u}{2} \sqrt{1-u^{2}}+\frac{1}{2} \operatorname{arcsen} u\right] \\
& =-u \sqrt{1-u^{2}}-\operatorname{arcsen} u \\
& =-\sqrt{1-x} \sqrt{x}-\operatorname{arcsen} \sqrt{1-x} \\
I & =-2 \sqrt{1-x}+\sqrt{x-x^{2}}+\operatorname{arcsen} \sqrt{1-x}+C
\end{aligned}
$$

102. $\operatorname{Si} \int \frac{1}{x^{2} \sqrt{x^{2}+4}} d x=F_{(x)}+C$, halle $F_{(2)}$.

Solución:

Sea:

$$
x=\frac{1}{t} \Rightarrow d x=-\frac{1}{t^{2}} d t
$$


Entonces:

$$
\begin{gathered}
\int \frac{d x}{x^{2} \sqrt{x^{2}+4}}=\int \frac{-\frac{1}{t^{2}} d t}{\frac{1}{t^{2}} \sqrt{\frac{1}{t^{2}}+4}}=-\int \frac{t d t}{\sqrt{1+4 t^{2}}} \\
\int \frac{d x}{x^{2} \sqrt{x^{2}+4}}=-\frac{1}{8} \int\left(1+4 t^{2}\right)^{-1 / 2}(8 t d t)=-\frac{1}{8} \frac{\left(1+4 t^{2}\right)^{1 / 2}}{1 / 2}+C \\
\int \frac{d x}{x^{2} \sqrt{x^{2}+4}}=\underbrace{-\frac{1}{4} \sqrt{1+4 t^{2}}}_{F_{(t)}}+C
\end{gathered}
$$

De donde:

$$
F_{(x)}=\frac{-1}{4} \sqrt{1+\frac{4}{x^{2}}}
$$

Con lo cual:

202

$$
F_{(2)}=\frac{-1}{4} \sqrt{1+1}=\frac{-\sqrt{2}}{4}
$$

103. $\int \frac{x^{3}}{\sqrt{x^{2}+1}} d x=\frac{\left(x^{2}+1\right)^{m}}{n}-\left(x^{2}+1\right)^{p}+c$, halle $m+p+n$.

\section{Solución:}

Sea $x^{2}+1=u^{2} \Rightarrow 2 x d x=2 u d u$

Entonces:

$$
\begin{gathered}
\int \frac{x^{3}}{\sqrt{x^{2}+1}} d x=\frac{1}{2} \int \frac{x^{2}(2 x d x)}{\sqrt{x^{2}+1}}=\frac{1}{2} \int \frac{\left(u^{2}-1\right)(2 u d u)}{u} \\
\int \frac{x^{3}}{\sqrt{x^{2}+1}} d x=\int\left(u^{2}-1\right) d u=\frac{u^{3}}{3}-u+C
\end{gathered}
$$

Es decir:

$$
\int \frac{x^{3}}{\sqrt{x^{2}+1}} d x=\frac{\left(x^{2}+1\right)^{3 / 2}}{3}-\left(x^{2}+1\right)^{1 / 2}+C
$$


De donde:

$$
m=3 / 2, n=3 \text { y } p=1 / 2
$$

Con lo cual:

$$
m+p-n=\frac{3}{2}+\frac{1}{2}-3=-1
$$

104. Si $\int \frac{\ln x}{x^{3}(\ln x-1)\left(2 \ln x-\ln ^{2} x-1\right)} d x=f_{(x)}+C$, halle $f_{\left(e^{2}\right)}$.

\section{Solución:}

Se tiene:

$$
\begin{gathered}
I=\int \frac{\ln x d x}{x^{3}(\ln x-1)\left(2 \ln x-\ln ^{2} x-1\right)}=\int \frac{\ln x d x}{x^{3}(\ln x-1)\left[-(\ln x-1)^{2}\right]} \\
I=-\int \frac{\ln x d x}{x^{3}(\ln x-1)^{3}}=-\int[x(\ln x-1)]^{-3}[\ln x] d x
\end{gathered}
$$

Si $u=x(\ln x-1) \Rightarrow d u=\left[1(\ln x-1)+x\left(\frac{1}{x}\right)\right] d x=\ln x d x$

Entonces:

$$
I=-\int u^{-3} d u=-\frac{u^{-2}}{-2}+C=\underbrace{\frac{1}{2 u^{2}}}_{f_{(u)}}+C
$$

De donde:

$$
f(x)=\frac{1}{2[x(\ln x-1)]^{2}}
$$

Con lo cual:

$$
f\left(e^{2}\right)=\frac{1}{2\left[e^{2}(2-1)\right]^{2}}=\frac{1}{2 e^{4}}
$$

105. Si $\int e^{x} \sqrt{a-b e^{x}} d x=m\left(a-b e^{x}\right)^{n}+C$, calcular $m b+n$.

\section{Solución:}

Sea:

$$
w^{2}-a-b e^{x} \Rightarrow b e^{x}=a-w^{2} \Rightarrow b e^{x} d x=-2 w d w
$$


Entonces:

$$
\begin{gathered}
\int e^{x} \sqrt{a-b e^{x}} d x=\frac{1}{b} \int \sqrt{a-b e^{x}}\left(b e^{x} d x\right)=\frac{1}{b} \int w(-2 w d w) \\
\int e^{x} \sqrt{a-b e^{x}} d x=\frac{-2}{b} \int w^{2} d w=\frac{-2}{b} \cdot \frac{w^{3}}{3}+C
\end{gathered}
$$

De donde:

$$
-\frac{2}{3 b}\left(a-b e^{x}\right)^{3 / 2}=m\left(a-b e^{x}\right)^{n}
$$

Con lo cual:

$$
m=\frac{-2}{3 b} \text { y } n=\frac{3}{2}
$$

$y:$

$$
m b+n=\frac{-2}{3 b} b+\frac{3}{2}=\frac{-2}{3}+\frac{3}{2}=\frac{5}{6}
$$

204 106. Hallar $\int \frac{\ln (\ln x)}{x \ln x} d x$.

\section{Solución:}

Sea:

$$
\ln x=u \Rightarrow x=e^{u} \Rightarrow d x=e^{u} d u
$$

Entonces:

$$
\begin{gathered}
\int \frac{\ln (\ln x)}{x \ln x} d x=\int \frac{\ln u}{e^{u} u} e^{u} d u=\int \frac{\ln u}{u} d u \\
\int \frac{\ln (\ln x)}{x \ln x} d x=\int(\ln u)\left(\frac{d u}{u}\right)=\frac{(\ln u)^{2}}{2}+C
\end{gathered}
$$

De donde:

$$
\int \frac{\ln (\ln x)}{x \ln x} d x=\frac{1}{2}[\ln (\ln x)]^{2}+C
$$

107. Integrar: $I=\int \frac{d x}{x \sqrt{x+1}}$.

Solución:

Sea $u^{2}=x+1 \Rightarrow 2 u d u=d x$ 
Entonces:

$$
\begin{gathered}
I=\int \frac{2 u d u}{\left(u^{2}-1\right) \sqrt{u^{2}}}=2 \int \frac{d u}{u^{2}-1} \\
I=2\left(\frac{1}{2(1)}\right) \ln \frac{u-1}{u+1}
\end{gathered}
$$

Es decir:

$$
I=\ln \frac{\sqrt{x+1}-1}{\sqrt{x+1}+1}+C
$$

108. Integrar: $I=\int f^{\prime}(\ln x) \frac{d x}{x}$.

\section{Solución:}

Si $u=\ln x \Rightarrow d u=\frac{1}{x} d x$

$$
I=\int f^{\prime}(u) d u=f_{(u)}+C
$$

Es decir:

$$
I=f_{(\ln x)}+C
$$

109. Integrar: $I=\int \frac{\sqrt{x+9}}{x} d x$.

\section{Solución:}

Sea $x+9=u^{2} \Rightarrow x=u^{2}-9 \Rightarrow d x=2 u d u$

Entonces:

$$
\begin{gathered}
I=\int \frac{\sqrt{u^{2}}}{u^{2}-9}(2 u d u)=2 \int \frac{u^{2} d u}{u^{2}-9} \\
I=2 \int \frac{\left(u^{2}-9\right)+9}{u^{2}-9} d u \\
I=2 \int\left[1+\frac{9}{u^{2}-9}\right] d u \\
I=2\left[u+9\left(\frac{1}{2(3)}\right) \ln \frac{u-3}{u+3}\right]+C
\end{gathered}
$$


De donde:

$$
I=2 \sqrt{x+9}+3 \cdot \ln \frac{\sqrt{x+9}-3}{\sqrt{x+9}+3}+C
$$

110. Obtener $\int \frac{x d x}{a+b x}$ de dos formas distintas y comprobar que la diferencia de los dos resultados es una constante.

\section{Solución:}

Primera forma:

Sea $a+b x=u \Rightarrow b d x=d u \quad x=\frac{u-a}{b}$

Entonces:

$$
\begin{gathered}
\int \frac{x d x}{a+b x}=\int \frac{\frac{u-a}{b} \cdot \frac{d u}{b}}{u}=\frac{1}{b^{2}} \int \frac{u-a}{u} d u \\
\int \frac{x d x}{a+b x}=\frac{1}{b^{2}} \int\left[1-\frac{a}{u}\right] d u=\frac{1}{b^{2}}[u-a \ln u]+C_{1}
\end{gathered}
$$

La respuesta es:

$$
\frac{u}{b^{2}}-\frac{a}{b^{2}} \ln u+C_{1}
$$

Es decir:

$$
\begin{aligned}
& \frac{a+b x}{b^{2}}-\frac{a}{b^{2}} \ln (a+b x)+C_{1} \\
& \frac{a}{b^{2}}+\frac{x}{b}-\frac{a}{b^{2}} \ln (a+b x)+C_{1}
\end{aligned}
$$

Segunda forma:

Dividamos:

$$
\frac{x-\frac{a}{b}}{-\frac{a}{b}}
$$


De donde:

$$
\frac{x}{a+b x}=\frac{1}{b}-\frac{a / b}{a+b x}
$$

Entonces:

$$
\begin{gathered}
\int \frac{x d x}{a+b x}=\int\left[\frac{1}{b}-\frac{a / b}{a+b x}\right] d x \\
\int \frac{x d x}{a+b x}=\frac{1}{b} x-\frac{a}{b^{2}} \ln (a+b x)+C_{2}
\end{gathered}
$$

De $(\alpha)$ :

$$
\frac{x}{b}-\frac{a}{b^{2}} \ln (a+b x)+\left[\frac{a}{b^{2}}+C_{1}\right]
$$

$\operatorname{De}(\beta)$ :

$$
\frac{x}{b}-\frac{a}{b^{2}} \ln (a+b x)+\left[C_{2}\right]
$$

La diferencia de los dos resultados es:

$$
\frac{a}{b^{2}}+C_{1}-C_{2}=\text { constante }
$$

111. Hallar: $\int \frac{x^{2} d x}{(a+b x)}$.

\section{Solución:}

Sea:

$$
u=a+b x \rightarrow x=\frac{u-a}{b} \Rightarrow d x=\frac{1}{b} d u
$$

Entonces:

$$
\begin{gathered}
\int \frac{x^{2} d x}{(a+b x)}=\int \frac{\left(\frac{u-a}{b}\right)^{2}\left(\frac{1}{b} d u\right)}{u}=\frac{1}{b^{3}} \int \frac{(u-a)^{2}}{u} d u \\
\int \frac{x^{2} d x}{(a+b x)}=\frac{1}{b^{3}} \int\left[u-2 a+\frac{a^{2}}{u}\right] d u \\
\int \frac{x^{2} d x}{(a+b x)}=\frac{1}{b^{3}}\left[\frac{u^{2}}{2}-2 a u+a^{2} \ln u\right]+C
\end{gathered}
$$


De donde:

$$
\int \frac{x^{2} d x}{(a+b x)}=\frac{1}{2 b^{3}}(a+b x)^{2}-\frac{2 a}{b^{3}}(a+b x)+\frac{a^{2}}{b^{3}} \ln (a+b x)+C
$$

112. Integrar: $I=\int \frac{x}{\sqrt{1+x^{4}}} d x$.

Solución:

Escribamos:

$$
I=\int \frac{x}{\sqrt{1+\left(x^{2}\right)^{2}}} d x
$$

Sea $u=x^{2} \Rightarrow d u=2 x d x$

Entonces:

$$
I=\frac{1}{2} \int \frac{2 x d x}{\sqrt{1+\left(x^{2}\right)^{2}}}=\frac{1}{2} \int \frac{d u}{\sqrt{1+u^{2}}}
$$

208

$$
I=\frac{1}{2} \ln \left(u+\sqrt{1+u^{2}}\right)+C
$$

Finalmente:

$$
I=\frac{1}{2} \ln \left(x^{2}+\sqrt{1+x^{4}}\right)+C
$$

113. Integrar: $I=\int x^{2} \cdot \ln \left(x+\sqrt{x^{2}+1}\right) \cdot d x$.

Solución:

Por partes:

$$
\begin{array}{cc}
u=\ln \left(x+\sqrt{x^{2}+1}\right) & v=\frac{x^{3}}{3} \\
\downarrow & \uparrow \\
d u=\frac{x}{x+\frac{x}{\sqrt{x^{2}+1}}} d x=\frac{1}{\sqrt{x^{2}+1}} d x & d v=x^{2} d x
\end{array}
$$

Entonces:

$$
\int x^{2} \cdot \ln \left(x+\sqrt{x^{2}+1}\right) d x=\frac{x^{3}}{3} \ln \left(x+\sqrt{x^{2}+1}\right)-\frac{1}{3} \int \frac{x^{3}}{\sqrt{x^{2}+1}} d x
$$


Calculemos:

$$
\int \frac{x^{3}}{\sqrt{x^{2}+1}} d x=\int\left(x^{2}+1\right)^{-1 / 2}\left(x^{2}\right)(x d x)
$$

Si $u^{2}=x^{2}+1 \Rightarrow 2 u d u=2 x d x \Rightarrow u d u=x d x$

Entonces:

$$
\begin{aligned}
& \int \frac{x^{3}}{\sqrt{x^{2}+1}} d x=\int \frac{1}{u}\left(u^{2}-1\right)(u d u)=\int\left(u^{2}-1\right) d u \\
& \int \frac{x^{3}}{\sqrt{x^{2}+1}} d x=\frac{1}{3} u^{3}-u+C^{\prime}=\frac{1}{3}\left(x^{2}+1\right)^{3 / 2}-\left(x^{2}+1\right)^{1 / 2}+C^{\prime}
\end{aligned}
$$

(2) en (1):

$$
\int x^{2} \cdot \ln \left(x+\sqrt{x^{2}+1}\right) d x=\frac{x^{3}}{3} \ln \left(x+\sqrt{x^{2}+1}\right)-\frac{1}{9}\left(x^{2}+1\right)^{3 / 2}+\frac{1}{3}\left(x^{2}+1\right)^{1 / 2}+C
$$

114. Calcular:

$$
\int e^{a \theta} \operatorname{sen} b \theta d \theta
$$

\section{Solución:}

Por partes:

$$
\begin{array}{ccc}
u=e^{a \theta} & v=\frac{-1}{b} \cos b \theta \\
\downarrow & \uparrow \\
d u=a e^{a \theta} d \theta & d v=\operatorname{sen} b \theta d \theta
\end{array}
$$

Entonces:

$$
\int e^{a \theta} \operatorname{sen} b \theta d \theta=-\frac{e^{a \theta}}{b} \cos b \theta+\frac{a}{b} \int e^{a \theta} \cos b \theta d \theta
$$

Calculemos $\int e^{a \theta} \cos b \theta d \theta$

$$
\begin{array}{ccc}
u=e^{a \theta} & v=\frac{1}{b} \operatorname{sen} b \theta \\
\downarrow & \uparrow \\
d u=a e^{a \theta} d \theta & d v=\cos b \theta d \theta
\end{array}
$$


De donde:

$$
\int e^{a \theta} \cos b \theta d \theta=\frac{e^{a \theta}}{b} \operatorname{sen} b \theta-\frac{a}{b} \int e^{a \theta} \operatorname{sen} b \theta d \theta
$$

(2) en (1)

$$
\begin{gathered}
\int e^{a \theta} \operatorname{sen} b \theta d \theta=-\frac{e^{a \theta}}{b} \cos b \theta+\frac{a e^{a \theta}}{b^{2}} \operatorname{sen} b \theta-\frac{a^{2}}{b^{2}} \int e^{a \theta} \operatorname{sen} b \theta d \theta \\
\left(1+\frac{a^{2}}{b^{2}}\right) \int e^{a \theta} \operatorname{sen} b \theta d \theta=\frac{e^{a \theta}}{b^{2}}(a \operatorname{sen} b \theta-b \cos b \theta)
\end{gathered}
$$

Finalmente:

$$
\int e^{a \theta} \operatorname{sen} b \theta d \theta=\frac{e^{a \theta}(a \operatorname{sen} b \theta-b \cos b \theta)}{a^{2}+b^{2}}+C
$$

115. Hallar $g_{\left(e^{2}\right)}$ si se sabe que:

$$
\int \operatorname{sen}(\ln x) d x=g_{(x)}+C
$$

210

\section{Solución:}

Sea $\left\{\begin{array}{l}w=\ln x \Rightarrow d w=\frac{d x}{x} \Rightarrow d x=e^{w} d w \\ x=e^{w}\end{array}\right.$

Entonces:

$$
I=\int \operatorname{sen}(\ln x) d x=\int \operatorname{sen} w \cdot e^{w} \cdot d w
$$

Por partes:

$$
\begin{array}{cc}
u=\operatorname{sen} w & v=e^{w} \\
\downarrow & \uparrow \\
d u=\cos w d w & d v=e^{w} d w
\end{array}
$$

De donde:

$$
I=\int \operatorname{sen}(\ln x) d x=e^{w} \operatorname{sen} w-\underbrace{\int e^{w} \cos w d w}_{\substack{u=\cos w \\
\downarrow \\
d u=-\operatorname{sen} w d w \quad} \quad \begin{array}{c}
v=e^{w} \\
d v=e^{w} d w
\end{array}}
$$




$$
\begin{gathered}
I=e^{w} \operatorname{sen} w-[e^{w} \cos w+\underbrace{\int \operatorname{sen} w \cdot e^{w} \cdot d w}_{l}] \\
2 I=e^{w}(\operatorname{sen} w-\cos w)+2 C \\
I=\underbrace{\frac{1}{2} e^{w}(\operatorname{sen} w-\cos w)+C}_{g_{(w)}} \\
g_{(x)}=\frac{1}{2} e^{\ln x}[\operatorname{sen}(\ln x)-\cos (\ln x)] \\
g_{(x)}=\frac{x}{2}[\operatorname{sen}(\ln x)-\cos (\ln x)]
\end{gathered}
$$

Con lo cual:

$$
g_{\left(e^{2}\right)}=\frac{e^{2}}{2}[\operatorname{sen} 2-\cos 2]
$$

116. $\operatorname{Si}_{n}(x)=\int \frac{e^{a x}}{x^{n}} d x$, al integrar por partes se deduce que $I_{n}(x)=f(x)+g(n, a) I_{n-1}(x)$. Halle $g_{(3,2)}$.

\section{Solución:}

Se tiene que: $I_{n}(x)=\int \frac{e^{a x}}{x^{n}} d x$

$$
\begin{aligned}
& u=e^{a x} \quad v=\frac{x^{-n+1}}{-n+1} \\
& \downarrow \quad \uparrow \\
& d u=a e^{a x} d x \quad d v=\frac{d x}{x^{n}}=x^{-n} d x
\end{aligned}
$$

Entonces:

$$
\begin{aligned}
& I_{n}(x)=\frac{e^{a x}\left(x^{-n+1}\right)}{1-n}-\int \frac{a}{1-n} \frac{e^{a x}}{x^{n-1}} d x \\
& I_{n}(x)=\frac{e^{a x}\left(x^{-n+1}\right)}{1-n}-\frac{a}{1-n} I_{n-1}(x)
\end{aligned}
$$

De donde:

$$
g(n, a)=\frac{-a}{1-n}
$$


Con lo cual:

$$
g(3,2)=\frac{-2}{1-3}=\frac{-2}{-2}=1
$$

117. Se sabe que $\int \frac{1}{x+2 \sqrt{x-1}} d x=F_{(x)}+C$. Halle el valor de $F_{(2)}-F_{(1)}$.

Solución:

Sea $x-1=u^{2} \Rightarrow d x=2 u d u$

Entonces:

$$
\int \frac{1}{x+2 \sqrt{x-1}} d x=\int \frac{2 u d u}{u^{2}+1+2 u}=2 \int \frac{u d u}{(u+1)^{2}}
$$

Integrando por partes:

$$
\begin{array}{ccc}
w=u & v=\frac{-1}{u+1} \\
\downarrow & \uparrow \\
d w=d u & d v=\frac{d u}{(u+1)^{2}}
\end{array}
$$

De donde:

$$
\begin{aligned}
& \int \frac{1}{x+2 \sqrt{x-1}} d x=2\left[\frac{-u}{u+1}+\int \frac{d u}{u+1}\right] \\
& \int \frac{1}{x+2 \sqrt{x-1}} d x=2\left[\frac{-u}{u+1}+\ln (u+1)\right]+C
\end{aligned}
$$

Entonces:

$$
F_{(x)}=2\left[\frac{-\sqrt{x-1}}{\sqrt{x-1}+1}+\ln (\sqrt{x-1}+1)\right]
$$

Con lo cual:

$$
F_{(2)}-F_{(1)}=2\left[\frac{-1}{2}+\ln 2-(0+0)\right]=2 \ln 2-1
$$

118. Obtener $f_{(x)}$ si $\int(x \arctan x)(x d x)=f_{(x)}+C$.

Solución:

Sea:

$$
I=\int(x \arctan x)(x d x)=\int x^{2} \arctan x d x
$$


Por partes:

$$
\begin{array}{cc}
u=x \arctan x & v=\frac{x^{2}}{2} \\
\downarrow & \uparrow \\
d u=\left(\arctan x+\frac{x}{1+x^{2}}\right) d x & d v=x d x
\end{array}
$$

Entonces:

$$
\begin{gathered}
I=(x \arctan x)\left(\frac{x^{2}}{2}\right)-\frac{1}{2} \int x^{2}\left(\arctan x+\frac{x}{1+x^{2}}\right) d x \\
I=\frac{x^{3}}{2} \arctan x-\frac{1}{2} \int x^{2} \arctan x d x-\frac{1}{2} \int \frac{x^{3}}{1+x^{2}} d x \\
I=\frac{x^{3}}{2} \arctan x-\frac{1}{2} I-\frac{1}{2} \int\left[x-\frac{x}{1+x^{2}}\right] d x \\
\frac{3}{2}=\frac{x^{3}}{2} \arctan x-\frac{1}{2}\left[\frac{x^{2}}{2}-\frac{1}{2} \ln \left(1+x^{2}\right)\right]+\frac{3}{2} C \\
I=\frac{x^{3}}{3} \arctan x-\frac{x^{2}}{6}+\frac{1}{6} \ln \left(1+x^{2}\right)+C
\end{gathered}
$$

119. Integrar por partes $\int \frac{x^{2}}{\sqrt{1-x^{2}}} d x$.

\section{Solución:}

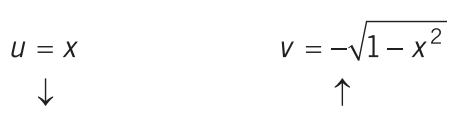

$$
d u=d x \quad d v=\frac{x d x}{\sqrt{1-x^{2}}}
$$

Entonces:

$$
\begin{gathered}
\int \frac{x^{2}}{\sqrt{1-x^{2}}} d x=-x \sqrt{1-x^{2}}+\int \sqrt{1-x^{2}} d x \\
\int \frac{x^{2}}{\sqrt{1-x^{2}}} d x=-x \sqrt{1-x^{2}}+\frac{1}{2} x \sqrt{1-x^{2}}+\frac{1}{2} \operatorname{arcsen} x+C
\end{gathered}
$$


120. Si $I_{n}(x)=\int \frac{e^{a x}}{x^{n}} d x$, al integrar por partes se deduce que $I_{n}(x)=f(x)+g(a, n) I_{n-1}$.

Halle $g_{(3,2)}$.

Solución:

$$
\begin{array}{cc} 
& I_{n}(x)=\int \frac{e^{a x}}{x^{n}} d x \\
u=e^{a x} & v=\frac{x^{-n+1}}{-n+1} \\
\downarrow & \uparrow \\
d u=a e^{a x} d x & d v=x^{-n} d x
\end{array}
$$

Entonces:

$$
\begin{aligned}
& I_{n}(x)=\frac{x^{-n+1}}{1-n} e^{a x}-\frac{a}{1-n} \int \frac{e^{a x}}{x^{n-1}} d x \\
& I_{n}(x)=\frac{x^{-n+1}}{1-n} e^{a x}-\frac{a}{1-n} I_{n-1}(x)
\end{aligned}
$$

214 De donde:

$$
g_{(a, n)}=\frac{-a}{1-n}
$$

Con lo cual:

$$
g_{(3,2)}=\frac{-3}{1-2}=3
$$

121. Hallar de dos maneras diferentes $\int x^{n} \ln x d x(n \neq-1)$.

Solución:

$$
I=\int x^{n} \ln x d x \quad(n \neq-1)
$$

Por partes:

$$
\begin{array}{cc}
u=x^{n} & v=x \ln x-x \\
\downarrow & \uparrow \\
d u=n x^{n-1} & d v=\ln x d x
\end{array}
$$


Entonces:

$$
\begin{gathered}
I=\int x^{n} \ln x d x=x^{n}(x \ln x-x)-n \int x^{n-1}(x \ln x-x) d x \\
I=\int x^{n} \ln x d x=x^{n+1}(\ln x-1)-n \int x^{n} \ln x d x+n \int x^{n} d x \\
I=x^{n+1}(\ln x-1)-n I+n \frac{x^{n+1}}{n+1}+C^{\prime} \\
(n+1) I=x^{n+1}(\ln x-1)+\frac{n x^{n+1}}{n+1}+C^{\prime}
\end{gathered}
$$

De donde:

$$
\begin{gathered}
I=\int x^{n} \ln x d x=\frac{x^{n+1}(\ln x-1)}{n+1}+\frac{n x^{n+1}}{(n+1)^{2}}+C \\
I=\frac{x^{n+1} \ln x}{n+1}-\frac{x^{n+1}}{n+1}+\frac{n x^{n+1}}{(n+1)^{2}}+C \\
I=\frac{x^{n+1} \ln x}{n+1}-\frac{x^{n+1}}{(n+1)^{2}}+C
\end{gathered}
$$

Otro modo:

$$
\begin{aligned}
& I=\int x^{n} \ln x d x \\
& \begin{array}{cc}
u=\ln x \\
\downarrow & v=\frac{x^{n+1}}{n+1} \\
\uparrow &
\end{array} \\
& d u=\frac{1}{x} d x \quad d v=x^{n} d x
\end{aligned}
$$

Entonces:

$$
\begin{aligned}
& I=\frac{x^{n+1} \ln x}{n+1}-\int \frac{x^{n}}{n+1} d x \\
& I=\frac{x^{n+1} \ln x}{n+1}-\frac{x^{n+1}}{(n+1)^{2}}+C
\end{aligned}
$$

122. Si $\int\left[x f^{\prime \prime}(x)+x f^{\prime}(x)+f_{(x)}\right] d x=g_{(x)}+C \wedge f_{(2)}=f^{\prime}(2)=-1$, halle $g_{(2)}$. 
Solución:

$$
\underbrace{\int x f^{\prime \prime}(x)}_{I_{1}} d x+\underbrace{\int x f^{\prime}(x) d x}_{I_{2}}+\int f_{(x)} d x=g_{(x)}+C
$$

Tenemos que:

$$
\begin{aligned}
& I_{1}=\int x f^{\prime \prime}(x) d x \\
& u=x \quad v=f^{\prime}(x) \\
& \begin{array}{cc}
\downarrow & \uparrow \\
d u=d x & d v=f^{\prime \prime}(x) d x
\end{array} \\
& \therefore I_{1}=x f^{\prime}(x)-\int f^{\prime}(x) d x \\
& I_{1}=x f^{\prime}(x)-f_{(x)}+C
\end{aligned}
$$

Análogamente:

$$
I_{2}=\int x f_{(x)}^{\prime} d x
$$

$$
\begin{array}{cc}
u=x & v=f_{(x)} \\
\downarrow & \uparrow \\
d u=d x & d v=f^{\prime}(x) d x \\
\therefore I_{2}=x f_{(x)}-\int f_{(x)} d x
\end{array}
$$

Entonces:

$$
\begin{gathered}
{\left[x f^{\prime}(x)-f_{(x)}+C\right]+\left[x f_{(x)}-\int f_{(x)} d x\right]+\int f_{(x)} d x=g_{(x)}+C} \\
x f^{\prime}{ }_{(x)}+x f_{(x)}-f_{(x)}=g_{(x)}
\end{gathered}
$$

En $x=2$

$$
2 f_{(2)}^{\prime}+2 f_{(2)}-f_{(2)}=g_{(2)}
$$

Con lo cual:

$$
g_{(2)}=2(-1)+2(-1)-(-1)=-3
$$


123. Integrar: $I=\int \operatorname{arcsen} x d x$.

\section{Solución:}

Sea:

$$
\begin{aligned}
& \theta=\operatorname{arcsen} x \rightarrow \operatorname{sen} \theta=x \rightarrow \cos \theta=\sqrt{1-x^{2}} \\
& d x=\cos \theta d \theta
\end{aligned}
$$

Entonces:

$$
\begin{array}{cc}
I=\int \theta \cos \theta d \theta \\
u=\theta \quad v=\operatorname{sen} \theta \\
\downarrow & \uparrow \\
d u=d \theta & d v=\cos \theta d \theta
\end{array}
$$

De donde:

$$
\begin{aligned}
& I=\theta \operatorname{sen} \theta-\int \operatorname{sen} \theta d \theta \\
& I=\theta \operatorname{sen} \theta+\cos \theta+C \\
& I=x \operatorname{arcsen} x+\sqrt{1-x^{2}}+C
\end{aligned}
$$

124. Integrar: $I=\int x \cdot \operatorname{arcsen} x \cdot d x$.

\section{Solución:}

Por partes:

Entonces:

$$
\begin{array}{cc}
u=x & v=x \operatorname{arcsen} x+\sqrt{1-x^{2}} \\
\downarrow & \uparrow \\
d u=d x & d v=\operatorname{arcsen} x d x
\end{array}
$$

$$
\begin{aligned}
& I=x^{2} \operatorname{arcsen} x+x \sqrt{1-x^{2}}-\int(x \operatorname{arcsen} x) d x-\int \sqrt{1-x^{2}} d x \\
& I=x^{2} \operatorname{arcsen} x+x \sqrt{1-x^{2}}-I-\int \sqrt{1-x^{2}} d x+2 C \\
& 2 I=x^{2} \operatorname{arcsen} x+x \sqrt{1-x^{2}}-\left[\frac{1}{2} x \sqrt{1-x^{2}}+\frac{1}{2} \operatorname{arcsen} x\right]+2 C \\
& I=\frac{x^{2}}{2} \operatorname{arcsen} x+\frac{1}{4} x \sqrt{1-x^{2}}-\frac{1}{4} \operatorname{arcsen} x+C
\end{aligned}
$$


125. Integrar: $I=\int x^{5} e^{x^{3}} d x$.

\section{Solución:}

Sea $u=x^{3} \Rightarrow d u=3 x^{2} d x$

Entonces:

$$
\begin{gathered}
I=\int x^{3} x^{2} e^{x^{3}} d x=\frac{1}{3} \int x^{3} e^{x^{3}} 3 x^{2} d x \\
I=\frac{1}{3} \int u e^{u} d u
\end{gathered}
$$

Integrando por partes, se obtiene:

$$
\begin{gathered}
I=\frac{1}{3}\left[u e^{u}-\int e^{u} d u\right] \\
I=\frac{1}{3}\left[u e^{u}-e^{u}\right]=\frac{1}{3} e^{u}(u-1)
\end{gathered}
$$

De donde:

$$
I=\frac{1}{3} e^{x^{3}}\left(x^{3}-1\right)+C
$$

126. Integrar: $I=\int \frac{\ln (x+3)}{x^{2}-2 x+1} d x$.

Solución:

$$
I=\int \frac{\ln (x+3)}{(x-1)^{2}} d x
$$

Como

$$
\begin{array}{cc}
u=\ln (x+3) & v=\frac{-1}{x-1} \\
\downarrow & \uparrow \\
d u=\frac{1}{x+3} d x & d v=\frac{d x}{(x-1)^{2}}
\end{array}
$$

Entonces:

$$
\begin{aligned}
& I=\frac{-\ln (x+3)}{x-1}+\int \frac{d x}{(x-1)(x+3)} \\
& I=\frac{-\ln (x+3)}{x-1}+\underbrace{\int \frac{d x}{x^{2}+2 x-3}}_{I_{1}}
\end{aligned}
$$


Calculemos $I_{1}$ :

$$
\begin{gathered}
I_{1}=\int \frac{d x}{x^{2}+2 x-3}=\int \frac{d x}{(x+1)^{2}-2^{2}} \\
I_{1}=\frac{1}{2(2)} \ln \frac{x+1-2}{x+1+2}+C \\
I_{1}=\frac{1}{4} \ln \frac{x-1}{x+3}+C
\end{gathered}
$$

Finalmente:

$$
I=\frac{-\ln (x+3)}{x-1}+\frac{1}{4} \ln \left[\frac{x-1}{x+3}\right]+C
$$

127. Integrar: $I=\int x \ln (x+1) d x$.

\section{Solución:}

Sea:

$$
\begin{array}{cc}
u=\ln (x+1) & v=\frac{x^{2}}{2} \\
\downarrow & \uparrow \\
d u=\frac{d x}{x+1} & d v=x d x
\end{array}
$$

Entonces:

$$
I=\frac{x^{2}}{2} \ln (x+1)-\frac{1}{2} \underbrace{\int \frac{x^{2}}{x+1} d x}_{l_{1}}
$$

Calculemos $I_{1}$ :

$$
\begin{gathered}
I_{1}=\int \frac{x^{2}}{x+1} d x=\int\left[x-1+\frac{1}{x+1}\right] d x \\
I_{1}=\frac{x^{2}}{2}-x+\ln (x+1)
\end{gathered}
$$

De donde:

$$
I=\frac{x^{2}}{2} \ln (x+1)-\frac{x^{2}}{4}+\frac{x}{2}-\frac{\ln (x+1)}{2}+C
$$


128. Integrar: $I=\int \frac{\ln ^{2} x}{x^{3}} d x$.

Solución:

Sea:

$$
\begin{array}{rlrl}
u & =\ln ^{2} x & v=\frac{x^{-2}}{-2} \\
\downarrow & \uparrow & \uparrow^{\uparrow} & d v=\frac{d x}{x^{3}}
\end{array}
$$

Entonces:

$$
I=-\frac{1}{2} \cdot \frac{\ln ^{2} x}{x^{2}}+\underbrace{\int \frac{\ln x}{x^{3}} d x}_{l_{1}}
$$

Calculemos $I_{1}$ :

$$
I_{1}=\int \frac{\ln x}{x^{3}} d x
$$

Sea:

$$
\begin{array}{crl}
u & =\ln x & v=\frac{x^{-2}}{-2} \\
\downarrow & \uparrow \\
d u=\frac{1}{x} d x & d v=\frac{d x}{x^{3}}
\end{array}
$$

Entonces:

$$
I_{1}=\frac{-1}{2} \cdot \frac{\ln x}{x^{2}}+\frac{1}{2} \underbrace{\frac{d x}{x^{3}}}_{\frac{x^{-2}}{-2}}
$$

De donde:

$$
I=-\frac{1}{2} \cdot \frac{\ln ^{2} x}{x^{2}}-\frac{1}{2} \cdot \frac{\ln x}{x^{2}}-\frac{1}{4 x^{2}}+C
$$

129. Integrar: $I=\int 2 x \ln ^{2}\left(x^{2}+1\right) d x$.

Solución:

Sea:

$$
\begin{array}{cc}
u=\ln \left(x^{2}+1\right) & v=\left(x^{2}+1\right) \ln \left(x^{2}+1\right)-\left(x^{2}+1\right) \\
\downarrow & \uparrow \\
d u=\frac{2 x}{x^{2}+1} d x & d v=\left[\ln \left(x^{2}+1\right)\right](2 x d x)
\end{array}
$$


Entonces:

$$
\begin{gathered}
I=\ln \left(x^{2}+1\right)\left[\left(x^{2}+1\right) \ln \left(x^{2}+1\right)-\left(x^{2}+1\right)\right]-\int 2 x\left[\ln \left(x^{2}+1\right)-1\right] d x \\
I=\left(x^{2}+1\right) \ln ^{2}\left(x^{2}+1\right)-\left(x^{2}+1\right) \ln \left(x^{2}+1\right)-\left[\left(x^{2}+1\right) \ln \left(x^{2}+1\right)-\left(x^{2}+1\right)-x^{2}\right]+C \\
I=\left(x^{2}+1\right) \ln ^{2}\left(x^{2}+1\right)-2\left(x^{2}+1\right) \ln \left(x^{2}+1\right)+2 x^{2}-1+C
\end{gathered}
$$

130. Integrar: $I=\int 2 x \ln ^{2}\left(x^{2}+1\right) d x$.

\section{Solución:}

Sea:

$$
\begin{array}{cc}
u=\ln ^{2}\left(x^{2}+1\right) & v=x^{2} \\
\downarrow & \uparrow \\
d u=[2]\left[\ln \left(x^{2}+1\right)\right] \frac{2 x}{x^{2}+1} d x & d v=2 x d x
\end{array}
$$

Entonces:

$$
\begin{aligned}
& I=x^{2} \ln ^{2}\left(x^{2}+1\right)-4 \int \frac{x^{3} \ln \left(x^{2}+1\right)}{x^{2}+1} d x \\
& I=x^{2} \ln ^{2}\left(x^{2}+1\right)-4 \int\left[x-\frac{x}{x^{2}+1}\right] \ln \left(x^{2}+1\right) d x \\
& I=x^{2} \ln ^{2}\left(x^{2}+1\right)-4 \underbrace{\int x \ln \left(x^{2}+1\right) d x}_{I_{1}}+4 \underbrace{\int \frac{x}{x^{2}+1} \ln \left(x^{2}+1\right) d x}_{I_{2}}
\end{aligned}
$$

Calculemos $I_{1}$ :

$$
I_{1}=\int x \ln \left(x^{2}+1\right) d x=\frac{1}{2} \int[\ln \underbrace{\left(x^{2}+1\right)}_{u}] \underbrace{[2 x d x]}_{d u}
$$

Como $\int \ln u d u=u \ln u-u$,

$$
I_{1}=\frac{1}{2}\left[\left(x^{2}+1\right) \ln \left(x^{2}+1\right)-\left(x^{2}+1\right)\right]
$$

Calculemos $I_{2}$ :

$$
I_{2}=\int \frac{x}{x^{2}+1} \ln \left(x^{2}+1\right) d x
$$

Sea:

$$
\begin{array}{cc}
u=\ln \left(x^{2}+1\right) & v=\frac{1}{2} \ln \left(x^{2}+1\right) \\
\downarrow & \uparrow \\
d u=\frac{2 x d x}{x^{2}+1} & d v=\frac{x d x}{x^{2}+1}
\end{array}
$$




$$
I_{2}=\frac{1}{2} \ln ^{2}\left(x^{2}+1\right)-\int \frac{x}{x^{2}+1} \ln \left(x^{2}+1\right) d x
$$

De donde:

$$
\begin{gathered}
I_{2}=\frac{1}{2} \ln ^{2}\left(x^{2}+1\right)-I_{2} \\
2 I_{2}=\frac{1}{2} \ln ^{2}\left(x^{2}+1\right) \Rightarrow I_{2}=\frac{1}{4} \ln ^{2}\left(x^{2}+1\right)
\end{gathered}
$$

En (1) :

$$
\begin{gathered}
I=x^{2} \ln ^{2}\left(x^{2}+1\right)-4\left[\frac{1}{2}\right]\left[\left(x^{2}+1\right) \ln \left(x^{2}+1\right)-\left(x^{2}+1\right)\right]+4\left(\frac{1}{4}\right) \ln ^{2}\left(x^{2}+1\right)+C^{\prime} \\
I=x^{2} \ln ^{2}\left(x^{2}+1\right)-2\left(x^{2}+1\right) \ln \left(x^{2}+1\right)+2\left(x^{2}+1\right)+\ln ^{2}\left(x^{2}+1\right)+C^{\prime} \\
I=\left(x^{2}+1\right) \ln ^{2}\left(x^{2}+1\right)-2\left(x^{2}+1\right) \ln \left(x^{2}+1\right)+2 x^{2}+2+C^{\prime}
\end{gathered}
$$

La respuesta coincide con la del ejercicio anterior.

131. Encontrar $K$ en la fórmula siguiente:

$$
\int \frac{\cos x}{x^{m}} d x=-\frac{\cos x}{(m-1) x^{m-1}}-K \int \frac{\operatorname{sen} x}{x^{m-1}} d x
$$

\section{Solución:}

Por partes:

$$
\begin{array}{cc}
u=\cos x & v=\frac{x^{-m+1}}{-m+1} \\
\downarrow & \uparrow \\
d u=-\operatorname{sen} x d x & d v=x^{-m} d x
\end{array}
$$

Entonces:

$$
\int \frac{\cos x}{x^{m}} d x=-\frac{\cos x}{(m-1) x^{m-1}}-\frac{1}{m-1} \int \frac{\operatorname{sen} x}{x^{m-1}} d x
$$

De donde:

$$
K=\frac{1}{m-1}
$$


132. Calcular: $\int \frac{x^{2} d x}{\sqrt{3+4 x}}$.

Solución:

Por partes:

$$
\begin{array}{ccc}
u=x^{2} & v=\frac{\sqrt{3+4 x}}{2} \\
\downarrow & \uparrow \\
d u=2 x d x & d v=\frac{d x}{\sqrt{3+4 x}}
\end{array}
$$

Entonces:

$$
\int \frac{x^{2} d x}{\sqrt{3+4 x}}=\frac{1}{2} x^{2} \sqrt{3+4 x}-\int x \sqrt{3+4 x} d x
$$

Calculemos $\int x \sqrt{3+4 x} d x$

Sea $u^{2}=3+4 x \rightarrow x=\frac{u^{2}-3}{4} \Rightarrow d x=\frac{2 u d u}{4}=\frac{u d u}{2}$

Entonces:

$$
\begin{gathered}
\int x \sqrt{3+4 x} d x=\int \frac{u^{2}-3}{4} \sqrt{u^{2}} \frac{u d u}{2}=\frac{1}{8} \int\left(u^{4}-3 u^{2}\right) d u \\
\int x \sqrt{3+4 x} d x=\frac{1}{8}\left[\frac{u^{5}}{5}-u^{3}\right]+C
\end{gathered}
$$

De donde:

$$
\begin{gathered}
\int \frac{x^{2} d x}{\sqrt{3+4 x}}=\frac{1}{2} x^{2} \sqrt{3+4 x}-\frac{1}{40}(3+4 x)^{5 / 2}+\frac{1}{8}(3+4 x)^{3 / 2}+C \\
\int \frac{x^{2} d x}{\sqrt{3+4 x}}=\left[\frac{x^{2}}{2}-\frac{1}{40}(3+4 x)^{2}+\frac{1}{8}(3+4 x)\right] \sqrt{3+4 x}+C \\
\int \frac{x^{2} d x}{\sqrt{3+4 x}}=\frac{2 x^{2}-2 x+3}{20} \sqrt{3+4 x}+C
\end{gathered}
$$


133. Encontrar $K$ en la siguiente expresión:

$$
\int \frac{x d x}{\sqrt{a+b x}}=K(2 a-b x) \sqrt{a+b x}+C
$$

\section{Solución:}

Por partes:

$$
\begin{array}{cc}
u=x & v=\frac{1(a+b x)^{1 / 2}}{b(1 / 2)}=\frac{2}{b}(a+b x)^{1 / 2} \\
\downarrow & \uparrow \\
d u=d x & d v=\frac{d x}{\sqrt{a+b x}}
\end{array}
$$

Entonces:

$$
\int \frac{x d x}{\sqrt{a+b x}}=\frac{2 x}{b}(a+b x)^{1 / 2}-\frac{2}{b} \int(a+b x)^{1 / 2} d x
$$

$$
\begin{gathered}
\int \frac{x d x}{\sqrt{a+b x}}=\frac{2 x}{b} \sqrt{a+b x}-\frac{2}{b^{2}} \cdot \frac{(a+b x)^{3 / 2}}{3 / 2}+C \\
\int \frac{x d x}{\sqrt{a+b x}}=\frac{2}{b} \sqrt{a+b x}\left[x-\frac{2}{3 b}(a+b x)\right]+C \\
\int \frac{x d x}{\sqrt{a+b x}}=\frac{2}{b} \sqrt{a+b x}\left[\frac{3 b x-2 a-2 b x}{3 b}\right]+C \\
\int \frac{x d x}{\sqrt{a+b x}}=\frac{2}{b}\left(\frac{b x-2 a}{3 b}\right) \sqrt{a+b x}+C
\end{gathered}
$$

De donde:

$$
\int \frac{x d x}{\sqrt{a+b x}}=\frac{-2}{3 b^{2}}(2 a-b x) \sqrt{a+b x}+C
$$

Con lo cual:

$$
K=-\frac{2}{3 b^{2}}
$$


134. Encontrar la constante $K$ en la siguiente fórmula de recurrencia:

$$
I=\int x^{m}\left(a+b x^{2}\right) d x=\frac{x^{m-1}\left(a+b x^{2}\right)^{2}}{b(m+3)}-K \int x^{m-2}\left(a+b x^{2}\right) d x
$$

\section{Solución:}

Por partes:

$$
\begin{array}{cc}
u=x^{m-1} & v=\frac{1}{4 b}\left(a+b x^{2}\right)^{2} \\
\downarrow & \uparrow \\
d u=(m-1) x^{m-2} d x & d v=\left(a+b x^{2}\right) x d x
\end{array}
$$

Entonces:

$$
I=\frac{1}{4 b} x^{m-1}\left(a+b x^{2}\right)^{2}-\frac{m-1}{4 b} \int\left(a+b x^{2}\right)^{2} x^{m-2} d x
$$

Escribamos:

$$
\begin{aligned}
& I=\frac{1}{4 b} x^{m-1}\left(a+b x^{2}\right)^{2}-\frac{m-1}{4 b} \int\left[\left(a+b x^{2}\right) x^{m-2}\right]\left[a+b x^{2}\right] d x \\
& I=\frac{1}{4 b} x^{m-1}\left(a+b x^{2}\right)^{2}-\frac{m-1}{4 b} \int\left(a x^{m-2}+b x^{m}\right)\left(a+b x^{2}\right) d x
\end{aligned}
$$

O mejor:

$$
\begin{gathered}
I=\frac{1}{4 b} x^{m-1}\left(a+b x^{2}\right)^{2}-\frac{m-1}{4 b}[\int a x^{m-2}\left(a+b x^{2}\right) d x+b \underbrace{\int x^{m}\left(a+b x^{2}\right) d x}_{I}] \\
I=\frac{1}{4 b} x^{m-1}\left(a+b x^{2}\right)^{2}-\frac{a(m-1)}{4 b} \int x^{m-2}\left(a+b x^{2}\right) d x-\frac{m-1}{4} I
\end{gathered}
$$

De donde:

$$
\underbrace{\left[1+\frac{m-1}{4}\right]}_{\frac{m+3}{4}} I=\frac{1}{4 b} x^{m-1}\left(a+b x^{2}\right)^{2}-\frac{a(m-1)}{4 b} \int x^{m-2}\left(a+b x^{2}\right) d x
$$


Con lo cual:

$$
I=\frac{x^{m-1}\left(a+b x^{2}\right)^{2}}{b(m+3)}-\frac{a(m-1)}{b(m+3)} \int x^{m-2}\left(a+b x^{2}\right) d x
$$

Finalmente:

$$
K=\frac{a(m-1)}{b(m+3)}
$$

135. Integrar: $I=\int \frac{x^{3}}{\sqrt[3]{1-x^{2}}} d x$.

Solución:

Escribamos:

$$
I=\frac{1}{2} \int\left(1-x^{2}\right)^{-1 / 3} x^{2}(2 x d x)
$$

Sea $w=x^{2} \Rightarrow d w=2 x d x$

$$
I=\frac{1}{2} \int(1-w)^{-1 / 3}(w)(d w)
$$

Por partes:

$$
\begin{array}{cc}
u=w & v=\frac{-3(1-w)^{2 / 3}}{2} \\
\downarrow & \uparrow \\
d u=d w & d v=(1-w)^{-1 / 3} d w
\end{array}
$$

Entonces:

$$
\begin{gathered}
I=\frac{1}{2}\left[\frac{-3 w(1-w)^{2 / 3}}{2}-\frac{3}{2} \int(1-w)^{2 / 3}(-d w)\right] \\
I=\frac{-3}{4}\left(w(1-w)^{2 / 3}+\frac{3(1-w)^{5 / 3}}{5}\right)
\end{gathered}
$$

Finalmente:

$$
I=\frac{-3}{4}\left(1-x^{2}\right)^{2 / 3}\left[x^{2}+\frac{3}{5}\left(1-x^{2}\right)\right]
$$




$$
I=\frac{-3}{20}\left(1-x^{2}\right)^{2 / 3}\left(2 x^{2}+3\right)+C
$$

136. Demostrar que $I=\int\left[\left(f^{\prime}(\ln x)\right)^{2}+f_{(\ln x)} \cdot f^{\prime \prime}{ }_{(\ln x)}\right] x^{-1} d x=f_{(\ln x)} \cdot f^{\prime}{ }_{(\ln x)}+C$.

\section{Solución:}

Sea $y=\ln x \Rightarrow d y=\frac{1}{x} d x$

Entonces:

$$
\begin{aligned}
& I=\int\left[\left(f^{\prime}(y)\right)^{2}+f_{(y)} \cdot f^{\prime \prime}(y)\right] d y \\
& I=\underbrace{\int\left[f^{\prime}(y)\right]^{2} d y+\int f_{(y)} \cdot f^{\prime \prime}(y)}_{I_{1}} d y
\end{aligned}
$$

Calculemos $I_{1}$ :

$$
\begin{array}{cc}
u=f^{\prime}(y) & v=f_{(y)} \\
\downarrow & \uparrow \\
d u=f^{\prime \prime}(y) d y & d v=f^{\prime}(y) d y
\end{array}
$$

De donde:

$$
I_{1}=f^{\prime}(y) f_{(y)}-\int f_{(y)} \cdot f^{\prime \prime}(y) d y
$$

Entonces:

$$
\begin{gathered}
I=f^{\prime}(y) f_{(y)}-\int f_{(y)} \cdot f^{\prime \prime}(y) d y+\int f_{(y)} \cdot f^{\prime \prime}(y) d y \\
I=f^{\prime}(y) f_{(y)}+C=f_{(\ln x)} f_{(\ln x)}^{\prime}+C
\end{gathered}
$$

137. Comprobar que la fórmula del problema anterior se verifica para $f_{(x)}=2 x^{2}-3 x+4$.

\section{Solución:}

Se tiene que:

$$
\begin{gathered}
f_{(x)}=2 x^{2}-3 x+4 \Rightarrow f_{(\ln x)}=2 \ln ^{2} x-3 \ln x+4 \\
f^{\prime}(x)=4 x-3 \rightarrow f^{\prime}{ }_{(\ln x)}=4 \ln x-3 \\
f_{(x)}^{\prime \prime}=4 \rightarrow f^{\prime \prime}{ }_{(\ln x)}=4
\end{gathered}
$$


Entonces, el primer miembro es:

$$
\int\left[\left(f^{\prime}(\ln x)\right)^{2}+f_{(\ln x)} \cdot f^{\prime \prime}(\ln x)\right] \frac{d x}{x}=\int\left[(4 \cdot \ln x-3)^{2}+\left(2 \cdot \ln ^{2} x-3 \cdot \ln x+4\right)(4)\right] \frac{d x}{x}
$$

O sea:

$$
\begin{gathered}
\int\left[16 \cdot \ln ^{2} x-24 \cdot \ln x+9+8 \cdot \ln ^{2} x-12 \cdot \ln x+16\right] \frac{d x}{x} \\
\int\left(24 \cdot \ln ^{2} x-36 \cdot \ln x+25\right) \frac{d x}{x}=24 \int \frac{\ln ^{2} x}{x} d x-36 \int \frac{\ln x}{x} d x+25 \int \frac{d x}{x} \\
24 \int(\ln x)^{2}\left(\frac{d x}{x}\right)-36 \int(\ln x)^{1}\left(\frac{d x}{x}\right)+25 \int \frac{d x}{x}=24 \frac{\ln ^{3} x}{3}-36 \frac{\ln ^{2} x}{2}+25 \ln x+C_{1}
\end{gathered}
$$

El primer miembro resulta ser:

$$
8 \ln ^{3} x-18 \ln ^{2} x+25 \ln x+C_{1}
$$

Calculemos el segundo miembro:

$$
f_{(\ln x)^{\prime}} f_{(\ln x)}^{\prime}=\left(2 \ln ^{2} x-3 \ln x+4\right)(4 \ln x-3)
$$

Es decir:

$$
8 \cdot \ln ^{3} x-6 \cdot \ln ^{2} x-12 \cdot \ln ^{2} x+9 \cdot \ln x+16 \cdot \ln x-12+C_{2}
$$

El segundo miembro resulta ser:

$$
8 \cdot \ln ^{3} x-18 \cdot \ln ^{2} x+25 \cdot \ln x-12+C_{2}
$$

Comparando $(\alpha)$ con $(\beta)$, se verifica la fórmula del problema anterior para $C_{1}=C_{2}-12$.

138. Resolver: $\int \frac{d x}{\left(2+e^{\tan x}\right) \cos ^{2} x}$.

\section{Solución:}

Escribamos:

$$
\int \frac{d x}{\left(2+e^{\tan x}\right) \cos ^{2} x}=\int \frac{\sec ^{2} x d x}{2+e^{\tan x}}
$$

Sea $u=\tan x \rightarrow d u=\sec ^{2} x d x$ 
Entonces:

$$
\begin{gathered}
\int \frac{d x}{\left(2+e^{\tan x}\right) \cos ^{2} x}=\int \frac{d u}{2+e^{u}}=\int \frac{e^{-u} d u}{2 e^{-u}+1} \\
\int \frac{d x}{\left(2+e^{\tan x}\right) \cos ^{2} x}=\frac{-1}{2} \int \frac{-2 e^{-u} d u}{2 e^{-u}+1}=\frac{-1}{2} \ln \left(2 e^{-u}+1\right)+C
\end{gathered}
$$

De donde:

$$
\frac{-1}{2} \ln \left(2 e^{-\tan x}+1\right)+C
$$

139. Resolver la siguiente integral: $\int \frac{\left(2 a^{2}-x^{2}\right) x^{3}}{\left(a^{2}-x^{2}\right)^{3 / 2}} d x$.

\section{Solución:}

Sea $x=\operatorname{acos} \theta \rightarrow d x=\operatorname{acos} \theta d \theta$

Entonces:

$$
\begin{aligned}
\int \frac{\left(2 a^{2}-x^{2}\right) x^{3}}{\left(a^{2}-x^{2}\right)^{3 / 2}} d x= & \int \frac{\left[\left(2-\operatorname{sen}^{2} \theta\right) a^{2}\right]\left(a^{3} \operatorname{sen}^{3} \theta\right)}{a^{3} \cos ^{3} \theta}(a \cos \theta) d \theta=a^{3} \int \frac{\left(2-\operatorname{sen}^{2} \theta\right) \operatorname{sen}^{3} \theta}{\cos ^{2} \theta} d \theta \\
& \int \frac{\left(2 a^{2}-x^{2}\right) x^{3}}{\left(a^{2}-x^{2}\right)^{3 / 2}} d x=a^{3} \int \frac{\left(2-\operatorname{sen}^{2} \theta\right) \operatorname{sen}^{3} \theta}{1-\operatorname{sen}^{2} \theta} d \theta
\end{aligned}
$$

Dividamos:

$$
\frac{\left(2-\operatorname{sen}^{2} \theta\right) \operatorname{sen}^{3} \theta}{1-\operatorname{sen}^{2} \theta}=\frac{\operatorname{sen}^{5} \theta-2 \operatorname{sen}^{3} \theta}{\operatorname{sen}^{2} \theta-1}=\operatorname{sen}^{3} \theta-\operatorname{sen} \theta-\frac{\operatorname{sen} \theta}{\operatorname{sen}^{2} \theta-1}
$$

De donde:

$$
\int \frac{\left(2 a^{2}-x^{2}\right) x^{3}}{\left(a^{2}-x^{2}\right)^{3 / 2}} d x=a^{3} \int\left[\operatorname{sen}^{3} \theta-\operatorname{sen} \theta+\frac{\operatorname{sen} \theta}{\cos ^{2} \theta}\right] d \theta
$$

Tendremos:

$$
\int \frac{\left(2 a^{2}-x^{2}\right) x^{3}}{\left(a^{2}-x^{2}\right)^{3 / 2}} d x=a^{3} \int\left[\operatorname{sen} \theta\left(\operatorname{sen}^{2} \theta-1\right)+(\cos \theta)^{-2} \operatorname{sen} \theta\right] d \theta
$$




$$
\begin{gathered}
\int \frac{\left(2 a^{2}-x^{2}\right) x^{3}}{\left(a^{2}-x^{2}\right)^{3 / 2}} d x=a^{3} \int\left[(\cos \theta)^{2}(-\operatorname{sen} \theta)-(\cos \theta)^{-2}(-\operatorname{sen} \theta)\right] d \theta \\
\int \frac{\left(2 a^{2}-x^{2}\right) x^{3}}{\left(a^{2}-x^{2}\right)^{3 / 2}} d x=a^{3}\left[\frac{\cos ^{3} \theta}{3}+\frac{1}{\cos \theta}\right]+C
\end{gathered}
$$

Como $x=a \operatorname{sen} \theta \rightarrow \operatorname{sen} \theta=\frac{x}{a} \rightarrow \cos \theta=\sqrt{1-\left(\frac{x}{a}\right)^{2}}=\frac{\sqrt{a^{2}-x^{2}}}{a}$

Se tendrá que:

$$
\int \frac{\left(2 a^{2}-x^{2}\right) x^{3}}{\left(a^{2}-x^{2}\right)^{3 / 2}} d x=a^{3}\left[\frac{\left(a^{2}-x^{2}\right)^{3 / 2}}{3 a^{3}}+\frac{a}{\sqrt{a^{2}-x^{2}}}\right]+C
$$

Finalmente:

$$
\frac{1}{3}\left(a^{2}-x^{2}\right)^{3 / 2}+\frac{a^{4}}{\sqrt{a^{2}-x^{2}}}+C
$$

230 140. Si $\int \frac{a^{x}}{1+a^{2 x}} d x=k \arctan n^{x}+C$, encontrar $k \ln n^{4}$.

\section{Solución:}

Sea $u=a^{x} \Rightarrow d u=a^{x} \cdot \ln a d x$

Entonces:

$$
\begin{gathered}
\int \frac{a^{x}}{1+a^{2 x}} d x=\frac{1}{\ln a} \int \frac{a^{x} \cdot \ln a \cdot d x}{1+\left(a^{x}\right)^{2}}=\frac{1}{\ln a} \int \frac{d u}{1+u^{2}} \\
\int \frac{a^{x}}{1+a^{2 x}} d x=\frac{1}{\ln a} \arctan u+C
\end{gathered}
$$

Si:

$$
\begin{gathered}
\frac{1}{\ln a} \arctan a^{x}=k \arctan n^{x} \\
k=\frac{1}{\ln a} \quad \text { y } n=a
\end{gathered}
$$

Entonces:

$$
k \ln n^{4}=\frac{1}{\ln a} \ln a^{4}=4
$$


141. Encontrar: $\int \arctan x d x$.

\section{Solución:}

Sea $\theta=\arctan x \rightarrow \tan \theta=x \rightarrow \sec ^{2} \theta d \theta=d x$

Entonces:

$$
\begin{aligned}
& \int \arctan x d x=\int \theta \sec ^{2} \theta d \theta \\
& u=\theta \quad v=\tan \theta \\
& \downarrow \quad \uparrow \\
& d u=d \theta \quad d v=\sec ^{2} \theta d \theta \\
& \int \arctan x d x=\theta \tan \theta-\int \tan \theta d \theta \\
& \int \arctan x d x=\theta \tan \theta+\int \frac{(-\operatorname{sen} \theta d \theta)}{\cos \theta} \\
& \int \arctan x d x=\theta \tan \theta+\ln \cos \theta
\end{aligned}
$$

A partir de $\tan \theta=x$ :

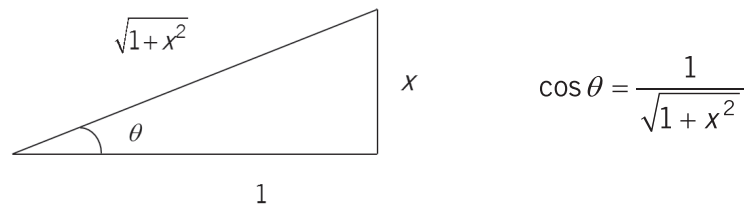

De donde:

$$
\begin{gathered}
\int \arctan x d x=(\arctan x) x+\ln \frac{1}{\sqrt{1+x^{2}}} \\
\int \arctan x d x=x(\arctan x)-\frac{1}{2} \ln \left(1+x^{2}\right)+C
\end{gathered}
$$

142. Si $\int \arccos 2 x d x=q_{(x)}+k$, hallar $q(1 / 3)$.

\section{Solución:}

Sea $\theta=\arccos 2 x \rightarrow \cos \theta=2 x \rightarrow-\operatorname{sen} \theta d \theta=2 d x$

Entonces:

$$
\int \arccos 2 x d x=\int \theta\left(\frac{-\operatorname{sen} \theta d \theta}{2}\right)=-\frac{1}{2} \int \theta \operatorname{sen} \theta d \theta
$$


Por partes:

De donde:

$$
\begin{array}{ccc} 
& \int \theta \operatorname{sen} \theta d \theta \\
\begin{array}{c}
\downarrow=\theta \\
\downarrow \\
d u=d \theta
\end{array} & v=-\cos \theta \\
& d v=\operatorname{sen} \theta d \theta
\end{array}
$$

$$
\begin{gathered}
\int \arccos 2 x d x=\frac{-1}{2}\left[-\theta \cos \theta-\int(-\cos \theta) d \theta\right] \\
\int \arccos 2 x d x=\frac{1}{2} \theta \cos \theta-\frac{1}{2} \operatorname{sen} \theta+C
\end{gathered}
$$

Con lo cual:

$$
\begin{gathered}
q_{(x)}=\frac{1}{2}(\arccos 2 x)(2 x)-\frac{1}{2} \sqrt{1-(2 x)^{2}} \\
q_{(x)}=x \arccos 2 x-\frac{1}{2} \sqrt{1-4 x^{2}}
\end{gathered}
$$

Finalmente:

$$
q_{(1 / 3)}=\frac{1}{3} \arccos \frac{2}{3}-\frac{1}{2} \sqrt{1-\frac{4}{9}}
$$

$$
q_{(1 / 3)}=0.2804-0.3727=-0.0923
$$

143. Si $\int \frac{x-1}{\sqrt{1-x^{2}}} d x=g_{(x)}+k$, hallar $g_{(1 / 4)}$.

Solución:

Escribamos:

$$
\begin{gathered}
\int \frac{x-1}{\sqrt{1-x^{2}}} d x=\int \frac{x d x}{\sqrt{1-x^{2}}}-\int \frac{d x}{\sqrt{1-x^{2}}}=\frac{1}{-2} \int \frac{-2 x d x}{\left(1-x^{2}\right)^{1 / 2}}-\int \frac{d x}{\sqrt{1-x^{2}}} \\
\int \frac{x-1}{\sqrt{1-x^{2}}} d x=\frac{1}{-2} \cdot \frac{\left(1-x^{2}\right)^{-1 / 2+1}}{-1 / 2+1}-\operatorname{arcsen} x+C \\
\int \frac{x-1}{\sqrt{1-x^{2}}} d x=-\sqrt{1-x^{2}}-\operatorname{arcsen} x+C
\end{gathered}
$$

De donde:

$$
g_{(x)}=-\sqrt{1-x^{2}}-\operatorname{arcsen} x
$$


Con lo cual:

$$
\begin{gathered}
g_{(1 / 4)}=-\sqrt{1-\frac{1}{16}}-\operatorname{arcsen} \frac{1}{4} \\
g_{(1 / 4)}=-0.9682-0.2527=-1.2209
\end{gathered}
$$

144. Resolver la siguiente integral: $\int \frac{\cos x}{5+4 \cos x} d x$.

\section{Solución:}

Hagamos $z=\tan \frac{x}{2} \Rightarrow x=2 \arctan z \Rightarrow d x=\frac{2 d z}{1+z^{2}}$

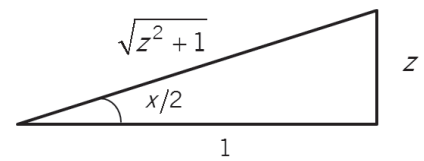

$$
\begin{aligned}
& \cos x / 2=\frac{1}{\sqrt{z^{2}+1}} \\
& \cos x=2 \cos ^{2} \frac{x}{2}-1=\frac{2}{z^{2}+1}-1=\frac{1-z^{2}}{z^{2}+1}
\end{aligned}
$$

Reemplacemos en la integral dada:

$$
\int \frac{\frac{1-z^{2}}{z^{2}+1}}{5+\frac{4-4 z^{2}}{z^{2}+1}} \cdot \frac{2 d z}{1+z^{2}}=2 \int \frac{1-z^{2}}{\left(z^{2}+9\right)\left(z^{2}+1\right)} d z
$$

Descompongamos en fracciones parciales:

$$
\begin{gathered}
\frac{1-z^{2}}{\left(z^{2}+9\right)\left(z^{2}+1\right)}=\frac{A z+B}{z^{2}+9}+\frac{C z+D}{z^{2}+1} \\
1-z^{2}=(A z+B)\left(z^{2}+1\right)+(C z+D)\left(z^{2}+9\right) \\
1-z^{2}=(A+C) z^{3}+(B+D) z^{2}+(A+9 C) z+(B+9 D)
\end{gathered}
$$

De donde:

$$
\begin{cases}A+C=0 & A=0 \\ B+D=-1 & C=0 \\ A+9 C=0 & D=1 / 4 \\ B+9 D=1 & B=-5 / 4\end{cases}
$$


Entonces:

$$
\begin{gathered}
2 \int \frac{1-z^{2}}{\left(z^{2}+9\right)\left(z^{2}+1\right)} d z=2 \int\left[\frac{-5 / 4}{z^{2}+9}+\frac{1 / 4}{z^{2}+1}\right] d z \\
2\left[\frac{-5}{4}\left(\frac{1}{3}\right) \arctan \frac{z}{3}+\frac{1}{4}(1) \arctan z\right]+C \\
\frac{-5}{6} \arctan \frac{z}{3}+\frac{1}{2} \arctan z+C
\end{gathered}
$$

145. Integrar: $\int \frac{d x}{3 \operatorname{sen}^{2} x+5 \cos ^{2} x}$.

\section{Solución:}

Dividamos ambos términos de la fracción entre $\cos ^{2} x$ :

$$
\begin{aligned}
& \int \frac{d x}{3 \operatorname{sen}^{2} x+5 \cos ^{2} x}=\int \frac{\frac{d x}{\cos ^{2} x}}{3 \tan ^{2} x+5}=\int \frac{\sec ^{2} x d x}{3 \tan ^{2} x+5} \\
& \int \frac{d x}{3 \operatorname{sen}^{2} x+5 \cos ^{2} x}=\frac{1}{3} \int \frac{\sec ^{2} x d x}{(\tan x)^{2}+\left(\frac{\sqrt{5}}{\sqrt{3}}\right)^{2}}
\end{aligned}
$$

Empleemos la fórmula:

$$
\int \frac{d u}{u^{2}+a^{2}}=\frac{1}{a} \arctan \frac{u}{a}+C
$$

Entonces:

$$
\begin{aligned}
& \int \frac{d x}{3 \operatorname{sen}^{2} x+5 \cos ^{2} x}=\frac{1}{3} \cdot \frac{1}{\sqrt{5} / \sqrt{3}} \arctan \left(\frac{\tan x}{\sqrt{5} / \sqrt{3}}\right)+C \\
& \int \frac{d x}{3 \operatorname{sen}^{2} x+5 \cos ^{2} x}=\frac{\sqrt{15}}{15} \arctan \left(\frac{\sqrt{3} \tan x}{\sqrt{5}}\right)+C
\end{aligned}
$$

146. Integrar: $\int \frac{d x}{8-4 \operatorname{sen} x+7 \cos x}$.

Solución:

Sean $\theta=\tan \frac{x}{2} \rightarrow \operatorname{sen} x=\frac{2 \theta}{1+\theta^{2}}, \quad \cos x=\frac{1-\theta^{2}}{1+\theta^{2}}, \quad d x=\frac{2 d \theta}{1+\theta^{2}}$ 
Reemplazándolos en la expresión dada:

$$
\begin{aligned}
& \int \frac{d x}{8-4 \operatorname{sen} x+7 \cos x}=\int \frac{\frac{2 \theta}{1+\theta^{2}}}{8-\frac{8 \theta}{1+\theta^{2}}+\frac{7-7 \theta^{2}}{1+\theta^{2}}}=2 \int \frac{d \theta}{\theta^{2}-8 \theta+15} \\
& \int \frac{d x}{8-4 \operatorname{sen} x+7 \cos x}=2 \int \frac{d \theta}{(\theta-3)(\theta-5)}=2 \int \frac{1}{2}\left[\frac{-1}{\theta-3}+\frac{1}{\theta-5}\right] d \theta \\
& \int \frac{d x}{8-4 \operatorname{sen} x+7 \cos x}=-\ln (\theta-3)+\ln (\theta-5)+C=\ln \frac{\theta-5}{\theta-3}+C
\end{aligned}
$$

De donde, la integral pedida es:

$$
\ln \frac{\tan \frac{x}{2}-5}{\tan \frac{x}{2}-3}+C
$$

147. Integrar: $\int \frac{\cos x}{1+\cos x} d x$.

Solución:

Hagamos:

$$
t=\tan \frac{x}{2} \rightarrow \cos x=\frac{1-t^{2}}{1+t^{2}} \rightarrow d x=\frac{2 d t}{1+t^{2}}
$$

Entonces:

$$
\begin{aligned}
& \int \frac{\cos x}{1+\cos x} d x=\int \frac{\frac{1-t^{2}}{1+t^{2}}\left(\frac{2 d t}{1+t^{2}}\right)}{1+\frac{1-t^{2}}{1+t^{2}}}=\int \frac{1-t^{2}}{1+t^{2}} d t \\
& \int \frac{\cos x}{1+\cos x} d x=-\int \frac{t^{2}-1}{t^{2}+1} d t=-\int\left[1-\frac{2}{t^{2}+1}\right] d t \\
& \int \frac{\cos x}{1+\cos x} d x=-\int d t+2 \int \frac{d t}{t^{2}+1}=-t+2 \arctan t+c
\end{aligned}
$$

De donde:

$$
\int \frac{\cos x}{1+\cos x} d x=-\tan \frac{x}{2}+2\left(\frac{x}{2}\right)+C=x-\tan \frac{x}{2}+C
$$


148. Resuelva la integral siguiente:

$$
\int \frac{\left(2+\tan ^{2} \theta\right)\left(1+\tan ^{2} \theta\right)}{1+\tan ^{3} \theta} d \theta
$$

Solución:

Sea $\tan \theta=u \Rightarrow \theta=\arctan u \Rightarrow d \theta=\frac{1}{1+u^{2}} d u$

Entonces:

$$
\int \frac{\left(2+\tan ^{2} \theta\right)\left(1+\tan ^{2} \theta\right)}{1+\tan ^{3} \theta} d \theta=\int \frac{\left(2+u^{2}\right)\left(1+u^{2}\right)}{1+u^{3}} \cdot \frac{d u}{1+u^{2}}=\int \frac{2+u^{2}}{1+u^{3}} d u
$$

Descompongamos:

$$
\begin{aligned}
& \frac{u^{2}+2}{u^{3}+1}=\frac{A}{u+1}+\frac{B u+C}{u^{2}-u+1} \\
& u^{2}+2=A\left(u^{2}-u+1\right)+(B u+C)(u+1) \\
& u=-1 \quad 3=A(3)+(C-B)(0) \Rightarrow A=1 \\
& u=0 \quad 2=A(1)+(C)(1) \quad \Rightarrow C=1 \\
& \text { Coeficiente de } u^{2}: \quad 1=A+B \Rightarrow B=0
\end{aligned}
$$

De donde:

$$
\begin{aligned}
& \int \frac{\left(2+\tan ^{2} \theta\right)\left(1+\tan ^{2} \theta\right)}{1+\tan ^{3} \theta} d \theta=\int\left[\frac{1}{u+1}+\frac{1}{\left(u-\frac{1}{2}\right)^{2}+\left(\frac{\sqrt{3}}{2}\right)^{2}}\right] d u \\
& \int \frac{\left(2+\tan ^{2} \theta\right)\left(1+\tan ^{2} \theta\right)}{1+\tan ^{3} \theta} d \theta=\ln (u+1)+\frac{1}{\sqrt{3} / 2} \arctan \frac{u-1 / 2}{\sqrt{3} / 2}+C \\
& \int \frac{\left(2+\tan ^{2} \theta\right)\left(1+\tan ^{2} \theta\right)}{1+\tan ^{3} \theta} d \theta=\ln (1+\tan \theta)+\frac{2 \sqrt{3}}{3} \arctan \frac{2 \tan \theta-1}{\sqrt{3}}+C
\end{aligned}
$$

149. Integrar: $\int \frac{\sqrt{\operatorname{sen} x}}{\cos x} d x$. 


\section{Solución:}

Sea:

$$
\operatorname{sen} x=u^{2} \rightarrow \cos x d x=2 u d u \rightarrow \cos x=\sqrt{1-\left(u^{2}\right)^{2}}
$$

Entonces:

$$
\int \frac{\sqrt{\operatorname{sen} x}}{\cos x} d x=\int \frac{u}{\sqrt{1-\left(u^{2}\right)^{2}}} \cdot \frac{2 u d u}{\sqrt{1-\left(u^{2}\right)^{2}}}=2 \int \frac{u^{2} d u}{1-u^{4}}
$$

Calculemos $2 \int \frac{u^{2} d u}{1-u^{4}}$

$$
\begin{gathered}
\frac{u^{2}}{1-u^{4}}=\frac{A}{1+u}+\frac{B}{1-u}+\frac{C u+D}{1+u^{2}} \\
u^{2}=A(1-u)\left(1+u^{2}\right)+B(1+u)\left(1+u^{2}\right)+(C u+D)(1+u)(1-u)
\end{gathered}
$$

Si

$$
\begin{array}{ll}
u=1 & 1=\mathrm{B}(2)(2) \rightarrow B=1 / 4 \\
u=-1 & 1=\mathrm{A}(2)(2) \rightarrow A=1 / 4 \\
u=0 & 0=\frac{1}{4}(1)+\frac{1}{4}(1)+D(1) \rightarrow D=-1 / 2
\end{array}
$$

Coeficiente de $u^{3}: 0=-A+B-C \rightarrow C=0$

Entonces:

$$
\begin{aligned}
& \int \frac{\sqrt{\operatorname{sen} x}}{\cos x} d x=2 \int\left[\frac{1 / 4}{1+u}+\frac{1 / 4}{1-u}-\frac{1 / 2}{1+u^{2}}\right] d u \\
& \int \frac{\sqrt{\operatorname{sen} x}}{\cos x} d x=\frac{1}{2} \ln (1+u)-\frac{1}{2} \ln (1-u)-\arctan u+C
\end{aligned}
$$

De donde:

$$
\begin{gathered}
\int \frac{\sqrt{\operatorname{sen} x}}{\cos x} d x=\frac{1}{2} \ln \left(\frac{1+u}{1-u}\right)-\arctan u+C \\
\int \frac{\sqrt{\operatorname{sen} x}}{\cos x} d x=\frac{1}{2} \ln \left(\frac{1+\sqrt{\operatorname{sen} x}}{1-\sqrt{\operatorname{sen} x}}\right)-\arctan \sqrt{\operatorname{sen} x}+C
\end{gathered}
$$


150. Calcular la siguiente integral:

$$
\int \frac{(x+1)}{x\left(1+x e^{x}\right)} d x
$$

\section{Solución:}

Sea:

$$
1+x e^{x}=u \Rightarrow\left(0+1 e^{x}+x e^{x}\right) d x=d u \Rightarrow e^{x}(x+1) d x=d u
$$

Entonces:

$$
\int \frac{(x+1)}{x\left(1+x e^{x}\right)} d x=\int \frac{\frac{d u}{e^{x}}}{x(u)}=\int \frac{d u}{\left(x e^{x}\right) u}=\int \frac{d u}{(u-1) u}
$$

Como

$$
\frac{1}{(u-1) u}=\frac{1}{u-1}-\frac{1}{u}
$$

Entonces:

$$
\int \frac{(x+1)}{x\left(1+x e^{x}\right)} d x=\int\left[\frac{1}{u-1}-\frac{1}{u}\right] d u=\ln (u-1)-\ln u+C
$$

El resultado es:

$$
\begin{gathered}
\ln x e^{x}-\ln 1+x e^{x}+C \\
\ln \frac{x e^{x}}{1+x e^{x}}+C
\end{gathered}
$$

151. Calcular: $\int \frac{16}{\sqrt{t}\left(16-t^{2}\right)} d t$.

\section{Solución:}

Sea $t=u^{2} \Rightarrow d t=2 u d u$

Entonces:

$$
I=\int \frac{16}{\sqrt{t}\left(16-t^{2}\right)} d t=16 \int \frac{2 u d u}{u\left(16-u^{4}\right)}=-32 \int \frac{d u}{u^{4}-16}
$$

Como:

$$
\begin{gathered}
\frac{1}{u^{4}-16}=\frac{1}{\left(u^{2}+4\right)(u+2)(u-2)}=\frac{A u+B}{u^{2}+4}+\frac{C}{u+2}+\frac{D}{u-2} \\
1=(A u+B)(u+2)(u-2)+C\left(u^{2}+4\right)(u-2)+D\left(u^{2}+4\right)(u+2)
\end{gathered}
$$




$$
\text { Si } \begin{array}{lll}
u=2 & 1=D(8)(4) \quad \rightarrow \quad D=1 / 32 \\
u=-2 & 1=C(8)(-4) \quad \rightarrow \quad C=-1 / 32 \\
u=0 & 1=B(-4)-\frac{1}{32}(-8)+\frac{1}{32}(8) \rightarrow B=\frac{-1}{8}
\end{array}
$$

Coeficiente de $u^{3}: \quad 0=A+C+D \rightarrow A=0$

Se tiene que:

$$
\begin{gathered}
I=-32 \int\left[\frac{-(1 / 8)}{u^{2}+4}-\frac{(1 / 32)}{u+2}+\frac{(1 / 32)}{u-2}\right] d u \\
I=4\left(\frac{1}{2}\right) \arctan \frac{u}{2}+\ln (u+2)-\ln (u-2)+C \\
I=\ln \frac{u+2}{u-2}+2 \arctan \frac{u}{2}+C
\end{gathered}
$$

Es decir:

$$
I=\ln \frac{\sqrt{t}+2}{\sqrt{t}-2}+2 \arctan \frac{\sqrt{t}}{2}+C
$$

152. Calcule $f_{(2)}$ si se sabe que $\int \frac{1}{x^{4} \sqrt{x^{2}-1}} d x=f_{(x)}+C$.

\section{Solución:}

Sea:

$$
\begin{gathered}
x=\csc \theta \rightarrow d x=-\csc \theta \cdot \cot \theta \cdot d \theta \\
x^{2}-1=\csc ^{2} \theta-1=\cot ^{2} \theta
\end{gathered}
$$

Entonces:

$$
\begin{gathered}
\int \frac{1}{x^{4} \sqrt{x^{2}-1}} d x=\int \frac{-\csc \theta \cdot \cot \theta \cdot d \theta}{\csc ^{4} \theta \cdot \cot \theta}=-\int \frac{d \theta}{\csc ^{3} \theta} \\
\int \frac{1}{x^{4} \sqrt{x^{2}-1}} d x=-\int \operatorname{sen}^{3} \theta d \theta=-\int \operatorname{sen} \theta\left(\operatorname{sen}^{2} \theta\right) d \theta \\
\int \frac{1}{x^{4} \sqrt{x^{2}-1}} d x=-\int \operatorname{sen} \theta\left(1-\cos ^{2} \theta\right) d \theta=+\underbrace{\cos \theta-\frac{\cos ^{3} \theta}{3}}_{f_{(\theta)}}+C
\end{gathered}
$$


De donde:

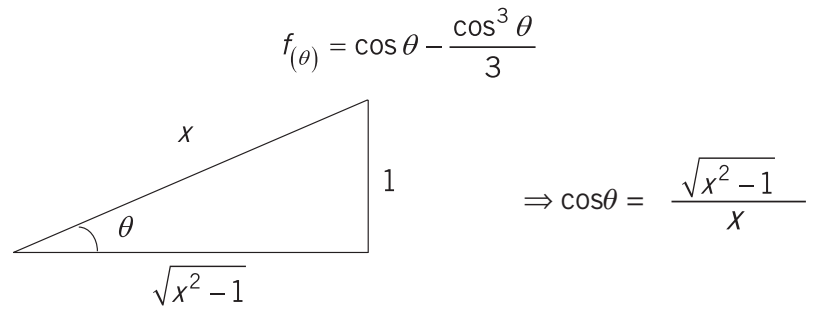

Entonces:

$$
f(x)=\frac{\sqrt{x^{2}-1}}{x}-\frac{\left(x^{2}-1\right)^{3 / 2}}{3 x^{3}}
$$

Con lo cual:

$$
f_{(x)}=\frac{\sqrt{3}}{2}-\frac{3 \sqrt{3}}{24}=\frac{3 \sqrt{3}}{8}
$$

153. Dada : $I=\int \frac{\sqrt{a^{2}+x^{2}}}{x^{4}} d x=G_{(x)}+C$
a) Calcular $24 a^{2} G(2 a)$.
b) Evaluar $81 a^{2} G(3 a)$.

\section{Solución:}

Sea:

$$
\begin{aligned}
& x=a \tan \theta \\
& d x=a \sec ^{2} \theta d \theta
\end{aligned}
$$

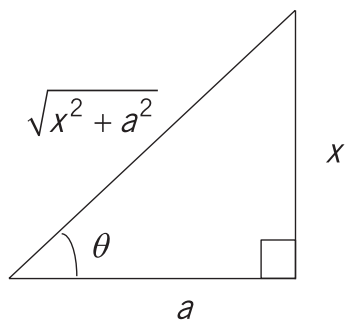

Entonces:

$$
\begin{aligned}
& I=\int \frac{\sqrt{a^{2}+a^{2} \tan ^{2} \theta} \cdot a \sec ^{2} \theta d \theta}{a^{4} \tan ^{4} \theta} \\
& I=\int \frac{a \sec \theta \cdot a \sec ^{2} \theta \cdot d \theta}{a^{4} \tan ^{4} \theta}
\end{aligned}
$$




$$
\begin{aligned}
& I=\frac{1}{a^{2}} \int \frac{\frac{1}{\cos ^{3} \theta}}{\frac{\operatorname{sen}^{4} \theta}{\cos ^{4} \theta}} d \theta \\
& I=\frac{1}{a^{2}} \int(\operatorname{sen} \theta)^{-4}(\cos \theta d \theta) \\
& I=\frac{1}{a^{2}} \cdot \frac{(\operatorname{sen} \theta)^{-3}}{-3}+C \\
& I=-\frac{1}{3 a^{2}}\left[\frac{x}{\left(x^{2}+a^{2}\right)^{1 / 2}}\right]^{-3}+C \\
& I=\underbrace{-\frac{1}{3 a^{2}} \cdot \frac{\left(x^{2}+a^{2}\right)^{3 / 2}}{x^{3}}}_{G_{(x)}}+C
\end{aligned}
$$

De donde:

$$
\begin{aligned}
& 24 a^{2} \cdot G_{(2 a)}=24 a^{2}\left[-\frac{1}{3 a^{2}} \cdot \frac{\left(5 a^{2}\right)^{3 / 2}}{8 a^{3}}\right]=-\frac{1}{a^{3}}\left(5^{3 / 2} a^{3}\right)=-5 \sqrt{5} \\
& 81 a^{2} \cdot G_{(3 a)}=81 a^{2}\left[-\frac{1}{3 a^{2}} \cdot \frac{\left(10 a^{2}\right)^{3 / 2}}{27 a^{3}}\right]=-\frac{1}{a^{3}}\left(10^{3 / 2} a^{3}\right)=-10 \sqrt{10}
\end{aligned}
$$

154. Encontrar: $\int \frac{d x}{(a+b x)(p+q x)}$.

\section{Solución:}

Escribamos:

$$
\begin{gathered}
\frac{1}{(a+b x)(p+q x)}=\frac{A}{a+b x}+\frac{B}{p+q x} \\
1=A(p+q x)+B(a+b x)
\end{gathered}
$$

Si $\quad x=-\frac{a}{b} \Rightarrow 1=A\left[p-\frac{a q}{b}\right] \Rightarrow A=\frac{b}{b p-a q}$

Si $x=-\frac{p}{q} \Rightarrow 1=B\left[a-\frac{b p}{q}\right] \Rightarrow B=\frac{q}{a q-b p}$ 
Entonces:

$$
\begin{gathered}
\int \frac{d x}{(a+b x)(p+q x)}=\frac{b}{b p-a q} \int \frac{d x}{a+b x}+\frac{q}{a q-b p} \int \frac{d x}{p+q x} \\
\int \frac{d x}{(a+b x)(p+q x)}=\frac{1}{b p-a q} \ln (a+b x)+\frac{1}{a q-b p} \ln (p+q x)+C
\end{gathered}
$$

De donde:

$$
\int \frac{d x}{(a+b x)(p+q x)}=\frac{1}{b p-a q} \ln \frac{a+b x}{p+q x}+C
$$

155. Integrar: $I=\int \frac{1}{(x+a)(x+b)} d x$.

\section{Solución:}

Descompongámoslo en fracciones parciales:

$$
\frac{1}{(x+a)(x+b)}=\frac{A}{x+a}+\frac{B}{x+b}=\frac{A(x+b)+B(x+a)}{(x+a)(x+b)}
$$

242

De donde:

$$
\begin{aligned}
& 1=A(x+b)+B(x+a) \\
& 1=(A+B) x+(A b+B a) \\
& \left\{\begin{array}{l}
0=A+B \rightarrow 0=A a+B a \\
1=A b+B a \rightarrow 1=A b+B a
\end{array}\right.
\end{aligned}
$$

Restándolas:

$$
\begin{aligned}
1=A b-A a=A(b-a) \Rightarrow A & =\frac{1}{b-a} \\
B & =-A=\frac{1}{a-b}
\end{aligned}
$$

Entonces:

$$
\begin{gathered}
I=\int\left[\frac{1 /(b-a)}{x+a}+\frac{1 /(a-b)}{x+b}\right] d x \\
I=\frac{1}{b-a} \ln (x+a)+\frac{1}{a-b} \ln (x+b)+C
\end{gathered}
$$


156. Integrar: $I=\int \frac{1}{(x+a)(x+b)} d x$.

Solución:

$$
\begin{gathered}
I=\int \frac{1}{x^{2}+(a+b) x+a b} d x \\
I=\int \frac{1}{\left[x+\frac{a+b}{2}\right]^{2}+a b-\left(\frac{a+b}{2}\right)^{2}} d x \\
I=\int \frac{1}{2\left(\frac{a-b}{2}\right)} \ln \frac{\left(x+\frac{a+b}{2}\right)-\left(\frac{a-b}{2}\right)}{\left(x+\frac{a+b}{2}\right)+\left(\frac{a-b}{2}\right)}+C \\
I=\frac{1}{a-b} \ln \frac{x+b}{x+a}+C
\end{gathered}
$$

Si lo escribiéramos así:

$$
\begin{aligned}
& I=\frac{1}{a-b} \ln (x+b)-\frac{1}{a-b} \ln (x+a)+C \\
& I=\frac{1}{a-b} \ln (x+b)+\frac{1}{b-a} \ln (x+a)+C,
\end{aligned}
$$

coincidiría con la respuesta del ejercicio anterior.

157. Encontrar $K$ si se tiene que $\int \frac{\left(2 x^{-2 / 3}+4\right)^{-1 / 4}}{\sqrt[3]{x^{5}}} d x=K\left(2 x^{-2 / 3}+4\right)^{3 / 4}+C$.

Solución:

$$
\int\left(2 x^{-2 / 3}+4\right)^{-1 / 4} x^{-5 / 3} d x=\frac{-3}{4} \int \underbrace{\left(2 x^{-2 / 3}+4\right)^{-1 / 4}}_{u} \underbrace{\left(-\frac{4}{3} x^{-5 / 3} d x\right)}_{d u}
$$

De donde:

$$
\begin{aligned}
\frac{-3}{4} \cdot \frac{\left(2 x^{-2 / 3}+4\right)^{3 / 4}}{3 / 4} & =K\left(2 x^{-2 / 3}+4\right)^{3 / 4} \\
-1 & =K
\end{aligned}
$$


158. Si $\int \frac{d x}{\sqrt{x^{3} \sqrt[3]{4+5 \sqrt[4]{x^{3}}}}}=F_{(x)}+C$, encontrar a tal que $F_{(a)}=-\frac{3 \sqrt[3]{3}}{2}$. (Sugerencia: $\left.z^{3}=4 x^{-3 / 4}+5\right)$.

Solución:

Sea $z^{3}=4 x^{-3 / 4}+5 \Rightarrow x^{-3 / 4}=\frac{z^{3}-5}{4} \Rightarrow x=\left(\frac{z^{3}-5}{4}\right)^{-4 / 3}$

De donde:

$$
d x=\frac{-4}{3}\left(\frac{z^{3}-5}{4}\right)^{-7 / 3}\left(\frac{3 z^{2}}{4}\right) d z=-\left(\frac{z^{3}-5}{4}\right)^{-7 / 3} z^{2} d z
$$

Entonces:

$$
\begin{aligned}
& \int \frac{d x}{\sqrt{x^{3} \sqrt[3]{4+5 \sqrt[4]{x^{3}}}}}=\int \frac{x^{-1 / 4} d x}{x^{3 / 2} x^{-1 / 4} \sqrt[3]{4+5 x^{3 / 4}}}=\int \frac{x^{-7 / 4} d x}{\sqrt[3]{4 x^{-3 / 4}+5}} \\
& \int \frac{d x}{\sqrt{x^{3} \sqrt[3]{4+5 \sqrt[4]{x^{3}}}}}=-\int \frac{\left(\frac{z^{3}-5}{4}\right)^{7 / 3}\left(\frac{z^{3}-5}{4}\right)^{-7 / 3} z^{2} d z}{\sqrt[3]{z^{3}}}=-\int z d z
\end{aligned}
$$

De donde:

$$
\int \frac{d x}{\sqrt{x^{3}} \sqrt[3]{4+5 \sqrt[4]{x^{3}}}}=-\frac{z^{2}}{2}+C=\underbrace{\frac{-1}{2}\left[4 x^{-3 / 4}+5\right]^{2 / 3}}_{F_{(x)}}+C
$$

Entonces:

$$
\begin{gathered}
F_{(a)}=\frac{-1}{2}\left(4 a^{-3 / 4}+5\right)^{2 / 3}=\frac{-3 \sqrt[3]{3}}{2} \\
\left(4 a^{-3 / 4}+5\right)^{2 / 3}=3^{4 / 3}=9^{2 / 3} \\
4 a^{-3 / 4}+5=9 \\
4 a^{-3 / 4}=4 \\
a^{-3 / 4}=1
\end{gathered}
$$

De donde:

$$
a=1
$$


159. Integrar: $\int \sqrt[3]{\frac{x+1}{x-1}} d x$.

\section{Solución:}

Sea

Entonces:

$$
\frac{x+1}{x-1}=u^{3} \rightarrow x=\frac{u^{3}+1}{u^{3}-1} \rightarrow d x \frac{-6 u^{2}}{\left(u^{3}-1\right)^{2}} d u
$$

$$
\int \sqrt[3]{\frac{x+1}{x-1}} d x=\int u \cdot\left(\frac{-6 u^{2}}{\left(u^{3}-1\right)^{2}}\right) d u=-6 \int \frac{u^{3}}{\left(u^{3}-1\right)^{2}} d u
$$

Por partes:

$$
\begin{array}{ccc}
U=u & V=\frac{-1}{3\left(u^{3}-1\right)} \\
\downarrow & \quad \begin{array}{c}
\uparrow \\
d U=d u
\end{array} & \frac{u^{2}}{\left(u^{3}-1\right)^{2}} d u
\end{array}
$$

De donde:

$$
\int \sqrt[3]{\frac{x+1}{x-1}} d x=-6\left[\frac{-u}{3\left(u^{3}-1\right)}+\frac{1}{3} \int \frac{d u}{u^{3}-1}\right]
$$

Como

$$
\int \frac{d u}{u^{3}-1}=\int\left[\frac{1 / 3}{u-1}-\frac{u / 3+2 / 3}{u^{2}+u+1}\right] d u
$$

Se tiene que:

$$
\begin{gathered}
\int \frac{d u}{u^{3}-1}=\frac{1}{3} \ln (u-1)-\frac{1}{3} \cdot \frac{1}{2}\left[\int \frac{2 u+1}{u^{2}+u+1} d u+\int \frac{3 d u}{\left(u+\frac{1}{2}\right)^{2}+\left(\frac{\sqrt{2}}{2}\right)^{2}}\right] \\
\int \frac{d u}{u^{3}-1}=\frac{1}{3} \ln (u-1)-\frac{1}{6} \ln \left(u^{2}+u+1\right)-\frac{1}{2}\left(\frac{2}{\sqrt{3}}\right) \arctan \frac{u+1 / 2}{\sqrt{3} / 2} \\
\int \frac{d u}{u^{3}-1}=\frac{1}{3} \ln (u-1)-\frac{1}{6} \ln \left(u^{2}+u+1\right)-\frac{\sqrt{3}}{3} \arctan \frac{2 u+1}{\sqrt{3}}+C^{\prime}
\end{gathered}
$$


En (1) :

$$
\begin{gathered}
\int \sqrt[3]{\frac{x+1}{x-1}} d x=\frac{2 u}{u^{3}-1}-\frac{2}{3} \ln (u-1)+\frac{1}{3} \ln \left(u^{2}+u+1\right)+\frac{2 \sqrt{3}}{3} \arctan \frac{2 u+1}{\sqrt{3}}+C \\
\int \sqrt[3]{\frac{x+1}{x-1}} d x=\frac{2 u}{u^{3}-1}+\frac{1}{3} \ln \frac{u^{2}+u+1}{(u-1)^{2}}+\frac{2 \sqrt{3}}{3} \arctan \frac{2 u+1}{\sqrt{3}}+C
\end{gathered}
$$

160. Integrar: $\int \frac{\sqrt{x}-1}{\sqrt[3]{x}+1} d x$.

\section{Solución:}

Sea $x=u^{6} \rightarrow d x=6 u^{5} d u$

Entonces:

$$
\int \frac{\sqrt{x}-1}{\sqrt[3]{x}+1} d x=\int \frac{u^{3}-1}{u^{2}+1}\left(6 u^{5} d u\right)=6 \int \frac{u^{8}-u^{5}}{u^{2}+1} d x
$$

Dividamos $\frac{u^{8}-u^{5}}{u^{2}+1}$

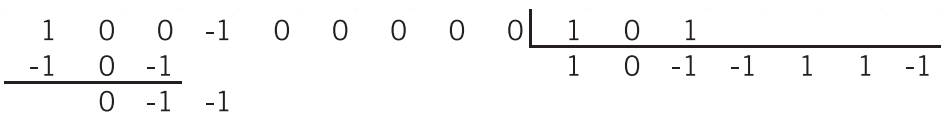

$$
\begin{aligned}
& \begin{array}{rrr}
+1 & 0 & 1 \\
\hline 0 & -1 & 1
\end{array} \\
& \begin{array}{llll}
1 & 0 & 1 & \\
\hline 0 & 1 & 1 & 0
\end{array} \\
& \begin{array}{rrrr}
-1 & 0 & -1 & \\
\hline 0 & 1 & -1 & 0
\end{array} \\
& \begin{array}{rrrr}
-1 & 0 & -1 & \\
\hline 0 & -1 & -1 & 0
\end{array} \\
& \begin{array}{lll}
1 & 0 & 1
\end{array} \\
& \begin{array}{lll}
0 & -1 & 1
\end{array}
\end{aligned}
$$

Se llega a que:

$$
\begin{aligned}
& \frac{u^{8}-u^{5}}{u^{2}+1}=u^{6}-u^{4}-u^{3}+u^{2}+u-1+\frac{-u+1}{u^{2}+1} \\
& \frac{u^{8}-u^{5}}{u^{2}+1}=u^{6}-u^{4}-u^{3}+u^{2}+u-1-\frac{1}{2} \cdot \frac{2 u}{u^{2}+1}+\frac{1}{u^{2}+1}
\end{aligned}
$$


De donde:

$$
\begin{aligned}
& \int \frac{\sqrt{x}-1}{\sqrt[3]{x}+1} d x=6 \int\left[u^{6}-u^{4}-u^{3}+u^{2}+u-1-\frac{1}{2} \cdot \frac{2 u}{u^{2}+1}+\frac{1}{u^{2}+1}\right] d u \\
& \int \frac{\sqrt{x}-1}{\sqrt[3]{x}+1} d x=\frac{6 u^{7}}{7}-\frac{6 u^{5}}{5}-\frac{6 u^{4}}{4}+\frac{6 u^{3}}{3}+\frac{6 u^{2}}{2}-6 u-3 \ln \left(u^{2}+1\right)+6 \arctan u+C \\
& \int \frac{\sqrt{x}-1}{\sqrt[3]{x}+1} d x=\frac{6 x \sqrt[6]{x}}{7}-\frac{6}{5} \sqrt[6]{x^{5}}-\frac{3}{2} \sqrt[3]{x^{2}}+2 \sqrt{x}+3 \sqrt[3]{x}-6 \sqrt[6]{x}-3 \ln (\sqrt[3]{x}+1)+6 \arctan \sqrt[6]{x}+C
\end{aligned}
$$

161. Obtener: $\int e^{a u} \cdot \cos b u \cdot d u$.

$$
\mathrm{R} .: \frac{e^{a u}(a \cos b u+b \operatorname{sen} b u)}{a^{2}+b^{2}}
$$

162. Integrar $\int \frac{d x}{\sqrt[3]{(1+x)^{2}}+\sqrt{1+x}}$. Sugerencia: haga $1+x=u^{6}$.

$$
\text { R.: } 3 \sqrt[3]{1+x}-6 \sqrt[6]{1+x}+\ln [\sqrt[6]{1+x}-1]+C
$$

163. Integrar: $I=\int \sqrt{x} \ln ^{2}(\sqrt{x}) \cdot d x$.

$$
\text { R.: } \frac{2}{3} x^{3 / 2}(\ln \sqrt{x})^{2}-\frac{4}{9} x^{3 / 2}(\ln \sqrt{x})+\frac{4}{27} x^{3 / 2}+C
$$

164. Integrar: $I=\int \sqrt{\frac{x-1}{x+2}} d x$.

$$
\text { R.: } \sqrt{(x+2)(x-1)}-3 \ln [\sqrt{x+2}+\sqrt{x-1}]+C
$$

165. Integrar: $I=\int \frac{\left(e^{2 x}+1\right)}{\left(e^{2 x}-1\right)\left(e^{x}-1\right)} d x$.

$$
\text { R.: } x-\frac{1}{2} \ln \left(e^{2 x}-1\right)-\frac{1}{e^{x}-1}+C
$$

166. Integrar: $\int x \cdot \ln \frac{x}{x+1} d x$.

$$
\text { R.: } \frac{x^{2}}{2} \ln \frac{x}{x+1}-\frac{x}{2}+\frac{1}{2} \ln (x+1)+C
$$


167. Integrar: $\int \frac{e^{4 x}}{\left(e^{2 x}+1\right)\left(e^{x}+1\right)} d x$.

R.: $e^{x}-\frac{1}{4} \ln \left(e^{2 x}+1\right)-\frac{1}{2} \arctan e^{x}-\frac{1}{2} \ln \left(e^{x}+1\right)+C$

168. Calcular la integral: $\int \frac{x^{4}+x^{3}+2 x^{2}-x+1}{\left(x^{2}+1\right)^{2}} d x$.

R.: $x+\frac{1}{2} \ln \left(x^{2}+1\right)+\frac{1}{2} \cdot \frac{1-x^{2}}{1+x^{2}}+C$

169. Encontrar el valor de $k+\frac{c}{2}$ si se cumple lo siguiente:

$$
\int \frac{d x}{(x-a) \sqrt{(x-a)(x-b)}}=\frac{\sqrt{\frac{x-b}{x-c}}}{k}+d
$$

R.: $a-\frac{b}{2}$

248

170. Si $\int\left(x^{2}-1\right) f^{\prime \prime \prime \prime}{ }_{(x)} d x=g_{(x)} f^{\prime \prime}{ }_{(x)}+h_{(x)} f^{\prime \prime}(x)+m f_{(x)}+C$, donde $m$ es una constante, hallar $E_{(x)}=\sqrt{g_{(x)}+h_{(x)}+m}$.

R.: $x-1$

171. $\mathrm{Si} \int(2 x+3) e^{-x} d x=-e^{-x} F_{(x)}+C$, calcule $F_{(2)}$.

R.: 9

172. Integrar: $\int \frac{d x}{a+b e^{p x}}$.

R.: $\frac{x}{a}-\frac{1}{a p} \log \left(a+b e^{p x}\right)+C$

173. Si $\int \frac{5 x^{2}-3 x+1}{x^{3}+x} d x=F_{(x)}+C$, halle $F_{(\sqrt{3})}$.

R.: 0.1803 
174. Si $\int \frac{d u}{e^{2 u}-3 e^{-2 u}+2}=F_{(u)}+C$, halle $F_{(\ln 2)}$.

R.: -0.1059

175. Haciendo el cambio de variable $u=\sqrt{\frac{x-b}{x-a}}$, se obtiene:

$\int \frac{d x}{(x-a) \sqrt{(x-a)(x-b)}}=F_{(x)}+C$. Halle $F_{(a+b)}$.

R.: $\frac{2 \sqrt{a b}}{b(b-a)}$

176. Si $\int x \ln \left(1+\frac{a}{x}\right) d x=F_{(x)}+C$, halle $\frac{2}{a^{2}} F_{(a)}$.

R.: 1 - Ina

177. Si $\int \frac{(x-1)^{n}-x+3}{x-1} d x=F_{(x)}$, entonces $F_{(2)}$ es igual a:

R.: $\frac{1-2 n}{n}$

178. Si $f_{(x)}=-5 x e^{-\frac{x}{3}}+15 e^{-\frac{x}{3}}$, y $\int f_{(x)} d x=F_{(x)}+C$, halle $F_{(6)}$.

R.: 12.180

179. Si $\int \frac{1}{x^{2} \sqrt{x^{2}+4}} d x=F_{(x)}+C$, halle $F_{(4)}$.
R.: $-\frac{\sqrt{5}}{8}$

180. Si $\int x^{m} \ln ^{n} x d x=\frac{x^{m+1} \ln ^{n} x}{m+1}+k \int x^{m} \ln ^{n-1} x d x$, halle el valor de $k$ para $m=1$ y $n=8$.

R.: -4

181. Si $\int x^{m} e^{n x} d x=\frac{1}{n} x^{m} e^{n x}+k \int x^{m-1} e^{n x} d x$, halle el valor de $k$ cuando $m=n$.

R.: -1 
182. Integrar: $\int t^{2} \operatorname{sen} t d t$.

R.: $2 t \operatorname{sen} t-\left(t^{2}-2\right) \cos t+C$

183. Obtener: $\int \frac{1 d z}{\sqrt{z}(1-\sqrt{z})^{2}}$.

R.: $\frac{2}{1-\sqrt{z}}+C$

184. Hallar: $\int\left(\cot x-\frac{x}{\operatorname{sen}^{2} x}\right) d x$.

R.: $x \cot x$

185. Determinar: $\int \frac{b x^{2}}{\sqrt[3]{\left(a+b x^{3}\right)^{2}}} d x$.

R.: $\sqrt[3]{a+b x^{3}}$

250

186. Integrar: $\int \frac{1+\tan x}{1-\tan x} d x$.

R.: $-\ln (\cos x-\operatorname{sen} x)+C$

187. Hallar: $\int \frac{d x}{\operatorname{sen}^{2} x-5 \operatorname{sen} x \cos x}$. (Sugerencia: divida ambos términos entre $\cos ^{2} x$ ).

R.: $\frac{1}{5} \ln \frac{\tan x-5}{\tan x}+C$

188. Obtener: $\int \frac{\cos x}{\operatorname{sen}^{2} x-6 \operatorname{sen} x+5} d x$.

R.: $\frac{1}{4} \ln \frac{5-\operatorname{sen} x}{1-\operatorname{sen} x}+C$

189. Integrar: $\int \frac{d x}{\sqrt{x}+\sqrt[3]{x}}$.

R.: $6 \sqrt[6]{x}-3 \sqrt[3]{x}+2 \sqrt{x}-6 \ln (\sqrt[6]{x}+1)+C$ 
190. Hallar: $\int \frac{\sqrt{x}}{x+2} d x$.

R.: $2 \sqrt{x}-2 \sqrt{2} \arctan \sqrt{\frac{x}{2}}+C$

191. Si $\int \frac{2 x+7}{(x-1)\left(x^{2}-x-6\right)} d x=G_{(x)}+C$, halle el valor de $G_{(2)}$.

R.: 0.2773

192. Si $\int \frac{1+5 x^{2}}{x^{2}\left(1+x^{2}\right)} d x=-\frac{1}{x}+f_{(x)}+C$, halle $f_{(1)}$.

R.: $\pi$

193. Si $\int \frac{\operatorname{sen} a x}{x^{n}} d x=-\frac{\operatorname{sen} a x}{k x^{n-1}}+\frac{a}{k} \int \frac{\cos a x}{x^{n-1}} d x, k \neq 0$, halle la constante $k$ cuando $n=8$.

R.: 7

194. Si $\int \frac{d x}{7 x^{2}-8}=G_{(x)}+C$, calcular $G_{(2)}$.

R.: -0.0797

195. Si $\int\left(a^{2}-x^{2}\right)^{3 / 2} d x=F_{(x)}+C$, determinar $F_{(x)}$ y obtener $F_{(a)}$.
R.: $\frac{3 \pi a^{4}}{16}$

196. Evaluar $\int \frac{x^{3} e^{\arccos x}}{\sqrt{1-x^{2}}} d x$ con el cambio de variable $x=\cos \theta$ e integrar por partes. Dar el resultado en función de $\theta$.

R.: $\frac{-e^{\theta}}{10}\left[\cos ^{3} \theta-3 \operatorname{sen}^{3} \theta+6 \operatorname{sen} \theta+3 \cos \theta\right]+C$

197. Si $\int \frac{x^{5}}{\sqrt{2 x^{2}-1}} d x=m_{(x)}+k$, hallar $m_{(1)}$.

R.: $7 / 30$ 
198. Si $\int \arccos 2 x d x=q_{(x)}+k$, hallar $q_{(14)}$.

R.: -0.1712

199. Si $\int \frac{\ln x}{(x+1)^{2}} d x=f_{(x)}+k$, hallar $f_{(4)}$.

R.: -0.5004

200. Si $\int \frac{e^{x}}{e^{2 x}+2 e^{x}+3} d x=n_{(x)}+k$, hallar $n_{(\ln 3)}$.

R.: 0.8704

201. Si $\int \frac{\ln x}{x\left(1+\ln ^{2} x\right)} d x=p_{(x)}+k$, hallar $p_{\left(e^{2}\right)}$.

R.: 0.8047

252

202. Si $\int \frac{e^{2 a t}}{\sqrt{e^{a t}+1}} d t=G_{(t)}+C$, halle $3 a G_{(0)}$.

R.: $-2 \sqrt{2}$

203. Si $\int \frac{x}{x^{3}-1} d x=F_{(x)}+C$, evalúe $F_{(0)}$.

R.: 0.3023

204. Halle $F_{(0)}$ si $\int \frac{e^{x}}{e^{2 x}+2 e^{x}+3} d x=F_{(x)}+C$.

R.: 0.6755

205. Si $\int \sqrt{\frac{x+1}{x+2}} d x=G_{(x)}+C$, encontrar $G_{(5)}$.

R.: 4.8524

206. Si $\int \sqrt{4+3 x-9 x^{2}} d x=F_{(x)}+C$, averiguar $F_{(x)}$ y obtener $F_{(0.5)}$.

R.: 0.6592 
207. Si $\int \frac{\sin ^{3 / 2} x}{\cos ^{11 / 2} x} d x=f_{(x)}+k$, hallar $f_{(\pi / 4)}$.

R.: $28 / 45$

208. Si $\int \frac{3 x-1}{x^{2}+9} d x=h_{(x)}+k$, hallar $h_{(4)}$.

R.: 4.5192

209. Encontrar: $f_{(1 / 3)}$ si $\int \frac{x-1}{\sqrt{1-x^{2}}} d x=f_{(x)}+C$.

R.: -1.2826

210. Demostrar que $\int \frac{x^{2} d x}{\sqrt{a x+b}}=\frac{2\left(8 b^{2}-4 a b x+3 a^{2} x^{2}\right)}{15 a^{3}} \sqrt{a x+b}+C$. 


\section{PROBLEMAS RESUELTOS Y PROPUESTOS DEL CAPÍTULO V}

211. Sea $f_{(x)}=a x^{2}+b x+c$.

a) Sabiendo que $\int_{m-h}^{m+h} f_{(x)} d x=k\left[f_{(m-h)}+4 f_{(m)}+f_{(m+h)}\right]$, hallar $k$.

b) Use el resultado de la parte a) para calcular $\int_{2}^{4}\left(2 x^{2}+3 x+1\right) d x$.

\section{Solución:}

a) Se tiene que:

$$
\begin{aligned}
& \int_{m-h}^{m+h} f_{(x)} d x=\int_{m-h}^{m+h}\left(a x^{2}+b x+c\right) d x=\frac{a x^{3}}{3}+\frac{b x^{2}}{2}+\left.c x\right|_{m-h} ^{m+h} \\
& \int_{m-h}^{m+h} f_{(x)} d x= \\
& =\frac{a}{3}\left[(m+h)^{3}-(m-h)^{3}\right]+\frac{b}{2}\left[(m+h)^{2}-(m-h)^{2}\right]+c[(m+h)-(m-h)] \\
& \int_{m-h}^{m+h} f_{(x)} d x=\frac{a}{3}\left[6 m^{2} h+2 h^{3}\right]+\frac{b}{2}[4 m h]+c[2 h] \\
& \int_{m-h}^{m+h} f_{(x)} d x=2 a m^{2} h+\frac{2 a}{3} h^{3}+2 b m h+2 c h \\
& \int_{m-h}^{m+h} f_{(x)} d x=\frac{h}{3}\left[6 a m^{2}+2 a h^{2}+6 b m+6 c\right]
\end{aligned}
$$

Por otro lado:

$$
\begin{aligned}
& k\left[f_{(m-h)}+4 f_{(m)}+f_{(m+h)}\right]= \\
& \quad=k\left[a(m-h)^{2}+b(m-h)+c+4 a m^{2}+4 b m+4 c+a(m+h)^{2}+b(m+h)+c\right] \\
& k\left[f_{(m-h)}+4 f_{(m)}+f_{(m+h)}\right]=k\left[6 a m^{2}+2 a h^{2}+6 b m+6 c\right]
\end{aligned}
$$

Comparando $(\alpha)$ con $(\beta)$ :

$$
K=\frac{h}{3}
$$

b) Si $\left\{\begin{array}{l}m+h=4 \\ m-h=2\end{array} \rightarrow m=3 \wedge h=1\right.$ 
$K=\frac{h}{3}=\frac{1}{3}$

Entonces:

$$
\int_{2}^{4} f_{(x)} d x=\frac{1}{3}\left[f_{(2)}+4 f_{(3)}+f_{(4)}\right]
$$

Como: $f_{(x)}=2 x^{2}+3 x+1$

$$
\begin{aligned}
& f_{(2)}=15 \\
& f_{(3)}=28 \\
& f_{(4)}=45
\end{aligned}
$$

De donde:

$$
\int_{2}^{4} f_{(x)} d x=\frac{1}{3}[15+4(28)+45]=\frac{172}{3}
$$

212. Efectuar el desarrollo de $f_{(x)}=-x^{4}+8 x^{3}-12 x^{2}+24 x-16$ en potencias de $(x-2)$ y calcular $\int_{x=2}^{3} f_{(x)} d x$

Solución:

Escribamos:

\begin{tabular}{c|ccccc} 
& -1 & 8 & -12 & 24 & -16 \\
2 & $\downarrow$ & -2 & 12 & 0 & 48 \\
\hline & -1 & 6 & 0 & 24 & 32 \\
2 & $\downarrow$ & -2 & 8 & 16 & \\
\hline & -1 & 4 & 8 & 40 & \\
2 & $\downarrow$ & -2 & 4 & & \\
\hline & -1 & 2 & 12 & & \\
2 & $\downarrow$ & -2 & & & \\
\hline & -1 & 0 & & &
\end{tabular}

Entonces:

$$
\begin{gathered}
f_{(x)}=-x^{4}+8 x^{3}-12 x^{2}+24 x-16 \\
f_{(x-2)}=-1(x-2)^{4}+0(x-2)^{3}+12(x-2)^{2}+40(x-2)+32
\end{gathered}
$$


Sea: $x-2=u \rightarrow f_{(u)}=-1 u^{4}+12 u^{2}+40 u+32$

$d x=d u$

Si: $x=3 \rightarrow u=1$

$x=2 \rightarrow u=0$

De donde:

$$
\begin{gathered}
\int_{2}^{3} f_{(x)} d x=\int_{0}^{1} f_{(u)} d u=\int_{0}^{1}\left[-u^{4}+12 u^{2}+40 u+32\right] d u \\
\int_{2}^{3} f_{(x)} d x=\frac{-u^{5}}{5}+4 u^{3}+20 u^{2}+\left.32 u\right|_{0} ^{1}
\end{gathered}
$$

Con lo cual:

$$
\int_{2}^{3} f_{(x)} d x=\frac{-1}{5}+4+20+32=554 / 5
$$

213. Encuentre la razón geométrica de $A$ y $D$ que corresponda a la expresión siguiente:

$$
\int_{1}^{3} x^{2} d x=\lim _{n \rightarrow \infty} \frac{\left(A n^{2}+B n+C\right)}{D n^{2}}
$$

Solución:

Como $\lim _{n \rightarrow \infty} \frac{\left(A n^{2}+B n+C\right)}{D n^{2}}=\lim _{n \rightarrow \infty} \frac{A+\frac{B}{n}+\frac{C}{n^{2}}}{D}=\frac{A}{D}$

$y \int_{1}^{3} x^{2} d x=\left.\frac{x^{3}}{3}\right|_{1} ^{3}=\frac{27}{3}-\frac{1}{3}=\frac{26}{3}$

Se concluye con que:

$$
\frac{A}{D}=\frac{26}{3}
$$

214. Calcular: $\int_{x=-1}^{5}\left|x^{3}-4 x\right| d x$

Solución:

$$
\int_{-1}^{5}\left|x^{3}-4 x\right| d x=\int_{-1}^{5}|x(x+2)(x-2)| d x
$$

El signo de $x(x+2)(x-2)$ es: 


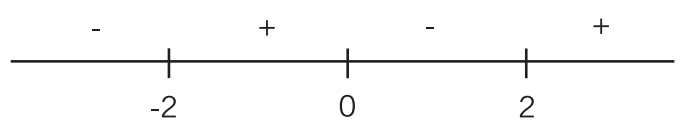

Entonces:

De donde:

$$
x(x+2)(x-2)= \begin{cases}x^{3}-4 x & x \in[-2,0] \cup[2, \infty] \\ -x^{3}+4 x & x \in[-\infty,-2] \cup[0,2]\end{cases}
$$

$$
\begin{aligned}
\int_{-1}^{5}\left|x^{3}-4 x\right| d x= & \int_{-1}^{0}\left(x^{3}-4 x\right) d x+\int_{0}^{2}\left(-x^{3}+4 x\right) d x+\int_{2}^{5}\left(x^{3}-4 x\right) d x \\
& \int_{-1}^{5}\left|x^{3}-4 x\right| d x=\frac{7}{4}+4+\frac{441}{4}=116
\end{aligned}
$$

215. Calcular la siguiente integral: $\int_{x=2}^{3} x \ln \frac{x-1}{x+1} d x$.

Solución:

$$
\begin{gathered}
I=\int x \cdot \ln \frac{x-1}{x+1} d x=\underbrace{\int x \cdot \ln (x-1) d x}_{I_{1}}-\underbrace{\int x \cdot \ln (x+1) d x}_{I_{2}} \\
I_{1}=\int x \cdot \ln (x-1) d x \quad \quad \quad \uparrow=\frac{x^{2}}{2} \\
u=\ln (x-1) \quad d v=x d x \\
\quad d u=\frac{d x}{x-1} \quad I_{1}=\frac{x^{2}}{2} \ln (x-1)-\frac{1}{2} \int \frac{x^{2}}{x-1} d x \\
I_{1}=\frac{x^{2}}{2} \ln (x-1)-\frac{1}{2} \int\left(x+1+\frac{1}{x-1}\right) d x \\
I_{1}=\frac{x^{2}}{2} \ln (x-1)-\frac{1}{2}\left(\frac{x^{2}}{2}+x+\ln (x-1)\right)+C_{1}
\end{gathered}
$$

Análogamente:

$$
I_{2}=\frac{x^{2}}{2} \ln (x+1)-\frac{1}{2}\left(\frac{x^{2}}{2}-x+\ln (x+1)\right)+C_{2}
$$


De donde:

$$
I=\frac{x^{2}}{2} \ln \frac{x-1}{x+1}-x-\frac{1}{2} \ln \frac{x-1}{x+1}+C
$$

Finalmente:

$$
\int_{2}^{3} x \cdot \ln \frac{x-1}{x+1} d x=\left.I\right|_{2} ^{3}=\left(4 \ln \frac{1}{2}-3\right)-\left(\frac{3}{2} \ln \frac{1}{3}-2\right)
$$

La respuesta es: $-4 \ln 2+\frac{3}{2} \ln 3-1$.

216. Calcular: $\int_{x=0}^{1} \frac{\sqrt{x}}{1+x \sqrt{x}} d x$.

Solución:

Sea $x=u^{2} \rightarrow d x=2 u d u$

Si: $\quad x=0 \Rightarrow u=0$

$$
x=1 \Rightarrow u=1
$$

Entonces:

$$
\begin{gathered}
I=\int_{0}^{1} \frac{u}{1+u^{2}(u)}(2 u d u) \\
I=2 \int_{0}^{1} \frac{u^{2} d u}{1+u^{3}} \\
I=\frac{2}{3} \int_{0}^{1} \frac{3 u^{2} d u}{1+u^{3}} \\
I=\left.\frac{2}{3} \ln \left(1+u^{3}\right)\right|_{0} ^{1} \\
I=\frac{2}{3}(\ln 2-\ln 1)=\frac{2}{3} \ln 2
\end{gathered}
$$

217. Calcular: $\int_{x=0}^{\frac{\pi}{4}} \sec ^{2} x \cdot \ln (\sec x) \cdot d x$.

Solución: 
Por partes:

$$
\begin{array}{cc}
u=\ln (\sec x) & v=\tan x \\
\downarrow & \uparrow \\
d u=\frac{\sec x \tan x d x}{\sec x} & d v=\sec ^{2} x d x \\
\int \sec ^{2} x \cdot \ln (\sec x) d x=\tan x \cdot \ln (\sec x)-\int \tan ^{2} x d x \\
\int \tan ^{2} x d x=\int\left(\sec ^{2} x-1\right) d x=\tan x-x+C^{\prime}
\end{array}
$$

De donde:

$$
\begin{gathered}
\int \sec ^{2} x \cdot \ln (\sec x) d x=\tan x \cdot \ln (\sec x)-\tan x+x+C \\
\int_{0}^{\frac{\pi}{4}} \sec ^{2} x \cdot \ln (\sec x) d x=1 \ln \sqrt{2}-1+\frac{\pi}{4}-0=\ln \sqrt{2}+\frac{\pi}{4}-1
\end{gathered}
$$

218. Calcular: $\int_{x=0}^{4} \ln (1+\sqrt{x}) d x$.

Solución:

Sea $e^{u}=1+\sqrt{x} \rightarrow \sqrt{x}=e^{u}-1 \rightarrow x=\left(e^{u}-1\right)^{2} \rightarrow d x=2\left(e^{u}-1\right) e^{u} d u$

Si: $\quad x=4 \rightarrow e^{u}=3 \rightarrow u=\ln 3$

$x=0 \rightarrow e^{u}=1 \rightarrow u=0$

Entonces:

$$
\begin{gathered}
I=\int_{0}^{4} \ln (1+\sqrt{x}) d x=\int_{0}^{\ln 3} 2 \underbrace{\left(\ln e^{u}\right)}_{u}\left(e^{u}-1\right) e^{u} d u \\
I=2 \int_{0}^{\ln 3} u e^{2 u} d u-2 \int_{0}^{\ln 3} u e^{u} d u
\end{gathered}
$$

De acuerdo con la fórmula:

$$
\begin{gathered}
\int u e^{a u} d u=\frac{e^{a u}}{a^{2}}(a u-1) \\
\int u e^{2 u} d u=\frac{e^{2 u}}{2^{2}}(2 u-1) \\
\int u e^{u} d u=\frac{e^{u}}{1^{2}}(u-1)
\end{gathered}
$$


De donde:

$$
I=\left.2 \frac{e^{2 u}}{4}(2 u-1)\right|_{0} ^{\ln 3}-\left.2 \frac{e^{u}}{1}(u-1)\right|_{0} ^{\ln 3}
$$

La respuesta es:

$$
\begin{gathered}
I=\frac{1}{2}(9)(2 \ln 3-1-0+1)-2(3)(\ln 3-1-0+1) \\
I=9 \ln 3-6 \ln 3=3 \ln 3
\end{gathered}
$$

219. Calcular: $\int_{x=0}^{\frac{\pi}{3}} \frac{x \operatorname{sen} x}{\cos ^{2} x} d x$.

Solución:

$$
I=\int_{0}^{\frac{\pi}{3}} x \frac{1}{\cos x} \cdot \frac{\operatorname{sen} x}{\cos x} d x=\int_{0}^{\frac{\pi}{3}} x \cdot \sec x \cdot \tan x \cdot d x
$$

Por partes:

260

$$
\begin{array}{cc}
u=x & v=\sec x \\
\downarrow & \uparrow \\
d u=d x & d v=\sec x \cdot \tan x d x
\end{array}
$$

Entonces:

$$
\begin{gathered}
I=\left.x \sec x\right|_{0} ^{\pi / 3}-\int_{0}^{\frac{\pi}{3}} \sec x d x \\
I=\left.x \sec x\right|_{0} ^{\pi / 3}-\int_{0}^{\frac{\pi}{3}} \frac{\sec ^{2} x+\sec x \cdot \tan x}{\sec x+\tan x} d x \\
I=\left.x \sec x\right|_{0} ^{\pi / 3}-\left.\ln (\sec x+\tan x)\right|_{0} ^{\pi / 3}
\end{gathered}
$$

De donde:

$$
\begin{gathered}
I=\frac{\pi}{3}(2)-0-\ln (2+\sqrt{3})+\ln (1+0) \\
I=\frac{2 \pi}{3}-\ln (2+\sqrt{3})
\end{gathered}
$$


220. Calcular: $\int_{x=0}^{\frac{2 \pi}{3}} \frac{d x}{1+\operatorname{sen} x+\cos x}$.

Solución:

Sea $z=\tan \frac{x}{2} \Rightarrow x=2 \arctan z \Rightarrow d x=\frac{2 d z}{1+z^{2}}$

Si: $\quad x=\frac{2 \pi}{3} \rightarrow z=\tan \frac{\pi}{3}=\sqrt{3}$

$$
x=0 \rightarrow z=\tan 0^{\circ}=0
$$

Entonces:

$$
\begin{aligned}
& \int_{0}^{\frac{2 \pi}{3}} \frac{d x}{1+\operatorname{sen} x+\cos x}=\int_{0}^{\sqrt{3}} \frac{\frac{2 d z}{1+z^{2}}}{1+\frac{2 z}{1+z^{2}}+\frac{1-z^{2}}{1+z^{2}}} \\
& \int_{0}^{\sqrt{3}} \frac{d x}{1+\operatorname{sen} x+\cos x}=2 \int_{0}^{\sqrt{3}} \frac{d z}{2(z+1)}=\int_{0}^{\sqrt{3}} \frac{d z}{z+1} \\
& \int_{0}^{\sqrt{3}} \frac{d x}{1+\operatorname{sen} x+\cos x}=\left.\ln (z+1)\right|_{0} ^{\sqrt{3}}=\ln (\sqrt{3}+1)
\end{aligned}
$$

221. Integrar: $I=\int_{x=0}^{\ln 2} \sqrt{1+e^{2 x}} d x$.

\section{Solución:}

Sea: $1+e^{2 x}=u^{2} \Rightarrow 2 e^{2 x} d x=2 u d u$

$$
d x=\frac{u d u}{e^{2 x}}=\frac{u d u}{u^{2}-1}
$$

Si: $x=\ln 2 \rightarrow u^{2}=1+4 \rightarrow u=\sqrt{5}$

$$
x=0 \rightarrow u^{2}=1+1 \rightarrow u=\sqrt{2}
$$

Entonces:

$$
\begin{gathered}
I=\int_{\sqrt{2}}^{\sqrt{5}} \sqrt{u^{2}} \frac{u d u}{u^{2}-1}=\int_{\sqrt{2}}^{\sqrt{5}} \frac{u^{2} d u}{u^{2}-1} \\
I=\int_{\sqrt{2}}^{\sqrt{5}}\left(\frac{u^{2}-1}{u^{2}-1}+\frac{1}{u^{2}-1}\right) d u
\end{gathered}
$$




$$
I=u+\left.\frac{1}{2} \ln \frac{u-1}{u+1}\right|_{\sqrt{2}} ^{\sqrt{5}}
$$

La respuesta es:

$$
I=1.222
$$

222. Calcular: $\int_{x=2}^{4}\left|\frac{x+1}{x+6}\right| d x$.

Solución:

Como $2 \leq x \leq 4 \rightarrow 3 \leq x+1 \leq 5 \wedge 8 \leq x+6 \leq 10$

De donde:

$$
\left|\frac{x+1}{x+6}\right|=\frac{x+1}{x+6}
$$

Entonces:

$$
\begin{gathered}
\int_{2}^{4}\left|\frac{x+1}{x+6}\right| d x=\int_{2}^{4} \frac{x+1}{x+6} d x=\int_{2}^{4} \frac{x+6-5}{x+6} d x \\
\int_{2}^{4}\left|\frac{x+1}{x+6}\right| d x=\int_{2}^{4}\left(1-\frac{5}{x+6}\right) d x=x-\left.5 \ln (x+6)\right|_{2} ^{4}
\end{gathered}
$$

La respuesta es:

$$
4-5 \ln 10-2+5 \ln 8=2+5 \ln 0.8
$$

223. Calcular la siguiente integral definida: $\int_{-a}^{a} \frac{d x}{\left(a^{2}+x^{2}\right)^{3 / 2}}$.
Solución:

Sea $x=a \tan \theta \Rightarrow d x=a \sec ^{2} \theta d \theta$

$$
\int \frac{d x}{\left(a^{2}+x^{2}\right)^{3 / 2}}=\int \frac{a \sec ^{2} \theta d \theta}{a^{3} \sec ^{3} \theta}=\frac{1}{a^{2}} \int \cos \theta d \theta=\frac{1}{a^{2}} \operatorname{sen} \theta+C
$$

Entonces:

$$
\int_{-a}^{a} \frac{d x}{\left(a^{2}+x^{2}\right)^{3 / 2}}=\left.\frac{1}{a^{2}} \operatorname{sen} \theta\right|_{\arctan -1} ^{\arctan 1}=\left.\frac{1}{a^{2}} \operatorname{sen} \theta\right|_{-\pi / 4} ^{\pi / 4}=\frac{\sqrt{2}}{a^{2}}
$$


224. Calcular la siguiente integral definida: $\int_{x=0}^{4} \frac{d x}{2+\sqrt{x}}$.

Solución:

Sea $x=u^{2} \rightarrow d x=2 u d u$

Si: $\quad x=4 \rightarrow u=2$

$$
x=0 \rightarrow u=0
$$

Entonces:

$$
\begin{gathered}
\int_{0}^{4} \frac{d x}{2+\sqrt{x}}=\int_{0}^{2} \frac{2 u d u}{2+u}=2 \int_{0}^{2} \frac{(u+2)-2}{u+2} d u \\
\int_{0}^{4} \frac{d x}{2+\sqrt{x}}=\left.2[u-2 \ln (u+2)]\right|_{0} ^{2} \\
\int_{0}^{4} \frac{d x}{2+\sqrt{x}}=2[2-2 \ln 4-0+2 \ln 2] \\
\int_{0}^{4} \frac{d x}{2+\sqrt{x}}=2(2-2 \ln 2)=4-4 \ln 2
\end{gathered}
$$

225. Calcular la siguiente integral definida: $\int_{x=0}^{16} \sqrt{1+\sqrt{x}} d x$.

\section{Solución:}

Sea $1+\sqrt{x}=u^{2} \rightarrow \sqrt{x}=u^{2}-1 \rightarrow \frac{d x}{2 \sqrt{x}}=2 u d u$

$$
d x=4 u \sqrt{x} d u=4 u\left(u^{2}-1\right) d u
$$

Si: $\quad x=16 \rightarrow u^{2}=5 \rightarrow u=\sqrt{5}$

$x=0 \rightarrow u^{2}=1 \rightarrow u=1$

Entonces:

$$
\begin{gathered}
\int_{0}^{16} \sqrt{1+\sqrt{x}} d x=4 \int_{1}^{\sqrt{5}} u^{2}\left(u^{2}-1\right) d u \\
\int_{0}^{16} \sqrt{1+\sqrt{x}} d x=\left.4\left(\frac{u^{5}}{5}-\frac{u^{3}}{3}\right)\right|_{1} ^{\sqrt{5}}=\frac{40 \sqrt{5}}{3}+\frac{8}{15}
\end{gathered}
$$


226. Calcular la siguiente integral definida: $\int_{x=0}^{\pi^{2}} \operatorname{sen} \sqrt{x} d x$.

\section{Solución:}

Sea $x=u^{2} \rightarrow d x=2 u d u$

$$
\int_{x=0}^{\pi^{2}} \operatorname{sen} \sqrt{x} d x=\int_{0}^{\pi}(\operatorname{sen} u)(2 u d u)=2 \int_{0}^{\pi} u \operatorname{sen} u d u
$$

Integrando por partes:

$$
2 \int_{0}^{\pi} u \operatorname{sen} u d u=\left.2(\operatorname{sen} u-u \cos u)\right|_{0} ^{\pi}=2(0-\pi(-1)-0+0)=2 \pi
$$

227. Calcular la siguiente integral definida: $\int_{x=0}^{\ln 5} \sqrt{e^{x}-1} d x$.

\section{Solución:}

Sea $e^{x}-1=u^{2} \rightarrow e^{x} d x=2 u d u \rightarrow d x=\frac{2 u d u}{u^{2}+1}$

264

Si: $\quad x=\ln 5 \rightarrow u^{2}=5-1=4 \rightarrow u=2$

$$
x=0 \rightarrow u^{2}=1-1=0 \rightarrow u=0
$$

Entonces:

$$
\begin{aligned}
& \int_{0}^{\ln 5} \sqrt{e^{x}-1} d x=\int_{0}^{2} u\left(\frac{2 u d u}{u^{2}+1}\right)=2 \int_{0}^{2} \frac{\left(u^{2}+1\right)-1}{u^{2}+1} d u \\
& \int_{0}^{\ln 5} \sqrt{e^{x}-1} d x=\left.2(u-\arctan u)\right|_{0} ^{2}=4-2 \arctan 2
\end{aligned}
$$

228. Resolver la siguiente integral definida: $\int_{\pi / 2}^{\pi} \frac{d x}{1-\cos x}$.

Solución:

$$
\begin{gathered}
\int_{\pi / 2}^{\pi} \frac{d x}{1-\cos x}=\int_{\pi / 2}^{\pi} \frac{d x}{2 \operatorname{sen}^{2} \frac{x}{2}}=\frac{1}{2} \int_{\pi / 2}^{\pi} \csc ^{2} \frac{x}{2} d x \\
\int_{\pi / 2}^{\pi} \frac{d x}{1-\cos x}=\left.\frac{1}{2}(2)\left(-\cot \frac{x}{2}\right)\right|_{\pi / 2} ^{\pi}
\end{gathered}
$$


La respuesta es: $-\left.\cot \frac{x}{2}\right|_{\pi / 2} ^{\pi}=-(0-1)=1$.

229. Resolver la siguiente integral definida: $\int_{-1}^{1} \frac{|x|}{1+x^{2}} d x$.

\section{Solución:}

$$
\begin{gathered}
\int_{-1}^{1} \frac{|x|}{1+x^{2}} d x=\int_{1}^{0} \frac{-x}{1+x^{2}} d x+\int_{0}^{1} \frac{x}{1+x^{2}} d x \\
\int_{-1}^{1} \frac{|x|}{1+x^{2}} d x=-\frac{1}{2} \int_{-1}^{0} \frac{2 x d x}{1+x^{2}}+\frac{1}{2} \int_{0}^{1} \frac{2 x d x}{1+x^{2}} \\
\int_{-1}^{1} \frac{|x|}{1+x^{2}} d x=-\left.\frac{1}{2} \ln \left(1+x^{2}\right)\right|_{-1} ^{0}+\left.\frac{1}{2} \ln \left(1+x^{2}\right)\right|_{0} ^{1} \\
\int_{-1}^{1} \frac{|x|}{1+x^{2}} d x=-\frac{1}{2}(\ln 1-\ln 2)+\frac{1}{2}(\ln 2-\ln 1)=\ln 2
\end{gathered}
$$

230. Calcular: $I=\int_{x=0}^{1 / 3} \operatorname{arcsen} \sqrt{3 x} d x$.

Solución:

Sea: $\quad \theta=\operatorname{arcsen} \sqrt{3 x} \rightarrow \operatorname{sen} \theta=\sqrt{3 x} \rightarrow \cos \theta d \theta=\frac{3 d x}{2 \sqrt{3 x}}$

$$
d x=\frac{2}{3} \sqrt{3 x} \cos \theta d \theta=\frac{2}{3} \operatorname{sen} \theta \cos \theta d \theta=\frac{\operatorname{sen} 2 \theta}{3} d \theta
$$

Si: $\quad x=1 / 3 \rightarrow \theta=\operatorname{arcsen} 1=\pi / 2$

$$
x=0 \quad \rightarrow \quad \theta=\operatorname{arc} \operatorname{sen} 0=0
$$

Entonces:

$$
I=\int_{0}^{\pi / 2} \theta \frac{\operatorname{sen} 2 \theta}{3} d \theta=\frac{1}{3} \int_{0}^{\pi / 2} \theta \operatorname{sen} 2 \theta d \theta
$$

Por partes:

$$
\begin{array}{cc}
u=\theta & v=\frac{-1}{2} \cos 2 \theta \\
\downarrow & \uparrow \\
d u=d \theta & d v=\sec 2 \theta d \theta
\end{array}
$$




$$
I=\left.\frac{1}{3}\left[\frac{1}{2} \theta \cos 2 \theta+\frac{1}{2} \cdot \frac{1}{2} \operatorname{sen} 2 \theta\right]\right|_{0} ^{\pi / 2}=\frac{\pi}{12}
$$

231. Evaluar: $\int_{0}^{1} \frac{x}{(x+1)^{2}\left(x^{2}+1\right)} d x$.

Solución:

Por descomposición en fracciones parciales:

$$
\frac{x}{(x+1)^{2}\left(x^{2}+1\right)}=\frac{-1 / 2}{(x+1)^{2}}+\frac{1 / 2}{x^{2}+1}
$$

Entonces:

$$
\begin{aligned}
& \int_{0}^{1} \frac{x d x}{(x+1)^{2}\left(x^{2}+1\right)}=-\frac{1}{2} \int_{0}^{1} \frac{d x}{(x+1)^{2}}+\frac{1}{2} \int_{0}^{1} \frac{d x}{x^{2}+1} \\
& \int_{0}^{1} \frac{x d x}{(x+1)^{2}\left(x^{2}+1\right)}=-\frac{1}{2}\left(\frac{-1}{x+1}\right)+\left.\frac{1}{2} \arctan x\right|_{0} ^{1} \\
& \int_{0}^{1} \frac{x d x}{(x+1)^{2}\left(x^{2}+1\right)}=\frac{1}{2}\left(\frac{1}{2}+\frac{\pi}{4}-1-0\right)=\frac{\pi}{8}-\frac{1}{4}
\end{aligned}
$$

232. Calcular: $\int_{0}^{1} x^{2} \arctan x d x$.

Solución:

Sea:

$$
\begin{array}{cc}
u=\arctan x & v=\frac{x^{3}}{3} \\
\downarrow & \uparrow \\
d u=\frac{d x}{1+x^{2}} & d v=x^{2} d x
\end{array}
$$

Entonces:

$$
\int x^{2} \cdot \arctan x \cdot d x=\frac{x^{3}}{3} \arctan x-\frac{1}{3} \int \frac{x^{3}}{1+x^{2}} d x
$$




$$
\begin{aligned}
& \text { Como } \frac{x^{3}}{1+x^{2}}=x-\frac{x}{1+x^{2}}, \\
& \qquad \int_{0}^{1} x^{2} \arctan x d x=\frac{x^{3}}{3} \arctan x-\left.\frac{1}{3}\left\{\frac{x^{2}}{2}-\frac{1}{2} \ln \left(1+x^{2}\right)\right\}\right|_{0} ^{1}
\end{aligned}
$$

De donde:

$$
\begin{gathered}
\int_{0}^{1} x^{2} \arctan x d x=\left\{\frac{1}{3} \cdot \frac{\pi}{4}-\frac{1}{3}\left(\frac{1}{2}-\frac{1}{2} \ln 2\right)\right\}-\left\{0-\frac{1}{3}(0)\right\} \\
\int_{0}^{1} x^{2} \arctan x d x=\frac{\pi}{12}-\frac{1}{6}+\frac{1}{6} \ln 2
\end{gathered}
$$

233. Si $\int_{0}^{1} x^{2} f^{\prime \prime}(x) d x=3$, halle $\int_{0}^{1} f_{(x)} d x$ si en $(1,1) \in f_{(x)}$ ocurre un máximo relativo.

\section{Solución:}

Encontremos:

$$
\begin{gathered}
I_{1}=\int x^{2} f^{\prime \prime}{ }_{(x)} d x \\
u=x^{2} \quad \quad v=f^{\prime}(x) \\
\downarrow \quad \uparrow^{\uparrow} \\
d u=2 x d x \quad d v=f^{\prime \prime}{ }_{(x)} d x \\
\therefore \quad l_{1}=x^{2} f^{\prime}(x)-2 \underbrace{\int x f^{\prime}(x)}_{I_{2}} d x
\end{gathered}
$$

Calculemos:

$$
\begin{aligned}
& I_{2}=\int x f^{\prime}(x) d x \\
& u=x \quad v=f_{(x)} \\
& \downarrow \quad \uparrow \\
& d u=d x \quad d v=f^{\prime}(x) d x \\
& \therefore \quad I_{2}=x f_{(x)}-\int f_{(x)} d x
\end{aligned}
$$


Entonces:

$$
\begin{gathered}
\int_{0}^{1} x^{2} f^{\prime \prime}{ }_{(x)} d x=\left.x^{2} f^{\prime}(x)\right|_{0} ^{1}-\left.2 x f_{(x)}\right|_{0} ^{1}+2 \int_{0}^{1} f_{(x)} d x \\
3=\left(f^{\prime}{ }_{(1)}-0\right)-2\left(f_{(1)}-0\right)+2 \int_{0}^{1} f_{(x)} d x
\end{gathered}
$$

Como $(1,1)$ es máximo relativo de $f_{(x)}: f_{(1)}=1 \wedge f_{(1)}^{\prime}=0$.

En (1):

$$
3=(0-0)-2(1-0)+2 \int_{0}^{1} f_{(x)} d x
$$

Con lo cual:

$$
\int_{0}^{1} f_{(x)} d x=\frac{5}{2}
$$

234. Encontrar el valor de la siguiente integral definida $\int x^{r} \ln x d x$ comprendida en el intervalo $\ln x \in[0,1]$, con $r^{2}=1$

\section{Solución:}

Si $r=1 \rightarrow I_{1}=\int x^{1} \ln x \cdot d x$

$$
\begin{array}{cc}
u=\ln x & v=\frac{x^{2}}{2} \\
\downarrow & \uparrow \\
d u=\frac{d x}{x} & d v=x d x
\end{array}
$$

Entonces:

$$
I_{1}=\frac{x^{2}}{2} \ln x-\int \frac{x}{2} d x=\frac{x^{2}}{2} \ln x-\frac{x^{2}}{4}+C_{1}
$$

Los límites de integración son: $\ln x=1 \rightarrow x=e$

$$
\ln x=0 \rightarrow x=1
$$

Con lo cual:

$$
\int_{1}^{e} x \ln x d x=\left(\frac{e^{2}}{2}-\frac{e^{2}}{4}\right)-\left(0-\frac{1}{4}\right)=2.097
$$


Si: $r=-1 \rightarrow I_{2}=\int x^{-1} \ln x \cdot d x$

$$
\begin{array}{cc}
u=\ln x & v=\ln x \\
\downarrow & \uparrow \\
d u=\frac{d x}{x} & d v=\frac{d x}{x}
\end{array}
$$

Entonces:

Finalmente:

$$
\begin{gathered}
I_{2}=(\ln x)^{2}-\int x^{-1} \ln x d x \\
I_{2}=(\ln x)^{2}-I_{2} \rightarrow I_{2}=\frac{(\ln x)^{2}}{2}+C_{2}
\end{gathered}
$$

$$
\int_{1}^{e} x^{-1} \ln x d x=\left.\frac{1}{2}(\ln x)^{2}\right|_{1} ^{e}=\frac{1}{2}-0=0.5
$$

235. Determinar: $\int_{0}^{1} \frac{d x}{\left(2^{x}+3\right)}$.

\section{Solución:}

Multipliquemos y dividamos por $2^{-x}$ :

$$
\int \frac{d x}{2^{x}+3}=\int \frac{2^{-x} d x}{1+3\left(2^{-x}\right)}
$$

Si: $u=1+3\left(2^{-x}\right)$

$$
d u=3\left(2^{-x}\right)(\ln 2)(-1) d x \rightarrow 2^{-x} d x=\frac{d u}{-3 \ln 2}
$$

Entonces:

$$
\begin{aligned}
& \int \frac{d x}{2^{x}+3}=\int \frac{\frac{d u}{-3 \ln 2}}{u}=\frac{-1}{3 \ln 2} \int \frac{d u}{u}=\frac{-1}{\ln 8} \ln u+C \\
& \int_{0}^{1} \frac{d x}{2^{x}+3}=\left.\frac{-1}{\ln 8} \ln \left(1+3\left(2^{-x}\right)\right)\right|_{0} ^{1}=\frac{-1}{\ln 8} \ln 5 / 8=\frac{\ln 8 / 5}{\ln 8}
\end{aligned}
$$

236. Hallar $A, B$ y $C$ si: $\quad \int_{1}^{5} \frac{x-1}{x^{2}(x+1)} d x=A \ln B+C$

\section{Solución:}

Se descompone el integrando en fracciones parciales: 


$$
\frac{x-1}{x^{2}(x+1)}=\frac{2}{x}-\frac{1}{x^{2}}-\frac{2}{x+1}
$$

Entonces:

$$
\begin{gathered}
\int_{1}^{5} \frac{x-1}{x^{2}(x+1)} d x=2 \int_{1}^{5} \frac{d x}{x}-\int_{1}^{5} \frac{d x}{x^{2}}-2 \int_{1}^{5} \frac{d x}{x+1} \\
\int_{1}^{5} \frac{x-1}{x^{2}(x+1)} d x=2 \ln x+\frac{1}{x}-\left.2 \ln (x+1)\right|_{1} ^{5} \\
\int_{1}^{5} \frac{x-1}{x^{2}(x+1)} d x=2 \ln \frac{5}{1}+\left(\frac{1}{5}-1\right)-2 \ln \frac{6}{2}
\end{gathered}
$$

La respuesta es:

$$
2 \ln \frac{5}{3}-\frac{4}{5} \Rightarrow\left\{\begin{array}{l}
A=2 \\
B=5 / 3 \\
C=-4 / 5
\end{array}\right.
$$

237. Evaluar la integral: $\int_{0}^{1} \frac{x^{2}}{\left(4-x^{2}\right)^{3 / 2}} d x$.

Solución:

Por partes:

$$
\begin{array}{cc}
u=x & v=\frac{1}{-2} \frac{\left(4-x^{2}\right)^{-1 / 2}}{-1 / 2} \\
\downarrow & \uparrow \\
d u=d x & d v=\frac{x d x}{\left(4-x^{2}\right)^{3 / 2}}
\end{array}
$$

Entonces:

$$
\begin{aligned}
& \int_{0}^{1} \frac{x^{2} d x}{\left(4-x^{2}\right)^{3 / 2}}=\left.\frac{x}{\sqrt{4-x^{2}}}\right|_{0} ^{1}-\int_{0}^{1} \frac{d x}{\sqrt{4-x^{2}}} \\
& \int_{0}^{1} \frac{x^{2} d x}{\left(4-x^{2}\right)^{3 / 2}}=\left.\left(\frac{x}{\sqrt{4-x^{2}}}-\operatorname{arcsen} \frac{x}{2}\right)\right|_{0} ^{1}
\end{aligned}
$$




$$
\int_{0}^{1} \frac{x^{2} d x}{\left(4-x^{2}\right)^{3 / 2}}=\left(\frac{1}{\sqrt{3}}-\operatorname{arcsen} \frac{1}{2}\right)-(0-0)=\frac{\sqrt{3}}{3}-\frac{\pi}{6}
$$

238. Evaluar: $I=\int_{0}^{3 / 4} \frac{b}{\sqrt{1-y}} d y$.

\section{Solución:}

Sea $u^{2}=1-y \Rightarrow 2 u d u=-d y$

Si: $y=3 / 4 \quad \rightarrow u^{2}=1 / 4 \quad \rightarrow u=1 / 2$

$$
y=0 \quad \rightarrow u^{2}=1 \quad \rightarrow u=1
$$

Entonces:

$$
\begin{aligned}
& I=\int_{1}^{1 / 2} \frac{b}{u}(-2 u d u)=-2 \int_{1}^{1 / 2} b d u \\
& I=-\left.2 b u\right|_{1} ^{1 / 2}=-2 b(1 / 2-1)=b
\end{aligned}
$$

239. Calcular: $\int_{2}^{2.6} x 7^{x^{2}} d x$.

Solución:

Escribamos:

$$
\int_{2}^{2.6} 7^{x^{2}} x d x=\frac{1}{2} \int_{2}^{2.6} 7^{x^{2}}(2 x d x)
$$

Si:

$$
\begin{gathered}
u=x^{2} \rightarrow d u=2 x d x<\begin{array}{l}
x=2.6 \rightarrow u=6.76 \\
x=2 \quad \rightarrow u=4
\end{array} \\
\int_{2}^{2.6} 7^{x^{2}} x d x=\frac{1}{2} \int_{4}^{6.76} 7^{u} d u=\left.\frac{1}{2 \ln 7} 7^{u}\right|_{4} ^{6.76} \\
\int_{2}^{2.6} 7^{x^{2}} x d x=\frac{1}{2 \ln 7}\left(7^{6.76}-7^{4}\right)=132,034
\end{gathered}
$$

240. Calcular: $\int_{0}^{1 / 2} \frac{4 x^{2}-2 x-2}{\left(x^{2}-x+1\right)(x-1)} d x$. 


\section{Solución:}

Factoricemos y simplifiquemos:

$$
\frac{4 x^{2}-2 x-2}{\left(x^{2}-x+1\right)(x-1)}=\frac{(4 x+2)(x-1)}{\left(x^{2}-x+1\right)(x-1)}=\frac{4 x+2}{x^{2}-x+1}
$$

Entonces:

$$
\begin{gathered}
\int_{0}^{1 / 2} \frac{4 x^{2}-2 x-2}{\left(x^{2}-x+1\right)(x-1)} d x=2 \int_{0}^{1 / 2} \frac{2 x-1}{x^{2}-x+1} d x+2 \int_{0}^{1 / 2} \frac{2 d x}{(x-1 / 2)^{2}+(\sqrt{3} / 2)^{2}} \\
\int_{0}^{1 / 2} \frac{4 x^{2}-2 x-2}{\left(x^{2}-x+1\right)(x-1)} d x=2 \ln \left(x^{2}-x+1\right)+\left.\frac{4}{\sqrt{3} / 2} \arctan \frac{x-1 / 2}{\sqrt{3} / 2}\right|_{0} ^{1 / 2} \\
\int_{0}^{1 / 2} \frac{4 x^{2}-2 x-2}{\left(x^{2}-x+1\right)(x-1)} d x=2 \ln 3 / 4+\frac{8}{\sqrt{3}}(0)-2 \ln 1-\frac{8}{\sqrt{3}}\left(\frac{-\pi}{6}\right)
\end{gathered}
$$

La respuesta es:

$$
2 \ln 3 / 4+\frac{4 \sqrt{3}}{9} \pi
$$

241. Determine el valor de $b$, de manera que $\int_{0}^{b}(3 x+b)(x-b) d x=8$.

\section{Solución:}

$$
\int_{0}^{b}(3 x+b)(x-b) d x=\int_{0}^{b}\left(3 x^{2}-2 b x-b^{2}\right) d x=x^{3}-b x^{2}-\left.b^{2} x\right|_{0} ^{b}
$$

Entonces:

$$
\begin{gathered}
b^{3}-b\left(b^{2}\right)-b^{2}(b)-0=8 \\
-b^{3}=8 \Rightarrow b=-2
\end{gathered}
$$

242. Halle el valor de $I=\int_{0}^{\sqrt{2}} 2 x^{3} e^{x^{2}} d x$.

\section{Solución:}

Escribámoslo así:

$$
\int 2 x^{3} e^{x^{2}} d x=\int x^{2} e^{x^{2}}(2 x d x)
$$




$$
\begin{aligned}
& u=x^{2} \rightarrow d u=2 x d x \\
& \int 2 x^{3} e^{x^{2}} d x=\int u e^{u} d u
\end{aligned}
$$

Integrando por partes:

$$
\int u e^{u} d u=u e^{u}-e^{u}+C
$$

Entonces:

$$
\begin{gathered}
I=\int_{0}^{2} u e^{u} d u=u e^{u}-\left.e^{u}\right|_{0} ^{2} \\
I=\left(2 e^{2}-e^{2}\right)-(0-1)=e^{2}+1
\end{gathered}
$$

243. Sea $f_{(n)}=\int_{0}^{\pi / 4} \tan ^{n} x d x \quad(n \geq 1)$.

Demostrar que $f_{(n)}+f_{(n-2)}=\frac{1}{n-1} \quad(n>2)$.

Solución:

Escribámoslo así:

$$
\begin{gathered}
\int_{0}^{\pi / 4} \tan ^{n} x d x=\int_{0}^{\pi / 4} \tan ^{n-2} x \cdot \tan ^{2} x \cdot d x=\int_{0}^{\pi / 4} \tan ^{n-2} x \cdot\left(\sec ^{2} x-1\right) \cdot d x \\
\int_{0}^{\pi / 4} \tan ^{n} x d x=\int_{0}^{\pi / 4} \tan ^{n-2} x \cdot\left(\sec ^{2} x \cdot d x\right)-\int_{0}^{\pi / 4} \tan ^{n-2} x d x
\end{gathered}
$$

De donde:

$$
\begin{gathered}
\int_{0}^{\pi / 4} \tan ^{n} x d x+\int_{0}^{\pi / 4} \tan ^{n-2} x d x=\int_{0}^{\pi / 4} \tan ^{n-2} x \cdot\left(\sec ^{2} x \cdot d x\right) \\
f_{(n)}+f_{(n-2)}=\left.\frac{(\tan x)^{n-1}}{n-1}\right|_{0} ^{\pi / 4} \\
f_{(n)}+f_{(n-2)}=\frac{1^{n-1}-0^{n-1}}{n-1}=\frac{1}{n-1}
\end{gathered}
$$

244. Evaluar: $\int_{2}^{5} \frac{1}{x \sqrt{1+x^{2}}} d x$. 


\section{Solución:}

$$
I=\int \frac{d x}{x \sqrt{1+x^{2}}}
$$

Sea: $x=\frac{1}{z} \Rightarrow d x=\frac{-1}{z^{2}} d z$

$$
\begin{gathered}
I=\int \frac{-d z / z^{2}}{\frac{1}{z} \sqrt{1+\frac{1}{z^{2}}}}=-\int \frac{d z}{\sqrt{z^{2}+1}} \\
I=-\ln \left(z+\sqrt{z^{2}+1}\right)+C
\end{gathered}
$$

Entonces:

$$
\int_{2}^{5} \frac{1}{x \sqrt{1+x^{2}}} d x=-\int_{1 / 2}^{1 / 5} \frac{d z}{\sqrt{z^{2}+1}}=-\left.\ln \left(z+\sqrt{z^{2}+1}\right)\right|_{1 / 2} ^{1 / 5}
$$

De donde:

$$
-\ln (1 / 5+\sqrt{26 / 25})+\ln (1 / 2+\sqrt{5 / 4})
$$$$
-\ln 1.2198+\ln 1.618=0.2825
$$

245. Calcular: $\int_{x=2.25}^{1+\sqrt{2}} \frac{d x}{(x-1)^{2} \sqrt{x^{2}-2 x}}$

\section{Solución:}

Escribamos:

$$
\int \frac{d x}{(x-1)^{2} \sqrt{x^{2}-2 x}}=\int \frac{d x}{(x-1)^{2} \sqrt{(x-1)^{2}-1}}
$$

Sea $u^{2}=(x-1)^{2}-1 \rightarrow 2 u d u=2(x-1) d x$

Entonces:

$$
\begin{gathered}
\int \frac{d x}{(x-1)^{2} \sqrt{x^{2}-2 x}}=\int \frac{\frac{u d u}{\sqrt{u^{2}+1}}}{\left(u^{2}+1\right) \sqrt{u^{2}}}=\int \frac{d u}{\left(u^{2}+1\right) \sqrt{u^{2}+1}} \\
\int \frac{d x}{(x-1)^{2} \sqrt{x^{2}-2 x}}=\int \frac{d u}{\left(u^{2}+1\right)^{3 / 2}}
\end{gathered}
$$


Hagamos que: $u=\tan \theta \rightarrow d u=\sec ^{2} \theta d \theta$

De donde:

$$
\begin{gathered}
\int \frac{d x}{(x-1)^{2} \sqrt{x^{2}-2 x}}=\int \frac{\sec ^{2} \theta d \theta}{\left(\sec ^{2} \theta\right)^{3 / 2}}=\int \frac{1}{\sec \theta} d \theta=\int \cos \theta d \theta \\
\int \frac{d x}{(x-1)^{2} \sqrt{x^{2}-2 x}}=\operatorname{sen} \theta+C
\end{gathered}
$$

Si:

$$
\begin{gathered}
x=1+\sqrt{2} \rightarrow u^{2}=(\sqrt{2})^{2}-1=1 \rightarrow u=1 \\
\tan \theta=1 \rightarrow \theta=\pi / 4
\end{gathered}
$$

Si:

$$
\begin{gathered}
x=2.25 \rightarrow u^{2}=1.25^{2}-1=0.5625 \rightarrow u=0.75 \\
\tan \theta=0.75 \rightarrow \theta=0.6435
\end{gathered}
$$

De donde:

$$
\int_{2.25}^{1+\sqrt{2}} \frac{d x}{(x-1)^{2} \sqrt{x^{2}-2 x}}=\left.\operatorname{sen} \theta\right|_{0.6435} ^{\pi / 4}=\operatorname{sen} \frac{\pi}{4}-\operatorname{sen} 0.6435
$$

La respuesta es:

$$
\frac{\sqrt{2}}{2}-0.6=0.1071
$$

246. Calcular la integral: $\int_{1.25}^{2} \frac{x^{3}}{\sqrt{x-1}} d x$.

\section{Solución:}

Sea $x-1=u^{2} \rightarrow d x=2 u d u$

Si: $x=2 \rightarrow u^{2}=1 \quad \rightarrow \quad u=1$

$$
x=1.25 \rightarrow u^{2}=0.25 \rightarrow u=0.5
$$

Entonces:

$$
\int_{1.25}^{2} \frac{x^{3}}{\sqrt{x-1}} d x=\int_{0.5}^{1} \frac{\left(u^{2}+1\right)^{3}}{u}(2 u d u)=2 \int_{0.5}^{1}\left(u^{2}+1\right)^{3} d u
$$




$$
\begin{gathered}
\int_{1.25}^{2} \frac{x^{3}}{\sqrt{x-1}} d x=2 \int_{0.5}^{1}\left(u^{6}+3 u^{4}+3 u^{2}+1\right) d u \\
\int_{1.25}^{2} \frac{x^{3}}{\sqrt{x-1}} d x=\left.2\left[\frac{u^{7}}{7}+\frac{3 u^{5}}{5}+u^{3}+u\right]\right|_{0.5} ^{1} \\
\int_{1.25}^{2} \frac{x^{3}}{\sqrt{x-1}} d x=2\left[\frac{1}{7}+\frac{3}{5}+1+1\right]-2\left[\frac{1}{896}+\frac{3}{160}+\frac{1}{8}+\frac{1}{2}\right]=4.19598
\end{gathered}
$$

247. Calcular: $\int_{1}^{8} \frac{\ln (2+\sqrt[3]{x})}{\sqrt[3]{x}} d x$

\section{Solución:}

Sea $u=\sqrt[3]{x} \rightarrow x=u^{3} \rightarrow d x=3 u^{2} d u$

Si: $x=8 \rightarrow u=2$

$$
x=1 \rightarrow u=1
$$

Entonces:

$$
\int_{1}^{8} \frac{\ln (2+\sqrt[3]{x})}{\sqrt[3]{x}} d x=\int_{1}^{2} \frac{\ln (2+u)}{u}\left(3 u^{2} d u\right)=3 \int_{1}^{2} u \ln (2+u) d u
$$

Por partes:

$$
\begin{gathered}
\int u \ln (2+u) d u \\
v=\ln (u+2) \quad w=\frac{u^{2}}{2} \\
\downarrow \quad \uparrow \quad d w=u d u \\
d v=\frac{d u}{u+2} \quad d w \ln (2+u) d u=\frac{u^{2}}{2} \ln (u+2)-\frac{1}{2} \int \frac{u^{2}}{u+2} d u \\
\int u \ln (2+u) d u=\frac{u^{2}}{2} \ln (u+2)-\frac{1}{2} \int\left[u-2+\frac{4}{u+2}\right] d u \\
\int u \ln (2+u) d u=\frac{u^{2}}{2} \ln (u+2)-\frac{1}{2}\left[\frac{u^{2}}{2}-2 u+4 \ln (u+2)\right]+C \\
\int_{1}^{2} u \ln (2+u) d u=[2 \ln 4-1+2-2 \ln 4]-\left[\frac{1}{2} \ln 3-\frac{1}{4}+1-2 \ln 3\right]=\frac{1}{4}+\frac{3}{2} \ln 3
\end{gathered}
$$


De donde:

$$
\int_{1}^{8} \frac{\ln (2+\sqrt[3]{x})}{\sqrt[3]{x}} d x=3 \int_{1}^{2} u \ln (2+u) d u=3\left[\frac{1}{4}+\frac{3}{2} \ln 3\right]=\frac{3}{4}+\frac{9}{2} \ln 3
$$

248. Calcular: $\int_{-1 / 2}^{1 / 2}\left(\frac{1+x}{1-x}\right)^{1 / 2} d x$.

\section{Solución:}

Sea: $\frac{1+x}{1-x}=u^{2} \Rightarrow 1+x=u^{2}-u^{2} x \Rightarrow x=\frac{u^{2}-1}{u^{2}+1}$

$$
d x=\frac{2 u\left(u^{2}+1\right)-2 u\left(u^{2}-1\right)}{\left(u^{2}+1\right)^{2}} d u=\frac{4 u}{\left(u^{2}+1\right)^{2}} d u
$$

Si: $\quad x=1 / 2 \rightarrow u^{2}=\frac{1.5}{0.5}=3 \rightarrow u=\sqrt{3}$

$$
x=-1 / 2 \rightarrow u^{2}=\frac{0.5}{1.5}=\frac{1}{3} \rightarrow u=\frac{\sqrt{3}}{3}
$$

Entonces:

$$
\int_{-1 / 2}^{1 / 2}\left(\frac{1+x}{1-x}\right)^{1 / 2} d x=\int_{\sqrt{3} / 3}^{\sqrt{3}}\left(u^{2}\right)^{1 / 2}\left(\frac{4 u d u}{\left(u^{2}+1\right)^{2}}\right)=4 \int_{\sqrt{3} / 3}^{\sqrt{3}} \frac{u^{2}}{\left(u^{2}+1\right)^{2}} d u
$$

Calculemos:

$$
\begin{gathered}
\int_{\sqrt{3} / 3}^{\sqrt{3}} \frac{u^{2}}{\left(u^{2}+1\right)^{2}} d u \\
u=\tan \theta \rightarrow d u=\sec ^{2} \theta d \theta
\end{gathered}
$$

Si: $u=\sqrt{3} \Rightarrow \tan \theta=\sqrt{3} \Rightarrow \theta=\frac{\pi}{3}$

$$
u=\frac{\sqrt{3}}{3} \Rightarrow \tan \theta=\frac{\sqrt{3}}{3} \Rightarrow \theta=\frac{\pi}{6}
$$

Entonces:

$$
\begin{aligned}
& \int_{\sqrt{3} / 3}^{\sqrt{3}} \frac{u^{2}}{\left(u^{2}+1\right)^{2}} d u=\int_{\pi / 6}^{\pi / 3} \frac{\tan ^{2} \theta \sec ^{2} \theta d \theta}{\left(\sec ^{2} \theta\right)^{2}}=\int_{\pi / 6}^{\pi / 3} \frac{\tan ^{2} \theta}{\sec ^{2} \theta} d \theta \\
& \int_{\sqrt{3} / 3}^{\sqrt{3}} \frac{u^{2}}{\left(u^{2}+1\right)^{2}} d u=\int_{\pi / 6}^{\pi / 3} \operatorname{sen}^{2} \theta d \theta=\int_{\pi / 6}^{\pi / 3}\left[\frac{1-\cos 2 \theta}{2}\right] d \theta
\end{aligned}
$$




$$
\int_{\sqrt{3} / 3}^{\sqrt{3}} \frac{u^{2}}{\left(u^{2}+1\right)^{2}} d u=\frac{\theta}{2}-\left.\frac{1}{4} \operatorname{sen} 2 \theta\right|_{\pi / 6} ^{\pi / 3}=\left(\frac{\pi}{6}-\frac{1}{4} \frac{\sqrt{3}}{2}\right)-\left(\frac{\pi}{12}-\frac{1}{4} \frac{\sqrt{3}}{2}\right)=\frac{\pi}{12}
$$

Finalmente, en (1):

$$
\int_{-1 / 2}^{1 / 2}\left(\frac{1+x}{1-x}\right)^{1 / 2} d x=4\left(\frac{\pi}{12}\right)=\frac{\pi}{3}=1.047198
$$

249. Indicar: $\int_{0}^{1} \frac{x}{\sqrt{1+x^{4}}} d x$

\section{Solución:}

Escribamos:

$$
\begin{gathered}
\int_{0}^{1} \frac{x}{\sqrt{1+x^{4}}} d x=\int_{0}^{1} \frac{x d x}{\sqrt{1+\left(x^{2}\right)^{2}}}=\frac{1}{2} \int_{0}^{1}\left(1+\left(x^{2}\right)^{2}\right)^{-1 / 2}(2 x d x) \\
\int_{0}^{1} \frac{x}{\sqrt{1+x^{4}}} d x=\left.\frac{1}{2} \ln \left[x^{2}+\sqrt{1+x^{4}}\right]\right|_{0} ^{1}
\end{gathered}
$$

La respuesta es:

$$
\frac{1}{2}[\ln (1+\sqrt{2})-\ln (0+\sqrt{1})]=\frac{1}{2} \ln (1+\sqrt{2})
$$

250. $S i \int_{0}^{\frac{\pi}{2}}\left(f^{\prime}(x)+f^{\prime \prime \prime}(x)\right) \cos x d x=8$ y $f^{\prime \prime}(0)=7$, halle $f^{\prime}\left(\frac{\pi}{2}\right)$.

\section{Solución:}

Se tiene que:

$$
\begin{array}{cccc} 
& \int_{0}^{\pi / 2} f^{\prime}(x) \\
u=f^{\prime}(x) & v=\cos x d x+\int_{0}^{\pi / 2} f^{\prime \prime \prime}(x) & \cos x d x=8 \\
\downarrow & \uparrow & u=\cos x & v=f^{\prime \prime}(x) \\
d u=f^{\prime \prime}(x) & \downarrow & \downarrow & \uparrow \\
& d v=\cos x d x & d u=-\operatorname{sen} x d x & d v=f^{\prime \prime \prime}(x)
\end{array}
$$


Entonces:

$$
\begin{gathered}
\left.f^{\prime}(x) \operatorname{sen} x\right|_{0} ^{\pi / 2}-\int_{0}^{\pi / 2} f^{\prime \prime}(x) \operatorname{sen} x d x+\left.f^{\prime \prime}{ }_{(x)} \cos x\right|_{0} ^{\pi / 2}+\int_{0}^{\pi / 2} f^{\prime \prime}(x) \operatorname{sen} x d x=8 \\
{\left[f^{\prime}{ }_{(\pi / 2)}(1)-f^{\prime}(0)(0)\right]+\left[f^{\prime \prime}{ }_{(\pi / 2)}(0)-f^{\prime \prime}{ }_{(0)}(1)\right]=8} \\
f^{\prime}{ }_{(\pi / 2)}-7=8 \Rightarrow f^{\prime}{ }_{(\pi / 2)}=15
\end{gathered}
$$

251. Si $\frac{4 b}{a} \int_{0}^{a} \sqrt{a^{2}-x^{2}} d x=F_{(a, b)}$, halle $F_{(8,2)}$

\section{Solución:}

Se tiene que:

$$
\begin{gathered}
\frac{4 b}{a} \int_{0}^{a} \sqrt{a^{2}-x^{2}} d x=\left.\frac{4 b}{a}\left[\frac{1}{2} x \sqrt{a^{2}-x^{2}}+\frac{1}{2} a^{2} \operatorname{arcsen} \frac{x}{a}\right]\right|_{0} ^{a} \\
\frac{4 b}{a} \int_{0}^{a} \sqrt{a^{2}-x^{2}} d x=F_{(a, b)}=\frac{4 b}{a}\left[0+\frac{a^{2}}{2} \operatorname{arcsen} 1-0-\frac{a^{2}}{2} \operatorname{arcsen} 0\right] \\
\frac{4 b}{a} \int_{0}^{a} \sqrt{a^{2}-x^{2}} d x=F_{(a, b)}=\frac{4 b}{a}\left[\frac{a^{2}}{2} \cdot \frac{\pi}{2}-0\right]=\pi a b
\end{gathered}
$$

Si $F_{(a, b)}=\pi a b \rightarrow F_{(8,2)}=\pi(8)(2)=16 \pi$

252. Calcule $\int_{a}^{b}\left[f_{(x)} g^{\prime \prime}(x)-g_{(x)} f^{\prime \prime}(x)\right] d x$ si $f_{(x)}^{\prime}=-f_{(x)}, g^{\prime}(x)=2 g_{(x)}, f_{(a)} g_{(a)}=3, f_{(b)} g_{(b)}=4$

Solución:

Se pide:

$$
\begin{array}{cc|cc} 
& \int_{a}^{b} f_{(x)} g^{\prime \prime}{ }_{(x)} d x-\int_{a}^{b} g_{(x)} f^{\prime \prime}(x) \\
u=f_{(x)} & v=g^{\prime}(x) & u=g_{(x)} & v=f^{\prime}(x) \\
\downarrow & \uparrow & \downarrow & \uparrow \\
d u=f_{(x)}^{\prime} d x & d v=g^{\prime \prime}(x) & d u=g_{(x)}^{\prime} d x & d v=f^{\prime \prime}{ }_{(x)} d x
\end{array}
$$

Entonces, se pide:

$$
\left.f_{(x)} g^{\prime}(x)\right|_{a} ^{b}-\int_{a}^{b} f^{\prime}{ }_{(x)} g^{\prime}(x) d x-\left.f^{\prime}(x) g_{(x)}\right|_{a} ^{b}+\int_{a}^{b} f^{\prime}{ }_{(x)} g^{\prime}{ }_{(x)} d x
$$


Es decir:

$$
\left.f_{(x)} g^{\prime}(x)\right|_{a} ^{b}-\left.f^{\prime}(x) g_{(x)}\right|_{a} ^{b}=f_{(b)} g^{\prime_{(b)}}-f_{(a)} g^{\prime_{(a)}}-f^{\prime}{ }_{(b)} g_{(b)}+f^{\prime}{ }_{(a)} g_{(a)}
$$

La respuesta es:

$$
\begin{gathered}
f_{(b)}\left[2 g_{(b)}\right]-f_{(a)}\left[2 g_{(a)}\right]-\left(-f_{(b)}\right) g_{(b)}+\left(-f_{(a)}\right) g_{(a)} \\
3 f_{(b)} g_{(b)}-3 f_{(a)} g_{(a)}=3(4)-3(3)=3
\end{gathered}
$$

253. Calcular: $\int_{0}^{1} \frac{\operatorname{arccot} e^{x}}{e^{x}} d x$.

Solución:

Por partes:

$$
\begin{array}{cc}
u=\underset{\downarrow}{\operatorname{arccot} e^{x}} & v=-e^{-x} \\
\downarrow & \uparrow \\
d u=\frac{-1}{1+\left(e^{x}\right)^{2}} e^{x} d x & d v=\frac{d x}{e^{x}}
\end{array}
$$

Entonces:

$$
\begin{gathered}
\int \frac{\operatorname{arccot} e^{x}}{e^{x}} d x=-\frac{\operatorname{arccot} e^{x}}{e^{x}}-\int \frac{d x}{1+\left(e^{x}\right)^{2}} \\
\int \frac{\operatorname{arccot} e^{x}}{e^{x}} d x=-\frac{\operatorname{arccot} e^{x}}{e^{x}}+\int \frac{-e^{-2 x} d x}{e^{-2 x}+1} \\
\int \frac{\operatorname{arccot} e^{x}}{e^{x}} d x=-\frac{\operatorname{arccot} e^{x}}{e^{x}}+\frac{1}{2} \ln \left(e^{-2 x}+1\right)+C
\end{gathered}
$$

De donde:

$$
\begin{gathered}
\int_{0}^{1} \frac{\operatorname{arccot} e^{x}}{e^{x}} d x=\left[-\frac{\operatorname{arccot} e}{e}+\frac{1}{2} \ln \left(e^{-2}+1\right)\right]-\left[-\frac{\operatorname{arccot} 1}{1}+\frac{1}{2} \ln 2\right] \\
\int_{0}^{1} \frac{\operatorname{arccot} e^{x}}{e^{x}} d x=[-0.1297+0.0635]-[-0.7854+0.3466]=0.3726
\end{gathered}
$$


254. Evaluar: $\int_{0}^{2} \frac{x^{6}+6 x^{4}+x^{3}+8 x^{2}+x+5}{x^{3}+5 x+1} d x$.

\section{Solución:}

Se tiene que:

$$
\begin{gathered}
N=x^{6}+6 x^{4}+x^{3}+8 x^{2}+x+5=x^{3}\left(x^{3}+5 x+1\right)+x^{4}+8 x^{2}+x+5 \\
N=x^{3}\left(x^{3}+5 x+1\right)+x\left(x^{3}+5 x+1\right)+3 x^{2}+5
\end{gathered}
$$

Entonces:

$$
\frac{N}{x^{3}+5 x+1}=x^{3}+x+\frac{3 x^{2}+5}{x^{3}+5 x+1}
$$

De donde:

$$
\begin{aligned}
& \int_{0}^{2} \frac{N}{x^{3}+5 x+1} d x=\int_{0}^{2}\left[x^{3}+x+\frac{3 x^{2}+5}{x^{3}+5 x+1}\right] d x \\
& \int_{0}^{2} \frac{N}{x^{3}+5 x+1} d x=\frac{x^{4}}{4}+\frac{x^{2}}{2}+\left.\ln \left(x^{3}+5 x+1\right)\right|_{0} ^{2}
\end{aligned}
$$

La respuesta es:

$$
\frac{2^{4}}{4}+\frac{2^{2}}{2}+\ln (8+10+1)-0-0-\ln 1=4+2+\ln 19=8.9444
$$

255. Calcular a si se cumple que $\int_{a}^{0} \frac{d x}{x^{2}+2 x+2}=\frac{\pi}{3}$.

\section{Solución:}

Escribamos:

$$
\int \frac{d x}{x^{2}+2 x+2}=\int \frac{d x}{(x+1)^{2}+1^{2}}=\arctan (x+1)+C
$$

Entonces:

$$
\int_{a}^{0} \frac{d x}{x^{2}+2 x+2}=\left.\arctan (x+1)\right|_{a} ^{0}=\arctan 1-\arctan (a+1)
$$

De donde:

$$
\begin{gathered}
\arctan 1-\arctan (a+1)=\pi / 3 \\
\frac{\pi}{4}-\arctan (a+1)=\frac{\pi}{3}
\end{gathered}
$$




$$
\begin{gathered}
\arctan (a+1)=\frac{-\pi}{12}=-15^{\circ} \\
a+1=\tan \left(-15^{\circ}\right)=-0.2679 \\
\therefore \quad a=-1.2679
\end{gathered}
$$

256. Resolver la siguiente integral definida: $\int_{-4}^{4}\left|x^{2}+x-6\right| d x$.

$$
\text { R.: } \frac{109}{3}
$$

257. Determinar: $\int_{a+b}^{a+2 b}\left[a+\frac{b}{(x-a)^{2}}\right]^{2} d x$.

$$
\text { R.: } a^{2} b+a+\frac{7}{24 b}
$$

258. Evaluar: $\int_{0}^{1} x \ln (x+3) d x$.

$$
\text { R.: } \frac{5}{4}-4 \ln 4+\frac{9}{2} \ln 3
$$

259. Calcular: $\int_{2}^{2.4} x 7^{x^{2}} d x$.
R.: 18,333

260. Determinar: $\int_{1}^{\sqrt{2}} \frac{\ln x}{x^{3}} d x$.

$$
\text { R.: } \frac{1}{8}-\frac{\ln \sqrt{2}}{4}
$$

261. Encontrar: $\int_{5}^{9} \frac{x^{2}-5 x+9}{x^{2}-5 x+6} d x$.

$$
\text { R.: } 4+3 \ln \frac{9}{7}
$$

262. Integrar: $\frac{d x}{x^{3}+x}$ en $[3,7]$.

$$
\text { R.: } \ln \frac{7 \sqrt{5}}{15}
$$


263. Evaluar: $\int_{0}^{1 / 2} \frac{b}{\sqrt{1-y}} d y$.

$$
\text { R.: }(2-\sqrt{2}) b
$$

264. Integrar: $\int_{0}^{2} \frac{x}{\sqrt{1+x^{4}}} d x$.

$$
\text { R.: } \frac{1}{2} \ln (4+\sqrt{17})
$$

265. Determinar: $\int_{1}^{\sqrt{3}} \frac{\ln x}{x^{3}} d x$.

$$
\text { R.: } \frac{1}{6}-\frac{\ln \sqrt{3}}{6}
$$

266. Calcular: $\int_{0}^{\pi / 3} \tan ^{2} x \cdot \operatorname{sen} x \cdot d x$.

$$
\text { R.: } \frac{1}{2}
$$

267. Calcular: $\int_{0}^{r} \sqrt{r^{2}-x^{2}} d x$.

$$
\text { R.: } \frac{\pi r^{2}}{4}
$$

268. Calcular: $\int_{0}^{16} \sqrt{4-\sqrt{x}} d x$.
R.: $\frac{256}{15}$

269. Calcular: $\int_{e}^{e^{2}} \frac{\ln (\ln x)}{x \ln x} d x$.

$$
\text { R.: } \frac{(\ln 2)^{2}}{2}
$$

270. Calcular: $\int_{0}^{4} \frac{d x}{\left(x^{2}+9\right)^{3 / 2}}$.

$$
\text { R.: } \frac{4}{45}
$$


271. Calcular: $\int_{0}^{1} x \arctan x d x$.

R.: $\frac{\pi-2}{4}$

272. Calcular: $\int_{-1}^{-1 / 2} \frac{d x}{4 x^{2}+8 x+5}$.

R.: $\frac{\pi}{8}$

273. Calcular: $\int_{0}^{\sqrt[3]{6}} \frac{x^{5}}{\sqrt{2 x^{3}+4}} d x$.

R.: $\frac{16}{9}$

274. Calcular: $I=4 \int_{0}^{\pi / 4} \operatorname{sen}^{3} x \cdot \sec x \cdot d x$.

R.: $2 \ln 2-1$

284 275. Calcular: $\int_{0}^{\pi / 3} \sec ^{2} x \cdot \tan ^{3} x \cdot d x$
R.: $\frac{9}{4}$

276. Calcular: $\int_{3}^{4} \frac{x^{2}-2 x+3}{x^{3}-2 x^{2}-x+2} d x$.
R.: $\ln \left(\frac{5}{3}\right)$

277. Calcular: $\ln 2+\int_{0}^{1} \ln \sqrt{2-x} d x$.
R.: $\ln 4-\frac{1}{2}$

278. Calcular: $\int_{0}^{9} x \sqrt{1+x} d x$.

R.: 105.6759 
279. Encontrar: $\int_{0}^{30^{\circ}} \arctan x d x$.

R.: 0.1314

280. Evaluar: $\int_{0}^{60^{\circ}} \operatorname{sen}^{3} x \cdot d x$.

R.: 0.2083

281. Si $\frac{4 b}{a} \int_{0}^{a} \sqrt{a^{2}-x^{2}} d x=F_{(a, b)}$, halle $F(3,2)$.

R.: $6 \pi$

282. Calcular: $\int_{0}^{6} x \sqrt{1+x} d x$.

R.: 39.7766

283. Encontrar: $\int_{0}^{60^{\circ}} \arctan x d x$.

R.: 0.4764

284. Evaluar: $\int_{0}^{30^{\circ}} \operatorname{sen}^{3} x \cdot d x$.

R.: 0.01715

285. Calcular: $\int_{0}^{3} \frac{x+3}{\sqrt{x+1}} d x$.

R.: $26 / 3$

286. Calcular: $\int_{0}^{2} \frac{x^{3}}{x^{8}+4} d x$.

R.: 0.1808

287. Calcular: $\int_{1}^{e} 2 \ln ^{2} x d x$.

R.: $2 e-4$ 
288. Calcular: $\int_{1}^{e} \ln ^{2} x d x$.
R.: $e-2$

289. Calcular: $\int_{0}^{1} \frac{x^{2}}{x+1} d x$.

R.: 0.193

290. Calcular: $\int_{0}^{\frac{\pi}{4}} 4 x \sec ^{2} x d x+\ln 4$.

R.: $\pi$

291. Si $\int_{0}^{a} \frac{e^{x}}{1+e^{x}} d x=\ln 3$, halle el valor de a.

R.: 1.61

286 292. Si $\int(2 x+3) e^{-x} d x=-e^{-x} F_{(x)}+C$ :
a) Calcule $F_{(1)}$.
b) Evalúe $\int_{1}^{2} F_{(x)} \ln x d x$.

R.: a) 7

b) 3.204

293. Calcular: $\int_{0}^{2} \frac{x^{2}}{x+1} d x$.
R.: 1.098

294. Calcular: $\int_{0}^{\frac{\pi}{4}} 8 x \sec ^{2} x d x+\ln 16$.

R.: $2 \pi$

295. Si $\int_{0}^{a} \frac{e^{x}}{1+e^{x}} d x=\ln 4$, halle el valor de $a$.

R.: $\ln 7$ 
296. Calcular: $\int_{0}^{1} \frac{x+\arctan x}{1+x^{2}} d x$.

R.: 0.655

297. Evaluar: $\int_{0}^{2 \sqrt{2}} \frac{x^{3}}{\sqrt{x^{2}+1}} d x$.

R.: $20 / 3$.

298. Evaluar: $\int_{e}^{e^{2}} \frac{\sqrt{x}+\ln x}{x} d x$.

R.: 3.6392

299. Evaluar: $\int_{-2}^{-1.9} x(2 x+5)^{10} d x$.

R.: -0.5656

300. Hallar: $\int_{2}^{4} \frac{d x}{1+e^{x}}$. (Sugerencia: $e^{x}=\frac{1}{t}$ ).

R.: 0.1088

301. Calcular: $\int_{0}^{1} \frac{\ln (x+1)}{(x+1)^{3 / 2}} d x$.

R.: 0.1913

302. Calcular: $\int_{1}^{2} \frac{x+2}{x\left(1+x^{2} e^{x}\right)} d x$. (Sugerencia: $u=1+x^{2} e^{x}$ ).

R.: 0.28

303. Calcular: $\int_{2}^{3} \frac{x^{4}+1}{x^{3}-x^{2}+x-1} d x$.

R.: 3.7047

304. Calcular: $\int_{0}^{1} \frac{\ln (x+2)}{(x+2)^{3 / 2}} d x$.

R.: 0.2307 
305. Evaluar: $\int_{2}^{6} \frac{1}{x \sqrt{1+x^{2}}} d x$. Sugerencia: haga $x=\frac{1}{z}$.

R.: 0.3153

306. Calcular: $\int_{0}^{\sqrt{7}} \frac{x^{3}}{\sqrt{x^{2}+1}} d x$.

R.: 5.3807

307. Hallar: $\int_{0}^{1}(\arctan x) x^{2} d x$.

R.: 0.2107

308. Evaluar: $\int_{0}^{1 / 2} \frac{\operatorname{arcsen} x}{\left(1-x^{2}\right)^{3 / 2}} d x$

R.: 0.1585

288 309. Determinar: $\int_{5}^{8} \frac{2 x^{2}-3 x+7}{x(x-3)(x+4)} d x$.

R.: 0.94795

310. Determinar: $\int_{6}^{8} \frac{4 x^{2}-6 x+14}{x^{3}-7 x^{2}+12 x} d x$.

R.: 4.2443

311. Calcular: $\int_{0}^{\sqrt{6}} \frac{x^{3}}{\sqrt{x^{2}+1}} d x$.

R.: 4.1944

312. Encontrar: $\int_{1}^{2} x^{2} \arctan x d x$.

R.: 2.3433

313. Evaluar: $\int_{0}^{\sqrt{2} / 2} \frac{\operatorname{arcsen} x}{\left(1-x^{2}\right)^{3 / 2}} d x$.

R.: 0.4388 
314. Determinar: $\int_{5}^{7} \frac{2 x^{2}-3 x+7}{x(x-3)(x-4)} d x$.

R.: 3.9151

315. Evaluar: $\int_{2}^{4} \frac{1}{x \sqrt{1+x^{2}}} d x$. Sugerencia: haga $x=\frac{1}{z}$.

R.: 0.2337

316. Determinar: $\int_{6}^{7} \frac{4 x^{2}-6 x+14}{x^{3}-7 x^{2}+12 x} d x$.

R.: 2.5850

317. Si $f^{\prime \prime}$ es continua, $\int_{0}^{\pi}\left[f_{(x)}+f^{\prime \prime}(x)\right] \operatorname{sen} x d x=2$ y $f_{(\pi)}=1$, hallar $f_{(0)}$.

R.: 1

318. Si $\int_{0}^{a^{2}} \sqrt{x}\left(1+\frac{1}{x}\right) d x=\frac{8}{3} a, a>0$, halle el valor de $a$.

R.: 1

319. Calcular: $\int_{0}^{1} \frac{x-\arctan x}{1+x^{2}} d x$.

R.: 0.03815

320. Calcular: $\int_{4}^{5} \frac{d x}{(x-2) \sqrt{x^{2}-4 x+1}}$.

R.: 0.24925 


\section{PROBLEMAS RESUELTOS Y PROPUESTOS DEL CAPÍTULO VI}

321. Calcular: $\int_{-\infty}^{+\infty} \frac{d x}{e^{x}+e^{-x}}$.

\section{Solución:}

Sea $e^{x}=u \Rightarrow x=\ln u \Rightarrow d x=\frac{d u}{u}$

Si

$$
\begin{array}{ll}
x \rightarrow \infty & u \rightarrow \infty \\
x \rightarrow-\infty & u \rightarrow 0
\end{array}
$$

Entonces:

$$
\int \frac{d x}{e^{x}+e^{-x}}=\int \frac{\frac{d u}{u}}{u+u^{-1}}=\int \frac{d u}{u^{2}+1}=\arctan u+C
$$

De donde:

$$
\begin{gathered}
\int_{-\infty}^{+\infty} \frac{d x}{e^{x}+e^{-x}}=\int_{0}^{+\infty} \frac{d u}{u^{2}+1}=\lim _{b \rightarrow \infty} \int_{0}^{b} \frac{d u}{u^{2}+1}=\left.\lim _{b \rightarrow \infty} \arctan u\right|_{0} ^{b} \\
\int_{-\infty}^{+\infty} \frac{d x}{e^{x}+e^{-x}}=\lim _{b \rightarrow \infty}(\arctan b-\arctan 0)=\frac{\pi}{2}-0=\frac{\pi}{2}
\end{gathered}
$$

322. Dada la integral impropia $\int_{x=2}^{+\infty} \frac{d x}{x \ln ^{m} x}$, determinar para qué valores de $m$ converge.

\section{Solución:}

Se tiene que:

$$
\begin{gathered}
\int_{x=2}^{+\infty} \frac{d x}{x \ln ^{m} x}=\lim _{b \rightarrow \infty} \int_{2}^{b} \frac{d x}{x \ln ^{m} x}=\lim _{b \rightarrow \infty} \int_{2}^{b} \frac{d x / x}{(\ln x)^{m}} \\
\int_{x=2}^{+\infty} \frac{d x}{x \ln ^{m} x}=\left.\lim _{b \rightarrow \infty} \frac{(\ln x)^{-m+1}}{-m+1}\right|_{2} ^{b}=\lim _{b \rightarrow \infty} \frac{1}{1-m}\left[\frac{1}{(\ln b)^{m-1}}-\frac{1}{(\ln 2)^{m-1}}\right]
\end{gathered}
$$

Como $\lim _{b \rightarrow \infty} \frac{1}{(\ln b)^{m-1}}=\lim _{b \rightarrow \infty}\left[\frac{1}{\ln b}\right]^{m-1}=0^{m-1}$ debe existir, se concluye con que $\mathrm{m}-1>0 \rightarrow \mathrm{m}>1$. 
323. Evaluar la siguiente integral:

\section{Solución:}

$$
I=\int_{0}^{+\infty} e^{-x} \operatorname{sen} 3 x d x
$$

Por partes:

$$
\begin{array}{cc}
I=\int e^{-x} \operatorname{sen} 3 x d x & \\
u=\operatorname{sen} 3 x & v=-e^{-x} \\
\downarrow & \uparrow \\
d u=3 \cos 3 x d x & d v=e^{-x} d x
\end{array}
$$

Entonces:

\section{Entonces:}

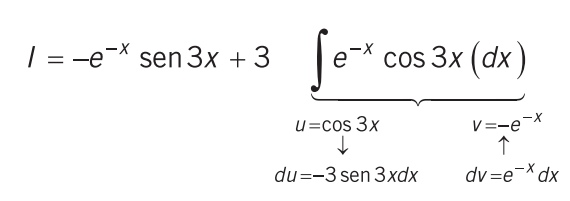

De donde:

Con lo cual:

$$
\begin{gathered}
I=-e^{-x} \operatorname{sen} 3 x-3 e^{-x} \cos 3 x-3(3) \underbrace{\int e^{-x} \operatorname{sen} 3 x d x}_{I} \\
10 I=-e^{-x} \operatorname{sen} 3 x-3 e^{-x} \cos 3 x+10 C \\
I=\frac{-e^{-x} \operatorname{sen} 3 x}{10}-\frac{3 e^{-x} \cos 3 x}{10}+C
\end{gathered}
$$

$$
\int_{0}^{\infty} e^{-x} \operatorname{sen} 3 x d x=\left.\lim _{b \rightarrow \infty}\left[-\frac{1}{10} \cdot \frac{\operatorname{sen} 3 x}{e^{x}}-\frac{3}{10} \cdot \frac{\cos 3 x}{e^{x}}\right]\right|_{0} ^{b}
$$

Tenemos que: $\int_{0}^{\infty} e^{-x} \operatorname{sen} 3 x d x=\frac{-1}{10} \lim _{b \rightarrow \infty}\left[\frac{\operatorname{sen} 3 b}{e^{b}}+\frac{3 \cos 3 b}{e^{b}}-\frac{0}{1}-3\right]$

$$
\left.\begin{array}{l}
\lim _{b \rightarrow \infty} \frac{\operatorname{sen} 3 b}{e^{b}}=0 \\
\lim _{b \rightarrow \infty} \frac{3 \cos 3 b}{e^{b}}=0
\end{array}\right\} \begin{aligned}
& \text { Ya que el numerador está } \\
& \text { comprendido entre }-1 \text { y } 1 \text { pero el } \\
& \text { denominador tiende a } \infty .
\end{aligned}
$$

Con lo cual:

$$
\int_{0}^{\infty} e^{-x} \operatorname{sen} 3 x d x=\frac{-1}{10}[0+0-0-3]=\frac{3}{10}=0.3
$$


324. Calcular la integral impropia

$$
\int_{x=0}^{+\infty} e^{-\sqrt{x}} d x
$$

\section{Solución:}

Sea $u=e^{-\sqrt{x}} \rightarrow-\sqrt{x}=\ln u \rightarrow x=\ln ^{2} u \rightarrow d x=2 \ln u\left(\frac{d u}{u}\right)$

Si:

$$
\begin{array}{ll}
x \rightarrow \infty & u \rightarrow 0 \\
x \rightarrow 0 & u \rightarrow 1
\end{array}
$$

Entonces:

$$
\begin{gathered}
\int_{0}^{+\infty} e^{-\sqrt{x}} d x=\int_{1}^{0} u(2 \ln u) \frac{d u}{u}=2 \int_{1}^{0} \ln u d u \\
\int_{0}^{+\infty} e^{-\sqrt{x}} d x=2 \lim _{b \rightarrow 0} \int_{1}^{b} \ln u d u=\left.2 \lim _{b \rightarrow 0}[u \ln u-u]\right|_{1} ^{b}
\end{gathered}
$$

$$
\int_{0}^{+\infty} e^{-\sqrt{x}} d x=2 \lim _{b \rightarrow 0}[b \ln b-b-0+1]=2 \lim _{b \rightarrow 0} b \ln b+2
$$

Calculemos con la regla de L'Hopital:

$$
\lim _{b \rightarrow 0} b \ln b=\lim _{b \rightarrow 0} \frac{\ln b}{\frac{1}{b}}=\lim _{b \rightarrow 0} \frac{\frac{1}{b}}{\frac{-1}{b^{2}}}=\lim _{b \rightarrow 0}(-b)=0
$$

De donde:

$$
\int_{0}^{+\infty} e^{-\sqrt{x}} d x=2(0)+2=2
$$

325. Calcular: $\int_{-\infty}^{+\infty} \frac{d x}{x^{2}+2 x+2}$.

Solución:

Se pide:

$$
\int_{-\infty}^{\infty} \frac{d x}{x^{2}+2 x+2}=\int_{-\infty}^{0} \frac{d x}{x^{2}+2 x+2}+\int_{0}^{\infty} \frac{d x}{x^{2}+2 x+2}
$$




$$
\begin{gathered}
\int_{-\infty}^{\infty} \frac{d x}{x^{2}+2 x+2}=\lim _{a \rightarrow-\infty} \int_{a}^{0} \frac{d x}{x^{2}+2 x+2}+\lim _{b \rightarrow \infty} \int_{0}^{b} \frac{d x}{x^{2}+2 x+2} \\
\int_{-\infty}^{\infty} \frac{d x}{x^{2}+2 x+2}=\lim _{a \rightarrow-\infty} \int_{a}^{0} \frac{d x}{(x+1)^{2}+1^{2}}+\lim _{b \rightarrow \infty} \int_{0}^{b} \frac{d x}{(x+1)^{2}+1^{2}} \\
\int_{-\infty}^{\infty} \frac{d x}{x^{2}+2 x+2}=\left.\lim _{a \rightarrow-\infty} \arctan (x+1)\right|_{a} ^{0}+\left.\lim _{b \rightarrow \infty} \arctan (x+1)\right|_{0} ^{b} \\
\int_{-\infty}^{\infty} \frac{d x}{x^{2}+2 x+2}=\lim _{a \rightarrow-\infty}(\arctan 1-\arctan (a+1))+\lim _{b \rightarrow \infty}(\arctan (b+1)-\arctan 1) \\
\int_{-\infty}^{\infty} \frac{d x}{x^{2}+2 x+2}=\left[\frac{\pi}{4}-\left(\frac{-\pi}{2}\right)\right]+\left[\frac{\pi}{2}-\frac{\pi}{4}\right]=\pi
\end{gathered}
$$

326. Halle el valor de la siguiente integral impropia $\int_{1}^{2}(x+1) \ln (x-1) d x$.

\section{Solución:}

Se tiene que:

$$
\int_{1}^{2}(x+1) \ln (x-1) d x=\lim _{\varepsilon \rightarrow 0} \int_{1+\varepsilon}^{2}(x+1) \ln (x-1) d x
$$

Integremos:

$$
\begin{array}{cc}
\int(x+1) \ln (x-1) d x & v=\frac{x^{2}}{2}+x \\
\downarrow=\ln (x-1) & \uparrow \\
d u=\frac{d x}{x-1} & d v=(x+1) d x
\end{array}
$$

Entonces:

$$
\begin{gathered}
\int(x+1) \ln (x-1) d x=\left(\frac{x^{2}}{2}+x\right) \ln (x-1)-\int \frac{\frac{x^{2}}{2}+x}{x-1} d x \\
\int(x+1) \ln (x-1) d x=\left(\frac{x^{2}}{2}+x\right) \ln (x-1)-\frac{1}{2} \int\left(x+3+\frac{3}{x-1}\right) d x \\
\int(x+1) \ln (x-1) d x=\left(\frac{x^{2}}{2}+x\right) \ln (x-1)-\frac{1}{2}\left(\frac{x^{2}}{2}+3 x+3 \ln (x-1)\right)+C
\end{gathered}
$$




$$
\int(x+1) \ln (x-1) d x=\left(\frac{x^{2}}{2}+x-\frac{3}{2}\right) \ln (x-1)-\frac{x^{2}}{4}-\frac{3 x}{2}+C
$$

De donde:

$$
\begin{gathered}
\int_{1}^{2}(x+1) \ln (x-1) d x=\lim _{\varepsilon \rightarrow 0}\left[\left(\frac{x^{2}}{2}+x-\frac{3}{2}\right) \ln (x-1)-\frac{x^{2}}{4}-\frac{3 x}{2}\right]_{1+\varepsilon}^{2} \\
\int_{1}^{2}(x+1) \ln (x-1) d x=\lim _{\varepsilon \rightarrow 0}\left[\left(\frac{5}{2} \ln 1-1-3\right)-\left(\frac{(1+\varepsilon)^{2}}{2}+1+\varepsilon-\frac{3}{2}\right) \ln \varepsilon+\frac{(1+\varepsilon)^{2}}{4}+\frac{3(1+\varepsilon)}{2}\right]
\end{gathered}
$$

Calculemos:

$$
\lim _{\varepsilon \rightarrow 0}\left[\frac{(1+\varepsilon)^{2}}{2}+1+\varepsilon-\frac{3}{2}\right] \ln \varepsilon=\lim _{\varepsilon \rightarrow 0} \frac{\varepsilon^{2}+4 \varepsilon}{2} \ln \varepsilon=\frac{1}{2} \lim _{\varepsilon \rightarrow 0} \frac{\ln \varepsilon}{\left(\varepsilon^{2}+4 \varepsilon\right)^{-1}}
$$

$$
\begin{gathered}
\frac{1}{2} \lim _{\varepsilon \rightarrow 0} \frac{\ln \varepsilon}{\left(\varepsilon^{2}+4 \varepsilon\right)^{-1}}=\frac{1}{2} \lim _{\varepsilon \rightarrow 0} \frac{\frac{1}{\varepsilon}-\left(\varepsilon^{2}+4 \varepsilon\right)^{-2}(2 \varepsilon+4)}{2}=-\frac{1}{2} \lim _{\varepsilon \rightarrow 0} \frac{\frac{\left(\varepsilon^{2}+4 \varepsilon\right)^{2}}{\varepsilon}}{2 \varepsilon+4} \\
\frac{1}{2} \lim _{\varepsilon \rightarrow 0} \frac{\ln \varepsilon}{\left(\varepsilon^{2}+4 \varepsilon\right)^{-1}}=-\frac{1}{2} \lim _{\varepsilon \rightarrow 0} \frac{\varepsilon(\varepsilon+4)^{2}}{2 \varepsilon+4}=-\frac{1}{2}\left(\frac{0}{4}\right)=0
\end{gathered}
$$

Entonces:

$$
\int_{1}^{2}(x+1) \ln (x-1) d x=0-4-0+\frac{1}{4}+\frac{3}{2}=\frac{-9}{4}
$$

327. Si $k>0$, determine el valor de $\int_{0}^{+\infty} k e^{-k x} d x-\int_{1}^{+\infty} \frac{k}{x^{k+1}} d x$.

\section{Solución:}

Se tiene que:

$$
\begin{gathered}
\int_{0}^{\infty} k e^{-k x} d x=\lim _{b \rightarrow \infty}-\int_{0}^{b}-k e^{-k x} d x=-\left.\lim _{b \rightarrow \infty} e^{-k x}\right|_{0} ^{b} \\
\int_{0}^{\infty} k e^{-k x} d x=-\lim _{b \rightarrow \infty}\left(e^{-k b}-e^{0}\right)=-\lim _{b \rightarrow \infty} \frac{1}{e^{k b}}-1=-(0-1)=1
\end{gathered}
$$


Además:

$$
\begin{gathered}
\int_{1}^{\infty} \frac{k}{x^{k+1}} d x=\lim _{b \rightarrow \infty} k \int_{1}^{b} x^{-k-1} d x=\left.\lim _{b \rightarrow \infty} \frac{k x^{-k}}{-k}\right|_{1} ^{b} \\
\int_{1}^{\infty} \frac{k}{x^{k+1}} d x=-\left.\lim _{b \rightarrow \infty} x^{-k}\right|_{1} ^{b}=-\lim _{b \rightarrow \infty}\left(\frac{1}{b^{k}}-1\right)=-(0-1)=1
\end{gathered}
$$

De donde:

$$
+1-(1)=0
$$

328. Obtener: $\int_{-\infty}^{\infty} \frac{d x}{x^{2}+2 a x+b^{2}}$.

\section{Solución:}

Tenemos que:

$$
\begin{aligned}
& \int_{-\infty}^{\infty} \frac{d x}{x^{2}+2 a x+b^{2}}=\int_{-\infty}^{0} \frac{d x}{x^{2}+2 a x+b^{2}}+\int_{0}^{\infty} \frac{d x}{x^{2}+2 a x+b^{2}} \\
& \int_{-\infty}^{\infty} \frac{d x}{x^{2}+2 a x+b^{2}}=\lim _{c \rightarrow-\infty} \int_{c}^{0} \frac{d x}{x^{2}+2 a x+b^{2}}+\lim _{d \rightarrow \infty} \int_{0}^{d} \frac{d x}{x^{2}+2 a x+b^{2}} \\
& \int_{-\infty}^{\infty} \frac{d x}{x^{2}+2 a x+b^{2}}=\lim _{c \rightarrow-\infty} \int_{c}^{0} \frac{d x}{(x+a)^{2}+\left(\sqrt{b^{2}-a^{2}}\right)^{2}}+\lim _{d \rightarrow \infty} \int_{0}^{d} \frac{d x}{(x+a)^{2}+\left(\sqrt{b^{2}-a^{2}}\right)^{2}} \\
& \int_{-\infty}^{\infty} \frac{d x}{x^{2}+2 a x+b^{2}}=\left.\lim _{c \rightarrow-\infty} \frac{1}{\sqrt{b^{2}-a^{2}}} \arctan \frac{x+a}{\sqrt{b^{2}-a^{2}}}\right|_{c} ^{0}+\left.\lim _{d \rightarrow \infty} \frac{1}{\sqrt{b^{2}-a^{2}}} \arctan \frac{x+a}{\sqrt{b^{2}-a^{2}}}\right|_{0} ^{d} \\
& \int_{-\infty}^{\infty} \frac{d x}{x^{2}+2 a x+b^{2}}=\frac{1}{\sqrt{b^{2}-a^{2}}}\left[\begin{array}{c}
\lim _{c \rightarrow-\infty}\left(\arctan \frac{a}{\sqrt{b^{2}-a^{2}}}-\arctan \frac{c+a}{\sqrt{b^{2}-a^{2}}}\right)+ \\
\lim _{d \rightarrow \infty}\left(\arctan \frac{d+a}{\sqrt{b^{2}-a^{2}}}-\arctan \frac{a}{\sqrt{b^{2}-a^{2}}}\right)
\end{array}\right] \\
& \int_{-\infty}^{\infty} \frac{d x}{x^{2}+2 a x+b^{2}}=\frac{1}{\sqrt{b^{2}-a^{2}}}\left[-\left(\frac{-\pi}{2}\right)+\left(\frac{\pi}{2}\right)\right]=\frac{\pi}{\sqrt{b^{2}-a^{2}}}
\end{aligned}
$$


329. Calcular la integral impropia $\int_{0}^{\infty} \frac{d x}{x^{2}+a}$.

\section{Solución:}

Se pide:

$$
\lim _{b \rightarrow \infty} \int_{0}^{b} \frac{d x}{x^{2}+a}=\lim _{b \rightarrow \infty} \int_{0}^{b} \frac{d x}{x^{2}+(\sqrt{a})^{2}}=\left.\lim _{b \rightarrow \infty} \frac{1}{\sqrt{a}} \arctan \frac{x}{\sqrt{a}}\right|_{0} ^{b}
$$

Entonces:

$$
\int_{0}^{\infty} \frac{d x}{x^{2}+a}=\frac{1}{\sqrt{a}} \lim _{b \rightarrow \infty}\left[\arctan \frac{b}{\sqrt{a}}-\arctan 0\right]=\frac{1}{\sqrt{a}}\left[\frac{\pi}{2}-0\right]=\frac{\pi}{2 \sqrt{a}}
$$

330. Obtener: $\int_{0}^{1} x \ln (1-x) d x$.

Solución:

Encontremos:

$$
\begin{array}{cc}
\int x \ln (1-x) d x & \\
u=\ln (1-x) & v=\frac{x^{2}}{2} \\
\downarrow & \uparrow \\
d u=\frac{-1}{1-x} d x & d v=x d x
\end{array}
$$

Entonces:

$$
\begin{array}{r}
\int x \ln (1-x) d x=\frac{x^{2}}{2} \ln (1-x)+\frac{1}{2} \int \frac{x^{2}}{1-x} d x \\
\text { Como } \int \frac{x^{2}}{1-x} d x=\int\left[-x-1+\frac{1}{1-x}\right] d x=\frac{-x^{2}}{2}-x-\ln (1-x)+2 C
\end{array}
$$

se tendrá que:

$$
\begin{gathered}
\int x \ln (1-x) d x=\frac{x^{2}}{2} \ln (1-x)+\frac{1}{2}\left[-\frac{x^{2}}{2}-x-\ln (1-x)\right]+C \\
\int x \ln (1-x) d x=\left(\frac{x^{2}}{2}-\frac{1}{2}\right) \ln (1-x)-\frac{x^{2}}{4}-\frac{x}{2}+C
\end{gathered}
$$


APUNTES DE ESTUDIO

De donde:

$$
\begin{gathered}
\int_{0}^{1} x \ln (1-x) d x=\lim _{\varepsilon \rightarrow 0^{+}} \int_{0}^{1-\varepsilon} x \ln (1-x) d x=\left.\lim _{\varepsilon \rightarrow 0^{+}}\left[\left(\frac{x^{2}}{2}-\frac{1}{2}\right) \ln (1-x)-\frac{x^{2}}{4}-\frac{x}{2}\right]\right|_{0} ^{1-\varepsilon} \\
\int_{0}^{1} x \ln (1-x) d x=\lim _{\varepsilon \rightarrow 0^{+}}\left[\left(\frac{(1-\varepsilon)^{2}}{2}-\frac{1}{2}\right) \ln \varepsilon-\frac{(1-\varepsilon)^{2}}{4}-\frac{(1-\varepsilon)}{2}-0+0+0\right] \\
\int_{0}^{1} x \ln (1-x) d x=\lim _{\varepsilon \rightarrow 0^{+}}\left[\left(-\varepsilon+\frac{\varepsilon^{2}}{2}\right) \ln \varepsilon-\frac{1}{4}-\frac{1}{2}\right] \\
\int_{0}^{1} x \ln (1-x) d x=\lim _{\varepsilon \rightarrow 0^{+}}\left(\frac{\varepsilon^{2}-2 \varepsilon}{2}\right) \ln \varepsilon-\frac{3}{4} \quad \ldots(\alpha)
\end{gathered}
$$

Calculemos con la regla de L'Hopital:

$$
\begin{aligned}
& \qquad \lim _{\varepsilon \rightarrow 0^{+}}\left(\frac{\varepsilon^{2}-2 \varepsilon}{2}\right) \ln \varepsilon=\frac{1}{2} \lim _{\varepsilon \rightarrow 0^{+}} \frac{\ln \varepsilon}{\left(\varepsilon^{2}-2 \varepsilon\right)^{-1}}=\frac{1}{2} \lim _{\varepsilon \rightarrow 0^{+}} \frac{1 / \varepsilon}{-\left(\varepsilon^{2}-2 \varepsilon\right)^{-2}(2 \varepsilon-2)} \\
& \lim _{\varepsilon \rightarrow 0^{+}}\left(\frac{\varepsilon^{2}-2 \varepsilon}{2}\right) \ln \varepsilon=-\frac{1}{2} \lim _{\varepsilon \rightarrow 0^{+}} \frac{\left(\varepsilon^{2}-2 \varepsilon\right)^{2}}{2 \varepsilon^{2}-2 \varepsilon}=-\frac{1}{2} \lim _{\varepsilon \rightarrow 0^{+}} \frac{\varepsilon^{3}-4 \varepsilon^{2}+4 \varepsilon}{2 \varepsilon-2}=\frac{-1}{2} \cdot \frac{0-0+0}{0-2}=0 \\
& \text { Reemplazando en }(\alpha): \\
& \qquad \int_{0}^{1} x \ln (1-x) d x=0-\frac{3}{4}=\frac{-3}{4}
\end{aligned}
$$

297

331. Calcular: $\int_{0}^{\infty} x e^{-x^{2}} d x$.

Solución:

Se tiene que:

$$
\int x e^{-x^{2}} d x=\frac{1}{2} \int e^{-x^{2}}(2 x d x)=\frac{-1}{2} \int e^{-x^{2}}(-2 x d x)=\frac{-1}{2} e^{-x^{2}}+C
$$

Entonces:

$$
\begin{gathered}
\int_{0}^{\infty} x e^{-x^{2}} d x=\lim _{b \rightarrow \infty} \int_{0}^{b} x e^{-x^{2}} d x=\left.\lim _{b \rightarrow \infty} \frac{-1}{2} e^{-x^{2}}\right|_{0} ^{b} \\
\int_{0}^{\infty} x e^{-x^{2}} d x=\lim _{b \rightarrow \infty} \frac{-1}{2}\left[e^{-b^{2}}-e^{0}\right]=\frac{-1}{2} \lim _{b \rightarrow \infty}\left[\frac{1}{e^{b^{2}}}-1\right]=\frac{1}{2}
\end{gathered}
$$


332. Calcular: $\int_{0}^{\infty} e^{-a x} \operatorname{sen} m x d x \quad(a>0)$.

\section{Solución:}

Sea:

$$
\begin{array}{cc}
u=e^{-a x} & v=-\frac{1}{m} \cos m x \\
\downarrow & \uparrow \\
d u=-a e^{-a x} d x & d v=\operatorname{sen} m x d x
\end{array}
$$

Entonces:

$$
I=\int e^{-a x} \operatorname{sen} m x d x=\frac{-e^{-a x}}{m} \cos m x-\frac{a}{m} \int e^{-a x} \cos m x d x
$$

Por otro lado:

$$
\begin{array}{ccc}
\int e^{-a x} \cos m x d x & \\
u=e^{-a x} & v=\frac{1}{m} \operatorname{sen} m x \\
\downarrow & \uparrow \\
d u=-a e^{-a x} d x & d v=\cos m x d x
\end{array}
$$

De donde:

$$
\int e^{-a x} \cos m x d x=\frac{1}{m} e^{-a x} \operatorname{sen} m x+\frac{a}{m} \int e^{-a x} \operatorname{sen} m x d x
$$

Con lo cual, de (2) en (1):

$$
\begin{aligned}
& I=\frac{-e^{-a x}}{m} \cos m x-\frac{a}{m}\left[\frac{1}{m} e^{-a x} \operatorname{sen} m x+\frac{a}{m} I\right] \\
& I\left[1+\frac{a^{2}}{m^{2}}\right]=\frac{-e^{-a x}}{m} \cos m x-\frac{a}{m^{2}} e^{-a x} \operatorname{sen} m x \\
& I=\frac{-m^{2}}{a^{2}+m^{2}}\left[\frac{m e^{-a x} \cos m x+a e^{-a x} \operatorname{sen} m x}{m^{2}}\right]
\end{aligned}
$$

Entonces:

$$
\int_{0}^{\infty} e^{-a x} \operatorname{sen} m x d x=\lim _{b \rightarrow \infty} \int_{0}^{b} e^{-a x} \operatorname{sen} m x d x=\left.\lim _{b \rightarrow \infty} \frac{-m^{2}}{a^{2}+m^{2}}\left[\frac{m e^{-a x} \cos m x+a e^{-a x} \operatorname{sen} m x}{m^{2}}\right]\right|_{0} ^{b}
$$




$$
\begin{gathered}
\int_{0}^{\infty} e^{-a x} \operatorname{sen} m x d x=\frac{-1}{a^{2}+m^{2}} \lim _{b \rightarrow \infty}\left[m e^{-a b} \cos m b+a e^{-a b} \operatorname{sen} m b-m e^{0} \cos 0+a e^{0} \operatorname{sen} 0\right] \\
\int_{0}^{\infty} e^{-a x} \operatorname{sen} m x d x=\frac{-1}{a^{2}+m^{2}}\left\{\lim _{b \rightarrow \infty}\left(\frac{m \cos m b+a \operatorname{sen} m b}{e^{a b}}-m-0\right)\right\} \\
\int_{0}^{\infty} e^{-a x} \operatorname{sen} m x d x=\frac{-1}{a^{2}+m^{2}}[0-m]=\frac{m}{a^{2}+m^{2}}
\end{gathered}
$$

333. Calcular: $\int_{0}^{\infty} \frac{\arctan x}{1+x^{2}} d x$.

\section{Solución:}

Se tiene que:

$$
\int \frac{\arctan x}{1+x^{2}} d x=\int(\arctan x)\left(\frac{d x}{1+x^{2}}\right)=\frac{(\arctan x)^{2}}{2}+C
$$

Entonces:

$$
\begin{gathered}
\int_{0}^{\infty} \frac{\arctan x}{1+x^{2}} d x=\lim _{b \rightarrow \infty} \int_{0}^{b} \frac{\arctan x}{1+x^{2}} d x=\left.\lim _{b \rightarrow \infty} \frac{(\arctan x)^{2}}{2}\right|_{0} ^{b} \\
\int_{0}^{\infty} \frac{\arctan x}{1+x^{2}} d x=\frac{1}{2} \lim _{b \rightarrow \infty}\left[(\arctan b)^{2}-(\arctan 0)^{2}\right] \\
\int_{0}^{\infty} \frac{\arctan x}{1+x^{2}} d x=\frac{1}{2}\left[\left(\frac{\pi}{2}\right)^{2}-0\right]=\frac{\pi^{2}}{8}
\end{gathered}
$$

334. Calcular: $\int_{1}^{2} \frac{d s}{s \sqrt{s^{2}-1}}$.

\section{Solución:}

Efectuemos el cambio de variable $s=\sec \theta \Rightarrow d s=\sec \theta \tan \theta d \theta$.

Entonces:

$$
\begin{gathered}
\int \frac{d s}{s \sqrt{s^{2}-1}}=\int \frac{\sec \theta \tan \theta d \theta}{\sec \theta \tan \theta}=\int d \theta=\theta=\operatorname{arcsec} s+C \\
\int_{1}^{2} \frac{d s}{s \sqrt{s^{2}-1}}=\lim _{\varepsilon \rightarrow 0^{+}} \int_{1+\varepsilon}^{2} \frac{d s}{s \sqrt{s^{2}-1}}=\lim _{\varepsilon \rightarrow 0^{+}}[\operatorname{arcsec} 2-\operatorname{arcsec}(1+\varepsilon)]
\end{gathered}
$$




$$
\int_{1}^{2} \frac{d s}{s \sqrt{s^{2}-1}}=\lim _{\varepsilon \rightarrow 0^{+}}\left[\frac{\pi}{3}-0\right]=\frac{\pi}{3}
$$

335. Calcular: $\int_{0}^{\infty} \frac{d x}{(1+x) \sqrt{x}}$.

Solución:

Sea $x=t^{2} \Rightarrow d x=2 t d t$

Entonces:

$$
\int \frac{d x}{(1+x) \sqrt{x}}=\int \frac{2 t d t}{t\left(1+t^{2}\right)}=2 \int \frac{d t}{\left(1+t^{2}\right)}=2 \arctan t+C=2 \arctan \sqrt{x}+C
$$

De donde:

$$
\int_{0}^{\infty} \frac{d x}{\sqrt{x}(1+x)}=\lim _{\substack{\varepsilon \rightarrow 0^{+} \\ b \rightarrow \infty}} \int_{\varepsilon}^{b} \frac{d x}{\sqrt{x}(1+x)}=\left.2 \lim _{\substack{\varepsilon \rightarrow 0^{+} \\ b \rightarrow \infty}} \arctan \sqrt{x}\right|_{\varepsilon} ^{b}
$$

300

$$
\int_{0}^{\infty} \frac{d x}{\sqrt{x}(1+x)}=2 \lim _{\substack{\varepsilon \rightarrow 0^{+} \\ b \rightarrow \infty}}(\arctan \sqrt{b}-\arctan \varepsilon)=2\left[\frac{\pi}{2}-0\right]=\pi
$$

336. Sea $f_{(n+1)}=\int_{0}^{\infty} x^{n} e^{-x} d x \quad(n \geq 0)$. Demuestre que $f_{(n+1)}=n f_{(n)}$.

\section{Solución:}

Integremos $\int x^{n} e^{-x} d x$ por partes:

$$
\begin{array}{cc}
u=x^{n} & v=-e^{-x} \\
\downarrow & \uparrow \\
d u=n x^{n-1} d x & d v=e^{-x} d x
\end{array}
$$

Entonces:

$$
\begin{gathered}
\int x^{n} e^{-x} d x=-x^{n} e^{-x}+n \int x^{n-1} e^{-x} d x \\
\int_{0}^{\infty} x^{n} e^{-x} d x=\lim _{b \rightarrow \infty} \int_{0}^{b} x^{n} e^{-x} d x=\lim _{b \rightarrow \infty}\left[-x^{n} e^{-x}+\left.n \int x^{n-1} e^{-x} d x\right|_{0} ^{b}\right. \\
\int_{0}^{\infty} x^{n} e^{-x} d x=\lim _{b \rightarrow \infty}\left(\frac{-b^{n}}{e^{b}}+\frac{0^{n}}{e^{0}}\right)+n \int_{0}^{\infty} x^{n-1} e^{-x} d x \\
\int_{0}^{\infty} x^{n} e^{-x} d x=-\lim _{b \rightarrow \infty} \frac{b^{n}}{e^{b}}+0+n f_{(n)}
\end{gathered}
$$


Calculemos con la regla de L'Hopital:

$$
\lim _{b \rightarrow \infty} \frac{b^{n}}{e^{b}}=\lim _{b \rightarrow \infty} \frac{n b^{n-1}}{e^{b}}=\lim _{b \rightarrow \infty} \frac{n(n-1) b^{n-2}}{e^{b}}=\lim _{b \rightarrow \infty} \frac{n(n-1)(n-2) \ldots(1)}{e^{b}}=0
$$

De donde:

$$
f_{(n+1)}=\int_{0}^{\infty} x^{n} e^{-x} d x=-0+0+n f_{(n)}=n f_{(n)}
$$

337. Calcular: $\int_{e}^{\infty} \frac{d x}{x(\ln x)^{2}}$.

\section{Solución:}

Sea $\ln x=u \Rightarrow \frac{d x}{x}=d u$.

Si: $\mathrm{x} \rightarrow \infty$ entonces $\mathrm{u} \rightarrow \infty$

$$
\mathrm{x}=\theta \quad \text { entonces } \quad \mathrm{u}=1
$$

Con lo cual:

$$
\begin{gathered}
\int_{e}^{\infty} \frac{d x / x}{(\ln x)^{2}}=\int_{1}^{\infty} \frac{d u}{u^{2}}=\lim _{b \rightarrow \infty} \int_{1}^{b} \frac{d u}{u^{2}} \\
\int_{e}^{\infty} \frac{d x / x}{(\ln x)^{2}}=\left.\lim _{b \rightarrow \infty}\left[-\frac{1}{u}\right]\right|_{1} ^{b}=\lim _{b \rightarrow \infty}\left(-\frac{1}{b}+\frac{1}{1}\right)=1
\end{gathered}
$$

338. Calcular: $\int_{2}^{3} \frac{d y}{\sqrt[3]{y-2}}$.

\section{Solución:}

Se tiene que:

$$
\int \frac{d y}{\sqrt[3]{y-2}}=\int(y-2)^{-1 / 3} d y=\frac{(y-2)^{2 / 3}}{2 / 3}+C
$$

Entonces:

$$
\begin{gathered}
\int_{2}^{3} \frac{d y}{\sqrt[3]{y-2}}=\lim _{\varepsilon \rightarrow 0^{+}} \int_{2+\varepsilon}^{3} \frac{d y}{\sqrt[3]{y-2}}=\left.\frac{3}{2} \lim _{\varepsilon \rightarrow 0^{+}}(y-2)^{2 / 3}\right|_{2+\varepsilon} ^{3} \\
\int_{2}^{3} \frac{d y}{\sqrt[3]{y-2}}=\frac{3}{2} \lim _{\varepsilon \rightarrow 0^{+}}\left[\sqrt[3]{1^{2}}-\sqrt[3]{\varepsilon^{2}}\right]=\frac{3}{2}(1-0)=\frac{3}{2}
\end{gathered}
$$


339. Calcular: $\int_{0}^{\infty} \frac{3^{-\sqrt{r}}}{\sqrt{r}} d r$.

Solución:

Hagamos que $r=u^{2} \rightarrow d r=2 u d u$.

Entonces:

$$
\begin{gathered}
\int \frac{3^{-\sqrt{r}}}{\sqrt{r}} d r=\int \frac{3^{-u}}{u}(2 u d u)=2 \int 3^{-u} d u \\
\int \frac{3^{-\sqrt{r}}}{\sqrt{r}} d r=-2 \int 3^{-u}(-d u)=\frac{-2\left(3^{-u}\right)}{\ln 3}+C=\frac{-2}{(\ln 3) 3^{\sqrt{r}}}+C
\end{gathered}
$$

De donde:

302

$$
\begin{gathered}
\int_{0}^{\infty} \frac{3^{-\sqrt{r}}}{\sqrt{r}} d r=\lim _{\substack{\varepsilon \rightarrow 0^{+} \\
b \rightarrow \infty}} \int_{\varepsilon}^{b} \frac{3^{-\sqrt{r}}}{\sqrt{r}} d r=\left.\lim _{\substack{\varepsilon \rightarrow 0^{+} \\
b \rightarrow \infty}} \frac{-2}{(\ln 3) 3^{\sqrt{r}}}\right|_{\varepsilon} ^{b} \\
\int_{0}^{\infty} \frac{3^{-\sqrt{r}}}{\sqrt{r}} d r=\frac{-2}{\ln 3} \lim _{\substack{\varepsilon \rightarrow 0^{+} \\
b \rightarrow \infty}}\left[\frac{1}{3^{\sqrt{b}}}-\frac{1}{3^{\sqrt{\varepsilon}}}\right]=\frac{-2}{\ln 3}\left(0-\frac{1}{1}\right)=\frac{2}{\ln 3}
\end{gathered}
$$

340. Calcular: $\int_{-\infty}^{0} x \cdot 5^{-x^{2}} \cdot d x$.

\section{Solución:}

Escribamos:

$$
\int x \cdot 5^{-x^{2}} \cdot d x=-\frac{1}{2} \int 5^{-x^{2}} \cdot(-2 x) \cdot d x=-\frac{1}{2} \cdot \frac{5^{-x^{2}}}{\ln 5}+C
$$

De donde:

$$
\begin{gathered}
\int_{-\infty}^{0} x \cdot 5^{-x^{2}} \cdot d x=\lim _{a \rightarrow-\infty} \int_{a}^{0} x \cdot 5^{-x^{2}} \cdot d x=\left.\lim _{a \rightarrow-\infty}\left(-\frac{1}{2 \ln 5}\right)\left(\frac{1}{5^{x^{2}}}\right)\right|_{a} ^{0} \\
\int_{-\infty}^{0} x \cdot 5^{-x^{2}} \cdot d x=-\frac{1}{2 \ln 5} \lim _{a \rightarrow-\infty}\left(\frac{1}{5^{0}}-\frac{1}{5^{a^{2}}}\right)=-\frac{1}{2 \ln 5}[1-0]=-\frac{1}{2 \ln 5}
\end{gathered}
$$

341. Si $G_{(a)}=\int_{0}^{\infty} \frac{1}{\left(1+x^{a}\right)\left(1+x^{2}\right)} d x$, calcular $G_{(0)}, G_{(1)} y G_{(2)}$. 
a) Calculemos $G_{(0)}$ :

$$
\begin{gathered}
G_{(0)}=\int_{0}^{\infty} \frac{1}{(1+1)\left(1+x^{2}\right)} d x=\int_{0}^{\infty} \frac{d x}{2\left(1+x^{2}\right)}=\lim _{b \rightarrow \infty} \frac{1}{2} \int_{0}^{b} \frac{d x}{1+x^{2}} \\
G_{(0)}=\left.\lim _{b \rightarrow \infty} \frac{1}{2} \arctan x\right|_{0} ^{b}=\frac{1}{2} \lim _{b \rightarrow \infty}(\arctan b-\arctan 0)=\frac{1}{2}\left(\frac{\pi}{2}-0\right)=\frac{\pi}{4}
\end{gathered}
$$

b) Por fracciones parciales, calculemos $G_{(1)}$ :

$$
\begin{aligned}
& G_{(1)}=\int_{0}^{\infty} \frac{1}{(1+x)\left(1+x^{2}\right)} d x=\lim _{b \rightarrow \infty} \int_{0}^{b}\left[\frac{1}{2} \cdot \frac{1}{1+x}-\frac{1}{2} \cdot \frac{x-1}{x^{2}+1}\right] d x \\
& G_{(1)}=\frac{1}{2} \lim _{b \rightarrow \infty} \int_{0}^{b}\left[\frac{1}{1+x}-\frac{1}{2} \cdot \frac{2 x}{x^{2}+1}+\frac{1}{x^{2}+1}\right] d x \\
& G_{(1)}=\frac{1}{2} \lim _{b \rightarrow \infty} \ln (1+x)-\frac{1}{2} \ln \left(x^{2}+1\right)+\left.\arctan x\right|_{0} ^{b} \\
& G_{(1)}=\frac{1}{2} \lim _{b \rightarrow \infty} \ln \frac{1+x}{\sqrt{x^{2}+1}}+\left.\arctan x\right|_{0} ^{b} \\
& G_{(1)}=\frac{1}{2} \lim _{b \rightarrow \infty} \ln \frac{\frac{1}{x}+1}{\sqrt{1+\frac{1}{x^{2}}}}+\left.\arctan x\right|_{0} ^{b} \\
& G_{(1)}=\frac{1}{2} \lim _{b \rightarrow \infty}\left(\ln \frac{\frac{1}{b}+1}{\sqrt{1+\frac{1}{b^{2}}}}+\arctan b-\ln \frac{1}{1}-0\right) \\
& G_{(1)}=\frac{1}{2}\left[\ln 1+\frac{\pi}{2}-0\right]=\frac{\pi}{4}
\end{aligned}
$$

c) Calculemos $G_{(2)}$ :

$$
G_{(2)}=\int_{0}^{\infty} \frac{1}{\left(1+x^{2}\right)\left(1+x^{2}\right)} d x=\int_{0}^{\infty} \frac{1}{\left(1+x^{2}\right)^{2}} d x
$$

Hagamos: $\quad x=\tan \theta \rightarrow d x=\sec ^{2} \theta d \theta$ 
Si: $x \Rightarrow \infty \rightarrow \theta=\frac{\pi}{2}$

$$
\mathrm{x}=\theta \quad \rightarrow \theta=0
$$

Entonces:

$$
\begin{gathered}
G_{(2)}=\int_{0}^{\infty} \frac{1}{\left(1+x^{2}\right)^{2}} d x=\int_{0}^{\pi / 2} \frac{\sec ^{2} \theta d \theta}{\left(1+\tan ^{2} \theta\right)^{2}}=\int_{0}^{\pi / 2} \frac{d \theta}{\sec ^{2} \theta}=\int_{0}^{\pi / 2} \cos ^{2} \theta d \theta \\
G_{(2)}=\int_{0}^{\pi / 2} \frac{1+\cos 2 \theta}{2} d \theta=\frac{1}{2} \theta+\left.\frac{1}{4} \operatorname{sen} 2 \theta\right|_{0} ^{\pi / 2} \\
G_{(2)}=\frac{1}{2}\left(\frac{\pi}{2}-0\right)+\frac{1}{4}(\operatorname{sen} \pi-\operatorname{sen} 0)=\frac{\pi}{4}
\end{gathered}
$$

342. Encontrar para cuáles valores de $p$ converge la integral $\int_{1}^{\infty} \frac{d x}{x^{p}}$. Averiguar el valor de la
integral cuando sea convergente.

\section{Solución:}

Se tiene que:

$$
\begin{gathered}
\int_{1}^{\infty} \frac{d x}{x^{p}}=\lim _{b \rightarrow \infty} \int_{1}^{b} \frac{d x}{x^{p}}=\left.\lim _{b \rightarrow \infty} \frac{x^{-p+1}}{-p+1}\right|_{1} ^{b} \\
\int_{1}^{\infty} \frac{d x}{x^{p}}=\frac{1}{1-p} \lim _{b \rightarrow \infty}\left[\frac{1}{b^{p-1}}-1\right]
\end{gathered}
$$

La integral será convergente cuando $p>1$ y su valor será $\frac{1}{1-p}[0-1]=\frac{1}{p-1}$.

Si $p<1$, la integral será divergente.

$$
\begin{aligned}
\text { Si } p=1, & \int_{1}^{\infty} \frac{d x}{x}=\lim _{b \rightarrow \infty} \int_{1}^{b} \frac{d x}{x}=\left.\lim _{b \rightarrow \infty} \ln x\right|_{1} ^{b} \\
& \int_{1}^{\infty} \frac{d x}{x}=\lim _{b \rightarrow \infty}(\ln b-0) \text { no es finito. }
\end{aligned}
$$

Por lo tanto, es divergente para $\mathrm{p} \leq 1$. 
343. Determinar si la integral impropia $\int_{-\infty}^{\infty} \frac{d x}{e^{x}+e^{-x}}$ converge o diverge.

Solución:

Escribamos:

$$
\frac{1}{e^{x}+e^{-x}}=\frac{e^{x}}{e^{2 x}+1}
$$

Entonces:

$$
\int \frac{d x}{e^{x}+e^{-x}}=\int \frac{e^{x}}{e^{2 x}+1} d x
$$

Si $u=e^{x} \Rightarrow d u=e^{x} d x$ :

$$
\int \frac{d x}{e^{x}+e^{-x}}=\int \frac{d u}{u^{2}+1}=\arctan u+C=\arctan e^{x}+C
$$

De donde:

$$
\begin{gathered}
\int_{-\infty}^{\infty} \frac{d x}{e^{x}+e^{-x}}=\int_{-\infty}^{0} \frac{d x}{e^{x}+e^{-x}}+\int_{0}^{\infty} \frac{d x}{e^{x}+e^{-x}} \\
\int_{-\infty}^{\infty} \frac{d x}{e^{x}+e^{-x}}=\lim _{b \rightarrow-\infty} \int_{b}^{0} \frac{d x}{e^{x}+e^{-x}}+\lim _{b^{\prime} \rightarrow \infty} \int_{0}^{b^{\prime}} \frac{d x}{e^{x}+e^{-x}} \\
\int_{-\infty}^{\infty} \frac{d x}{e^{x}+e^{-x}}=\lim _{b \rightarrow-\infty}\left(\arctan e^{0}-\arctan e^{b}\right)+\lim _{b^{\prime} \rightarrow \infty}\left(\arctan e^{b^{\prime}}-\arctan e^{0}\right) \\
\int_{-\infty}^{\infty} \frac{d x}{e^{x}+e^{-x}}=\lim _{b \rightarrow-\infty}\left(\frac{\pi}{4}-\arctan e^{b}\right)+\lim _{b^{\prime} \rightarrow \infty}\left(\arctan e^{b^{\prime}}-\frac{\pi}{4}\right)
\end{gathered}
$$

Con lo cual:

$$
\int_{-\infty}^{\infty} \frac{d x}{e^{x}+e^{-x}}=\left(\frac{\pi}{4}-0\right)+\left(\frac{\pi}{2}-\frac{\pi}{4}\right)=\frac{\pi}{2}
$$

La integral dada converge al valor $\pi / 2$.

344. Analizar si $\int_{0}^{1} \frac{3 \operatorname{sen}^{2} x+4 \cos ^{2} x}{\sqrt{x(1-x)}} d x$ converge.

\section{Solución:}

Se tiene que para todo $x \in R$ (por ende, para $0<x<1$ ) se cumple que: 


$$
0<\frac{3 \operatorname{sen}^{2} x+4 \cos ^{2} x}{\sqrt{x(1-x)}}<\frac{7}{\sqrt{x(1-x)}}
$$

Según el criterio de comparación directa, $\int_{0}^{1} \frac{3 \operatorname{sen}^{2} x+4 \cos ^{2} x}{\sqrt{x(1-x)}} d x$ será convergente siempre y cuando $\int_{0}^{1} \frac{7 d x}{\sqrt{x(1-x)}}$ lo sea.

Analicemos $\int_{0}^{1} \frac{7 d x}{\sqrt{x(1-x)}}$ reescribiéndolo como $7 \int_{0}^{1} \frac{1}{x} \sqrt{\frac{x}{1-x}} d x$.

Hagamos un cambio de variable:

$$
\begin{gathered}
t=\sqrt{\frac{x}{1-x}} \Rightarrow t \sqrt{1-x}=\sqrt{x} \Rightarrow t^{2}(1-x)=x \\
\frac{1-x}{x}=\frac{1}{x}-1=\frac{1}{t^{2}} \Rightarrow \frac{1}{x}=1+\frac{1}{t^{2}}=\frac{1+t^{2}}{t^{2}} \Rightarrow x=\frac{t^{2}}{t^{2}+1} \\
x=\frac{t^{2}+1-1}{t^{2}+1}=1-\frac{1}{t^{2}+1} \Rightarrow d x=\frac{2 t}{\left(t^{2}+1\right)^{2}} d t
\end{gathered}
$$

Si $x=0$, entonces $t=0$ y si $x=1$, entonces $t \rightarrow \infty$.

De donde:

$$
\begin{aligned}
& 7 \int_{0}^{1} \frac{1}{x} \sqrt{\frac{x}{1-x}} d x=7 \int_{0}^{\infty}\left(\frac{1+t^{2}}{t^{2}}\right)(t)\left(\frac{2 t}{\left(t^{2}+1\right)^{2}}\right) d t \\
& 7 \int_{0}^{1} \frac{1}{x} \sqrt{\frac{x}{1-x}} d x=14 \int_{0}^{\infty} \frac{d t}{1+t^{2}}=14 \lim _{b \rightarrow \infty} \int_{0}^{b} \frac{d t}{1+t^{2}}
\end{aligned}
$$

$$
7 \int_{0}^{1} \frac{1}{x} \sqrt{\frac{x}{1-x}} d x=\left.14 \lim _{b \rightarrow \infty} \arctan t\right|_{0} ^{b}=14 \lim _{b \rightarrow \infty}(\arctan b-0)=14\left(\frac{\pi}{2}\right)=7 \pi
$$

Como $\int_{0}^{1} \frac{7 d x}{\sqrt{x(1-x)}}$ es convergente, la integral impropia $\int_{0}^{1} \frac{3 \operatorname{sen}^{2} x+4 \cos ^{2} x}{\sqrt{x(1-x)}} d x$ será convergente. 
345. Analizar la convergencia de la siguiente integral impropia:

$$
\int_{1}^{\infty} \frac{x^{3}+2}{x^{6}+1} d x
$$

\section{Solución:}

Consideremos $f_{(x)}=\frac{x^{3}+2}{x^{6}+1}$ y $g_{(x)}=\frac{x^{3}}{x^{6}}=\frac{1}{x^{3}}$, ambas funciones positivas y continuas en $[1, \infty[$.

Calculemos:

$$
L=\lim _{x \rightarrow \infty} \frac{f_{(x)}}{g_{(x)}}=\lim _{x \rightarrow \infty} \frac{\frac{x^{3}+2}{x^{6}+1}}{\frac{1}{x^{3}}}=\lim _{x \rightarrow \infty} \frac{x^{6}+2 x^{3}}{x^{6}+1}=\lim _{x \rightarrow \infty} \frac{1+\frac{2}{x^{3}}}{1+\frac{1}{x^{6}}}=1
$$

Como $0<\mathrm{L}<\infty, \quad \int_{1}^{\infty} \frac{1}{x^{3}} d x$ y $\int_{1}^{\infty} \frac{x^{3}+2}{x^{6}+1} d x$ son integrales convergentes porque $\int_{1}^{\infty} \frac{1}{x^{3}} d x$ es convergente.

346. Calcular la siguiente integral impropia $\int_{x=0}^{+\infty} x e^{-x} d x$.

R.: 1

347. Si se sabe que la integral $\int_{2}^{\infty}\left(\frac{c x}{x^{2}+1}-\frac{1}{2 x+1}\right) d x$ es convergente, encontrar el valor de $\mathrm{y}$ el de la integral.
R.: $\frac{1}{2}$ y $\frac{1}{4} \ln \frac{5}{4}$.

348. Si $\int_{a}^{\infty} \frac{d x}{(1+x)^{3 / 2}}=\frac{2 \sqrt{5}}{5}$, encuentre $a$.

R.: 4

349. ¿Para qué valor de $k$, la integral impropia $\int_{0}^{+\infty}\left(\frac{2 x}{x^{2}+1}-\frac{k}{x+1}\right) d x$ converge a cero?

R.: 2

350. Halle el valor de la siguiente integral impropia: $\int_{1}^{2} x \ln (x-1) d x$.

$$
\text { R.: }-5 / 4
$$


351. Si $k>0$, determine el valor de $\int_{0}^{+\infty} k e^{-k x} d x+\int_{1}^{+\infty} \frac{k}{x^{k+1}} d x$.

R.: 2

352. ¿Para qué valor de $k$ la integral impropia $\int_{0}^{+\infty}\left(\frac{3 x^{2}}{x^{3}+1}-\frac{k}{x+1}\right) d x$ converge a cero?

R.: 3

353. Si la integral impropia $\int_{0}^{\infty} a e^{-a x} d x$ es convergente, ¿cómo debe ser a?

R.: $a>0$

354. Si $g_{(n)}=\int_{0}^{1 / a}\left(\ln \left[\frac{1}{a x}\right]\right)^{n-1} d x, a>0$, hallar $g_{\left(\frac{1}{2}\right)}$. Sugerencia: hacer la sustitución $u=\ln \left(\frac{1}{a x}\right)$.

R.: $\sqrt{\pi} / a$

308 355. Encontrar: $\int_{0}^{\infty} x^{p} e^{-2 x} d x \quad(p>0)$.

R.: $\frac{p !}{2^{p+1}}$

356. Calcular: $\int_{0}^{3} \frac{d x}{(x-1)^{2 / 3}}$.

R.: $3+3 \sqrt[3]{2}$

357. Calcular: $\int_{1}^{5} \frac{d x}{x-2}$.

R.: Diverge

358. Calcular: $\int_{-8}^{1} \frac{d x}{x^{1 / 3}}$.

R.: $\frac{-9}{2}$

359. Calcular: $\int_{-\infty}^{0} \theta e^{\theta} d \theta$.

R.: -1 
360. Encontrar el valor de $\int_{0}^{1} \frac{\theta+1}{\sqrt{\theta^{2}+2 \theta}} d \theta$.

R.: $\sqrt{3}$

361. Calcular: $\int_{-\infty}^{-2} \frac{2 d x}{x^{2}-1}$.

R.: $\ln 3$

362. Encontrar: $\int_{0}^{\infty} \frac{d x}{\sqrt{x} e^{\sqrt{x}}}+\int_{-\infty}^{\infty} x e^{-x^{2}} d x$.

R.: 2

363. Determinar si la integral $\int_{0}^{\ln 2} x^{-2} e^{1 / x} d x$ es convergente 0 divergente.

R.: Divergente

364. ¿Cuál de las dos integrales es divergente?
a. $\int_{0}^{\infty} \frac{d x}{\sqrt{e^{x}}}$
b. $\int_{1}^{\infty} \ln x d x$

R.: b

365. ¿Son divergentes $\int_{-1}^{1} \frac{d x}{x^{2}}$ y $\int_{-\infty}^{0} \frac{d x}{x+3}$ ?

R.: Sí

366. ¿Es convergente la integral $\int_{0}^{1} \frac{(\ln x)^{2}}{x} d x$ ?

R.: No

367. ¿Converge o diverge $\int_{-2}^{0} \frac{d x}{2 x+1}$ ?

R.: Diverge 
368. Dada $\int_{-\infty}^{-1} \frac{e^{x}}{x} d x$, pruebe que la integral converge. Compárela con $y=e^{x}$.

369. Estudiar la convergencia de $\int_{2}^{\infty} \frac{x^{2}-1}{\sqrt{x^{6}+16}} d x$, comparándola con $f_{(x)}=\frac{1}{x}$ para valores grandes de $x$.

R.: Diverge

370. ¿Es $\int_{0}^{\pi} \frac{1-\cos x}{x^{2}} d x$ una integral impropia? Calcularla.

R.: No. $1 / 2$. 


\section{PROBLEMAS RESUELTOS Y PROPUESTOS DEL CAPÍTULO VII}

371. Calcular el área limitada por la parábola $(x-2)^{2}=y-1$, la tangente a la parábola en el punto de abscisa igual a $\mathbf{3}$ y el eje de ordenadas.

\section{Solución:}

La ordenada del punto de tangencia $\left(3, y_{0}\right)$ es $y_{0}=(3-2)^{2}+1=2$.

La pendiente de la recta $L$ tangente a la parábola es:

$$
\left.m\right|_{(3,2)}=\left.y^{\prime}\right|_{(3,2)}=\left.2(x-2)\right|_{(3,2)}=2(3-2)=2
$$

Como $(3,2)$ es un punto de la recta tangente $y=2 x+b$ a la parábola, entonces: $2=2(3)+$ $b \Rightarrow b=-4 \Rightarrow \mathrm{L}: y=2 x-4$.

El gráfico es:

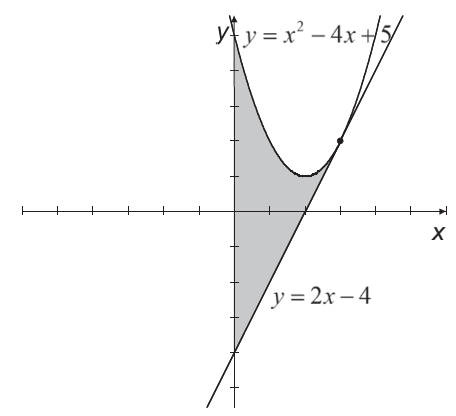

El área sombreada es:

$$
\begin{aligned}
& A=\int_{0}^{3}\left[(x-2)^{2}+1-(2 x-4)\right] d x \\
& A=\int_{0}^{3}\left[x^{2}-4 x+4+1-2 x+4\right] d x \\
& A=\int_{0}^{3}\left[x^{2}-6 x+9\right] d x \\
& A=\int_{0}^{3}(x-3)^{2} d x \\
& A=\left[\frac{(x-3)^{3}}{3}\right]_{0}^{3}=\left[\frac{(3-3)^{3}}{3}\right]-\left[\frac{(0-3)^{3}}{3}\right]=9 \mathrm{u}^{2}
\end{aligned}
$$


372. Calcular el área encerrada por las curvas: $y=\ln x, y=\ln ^{2} x$.

\section{Solución:}

Hallemos los puntos de intersección de las curvas: $\left\{\begin{array}{l}y=\ln x \\ y=\ln ^{2} x\end{array}\right.$

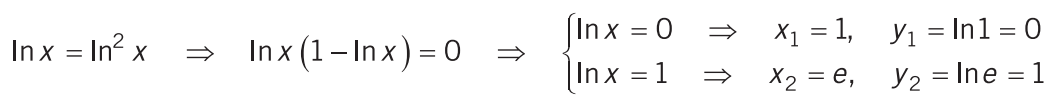

El gráfico es:

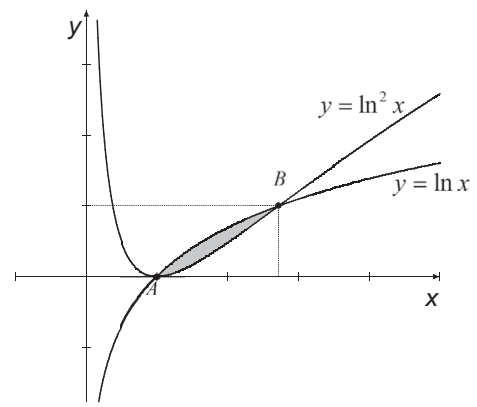

312

El área encerrada por las curvas está dada por:

$$
A=\int_{x_{A}}^{x_{B}}\left[\ln x-\ln ^{2} x\right] d x=\int_{1}^{e}\left[\ln x-\ln ^{2} x\right] d x
$$

Hallemos $I=\int\left(\ln x-\ln ^{2} x\right) d x$ :

$$
\begin{aligned}
I & =-\int\left[\ln ^{2} x-\ln x\right] d x \\
& =-\int\left[(\ln x-1 / 2)^{2}-1 / 4\right] d x \\
& =-\int(\ln x-1 / 2)^{2} d x+1 / 4 \int d x \\
& =-\int(\ln x-1 / 2)^{2} d x+x / 4
\end{aligned}
$$

Calculemos por partes:

$$
I_{1}=-\int(\ln x-1 / 2)^{2} d x:\left\{\begin{array}{l}
u=\left(\ln x-\frac{1}{2}\right)^{2} \Rightarrow d u=2\left(\ln x-\frac{1}{2}\right) \frac{1}{x} d x \\
d v=d x \Rightarrow v=x
\end{array}\right.
$$




$$
\begin{aligned}
& I_{1}=x\left(\ln x-\frac{1}{2}\right)^{2}-2 \int\left(\ln x-\frac{1}{2}\right) d x, \quad\left\{\begin{array}{c}
u=\left(\ln x-\frac{1}{2}\right) \Rightarrow d u=\frac{1}{x} d x \\
d v=d x \Rightarrow v=x
\end{array}\right. \\
& I_{1}=x\left(\ln x-\frac{1}{2}\right)^{2}-2\left[x\left(\ln x-\frac{1}{2}\right)-\int d x\right] \\
& I_{1}=x\left(\ln x-\frac{1}{2}\right)^{2}-2 x\left(\ln x-\frac{1}{2}\right)+2 x+C
\end{aligned}
$$

De (2) en (1):

$$
\begin{gathered}
I=-x\left(\ln x-\frac{1}{2}\right)^{2}+2 x\left(\ln x-\frac{1}{2}\right)-2 x+\frac{x}{4}+C \\
I=-x\left[\ln ^{2} x-\ln x+\frac{1}{4}-2 \ln x+1+2-\frac{1}{4}\right]+C \\
I=-x\left[\ln ^{2} x-3 \ln x+3\right]+C
\end{gathered}
$$

Entonces:

$$
\begin{gathered}
A=\left[-x\left(\ln ^{2} x-3 \ln x+3\right)\right]_{1}^{e} \\
A=\left[-e\left(\ln ^{2} e-3 \ln e+3\right)\right]-\left[-1\left(\ln ^{2} 1-3 \ln 1+3\right)\right] \\
A=[-e(1-3+3)]+[0-0+3] \\
A=3-e \quad u^{2}
\end{gathered}
$$

373. La región limitada por la curva: $4 y=8 x-x^{2}$ y el eje de abscisas es dividida en dos partes iguales por una recta que pasa por el origen de coordenadas. Hallar la ecuación de dicha recta.

\section{Solución:}

Los puntos de intersección de la parábola con el eje $\boldsymbol{x}$ son:

$$
\left\{\begin{array}{rl}
4 y & =8 x-x^{2} \\
y & =0
\end{array} \Rightarrow 8 x-x^{2}=0 \Rightarrow x(8-x)=0 \Rightarrow \begin{cases}x_{1}=0, & y_{1}=0 \\
x_{2}=8, & y_{2}=0\end{cases}\right.
$$

La abscisa del vértice de la parábola es $h=\frac{x_{1}+x_{2}}{2}=\frac{0+8}{2}=4$ y la ordenada: $k=\frac{8(4)-4^{2}}{4}=4$.

Sea $y=m x$ la ecuación de la recta que pasa por el origen de coordenadas y que divide a la región limitada por la parábola y el eje $x$ en dos regiones de igual área: 


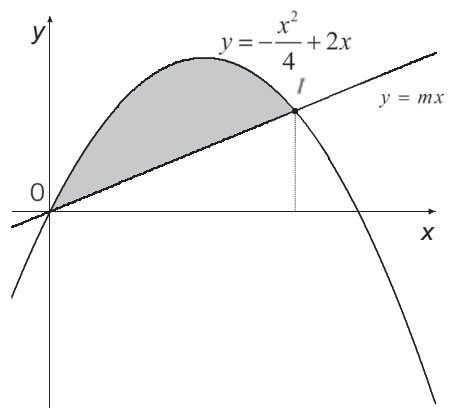

Debemos hallar las coordenadas del punto de intersección I $\left(x_{0}, y_{0}\right)$ de la recta con la parábola:

$\left\{\begin{array}{l}y=2 x-\frac{x^{2}}{4} \\ y=m x\end{array} \Rightarrow 2 x-\frac{x^{2}}{4}=m x \Rightarrow x=0 \vee 2-\frac{x}{4}=m \Rightarrow\left\{\begin{array}{l}x_{1}=0, \quad y_{1}=0 \\ x_{2}=4(2-m), \quad y_{2}=4 m(2-m)\end{array}\right.\right.$

Por lo tanto, la abscisa de / es $x^{\circ}=4(2-m)$

314 El área de la región sombreada es:

$$
\begin{gathered}
A_{1}=\int_{0}^{4(2-m)}\left[2 x-\frac{x^{2}}{4}-m x\right] d x \\
A_{1}=\int_{0}^{4(2-m)}\left[(2-m) x-\frac{x^{2}}{4}\right] d x \\
A_{1}=\left[(2-m) \cdot \frac{x^{2}}{2}-\frac{x^{3}}{12}\right]_{0}^{4(2-m)} \\
A_{1}=(2-m) \cdot \frac{16(2-m)^{2}}{2}-\frac{64(2-m)^{3}}{12} \\
A_{1}=\left[8-\frac{16}{3}\right](2-m)^{3} \\
A_{1}=\frac{8}{3}(2-m)^{3}
\end{gathered}
$$

El área de la región limitada por la parábola y el eje $x$ es:

$$
A=\int_{0}^{8}\left[2 x-\frac{x^{2}}{4}\right] d x=\left[x^{2}-\frac{x^{3}}{12}\right]_{0}^{8}=64-\frac{8^{3}}{12}=64-\frac{128}{3}=\frac{192-128}{3}=\frac{64}{3} u^{2}
$$


Se debe cumplir que $A_{1}=\frac{1}{2} A$, entonces:

$$
\begin{aligned}
\frac{8}{3}(2-m)^{3} & =\frac{32}{3} \\
(2-m)^{3} & =4 \\
2-m & =\sqrt[3]{4} \\
m & =2-\sqrt[3]{4}
\end{aligned}
$$

La ecuación de la recta es $y=(2-\sqrt[3]{4}) x$.

374. Calcular el área comprendida entre las curvas $y=e^{x}, y=\ln x y$ las rectas: $x=-1 ; x=2 ; y=0$.

\section{Solución:}

El gráfico correspondiente es:

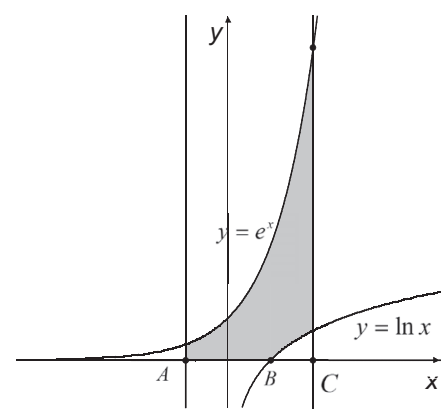

El área pedida está dada por:

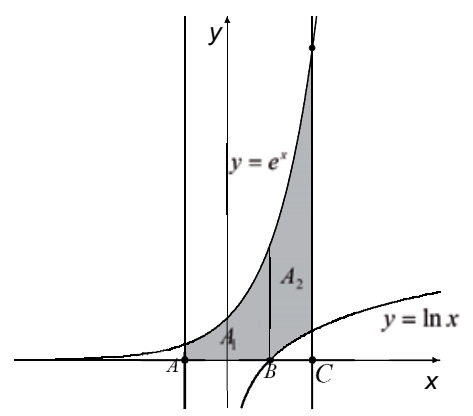

$$
\begin{gathered}
A=A_{1}+A_{2} \\
A=\int_{-1}^{1} e^{x} d x+\int_{1}^{2}\left[e^{x}-\ln x\right] d x
\end{gathered}
$$




$$
\begin{gathered}
A=\left[e^{x}\right]_{-1}^{1}+\left[e^{x}-x(\ln x-1)\right]_{1}^{2} \\
A=e^{1}-e^{-1}+\left[e^{2}-2(\ln 2-1)\right]-\left[e^{1}-1(\ln 1-1)\right] \\
A=e-\frac{1}{e}+e^{2}-2 \ln 2+2-e+0-1 \\
A=e^{2}-\frac{1}{e}-2 \ln 2+1 \mathrm{u}^{2}
\end{gathered}
$$

375. Calcular el área encerrada por las curvas: $y=\cos x$ e $y=-1+\operatorname{sen} x$ entre $x=0$ y $x=2 \pi$.

\section{Solución:}

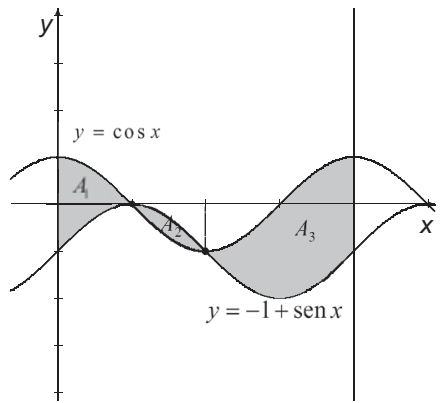

El área pedida es:

$$
\begin{gathered}
A=A_{1}+A_{2} \\
A=\int_{0}^{\pi / 2}[\cos x-(-1+\operatorname{sen} x)] d x+\int_{\pi / 2}^{\pi}[-1+\operatorname{sen} x-(\cos x)] d x+\int_{\pi}^{2 \pi}[\cos x-(-1+\operatorname{sen} x)] d x \\
A=[\operatorname{sen} x+x+\cos x]_{0}^{\pi / 2}+[-x-\cos x-\operatorname{sen} x]_{\pi / 2}^{\pi}+[\operatorname{sen} x+x+\cos x]_{\pi}^{2 \pi} \\
A=\left[\operatorname{sen} \frac{\pi}{2}+\frac{\pi}{2}+\cos \frac{\pi}{2}\right]-[\operatorname{sen} 0+0+\cos 0]+[-\pi-\cos \pi-\operatorname{sen} \pi]-\left[-\frac{\pi}{2}-\cos \frac{\pi}{2}-\operatorname{sen} \frac{\pi}{2}\right]+\ldots \\
+[\operatorname{sen} 2 \pi+2 \pi+\cos 2 \pi]-[\operatorname{sen} \pi+\pi+\cos \pi] \\
A=\left[1+\frac{\pi}{2}+0\right]-[0+0+1]+[-\pi+1-0]-\left[-\frac{\pi}{2}-0-1\right]+[0+2 \pi+1]-[0+\pi-1] \\
A=4+\pi u^{2}
\end{gathered}
$$


376. Calcular el área de la figura limitada por las curvas: $y=\frac{\ln x}{4 x}$ e $y=x \ln x$.

\section{Solución:}

$$
\begin{aligned}
& \text { Hallemos los puntos de intersección de las curvas: }\left\{\begin{array}{l}
y=\frac{\ln x}{4 x} \\
y=x \ln x
\end{array}\right. \\
& \frac{\ln x}{4 x}=x \ln x \Rightarrow \ln x=0 \vee \frac{1}{4 x}=x \Rightarrow \begin{cases}\ln x=0, & x_{1}=e^{0}=1, \quad y_{1}=0 \\
x^{2}=\frac{1}{4}, & x= \pm \frac{1}{2}, \quad x_{2}=\frac{1}{2}, \quad y_{2}=\frac{1}{2} \ln \frac{1}{2}\end{cases}
\end{aligned}
$$

El gráfico es:

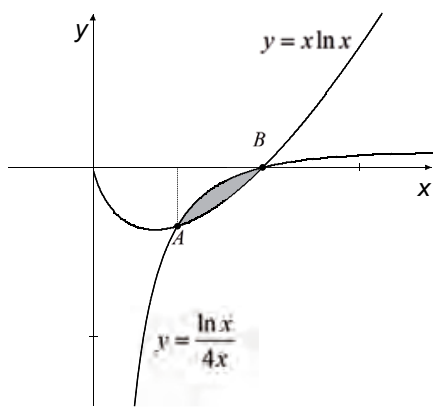

El área sombreada es:

$$
A=\int_{X_{A}}^{x_{B}}\left[\frac{\ln x}{4 x}-x \ln x\right] d x=\int_{0,5}^{1}\left[\frac{\ln x}{4 x}-x \ln x\right] d x
$$

Calculemos:

$$
I=\int \frac{\ln x}{4 x} d x=\frac{1}{4} \int \stackrel{4}{\ln x} \cdot \frac{d u}{\frac{1}{x}} d x=\frac{1}{4}\left[\frac{u^{2}}{2}\right]+C=\frac{\ln ^{2} x}{8}+C .
$$

Además:

$$
J=\int x \ln x d x=\left\{\begin{array}{lll}
u=\ln x & \Rightarrow & d u=\frac{1}{x} d x \\
d v=x d x & \Rightarrow & v=\frac{x^{2}}{2}
\end{array} \Rightarrow J=\frac{x^{2} \ln x}{2}-\frac{1}{2} \int x d x=\frac{x^{2} \ln x}{2}-\frac{x^{2}}{4}+C\right.
$$


De manera que el área pedida es:

$$
\begin{gathered}
A=\left[\frac{\ln x}{8}-\frac{x^{2} \ln x}{2}+\frac{x^{2}}{4}\right]_{0,5}^{1} \\
A=\left[\frac{\ln ^{2} 1}{8}-\frac{1^{2} \ln 1}{2}+\frac{1^{2}}{4}\right]-\left[\frac{\ln { }^{2} 0,5}{8}-\frac{0,5^{2} \ln 0,5}{2}+\frac{0,5^{2}}{4}\right] \\
A=\left[0+0+\frac{1}{4}\right]-\left[\frac{\ln ^{2} 0,5}{8}-\frac{\ln 0,5}{8}+\frac{1}{16}\right] \\
A=\frac{\ln 0,5}{8}-\frac{\ln ^{2} 0,5}{8}+\frac{3}{16} \mathrm{u}^{2}
\end{gathered}
$$

377. Calcular el área limitada por las curvas:

$$
\begin{aligned}
& y=\ln (x+2) \\
& y=2 \ln x
\end{aligned}
$$

y por el eje de las abscisas.

\section{Solución:}

318

Hallemos los puntos de intersección de las curvas: $\left\{\begin{array}{l}y=\ln (x+2) \\ y=2 \ln x\end{array}\right.$

$$
\begin{aligned}
& \ln (x+2)=2 \ln x \Rightarrow \ln (x+2)=\ln x^{2} \Rightarrow x^{2}-x-2=0 \Rightarrow(x-2)(x+1)=0 \\
& \quad \Rightarrow \quad x=2
\end{aligned}
$$

El único punto de intersección es $(2, \ln 4)$.

El gráfico es:

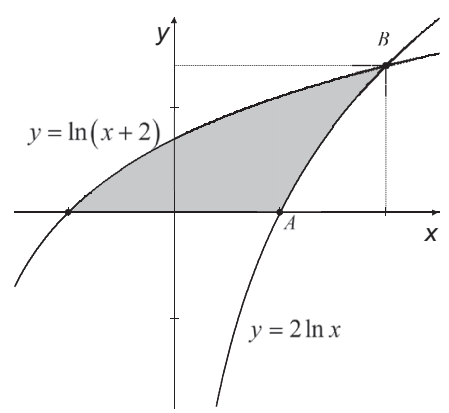


Calculemos el área sombreada integrando a lo largo del eje $y$ :

$$
\begin{aligned}
& y=\ln (x+2) \Rightarrow x+2=e^{y} \Rightarrow \underset{y}{x}=e^{y}-2 \\
& y=2 \ln x \Rightarrow \frac{y}{2}=\ln x \Rightarrow x=e^{\frac{y}{2}}
\end{aligned}
$$

El área está dada por:

$$
\begin{gathered}
A=\int_{y_{A}}^{y_{B}}\left[e^{\frac{y}{2}}-\left(e^{y}-2\right)\right] d y \\
A=\int_{0}^{\ln 4}\left[e^{\frac{y}{2}}-\left(e^{y}-2\right)\right] d y \\
A=\int_{0}^{\ln 4}\left[e^{\frac{y}{2}}-e^{y}+2\right] d y \\
A=\left[2 e^{\frac{y}{2}}-e^{y}+2 y\right]_{0}^{\ln 4} \\
\left.2 e^{\frac{\ln 4}{2}}-e^{\ln 4}+2(\ln 4)\right]-\left[2 e^{0}-e^{0}+2(0)\right] \\
A=2 e^{\ln 2}-4+2 \ln 4-2+1 \\
A=2(2)-4+2 \ln 4-2+1 \\
A=2 \ln 4-1 u^{2}
\end{gathered}
$$

378. Calcular el área encerrada en el primer cuadrante por las rectas:

$$
\begin{aligned}
& y=2 x \\
& 2 y=x
\end{aligned}
$$

y la parábola: $x^{2}=3-y$

\section{Solución:}

Hallemos los puntos de intersección de cada recta con la parábola: $\left\{\begin{array}{l}y=2 x \\ y=3-x^{2}\end{array}\right.$

$3-x^{2}=2 x \Rightarrow x^{2}+2 x-3=0 \Rightarrow(x+3)(x-1)=0 \Rightarrow\left\{\begin{array}{l}x_{1}=-3, y_{1}=-6 \\ x_{2}=1, y_{2}=2\end{array}\right.$

$\left\{\begin{array}{l}y=\frac{x}{2} \\ y=3-x^{2}\end{array}\right.$

$3-x^{2}=\frac{x}{2} \Rightarrow 2 x^{2}+x-6=0 \Rightarrow(2 x-3)(x+2)=0 \Rightarrow\left\{\begin{array}{l}x_{1}=\frac{3}{2}, y_{1}=\frac{3}{4} \\ x_{2}=-2, y_{2}=-1\end{array}\right.$ 
El gráfico respectivo es:

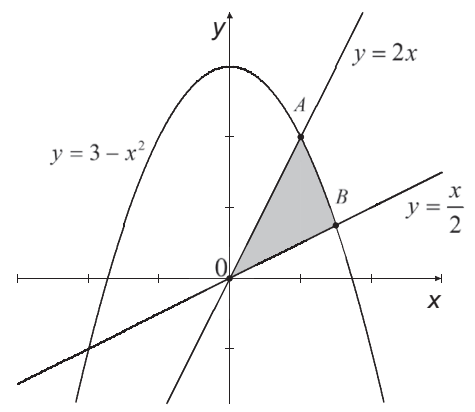

El área sombreada está dada por:

$$
\begin{gathered}
A=\int_{0}^{x_{A}}\left[2 x-\frac{x}{2}\right] d x+\int_{x_{A}}^{x_{B}}\left[3-x^{2}-\frac{x}{2}\right] d x \\
A=\int_{0}^{1}\left[\frac{3 x}{2}\right] d x+\int_{1}^{1,5}\left[3-x^{2}-\frac{x}{2}\right] d x
\end{gathered}
$$

320

Es decir:

$$
\begin{gathered}
A=\left[\frac{3}{4} x^{2}\right]_{0}^{1}+\left[3 x-\frac{1}{3} x^{3}-\frac{1}{4} x^{2}\right]_{1}^{1,5} \\
A=\left[\frac{3}{4}(1)^{2}\right]+\left[3\left(\frac{3}{2}\right)-\frac{1}{3}\left(\frac{3}{2}\right)^{3}-\frac{1}{4}\left(\frac{3}{2}\right)^{2}\right]-\left[3(1)-\frac{1}{3}(1)^{3}-\frac{1}{4}(1)^{2}\right] \\
A=\frac{3}{4}+\frac{9}{2}-\frac{9}{8}-\frac{9}{16}-3+\frac{1}{3}+\frac{1}{4} \\
A=\frac{55}{48} \mathrm{u}^{2}
\end{gathered}
$$

379. Encontrar el área de la región limitada por las curvas:

$$
\begin{gathered}
y=x^{3}-6 x^{2}+8 x \\
y=x^{2}-4 x
\end{gathered}
$$

Solución:

Hallemos los puntos de intersección de las curvas:

$$
\begin{gathered}
\left\{\begin{array}{l}
y=x^{3}-6 x^{2}+8 x \\
y=x^{2}-4 x
\end{array}\right. \\
x^{3}-6 x^{2}+8 x=x^{2}-4 x, \quad x^{3}-7 x^{2}+12 x=0, \quad x\left(x^{2}-7 x+12\right)=0, \quad x(x-3)(x-4)=0
\end{gathered}
$$




$$
\begin{cases}x_{1}=0, & y_{1}=0 \\ x_{2}=3, & y_{2}=-3 \\ x_{3}=4, & y_{3}=0\end{cases}
$$

El gráfico es:

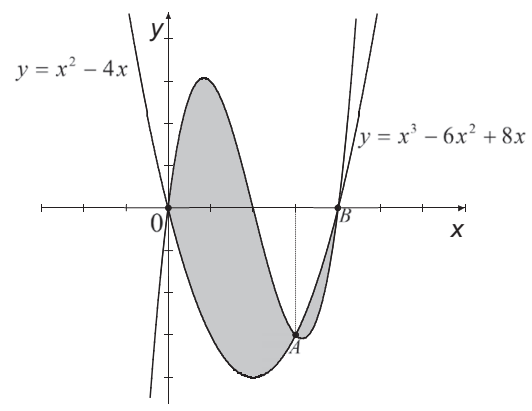

El área encerrada por las curvas es:

$$
\begin{gathered}
A=A_{1}+A_{2} \\
A=\int_{0}^{x_{A}}\left[x^{3}-6 x^{2}+8 x-\left(x^{2}-4 x\right)\right] d x+\int_{x_{A}}^{x_{B}}\left[x^{2}-4 x-\left(x^{3}-6 x^{2}+8 x\right)\right] d x \\
A=\int_{0}^{3}\left[x^{3}-6 x^{2}+8 x-\left(x^{2}-4 x\right)\right] d x+\int_{3}^{4}\left[x^{2}-4 x-\left(x^{3}-6 x^{2}+8 x\right)\right] d x \\
A=\int_{0}^{3}\left[x^{3}-7 x^{2}+12 x\right] d x+\int_{3}^{4}\left[-x^{3}+7 x^{2}-12 x\right] d x \\
A=\left[\frac{1}{4} x^{4}-\frac{7}{3} x^{3}+6 x^{2}\right]_{0}^{3}+\left[-\frac{1}{4} x^{4}+\frac{7}{3} x^{3}-6 x^{2}\right]_{3}^{4} \\
A=\left[\frac{1}{4} \cdot 81-63+54\right]+\left[-64+\frac{7}{3} \cdot 64-96\right]-\left[-\frac{1}{4} \cdot 81+63-54\right] \\
A=\frac{1}{2} \cdot 81+\frac{7}{3} \cdot 64-18-160 \\
A=\frac{71}{6} u^{2}
\end{gathered}
$$

380. Encontrar el área encerrada por las curvas:

$$
\begin{gathered}
x=y^{2} \\
x^{2}=-8 y
\end{gathered}
$$




\section{Solución:}

Hallemos los puntos de intersección de las curvas: $\left\{\begin{array}{c}x=y^{2} \\ x^{2}=-8 y\end{array}\right.$

$$
\left(y^{2}\right)^{2}=-8 y \Rightarrow y^{4}+8 y=0 \Rightarrow y\left(y^{3}+8\right)=0 \Rightarrow \begin{cases}y_{1}=0, & x_{1}=0 \\ y_{2}=-2, & x_{2}=4\end{cases}
$$

El gráfico correspondiente es:

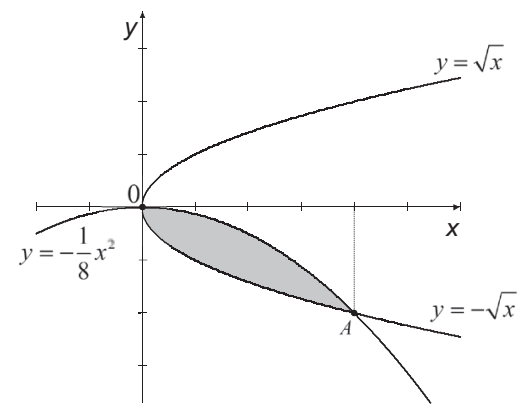

El área sombreada es:

$$
\begin{gathered}
A=\int_{0}^{4}\left[-\frac{1}{8} x^{2}-(-\sqrt{x})\right] d x \\
A=\int_{0}^{4}\left[-\frac{1}{8} x^{2}+\sqrt{x}\right] d x \\
A=\left[-\frac{1}{24} x^{3}+\frac{2}{3} \sqrt{x^{3}}\right]_{0}^{4} \\
A=\left[-\frac{1}{24} \cdot 4^{3}+\frac{2}{3} \sqrt{4^{3}}\right] \\
A=-\frac{8}{3}+\frac{16}{3} \\
A=\frac{8}{3} u^{2}
\end{gathered}
$$

381. Calcular el área encerrada por las curvas:

$$
\begin{gathered}
x y=1 \\
x y=4 \\
x-x y=1
\end{gathered}
$$

y por la recta: $y=x$. 


\section{Solución:}

Las ecuaciones de las curvas que limitan la región son:

$$
\begin{aligned}
y & =\frac{1}{x} \\
y & =\frac{4}{x} \\
y=\frac{x-1}{x} \Rightarrow y=1-\frac{1}{x} & \Rightarrow x
\end{aligned}
$$

El gráfico respectivo es:

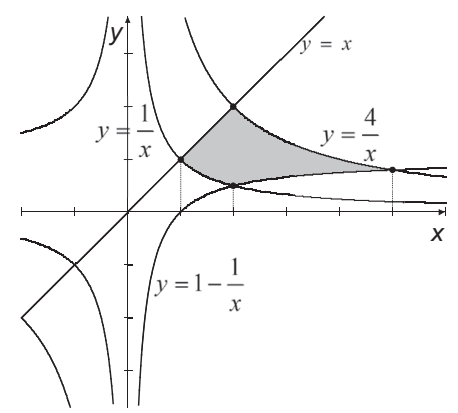

El área sombreada es:

$$
\begin{gathered}
A=A_{1}+A_{2} \\
A=\int_{1}^{2}\left[x-\frac{1}{x}\right] d x+\int_{2}^{5}\left[\frac{4}{x}-\left(1-\frac{1}{x}\right)\right] d x \\
A=\left[\frac{1}{2} x^{2}-\ln (x)\right]_{1}^{2}+\int_{2}^{5}\left[\frac{5}{x}-1\right] d x \\
A=[2-\ln 2]-\left[\frac{1}{2}-0\right]+[5 \ln x-x]_{2}^{5} \\
A=2-\ln 2-\frac{1}{2}+[5 \ln 5-5]-[5 \ln 2-2] \\
A=-\frac{3}{2}-6 \ln 2+5 \ln 5 \\
A=\ln \frac{5^{5}}{2^{6}}-\frac{3}{2} \mathrm{u}^{2}
\end{gathered}
$$


382. Calcular el área encerrada por las curvas $y=e^{x} ; y=e^{-x}$ y la recta $x=1$.

\section{Solución:}

Hallemos los puntos de intersección de las curvas: $\left\{\begin{array}{l}y=e^{x} \\ y=e^{-x}\end{array}\right.$

$$
e^{x}=e^{-x} \Rightarrow e^{2 x}=1 \Rightarrow x_{1}=0, \quad y_{1}=e^{0}=1
$$

El punto de intersección de la recta $x=1$ con la curva $y=e^{x}$ es $(1, e)$

El punto de intersección de la recta $x=1$ con la curva $y=e^{-x}$ es $\left(1, e^{-1}\right)$

Grafiquemos las curvas:

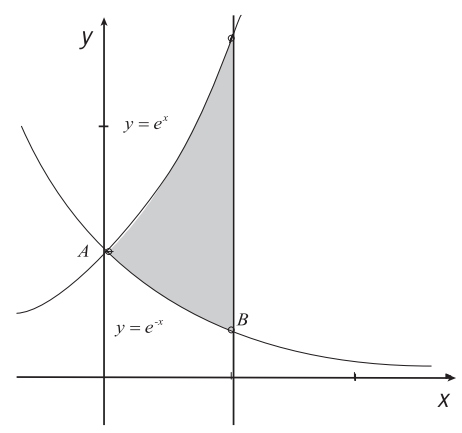

El área sombreada es:

$$
\begin{gathered}
A=\int_{x_{A}}^{x_{B}}\left[e^{x}-e^{-x}\right] d x \\
A=\int_{0}^{1}\left[e^{x}-e^{-x}\right] d x \\
A=\left[e^{x}+e^{-x}\right]_{0}^{1} \\
A=\left[e+e^{-1}\right]-\left[e^{0}+e^{0}\right] \\
A=e+\frac{1}{e}-2 u^{2}
\end{gathered}
$$


383. Calcular el área encerrada por la parábola: $y^{2}=x+1$ y la recta: $x-y-1=0$

\section{Solución:}

Hallemos los puntos de intersección de las curvas: $\left\{\begin{array}{r}y^{2}=x+1 \\ y=x-1\end{array}\right.$

$$
\begin{gathered}
(x-1)^{2}=x+1 \Rightarrow x^{2}-2 x+1=x+1 \\
x^{2}-3 x=0 \Rightarrow x(x-3)=0 \\
\left\{\begin{array}{lll}
x_{1}=0 & \Rightarrow y_{1}=-1 \\
x_{2}=3 & \Rightarrow y_{2}=2
\end{array}\right.
\end{gathered}
$$

Grafiquemos:

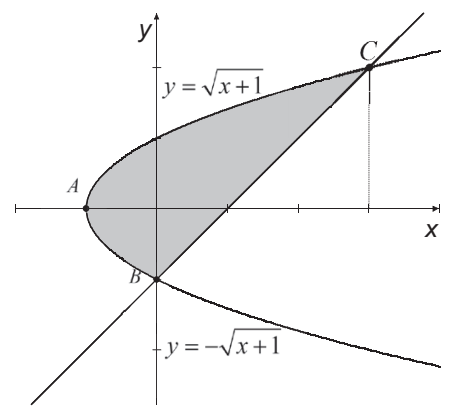

El área sombreada está dada por:

$$
\begin{gathered}
A=\int_{x_{A}}^{x_{B}}[\sqrt{x+1}-(-\sqrt{x+1})] d x+\int_{x_{B}}^{x_{C}}[\sqrt{x+1}-(x-1)] d x \\
A=\int_{-1}^{0}[\sqrt{x+1}-(-\sqrt{x+1})] d x+\int_{0}^{3}[\sqrt{x+1}-(x-1)] d x \\
A=\int_{-1}^{0}[2 \sqrt{x+1}] d x+\int_{0}^{3}[\sqrt{x+1}-x+1] d x \\
A=\left[\frac{4}{3}(x+1)^{\frac{3}{2}}\right]_{-1}^{0}+\left[\frac{2}{3}(x+1)^{\frac{3}{2}}-\frac{1}{2} x^{2}+x\right]_{0}^{3} \\
A=\left[\frac{4}{3}\right]+\left[\frac{2}{3} \cdot 8-\frac{9}{2}+3\right]-\left[\frac{2}{3}\right] \\
A=\frac{9}{2} u^{2}
\end{gathered}
$$


384. Calcular el área encerrada por las curvas: $y=x^{2}, y=\frac{1}{3} x^{3}$.

\section{Solución:}

Hallemos los puntos de intersección de las curvas: $\left\{\begin{array}{l}y=x^{2} \\ y=\frac{1}{3} x^{3}\end{array}\right.$

$$
x^{2}=\frac{1}{3} x^{3} \Rightarrow 3 x^{2}=x^{3} \Rightarrow x^{2}(3-x)=0 \Rightarrow \begin{cases}x_{1}=0, & y_{1}=0 \\ x_{2}=3, & y_{2}=9\end{cases}
$$

Grafiquemos las curvas:

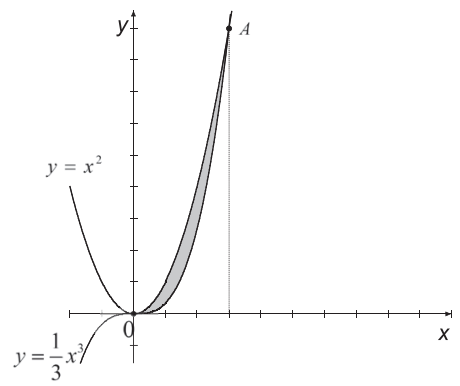

El área de la región encerrada por las curvas es:

$$
\begin{gathered}
A=\int_{0}^{3}\left[x^{2}-\frac{1}{3} x^{3}\right] d x \\
A=\left[\frac{1}{3} x^{3}-\frac{1}{12} x^{4}\right]_{0}^{3} \\
A=\left[\frac{1}{3} \cdot 27-\frac{1}{12} \cdot 81\right] \\
A=\frac{9}{4} u^{2}
\end{gathered}
$$

385. Calcular el área de la figura comprendida entre las curvas:

$$
\begin{gathered}
y=\frac{1}{x^{2}+1} \\
y=\frac{x^{2}}{2}
\end{gathered}
$$




\section{Solución:}

Hallemos los puntos de intersección de las curvas: $\left\{\begin{array}{l}y=\frac{1}{x^{2}+1} \\ y=\frac{1}{2} x^{2}\end{array}\right.$

$$
\begin{aligned}
& \frac{1}{x^{2}+1}=\frac{1}{2} x^{2} \Rightarrow 2=x^{4}+x^{2} \Rightarrow x^{4}+x^{2}-2=0 \Rightarrow\left(x^{2}+2\right)\left(x^{2}-1\right)=0 \Rightarrow \\
& \left\{\begin{array}{l}
x_{1}=-1, \quad y_{1}=\frac{1}{2} \\
x_{2}=1, \quad y_{2}=\frac{1}{2}
\end{array}\right.
\end{aligned}
$$

Grafiquemos las curvas:

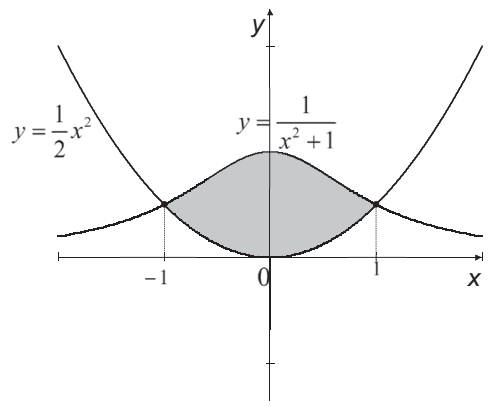

El área de la región limitada por las curvas es:

$$
A=\int_{-1}^{1}\left[\frac{1}{x^{2}+1}-\frac{1}{2} x^{2}\right] d x
$$

que por la simetría de la figura con relación al eje $y$, equivale a:

$$
\begin{gathered}
A=2 \int_{0}^{1}\left[\frac{1}{x^{2}+1}-\frac{1}{2} x^{2}\right] d x \\
A=2\left[\arctan x-\frac{1}{6} x^{3}\right]_{0}^{1} \\
A=2\left[\frac{\pi}{4}-\frac{1}{6}\right] \\
A=\frac{\pi}{2}-\frac{1}{3} \mathrm{u}^{2}
\end{gathered}
$$


386. Calcular el área encerrada por las curvas:

$$
\begin{aligned}
& y=\ln x \\
& y=\ln ^{2} x
\end{aligned}
$$

\section{Solución:}

Hallemos los puntos de intersección de las curvas: $\left\{\begin{array}{l}y=\ln x \\ y=\ln ^{2} x\end{array}\right.$

$$
\ln x=\ln ^{2} x \Rightarrow \ln ^{2} x-\ln x=0 \Rightarrow \ln x(\ln x-1)=0 \Rightarrow \begin{cases}x_{1}=1, & y_{1}=0 \\ x_{2}=e, & y_{2}=1\end{cases}
$$

Grafiquemos las ecuaciones:

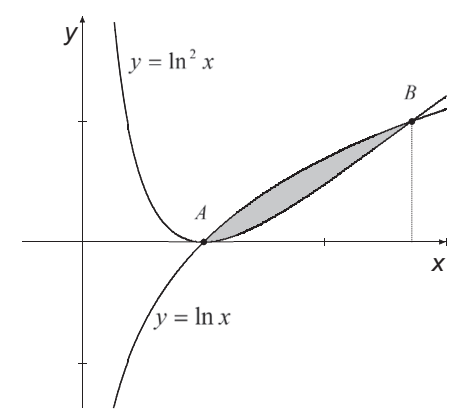

Calculemos el área de la región limitada por las curvas:

$$
\begin{aligned}
& A=\int_{x_{A}}^{x_{B}}\left[\ln x-\ln ^{2} x\right] d x \\
& A=\int_{1}^{e}\left[\ln x-\ln ^{2} x\right] d x
\end{aligned}
$$

Evaluemos:

$$
\begin{gathered}
\int \ln ^{2} x d x=\left\{\begin{array}{c}
u=\ln ^{2} x \Rightarrow d u=2 \ln x \cdot \frac{1}{x} d x \\
d v=d x \Rightarrow v=x
\end{array}\right. \\
\int \ln ^{2} x d x=x \ln ^{2} x-2 \int \ln x d x \quad(1) \\
\int \ln x d x=\left\{\begin{array}{l}
u=\ln x \quad \Rightarrow \quad d u=\frac{1}{x} d x \\
d v=d x \Rightarrow v=x
\end{array}\right. \\
\int \ln x d x=x \ln x-\int d x \\
\int \ln x d x=x \ln x-x
\end{gathered}
$$


De (2) en (1):

$$
\int \ln ^{2} x d x=x \ln ^{2} x-2 x \ln x+2 x
$$

De manera que:

$$
\begin{aligned}
& A=\left[x \ln x-x-x \ln ^{2} x+2 x \ln x-2 x\right]_{1}^{e} \\
& A=\left[3 x \ln x-x \ln ^{2} x-3 x\right]_{1}^{e} \\
& A=[3 e-e-3 e]-[-3] \\
& A=3-e u^{2}
\end{aligned}
$$

387. Calcular el área encerrada por las rectas y/o curvas siguientes:
a) $y=x^{3}+3 x^{2}+2 ; y=x^{3}+6 x^{2}-25$
b) $y=x^{2} ; y=2 x+1 ; y=x+2$

\section{Solución:}

a) Hallemos los puntos de intersección de las curvas: $\left\{\begin{array}{l}y=x^{3}+3 x^{2}+2 \\ y=x^{3}+6 x^{2}-25\end{array}\right.$
$x^{3}+3 x^{2}+2=x^{3}+6 x^{2}-25 \Rightarrow 3 x^{2}=27 \Rightarrow x^{2}=9 \Rightarrow \begin{cases}x_{1}=-3, & y_{1}=(-3)^{3}+3(-3)^{2}+2=2 \\ x_{2}=3, & y_{2}=3^{3}+3 \cdot 3^{2}+2=56\end{cases}$

Grafiquemos:

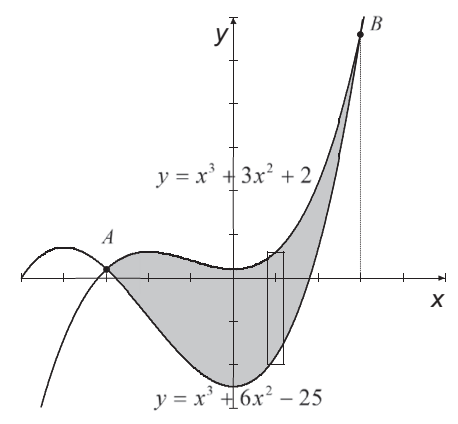

El área solicitada es: 


$$
\begin{aligned}
& A=\int_{-3}^{3}\left[x^{3}+3 x^{2}+2-\left(x^{3}+6 x^{2}-25\right)\right] d x \\
& A=\int_{-3}^{3}\left[-3 x^{2}+27\right] d x \\
& A=\left[-x^{3}+27 x\right]_{-3}^{3} \\
& A=\left[-3^{3}+27 \cdot 3\right]-\left[-(-3)^{3}+27 \cdot(-3)\right] \\
& A=54+54 \\
& A=108 \mathrm{u}^{2}
\end{aligned}
$$

b) Hallemos los puntos de intersección de las curvas: $\left\{\begin{array}{l}y=x^{2} \\ y=x+2\end{array}\right.$

$$
x^{2}=x+2 \Rightarrow x^{2}-x-2=0 \Rightarrow(x-2)(x+1)=0 \Rightarrow \begin{cases}x_{1}=2, & y_{1}=2+2=4 \\ x_{2}=-1, & y_{2}=-1+2=1\end{cases}
$$

El gráfico correspondiente es:

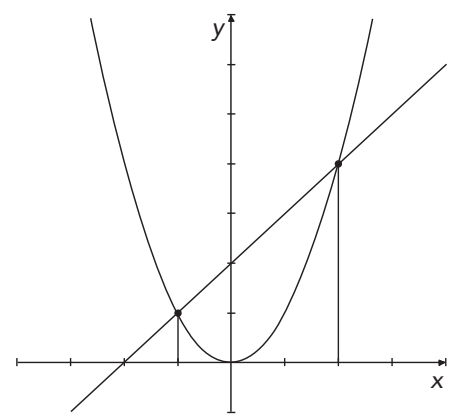

Si añadimos el gráfico de $y=2 x+1$ y sombreamos la región limitada por las dos rectas y la curva, obtenemos:

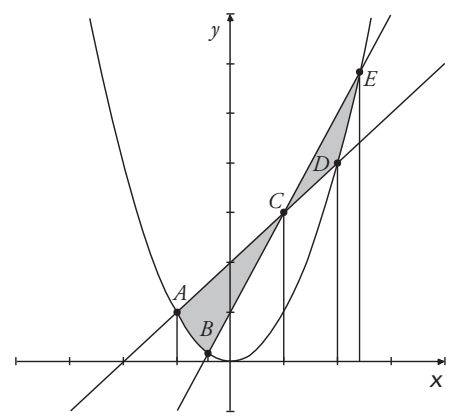


Para calcular el área sombreada $A_{1}$ comprendida entre $x=x_{A}=-1$ y $x=x_{C}=-1$, dividamos la región de integración en dos partes:

$$
\begin{aligned}
& A_{1}=\int_{x_{A}}^{x_{B}}\left[x+2-\left(x^{2}\right)\right] d x+\int_{x_{B}}^{x_{C}}[x+2-(2 x+1)] d x \\
& A_{1}=\int_{-1}^{x_{B}}\left[x+2-x^{2}\right] d x+\int_{x_{B}}^{1}[-x+1] d x \\
& A_{1}=\left[\frac{x^{2}}{2}+2 x-\frac{x^{3}}{3}\right]_{-1}^{x_{B}}+\left[-\frac{x^{2}}{2}+x\right]_{x_{B}}^{1} \\
& A_{1}=\left[\frac{x_{B}^{2}}{2}+2 x_{B}-\frac{x_{B}^{3}}{3}\right]-\left[\frac{(-1)^{2}}{2}+2(-1)-\frac{(-1)^{3}}{3}\right]+\left[-\frac{1^{2}}{2}+1\right]-\left[-\frac{x_{B}^{2}}{2}+x_{B}\right] \\
& A_{1}=x_{B}^{2}+x_{B}-\frac{x_{B}^{3}}{3}-\frac{1}{2}+2-\frac{1}{3}-\frac{1}{2}+1 \\
& A_{1}=x_{B}^{2}+x_{B}-\frac{x_{B}^{3}}{3}+\frac{5}{3}
\end{aligned}
$$

Como $B$ es el punto de intersección de la parábola $y=x^{2}$ con la recta $y=2 x+1$ :

$x^{2}=2 x+1 \Rightarrow x^{2}-2 x-1=0 \Rightarrow(x-1)^{2}-2=0 \Rightarrow x-1= \pm \sqrt{2} \Rightarrow x=1 \pm \sqrt{2}$

Entonces $x_{B}=1-\sqrt{2}, x_{E}=1+\sqrt{2}$. Si reemplazamos:

$$
\begin{aligned}
& A_{1}=x_{B}^{2}+x_{B}-\frac{x_{B}^{3}}{3}+\frac{5}{3} \\
& A_{1}=(1-\sqrt{2})^{2}+(1-\sqrt{2})-\frac{(1-\sqrt{2})^{3}}{3}+\frac{5}{3} u^{2} \\
& A_{1}=1+2-2 \sqrt{2}+1-\sqrt{2}-\frac{(1-\sqrt{2})^{3}}{3}+\frac{5}{3} u^{2} \\
& A_{1}=\frac{17}{3}-3 \sqrt{2}-\frac{(1-\sqrt{2})^{3}}{3} u^{2}
\end{aligned}
$$

El área sombreada $A_{2}$ comprendida entre $x=x_{C}=1$ y $x=x_{E}=1+\sqrt{2}$, es igual a:

$$
\begin{aligned}
& A_{2}=\int_{x_{C}}^{x_{D}}[2 x+1-(x+2)] d x+\int_{x_{D}}^{x_{E}}\left[2 x+1-\left(x^{2}\right)\right] d x \\
& A_{2}=\int_{1}^{2}[x-1] d x+\int_{2}^{1+\sqrt{2}}\left[2 x+1-x^{2}\right] d x
\end{aligned}
$$




$$
\begin{aligned}
& A_{2}=\left[\frac{x^{2}}{2}-x\right]_{1}^{2}+\left[x^{2}+x-\frac{x^{3}}{3}\right]_{2}^{1+\sqrt{2}} \\
& A_{2}=\left[\frac{2^{2}}{2}-2\right]-\left[\frac{1^{2}}{2}-1\right]+\left[(1+\sqrt{2})^{2}+(1+\sqrt{2})-\frac{(1+\sqrt{2})^{3}}{3}\right]-\left[2^{2}+2-\frac{2^{3}}{3}\right] \\
& A_{2}=\frac{1}{2}+1+2+2 \sqrt{2}+1+\sqrt{2}-\frac{(1+\sqrt{2})^{3}}{3}-\frac{10}{3} \\
& A_{2}=\frac{7}{6}+3 \sqrt{2}-\frac{(1+\sqrt{2})^{3}}{3} \mathrm{u}^{2}
\end{aligned}
$$

De manera que el área sombreada total es:

$$
\begin{aligned}
& A_{T}=A_{1}+A_{2} \\
& A_{T}=\frac{17}{3}-3 \sqrt{2}-\frac{(1-\sqrt{2})^{3}}{3}+\frac{7}{6}+3 \sqrt{2}-\frac{(1+\sqrt{2})^{3}}{3} \\
& A_{T}=\frac{41}{6}-\frac{1}{3}\left[(1-\sqrt{2})^{3}+(1+\sqrt{2})^{3}\right] \\
& A_{T}=\frac{41}{6}-\frac{1}{3}[2]\left[(1-\sqrt{2})^{2}-(1-\sqrt{2})(1+\sqrt{2})+(1+\sqrt{2})^{2}\right] \\
& A_{T}=\frac{41}{6}-\frac{2}{3}\left[2\left(1+\sqrt{2}^{2}\right)-(1-2)\right] \\
& A_{T}=\frac{41}{6}-\frac{2}{3}[7] \\
& A_{T}=\frac{13}{6} \mathrm{u}^{2}
\end{aligned}
$$

388. Encontrar el área encerrada por las rectas y curvas siguientes:

a) $y=x^{2} ; y^{3}=x ; x+y=2$

b) $y=|x-2| ; y+x^{2}=0 ; x=1 ; x=3$

\section{Solución:}

a) $y=x^{2}, y^{3}=x, x+y=2$

Los puntos de intersección de las curvas $y=x^{2}, y^{3}=x$ son:

$$
\left(x^{2}\right)^{3}=x \Rightarrow x^{6}-x=0 \Rightarrow x\left(x^{5}-1\right)=0 \Rightarrow \begin{cases}x_{1}=0, & y_{1}=0 \\ x_{2}=1, & y_{2}=1\end{cases}
$$


Los puntos de intersección de $y=x^{2}$ y $y=2-x$ son:

$2-x=x^{2} \Rightarrow x^{2}+x-2=0 \Rightarrow(x+2)(x-1)=0 \Rightarrow \begin{cases}x_{3}=-2, & y_{3}=2-(-2)=4 \\ x_{4}=1, & y_{4}=2-1=1\end{cases}$

Los puntos de intersección de $y^{3}=x$ y $y=2-x$ y son:

$$
\begin{aligned}
& (2-x)^{3}=x \Rightarrow 8-3(2)^{2} x+3(2) x^{2}-x^{3}=x \Rightarrow x^{3}-6 x^{2}+13 x-8=0 \Rightarrow \\
& (x-1)\left(x^{2}-5 x+8\right)=0 \Rightarrow x_{5}=1, \quad y_{5}=2-1=1
\end{aligned}
$$

El gráfico correspondiente es:

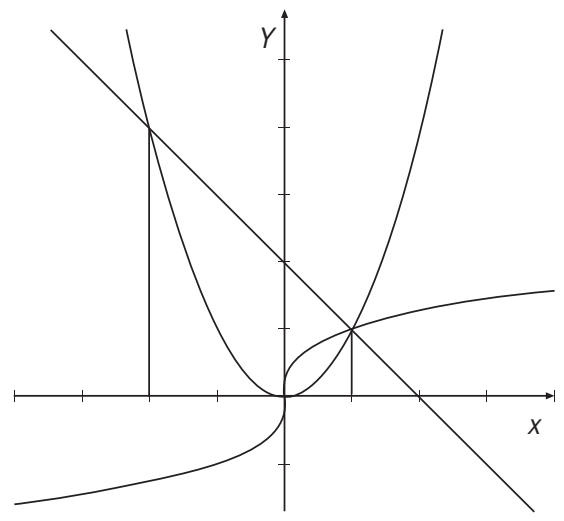

La región limitada por las curvas y la recta es:

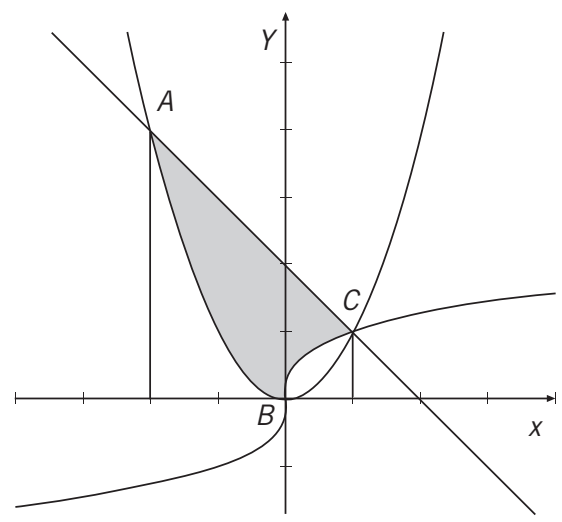


El área de dicha región es:

$$
\begin{aligned}
& A=\int_{x_{A}}^{x_{B}}\left[2-x-\left(x^{2}\right)\right] d x+\int_{x_{B}}^{x_{C}}[2-x-(\sqrt[3]{x})] d x \\
& A=\int_{-2}^{0}\left[2-x-x^{2}\right] d x+\int_{0}^{1}\left[2-x-x^{\frac{1}{3}}\right] d x \\
& A=\left[2 x-\frac{x^{2}}{2}-\frac{x^{3}}{3}\right]_{-2}^{0}+\left[2 x-\frac{x^{2}}{2}-\frac{3}{4} x^{\frac{4}{3}}\right]_{0}^{1} \\
& A=[0]-\left[2(-2)-\frac{(-2)^{2}}{2}-\frac{(-2)^{3}}{3}\right]+\left[2(1)-\frac{(1)^{2}}{2}-\frac{3}{4}(1)^{\frac{4}{3}}\right]-[0] \\
& A=4+2-\frac{8}{3}+2-\frac{1}{2}-\frac{3}{4} \\
& A=\frac{49}{12} \mathrm{u}^{2}
\end{aligned}
$$

b) $y=|x-2| ; y+x^{2}=0 ; x=1 ; x=3$

334

Por definición: $y=|x-2|=\left\{\begin{array}{ll}-(x-2), & x-2<0 \\ (x-2), & x-2 \geq 0\end{array}\right.$, es decir:

$$
\begin{cases}y=-x+2, & x<2 \\ y=x-2, & x \geq 2\end{cases}
$$

Grafiquemos las curvas y la región limitada por ellas:

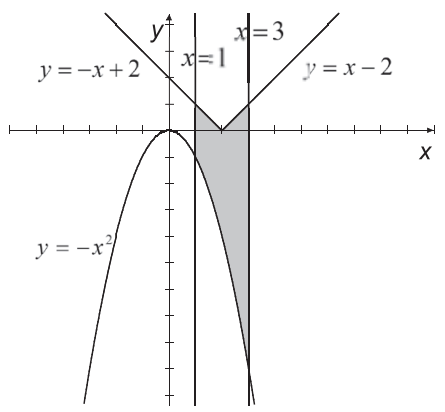

El área sombreada es: 


$$
\begin{aligned}
& A=\int_{1}^{2}\left[-x+2-\left(-x^{2}\right)\right] d x+\int_{2}^{3}\left[x-2-\left(-x^{2}\right)\right] d x \\
& A=\int_{1}^{2}\left[-x+2+x^{2}\right] d x+\int_{2}^{3}\left[x-2+x^{2}\right] d x \\
& A=\left[-\frac{x^{2}}{2}+2 x+\frac{x^{3}}{3}\right]_{1}^{2}+\left[\frac{x^{2}}{2}-2 x+\frac{x^{3}}{3}\right]_{2}^{3} \\
& A=\left[-\frac{(2)^{2}}{2}+2(2)+\frac{(2)^{3}}{3}\right]-\left[-\frac{(1)^{2}}{2}+2(1)+\frac{(1)^{3}}{3}\right]+\left[\frac{(3)^{2}}{2}-2(3)+\frac{(3)^{3}}{3}\right]-\left[\frac{(2)^{2}}{2}-2(2)+\frac{(2)^{3}}{3}\right] \\
& A=\left[-2+4+\frac{8}{3}\right]-\left[-\frac{1}{2}+2+\frac{1}{3}\right]+\left[\frac{9}{2}-6+9\right]-\left[2-4+\frac{8}{3}\right] \\
& A=\frac{14}{3}-\frac{11}{6}+\frac{15}{2}-\frac{2}{3} \\
& A=\frac{29}{3} u^{2}
\end{aligned}
$$

389. Calcular el área encerrada por la curva:

a) $x=a \cdot \cos \theta, y=b \cdot \operatorname{sen} \theta$

b) $x=2 \cos \theta-\cos 2 \theta-1, y=2 \operatorname{sen} \theta-\operatorname{sen} 2 \theta$

\section{Solución:}

a)

$$
\left.\begin{array}{l}
x=a \cdot \cos \theta \Rightarrow x^{2}=a^{2} \cdot \cos ^{2} \theta \Rightarrow \frac{x^{2}}{a^{2}}=\cos ^{2} \theta \\
y=b \cdot \operatorname{sen} \theta \Rightarrow y^{2}=b^{2} \cdot \operatorname{sen}^{2} \theta \Rightarrow \frac{y^{2}}{b^{2}}=\operatorname{sen}^{2} \theta
\end{array}\right\} \Rightarrow \frac{x^{2}}{a^{2}}+\frac{y^{2}}{b^{2}}=\underbrace{\cos ^{2} \theta+\operatorname{sen}^{2} \theta}_{1}
$$

La ecuación corresponde a una elipse con centro en el origen del sistema de coordenadas y semiejes $a$ y $b$. El gráfico siguiente corresponde al caso en que $a>b>0$ :

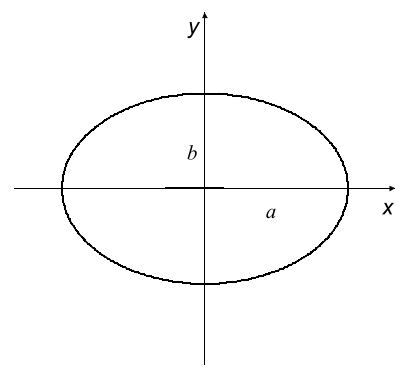


El área encerrada por la curva es cuatro veces el área de la región encerrada por la curva en el primer cuadrante:

$$
A=4 \int_{0}^{a} \frac{b}{a} \sqrt{a^{2}-x^{2}} d x
$$

Si usamos la fórmula de integración inmediata:

$$
\int \sqrt{a^{2}-u^{2}} d u=\frac{u}{2} \sqrt{a^{2}-u^{2}}+\frac{a^{2}}{2} \operatorname{arcsen} \frac{u}{a}+C
$$

Tenemos que:

$$
\begin{gathered}
A=4 \cdot \frac{b}{a}\left[\frac{x}{2} \sqrt{a^{2}-x^{2}}+\frac{a^{2}}{2} \operatorname{arcsen} \frac{x}{a}\right]_{0}^{a} \\
A=4 \cdot \frac{b}{a}\left[\frac{a}{2} \sqrt{a^{2}-a^{2}}+\frac{a^{2}}{2} \operatorname{arcsen} \frac{a}{a}\right]-4 \cdot \frac{b}{a}\left[\frac{0}{2} \sqrt{a^{2}-0^{2}}+\frac{a^{2}}{2} \operatorname{arcsen} \frac{0}{a}\right] \\
A=4 \cdot \frac{b}{a}\left[\frac{a^{2}}{2} \cdot \frac{\pi}{2}\right]-4[0] \\
A=\pi a b \mathrm{u}^{2}
\end{gathered}
$$

b) De las ecuaciones paramétricas $x=2 \cos \theta-\cos 2 \theta-1, y=2 \operatorname{sen} \theta-\operatorname{sen} 2 \theta$ se deduce que:

$$
\begin{aligned}
& x=2 \cos \theta-\left(\cos ^{2} \theta-\operatorname{sen}^{2} \theta\right)-\left(\operatorname{sen}^{2} \theta+\cos ^{2} \theta\right) \Rightarrow x=2 \cos \theta-2 \cos ^{2} \theta \Rightarrow x=2 \cos \theta(1-\cos \theta) \\
& y=2 \operatorname{sen} \theta-2 \operatorname{sen} \theta \cos \theta \Rightarrow y=2 \operatorname{sen} \theta(1-\cos \theta)
\end{aligned}
$$

Si elevamos al cuadrado y sumamos:

$$
\underbrace{x^{2}+y^{2}}_{\rho^{2}}=4 \cos ^{2} \theta(1-\cos \theta)^{2}+4 \operatorname{sen}^{2} \theta(1-\cos \theta)^{2}
$$

De donde:

$$
\begin{aligned}
\rho^{2} & =4(1-\cos \theta)^{2}\left(\cos ^{2} \theta+\operatorname{sen}^{2} \theta\right) \\
\rho^{2} & =4(1-\cos \theta)^{2} \\
\rho & = \pm 2(1-\cos \theta) \\
\rho & =2(1-\cos \theta)
\end{aligned}
$$

La gráfica de esta última ecuación es una cardioide, la gráfica de la otra ecuación (con el signo negativo) es el reflejo en el eje y de la primera gráfica. Si hacemos una tabulación obtenemos: 


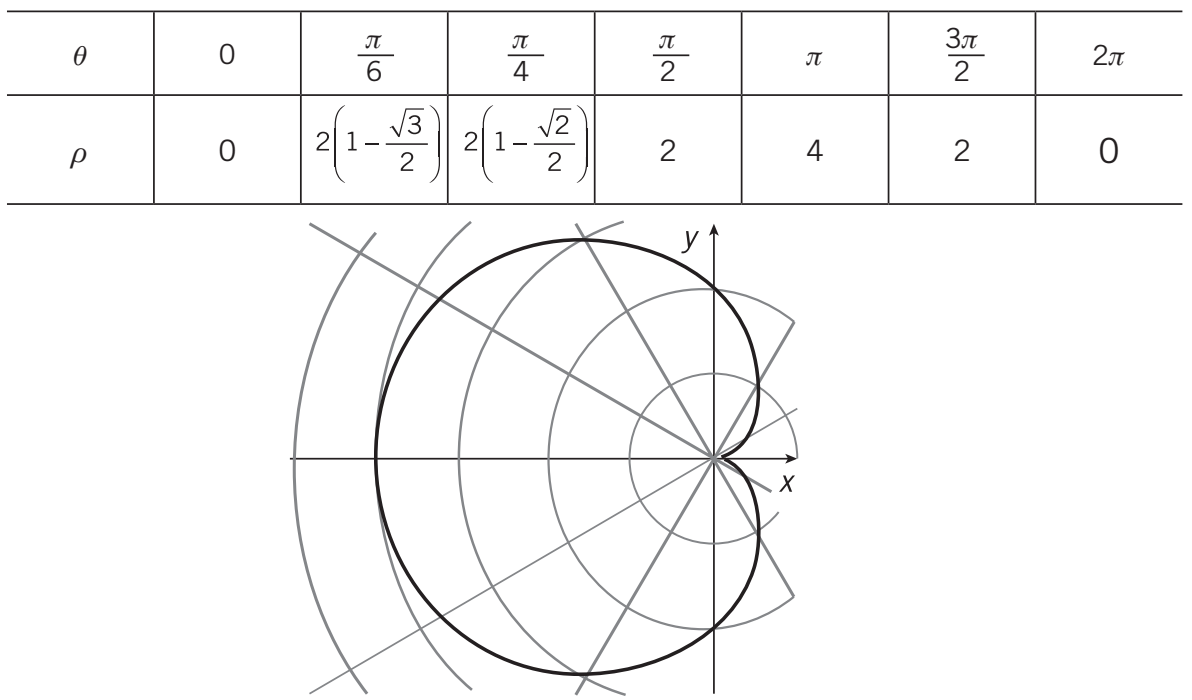

Sea $\rho=\rho(\theta)$ la ecuación de una curva en forma polar, si $\rho(\theta)$ es una función continua en el intervalo $\left[\theta_{1}, \theta_{2}\right]$, entonces el área del sector limitado por la curva y los radio vectores extremos está dada por:

$$
A=\frac{1}{2} \int_{\theta_{1}}^{\theta_{2}} \rho^{2}(\theta) d \theta
$$

Entonces, en el caso considerado, tenemos que:

$$
\begin{aligned}
& A=\frac{1}{2} \int_{0}^{2 \pi} 4(1-\cos \theta)^{2} d \theta \\
& A=2 \int_{0}^{2 \pi}\left(1-2 \cos \theta+\cos ^{2} \theta\right) d \theta
\end{aligned}
$$

Como $\cos ^{2} \theta=\frac{1+\cos 2 \theta}{2}$ :

$$
\begin{aligned}
& A=2 \int_{0}^{2 \pi}\left(1-2 \cos \theta+\frac{1+\cos 2 \theta}{2}\right) d \theta \\
& A=2 \int_{0}^{2 \pi}\left(\frac{3}{2}-2 \cos \theta+\frac{1}{2} \cos 2 \theta\right) d \theta \\
& A=2\left[\frac{3}{2} \theta-2 \operatorname{sen} \theta+\frac{1}{4} \operatorname{sen} 2 \theta\right]_{0}^{2 \pi} \\
& A=2\left[\frac{3}{2}(2 \pi)-2 \operatorname{sen}(2 \pi)+\frac{1}{4} \operatorname{sen}(4 \pi)\right]-2\left[\frac{3}{2}(0)-2 \operatorname{sen} 0+\frac{1}{4} \operatorname{sen} 0\right]
\end{aligned}
$$




$$
\begin{aligned}
& A=2[3 \pi] \\
& A=6 \pi \mathrm{u}^{2}
\end{aligned}
$$

390. Calcular el área encerrada por la curva: $y=\cos 2 x$ y el eje de abscisas, comprendida entre $\mathrm{x}=\pi / 4$ y $\mathrm{x}=\pi$.

\section{Solución:}

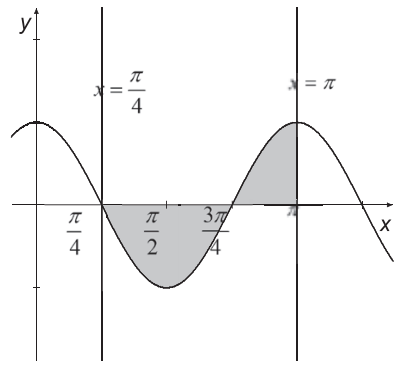

El área sombreada es:

$$
\begin{aligned}
& A=A_{1}+A_{2} \\
& A=\int_{\pi / 4}^{3 \pi / 4}[0-\cos 2 x] d x+\int_{3 \pi / 4}^{\pi}[\cos 2 x] d x \\
& A=\left[-\frac{\operatorname{sen} 2 x}{2}\right]_{\pi / 4}^{3 \pi / 4}+\left[\frac{\operatorname{sen} 2 x}{2}\right]_{3 \pi / 4}^{\pi} \\
& A=\left[-\frac{\operatorname{sen} \frac{3 \pi}{2}}{2}+\frac{\operatorname{sen} \frac{\pi}{2}}{2}\right]+\left[\frac{\operatorname{sen} 2 \pi}{2}-\frac{\operatorname{sen} \frac{3 \pi}{2}}{2}\right] \\
& A=\left[\frac{1}{2}+\frac{1}{2}\right]+\left[0+\frac{1}{2}\right] \\
& A=\frac{3}{2} \mathrm{u}^{2}
\end{aligned}
$$

391. Hallar el área encerrada entre la parábola: $y=-x^{2}+4 x-3$ y las tangentes a esta en los puntos: $(0,-3)$ y $(3,0)$.

\section{Solución:}

La ecuación de la recta tangente a la parábola en el punto $\left(x_{0}, y_{0}\right)$ está dada por:

$$
y-y_{0}=\left.y^{\prime}\right|_{\left(x_{0}, y_{0}\right)} \cdot\left(x-x_{0}\right)
$$


Como $y^{\prime}=-2 x+4$, la ecuación de la tangente a la parábola en el punto $A(0,-3)$ es:

$$
y-(-3)=(-2(0)+4) \cdot(x-0) \Rightarrow \ell_{1}: y=4 x-3
$$

Y la ecuación de la tangente a la parábola en el punto $C(3,0)$ es:

$$
y-0=(-2(3)+4) \cdot(x-3) \Rightarrow \ell_{2}: y=-2 x+6
$$

Las rectas tangentes se cortan en el punto $B$ :

$$
4 x-3=-2 x+6 \Rightarrow 6 x=9 \Rightarrow x_{1}=\frac{3}{2} \Rightarrow y_{1}=-2\left(\frac{3}{2}\right)+6=3 \Rightarrow B\left(\frac{3}{2}, 3\right)
$$

Grafiquemos la parábola, las rectas tangentes y la región limitada por la curva y las rectas:

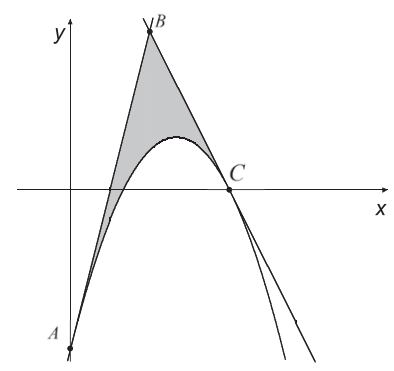

El área sombreada es:

$$
\begin{aligned}
& A=\int_{x_{A}}^{x_{B}}\left[4 x-3-\left(-x^{2}+4 x-3\right)\right] d x+\int_{x_{B}}^{x_{C}}\left[-2 x+6-\left(-x^{2}+4 x-3\right)\right] d x \\
& A=\int_{0}^{\frac{3}{2}}\left[x^{2}\right] d x+\int_{\frac{3}{2}}^{3}\left[(x-3)^{2}\right] d x \\
& A=\left[\frac{x^{3}}{3}\right]_{0}^{\frac{3}{2}}+\left[\frac{(x-3)^{3}}{3}\right]_{\frac{3}{2}}^{3} \\
& A=\left[\frac{(3 / 2)^{3}}{3}\right]-[0]+[0]-\left[\frac{(3 / 2-3)^{3}}{3}\right] \\
& A=\frac{9}{8}+\frac{9}{8} \\
& A=\frac{9}{4} u^{2}
\end{aligned}
$$


392. Calcular el área encerrada por las curvas:

$$
\begin{aligned}
& y=4 x-x^{2} \\
& y=4 x^{2}-x^{3}
\end{aligned}
$$

\section{Solución:}

Hallemos los puntos de intersección de las curvas:

$$
\begin{aligned}
& 4 x-x^{2}=4 x^{2}-x^{3} \Rightarrow x^{3}-5 x^{2}+4 x=0 \Rightarrow x\left(x^{2}-5 x+4\right)=0 \Rightarrow x(x-1)(x-4)=0 \\
& \begin{cases}x_{1}=0, \quad y_{1}=4(0)-(0)^{2}=0 \\
x_{2}=1, \quad y_{2}=4(1)-(1)^{2}=3 \\
x_{3}=4, \quad y_{3}=4(4)-(4)^{2}=0\end{cases}
\end{aligned}
$$

Podemos hallar el área de la región comprendida entre las curvas dadas que corresponden a funciones polinómicas, sin tener que dibujarlas.

Como las curvas se cortan únicamente en puntos de abscisas 0, 1 y 4, entonces las regiones a la izquierda de 0 y a la derecha de 4 son abiertas y, en consecuencia, la región "comprendida entre las curvas" debe ser solamente una parte de la zona $0 \leq x \leq 4$.

Hallemos la diferencia $d=y_{1}-y_{2}$, donde $y_{1}=4 x-x^{2}$ y $y_{2}=4 x^{2}-x^{3}$, es decir:

$$
\begin{aligned}
& d=\left(4 x-x^{2}\right)-\left(4 x^{2}-x^{3}\right) \\
& d=x^{3}-5 x^{2}+4 x \\
& d=x\left(x^{2}-5 x+4\right) \\
& d=x(x-1)(x-4)
\end{aligned}
$$

El signo que tenga $d$ servirá para conocer las posiciones relativas de las curvas: si $d>0$, entonces $y_{1}>y_{2}$ (la curva $y_{1}=4 x-x^{2}$ está por encima de la curva $y_{2}=4 x^{2}-x^{3}$ y si, por el contrario, $d<0$, entonces $y_{1}<y_{2}$ (la curva $y_{1}=4 x-x^{2}$ está por debajo de la curva $y_{2}=$ $\left.4 x^{2}-x^{3}\right)$.

Deducimos entonces que en la región $0<x<1$ : $d=(+)(-)(-)>0$, es decir $y_{1}>y_{2}$, mientras que en la región $1<x<4: d=(+)(+)(-)<0$, entonces $y_{1}<y_{2}$.

De lo anterior podemos estar seguros de que el área de la región comprendida entre las curvas está dada por: 


$$
\begin{aligned}
& A=\int_{0}^{1}\left[y_{1}-y_{2}\right] d x+\int_{1}^{4}\left[y_{2}-y_{1}\right] d x \\
& A=\int_{0}^{1}\left[\left(4 x-x^{2}\right)-\left(4 x^{2}-x^{3}\right)\right] d x+\int_{1}^{4}\left[\left(4 x^{2}-x^{3}\right)-\left(4 x-x^{2}\right)\right] d x \\
& A=\int_{0}^{1}\left[x^{3}-5 x^{2}+4 x\right] d x+\int_{1}^{4}\left[-x^{3}+5 x^{2}-4 x\right] d x \\
& A=\left[\frac{x^{4}}{4}-\frac{5 x^{3}}{3}+2 x^{2}\right]_{0}^{1}+\left[-\frac{x^{4}}{4}+\frac{5 x^{3}}{3}-2 x^{2}\right]_{1}^{4} \\
& A=\left[\frac{1}{4}-\frac{5}{3}+2\right]-[0]+\left[-64+\frac{320}{3}-32\right]-\left[-\frac{1}{4}+\frac{5}{3}-2\right] \\
& A=\frac{1}{4}-\frac{5}{3}+2-96+\frac{320}{3}+\frac{1}{4}-\frac{5}{3}+2 \\
& A=\frac{71}{6} \mathrm{u}^{2}
\end{aligned}
$$

El resultado anterior se puede comprobar si graficamos las curvas:

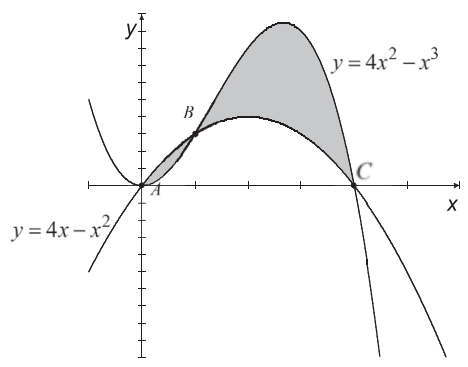

393. Calcular el área comprendida entre las curvas:

$$
\begin{gathered}
y^{2}=x \\
y^{2}=\frac{16}{27}\left(\frac{2 x-1}{2}\right)^{3}
\end{gathered}
$$

\section{Solución:}

Las gráficas de las curvas son simétricas con respecto al eje $x$ porque al sustituir $y$ por $-y$ en cualquiera de las ecuaciones resultan las mismas ecuaciones. La primera ecuación $y^{2}$ $=x$ corresponde a una parábola con vértice en $(0,0)$, cuyo eje de simetría es el eje $x$ y que se abre hacia la derecha $(x \geq 0)$. 
En el caso de la segunda ecuación $y^{2}=\frac{2}{27}(2 x-1)^{3}$, su gráfica se limita a la zona en que $\frac{2}{27}(2 x-1)^{3} \geq 0$ es decir en $x \geq \frac{1}{2}$. Considerando la simetría de la curva analicemos únicamente $y=\frac{\sqrt{2}}{3 \sqrt{3}}(2 x-1)^{\frac{3}{2}}$; se tiene que $y^{\prime}=\frac{\sqrt{2}}{3 \sqrt{3}} \cdot \frac{3}{2}(2 x-1)^{\frac{1}{2}} \cdot 2$, la derivada es igual a 0 únicamente en $x=\frac{1}{2}$ (la tangente a la curva es horizontal en $x=\frac{1}{2}$ ) y es positiva en todo el dominio de la función considerada, entonces la curva correspondiente debe ser creciente y su gráfica es de la forma:

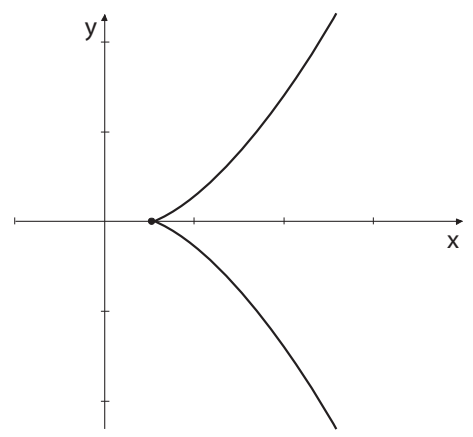

Hallemos los puntos de intersección de las curvas:

$$
\begin{aligned}
x & =\frac{2}{27}(2 x-1)^{3} \\
27 x & =2\left(8 x^{3}-12 x^{2}+6 x-1\right) \\
16 x^{3}-24 x^{2}-15 x-2 & =0
\end{aligned}
$$

Factoricemos el polinomio del primer miembro:

\begin{tabular}{r|rrrr} 
& 16 & -24 & -15 & -2 \\
$-\frac{1}{4}$ & & -4 & 7 & 2 \\
\hline & 16 & -28 & -8 & 0 \\
\hline 2 & & 32 & 8 & \\
\hline & 16 & 4 & 0 &
\end{tabular}


La ecuación anterior se puede escribir en la forma:

$$
\begin{aligned}
\left(x+\frac{1}{4}\right)(x-2)(16 x+4) & =0 \\
(4 x+1)(x-2)(4 x+1) & =0 \\
(4 x+1)^{2}(x-2) & =0
\end{aligned}
$$

Como $x$ solo puede tomar valores no menores que $\frac{1}{2}$, el único valor aceptable es $x=2$, al que le corresponde $y= \pm \sqrt{2}$; así, tenemos los puntos $(2,-\sqrt{2})$ y $(2, \sqrt{2})$ en los que se cortan las curvas.

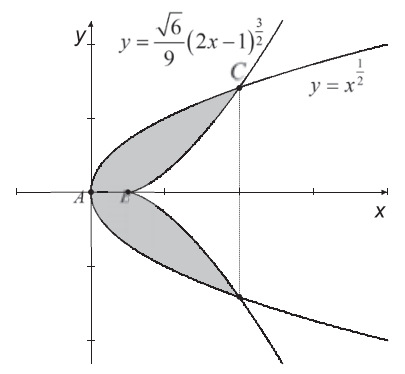

Hallemos el área $A$ de la región sombreada:

$$
\begin{aligned}
\frac{1}{2} A=\int_{x_{A}}^{x_{C}}\left[x^{\frac{1}{2}}\right] d x-\int_{x_{B}}^{x_{C}}\left[\frac{\sqrt{6}}{9}(2 x-1)^{\frac{3}{2}}\right] d x \\
\frac{1}{2} A=\int_{0}^{2}\left[x^{\frac{1}{2}}\right] d x-\int_{\frac{1}{2}}^{2}\left[\frac{\sqrt{6}}{9}(2 x-1)^{\frac{3}{2}}\right] d x \\
\frac{1}{2} A=\left[\frac{2}{3} \cdot x^{\frac{3}{2}}\right]_{0}^{2}-\left[\frac{\sqrt{6}}{9} \cdot \frac{1}{5} \cdot(2 x-1)^{\frac{5}{2}}\right]_{\frac{1}{2}}^{2} \\
\frac{1}{2} A=\left[\frac{2}{3} \cdot 2^{\frac{3}{2}}\right]-[0]-\left[\frac{\sqrt{6}}{45} \cdot(2 \cdot 2-1)^{\frac{5}{2}}\right]+\left[\frac{\sqrt{6}}{45} \cdot\left(2 \cdot \frac{1}{2}-1\right)^{\frac{5}{2}}\right] \\
\frac{1}{2} A=\frac{4}{3} \sqrt{2}-\frac{\sqrt{6}}{45} \cdot 9 \sqrt{3} \\
\frac{1}{2} A=\frac{4}{3} \sqrt{2}-\frac{3}{5} \sqrt{2} \\
A=\frac{22 \sqrt{2}}{15} u^{2}
\end{aligned}
$$


394. Hallar el área de la figura limitada por la curva $y=x(x-1)(x-2)$ y el eje OX.

\section{Solución:}

La curva corta al eje $x$ en los puntos de abscisas 0,1 y 2 únicamente.

La región encerrada por la curva y el eje $x$ debe ser solo una parte de la región $0 \leq x \leq 2$, porque los extremos ( $x \leq 0 \circ x \geq 2)$ corresponden a regiones abiertas.

Analicemos la región de interés: si $0<x<1$, ocurre que $y=(+)(-)(-)>0$, es decir la curva está por encima del eje $x$. Si $1<x<2$, tenemos que $y=(+)(+)(-)<0$; en este caso la curva está por debajo del eje $x$.

En consecuencia, el área de la región encerrada por la curva y el eje $x$ es:

$$
\begin{aligned}
& A=\int_{0}^{1}[y] d x+\int_{1}^{2}[-y] d x \\
& A=\int_{0}^{1}[y] d x-\int_{1}^{2}[y] d x \\
& A=\int_{0}^{1}\left[x^{3}-3 x^{2}+2 x\right] d x-\int_{1}^{2}\left[x^{3}-3 x^{2}+2 x\right] d x
\end{aligned}
$$

344

$$
\begin{aligned}
& A=\left[\frac{x^{4}}{4}-x^{3}+x^{2}\right]_{0}^{1}-\left[\frac{x^{4}}{4}-x^{3}+x^{2}\right]_{1}^{2} \\
& A=\left[\frac{1}{4}-1+1\right]-[0]-[4-8+4]+\left[\frac{1}{4}-1+1\right] \\
& A=\frac{1}{4}+\frac{1}{4} \\
& A=\frac{1}{2} \mathrm{u}^{2}
\end{aligned}
$$

El resultado obtenido se puede comprobar al graficar la curva $y=x^{3}-3 x^{2}+2 x$ :

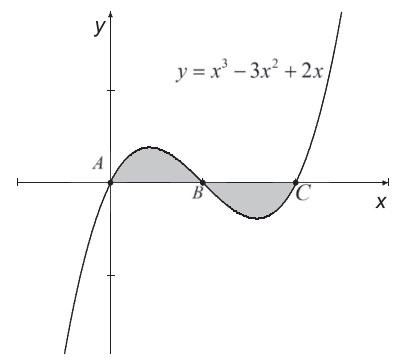


395. Hallar el área comprendida entre las curvas:

$$
\begin{gathered}
y^{2}=x^{3} \\
y^{2}=x
\end{gathered}
$$

\section{Solución:}

El gráfico de $y^{2}=x$ es una parábola con vértice $(0,0)$, con el eje $x$ como eje de simetría y que se abre hacia la derecha $(x>0)$.

El gráfico de $y^{2}=x^{3}$ es simétrico con respecto al eje $x$ y solo existe representación en $x^{3} \geq 0$, es decir en $x \geq 0$. Si consideramos solo la rama positiva de la curva: $y=x^{\frac{3}{2}}, y^{\prime}=\frac{3}{2} x^{\frac{1}{2}}$, vemos que la derivada es 0 (tangente horizontal) solo en $x=0$ y que es positiva (función creciente) para todo $x>0$. Entonces el gráfico de $y^{2}=x^{3}$ es de la forma:

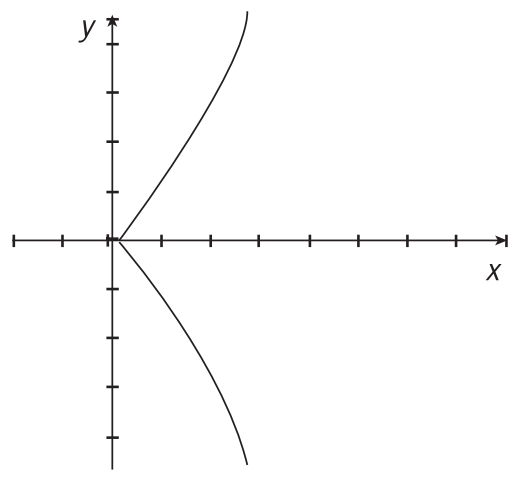

Hallemos los puntos de intersección de las curvas.

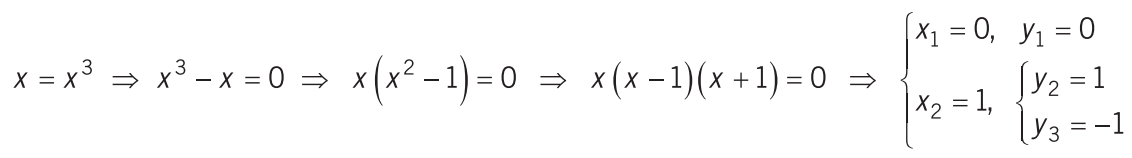

Incorporemos el gráfico de $y^{2}=x$ y determinemos la región comprendida por las curvas.

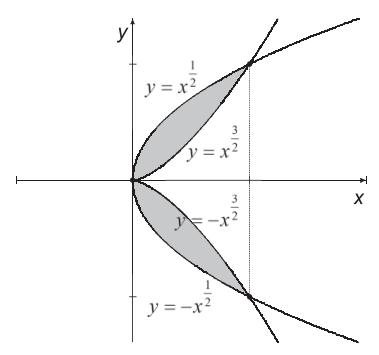


Si $A$ es el área de la región limitada por las curvas:

$$
\begin{aligned}
\frac{1}{2} A & =\int_{0}^{1}\left[x^{\frac{1}{2}}-x^{\frac{3}{2}}\right] d x \\
\frac{1}{2} A & =\left[\frac{2}{3} x^{\frac{3}{2}}-\frac{2}{5} x^{\frac{5}{2}}\right]_{0}^{1} \\
\frac{1}{2} A & =\left[\frac{2}{3}-\frac{2}{5}\right]-[0] \\
\frac{1}{2} A & =\frac{4}{15} \\
A & =\frac{8}{15} \mathrm{u}^{2}
\end{aligned}
$$

396. Hallar el área encerrada por la parábola: $y=x^{2}$ y las rectas $y=2 x-1, y=x+2$.

\section{Solución:}

Hallemos los puntos de intersección de $y=x^{2}$ y $y=2 x-1$ :

$$
x^{2}=2 x-1 \Rightarrow x^{2}-2 x+1=0 \Rightarrow(x-1)^{2}=0 \quad \Rightarrow \quad x_{1}=1, \quad y_{1}=2(1)-1=1
$$

Los puntos de intersección de $y=x^{2}$ e $y=x+2$ :

$$
x^{2}=x+2 \Rightarrow x^{2}-x-2=0 \Rightarrow(x-2)(x+1)=0 \Rightarrow \begin{cases}x_{2}=2, & y_{2}=2^{2}=4 \\ x_{3}=-1, & y_{3}=(-1)^{2}=1\end{cases}
$$

Los puntos de intersección de $y=2 x-1$ y $y=x+2$ :

$$
2 x-1=x+2 \Rightarrow x_{4}=3, \quad y_{4}=3+2=5
$$

Grafiquemos:

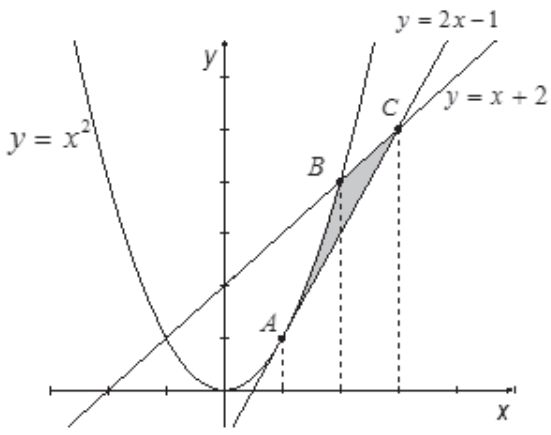


El área de la región sombreada es:

$$
\begin{aligned}
& A=\int_{x_{A}}^{x_{B}}\left[x^{2}-(2 x-1)\right] d x+\int_{x_{B}}^{x_{C}}[x+2-(2 x-1)] d x \\
& A=\int_{1}^{2}\left[x^{2}-2 x+1\right] d x+\int_{2}^{3}[-x+3] d x \\
& A=\int_{1}^{2}(x-1)^{2} d x-\int_{2}^{3}[x-3] d x \\
& A=\left[\frac{(x-1)^{3}}{3}\right]_{1}^{2}-\left[\frac{x^{2}}{2}-3 x\right]_{2}^{3} \\
& A=\left[\frac{1}{3}\right]-[0]-\left[\frac{9}{2}-9\right]+[2-6] \\
& A=\frac{1}{3}+\frac{9}{2}-4 \\
& A=\frac{5}{6} u^{2}
\end{aligned}
$$

397. Calcular el área limitada por las curvas:

$$
\begin{gathered}
y=4-\ln (x+1) \\
y=\ln (x+1) \\
x=0
\end{gathered}
$$

\section{Solución:}

Hallemos los puntos de intersección de las curvas $y=4-\ln (x+1)$ y $y=\ln (x+1)$ :

$$
4-\ln (x+1)=\ln (x+1) \Rightarrow \ln (x+1)=2 \Rightarrow x+1=e^{2} \Rightarrow x_{1}=e^{2}-1, \quad y_{1}=4-2=2
$$

El gráfico de $y=\ln (x+1)$ corresponde al de una función creciente (la base del logaritmo es $e>1$ ) en todo su dominio $(x>-1)$; además, la curva es cóncava hacia abajo y tiene la asíntota vertical: $x=-1$.

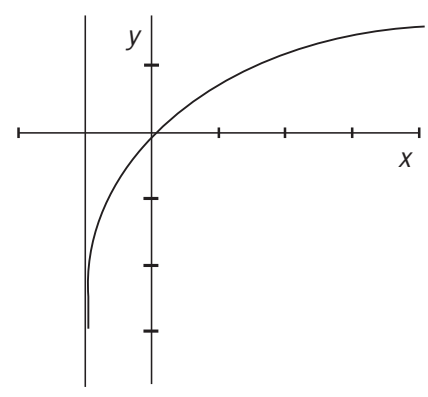


El gráfico de $y=4-\ln (x+1)$ se construye a partir del anterior, primero se dibuja el reflejo de la curva $y=\ln (x+1)$ en el eje $x$ y luego se desplaza la curva obtenida 4 unidades hacia arriba. El gráfico de $x=0$ es el eje $y$ :

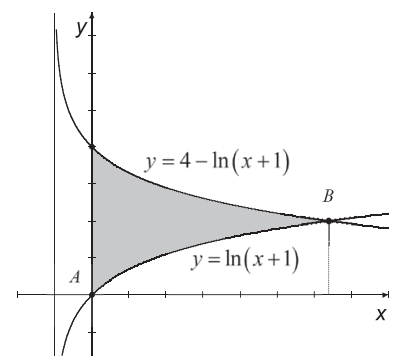

El área de la región sombreada es:

$$
\begin{aligned}
& A=\int_{x_{A}}^{x_{B}}[4-\ln (x+1)-\ln (x+1)] d x \\
& A=\int_{0}^{e^{2}-1}[4-2 \ln (x+1)] d x \\
& A=[4 x-2(x+1) \ln (x+1)+2 x]_{0}^{e^{2}-1} \\
& A=[6 x-2(x+1) \ln (x+1)]_{0}^{e^{2}-1} \\
& A=\left[6\left(e^{2}-1\right)-2\left(e^{2}-1+1\right) \ln \left(e^{2}-1+1\right)\right]-[0] \\
& A=6\left(e^{2}-1\right)-2 e^{2} \ln e^{2} \\
& A=6\left(e^{2}-1\right)-4 e^{2} \\
& A=\left[2 e^{2}-6\right] \mathrm{u}^{2}
\end{aligned}
$$

398. Calcular el área encerrada por las parábolas:

$$
\begin{gathered}
y=x^{2} \\
y=8-x^{2}
\end{gathered}
$$

y la recta: $4 x-y+12=0$

\section{Solucion:}

Hallemos los puntos de intersección de las parábolas:

$$
x^{2}=8-x^{2} \Rightarrow x^{2}=4 \Rightarrow \begin{cases}x_{1}=-2, & y_{1}=(-2)^{2}=4 \\ x_{2}=2, & y_{2}=(2)^{2}=4\end{cases}
$$


Los puntos de intersección de $y=x^{2}$ con $y-4 x+12$ :

$x^{2}=4 x+12 \Rightarrow x^{2}-4 x-12=0 \Rightarrow(x-6)(x+2)=0 \Rightarrow \begin{cases}x_{3}=6, & y_{3}=4(6)+12=36 \\ x_{4}=-2, & y_{4}=4(-2)+12=4\end{cases}$

Los puntos de intersección de $y=8-x^{2}$ con $y=4 x+12$ :

$8-x^{2}=4 x+12 \Rightarrow x^{2}+4 x+4=0 \Rightarrow(x+2)^{2}=0 \Rightarrow x_{5}=-2, \quad y_{5}=4(-2)+12=4$

Grafiquemos:

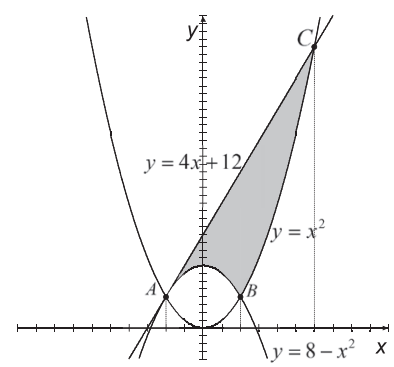

El área de la región sombreada es:

$$
\begin{aligned}
& A=\int_{x_{A}}^{x_{B}}\left[x^{2}-(2 x-1)\right] d x+\int_{x_{B}}^{x_{C}}[x+2-(2 x-1)] d x \\
& A=\int_{1}^{2}\left[x^{2}-2 x+1\right] d x+\int_{2}^{3}[-x+3] d x \\
& A=\int_{1}^{2}(x-1)^{2} d x-\int_{2}^{3}[x-3] d x \\
& A=\left[\frac{(x-1)^{3}}{3}\right]_{1}^{2}-\left[\frac{x^{2}}{2}-3 x\right]_{2}^{3} \\
& A=\left[\frac{1}{3}\right]-[0]-\left[\frac{9}{2}-9\right]+[2-6] \\
& A=\frac{1}{3}+\frac{9}{2}-4 \\
& A=\frac{5}{6} u^{2}
\end{aligned}
$$


399. Hallar el área comprendida entre las curvas:

$$
y x^{2}=2 ; x+y=4 ; x=1 ; x=2
$$

\section{Solución:}

Grafiquemos la curva $y=\frac{2}{x^{2}}$. El gráfico es simétrico con respecto al eje $y$ porque al sustituir $x$ por $-x$ no hay cambio en la ecuación. Además $y^{\prime}=-\frac{4}{x^{3}}$, la derivada no está definida en $x=0$ pero este valor no pertenece al dominio de la función y más bien corresponde a una asíntota vertical, si la derivada es negativa; es decir, la función es decreciente y si $x<0$, la derivada es positiva y en consecuencia la función es creciente. Su gráfico es de la forma:

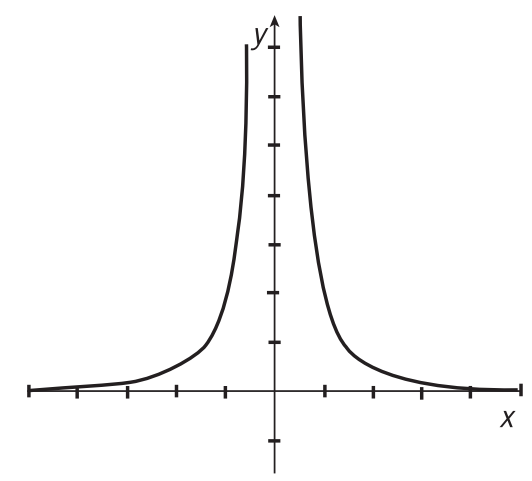

Hallemos los puntos de intersección de $y x^{2}=2$ y $y=4-x$ :

$$
\begin{gathered}
(4-x) x^{2}=2 \\
x^{3}-4 x^{2}+2=0
\end{gathered}
$$

Ocurre que esta ecuación no tiene soluciones racionales.

En este caso, conviene incorporar las rectas al gráfico anterior, y obtenemos:

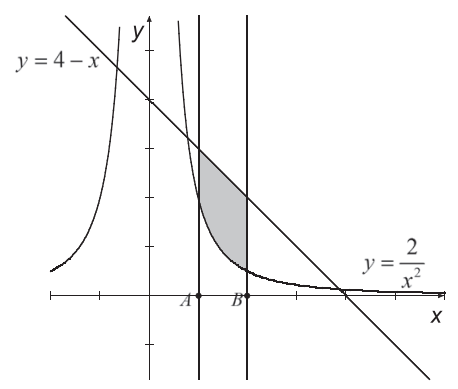


Entonces, el área de la región sombreada es:

$$
\begin{gathered}
A=\int_{x_{A}}^{x_{B}}\left[4-x-\left(\frac{2}{x^{2}}\right)\right] d x \\
A=\int_{1}^{2}\left[4-x-\frac{2}{x^{2}}\right] d x \\
A=\left[4 x-\frac{x^{2}}{2}+\frac{2}{x}\right]_{1}^{2} \\
A=\left[4(2)-\frac{(2)^{2}}{2}+\frac{2}{2}\right]-\left[4(1)-\frac{(1)^{2}}{2}+\frac{2}{1}\right] \\
A=8-2+1-4+\frac{1}{2}-2 \\
A=\frac{3}{2} u^{2}
\end{gathered}
$$

400. Calcular el área encerrada por las curvas:

$$
\begin{aligned}
& y=\sqrt{1-x}-\sqrt{x} \\
& y= \pm \sqrt{x}
\end{aligned}
$$

\section{Solución:}

El gráfico de $y=\sqrt{1-x}-\sqrt{x}$ se limita a $1-x \geq 0$ y $x \geq 0$, es decir a $0 \leq x \leq 1$. Hagamos una tabulación para tener una idea de la forma de la curva:

\begin{tabular}{c|c}
$x$ & $y=\sqrt{1-x}-\sqrt{x}$ \\
\hline 0,0 & 1,00 \\
0,1 & 0,63 \\
0,2 & 0,45 \\
0,3 & 0,29 \\
0,4 & 0,14 \\
0,5 & 0,00 \\
0,6 & $-0,14$ \\
0,7 & $-0,29$ \\
0,8 & $-0,45$ \\
0,9 & $-0,63$ \\
1,0 & $-1,00$ \\
\hline
\end{tabular}


Y tenemos que la gráfica es:

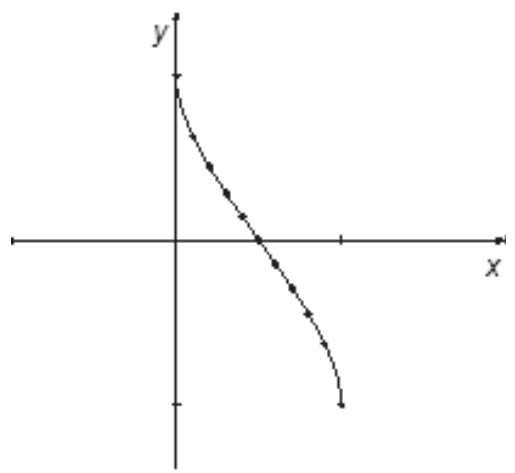

Hallemos la intersección de $y=\sqrt{1-x}-\sqrt{x}$ con $y=\sqrt{x}$ :

$\sqrt{1-x}-\sqrt{x}=\sqrt{x} \Rightarrow \sqrt{1-x}=2 \sqrt{x} \Rightarrow 1-x=4 x \Rightarrow x_{1}=\frac{1}{5}, \quad y_{1}=\sqrt{\frac{1}{5}}=\frac{\sqrt{5}}{5}$

Hallemos la intersección de $y=\sqrt{1-x}-\sqrt{x}$ con $y=-\sqrt{x}$ :

352 $\sqrt{1-x}-\sqrt{x}=-\sqrt{x} \Rightarrow \sqrt{1-x}=0 \Rightarrow x_{2}=1, \quad y_{2}=-\sqrt{1}=-1$

Grafiquemos:

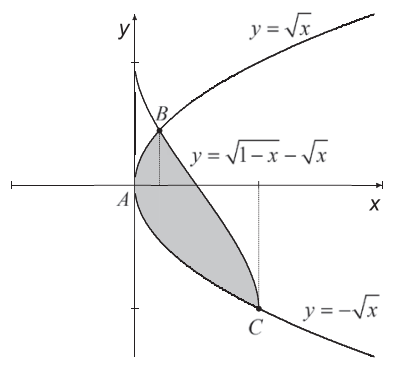

El área de la región sombreada es:

$$
\begin{aligned}
& A=\int_{x_{A}}^{x_{B}}[\sqrt{x}-(-\sqrt{x})] d x+\int_{x_{B}}^{x_{C}}[\sqrt{1-x}-\sqrt{x}-(-\sqrt{x})] d x \\
& A=\int_{0}^{1 / 5}[2 \sqrt{x}] d x+\int_{1 / 5}^{1}[\sqrt{1-x}] d x \\
& A=\left[\frac{4 x^{\frac{3}{2}}}{3}\right]_{0}^{1 / 5}+\left[-\frac{2}{3}(1-x)^{\frac{3}{2}}\right]_{1 / 5}^{1}
\end{aligned}
$$




$$
\begin{aligned}
& A=\left[\frac{4}{3} \cdot \frac{1}{5} \cdot \frac{\sqrt{5}}{5}\right]-[0]+[0]-\left[-\frac{2}{3} \cdot \frac{4}{5} \cdot \frac{2 \sqrt{5}}{5}\right] \\
& A=\frac{4 \sqrt{5}}{75}+\frac{16 \sqrt{5}}{75} \\
& A=\frac{4 \sqrt{5}}{15} u^{2}
\end{aligned}
$$

401. Responder lo presentado a continuación: a) La circunferencia $x^{2}+y^{2}=8$ está dividida por la parábola $y=\frac{x^{2}}{2}$. Hallar la menor área
encerrada por ambas curvas.

b) Hallar el área encerrada por las curvas:

$$
\begin{gathered}
f_{(x)}=x^{3}-3 x^{2}+2 x \\
g_{(x)}=-x^{3}+4 x^{2}-3 x
\end{gathered}
$$

$y$ las rectas $x=0 ; x=2$.

\section{Solución:}

a) Hallemos los puntos de intersección de $x^{2}+y^{2}=8$ con $y=\frac{x^{2}}{2}$ :

$$
2 y+y^{2}=8 \Rightarrow y^{2}+2 y-8=0 \Rightarrow(y+4)(y-2)=0 \Rightarrow\left\{\begin{array}{l}
y_{1}=-4, \quad x \notin R \\
y_{2}=2, \quad x_{2,3}= \pm 2
\end{array}\right.
$$

El gráfico correspondiente es:

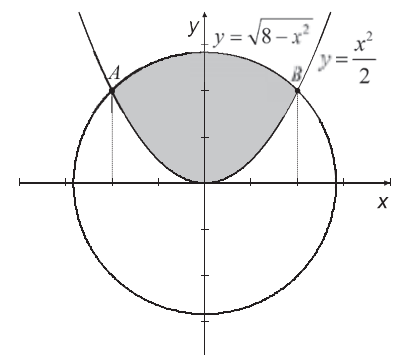

El área de la región sombreada es:

$$
\begin{aligned}
& A=\int_{x_{A}}^{x_{B}}\left[\sqrt{8-x^{2}}-\left(\frac{x^{2}}{2}\right)\right] d x \\
& A=2 \int_{0}^{2}\left[\sqrt{8-x^{2}}-\frac{x^{2}}{2}\right] d x
\end{aligned}
$$




$$
\text { Usemos: } \begin{aligned}
\int & \sqrt{a^{2}-u^{2}} d u=\frac{u}{2} \sqrt{a^{2}-u^{2}}+\frac{a^{2}}{2} \operatorname{arcsen} \frac{u}{a}+C \\
A & =\int_{x_{A}}^{x_{B}}\left[\sqrt{8-x^{2}}-\left(\frac{x^{2}}{2}\right)\right] d x \\
A & =2 \int_{0}^{2}\left[\sqrt{8-x^{2}}-\frac{x^{2}}{2}\right] d x \\
A & =2\left[\frac{x}{2} \sqrt{8-x^{2}}+4 \operatorname{arcsen} \frac{x}{2 \sqrt{2}}-\frac{x^{3}}{6}\right]_{0}^{2} \\
A & =2\left[\frac{2}{2} \sqrt{8-2^{2}}+4 \operatorname{arcsen} \frac{2}{2 \sqrt{2}}-\frac{2^{3}}{6}\right]-2[0] \\
A & =2\left[2+4 \cdot \frac{\pi}{4}-\frac{4}{3}\right] \\
A & =\left[\frac{4}{3}+2 \pi\right] u^{2}
\end{aligned}
$$

b) $f_{(x)}=x^{3}-3 x^{2}+2 x, \quad g_{(x)}=-x^{3}+4 x^{2}-3 x, \quad x=0, \quad x=2$

354

Hallemos los puntos de intersección de las curvas

$$
\begin{aligned}
& f_{(x)}=x^{3}-3 x^{2}+2 x, \quad g_{(x)}=-x^{3}+4 x^{2}-3 x: \\
& x^{3}-3 x^{2}+2 x=-x^{3}+4 x^{2}-3 x, \quad 2 x^{3}-7 x^{2}+5 x=0, \quad x\left(2 x^{2}-7 x+5\right)=0, \quad x(2 x-5)(x-1)=0 \\
& \left\{\begin{array}{l}
x_{1}=0, \quad y_{1}=0^{3}-3(0)^{2}+2(0)=0 \\
x_{2}=\frac{5}{2}, \quad y_{2}=\left(\frac{5}{2}\right)^{3}-3\left(\frac{5}{2}\right)^{2}+2\left(\frac{5}{2}\right)=\frac{15}{8} \\
x_{3}=1, \quad y_{3}=1^{3}-3(1)^{2}+2(1)=0
\end{array}\right.
\end{aligned}
$$

Como la región de integración debe ser $0 \leq x \leq 2$ y las curvas se cortan en $x=0, x=1$ y $x=\frac{5}{2}$, para construir las integrales que permitan calcular el área encerrada por las curvas bastará con determinar las posiciones relativas de las curvas únicamente en las regiones $0<x<1$ y $1<x<2$, para lo cual debemos determinar el signo que tiene $d$ $=f_{(x)}-g_{(x)}$ en dichas regiones:

$$
\begin{aligned}
& d=2 x^{3}-7 x^{2}+5 x \\
& d=2 x(x-1)\left(x-\frac{5}{2}\right)
\end{aligned}
$$


Vemos que si $0<x<1, d=(+)(-)(-)>0$, por lo tanto la curva $y=f_{(x)}$ está por encima de la curva $y=g_{(x)}$. Entonces, en este caso el área encerrada por las curvas está dada por:

$$
A_{1}=\int_{0}^{1}\left[f_{(x)}-g_{(x)}\right] d x
$$

De otro lado, si $1<x<2$, tenemos que $d=(+)(+)(-)<0$ y la curva $y=f_{(x)}$ está por debajo de la curva $y=g_{(x)}$; en consecuencia, el área encerrada por las curvas es:

$$
A_{2}=\int_{1}^{2}\left[g_{(x)}-f_{(x)}\right] d x
$$

El área total encerrada por las curvas es:

$$
\begin{aligned}
& A=\int_{0}^{1}\left[f_{(x)}-g_{(x)}\right] d x+\int_{1}^{2}\left[g_{(x)}-f_{(x)}\right] d x \\
& A=\int_{0}^{1}\left[2 x^{3}-7 x^{2}+5 x\right] d x-\int_{1}^{2}\left[2 x^{3}-7 x^{2}+5 x\right] d x \\
& A=\left[\frac{x^{4}}{2}-\frac{7 x^{3}}{3}+\frac{5 x^{2}}{2}\right]_{0}^{1}-\left[\frac{x^{4}}{2}-\frac{7 x^{3}}{3}+\frac{5 x^{2}}{2}\right]_{1}^{2} \\
& A=\left[\frac{1}{2}-\frac{7}{3}+\frac{5}{2}\right]-[0]-\left[8-\frac{56}{3}+10\right]+\left[\frac{1}{2}-\frac{7}{3}+\frac{5}{2}\right] \\
& A=2 \mathrm{u}^{2}
\end{aligned}
$$

Si hubiésemos graficado las curvas, habríamos obtenido:

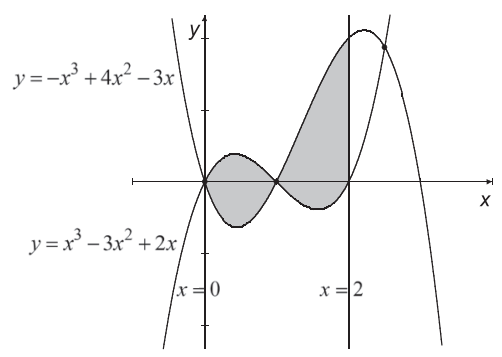

402. Hallar el área encerrada por el eje de ordenadas y las parábolas $x^{2}=9 y-81, x^{2}=4 y-16$, $x^{2}=y-1$.

\section{Solución:}

Las ecuaciones dadas:

$$
\begin{aligned}
& y=\frac{x^{2}}{9}+9 \Rightarrow(y-9)=\frac{1}{9}(x-0)^{2} \Rightarrow V(0,9) \\
& y=\frac{x^{2}}{4}+4 \Rightarrow(y-4)=\frac{1}{4}(x-0)^{2} \Rightarrow V(0,4) \\
& y=x^{2}+1 \Rightarrow(y-1)=(x-0)^{2} \Rightarrow V(0,1)
\end{aligned}
$$


Corresponden a parábolas cuyos vértices están en el eje $y$, todas se abren hacia arriba.

Los puntos de intersección de $x^{2}=9 y-81, x^{2}=4 y-16$, son:

$$
9 y-81=4 y-16 \Rightarrow 5 y=65 \Rightarrow y=13 \Rightarrow x^{2}=4(13)-16=36 \Rightarrow\left\{\begin{array}{l}
(6,13) \\
(-6,13)
\end{array}\right.
$$

Los puntos de intersección de $x^{2}=9 y-81, x^{2}=y-1$, son:

$$
9 y-81=y-1 \Rightarrow 8 y=80 \Rightarrow y=10 \Rightarrow x^{2}=10-1=9 \Rightarrow\left\{\begin{array}{l}
(3,10) \\
(-3,10)
\end{array}\right.
$$

Los puntos de intersección de $x^{2}=y-1, x^{2}=4 y-16$, son:

$$
y-1=4 y-16 \Rightarrow 3 y=15 \Rightarrow y=5 \Rightarrow x^{2}=5-1=4 \Rightarrow\left\{\begin{array}{l}
(2,5) \\
(-2,5)
\end{array}\right.
$$

Las parábolas y el eje y determinan la región señalada en seguida (también se podría haber considerado la región simétrica a la izquierda del eje $y$ ):

\section{6}

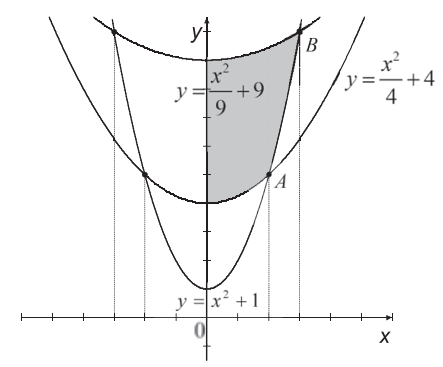

El área sombreada es igual a:

$$
\begin{aligned}
& A=\int_{0}^{x_{A}}\left[\frac{x^{2}}{9}+9-\left(\frac{x^{2}}{4}+4\right)\right] d x+\int_{x_{A}}^{x_{B}}\left[\frac{x^{2}}{9}+9-\left(x^{2}+1\right)\right] d x \\
& A=\int_{0}^{2}\left[-\frac{5 x^{2}}{36}+5\right] d x+\int_{2}^{3}\left[-\frac{8 x^{2}}{9}+8\right] d x \\
& A=\left[-\frac{5 x^{3}}{108}+5 x\right]_{0}^{2}+\left[-\frac{8 x^{3}}{27}+8 x\right]_{2}^{3} \\
& A=\left[-\frac{10}{27}+10\right]-[0]+[-8+24]-\left[-\frac{64}{27}+16\right] \\
& A=12 u^{2}
\end{aligned}
$$


403. Hallar el área de la parte sombreada, si $0<a \leq 1$.

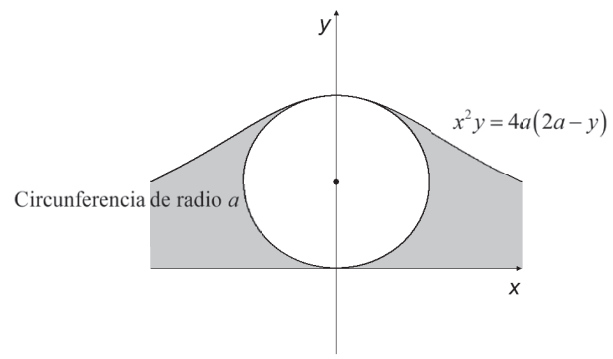

\section{Solución:}

$$
\begin{aligned}
& x^{2} y=8 a^{2}-4 a y \\
& \left(x^{2}+4 a\right) y=8 a^{2} \\
& y=\frac{8 a^{2}}{x^{2}+4 a}
\end{aligned}
$$

Por la simetría de la curva, el área sombreada es igual a dos veces el área bajo la curva $y=\frac{8 a^{2}}{x^{2}+4 a}, y$ a la derecha de $0(x>0)$, menos el área del círculo, es decir:

$$
\begin{aligned}
& A=2 \int_{0}^{\infty} \frac{8 a^{2}}{x^{2}+4 a} d x-\pi a^{2} \\
& A=16 \int_{0}^{\infty} \frac{a^{2}}{x^{2}+4 a} d x-\pi a^{2}
\end{aligned}
$$

Evaluemos la integral impropia $\int_{0}^{\infty} \frac{a^{2}}{x^{2}+4 a} d x$ :

$$
\int_{0}^{\infty} \frac{a^{2}}{x^{2}+4 a} d x=\lim _{b \rightarrow \infty} \int_{0}^{b} \frac{a^{2}}{x^{2}+4 a} d x
$$

Como $\int \frac{1}{u^{2}+a^{2}} d u=\frac{1}{2} \arctan \frac{u}{a}+C, \quad a>0$, tenemos que: 


$$
\begin{aligned}
\int_{0}^{\infty} \frac{a^{2}}{x^{2}+4 a} d x & =\lim _{b \rightarrow \infty} \int_{0}^{b} \frac{a^{2}}{x^{2}+4 a} d x \\
& =\lim _{b \rightarrow \infty}\left[\frac{a^{2}}{2} \arctan \frac{x}{2 \sqrt{a}}\right]_{0}^{b} \\
& =\lim _{b \rightarrow \infty}\left[\frac{a^{2}}{2} \arctan \frac{b}{2 \sqrt{a}}-0\right] \\
& =\frac{a^{2}}{2} \lim _{b \rightarrow \infty}\left[\arctan \frac{b}{2 \sqrt{a}}\right] \\
& =\frac{a^{2}}{2} \cdot \frac{\pi}{2} \\
& =\frac{\pi a^{2}}{4}
\end{aligned}
$$

Entonces:

$$
\begin{aligned}
& A=16\left[\frac{\pi a^{2}}{4}\right]-\pi a^{2} \\
& A=3 \pi a^{2} u^{2}
\end{aligned}
$$

358 404. Calcular el área de la región ubicada en el primer cuadrante encerrada por las curvas:

$$
x^{2}+y^{2}=9, x=0, y=3 x, x^{2}+\frac{3}{2} y=9 \text {. }
$$

\section{Solución:}

Grafiquemos las curvas:

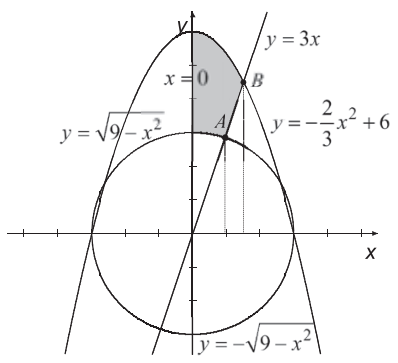

Hallemos las coordenadas del punto $A$ (en el primer cuadrante):

$$
\left\{\begin{array}{l}
y=3 x \\
y=\sqrt{9-x^{2}}
\end{array}\right.
$$




$$
3 x=\sqrt{9-x^{2}} \Rightarrow 9 x^{2}=9-x^{2} \Rightarrow 10 x^{2}=9 \Rightarrow x= \pm \sqrt{\frac{9}{10}} \Rightarrow\left\{\begin{array}{l}
x_{A}=\frac{3}{\sqrt{10}} \\
y_{A}=\frac{9}{\sqrt{10}}
\end{array}\right.
$$

Hallemos las coordenadas del punto $B$ (en el primer cuadrante):

$$
\left\{\begin{array}{l}
y=3 x \\
y=-\frac{2}{3} x^{2}+6
\end{array}\right.
$$

Entonces, el área de la región sombreada es:

$$
\begin{aligned}
& A=\int_{0}^{x_{A}}\left[-\frac{2}{3} x^{2}+6-\sqrt{9-x^{2}}\right] d x+\int_{x_{A}}^{x_{B}}\left[-\frac{2}{3} x^{2}+6-3 x\right] d x \\
& A=\int_{0}^{\frac{3}{\sqrt{10}}}\left[-\frac{2}{3} x^{2}+6-\sqrt{9-x^{2}}\right] d x+\int_{\frac{3}{\sqrt{10}}}^{\frac{3}{2}}\left[-\frac{2}{3} x^{2}+6-3 x\right] d x
\end{aligned}
$$

Como $\int \sqrt{a^{2}-u^{2}} d u=\frac{u}{2} \sqrt{a^{2}-u^{2}}+\frac{a^{2}}{2} \operatorname{arcsen} \frac{u}{a} \quad(a>0)$ :

$$
\begin{aligned}
& A=\int_{0}^{\frac{3}{\sqrt{10}}}\left[-\frac{2}{3} x^{2}+6-\sqrt{9-x^{2}}\right] d x+\int_{\frac{3}{\sqrt{10}}}^{\frac{3}{2}}\left[-\frac{2}{3} x^{2}+6-3 x\right] d x \\
& A=\left[-\frac{2}{9} x^{3}+6 x-\left(\frac{x}{2} \sqrt{9-x^{2}}+\frac{9}{2} \operatorname{arcsen} \frac{x}{3}\right)\right]_{0}^{\sqrt{10}}+\left[-\frac{2}{9} x^{3}+6 x-\frac{3}{2} x^{2}\right]_{\frac{3}{\sqrt{10}}}^{\frac{3}{\sqrt{10}}} \\
& A=\left[-\frac{3}{5 \sqrt{10}}+\frac{18}{\sqrt{10}}-\frac{27}{20}-\frac{9}{2} \operatorname{arcsen} \frac{1}{\sqrt{10}}\right]-[0]+\left[-\frac{3}{4}+9-\frac{27}{8}\right]-\left[-\frac{3}{5 \sqrt{10}}+\frac{18}{\sqrt{10}}-\frac{27}{20}\right] \\
& A=\left[\frac{87}{5 \sqrt{10}}-\frac{27}{20}-\frac{9}{2} \operatorname{arcsen} \frac{1}{\sqrt{10}}\right]+\frac{39}{8}-\frac{87}{5 \sqrt{10}}+\frac{27}{20} \\
& A=\frac{39}{8}-\frac{9}{2} \operatorname{arcsen} \frac{1}{\sqrt{10}} \mathrm{u}^{2}
\end{aligned}
$$


405. Hallar el área encerrada por la curva $y=\frac{x^{2}-4}{x^{2}-16}$ y la recta $y=1$, tal que $|x|>5$.

\section{Solución:}

$|x|>5$ implica que $x<-5 \circ x>5$, lo que corresponde a la zona sombreada siguiente:

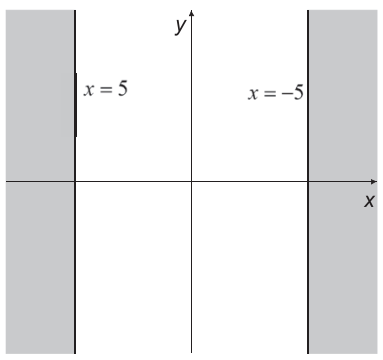

El gráfico respectivo es:

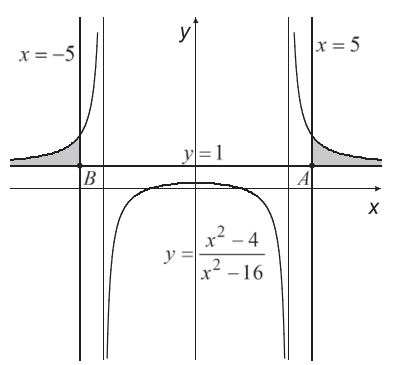

El área sombreada total es igual al doble del área sombreada ubicada en el primer cuadrante:

$$
\begin{aligned}
& A=2 \int_{5}^{\infty}\left[\frac{x^{2}-4}{x^{2}-16}-1\right] d x \\
& A=2 \int_{5}^{\infty}\left[\frac{x^{2}-4-x^{2}+16}{x^{2}-16}\right] d x \\
& A=24 \int_{5}^{\infty}\left[\frac{1}{x^{2}-16}\right] d x \\
& A=24 \lim _{b \rightarrow \infty} \int_{5}^{b}\left[\frac{1}{x^{2}-16}\right] d x
\end{aligned}
$$

Como $\int \frac{1}{u^{2}-a^{2}} d u=\frac{1}{2 a} \ln \left|\frac{u-a}{u+a}\right|+C$ 


$$
\begin{aligned}
& A=24 \lim _{b \rightarrow \infty}\left[\frac{1}{8} \ln \left|\frac{x-4}{x+4}\right|\right]_{5}^{b} \\
& A=\frac{24}{8} \lim _{b \rightarrow \infty}\left[\ln \left|\frac{b-4}{b+4}\right|-\ln \left|\frac{5-4}{5+4}\right|\right] \\
& A=3 \lim _{b \rightarrow \infty}\left[\ln \left|\frac{1-\frac{4}{b}}{1+\frac{4}{b}}\right|-\ln \frac{1}{9} \mid\right. \\
& A=3[\ln 1+\ln 9] \\
& A=3 \ln 9 \mathrm{u}^{2}
\end{aligned}
$$

406. Hallar el área comprendida entre la curva $y=\frac{a^{3}}{x^{2}+a^{2}},(a>0)$ y el eje de abscisas.

\section{Solución:}

Como a > 0, el gráfico correspondiente es:

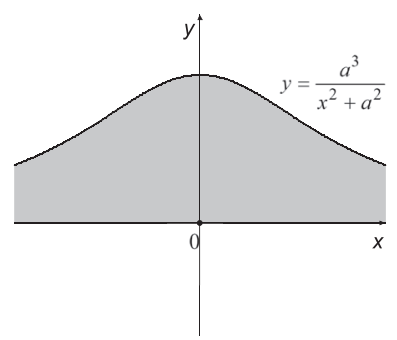

El área sombreada es igual a:

$$
\begin{aligned}
& A=2 \int_{0}^{\infty}\left[\frac{a^{3}}{x^{2}+a^{2}}\right] d x \\
& A=2 a^{3} \int_{0}^{\infty}\left[\frac{1}{x^{2}+a^{2}}\right] d x \\
& A=2 a^{3} \lim _{b \rightarrow \infty} \int_{0}^{b}\left[\frac{1}{x^{2}+a^{2}}\right] d x \\
& A=2 a^{3} \lim _{b \rightarrow \infty}\left[\frac{1}{a} \arctan \frac{x}{a}\right]_{0}^{b} \\
& A=\frac{2 a^{3}}{a} \lim _{b \rightarrow \infty}\left[\arctan \frac{b}{a}-\arctan \frac{0}{a}\right] \\
& A=2 a^{2}\left[\frac{\pi}{2}\right] \\
& A=\pi a^{2} u^{2}
\end{aligned}
$$


407. Calcular el área limitada por la curva $y=e^{x}$ (en el segundo cuadrante) y las rectas $x=0 \mathrm{e}$ $y=0$.

\section{Solución:}

La gráfica correspondiente es:

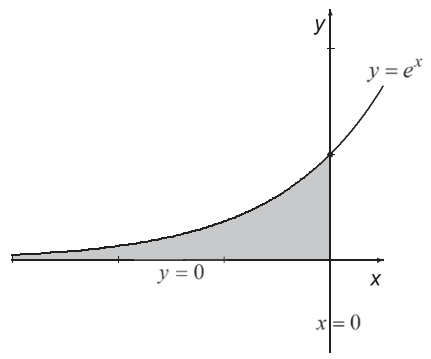

El área sombreada es igual a:

$$
A=\int_{-\infty}^{0}\left[e^{x}-0\right] d x
$$

362

$$
A=\int_{-\infty}^{0} e^{x} d x
$$

Que por simetría es igual a:

$$
\begin{aligned}
& A=\int_{0}^{\infty} e^{-x} d x \\
& A=\lim _{b \rightarrow \infty} \int_{0}^{b} e^{-x} d x \\
& A=\lim _{b \rightarrow \infty}\left[-e^{-x}\right]_{0}^{b} \\
& A=\lim _{b \rightarrow \infty}\left[-\frac{1}{e^{b}}+e^{0}\right] \\
& A=1 u^{2}
\end{aligned}
$$

408. Hallar el área de la menor región comprendida entre las curvas: $y^{2}=a x, y^{2}=2 a x-x^{2}$ en el primer cuadrante.

\section{Solución:}

El gráfico de $y^{2}=a x$ es una parábola de eje horizontal, con vértice en $(0,0)$ y que se abre hacia la derecha (si $a>0)$.

El gráfico de $y^{2}=2 a x-x^{2}$ es una circunferencia, con centro en $(a, 0)$ y radio a (si $\left.a>0\right)$. 
Los puntos que están en el primer cuadrante $(x \geq 0, y \geq 0)$ en los que se cortan las curvas $y^{2}=a x y y^{2}=-x^{2}+2 a x$ son:

$$
a x=-x^{2}+2 a x \Rightarrow x^{2}-a x=0 \Rightarrow x(x-a)=0 \Rightarrow \begin{cases}x_{1}=0, & y_{1}=0 \\ x_{2}=a, & y_{2}=a\end{cases}
$$

El gráfico correspondiente es:

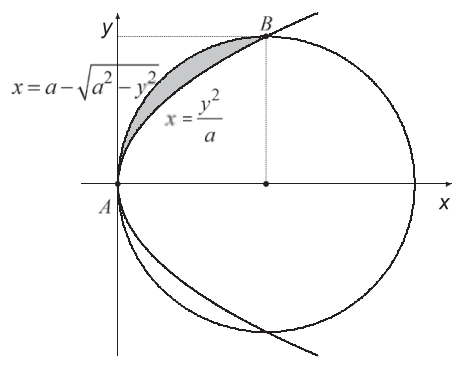

Calculemos el área sombreada integrando a lo largo del eje $y$, para lo cual a partir de cada ecuación debemos expresar $x$ en términos de $y$. De la ecuación $y^{2}=a x$ se tiene que de la ecuación $y^{2}=-x^{2}+a x$ tenemos $(x-a)^{2}-a^{2}=-y^{2}, x-a= \pm \sqrt{a^{2}-y^{2}}$; en este caso debemos seleccionar $x-a=-\sqrt{a^{2}-y^{2}}, x=a-\sqrt{a^{2}-y^{2}}$ porque es el valor de $x$, de los dos que corresponden a cada valor de $y$, que se encuentra a la izquierda de $x=a$. Entonces, el área sombreada está dada por:

$$
\begin{aligned}
& A=\int_{0}^{a}\left[\frac{y^{2}}{a}-\left(a-\sqrt{a^{2}-y^{2}}\right)\right] d y \\
& A=\int_{0}^{a}\left[\frac{y^{2}}{a}-a+\sqrt{a^{2}-y^{2}}\right] d y
\end{aligned}
$$

Como $\int \sqrt{a^{2}-u^{2}} d u=\frac{u}{2} \sqrt{a^{2}-u^{2}}+\frac{a^{2}}{2} \operatorname{arcsen} \frac{u}{a} \quad(a>0)$ :

$$
\begin{aligned}
& A=\int_{0}^{a}\left[\frac{y^{2}}{a}-\left(a-\sqrt{a^{2}-y^{2}}\right)\right] d y \\
& A=\int_{0}^{a}\left[\frac{y^{2}}{a}-a+\sqrt{a^{2}-y^{2}}\right] d y \\
& A=\left[\frac{y^{3}}{3 a}-a y+\frac{y}{2} \sqrt{a^{2}-y^{2}}+\frac{a^{2}}{2} \operatorname{arcsen} \frac{y}{a}\right]_{0}^{a} \\
& A=\left[\frac{a^{3}}{3 a}-a^{2}+\frac{a}{2} \sqrt{a^{2}-a^{2}}+\frac{a^{2}}{2} \operatorname{arcsen} \frac{a}{a}\right]-[0]
\end{aligned}
$$




$$
\begin{aligned}
& A=\frac{a^{2}}{3}-a^{2}+\frac{a^{2}}{2} \cdot \frac{\pi}{2} \\
& A=\left(\frac{\pi}{4}-\frac{2}{3}\right) a^{2} u^{2}
\end{aligned}
$$

409. Comprobar que el área de la región comprendida entre la gráfica de $f_{(x)}=\frac{1}{x}$ y las rectas que pasan por el origen y por los puntos $A\left(1, f_{(1)}\right)$ y $B\left(x_{1}, f_{(x 1)}\right)$ es igual a $\ln x_{1}$.

\section{Solución:}

Como $f_{(1)}=\frac{1}{1}=1$, la recta que pasa por los puntos $(0,0)$ y $A(1,1)$ tiene pendiente $m=1$ y su ecuación es:

$$
\begin{gathered}
y-0=1(x-0) \\
y=x
\end{gathered}
$$

$y=x$
$f_{\left(x_{1}\right)}=\frac{1}{x_{1}}$, la recta que pasa por los puntos $(0,0)$ y $B\left(x_{1}, \frac{1}{x_{1}}\right)$ tiene pendiente $m=\frac{\frac{1}{x_{1}}-0}{x_{1}-0}=\frac{1}{x_{1}^{2}}$ y su ecuación es:

$$
\begin{gathered}
y-0=\frac{1}{x_{1}^{2}}(x-0) \\
y=\frac{1}{x_{1}^{2}} x
\end{gathered}
$$

La curva $y=\frac{1}{x}$ y la recta $y=x$ se cortan en: $\frac{1}{x}=x, x^{2}=1$,

$$
\left\{\begin{array}{l}
x_{A}=1, \quad y_{A}=1 \\
x_{D}=-1, \quad y_{D}=-1
\end{array}\right.
$$

La curva $y=\frac{1}{x}$ y la recta $y=\frac{1}{x_{1}^{2}} x$ se cortan en: $\frac{1}{x}=\frac{1}{x_{1}^{2}} x, x^{2}=x_{1}^{2}$,

$$
\left\{\begin{array}{l}
x_{B}=x_{1}, \quad y_{B}=\frac{1}{x_{1}} \\
x_{C}=-x_{1}, \quad y_{C}=-\frac{1}{x_{1}}
\end{array}\right.
$$

Para que exista $\ln x_{1}$, se requiere que $x_{1}>0$. Por lo tanto, solo consideramos el punto de intersección $B\left(x_{1}, \frac{1}{x_{1}^{2}}\right)$ que está ubicado en el primer cuadrante. 
Grafiquemos:

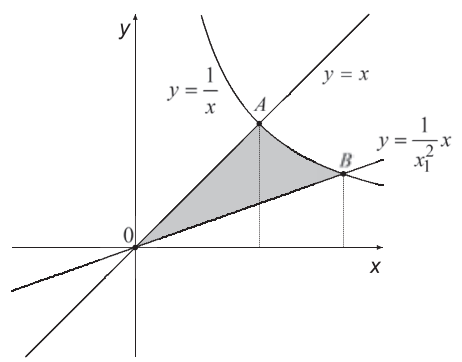

El área sombreada es igual a:

$$
\begin{aligned}
& A=\int_{0}^{x_{A}}\left[x-\frac{1}{x_{1}^{2}} x\right] d x+\int_{x_{A}}^{x_{B}}\left[\frac{1}{x}-\frac{1}{x_{1}^{2}} x\right] d x \\
& A=\int_{0}^{1}\left[x-\frac{1}{x_{1}^{2}} x\right] d x+\int_{1}^{x_{1}}\left[\frac{1}{x}-\frac{1}{x_{1}^{2}} x\right] d x \\
& A=\left[\frac{x^{2}}{2}-\frac{x^{2}}{2 x_{1}^{2}}\right]_{0}^{1}+\left[\ln x-\frac{x^{2}}{2 x_{1}^{2}}\right]_{1}^{x_{1}} \\
& A=\left[\frac{1}{2}-\frac{1}{2 x_{1}^{2}}\right]-[0]+\left[\ln x_{1}-\frac{x_{1}^{2}}{2 x_{1}^{2}}\right]-\left[\ln 1-\frac{1}{2 x_{1}^{2}}\right] \\
& A=\frac{1}{2}-\frac{1}{2 x_{1}^{2}}+\ln x_{1}-\frac{1}{2}+\frac{1}{2 x_{1}^{2}} \\
& A=\ln x_{1} u^{2}
\end{aligned}
$$

410. Calcular el área de la región limitada por la parábola $y=x^{2}-9 x+18$, el eje de abscisas y las rectas $x=2$ y $x=8$.

\section{Solución:}

La ecuación $y=x^{2}-9 x+18$ se puede escribir como $y+\frac{9}{4}=\left(x-\frac{9}{2}\right)^{2}$, que corresponde a una parábola cuyo vértice es $V\left(\frac{9}{2},-\frac{9}{4}\right)$, eje de simetría vertical, que se abre hacia arriba. Las abscisas de los puntos de intersección de la parábola con el eje de abscisas $(y=0)$ son:

$$
\begin{aligned}
0+\frac{9}{4} & =\left(x-\frac{9}{2}\right)^{2} \\
\pm \frac{3}{2} & =x-\frac{9}{2} \\
x & =\frac{9}{2} \pm \frac{3}{2}=\left\{\begin{array}{l}
6 \\
3
\end{array}\right.
\end{aligned}
$$


La recta vertical $x=2$ corta a la parábola en el punto de ordenada $y=2^{2}-9(2)+18=4$ y la recta vertical $x=8$ corta a la parábola en el punto de ordenada $y=8^{2}-9(8)+18=10$ El gráfico correspondiente es:

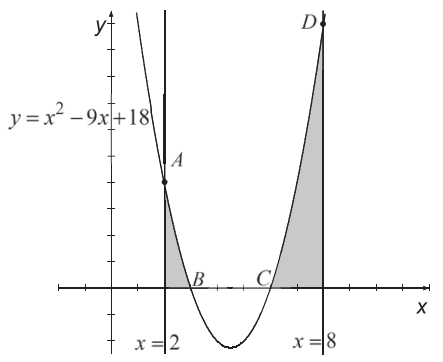

El área sombreada es:

$$
\begin{aligned}
& A=\int_{x_{A}}^{x_{B}}\left[x^{2}-9 x+18-0\right] d x+\int_{x_{C}}^{x_{D}}\left[x^{2}-9 x+18-0\right] d x \\
& A=\int_{2}^{3}\left[x^{2}-9 x+18\right] d x+\int_{6}^{8}\left[x^{2}-9 x+18\right] d x \\
& A=\left[\frac{x^{3}}{3}-\frac{9 x^{2}}{2}+18 x\right]_{2}^{3}+\left[\frac{x^{3}}{3}-\frac{9 x^{2}}{2}+18 x\right]_{6}^{8} \\
& A=\left[9-\frac{81}{2}+54\right]-\left[\frac{8}{3}-18+36\right]+\left[\frac{512}{3}-288+144\right]-[72-162+108] \\
& A=\frac{21}{2} \mathrm{u}^{2}
\end{aligned}
$$

411. ¿Qué área limitan las parábolas $y=x^{2}-2 x$ e $y=12-x^{2}$ ?

$$
\text { R.: } \frac{125}{3} u n^{2}
$$

412. Si $x \in[1,2]$, ¿qué área está debajo de la curva $y=\frac{x}{x^{2}+5 x+6}$ y encima del eje de abscisas?
R.: $\ln \frac{1125}{1024}=0.094 u^{2}$

413. Encontrar el área limitada por las gráficas de las parábolas $x=y^{2}$ y $x=2-y^{2}$.

$$
\text { R.: } \frac{8}{3} u n^{2}
$$


414. Calcular el área de la región $R$ del primer cuadrante acotada por $y=\sqrt{x}, y=0$ e $y=x=2$. Emplee elementos diferenciales horizontales.
R.: $\frac{10}{3} u n^{2}$

415. Encontrar el área de la región mostrada a continuación mediante una sola integral.

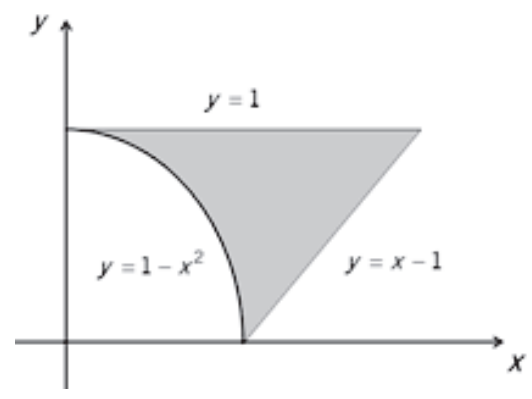

R.: $\frac{5}{6} u n^{2}$

416. ¿Cuál es el área de la figura sombreada?

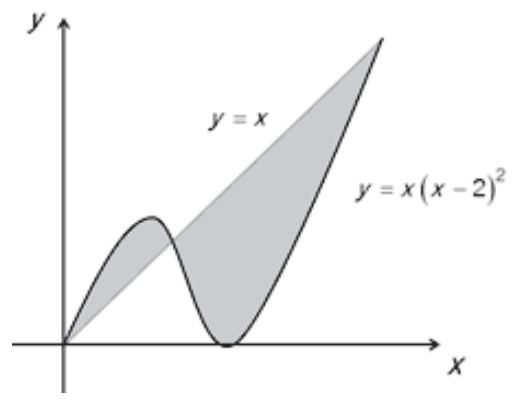
R.: $\frac{37}{12} u n^{2}$

417. Determinar el área comprendida entre las curvas $y=-x^{2}+3 x$ e $y=2 x^{3}-x^{2}-5 x$.

$$
\text { R.: } 16 u n^{2}
$$

418. ¿Qué área tiene la región comprendida por las curvas de ecuaciones $y=4-x^{2}, y=-x+2$, $x=-2$ y $x=3$ ?

$$
\text { R.: } \frac{49}{6} \mathrm{un}^{2}
$$


419. Dadas las curvas de ecuaciones $y=x^{3}$ e $y=3 x+2$, encontrar el área comprendida entre ambas.

R.: $6.75 u^{2}$

420. ¿Cuál es el área de la región bajo $y=-7 x+29$ y sobre $y=\frac{8}{\left(x^{2}-8\right)}$ que está en el primer
cuadrante? R.: $\frac{9}{2}-\sqrt{2} \ln (3+\sqrt{8})=2.007 u^{2}$

421. Encontrar el área de la región limitada por las curvas logarítmicas $y=\ln 2 x$ e $y=4-\ln x$, entre $x=1$ y $x=4$.

R.: $4.8302 u^{2}$

422. ¿Qué área encierran entre sí las curvas $y=e^{x}$ e $y=1-x^{2}$ ?

R.: $0.08235 u^{2}$

368

423. Una región está limitada por $y=\tan x, y=0$ y $x=\pi / 4$. Considere que $x \geq 0$ y encuentre el área de la región.

R.: $\ln \sqrt{2}$

424. ¿Cuál es el área comprendida entre $y=\operatorname{sen} x$ e $y=\sec ^{2} x$ siendo $x \in[0, \pi / 4]$ ?

R.: $\frac{\sqrt{2}}{2} u n^{2}$

425. Calcular el área bajo la curva $y=\arctan 2 x \operatorname{comprendida}$ entre las rectas $x=\frac{1}{2}$ y $x=\frac{\sqrt{3}}{3}$. R.: $\frac{\sqrt{3}}{3} \arctan \frac{2 \sqrt{3}}{3}-\frac{\pi}{8}-\frac{1}{4} \ln \frac{7}{6}=0.0636 \mathrm{un}^{2}$

426. Dadas las ecuaciones $q=\frac{90}{p}-2$ y $q=p-1$, calcular el área de la región encerrada entre ambas considerando que $q>0$ y $p>0$.

R.: 104.85 un $^{2}$

427. ¿Qué valor tiene el área encerrada por la elipse $\frac{x^{2}}{a^{2}}+\frac{y^{2}}{b^{2}}=1$ ?

R.: $\pi a b$ 
428. Demuestre que la parte sombreada de la figura adjunta corresponde a los $2 / 3$ del área del paralelogramo $A B C D$. ( $A D$ es tangente a la parábola).

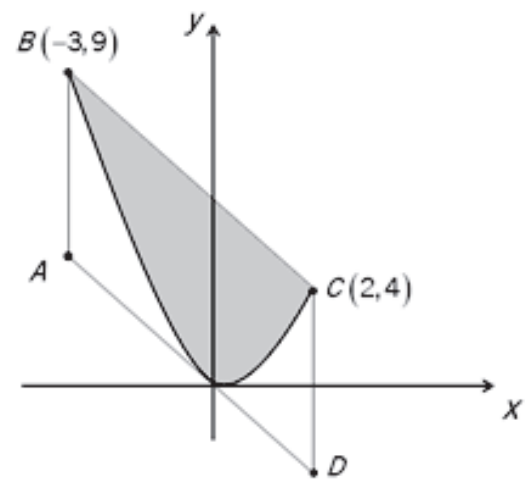

429. Sea $A_{(t)}$ el área de la región comprendida entre $y=x^{2}$ y $y=2 x^{2}$ en el primer cuadrante, y dentro del rectángulo limitado por $x=t, y=t^{2}$ y los ejes coordenados. Si $R_{(t)}$ es el área del rectángulo, hallar $\lim _{t \rightarrow 0} \frac{A_{(t)}}{R_{(t)}}$.

R.: $\frac{2-\sqrt{2}}{3}$

430. Una región $R$ está limitada por las curvas $y=4-x^{2}$ y el eje de abscisas. Calcular el momento de inercia de dicha región con respecto al eje de ordenadas. El momento de inercia de una región plana de densidad 1 , respecto de la recta $L$, se calcula como $\int_{R} d^{2} \cdot d A$, siendo $d$ la distancia del elemento $d A$ a la recta $L$. Considere las unidades $\mathrm{kg}$ y $\mathrm{m}$.

R.: $8.53 \mathrm{~kg} \mathrm{x} \mathrm{mt}^{2}$ 


\section{PROBLEMAS RESUELTOS Y PROPUESTOS DEL CAPÍTULO VIII}

431. El costo marginal en dólares por unidad de una compañía que fabrica cuadernos está dado por $C M_{(q)}=\frac{q}{1000} \sqrt{q^{2}+2500}$, en donde $q$ representa el número de cuadernos producidos. Encontrar el costo de producir 120 cuadernos si se sabe que los costos fijos son $\$ 200$.

\section{Solución:}

Por dato: $C M_{(q)}=\frac{d C}{d q}$, donde $C=C_{(q)}$.

Se tiene que: $\frac{d C}{d q}=\frac{1}{1,000}\left(q^{2}+2,500\right)^{1 / 2}(q)$

Integrando: $\int d C=\frac{1}{1,000} \int\left(q^{2}+2,500\right)^{1 / 2}(q d q)$

$$
\begin{aligned}
& \int d C=\frac{1}{2,000} \int\left(q^{2}+2,500\right)^{1 / 2}(2 q d q) \\
& C=\frac{1}{2,000} \frac{\left(q^{2}+2,500\right)^{3 / 2}}{3 / 2}+K
\end{aligned}
$$

Cuando $q=0 \rightarrow C=200:$

$$
\begin{aligned}
& 200=\frac{1}{3,000}(0+2,500)^{3 / 2}+K \\
& K=200-41.66=158.34
\end{aligned}
$$

De donde:

$$
C=\frac{1}{3,000}\left(q^{2}+2,500\right)^{3 / 2}+158.34
$$

Cuando $q=120$ :

$$
\begin{aligned}
& C=\frac{1}{3,000}\left(120^{2}+2,500\right)^{3 / 2}+158.34 \\
& C=732.33+158.34=\$ 890.67
\end{aligned}
$$

432. Durante la jornada laboral (8 a. m. a 5 p. m.), el número de llamadas telefónicas por minuto que pasan por un conmutador varía de acuerdo con la fórmula: 


$$
f_{(t)}= \begin{cases}5 t & 0 \leq t<1 \\ 5 & 1 \leq t<4 \\ 0 & 4 \leq t<5 \\ 3 & 5 \leq t<8 \\ 27-3 t & 8 \leq t \leq 9\end{cases}
$$

en donde $t$ es el tiempo en horas, medido a partir de las 8 a. m. Calcular el número total de llamadas durante la jornada laboral.

\section{Solución:}

Como $f_{(t)}$ está expresado en Ilamadas por minuto y $t$ está expresado en horas, al igual que

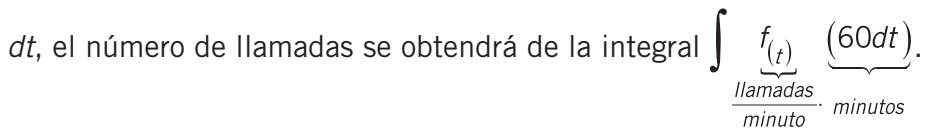

Es decir, el total de llamadas dentro de la jornada laboral viene dado por:

$$
\int_{0}^{1} 5 t(60 d t)+\int_{1}^{4} 5(60 d t)+\int_{4}^{5} 0(60 d t)+\int_{5}^{8} 3(60 d t)+\int_{8}^{9}(27-3 t) d t
$$

Efectuando operaciones:

$$
\left.60\left[\frac{5 t^{2}}{2}\right]\right|_{0} ^{1}+\left.5 t\right|_{1} ^{4}+\left.3 t\right|_{5} ^{8}+\left.\left(27 t-\frac{3}{2} t^{2}\right)\right|_{8} ^{9}
$$

Se obtiene:

$$
\begin{aligned}
& 60\left[\frac{5}{2}+5(4-1)+3(8-5)+27(9-8)-\frac{3}{2}\left(9^{2}-8^{2}\right)\right] \\
& 60[2.5+15+9+27-25.5]=60(28)=1,680 \text { Ilamadas }
\end{aligned}
$$

433. La utilidad marginal de cierta compañía es 100 - $2 q$ pesos por unidad cuando se producen y venden $q$ unidades. ¿Cuál es la utilidad máxima posible de la compañía si la utilidad es 700 pesos cuando $q=10$ ?

\section{Solución:}

A partir de:

$$
\frac{d U}{d q}=100-2 q \Rightarrow d U=(100-2 q) d q
$$


Se tiene que:

$$
\begin{gathered}
\int_{700}^{U} d U=\int_{10}^{q}(100-2 q) d q \\
\left.U\right|_{700} ^{U}=100 q-\left.q^{2}\right|_{10} ^{q} \\
U-700=100 q-q^{2}-(1,000-100)
\end{gathered}
$$

De donde:

$$
U=-q^{2}+100 q-200
$$

La máxima utilidad se produce cuando $\frac{d U}{d q}=-2 q+100=0$.

Si $q=50$ unidades $\Rightarrow U_{\text {máxima }}=-50^{2}+100(50)-200=2,300$ pesos

434. El valor de reventa de cierta maquinaria industrial disminuye a razón de $-960 e^{-t / 5}$ pesos por año cuando la antigüedad de la máquina es $t$ años. Si la máquina se compró nueva por $\$ 5,000$, ¿cuál será su valor al cabo de 10 años?

\section{Solución:}

372

Sea $V_{(t)}$ el valor de la máquina al cabo de $t$ años después de ser adquirida. Entonces:

$$
\frac{d V}{d t}=-960 e^{-t / 5} \Rightarrow d V=\frac{d V}{d t} d t=-960 e^{-t / 5} d t
$$

Con lo cual:

$$
\begin{aligned}
& V=\int d V=\int-960 e^{-t / 5} d t \\
& V=960(5) \int e^{-t / 5}\left(-\frac{1}{5} d t\right) \\
& V=4800 e^{-t / 5}+C
\end{aligned}
$$

Como $V=5000$ cuando $t=0$ :

$$
5,000=4,800(1)+C \rightarrow C=200
$$

De donde:

$$
V_{(t)}=4,800 e^{-t / 5}+200
$$

Con lo cual:

$$
V_{(10)}=4,800 e^{-10 / 5}+200=\$ 849.61
$$


435. Las estadísticas de población recopiladas desde 1990 indican que $t$ años después de dicho año, la población de cierto distrito crecía a razón de $\frac{1,500}{\sqrt{t}}$ personas por año aproximadamente.

a) ¿Cuál era la población en el año 1990 si en el año 1999 la población del distrito era 117,000 personas?

b) Si el patrón de crecimiento se mantuviera, ¿cuál será la población en el año 2015?

\section{Solución:}

Como

$$
\frac{d P_{(t)}}{d t}=\frac{1,500}{\sqrt{t}} \Rightarrow d P_{(t)}=\frac{1,500}{\sqrt{t}} d t
$$

Empleemos la integral definida:

$$
\begin{aligned}
& \int_{117,000}^{P_{(t)}} d P_{(t)}=\int_{9}^{t} \frac{1,500}{\sqrt{t}} d t \\
& \left.P\right|_{117,000} ^{P_{(t)}}=\left.\frac{1,500 \sqrt{t}}{1 / 2}\right|_{9} ^{t} \\
& P_{(t)}-117,000=3,000[\sqrt{t}-\sqrt{9}] \\
& P_{(t)}=3,000 \sqrt{t}+108,000
\end{aligned}
$$

a) En el año 1990, $t=0$, la población fue:

$$
P_{(0)}=3,000 \sqrt{0}+108,000=108,000 \text { habitantes }
$$

b) En el año 2015, $t=25$, la población será:

$$
P_{(25)}=3,000 \sqrt{25}+108,000=123,000 \text { habitantes }
$$

436. Determinado pozo de petróleo que produce 200 barriles de petróleo crudo al mes, se agotará en 3 años. El precio del petróleo crudo es $\$ 20$ por barril y se espera que aumente $\$ 0.05$ por mes. Asuma que el petróleo se vende tan pronto como se extrae del pozo y determine el ingreso total que se obtendrá de dicho pozo.

\section{Solución:}

Sea $I_{(t)}$ los ingresos totales acumulados durante los primeros $t$ meses. Entonces: 


$$
\begin{aligned}
& \frac{d l}{d t}=200[20+0.05 t] \frac{\text { pesos }}{\text { mes }} \\
& \int d I=\int \frac{d l}{d t} d t=200 \int(20+0.05 t) d t
\end{aligned}
$$

Cuando $t=0 \rightarrow I=0$ y cuando $t=36 \rightarrow I=I_{T}$

Entonces:

$$
\begin{aligned}
& \int_{0}^{I_{T}} d I=200 \int_{0}^{36}(20+0.05 t) d t \\
& I_{0}^{l_{T}}=\left.200\left(20 t+\frac{0.05 t^{2}}{2}\right)\right|_{0} ^{36}
\end{aligned}
$$

De donde:

$$
I_{T}-0=200\left[20(36)+\frac{0.05(36)^{2}}{2}-0-0\right]
$$

$$
I_{T}=\$ 150,480
$$

437. Resolver el problema anterior si se asume que el petróleo se venderá al final de cada: a) mes, b) quincena y c) día. Comparar los resultados.

\section{Solución:}

Sea $P_{(t)}=20+0.05 t$ el precio del barril de petróleo cuando al cabo de $t$ meses.

a) El precio de cada barril al cabo del primer mes será $P_{(1)}=\$ 20.05$. Al cabo de 2 meses, el barril costará $P_{(2)}=\$ 20.10$. Al cabo de 36 meses, el barril costará $P_{(36)}=\$ 21.80$

Los ingresos totales serán:

$$
\begin{gathered}
I_{T}=200[20.05+20.10+\ldots+21.80] \\
I_{T}=200(753.30)=\$ 150,660
\end{gathered}
$$

b) Si se lo vende cada quince días, se venderán $\frac{200}{2}=100$ barriles quincenales. Al cabo de los 15 primeros días, $t=\frac{15}{30}=0.5$ barriles. Al cabo de los 30 primeros días, $t=\frac{30}{30}=1$ barriles. 
De donde:

$$
\begin{gathered}
I_{T}=100\left[P_{(0.5)}+P_{(1)}+P_{(1.5)}+\ldots+P_{(36)}\right] \\
I_{T}=100[20.025+20.050+20.075+\ldots+21.80] \\
I_{T}=100(1,505.7)=\$ 150,570
\end{gathered}
$$

c) Cada día se venderán $\frac{200}{30}=6.66$ barriles. Al cabo del primer día, $t=\frac{1}{30}$; al cabo del segundo día, $t=\frac{2}{30}, \ldots$, y así sucesivamente. Los ingresos totales serán:

$$
\begin{gathered}
I_{T}=\frac{200}{30}\left[P_{(1 / 30)}+P_{(2 / 30)}+\ldots+P_{(36)}\right] \\
I_{T}=\frac{200}{30}[20.001666+20.003333+\ldots+21.80] \\
I_{T}=\frac{200}{30}[22,572.90]=\$ 150,486
\end{gathered}
$$

Los resultados se resumen en el siguiente cuadro:

\begin{tabular}{c|l}
\hline Ventas mensuales & $\$ 150,660$ \\
Ventas quincenales & $\$ 150,570$ \\
Ventas diarias & $\$ 150,486$ \\
Ventas continuas & $\$ 150,480$ \\
\hline
\end{tabular}

Como era de esperarse, el resultado del problema anterior es el valor límite de los ingresos totales obtenidos al mes, a la quincena, al día, etc.

438. A las 7:00 a. m. de cierto día, el nivel de ozono es 0.25 partes por millón (ppm). El pronóstico de las condiciones del aire en 12 horas indica que $t$ horas después el nivel de ozono estará variando a razón de $\frac{0.24-0.03 t}{\sqrt{36+16 t-t^{2}}}$ partes por millón por hora. ¿En qué momento se presentará el nivel máximo de ozono? ¿Cuál será el nivel de ozono en ese momento?

\section{Solución:}

Sea $O_{(t)}$ el nivel de ozono al cabo de $t$ horas después de las 7 a. m. Del dato:

$$
\frac{d O_{(t)}}{d t}=\frac{0.24-0.03 t}{\sqrt{36+16 t-t^{2}}}=\left(36+16 t-t^{2}\right)^{-1 / 2}(0.24-0.03 t)
$$


Entonces:

$$
d O_{(t)}=\left(36+16 t-t^{2}\right)^{-1 / 2}(0.24-0.03 t) d t
$$

O mejor:

$$
\begin{gathered}
d O_{(t)}=\left(36+16 t-t^{2}\right)^{-1 / 2}\left(\frac{\frac{2}{\frac{0.03}{2}}}{0.03}\right)(0.24-0.03 t) d t \\
d O_{(t)}=\frac{0.03}{2}\left(36+16 t-t^{2}\right)^{-1 / 2}(16-2 t) d t
\end{gathered}
$$

De donde:

$$
\begin{aligned}
& O_{(t)}=0.015 \int\left(36+16 t-t^{2}\right)^{-1 / 2}(16-2 t) d t \\
& O_{(t)}=0.015 \frac{\sqrt{36+16 t-t^{2}}}{1 / 2}+C
\end{aligned}
$$

$$
O_{(t)}=0.03 \sqrt{36+16 t-t^{2}}+C
$$

Cuando $t=0 \rightarrow O_{(0)}=0.25$

Entonces:

$$
\begin{gathered}
0.25=0.03 \sqrt{36+0-0}+C \\
C=0.07
\end{gathered}
$$

El nivel de ozono es:

$$
O_{(t)}=0.03 \sqrt{36+16 t-t^{2}}+0.07
$$

El máximo nivel de ozono se producirá cuando:

$$
\frac{d O_{(t)}}{d t}=0 \Rightarrow \frac{0.03(16-2 t)}{2 \sqrt{36+16 t-t^{2}}}=0 \Rightarrow t=8
$$

Es decir, se producirá a las $7+8=15$ horas. El máximo nivel de ozono es:

$$
\begin{aligned}
& O_{(8)}=0.03 \sqrt{36+16(8)-8^{2}}+0.07 \\
& O_{(8)}=0.03(10)+0.07=0.37 p p m
\end{aligned}
$$


439. Después de $t$ meses en el trabajo, un empleado postal puede clasificar las cartas a un ritmo de $Q_{(t)}=700-400 e^{-0.5 t}$ cartas por hora. ¿Cuál es el ritmo promedio al que el empleado clasifica las cartas durante los 3 primeros meses en el trabajo?

\section{Solución:}

A partir de $d Q=\frac{d Q}{d t} d t$ se obtiene que:

$$
d Q=\left(700-400 e^{-0.5 t}\right) d t
$$

Entonces:

$$
\begin{aligned}
& \int d Q=\int\left(700-400 e^{-0.5 t}\right) d t \\
& Q=700 t-\frac{400}{-0.5} e^{-0.5 t}+C \\
& Q=700 t+800 e^{-0.5 t}+C
\end{aligned}
$$

Cuando $t=0 \rightarrow Q=0$ :

$$
0=700(0)+800 e^{0}+C \Rightarrow C=-800
$$

De donde:

$$
Q_{(t)}=700 t+800 e^{-0.5 t}-800
$$

Al cabo de 3 meses, habrá clasificado:

$$
\begin{gathered}
Q_{(3)}=700(3)+800 e^{-1.5}-800 \\
Q_{(3)}=2,100+178.50-800 \\
Q_{(3)}=1,479 \text { cartas }
\end{gathered}
$$

El promedio de cartas clasificadas por mes es $\frac{1,479}{3}=493$.

440. Encontrar una función $y=f_{(x)}$ que satisfaga las siguientes condiciones:

$$
f^{\prime \prime}(x)=x^{-3 / 2} ; f^{\prime}(4)=2 ; f_{(0)}=8 .
$$

\section{Solución:}

Se tiene que:

$$
f^{\prime \prime}(x)=x^{-3 / 2} \rightarrow \frac{d f^{\prime}(x)}{d x}=x^{-3 / 2} \rightarrow d f^{\prime}(x)=x^{-3 / 2} d x
$$


Entonces:

$$
\begin{gathered}
\int d f^{\prime}(x)=\int x^{-3 / 2} d x \\
f_{(x)}^{\prime}=\frac{x^{-1 / 2}}{-1 / 2}+K_{1}=-\frac{2}{\sqrt{x}}+K_{1}
\end{gathered}
$$

Como $f_{(4)}^{\prime}=2$ :

$$
f^{\prime}(4)=-\frac{2}{\sqrt{4}}+K_{1}=-1+K_{1}=2 \rightarrow K_{1}=3
$$

Si

$$
\begin{gathered}
f_{(x)}^{\prime}=\frac{d f_{(x)}}{d x}=-\frac{2}{\sqrt{x}}+3 \rightarrow d f_{(x)}=\left(-\frac{2}{\sqrt{x}}+3\right) d x \\
\int d f_{(x)}=\int\left(-\frac{2}{\sqrt{x}}+3\right) d x=\frac{-2 x^{1 / 2}}{1 / 2}+3 x+K_{2} \\
f_{(x)}=-4 \sqrt{x}+3 x+K_{2}
\end{gathered}
$$

378

Como $f_{(0)}=8$ :

$$
f_{(0)}=-4 \sqrt{0}+3(0)+K_{2}=8 \Rightarrow K_{2}=8
$$

De donde:

$$
f_{(x)}=-4 \sqrt{x}+3 x+8
$$

441. Una compañía maderera determinó que la pérdida media de peso $(W)$ por tronco en función del número de días $t$ de tiempo de secado cumple con $\frac{d W}{d t}=\frac{12}{\sqrt{16 t+9}} \frac{\mathrm{Ibs}}{d i ́ a}$. Calcular la pérdida total del peso en 100 días.

\section{Solución:}

Encontremos la función $W_{(t)}$ :

$$
d W=\frac{d W}{d t} d t \Rightarrow \int d W=\int \frac{12}{\sqrt{16 t+9}} d t
$$


Cuando $t=0 \rightarrow w=0$ (no hay pérdida de peso apenas el tronco es talado):

$$
\begin{gathered}
\int_{0}^{W_{(t)}} d W=\int_{0}^{t} \frac{12}{\sqrt{16 t+9}} d t=\frac{12}{16} \int_{0}^{t}(16 t+9)^{-1 / 2}(16 d t) \\
W_{(t)}-0=\left.\frac{12 \sqrt{16 t+9}}{16(1 / 2)}\right|_{0} ^{t} \\
W_{(t)}=\frac{3}{2}[\sqrt{16 t+9}-\sqrt{16(0)+9}] \\
W_{(t)}=\frac{3}{2}[\sqrt{16 t+9}-3]
\end{gathered}
$$

Entonces, al cabo de 100 días, la pérdida total de peso es:

$$
W_{(100)}=\frac{3}{2}[\sqrt{1,609}-3]=55.668 \text { libras }
$$

442. La probabilidad de encontrar un porcentaje de hierro comprendido entre $a$ y $b$, en una muestra de mineral en cierta región geográfica, viene dada por $P_{a, b}=\int_{a}^{b} \frac{1,155}{32} x^{3}(1-x)^{3 / 2} d x$. Encuentre la probabilidad de que una muestra contenga entre 0\% y $25 \%$.

\section{Solución:}

Encontremos $/=\int x^{3}(1-x)^{3 / 2} d x$

Sea $x=\operatorname{sen}^{2} \theta \rightarrow d x=2 \operatorname{sen} \theta \cos \theta d \theta$

Entonces:

$$
\begin{gathered}
I=\int \operatorname{sen}^{6} \theta\left(\cos ^{2} \theta\right)^{3 / 2}(2 \operatorname{sen} \theta \cos \theta d \theta) \\
I=2 \int \operatorname{sen}^{6} \theta \cdot \cos ^{4} \theta \cdot \operatorname{sen} \theta d \theta \\
I=2 \int\left(1-\cos ^{2} \theta\right)^{3} \cdot \cos ^{4} \theta \cdot \operatorname{sen} \theta d \theta \\
I=2 \int\left[1-3 \cos ^{2} \theta+3 \cos ^{4} \theta-\cos ^{6} \theta\right] \cdot \cos ^{4} \theta \cdot \operatorname{sen} \theta d \theta \\
I=-2 \int\left(\cos ^{4} \theta-3 \cos ^{6} \theta+3 \cos ^{8} \theta-\cos ^{10} \theta\right)(-\operatorname{sen} \theta d \theta)
\end{gathered}
$$




$$
\begin{aligned}
& I=-2\left[\frac{\cos ^{5} \theta}{5}-\frac{3 \cos ^{7} \theta}{7}+\frac{3 \cos ^{9} \theta}{9}-\frac{\cos ^{11} \theta}{11}\right]+C \\
& I=-2 \cos ^{5} \theta\left(\frac{1}{5}-\frac{3}{7} \cos ^{2} \theta+\frac{1}{3} \cos ^{4} \theta-\frac{1}{11} \cos ^{6} \theta\right)+C
\end{aligned}
$$

De donde:

$$
P_{0,0.25}=\int_{0}^{0.25} \frac{1155}{32} x^{3}(1-x)^{3 / 2} d x
$$

Si $x=\operatorname{sen}^{2} \theta$, cuando $x=0 \rightarrow \theta=0$

$$
\text { cuando } x=0.25 \rightarrow \operatorname{sen}^{2} \theta=0.25 \rightarrow \operatorname{sen} \theta=0.5 \rightarrow \theta=30^{\circ}
$$

Entonces:

$$
P_{0,0.25}=\frac{1,155}{32} \cdot /\left.\right|_{0} ^{30^{\circ}}
$$

380

$$
\begin{aligned}
& ||_{0}^{30^{\circ}}=-2 \cos ^{5} 30^{\circ}\left[\frac{1}{5}-\frac{3}{7} \cos ^{2} 30^{\circ}+\frac{1}{3} \cos ^{4} 30^{\circ}-\frac{1}{11} \cos ^{6} 30^{\circ}\right]+ \\
& +2 \cos ^{5} 0^{\circ}\left[\frac{1}{5}-\frac{3}{7} \cos ^{2} 0^{\circ}+\frac{1}{3} \cos ^{4} 0^{\circ}-\frac{1}{11} \cos ^{6} 0^{\circ}\right] \\
& /\left.\right|_{0} ^{30^{\circ}}=-2\left(\frac{\sqrt{3}}{2}\right)^{5}\left[\frac{1}{5}-\frac{3}{7}\left(\frac{3}{4}\right)+\frac{1}{3}\left(\frac{9}{16}\right)-\frac{1}{11}\left(\frac{27}{64}\right)\right]+2(1)^{5}\left[\frac{1}{5}-\frac{3}{7}+\frac{1}{3}-\frac{1}{11}\right] \\
& \left.\right|_{0} ^{30^{\circ}}=-2\left(\frac{9}{16}\right) \frac{\sqrt{3}}{2}\left[\frac{2,049}{73,920}\right]+2\left[\frac{16}{1,155}\right] \\
& \left.\right|_{0^{30^{\circ}}} ^{3}=-0.027006179+0.027705627=0.000699448
\end{aligned}
$$

Con lo cual:

$$
P_{0,0.25}=\frac{1,155}{32} \cdot(0.000699448)=0.025246
$$

Es decir, la probabilidad de encontrar entre el $0 \%$ y el $25 \%$ de hierro en determinada muestra es $2.5246 \%$ 
443. La probabilidad de recordar, en un cierto experimento sobre la memoria, resulta ser $P_{a, b}=\int_{a}^{b} \frac{15}{4} x \sqrt{1-x} d x$, en donde representa el porcentaje de recuerdos (véase la figura adjunta).

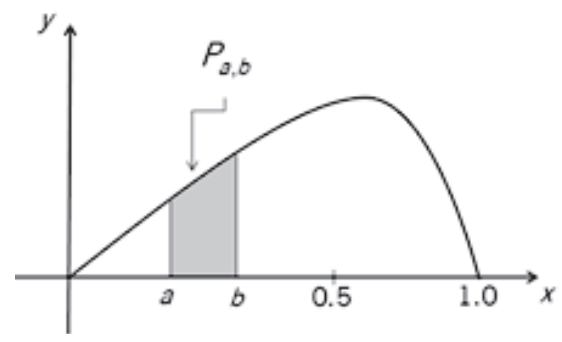

Para un individuo elegido al azar, ¿cuál es la probabilidad de que recuerde entre el 50\% y el $75 \%$ del material?

\section{Solución:}

Se pide encontrar:

$$
P_{a, b}=\int_{0.50}^{0.75} \frac{15}{4} x \sqrt{1-x} d x=\frac{15}{4} \int_{0.50}^{0.75} x \sqrt{1-x} d x
$$

Calculemos: $\quad I=\int x \sqrt{1-x} d x$

Sea: $1-x=t^{2} \Rightarrow d x=-2 t d t$

Si: $x=0.75 \rightarrow t^{2}=0.25 \Rightarrow t=\frac{1}{2}$

Si: $x=0.5 \rightarrow t^{2}=0.5 \Rightarrow t=\frac{\sqrt{2}}{2}$

Entonces:

$$
\begin{aligned}
& I=\int\left(1-t^{2}\right)(t)(-2 t d t)=-2 \int\left(t^{2}-t^{4}\right) d t \\
& I=-2\left(\frac{t^{3}}{3}-\frac{t^{5}}{5}\right)+C=\frac{-2 t^{3}}{15}\left[5-3 t^{2}\right]+C
\end{aligned}
$$


De donde:

$$
\begin{gathered}
P_{0.5,0.75}=\frac{15}{4} \int_{0.50}^{0.75} x \sqrt{1-x} d x=\frac{15}{4} \cdot /\left.\right|_{\sqrt{2} / 2} ^{1 / 2} \\
P_{0.5,0.75}=\frac{15}{4}\left[\frac{-2}{15}\right]\left\{\left(\frac{1}{2}\right)^{3}\left[5-3\left(\frac{1}{4}\right)\right]-\left(\frac{\sqrt{2}}{2}\right)^{3}\left[5-3\left(\frac{1}{2}\right)\right]\right\} \\
P_{0.5,0.75}=\frac{-1}{2}\left\{\frac{1}{8}\left(\frac{17}{4}\right)-\frac{\sqrt{2}}{4}\left(\frac{7}{2}\right)\right\}=\frac{-1}{64}\{17-28 \sqrt{2}\}=0.243528
\end{gathered}
$$

Es decir, la probabilidad de que recuerde entre el $50 \%$ y el $75 \%$ del material es $24.3528 \%$.

444. La probabilidad de recordar, en un cierto experimento sobre la memoria, resulta ser $P_{a, b}=\int_{a}^{b} \frac{15}{4} x \sqrt{1-x} d x$, en donde $x$ representa el porcentaje de recuerdos (véase la figura adjunta).

\section{2}

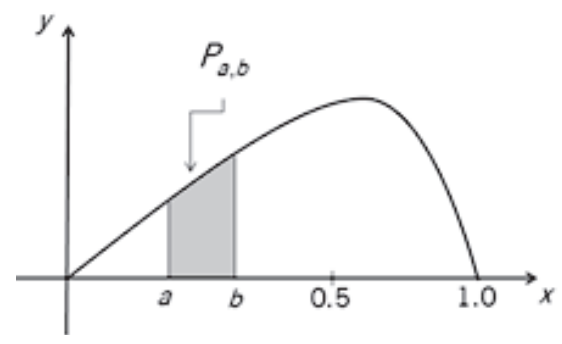

¿Cuál es el porcentaje medio de recuerdos? Es decir, ¿para qué valor de $b$ es cierto que la probabilidad desde 0 hasta $b$ es $50 \%$ ?

\section{Solución:}

Del problema anterior, considerando que $t^{2}=1-x$, se tiene que:

$$
\begin{gathered}
P_{a, b}=\left.\frac{15}{4}\left[\frac{-2}{15}(1-x)^{3 / 2}[5-3(1-x)]\right]\right|_{a} ^{b} \\
P_{a, b}=\left.\frac{-1}{2}(1-x)^{3 / 2}[2+3 x]\right|_{a} ^{b}
\end{gathered}
$$

Se requiere que:

$$
P_{0, b}=\left.\frac{-1}{2}(1-x)^{3 / 2}[2+3 x]\right|_{0} ^{b}=0.50
$$


Entonces:

$$
\begin{gathered}
\frac{-1}{2}\left[(1-b)^{3 / 2}[2+3 b]-1^{3 / 2}(2)\right]=0.50 \\
(1-b)^{3 / 2}[2+3 b]-2=-1 \\
(1-b)^{3 / 2}[2+3 b]=1
\end{gathered}
$$

Tabulamos para valores de b:

\begin{tabular}{c|c}
$b$ & $(1-b)^{3 / 2}(3 b+2)$ \\
\hline 0.50 & 1.237 \\
0.60 & 0.9613 \\
0.59 & 0.9897 \\
0.58 & 1.0180 \\
0.586 & 1.0010 \\
0.587 & 0.9982 \\
0.5865 & 0.9996 \\
0.5864 & 0.9999
\end{tabular}

Con lo cual, la probabilidad de recordar entre el $0 \%$ y el $58.64 \%$ del material es $50 \%$.

445. La pendiente de la normal (perpendicular a la tangente) en un punto cualquiera de una curva está dada por $-\frac{x^{2}}{\ln x}$. Hallar la ecuación de la curva, sabiendo que en el punto $(a,-a)$ la tangente a dicha curva es paralela al eje de abscisas.

\section{Solución:}

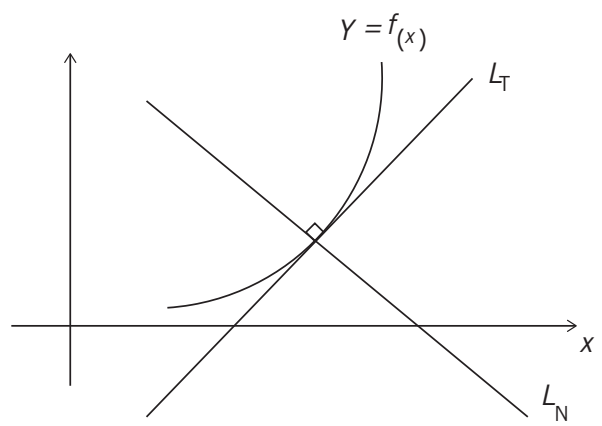


Se tiene que $L_{T} \perp L_{N}$ :

$$
\frac{d y}{d x} \cdot\left(\frac{-x^{2}}{\ln x}\right)=-1
$$

Por lo tanto:

$$
\frac{d y}{d x}=\frac{\ln x}{x^{2}}
$$

Entonces:

$$
\begin{aligned}
& d y=\frac{\ln x}{x^{2}} d x \\
& \int d y=\int \frac{\ln x}{x^{2}} d x \\
& u=\ln x \quad v=-\frac{1}{x} \\
& \downarrow \quad \uparrow \\
& d u=\frac{d x}{x} \quad d v=\frac{d x}{x^{2}}
\end{aligned}
$$

384 Con lo cual:

$$
\begin{aligned}
& y=-\frac{\ln x}{x}+\int \frac{d x}{x^{2}} \\
& y=-\frac{\ln x}{x}-\frac{1}{x}+C
\end{aligned}
$$

Calculemos $y^{\prime}$ :

$$
\begin{aligned}
& y^{\prime}=-\frac{\frac{1}{x} \cdot x-1 \cdot \ln x}{x^{2}}+\frac{1}{x^{2}} \\
& y_{(x=a)}^{\prime}=-\frac{1-\ln a}{a^{2}}+\frac{1}{a^{2}}=0
\end{aligned}
$$

De donde: $-1+\ln a+1=0 \Rightarrow \ln a=0 \Rightarrow a=1$

Por lo tanto, la curva pasará por el punto $(1,-1)$ :

$$
\begin{aligned}
(1,-1) \in y \Rightarrow-1 & =\frac{-\ln 1}{1}-\frac{1}{1}+C \\
-1 & =-1+C \Rightarrow C=0
\end{aligned}
$$


De donde, la ecuación de la curva es:

$$
y=\frac{-\ln x}{x}-\frac{1}{x}
$$

La gráfica de $f_{(x)}$ es:

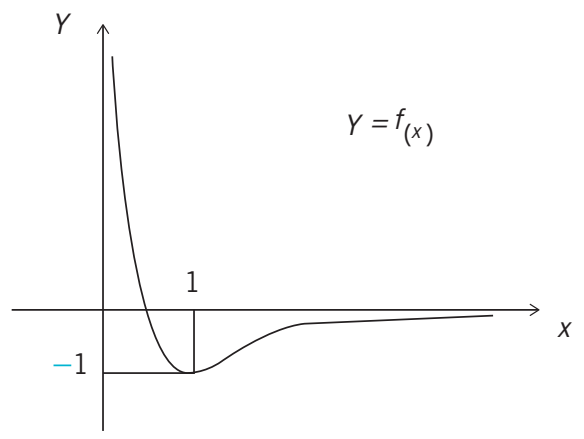

446. La rapidez de cambio de la pendiente de la recta tangente a una curva $y=g_{(x)}$ en cualquiera de sus puntos está dada por $f_{(x)}=18 x-4$. Encontrar $g_{(-1)}$ si la curva pasa por $(1 / 2,-13 / 8)$ y arctan6 es el ángulo que forma, con el eje $x$, la recta tangente a $g_{(x)}$ en el punto de intersección con el eje $y$.

\section{Solución:}

La pendiente de la recta tangente a la curva de ecuación $y=g_{(x)}$ en un punto $P$ de ella viene dada por $\left.\frac{d y}{d x}\right|_{P}$ y su rapidez de cambio es $\left.\frac{d^{2} y}{d x^{2}}\right|_{P}=g^{\prime \prime}\left(x_{P}\right)$.

Por lo tanto:

$$
\begin{aligned}
& g_{(x)}^{\prime \prime}=18 x-4 \\
& g_{(x)}^{\prime}=9 x^{2}-4 x+C_{1} \\
& g_{(x)}=3 x^{3}-2 x^{2}+C_{1} x+C_{2}
\end{aligned}
$$

$$
\text { Por lo tanto: }
$$

Se sabe que: $g_{(1 / 2)}=-13 / 8$

$$
\begin{aligned}
& -\frac{13}{8}=3\left(\frac{1}{2}\right)^{3}-2\left(\frac{1}{2}\right)^{2}+C_{1}\left(\frac{1}{2}\right)+C_{2} \\
& 4 C_{1}+8 C_{2}=-12
\end{aligned}
$$


Además: $g^{\prime}(x=0)=6$

$$
6=9(0)^{2}-4(0)+C_{1}
$$

Si $C_{1}=6$, en (1): $C_{2}=-4.5$.

Con lo cual, la ecuación de $y=g_{(x)}$ es $g_{(x)}=3 x^{3}-2 x^{2}+6 x-4.5$.

De donde:

$$
\begin{gathered}
g_{(-1)}=3(-1)^{3}-2(-1)^{2}+6(-1)-4.5 \\
g_{(-1)}=-15.5
\end{gathered}
$$

447. Asuma que un tractor adquirido en $\$ 60,000$ se deprecia mediante el método de equilibrio y doble balance durante un período de 10 años. Se puede suponer que la razón en la que disminuye su valor contable está dada por $R_{(t)}=13,388.61^{\mathrm{e}-0.22314 t}(0 \leq t \leq 10)$ dólares por año en el tiempo $t$. Encontrar el valor de mercado del tractor después de 5 años de haber sido adquirido.

\section{Solución:}

La depreciación total del tractor durante sus primeros 5 años de vida es:

$$
\begin{gathered}
\int_{0}^{5} d R=\int_{0}^{5} 13,388.61 e^{-0.22314 t} d t \\
\int_{0}^{5} d R=\left.\frac{13,388.61}{(-0.22314)} e^{-0.22314 t}\right|_{0} ^{5} \\
\int_{0}^{5} d R=\$ 40,339.48
\end{gathered}
$$

El valor de mercado del tractor al cabo de 5 años de haber sido adquirido es:

$$
60,000-40,339.48=\$ 19,660.52
$$

448. Se proyecta que el flujo neto de inversión (tasa de formación del capital) del conglomerado gigante LTF Incorporated será $t \sqrt{\frac{t^{2}}{2}+1}$ millones de dólares por año en el año $t$. Encuentre el capital acumulado por la compañía en el segundo año. 


\section{Solución:}

El capital acumulado por la compañía en el segundo año es:

$$
\begin{aligned}
& C=\int_{1}^{2}\left(t \sqrt{\frac{t^{2}}{2}+1}\right) d t=\int_{1}^{2}\left(\frac{t^{2}}{2}+1\right)^{1 / 2}(t d t) \\
& C=\left.\frac{\left(\frac{t^{2}}{2}+1\right)^{3 / 2}}{\frac{3}{2}}\right|_{1} ^{2}=\frac{2}{3}\left[\sqrt[2]{3^{3}}-\sqrt[2]{1.5^{3}}\right]
\end{aligned}
$$

De donde:

$$
C=\frac{2}{3}(5.196-1.837)=2.24 \text { millones de dólares }
$$

449. Si después de 100 segundos queda el 30\% de la cantidad inicial de una muestra radioactiva, encuentre la constante de decrecimiento y la vida media del elemento.

\section{Solución:}

La cantidad de muestra radioactiva $N_{(t)}$ al cabo de $t$ segundos viene dada por $N_{(t)}=N_{0} e^{-K t}$.

De acuerdo con el dato:

$$
N_{(100)}=N_{0} e^{-K(100)}=0.30 N_{0}
$$

De donde:

$$
\begin{aligned}
& e^{-100 K}=0.30 \\
& -100 K=\ln 0.30
\end{aligned}
$$

La constante de decrecimiento es $K=\frac{-\ln 0.30}{100}=0.01204$.

Por otro lado, la vida media $\left(t_{0}\right)$ de una sustancia radioactiva es el tiempo que se requiere para que la cantidad de la muestra se reduzca a la mitad. Entonces:

$$
N_{\left(t_{0}\right)}=N_{0} e^{-K\left(t_{0}\right)}=0.50 N_{0}
$$

De donde:

$$
e^{-K t_{0}}=0.50
$$




$$
\begin{gathered}
-K t_{0}=\ln 0.5 \\
t_{0}=\frac{\ln 1 / 2}{-K}=\frac{-\ln 2}{-K}=\frac{\ln 2}{K}
\end{gathered}
$$

Con lo cual:

$$
t_{0}=\frac{\ln 2}{0.01204}=57.57^{\prime \prime}
$$

450. Se encontró que un rollo de papiro egipcio tenía una relación ${ }^{14} \mathrm{C}$ a ${ }^{12} \mathrm{C}$ igual al $70 \%$ de la relación correspondiente a la de un material similar actual. Estime la antigüedad del rollo.

\section{Solución:}

Se debe tomar en cuenta que el carbono $14,{ }^{14} \mathrm{C}$, tiene una vida media de 5,600 años. Esto significa que al cabo de 5,600 años, la cantidad de átomos de carbono 14 contenida en restos de plantas fósiles o restos arqueológicos de origen orgánico se reduce a la mitad ya que la otra mitad se ha descompuesto.

De acuerdo con el problema anterior, la vida media de una sustancia es $t_{0}=\frac{\ln 2}{K}$.

Entonces:

$$
5,600=\frac{\ln 2}{K} \Rightarrow K=\frac{\ln 2}{5,600}
$$

Se tendrá que:

$$
N_{(t)}=N_{0} e^{-K t}=N_{0} e^{-\frac{\ln 2}{5,600} t}=0.70 N_{0}
$$

Es decir:

$$
\begin{aligned}
& e^{-\frac{\ln 2}{5,600} t}=0.70 \\
& -\frac{\ln 2}{5,600} t=\ln 0.70
\end{aligned}
$$

La antigüedad del rollo es:

$$
\begin{gathered}
t=-\frac{(\ln 0.70)(5,600)}{\ln 2} \\
t=2,882 \text { años }
\end{gathered}
$$


451. Encontrar el flujo promedio $\bar{F}$ en el intervalo $[0, T]$ si se asume que el flujo sanguíneo en el tiempo $t$ viene dado por $F_{(t)}=\frac{F_{1}}{(1+\alpha t)^{2}} \quad[0 \leq t \leq T]$.

\section{Solución:}

El flujo promedio $\bar{F}$ en el intervalo $[0, T]$ viene dado por:

$$
\bar{F}=\frac{\int_{0}^{T} F_{(t)} d t}{T-0}
$$

Es decir:

$$
\begin{gathered}
\bar{F}=\frac{1}{T} \int_{0}^{T} F_{1}(1+\alpha t)^{-2} d t \\
\bar{F}=\frac{F_{1}}{\alpha T} \int_{0}^{T}(1+\alpha t)^{-2}(\alpha d t) \\
\bar{F}=\left.\frac{F_{1}}{\alpha T}\left[\frac{(1+\alpha t)^{-1}}{-1}\right]\right|_{0} ^{T} \\
\bar{F}=\frac{F_{1}}{\alpha T}\left[\frac{-1}{(1+\alpha T)}+\frac{1}{(1+0)}\right] \\
\bar{F}=\frac{F_{1}}{\alpha T}\left[\frac{1+\alpha T-1}{1+\alpha T}\right]=\frac{F_{1}(\alpha T)}{(\alpha T)(1+\alpha T)}
\end{gathered}
$$

De donde:

$$
\bar{F}=\frac{F_{1}}{1+\alpha T}
$$

452. Considere que una población tiene un crecimiento exponencial dado por $\frac{d N}{d t}=K N$ para $t \geq t_{0}$. Suponga que $N=N_{0}$ cuando $t=t_{0}$. Determine el tamaño $N$ de la población al cabo de $t$ años.

\section{Solución:}

Se tiene que:

$$
d N=\frac{d N}{d t} d t
$$




$$
\begin{aligned}
& d N=K N d t \\
& \frac{d N}{N}=K d t
\end{aligned}
$$

Entonces:

$$
\begin{aligned}
& \int_{N_{0}}^{N} \frac{d N}{N}=\int_{t_{0}}^{t} K d t \\
& \left.\ln N\right|_{N_{0}} ^{N}=\left.K t\right|_{t_{0}} ^{t}
\end{aligned}
$$

De donde:

$$
\begin{gathered}
\ln N-\ln N_{0}=K\left(t-t_{0}\right) \\
\ln \frac{N}{N_{0}}=K\left(t-t_{0}\right) \\
\frac{N}{N_{0}}=e^{K\left(t-t_{0}\right)} \Rightarrow N=N_{0} e^{K\left(t-t_{0}\right)} \text { para } t \geq t_{0}
\end{gathered}
$$

453. Asuma que la población mundial era 2,000 millones en el año 1930 y que 30 años después aumentó en 50\%. Considere una ley de crecimiento exponencial y estime la población en el año 2020.

\section{Solución:}

Si $N_{(t)}$ es la población mundial en el tiempo $t$, se cumple que:

$$
\frac{d N}{d t}=K N \Rightarrow d N=K N d t \Rightarrow \frac{d N}{N}=K d t
$$

Sea $\quad t=0$ en el año $1930 \rightarrow N_{(0)}=2,000$ millones.

$$
\begin{aligned}
& t=30 \text { en el año } 1960 \rightarrow N_{(30)}=3,000 \text { millones. } \\
& t=90 \text { en el año } 2020 \rightarrow N_{(90)}=? .
\end{aligned}
$$

Calculemos la tasa de crecimiento $K$ :

$$
\begin{gathered}
\int_{2000^{\prime}}^{3000^{\prime}} \frac{d N}{N}=\int_{0}^{30} K d t \\
\left.\ln N\right|_{2000^{\prime}} ^{300^{\prime}}=\left.K t\right|_{0} ^{30} \\
\ln \frac{3,000}{2,000}=K(30-0) \Rightarrow K=\frac{1}{30} \ln \frac{3}{2}=0.00135=1.35 \%
\end{gathered}
$$


Entonces:

$$
\int_{2000}^{N(90)} \frac{d N}{N}=\int_{0}^{90} K d t=\int_{0}^{90}\left(\frac{1}{30} \ln \frac{3}{2}\right) d t
$$

De donde:

$$
\begin{aligned}
& \left.\ln N\right|_{2000} ^{N_{(90)}}=\left.\frac{1}{30} \ln \frac{3}{2}(t)\right|_{0} ^{90} \\
& \ln \frac{N_{(90)}}{2,000}=\frac{1}{30}\left(\ln \frac{3}{2}\right)(90-0) \\
& \frac{N_{(90)}}{2,000}=e^{\frac{1}{30}\left(\ln \frac{3}{2}\right) 90}=e^{3 \ln 1.5}
\end{aligned}
$$

De donde:

$$
\begin{aligned}
& N_{(90)}=2,000 e^{3 \ln 1.5} \text { millones } \\
& N_{(90)}=2,000 e^{\ln 1.5^{3}}=2,000(1.5)^{3}
\end{aligned}
$$

En el año 2020 se estima una población de 6,750 millones de personas.

Nótese que el aumento poblacional en 30 años (1930 a 1960) fue 50\%. Si se mantuviera ese aumento poblacional en cada período siguiente de 30 años (1960 a 1990 y 1990 a 2020), se llegaría a una población en el año 2020 de:

$$
2,000^{\prime}(1.5)(1.5)(1.5)=2,000^{\prime}(1.5)^{3}=6750^{\prime}
$$

(En el año 2011 se alcanzó la cantidad de 7,000 millones de personas en el mundo).

454. La velocidad de un auto (en pies/segundo), $t$ segundos después de partir del reposo, está dada por la función $f_{(t)}=2 \sqrt{t} \quad(0 \leq t \leq 30)$. Determine el espacio recorrido entre $t=9 \mathrm{y}$ $t=16$ segundos.

\section{Solución:}

A partir de $v=\frac{d s}{d t} \Rightarrow d s=v d t=2 \sqrt{t} d t$.

El espacio $s$ recorrido entre $t=9^{\prime \prime}$ y $t=16^{\prime \prime}$ es:

$$
s=\int_{0}^{s} d s=\int_{9}^{16} 2 \sqrt{t} d t
$$




$$
s=\left.\frac{4}{3} t^{3 / 2}\right|_{9} ^{16}=\frac{4}{3}\left[4^{3}-3^{3}\right]=49,33 \text { metros. }
$$

455. La razón de cambio del precio unitario $p$ (en dólares) de las botas para mujer está dada por $p_{(x)}^{\prime}=\frac{-250 x}{\left(16+x^{2}\right)^{3 / 2}}$, en donde $x$ representa la cantidad demandada diariamente en centenas. Determinar la función de demanda para estas botas si se sabe que el precio unitario es $\$ 50$ cuando se demandan 300 pares.

\section{Solución:}

Se tiene que:

$$
\frac{d p}{d x}=\frac{-250 x}{\left(16+x^{2}\right)^{3 / 2}}
$$

Cuando $x=3$ (centenas de pares) $\rightarrow p=50$

$x=x$ (centenas de pares) $\rightarrow p=p$

Entonces:

$$
\begin{gathered}
d p=\frac{d p}{d x} d x=\frac{-250 x}{\left(16+x^{2}\right)^{3 / 2}} d x \\
\int_{50}^{p} d p=\int_{3}^{x} \frac{-250 x}{\left(16+x^{2}\right)^{3 / 2}} d x \\
\left.p\right|_{50} ^{p}=-\left.\frac{250}{2} \cdot \frac{\left(16+x^{2}\right)^{-1 / 2}}{-1 / 2}\right|_{3} ^{x} \\
p-50=250\left[\frac{1}{\sqrt{16+x^{2}}}-\frac{1}{\sqrt{16+9}}\right]
\end{gathered}
$$

De donde:

$$
p-50=\frac{250}{\sqrt{16+x^{2}}}-50 \Rightarrow p=\frac{250}{\sqrt{16+x^{2}}}
$$


456. En cualquier punto $P_{(x, y)}$ de una curva se cumple que $\frac{d^{3} y}{d x^{3}}=2$. Encontrar la ecuación de la curva si se conoce que $(1,3)$ es un punto de inflexión en el que la pendiente de la recta tangente es -2 .

\section{Solución:}

Integramos en forma sucesiva la expresión $\frac{d^{3} y}{d x^{3}}=2$ :

$$
\begin{gathered}
\frac{d^{2} y}{d x^{2}}=\int \frac{d^{3} y d x}{d x^{3}}=\int 2 d x=2 x+C_{1} \\
\frac{d y}{d x}=\int \frac{d^{2} y d x}{d x^{2}}=\int\left(2 x+C_{1}\right) d x=x^{2}+C_{1} x+C_{2} \\
y=\int \frac{d y d x}{d x}=\int\left(x^{2}+C_{1} x+C_{2}\right) d x=\frac{x^{3}}{3}+\frac{C_{1} x^{2}}{2}+C_{2} x+C_{3}
\end{gathered}
$$

De los datos dados:

$(1,3)$ es punto de inflexión

$$
\left.\rightarrow \frac{d^{2} y}{d x^{2}}\right|_{(1)}=0
$$

la pendiente en $(1,3)$ es -2

$$
\left.\rightarrow \frac{d y}{d x}\right|_{(1)}=-2
$$

$(1,3)$ es un punto de la función

$$
\rightarrow y_{(1)}=3
$$

(1) en $(\alpha)$ :

$$
0=2(1)+C_{1}=-2
$$

(2) en $(\beta)$ :

$$
-2=1^{2}+(-2)(1)+C_{2} \rightarrow C_{2}=-1
$$

(3) en $(\gamma)$ :

$$
\begin{gathered}
3=\frac{1^{3}}{3}+\frac{(-2)(1)^{2}}{2}+(-1)(1)+C_{3} \\
3=\frac{1}{3}-1-1+C_{3} \rightarrow C_{3}=\frac{14}{3}
\end{gathered}
$$


Con lo cual:

$$
y=\frac{x^{3}}{3}+\frac{(-2) x^{2}}{2}+(-1) x+\frac{14}{3}
$$

Es decir:

$$
y=\frac{x^{3}}{3}-x^{2}-x+\frac{14}{3}
$$

457. La función de demanda de cartuchos de repuestos para un purificador de agua está dada por $p=-0.01 q^{2}-0.1 q+6$, en donde $p$ es el precio unitario en dólares y $q$ es la cantidad demandada cada semana, en millares. Determine el excedente de los consumidores si el precio de mercado se establece en $\$ 4$ por cartucho.

\section{Solución:}

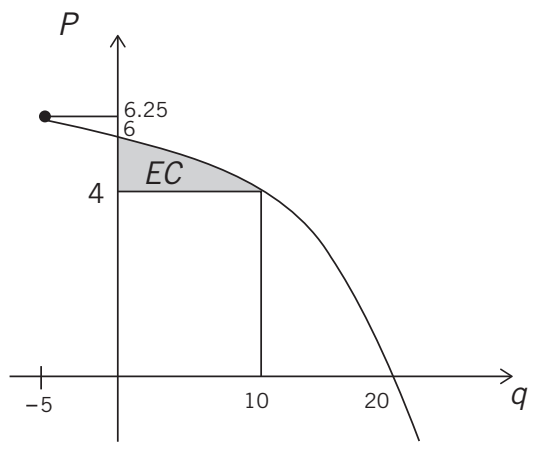

Dado:

$$
\begin{gathered}
p=-0.01 q^{2}-0.1 q+6 \\
-100 p=q^{2}+10 q-600 \\
-100 p=(q+5)^{2}-625 \\
(q+5)^{2}=-100(p-6.25)
\end{gathered}
$$

El vértice de la parábola es $V(-5,6.25)$.

Si $p=4(q+5)^{2}=-100(4-6.25)=225 \rightarrow q+5=15 \rightarrow q+10$,

el excedente de los consumidores es:

$$
E C=\int_{0}^{10}\left[\left(-0.01 q^{2}-0.1 q+6\right)-4\right] d q
$$




$$
\begin{gathered}
E C=-0.01 \frac{q^{3}}{3}-0.1 \frac{q^{2}}{2}+\left.2 q\right|_{0} ^{10} \\
E C=\frac{-0.001(1,000)}{3}-\frac{0.1(100)}{2}+20 \\
E C=-\frac{10}{3}-\frac{10}{2}+20=11.667 \text { millares de dólares }
\end{gathered}
$$

458. Para una determinada mercadería la ecuación de la demanda es $30 p=3600-q^{2}$ ( $p$ en dólares y $q$ en unidades). La función de costo total está dada por $C_{(q)}=(q+20)^{2}$ dólares. Calcular el excedente de los consumidores si la cantidad producida y, por lo tanto, el precio, en un monopolio, se determinaron de manera que se obtuvo la máxima utilidad total.

\section{Solución:}

La función utilidad es:

$$
\begin{gathered}
U=I-C=p q-C \\
U=\left(\frac{3,600-q^{2}}{30}\right) q-(q+20)^{2} \\
U=120 q-\frac{1}{30} q^{3}-q^{2}-40 q-400
\end{gathered}
$$

La utilidad será máxima cuando $U_{(q)}^{\prime}=0$.

$$
\begin{aligned}
U_{(q)}^{\prime}=80-\frac{1}{10} q^{2}-2 q & =0 \\
q^{2}+20 q-800 & =0 \\
(q+40)(q-20) & =0 \rightarrow q=20 \Rightarrow p=\frac{320}{3}
\end{aligned}
$$

Entonces:

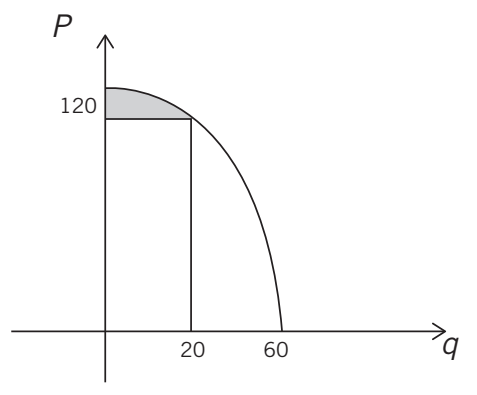

$$
\begin{aligned}
& E C=\int_{0}^{20}\left[120-\frac{q^{2}}{30}-\frac{320}{3}\right] d q \\
& E C=\int_{0}^{20}\left[\frac{40}{3}-\frac{q^{2}}{30}\right] d q
\end{aligned}
$$




$$
E C=\frac{40}{3} q-\left.\frac{q^{3}}{90}\right|_{0} ^{20}=\$ 177.78
$$

459. La curva de Lorenz se emplea en el estudio de la distribución del ingreso. Si $x$ es el porcentaje acumulado de receptores de ingresos, ordenados de los más pobres a los más ricos, e y es el porcentaje acumulado de ingresos, entonces la igualdad de la distribución de ingresos está dada por la recta $y=x$ (el $10 \%$ de las personas recibe el $10 \%$ de los ingresos totales, el $20 \%$ recibe el $20 \%$, etc). El grado de desviación con respecto a la igualdad se mide mediante el coeficiente de desigualdad $C D=\frac{\text { área entre la curva y la recta } y=x}{\text { área bajolarecta } y=x} .(C D=0$ si hay igualdad de distribución de ingresos).

Asuma que la distribución real está dada por la curva de Lorenz que se define por $y=\frac{20 x^{2}}{21}+\frac{x}{21}$ y determine el coeficiente de desigualdad.

\section{Solución:}

Grafiquemos la curva de Lorentz y la recta $y=x$ :

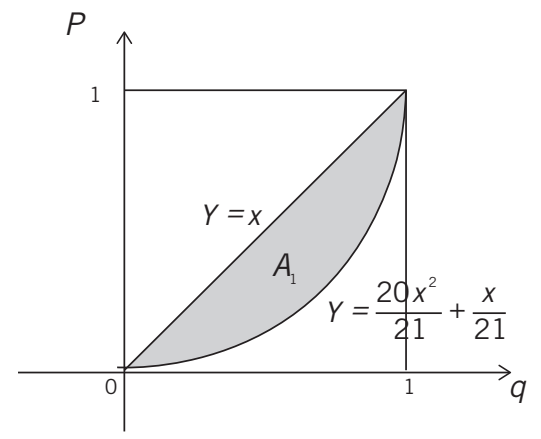

El coeficiente de desigualdad viene dado por:

$$
C D=\frac{A_{1}}{\frac{1(1)}{2}}=2 A_{1}
$$

Entonces:

$$
C D=2 \int_{0}^{1}\left[x-\left(\frac{20 x^{2}}{21}+\frac{x}{21}\right)\right] d x
$$




$$
\begin{gathered}
C D=\left.2\left(\frac{20 x^{2}}{(2) 21}-\frac{20 x^{3}}{(3) 21}\right)\right|_{0} ^{1} \\
C D=\frac{2}{21}\left[10-0-\frac{20}{3}+0\right]=\frac{20}{63}
\end{gathered}
$$

460. Si $\pi_{m}=\frac{a}{\sqrt{a x+b}}$ es la función de costo marginal y los costos fijos son iguales a cero, hallar el costo de producción de $\frac{8 b}{a}$ unidades.

\section{Solución:}

A partir de $C M=\frac{d C}{d x}=\frac{a}{\sqrt{a x+b}}=a(a x+b)^{-1 / 2}$, se tiene que:

$$
\begin{gathered}
\int d C=\int \frac{d C}{d x} d x=\int a(a x+b)^{-1 / 2} d x \\
\int_{0}^{C} d C=\int_{0}^{\frac{8 b}{a}}(a x+b)^{-1 / 2}(a d x)
\end{gathered}
$$

Es decir:

$$
\begin{gathered}
\left.C\right|_{0} ^{c}=\left.\frac{(a x+b)^{1 / 2}}{1 / 2}\right|_{0} ^{\frac{8 b}{a}} \\
C-0=2(8 b+b)^{1 / 2}-2(0+b)^{1 / 2} \\
C=2(3 \sqrt{b})-2 \sqrt{b}=4 \sqrt{b}
\end{gathered}
$$

461. Sea $p$ el precio de un bien; $q=130-3 p$, la demanda; $y=30+2 p$, la oferta. Supongamos que el precio $p=p_{(t)}$ varía con el tiempo y que la rapidez de variación de $p$ es proporcional al exceso de la cantidad demandada. Si inicialmente el precio es $\$ 10$ y al cabo de 5 años es $\$ 15$, determine el precio al cabo de 10 años.

\section{Solución:}

Grafiquemos las funciones de demanda $q_{d}=f_{(p)}$ y de oferta $q_{o}=f_{(p)}$ : 


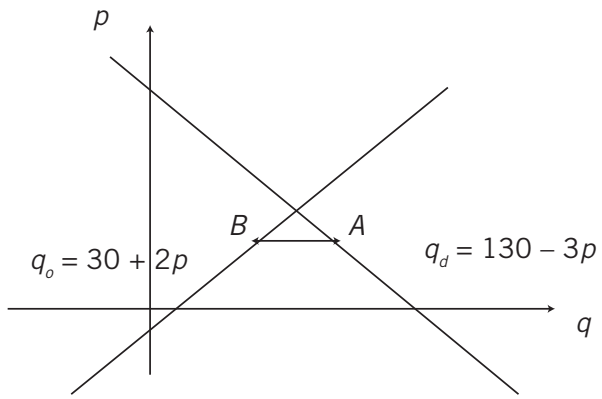

A partir del enunciado:

$$
\frac{d p}{d t}=K\left(q_{d}-q_{0}\right)=K[(130-3 p)-(30+2 p)]
$$

Si $\frac{d p}{d t}=K[100-5 p] \Rightarrow d p=\frac{d p}{d t} d t=K(100-5 p) d t$

Entonces:

$$
\frac{d p}{100-5 p}=K d t
$$

Como $p=10$ cuando $t=0$ y $p=15$ cuando $t=5$ :

$$
\begin{gathered}
\int_{10}^{15} \frac{d p}{100-5 p}=\int_{0}^{5} K d t \\
-\left.\frac{1}{5} \ln (100-5 p)\right|_{10} ^{15}=\left.K t\right|_{0} ^{5} \\
-\frac{1}{5} \ln \frac{100-5(15)}{100-5(10)}=K(5-0) \\
-\frac{1}{5} \ln \frac{25}{50}=-\frac{1}{5} \ln \frac{1}{2}=\frac{1}{5} \ln 2=5 K
\end{gathered}
$$

De donde:

$$
K=\frac{1}{25} \ln 2
$$

Análogamente:

$$
-\left.\frac{1}{5} \ln (100-5 p)\right|_{10} ^{P_{(10)}}=\left.\left(\frac{1}{25} \ln 2\right) t\right|_{0} ^{10}
$$




$$
\begin{aligned}
-\frac{1}{5} \ln \frac{100-5 p_{(10)}}{100-5(10)} & =\frac{1}{25}(\ln 2)(10-0) \\
\ln \frac{100-5 p_{(10)}}{50} & =-2 \ln 2=\ln \frac{1}{4}
\end{aligned}
$$

De donde:

$$
\frac{100-5 p_{(10)}}{50}=\frac{1}{4} \Rightarrow p_{(10)}=\$ 17.50
$$

462. Un estanque puede contener un máximo de 10,000 peces y la tasa de crecimiento de la población de peces es proporcional al número de peces presentes y a la diferencia entre 10,000 y el número presente. El estanque inicialmente contenía 400 peces y 6 semanas después había 3,000. ¿Cuántos peces tendrá el estanque después de 8 semanas?

\section{Solución:}

Sea $P_{(t)}$ la población de peces cuando han transcurrido $t$ semanas desde el inicio.

Se sabe que para:

$$
\begin{aligned}
& t=0 \rightarrow P_{(0)}=400 \\
& t=6 \rightarrow P_{(6)}=3,000 \\
& t=\infty \rightarrow P \Rightarrow 10,000
\end{aligned}
$$

Consideremos que $10,000=A$ y escribamos:

$$
\frac{d p}{d t}=K p(A-p)
$$

Entonces:

$$
\begin{gathered}
d p=K p(A-p) d t \\
\frac{d p}{p(A-p)}=K d t \\
\frac{1}{A}\left[\frac{1}{p}+\frac{1}{A-p}\right] d p=K d t \\
\frac{1}{A} \int\left[\frac{1}{p}+\frac{1}{10,000-p]}\right] d p=K \int d t=K t+C \\
\ln p-\ln (A-p)=A K t+A C \\
\ln \frac{p}{A-p}=A K t+A C
\end{gathered}
$$




$$
\begin{gathered}
\frac{p}{A-p}=e^{A K t} e^{A C}=A_{1} e^{A K t} \text { siendo } A_{1}=e^{A C} \\
p=A A_{1} e^{A K t}-A_{1} e^{A K t} p
\end{gathered}
$$

De donde:

$$
p=\frac{A A_{1} e^{A K t}}{1+A_{1} e^{A K t}}=\frac{A}{1+A_{1} e^{-A K t}}=\frac{10,000}{1+A_{1} e^{-10,000 K t}}
$$

Encontremos los valores de $A_{1}$ y de $K$ :

$$
\begin{aligned}
& p_{(0)}=400 \rightarrow 400=\frac{10,000}{1+A_{1} e^{0}} \Rightarrow A_{1}=24 \\
& p_{(6)}=3,000 \rightarrow 3,000=\frac{10,000}{1+24 e^{-60,000 K}}
\end{aligned}
$$

Con lo cual:

$$
e^{-60,000 K}=\frac{7}{72} \quad\left[e^{-10,000 K}=0.67810\right]
$$

400

Calculemos $p_{(8)}$ :

$$
\begin{aligned}
& p_{(8)}=\frac{10,000}{1+24 e^{-80,000 K}}=\frac{10,000}{1+24\left(e^{-60,000 K}\right)^{4 / 3}} \\
& p_{(8)}=\frac{10,000}{1+24(7 / 72)^{4 / 3}}=4,824 \text { peces }
\end{aligned}
$$

A continuación se muestra la gráfica de: $P_{(t)}=\frac{10,000}{1+24(0.6781)^{t}}$

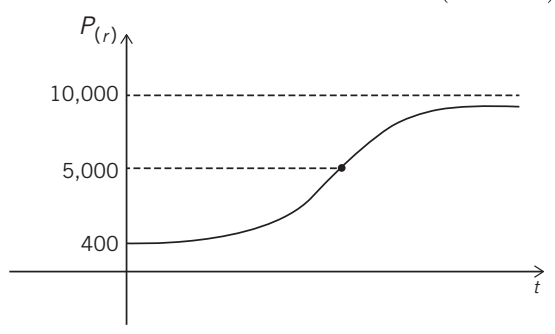

Obsérvese que el punto de inflexión ocurre cuando $P_{(t)}=\frac{A}{2}$ dado que la tasa de aumento de la población de peces crece de una manera más rápida cuando la población alcanza el $50 \%$ de la cantidad máxima de peces que puede contener el estanque. 
463. La ecuación de demanda para el producto de un fabricante está dada por:

$$
p=\frac{200(q+2)}{q^{2}+7 q+6}
$$

en donde $p$ es el precio por unidad (en dólares) cuando se demanda $q$ unidades. Si el precio de equilibrio es $\$ 2,700 / 403$, determine el excedente del consumidor cuando el mercado está en equilibrio.

\section{Solución:}

La gráfica de la ecuación de demanda es:

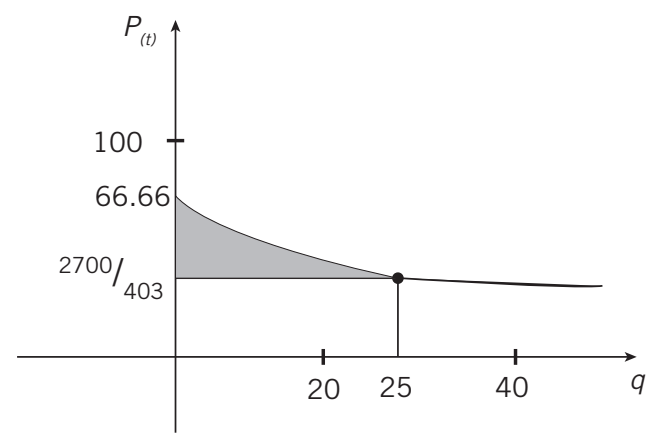

$$
\text { Si } p=\frac{2,700}{403} \Rightarrow \frac{2,700}{403}=\frac{200(q+2)}{q^{2}+7 q+6} \Rightarrow q=25 \text { unidades }
$$

Entonces:

$$
E C=\int_{0}^{25}\left[\frac{200(q+2)}{q^{2}+7 q+6}-\frac{2,700}{403}\right] d q
$$

Como:

$$
\begin{gathered}
\frac{q+2}{(q+1)(q+6)}=\frac{1 / 5}{q+1}+\frac{4 / 5}{q+6} \\
E C=200\left[\frac{1}{5} \int_{0}^{25} \frac{d q}{q+1}+\frac{4}{5} \int_{0}^{25} \frac{d q}{q+6}\right]-\frac{2,700}{403} \int_{0}^{25} d q \\
E C=\left.40 \ln (q+1)\right|_{0} ^{25}+\left.160 \ln (q+6)\right|_{0} ^{25}-\frac{2,700}{403} \times 25
\end{gathered}
$$


De donde:

$$
\begin{gathered}
E C=40 \ln 26+160 \ln \frac{31}{6}-\frac{67,500}{403} \\
E C=130.32+262.76-167.49=225.59 \text { dólares }
\end{gathered}
$$

464. Cuando el precio de un producto es $p$ dólares, las rapideces de cambio de la demanda y de la oferta son, respectivamente, $\frac{-p q}{\left(144-p^{2}\right)}$ y $\frac{p q}{\left(p^{2}-36\right)}$, en donde las cantidades $(q)$, demandadas y ofrecidas, están expresadas en miles de unidades. Considere que cuando $p=9$ dólares, la cantidad demandada es $2,000 \sqrt{7}$ unidades y la cantidad ofrecida es 3,000 unidades.

a) Encuentre la demanda cuando $p=6$ dólares.

b) Determine la demanda insatisfecha si $p=9.5$ dólares.

c) Encuentre el excedente de los consumidores si $p=11.2$ dólares.

\section{Solución:}

402

a) Se tiene que para la ecuación de demanda se cumple que:

$$
\begin{gathered}
\frac{d q}{d p}=\frac{-p q}{144-p^{2}} \Rightarrow d q=\frac{-p q}{144-p^{2}} d p \\
\int \frac{d q}{q}=\int \frac{-p d p}{144-p^{2}} \\
\ln q=\frac{1}{2} \ln \left(144-p^{2}\right)+\ln C
\end{gathered}
$$

Entonces:

$$
q=C \sqrt{144-p^{2}}
$$

Como $q_{(p=9)}=2,000 \sqrt{7} \Rightarrow 2,000 \sqrt{7}=C \sqrt{144-9^{2}} \Rightarrow C=\frac{2,000}{3}$, se obtiene:

$$
q_{d}=\frac{2,000}{3} \sqrt{144-p^{2}}
$$

Con lo cual:

$$
q_{(p=6)}=\frac{2,000}{3} \sqrt{144-6^{2}}=4,000 \sqrt{3} \text { miles de unidades }
$$


b) Con respecto a la ecuación de la oferta, se tiene que:

$$
\begin{gathered}
\frac{d p}{d p}=\frac{p q}{p^{2}-36} \Rightarrow d q=\frac{p q}{p^{2}-36} d p \\
\int \frac{d q}{q}=\int \frac{p d p}{p^{2}-36} \\
\ln q=\frac{1}{2} \ln \left(p^{2}-36\right)+\ln C^{\prime}
\end{gathered}
$$

Entonces:

$$
q=C^{\prime} \sqrt{p^{2}-36}
$$

Como $q_{(p=9)}=3,000 \Rightarrow 3,000=C^{\prime} \sqrt{9^{2}-36} \Rightarrow C^{\prime}=\frac{1,000}{\sqrt{5}}$, se obtiene:

$$
q_{0}=\frac{1,000}{\sqrt{5}} \sqrt{p^{2}-36}
$$

Para encontrar la demanda insatisfecha cuando $p=9.5$ :

$$
\begin{gathered}
\Delta q=q_{d_{(p=9.5)}}-q_{o_{(p=9.5)}} \\
\Delta q=\frac{2,000}{3} \sqrt{144-9.5^{2}}-\frac{1,000}{\sqrt{5}} \sqrt{9.5^{2}-36} \\
\Delta q=4887.63-3293.93=1593.70 \text { miles de unidades }
\end{gathered}
$$

Calculemos el excedente de los consumidores si $p=\$ 11.20$ :

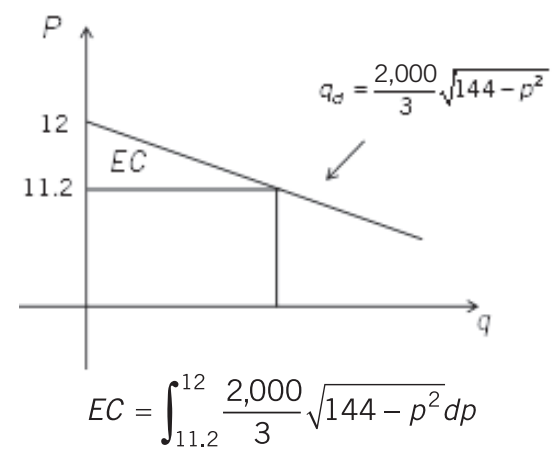

$$
\begin{gathered}
E C=\left.\frac{2,000}{3} \cdot \frac{1}{2}\left[p \sqrt{144-p^{2}}+144 \operatorname{arcsen} \frac{p}{12}\right]\right|_{11.2} ^{12} \\
E C=\frac{1,000}{3}[0+226.19-48.25-173.32]
\end{gathered}
$$




\section{$E C=1,540$ miles de dólares}

465. Una pelota se deja caer del reposo y después de $t$ segundos su velocidad es $V \frac{m t s}{s e g}$. Despreciando la resistencia del aire, demuestre que la velocidad promedio durante los primeros $\frac{T}{2}$ segundos es un tercio de la velocidad media durante el siguiente lapso de $\frac{T}{2}$ segundos.

\section{Solución:}

Se tiene que: $\frac{d V}{d t}=g \Rightarrow d V=g d t$

$V=\int d V=\int g d t=g t+C$

Cuando $t=0 \rightarrow V=0$ :

$$
0=g(0)+C \Rightarrow C=0
$$

Si $V=g t+0=g t$, la velocidad promedio en los primeros $\frac{T}{2}$ segundos es:

$$
\overline{V_{1}}=\frac{\int_{0}^{T / 2} g t d t}{T / 2}=\frac{\left.g \frac{t^{2}}{2}\right|_{0} ^{T / 2}}{T / 2}=\frac{\frac{g}{2}\left[\frac{T^{2}}{4}-0\right]}{T / 2}=\frac{g T}{4}
$$

En los siguientes $\frac{T}{2}$ segundos, la velocidad media es:

$$
\overline{V_{2}}=\frac{\int_{T / 2}^{T} g t d t}{T-\frac{T}{2}}=\frac{\left.g \frac{t^{2}}{2}\right|_{T / 2} ^{T}}{T / 2}=\frac{\frac{g}{2}\left[T^{2}-\frac{T^{2}}{4}\right]}{T / 2}=\frac{3 g T}{4}
$$

De donde: $\overline{V_{1}}=\frac{1}{3} \overline{V_{2}}$.

466. Durante una crisis económica reciente, el porcentaje de desempleados creció a razón de $P_{(t)}^{\prime}=\frac{0.4 e^{-0.1 t}}{\left(1+e^{-0.1 t}\right)^{2}}$, en donde $t$ es el tiempo en meses. Si en $t=0$ había $4 \%$ de desempleados, ¿qué porcentaje estará desempleado: a) 10 meses después, b) 20 meses después?
R.:
a) $4.924 \%$
b) $5.523 \%$ 
467. En el punto de abscisa $x$ de la gráfica de $y=f_{(x)}$ se cumple que la pendiente de la recta tangente es $f_{(x)}=4 x^{3}-3 \sqrt{x}$. Determinar $f_{(x)}$ si se sabe que el punto $(1,0)$ pertenece a la gráfica.

R.: $f_{(x)}=x^{4}-2 x^{3 / 2}+1$

468. Desde 1970, la razón de consumo de petróleo en cierto país ha sido dada, en millones de barriles por día, por la siguiente función:

$$
R_{(t)}= \begin{cases}1+0.1 t & 0 \leq t \leq 4 \\ 1.68-0.07 t & 4 \leq t \leq 12 \\ 0.24+0.05 t & 12 \leq t \leq 18\end{cases}
$$

en donde $t$ es el tiempo transcurrido en años desde 1970. Calcular el consumo total entre 1980 y 1985. Considere 365 días por año.

R.: 1,666.22 millones de barriles

469. La velocidad de producción de anticuerpos cuando han transcurrido $t$ horas después de haber sido inyectado un suero está dada por $f_{(t)}=\frac{10 t}{t^{2}+9}$. Encontrar el valor de $t$ para el cual $f_{(t)}$ es máximo y calcular el número total de anticuerpos producidos hasta ese momento.

R.: 3 horas

$5 \ln 2$

470. La productividad física marginal $\frac{d p}{d x}$ para una industria de relojes es $\frac{d p}{d q}=250(q+4)^{3 / 2}$. ¿Cuál será la productividad física $p$ cuando están en funcionamiento 5 máquinas?

R.: 21,000

471. El ingreso marginal de una empresa dedicada a comercializar zapatos es $R_{(q)}^{\prime}=10(20-q) e^{-q / 20}$. Determinar la ecuación de demanda del producto.

R.: $p=200 e^{-q / 20}$ 
472. El costo marginal de una empresa viene dado por $C_{(q)}^{\prime}=\frac{5000 \ln (q+20)}{(q+20)^{2}}$, en donde $q$ es el nivel de producción. Si los costos fijos ascienden a \$2,000, determinar la función costo. R.: $C_{(q)}=-\frac{5,000}{q+20}[1+\ln (q+20)]+2,250+250 \ln 20$

473. El volumen $V$ en litros del aire en los pulmones durante un ciclo respiratorio de 5 segundos viene dado aproximadamente por el modelo $V=0.1729 t+0.1522 t^{2}-0.0374 t^{3}$, en donde $t$ es el tiempo en segundos. Dar un valor aproximado del volumen medio del aire en los pulmones a lo largo de un ciclo.

R.: 0.5318 litros

474. Supongamos que el precio de la gasolina aumenta de acuerdo con la ecuación $P_{(t)}=1+$ $0.1 \mathrm{t}+0.02 t^{2}$ dólares por galón, en donde $t=0$ representa el año 1983. Si un automóvil recorre 15,000 millas en un año, con una media de $M$ millas por galón, encontrar el costo anual en combustible en los años: a) 1985 y b) 1990.

406
R.: a) $\frac{20,650}{M}$
b) $\frac{43,150}{M}$

475. La probabilidad de encontrar un porcentaje de hierro comprendido entre $a$ y $b$, en una muestra de mineral en cierta región geográfica, viene dada por $P_{a, b}=\frac{1,155}{32} \int_{a}^{b} x^{3}(1-x)^{3 / 2} d x$. Comprobar que $P_{0,1}=1$.

R.: $\frac{1,155}{32} \times \frac{32}{1,155}=1$

476. Respecto del problema anterior, ¿cuál es la probabilidad de que una muestra contenga no menos del $50 \%$ de hierro?
R.: $73.6109 \%$

477. En cierta universidad, la función de densidad de probabilidad de que la duración de una Ilamada telefónica sea $t$ minutos está dada por $f_{(t)}=0.4 e^{-0.4 t}(t>0)$. ¿Cuál es la probabilidad de que una llamada telefónica seleccionada al azar dure: a) entre 2 y 3 minutos, b) 2 minutos o menos y c) cuando mucho 3 minutos? 
Tenga presente que si $f$ es una función de densidad de probabilidad, entonces la probabilidad de que ocurra cierto suceso, en el intervalo $[c, d]$, es $\int_{c}^{d} f_{(x)} d x$. Además, toda función de densidad de probabilidad debe cumplir dos propiedades: i) $f_{(x)} \geq 0$ para todo $x$ en $[a, b]$ y ii) $\int_{a}^{b} f_{(x)} d x=10100 \%$. Verifíquelas.
R.: a) $14.81 \%$
b) $55.07 \%$
c) $69.88 \%$

478. En estadística, se define la función de densidad exponencial mediante $f_{(x)}=\lambda e^{-\lambda x}, x \geq 0 y$ $\lambda$ es una constante positiva. Determine:
a) El área bajo la gráfica de $f$ en $[0,+\infty[$.
b) El valor de $\int_{0}^{+\infty} x f_{(x)} d x$.
R.: a) 1
b) $1 / \lambda$

479. Las funciones de costo marginal son:

$$
\begin{gathered}
\text { CMg }=5,000 \frac{\ln (x+20)}{(x+20)^{2}}+10 x \\
I M g=180
\end{gathered}
$$

en donde $x$ es el nivel de producción. Si los costos fijos ascienden a $\$ 1,000$ y el nivel de producción actual es de 14 unidades, hallar la utilidad total.

\section{R.: \$ 206.71}

480. En cada punto de cierta curva se cumple que $y^{\prime \prime}=\frac{1}{x}$. La curva pasa por el punto $(1,0)$ siendo $135^{\circ}$ el ángulo de inclinación de la tangente en dicho punto. Encontrar el punto de abscisa $x=e$.

R.: $(e, 2-e)$ 
481. En cada punto de cierta curva se cumple que $y^{\prime \prime}=\frac{3}{\sqrt{x+3}}$. Hallar la ecuación de la curva sabiendo que pasa por el punto $(1,1)$ y tiene una inclinación de $45^{\circ}$ en dicho punto, y determinar la ordenada del punto cuyo $x=6$.

R.: $y=4(x+3)^{3 / 2}-11 x-20$

22

482. Un camión que viaja a lo largo de un camino recto tiene una velocidad (en pies/segundo) en el instante $t$ (en segundos) dada por:

$$
V_{(t)}=\frac{1}{12} t^{2}+2 t+44 \quad(0 \leq t \leq 5)
$$

Encontrar la velocidad promedio del camión durante el intervalo de tiempo desde $t=0$ hasta $t=5$.

R.: 49.69 pies/segundo

483. Las ventas de la compañía $X Y Z$ durante los primeros $t$ años de operación se aproximan mediante la función $S_{(t)}=t \sqrt{0.2 t^{2}+4}$, en donde $S_{(t)}$ se mide en millones de dólares. ¿Cuáles fueron las ventas anuales promedio de XYZ durante sus primeros 5 años de operación?

\section{R.: $\$ 6,333,333$}

484. Una inversión de $\$ 3,000$ gana intereses a una tasa anual de $5 \%$ compuesto continuamente. Después de $t$ años, su valor $S$ en dólares está dado por $\$ 3,000 e^{0.05 t}$. Encuentre el valor promedio de una inversión a 2 años.

\section{R.: \$ 3,155.13}

485. Suponga que se inyecta un líquido de contraste (tinte) en la corriente sanguínea a una razón $R$ constante. En el tiempo $t$, sea $C_{(t)}=\frac{R}{F_{(t)}}$ la concentración del tinte en un punto a cierta distancia del punto de inyección, en donde el flujo sanguíneo $F_{(t)}$ está dado por $F_{(t)}=\frac{F_{1}}{(1+\alpha t)^{2}} \quad[0 \leq t \leq T]$. Demostrar que la concentración promedio en el intervalo $[0, T]$ es $\bar{C}=\frac{R\left(1+\alpha T+\frac{1}{3} \alpha^{2} T^{2}\right)}{F_{1}}$. 
486. Se prevé que la población con 65 o más años (en millones) desde el año 2000 hasta el año 2050 será $f_{(t)}=\frac{85}{1+1,859 e^{-0.66 t}}(0 \leq t \leq 5)$, en donde $t$ se mide en décadas y $t=0$ corresponde al año 2000. ¿Cuál será la población promedio con 65 o más años entre los años 2000 y 2030 ?

$$
\text { R.: } 49.7 \text { millones/década }
$$

487. Encuentre la función $f_{(x)}$ si se conoce que la pendiente de la recta tangente a la gráfica de $f_{(x)}$ en cualquier punto $\left(x, f_{(x)}\right)$ es $e^{x}+x$ y que la gráfica pasa por $(0,3)$.

$$
\text { R.: } f_{(x)}=e^{x}+\frac{x^{2}}{2}+2
$$

488. El número de ciudadanos estadounidenses con edades entre 45 y 54 años (que era igual a 25 millones al principio de 1990) aumentará a una razón de $R_{(t)}=0.00933 t^{3}+0.019 t^{2}$ $-0.10833 t+1.3467$ millones de personas por año, $t$ años después del inicio de 1990 . ¿Cuántos ciudadanos entre 45 y 54 años se habrán agregado a la población entre el año 1990 y el año 2000?

\section{R.: 37.7085 millones}

489. Un estudio realizado por cierta compañía de televisión por cable estima que el número de suscriptores crecerá a razón de $100+210 t^{3 / 4}$ nuevos suscriptores por mes a $t$ meses del inicio del servicio. Si 5,000 suscriptores han solicitado el servicio antes de la fecha inicial, ¿cuántos suscriptores habrá a 16 meses de dicha fecha?

$$
\text { R.: } 21.960
$$

490. Durante una tormenta, la lluvia cayó a razón de $\frac{8}{(t+4)^{2}}(0 \leq t \leq 2)$ pulgadas por hora. ¿Cuánta Iluvia cayó durante la segunda hora?

$$
\text { R.: } \frac{4}{15} \text { pulgadas }
$$

491. ¿Cuál debe ser la desaceleración constante que debe imponerse a un automóvil que se mueve a lo largo de un camino recto para llevarlo al reposo desde una velocidad de 88 pies/segundo en 9 segundos? ¿Cuál sería la distancia recorrida durante el frenado?

$$
\text { R.: }-9.77 \mathrm{pies} / \mathrm{seg}^{2}
$$

396 pies 
492. Los puntos $(-1,3)$ y $(0,2)$ se encuentran en una curva y en cualquier punto $(x, y)$ sobre la curva se cumple que $\frac{d^{2} y}{d x^{2}}=2-4 x$. Determinar el punto de la curva que tenga abscisa $x=2$.

R.: $(2,2)$

493. La ecuación de la recta tangente a una curva en el punto $(1,3)$ es $y=x+2$. Si en cualquier punto $(x, y)$ de la curva se cumple que $y^{\prime \prime}(x, y)=6 x$, obtenga la ecuación de la curva.

R.: $y=x^{3}-2 x+4$

494. Determine el excedente de los consumidores y el de los productores si se conoce que la cantidad demandada ( $q$ centenas) se relaciona con el precio ( $p$ dólares) según $p=-0.2 q^{2}$ +80 , mientras que la cantidad ofertada ( $q$ centenas) se relaciona con el precio ( $p$ dólares) según $p=0.1 q^{2}+q+40$, y se sabe que el precio de mercado se establece como el precio de equilibrio.

$$
\text { R.: } \begin{aligned}
E C & =\$ 13,333 \\
E P & =\$ 11,667
\end{aligned}
$$

410

495. El ingreso marginal de una empresa por su producto es $/_{(x)}^{\prime}=10(20-x) e^{-x / 20}$. Determinar la ecuación de demanda del producto.

R.: $p=2,000 e^{-x / 20}$

496. Suponga que $f_{(x)}=\frac{1}{x}$, en donde $x \in\left[e, e^{2}\right]$, es una función de densidad de probabilidad. Encontrar la probabilidad de que $x \geq 3$.

R.: $2-\ln 3$

497. En el problema 461, ¿en cuánto tiende a estabilizarse el precio cuando el tiempo transcurrido es suficientemente grande $(t \rightarrow \infty)$ ?

R.: $\$ 20$ 
498. La rapidez instantánea de cambio del costo de la educación superior (miles de dólares/ año) es el $8 \%$ del costo actual $\left[C^{\prime}{ }_{(t)}=0.08 C_{(t)}\right]$. Si actualmente el costo de la educación superior es de $\$ 20,000$, ¿cuál será el costo dentro de 5 años?
R.: \$29,836.50

499. Las ecuaciones de oferta y demanda para una mercadería en particular son, respectivamente, $400 p=x^{2}+2,400$ y $100 p=1,600-x^{2}$. Calcule $3 E C-10 E P$ si prevalece el equilibrio.

\section{R.: $\$ 75.45$}

500. En un mercado monopólico se venden $q$ unidades de un producto al precio unitario de $p$ dólares, según $p=274-q^{2}$, siendo el costo marginal $3 q+4$.

a) Si el precio de mercado lo fija el monopolista de modo que se maximicen sus utilidades, ¿cuál es el precio de mercado?

b) Calcule el excedente del consumidor.
R.: a) 193
b) 486 


\section{PROBLEMAS RESUELTOS Y PROPUESTOS DEL CAPÍTULO X}

501. Calcular: $\int_{0}^{1} \int_{3 x}^{x^{2}} 14 x^{2} y d y d x$.

Solución:

Se pide:

$$
\begin{gathered}
I=\int_{0}^{1} d x \int_{3 x}^{x^{2}} 14 x^{2} y d y=\left.\int_{0}^{1} 14 x^{2} \frac{y^{2}}{2}\right|_{3 x} ^{x^{2}} d x \\
I=\int_{0}^{1} 7 x^{2}\left(x^{4}-9 x^{2}\right) d x=\left.7\left[\frac{x^{7}}{7}-\frac{9 x^{5}}{5}\right]\right|_{0} ^{1} \\
I=7\left[\frac{1}{7}-\frac{9}{5}\right]=7\left(-\frac{58}{35}\right)=-\frac{58}{5}
\end{gathered}
$$

412 502. Calcular: $\int_{0}^{2} \int_{0}^{1}(1+2 x+2 y) d y d x$.

Solución:

Se pide:

$$
I=\int_{0}^{2} d x \int_{0}^{1}(1+2 x+2 y) d y
$$

Es decir:

$$
\begin{gathered}
I=\left.\int_{0}^{2}\left(y+2 x y+y^{2}\right)\right|_{0} ^{1} d x \\
I=\int_{0}^{2}(1+2 x+1-0-0-0) d x \\
I=\int_{0}^{2}(2+2 x) d x=2 x+\left.x^{2}\right|_{0} ^{2}
\end{gathered}
$$

De donde:

$$
I=(4+4)-(0+0)=8
$$


503. Calcular: $\int_{1}^{e} \int_{0}^{\ln x} x y d x d y$.

Solución:

Se pide:

$$
\begin{gathered}
I=\int_{1}^{e} x d x \int_{0}^{\ln x} y d y=\left.\int_{1}^{e} x d x\left(\frac{y^{2}}{2}\right)\right|_{0} ^{\ln x} \\
I=\frac{1}{2} \int_{1}^{e} x\left(\ln ^{2} x-0\right) d x=\frac{1}{2} \int_{1}^{e} x \cdot \ln ^{2} x \cdot d x
\end{gathered}
$$

Calculemos: $\int x \cdot \ln ^{2} x \cdot d x$ :

$$
\begin{array}{cc}
u=\ln ^{2} x & v=\frac{x^{2}}{2} \\
\downarrow & \uparrow \\
d u=2 \frac{\ln x}{x} d x & d v=x \cdot d x
\end{array}
$$

Entonces:

$$
\int x \cdot \ln ^{2} x \cdot d x=\frac{x^{2}}{2} \ln ^{2} x-\int x \cdot \ln x \cdot d x
$$

Análogamente:

$$
\int x \cdot \ln x \cdot d x=\frac{x^{2}}{2} \ln x-\frac{1}{4} x^{2}
$$

Con lo cual:

$$
\begin{gathered}
I=\frac{1}{2} \int_{1}^{e} x \cdot \ln ^{2} x \cdot d x=\left.\frac{1}{2}\left[\frac{x^{2}}{2} \ln ^{2} x-\frac{x^{2}}{2} \ln x+\frac{1}{4} x^{2}\right]\right|_{1} ^{e} \\
I=\frac{1}{2}\left[\frac{e^{2}}{2}-\frac{e^{2}}{2}+\frac{e^{2}}{4}-0+0-\frac{1}{4}\right]=\frac{e^{2}-1}{8}
\end{gathered}
$$

504. Calcule la integral doble $\iint_{R} 4 x d A$ en donde $R$ es la región acotada por $y=4-x^{2}, y=3 x$ e
$y=0$.

\section{Solución:}

Se tiene que la región $R$ está conformada por $R_{1}$ y $R_{2}$ : 


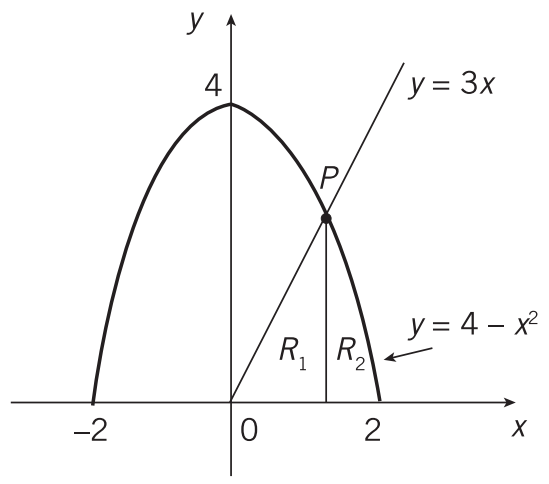

Encontramos el punto $P$ de intersección:

$$
\begin{gathered}
4-x^{2}=3 x \rightarrow x^{2}+3 x-4=0 \\
(x+4)(x-1)=0 \rightarrow x_{p}=1
\end{gathered}
$$

Se pide:

$$
\begin{gathered}
R_{1}+R_{2}=4 \iint_{R_{1}} x d y d x+4 \iint_{R_{2}} x d y d x \\
4 \int_{0}^{1} x d x \int_{0}^{3 x} d y+4 \int_{1}^{2} x d x \int_{0}^{4-x^{2}} d y \\
4 \int_{0}^{1} 3 x^{2} d x+4 \int_{1}^{2}\left(4 x-x^{3}\right) d x \\
\left.4 x^{3}\right|_{0} ^{1}+\left.4\left(2 x^{2}-\frac{x^{4}}{4}\right)\right|_{1} ^{2} \\
4(1-0)+4\left(8-4-2+\frac{1}{4}\right)
\end{gathered}
$$

La integral pedida vale $4+9=13$.

505. Calcule $\iint_{R} f_{(x, y)} d x d y$ si:

$$
\begin{aligned}
& f_{(x, y)}=x+y \\
& R: x^{2}+y^{2} \leq 4, x \geq 0, y \geq 0
\end{aligned}
$$




\section{Solución:}

Se tiene:
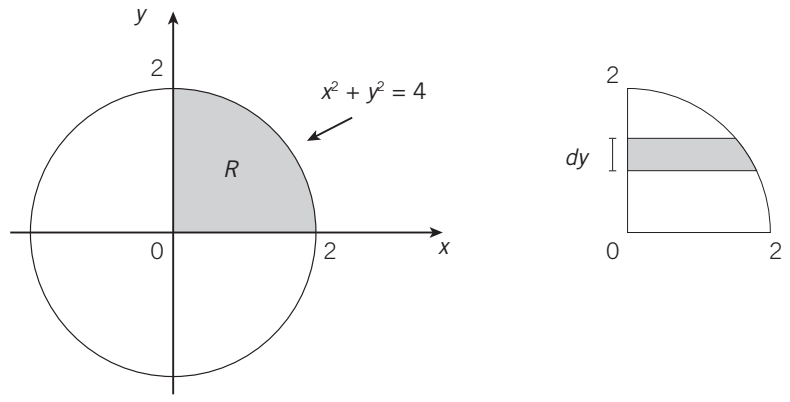

$y$ varía entre 0 y 2

$x$ varía desde $x=0$ hasta $x=\sqrt{4-y^{2}}$

Entonces:

$$
\begin{gathered}
I=\iint_{R} f_{(x, y)} d x d y=\int_{0}^{2} d y \int_{0}^{\sqrt{4-y^{2}}}(x+y) d x \\
I=\left.\int_{0}^{2} d y\left[\frac{x^{2}}{2}+x y\right]\right|_{0} ^{\sqrt{4-y^{2}}} \\
I=\int_{0}^{2}\left[\frac{4-y^{2}}{2}+\sqrt{4-y^{2}} y-0-0\right] d y \\
I=2 y-\left.\frac{y^{3}}{6}\right|_{0} ^{2}+\left(-\frac{1}{2}\right) \int_{0}^{2}(-2 y) \sqrt{4-y^{2}} d y \\
I=\left(4-\frac{8}{6}-0\right)+\left.\left(\frac{-1}{2}\right) \frac{\left(4-y^{2}\right)^{3 / 2}}{3 / 2}\right|_{0} ^{2}=\frac{8}{3}-\frac{1}{3}[0-8]=\frac{16}{3}
\end{gathered}
$$

506. Usando integrales dobles, calcular el área encerrada en el primer cuadrante por las curvas $y=4-\ln (x+1)$ e $y=\ln (x+1)$.

\section{Solución:}

La región cuya área se requiere calcular se muestra a continuación: 


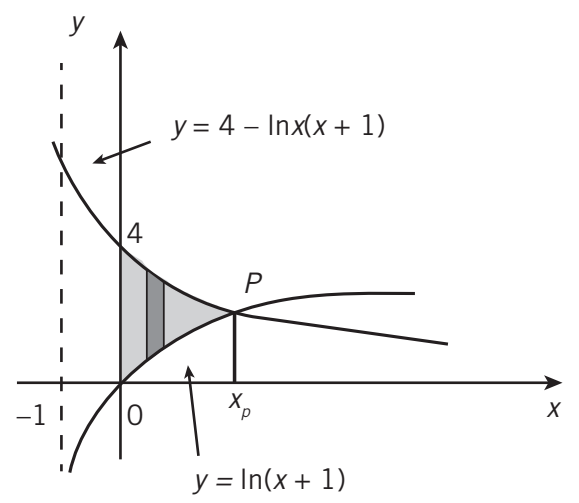

Si se consideran elementos diferenciales verticales, el área es:

$$
A=\int_{0}^{x_{p}} d x \int_{\ln (x+1)}^{4-\ln (x+1)} d y=\left.\int_{0}^{x_{p}} y\right|_{\ln (x+1)} ^{4-\ln (x-1)} d x
$$

Es decir:

$$
A=\int_{0}^{x_{p}}[4-\ln (x+1)-\ln (x+1)] d x
$$

La abscisa del punto $P$ se encuentra de igualar:

$$
\begin{gathered}
4-\ln \left(x_{p}+1\right)=\ln \left(x_{p}+1\right) \\
\ln \left(x_{p}+1\right)=2 \rightarrow x_{p}+1=e^{2}
\end{gathered}
$$

Con lo cual:

$$
x_{p}=e^{2}-1
$$

Entonces:

$$
\begin{gathered}
A=\int_{0}^{e^{2}-1}[4-2 \ln (x+1)] d x \\
A=4 x-\left.2(x+1)[\ln (x+1)-1]\right|_{0} ^{e^{2}-1} \\
A=4\left(e^{2}-1\right)-2\left(e^{2}\right)\left[\ln e^{2}-1\right]-0+2[\ln 1-1] \\
A=4 e^{2}-4-4 e^{2}+2 e^{2}-2=2 e^{2}-6 \mathrm{un}^{2}
\end{gathered}
$$


507. Dada $f_{(x)}=\left\{\begin{array}{ll}3 x^{2} & 0 \leq x \leq 2 \\ 16-2 x & x \geq 2\end{array}\right.$, determine el área de la región limitada por la gráfica de $f_{(x)}$, el eje de abscisas y la recta $x=3$.

\section{Solución:}

Se muestra un esbozo de la curva:

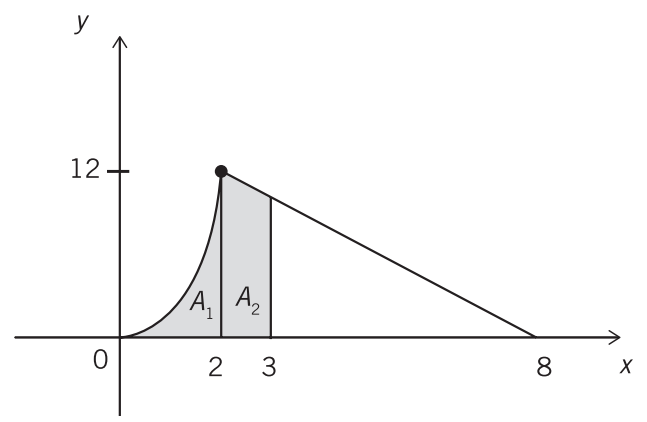

El área pedida se compone de:

$$
\begin{gathered}
A_{1}=\int_{0}^{2} d x \int_{0}^{3 x^{2}} d y=\left.\int_{0}^{2} y\right|_{0} ^{3 x^{2}} d x=\int_{0}^{2} 3 x^{2} d x \\
A_{2}=\int_{2}^{3} d x \int_{0}^{16-2 x} d y=\left.\int_{2}^{3} y\right|_{0} ^{16-2 x} d x=\int_{2}^{3}(16-2 x) d x
\end{gathered}
$$

De donde:

$$
\begin{gathered}
A_{T}=A_{1}+A_{2}=\left.x^{3}\right|_{0} ^{2}+\left.\left(16 x-x^{2}\right)\right|_{2} ^{3} \\
A_{T}=(8-0)+(48-9-32+4)=8+11=19 u^{2}
\end{gathered}
$$

508. Mediante el empleo de integrales dobles, hallar el área encerrada entre las curvas:

$$
\begin{gathered}
y=x^{3}-6 x^{2}+8 x \\
y=x^{2}-4 x
\end{gathered}
$$

\section{Solución:}

Las ecuaciones de las curvas son:

$$
\begin{gathered}
y=x^{3}-6 x^{2}+8 x=x\left(x^{2}-6 x+8\right)=x(x-2)(x-4) \\
y=x^{2}-4 x=x(x-4)
\end{gathered}
$$


La región encerrada por las curvas es:

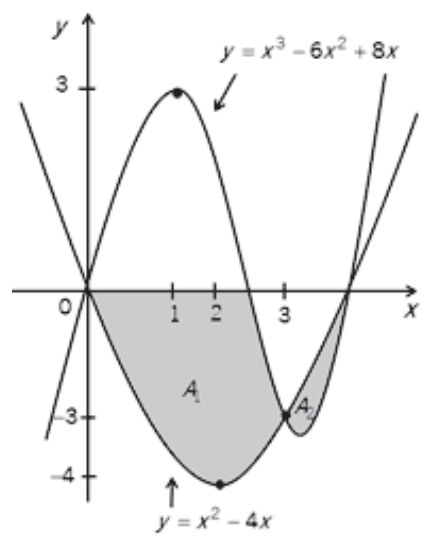

Los puntos de intersección se encuentran a partir de:

$$
\begin{gathered}
x^{3}-6 x^{2}+8 x=x^{2}-4 x \\
x^{3}-7 x^{2}+12 x=0
\end{gathered}
$$

$$
\begin{aligned}
x(x-3)(x-4)=0 & \rightarrow B(3,-3) \\
& \searrow C(4,0)
\end{aligned}
$$

Para el cálculo de $A_{1}$ y de $A_{2}$ :

$$
A_{T}=\int_{0}^{3} d x \int_{x^{2}-4 x}^{x^{3}-6 x^{2}+8 x} d y+\int_{3}^{4} d x \int_{x^{3}-6 x^{2}+8 x}^{x^{2}-4 x} d y
$$

Es decir:

$$
\begin{gathered}
A_{T}=\int_{0}^{3}\left[x^{3}-6 x^{2}+8 x-x^{2}+4 x\right] d x+\int_{3}^{4}\left[x^{2}-4 x-x^{3}+6 x^{2}-8 x\right] d x \\
A_{T}=\left.\left(\frac{x^{4}}{4}-\frac{7 x^{3}}{3}+6 x^{2}\right)\right|_{0} ^{3}+\left.\left(\frac{-x^{4}}{4}+\frac{7 x^{3}}{3}-6 x^{2}\right)\right|_{3} ^{4} \\
A_{T}=\left(\frac{81}{4}-63+54\right)+\left(-64+\frac{448}{3}-96+\frac{81}{4}-63+54\right)
\end{gathered}
$$

De donde:

$$
A_{T}=\frac{45}{4}+\left(\frac{-32}{3}+\frac{45}{4}\right)=\frac{71}{6} u^{2}
$$


509. Empleando integrales dobles, hallar el área encerrada entre las curvas $x y=1$ y $y x^{2}+y-x$ $=0$ a la derecha de la recta $x=1$.

\section{Solución:}

Las gráficas de las dos curvas se muestran en el gráfico siguiente:

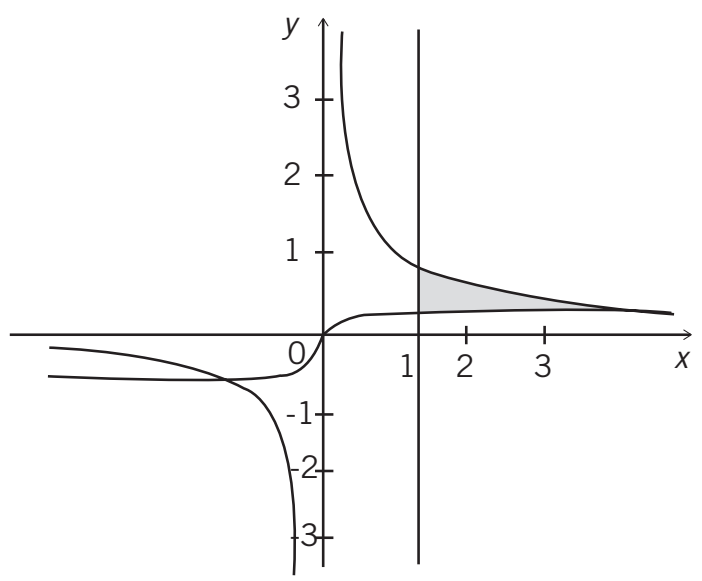

Analicemos si para $x>1$, las 2 gráficas se cortan o no:

$$
\begin{gathered}
x y=1 \rightarrow y_{1}=1 / x \\
y x^{2}+y-x=0 \rightarrow y_{2}=\frac{x}{x^{2}+1}
\end{gathered}
$$

Se cortarán si $y_{1}=y_{2}$.

$$
\begin{aligned}
& i \frac{1}{x}=\frac{x}{x^{2}+1} ? \\
& i x^{2}+1=x^{2} ?
\end{aligned}
$$

No se cortan.

Por lo tanto: $y_{1}>y_{2} \forall x>1$.

El área pedida es:

$$
A=\int_{1}^{\infty} d x \int_{y_{2}}^{y_{1}} d y=\lim _{b \rightarrow \infty} \int_{1}^{b} d x \int_{y_{2}}^{y_{1}} d y=\left.\lim _{b \rightarrow \infty} \int_{1}^{b} y\right|_{y_{2}} ^{y_{1}} d x
$$


De donde:

$$
\begin{gathered}
A=\lim _{b \rightarrow \infty} \int_{1}^{b}\left[y_{1}-y_{2}\right] d x \\
A=\lim _{b \rightarrow \infty} \int_{1}^{b}\left[\frac{1}{x}-\frac{x}{x^{2}+1}\right] d x \\
A=\left.\lim _{b \rightarrow \infty}\left[\ln x-\frac{1}{2} \ln \left(x^{2}+1\right)\right]\right|_{1} ^{b} \\
A=\left.\lim _{b \rightarrow \infty} \ln \frac{x}{\sqrt{x^{2}+1}}\right|_{1} ^{b} \\
A=\lim _{b \rightarrow \infty}\left(\ln \frac{b}{\sqrt{b^{2}+1}}-\ln \frac{1}{\sqrt{2}}\right)
\end{gathered}
$$

Como

se tendrá que:

$$
\lim _{b \rightarrow \infty}\left(\ln \frac{b}{\sqrt{b^{2}+1}}\right)=\ln \left(\lim _{b \rightarrow \infty} \frac{b}{\sqrt{b^{2}+1}}\right)=\ln \left(\lim _{b \rightarrow \infty} \frac{1}{\sqrt{1+\frac{1}{b^{2}}}}\right)=\ln 1=0,
$$

$$
A=0-\lim _{b \rightarrow \infty} \ln \frac{1}{\sqrt{2}}=-\ln \frac{1}{\sqrt{2}}=\frac{1}{2} \ln 2
$$

510. Suponga que una masa está distribuida sobre una región $R$ del plano $x y$ de forma que la densidad en todo punto es igual a la distancia desde el punto al origen $(0,0)$. Exprese la masa total de $R$ como una integral doble.

\section{Solución:}

Se tiene que:

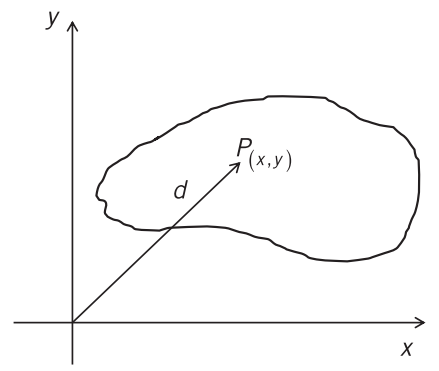




$$
\rho_{(x, y)}=d=\sqrt{x^{2}+y^{2}}
$$

Entonces:

$$
M=\iint_{R} \sqrt{x^{2}+y^{2}} d x d y
$$

511. Evaluar: $\int_{0}^{4} \int_{x^{2}}^{4} x^{2} y d y d x$

$$
\text { R.: } \frac{-20,992}{21}
$$

512. Calcular: $\int_{0}^{6} \int_{y / 2}^{3}(x+y) d x d y$.

$$
\text { R.: } 36
$$

513. Calcular: $\int_{0}^{1} \int_{y}^{1} y e^{x+y} d x d y$.

$$
\text { R.: }-\frac{1}{4} e^{2}+e-\frac{1}{4}
$$

514. Calcular: $\iint_{R} f_{(x, y)} d x d y$ si $f_{(x, y)}=9-x^{2}-y^{2}$ y $R: x^{2}+y^{2} \leq 9, x \geq 0, y \geq 0$.

$$
\text { R.: } \frac{81 \pi}{8}
$$

515. Calcular la siguiente integral: $\int_{1}^{2} \int_{x+1}^{x-1}\left(3 x^{2}-2 y\right) d y d x$.

$$
\text { R.: }-8
$$

516. Calcular $\iint_{R} \frac{1}{y^{2}+1} d A$ en donde $R$ es el triángulo acotado por las rectas $y=\frac{x}{2}, y=-x$ e $y=2$. Emplee elementos diferenciales horizontales.

$$
\text { R.: } \frac{3}{2} \ln 5
$$

517. Usando integrales dobles, calcular el área encerrada por las parábolas:

$$
\begin{gathered}
x=y^{2} \\
8 x^{2}=-y
\end{gathered}
$$
R.: $1 / 24$ 
518. Calcular el área comprendida entre las gráficas de $f_{(x)}=3 x^{3}-x^{2}-10 x$ y $g_{(x)}=-x^{2}+2 x$. R.: $24 u n^{2}$

519. Encontrar el área de la región limitada por las curvas, $y=x^{4}-2 x^{3}-2$, el eje de abscisas y las rectas $x=1$ y $x=3$.

R.: $11.41 u^{2}$

520. Asuma que $R$ es la región interior de los límites de cierto distrito, y sea $f_{(x, y)}$ la densidad de viviendas (número de casa por kilómetro cuadrado) en el punto $P_{(x, y)}$. Si el impuesto sobre la propiedad de cada casa del distrito es de $\$ 4,200$ por año, exprese el total de los impuestos sobre la propiedad recaudados en el distrito como una integral doble. (Asuma que $x$ e $y$ se miden en kilómetros).

R.: $\iint_{R} 4,200 f_{(x, y)} d x d y$ 


\section{AMENIDAD 2}

En la amenidad 2 del libro \#76 de la colección Apuntes de Estudio, titulado Geometría Analítica en dos dimensiones - de los mismos autores de este libro-, se planteó el siguiente problema cuya solución aparece a continuación. Se han empleado ocho maneras distintas de resolver la parte a) del mismo.

Una región $R$ está dentro de un cuadrado de lado 2a y está conformada por todos los puntos que se encuentran más cerca del centro que de sus lados.

a) Encontrar el área de $R$.

b) ¿Cuál es la probabilidad de que al escoger al azar un punto cualquiera del interior del cuadrado, se cumpla que la distancia al centro es menor que la distancia a los lados del cuadrado?

\section{Solución:}

a) Determinemos en primer lugar el área de la región $R$ mediante 8 formas diferentes.

Ubiquemos al cuadrado de lado $2 a$ de tal modo que el centro del cuadrado coincida con el origen de coordenadas:

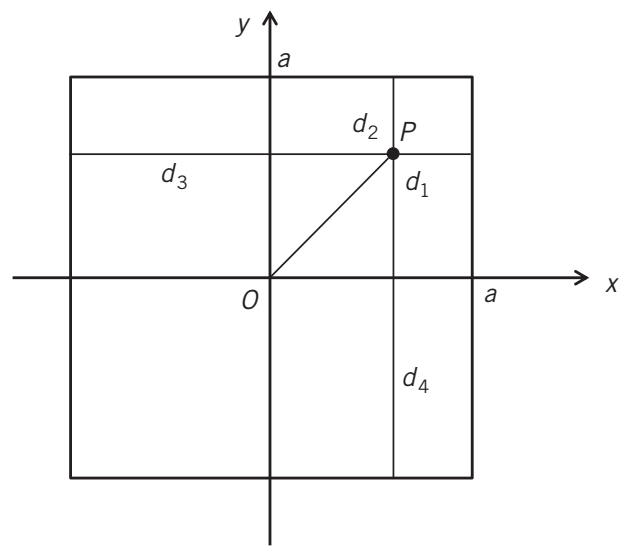


Sea $P_{(x, y)}$ un punto cualquiera dentro del cuadrado. Se debe cumplir que:

$$
\begin{aligned}
& d_{(P O)}<d_{1} \Rightarrow \sqrt{x^{2}+y^{2}}<a-x \\
& d_{(P O)}<d_{2} \Rightarrow \sqrt{x^{2}+y^{2}}<a-y . \\
& d_{(P O)}<d_{3} \Rightarrow \sqrt{x^{2}+y^{2}}<a+x \\
& d_{(P O)}<d_{4} \Rightarrow \sqrt{x^{2}+y^{2}}<a+y .
\end{aligned}
$$

A partir de (1): $\sqrt{x^{2}+y^{2}}<a-x$, se obtiene:

$$
x^{2}+y^{2}<(a-x)^{2} \Rightarrow y^{2}<-2 a\left(x-\frac{a}{2}\right)
$$

El vértice de la parábola $y^{2}=-2 a(x-a / 2)$ de eje horizontal es $V_{(a / 2,0)}$, se extiende hacia la izquierda y pasa por los puntos $(0, a)$ y $(0,-a)$.

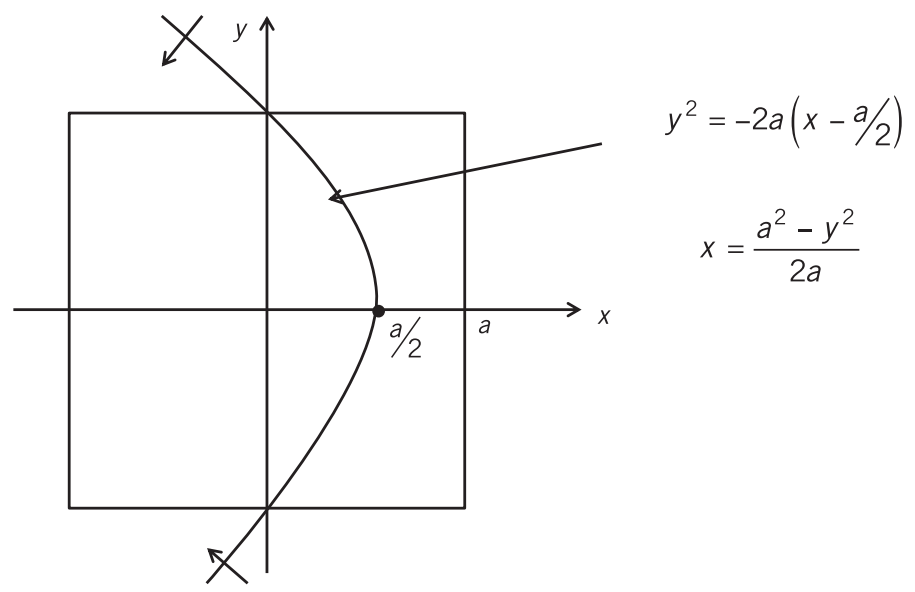


Como se trata de una inecuación y el punto $(0,0)$ satisface a $y^{2}<-2 a(x-a / 2) \Rightarrow 0<a^{2}$, los puntos situados a la izquierda de la parábola cumplen con la inecuación (1).

Análogamente, de (2), (3) y (4) se obtienen:

(2)

$(x-0)^{2}<-2 a(y-a / 2) \quad v(0, a / 2)$

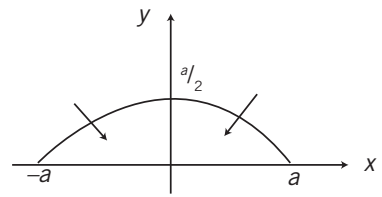

(3)

$$
(y-0)^{2}<2 a(x+a / 2) \quad v(-a / 2,0)
$$

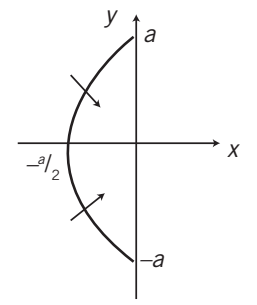

(4) $\quad(x-0)^{2}<2 a(y+a / 2) \quad v(0, a / 2)$

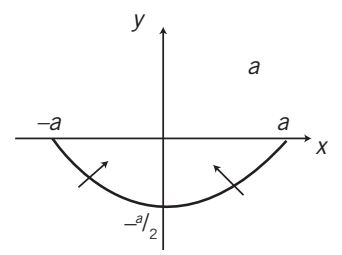

La región $R$ resulta ser la intersección de los conjuntos solución (1), (2), (3) y (4); vale decir:

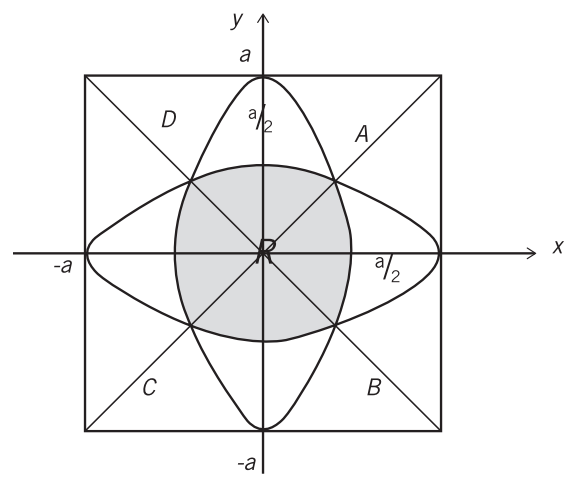

Para determinar el área de la región $R$ se requiere conocer las coordenadas de los puntos $A, B$, $C$ y $D$. De acuerdo con la simetría de la figura, $A$ es el punto de intersección de (1) o (2) con la recta $x=y$. Así pues: 


$$
\begin{gathered}
\left\{\begin{array}{l}
\sqrt{x^{2}+y^{2}}=a-x \Rightarrow \sqrt{x^{2}+y^{2}}=\sqrt{x^{2}+x^{2}}=a-x \\
x=y
\end{array}\right. \\
x \sqrt{2}=a-x \\
x=\frac{a}{\sqrt{2}+1}=(\sqrt{2}-1) a
\end{gathered}
$$

De donde se obtiene:

$$
\begin{aligned}
& A_{((\sqrt{2}-1) a,(\sqrt{2}-1) a)} \\
& B_{((\sqrt{2}-1) a,(1-\sqrt{2}) a)} \\
& C_{((1-\sqrt{2}) a,(1-\sqrt{2}) a)} \\
& D_{((1-\sqrt{2}) a,(\sqrt{2}-1) a)}
\end{aligned}
$$

\section{CÁLCULO DEL ÁREA DE LA REGIÓN R}

La calcularemos descomponiendo $R$ en formas distintas y empleando integrales simples o dobles, ya sea con coordenadas rectangulares o con coordenadas polares.

\section{FORMA \# 1}

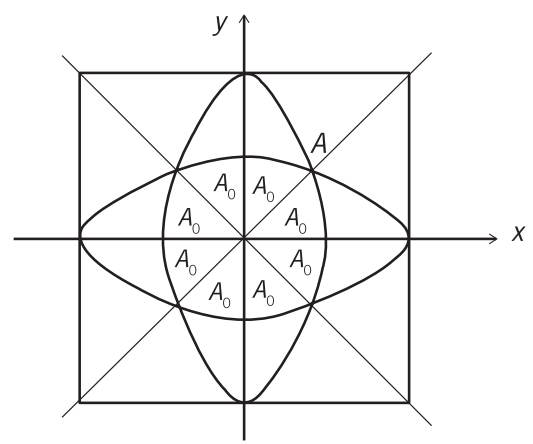

La región $R$ está conformada por 8 regiones de área $A_{0}$.

$$
A_{T}=8 A_{0}
$$

Para el cálculo de $A_{0}$ conviene considerar elementos diferenciales horizontales: 


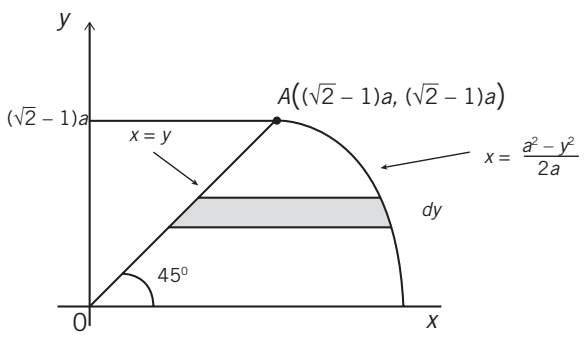

\section{Entonces:}

$$
\begin{gathered}
A_{T}=8 \int_{0}^{(\sqrt{2}-1) a}\left[\frac{a^{2}-y^{2}}{2 a}-y\right] d y \\
A_{T}=\left.8\left(\frac{1}{2 a}\right)\left[a^{2} y-\frac{y^{3}}{3}-a y^{2}\right]\right|_{0} ^{(\sqrt{2}-1) a} \\
A_{T}=\frac{4}{a}\left[a^{2}(\sqrt{2}-1) a-\frac{1}{3}(5 \sqrt{2}-7) a^{3}-(3-2 \sqrt{2}) a^{3}\right] \\
A_{T}=4 a^{2}\left[\frac{4 \sqrt{2}-5}{3}\right] \\
A_{T}=\frac{4}{3}(4 \sqrt{2}-5) a^{2}
\end{gathered}
$$

\section{FORMA \#2}

La región $R$ se compone de una unión de regiones:

$$
A_{T}=2\left(A_{1}+A_{2}\right)
$$
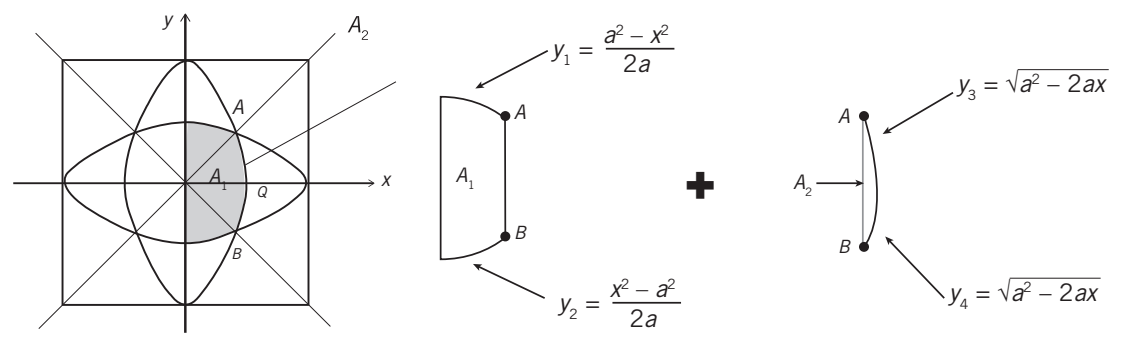

$$
A_{\left.((\sqrt{2}-1) a,(\sqrt{2}-1) a) \quad Q_{(a / 2}, 0\right)}
$$


Entonces:

$$
\begin{gathered}
A_{1}=\int_{0}^{(\sqrt{2}-1) a}\left[y_{1}-y_{2}\right] d x=\int_{0}^{(\sqrt{2}-1) a} \frac{a^{2}-x^{2}}{a} d x=a x-\left.\frac{x^{3}}{3 a}\right|_{0} ^{(\sqrt{2}-1) a}=\frac{4-2 \sqrt{2}}{3} \cdot a^{2} \\
A_{2}=\int_{(\sqrt{2}-1) a}^{0.5 a}\left[y_{3}-y_{4}\right] d x=2 \int_{(\sqrt{2}-1) a}^{0.5 a} \sqrt{a^{2}-2 a x} d x=\left.2\left(\frac{-1}{2 a}\right) \frac{\left(a^{2}-2 a x\right)^{3 / 2}}{3 / 2}\right|_{(\sqrt{2}-1) a} ^{0.5 a}=\frac{10 \sqrt{2}-14}{3} \cdot a^{2}
\end{gathered}
$$

Con lo cual:

$$
A_{T}=2\left(A_{1}+A_{2}\right)=2 \cdot \frac{4-2 \sqrt{2}+10 \sqrt{2}-14}{3} a^{2}=\frac{4}{3}(4 \sqrt{2}-5) a^{2}
$$

\section{FORMA \#3}

La región $R$ se compone de una unión de regiones:

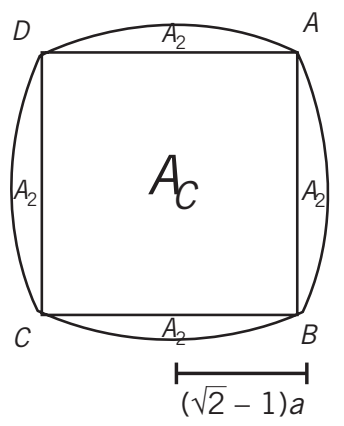

$A_{T}=A_{C}+4 A_{2}$

$A_{C}$ : área del cuadrado de lado $2(\sqrt{2}-1)$ a

$A_{2}$ : área del segmento parabólico calculado en la forma \#2.

Entonces:

$$
\begin{gathered}
A_{T}=[2(\sqrt{2}-1) a]^{2}+4\left(\frac{10 \sqrt{2}-14}{3}\right) a^{2} \\
A_{T}=4(3-2 \sqrt{2}) a^{2}+\frac{4}{3}(10 \sqrt{2}-14) a^{2} \\
A_{T}=\frac{4}{3}(4 \sqrt{2}-5) a^{2}
\end{gathered}
$$




\section{FORMA \#4}

La región $R$ se compone de una diferencia de regiones.

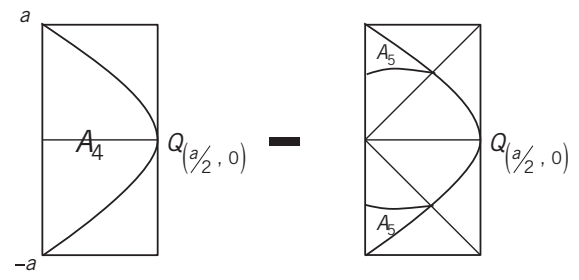

$$
\begin{aligned}
& A_{T}=2\left[A_{4}-2 A_{5}\right] \\
& A_{4}: \text { equivale a los } 2 /{ }_{3} \text { del área del rectángulo } \\
& \text { circunscrito. } \\
& A_{5} \text { : área comprendida entre } y=\sqrt{a^{2}-2 a x} \text { y } \\
& y=\frac{a^{2}-x^{2}}{2 a} .
\end{aligned}
$$

Entonces:

$$
\begin{gathered}
A_{4}=\frac{2}{3}\left(\frac{a}{2}\right)(2 a)=\frac{2 a^{2}}{3} \\
A_{5}=\int_{0}^{(\sqrt{2}-1) a}\left[\sqrt{a^{2}-2 a x}-\frac{a^{2}-x^{2}}{2 a}\right] d x \\
A_{5}=\frac{-1}{2 a} \frac{\left(a^{2}-2 a x\right)^{3 / 2}}{3 / 2}-\frac{a}{2} x+\left.\frac{x^{3}}{6 a}\right|_{0} ^{(\sqrt{2}-1) a} \\
A_{5}=\frac{-1}{3 a}\left[(\sqrt{2}-1)^{2} a^{2}\right]^{3 / 2}-\frac{a}{2}(\sqrt{2}-1) a+\frac{5 \sqrt{2}-7}{6 a} a^{3}-\left(-\frac{1}{3 a} a^{3}-0+0\right) \\
A_{5}=\left(\frac{12-8 \sqrt{2}}{6}\right) a^{2}=\frac{6-4 \sqrt{2}}{3} a^{2}
\end{gathered}
$$

Entonces:

$$
A_{T}=2\left[\frac{2 a^{2}}{3}-\frac{12-8 \sqrt{2}}{3} a^{2}\right]=\frac{4(4 \sqrt{2}-5)}{3} a^{2}
$$

\section{FORMA \#5}

Empleemos integrales dobles para el cálculo del área.

$$
A_{T}=4\left[A_{6}+A_{7}\right]
$$




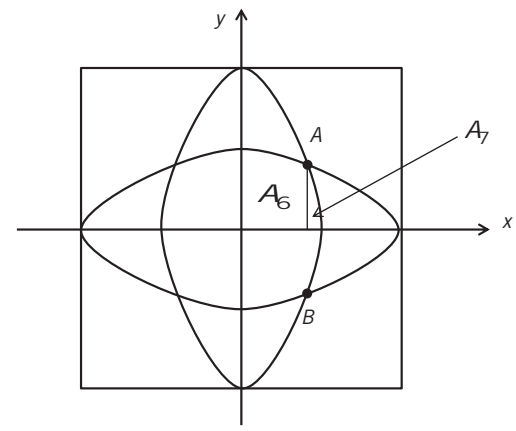

$A_{6}$ : área de la región limitada inferiormente por $y=0$ y superiormente por $y=\frac{a^{2}-x^{2}}{2 a}$, comprendida entre $x=0$ y $x=(\sqrt{2}-1) a$. $A_{T}$ : área de la región limitada inferiormente por $y=0$ y superiormente por $y=\sqrt{a^{2}-2 a x}$, comprendida entre $x=(\sqrt{2}-1)$ a y $x=0.5 a$.

De donde:

$$
\begin{gathered}
A_{6}=\iint_{R_{6}} d x d y=\int_{0}^{(\sqrt{2}-1) a} d x \int_{0}^{\frac{a^{2}-x^{2}}{2 a}} d y=\frac{1}{2 a} \int_{0}^{(\sqrt{2}-1) a}\left(a^{2}-x^{2}\right) d x \\
A_{6}=\frac{a x}{2}-\left.\frac{x^{3}}{6 a}\right|_{0} ^{(\sqrt{2}-1) a}=\frac{4-2 \sqrt{2}}{6} a^{2}
\end{gathered}
$$

430

$$
\begin{aligned}
A_{7}=\iint_{R_{7}} d x d y & =\int_{(\sqrt{2}-1) a}^{0.5 a} d x \int_{0}^{\sqrt{a^{2}-2 a x}} d y=\int_{(\sqrt{2}-1) a}^{0.5 a} \sqrt{a^{2}-2 a x} d x \\
A_{7} & =\left.\left(\frac{-1}{2 a}\right) \frac{\left(a^{2}-2 a x\right)^{3 / 2}}{3 / 2}\right|_{(\sqrt{2}-1) a} ^{0.5 a}=\frac{5 \sqrt{2}-7}{3} a^{2}
\end{aligned}
$$

Entonces:

$$
A_{T}=4\left[A_{6}+A_{7}\right]=4 \frac{4-2 \sqrt{2}+10 \sqrt{2}-14}{6} a^{2}=\frac{4}{3}(4 \sqrt{2}-5) a^{2}
$$

\section{FORMA \#6}

Consideremos elementos diferenciales horizontales:

$$
A_{T}=4\left[A_{8}+A_{9}\right]
$$




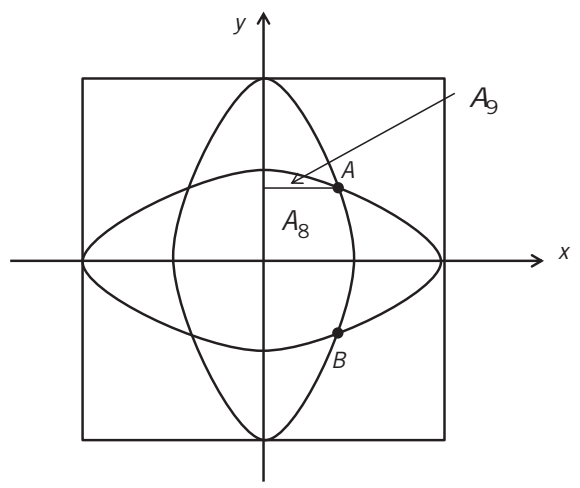

$A_{8}$ : sus límites son

- $x$ desde 0 hasta $\frac{a^{2}-y^{2}}{2 a}$

- $y$ desde 0 hasta $(\sqrt{2}-1) a$

$A_{9}$ : sus límites son:

- $x$ desde 0 hasta $\sqrt{a^{2}-2 a y}$

- $y$ desde $(\sqrt{2}-1) a$ hasta $0.5 a$

De donde:

$$
\begin{aligned}
& A_{8}=\iint_{R} d y d x=\int_{0}^{(\sqrt{2}-1) a} d y \int_{0}^{\frac{a^{2}-y^{2}}{2 a}} d x \\
& A_{9}=\iint_{R} d y d x=\int_{(\sqrt{2}-1) a}^{0.5 a} d y \int_{0}^{\sqrt{a^{2}-2 a y}} d x
\end{aligned}
$$

Las integrales $A_{8}$ y $A_{9}$ coinciden con las integrales $A_{6}$ y $A_{7}$, respectivamente, de la forma anterior dada la simetría de la región $R$ respecto de los ejes $x$ e $y$.

Por lo tanto:

$$
\begin{aligned}
& A_{8}=\frac{4-2 \sqrt{2}}{6} a^{2}=0.19526 a^{2} \\
& A_{9}=\frac{5 \sqrt{2}-7}{3} a^{2}=0.02369 a^{2} \\
& \therefore A_{T}=4\left[A_{8}+A_{9}\right]=0.8758 a^{2}
\end{aligned}
$$

\section{FORMA \#7}

En coordenadas polares y con integrales simples, el área de una región $R$ está dada por $\frac{1}{2} \int_{R} \rho^{2} d \theta$, siendo $\rho=f_{(\theta)}$. 


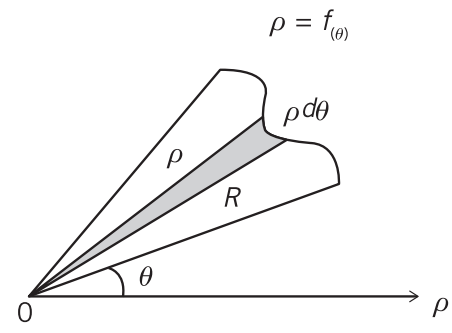

$$
\begin{gathered}
d A=\frac{1}{2}(\rho d \theta) \rho=\frac{\rho^{2}}{2} d \theta \\
\therefore \quad A=\frac{1}{2} \int_{R} \rho^{2} d \theta
\end{gathered}
$$

El área solicitada es $4 A_{10}$, siendo $A_{10}$ :
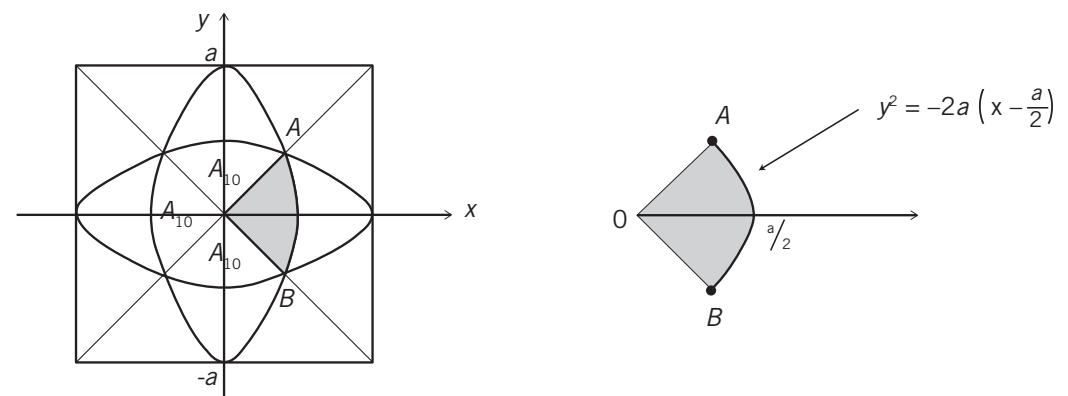

La ecuación de la parábola $y^{2}=-2 a\left(x-\frac{a}{2}\right)$ es $\rho=\frac{a}{1+\cos \theta}$ en coordenadas polares.
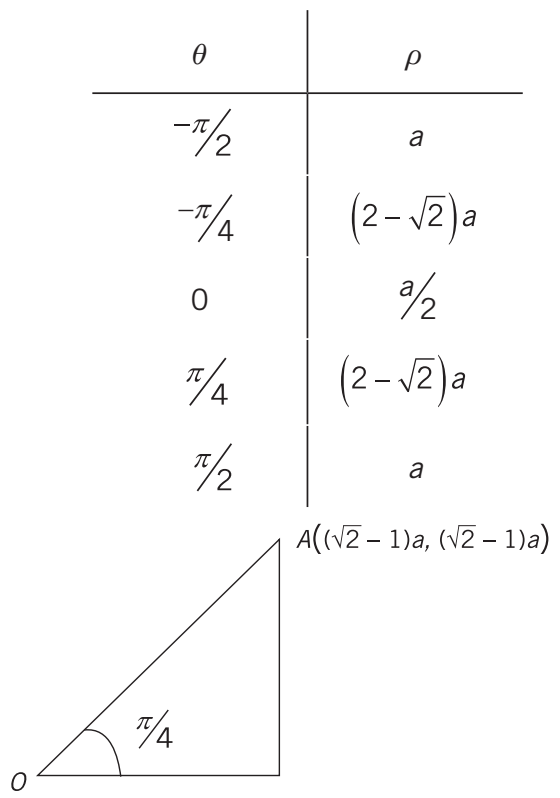


$$
O A=(\sqrt{2}-1) a \sqrt{2}=(2-\sqrt{2}) a
$$

Entonces:

$$
\begin{aligned}
& A_{T}=4 A_{10}=4\left[\frac{1}{2} \int_{-\pi / 4}^{\pi / 4}\left(\frac{a}{1+\cos \theta}\right)^{2} d \theta\right. \\
& A_{T}=2 a^{2} \int_{-\pi / 4}^{\pi / 4} \frac{d \theta}{(1+\cos \theta)^{2}}
\end{aligned}
$$

Como $\cos 2 \theta=2 \cos ^{2} \theta-1$,

$$
1+\cos 2 \theta=2 \cos ^{2} \theta \rightarrow 1+\cos \theta=2 \cos ^{2} \frac{\theta}{2}
$$

De donde:

$$
\begin{aligned}
& A_{T}=2 a^{2} \int_{-\pi / 4}^{\pi / 4} \frac{d \theta}{\left(2 \cos ^{2} \frac{\theta}{2}\right)^{2}}=\frac{a^{2}}{2} \int_{-\pi / 4}^{\pi / 4} \sec ^{4} \frac{\theta}{2} d \theta \\
& A_{T}=\frac{a^{2}}{2} \int_{-\pi / 4}^{\pi / 4}\left(\sec ^{2} \frac{\theta}{2}\right)\left(1+\tan ^{2} \frac{\theta}{2}\right) d \theta \\
& A_{T}=a^{2} \int_{-\pi / 4}^{\pi / 4} \sec ^{2} \frac{\theta}{2}\left(\frac{d \theta}{2}\right)+\int_{-\pi / 4}^{\pi / 4}\left(\tan \frac{\theta}{2}\right)^{2}\left(\sec ^{2} \frac{\theta}{2}\right) \frac{d \theta}{2} \\
& A_{T}=\left.a^{2}\left[\tan \frac{\theta}{2}+\frac{\tan ^{3} \theta / 2}{3}\right]\right|_{-\pi / 4} ^{\pi / 4} \\
& A_{T}=a^{2}\left[\tan \frac{\pi}{8}+\frac{\tan ^{3} \pi / 8}{3}-\tan \left(\frac{-\pi}{8}\right)-\frac{\tan ^{3}(-\pi / 8)}{3}\right] \\
& A_{T}=2 a^{2}\left[\tan \frac{\pi}{8}+\frac{\tan ^{3} \pi / 8}{3}\right] \\
& \text { Como } \tan 2 \theta=\frac{2 \tan \theta}{1-\tan ^{2} \theta} \Rightarrow \tan \frac{\pi}{4}=\frac{2 \tan \pi / 8}{1-\tan ^{2} \pi / 8}=1 \text {, } \\
& 1-\tan ^{2} \frac{\pi}{8}=2 \tan \frac{\pi}{8} \Rightarrow \tan ^{2} \frac{\pi}{8}+2 \tan \frac{\pi}{8}=1
\end{aligned}
$$


De donde:

$$
\begin{gathered}
\tan ^{2} \frac{\pi}{8}+2 \tan \frac{\pi}{8}+1=2 \\
\left(\tan \frac{\pi}{8}+1\right)^{2}=2 \rightarrow \tan \frac{\pi}{8}=\sqrt{2}-1
\end{gathered}
$$

Entonces:

$$
\begin{gathered}
A_{T}=2 a^{2}\left[(\sqrt{2}-1)+\frac{1}{3}(\sqrt{2}-1)^{3}\right] \\
A_{T}=2 a^{2}[\sqrt{2}-1]\left[1+\frac{1}{3}(3-2 \sqrt{2})\right] \\
A_{T}=2 a^{2}(\sqrt{2}-1) \frac{6-2 \sqrt{2}}{3}=\frac{2 a^{3}}{3}(8 \sqrt{2}-10) \\
\therefore A_{T}=\frac{4 a^{2}}{3}(4 \sqrt{2}-5)
\end{gathered}
$$

\section{FORMA \#8}

434

En coordenadas polares y como integrales dobles, el área está dada por $\iint_{R} \rho d \rho d \theta$, siendo $\rho=f_{(\theta)^{*}}$.

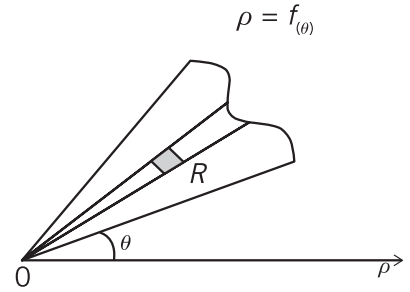

$$
\begin{aligned}
d A & =(\rho d \theta) d \rho \\
\diamond_{d \rho}^{\rho d \theta} & d A=\rho d \rho d \theta \\
\therefore A & =\iint_{R} \rho d \rho d \theta
\end{aligned}
$$

Por ejemplo, el área del círculo de radio $R$ es:

$$
A=\iint \rho d \rho d \theta=\int_{0}^{R} \rho d \rho \int_{0}^{2 \pi} d \theta
$$

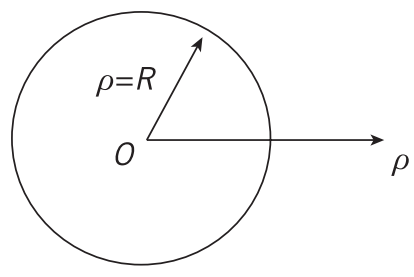

$$
\begin{gathered}
A=\left.\int_{0}^{R} \rho d \rho(\theta)\right|_{0} ^{2 \pi} \\
A=\int_{0}^{R} \rho d \rho(2 \pi-0)=2 \pi \int_{0}^{R} \rho d \rho \\
A=\left.2 \pi \frac{\rho^{2}}{2}\right|_{0} ^{R}=2 \pi\left(\frac{R^{2}}{2}-0\right)=\pi R^{2}
\end{gathered}
$$


Como en la forma anterior:

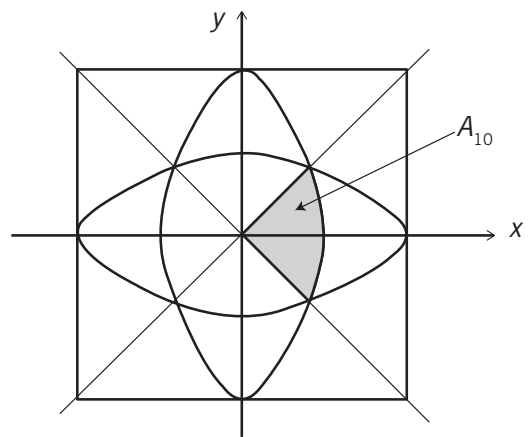

$$
\begin{aligned}
& A_{T}=4 A_{10}=4 \iint_{R} \rho d \rho d \theta \\
& A_{T}=4 \int_{-\pi / 4}^{\pi / 4} d \theta \int_{0}^{\frac{a}{1+\cos \theta}} \rho d \rho \\
& A_{T}=\left.4 \int_{-\pi / 4}^{\pi / 4} \frac{\rho^{2}}{2}\right|_{0} ^{\frac{a}{1+\cos \theta}} d \theta
\end{aligned}
$$

$$
A_{T}=2 a^{2} \int_{-\pi / 4}^{\pi / 4} \frac{1}{(1+\cos \theta)^{2}} d \theta
$$

Se obtiene lo mismo que en la forma anterior:

$$
A_{T}=\frac{4 a^{2}}{3}(4 \sqrt{2}-5)
$$

b) En resumidas cuentas, como el área de la región $R$ es $\frac{4 a^{2}}{3}(4 \sqrt{2}-5)=0.8758 a^{2}$ y el área del cuadrado de lado $2 a$ es $4 a^{2}$, la probabilidad de que un punto cualquiera del interior del cuadrado pertenezca a la región $R$ es:

$$
\frac{0.8758 a^{2}}{4 a^{2}}=0.21895 ; \text { vale decir, } 21.895 \%
$$


